UCRL-53327

Distribution Category UC- 20

UCRT --53327

0883001915

\title{
Transport and Equilibrium in Field-Reversed Mirrors
}

\author{
John K. Boyd
}

Manuscript date: September 1982

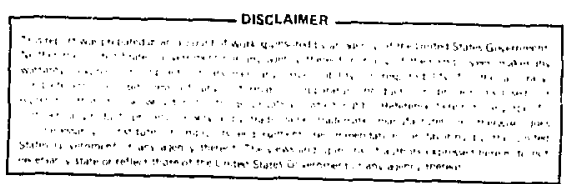

IOTICE

XOATYORS OR THIS REPORT ARE ILLEGIBLE. It hes been reproduced from the best available oopy to pormit the broadeat possible availab111ky.

LAWRENCE LIVERMORE LABORATORY University of California - Livermore, California 04550 


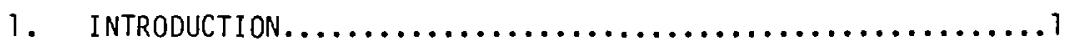

1.1 Background................................

1.1 .1 Energy Requirements.....................2

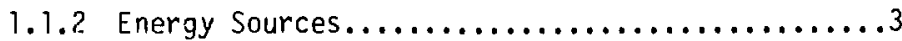

1.1.3 Fusion Energy.........................

1.2 Previous Work................................ 1

?.2.1 Equilibrium........................23

1.2.2 Transport.............................29

1.3 Scope of the Present Work....................40

2. ADIABATIC SMALL LARMOR RADIUS MODEL................. 45

2. 1 Introduction.............................45

2.2 Equations to be Solved......................46

2.2.1 Magnetohydrodynamic Equations.............46

2.2.2 Grad-Shafranov Equation..................49

2.2.3 Boundary Condition for the Grad-Shafranov

Equation.............................

2.2.4 Transport Equations.......................54

2.3 Numerical Methods for the ADB Code.................61

2.3.1 Flux Surface Average...................61

2.3.2 Averaged Grad-Shafranov Equation............65

2.3.3 Grad-Shafranov Equation.................68 
2.3.4 Boundary Condition for the Grad-Shafranov

Equation...........................

2.3.5 Transport Equations.....................

2.4 Initial Conditions.......................... 75

2.4.1 Weitzner Magnetic Field Model.............76

2.4.2 Spherical Hill's Vortex...................78

2.4.3 Elliptical Hill's Vartex....................

2.4.4 Variable Coefficient Model................84

2.5 ADB Code Results............................. 85

2.5.1 Simulation of a Neutral Beam Injected Plasma....87

2.5.2 Compressed Prolate Hill's Vortex..............109

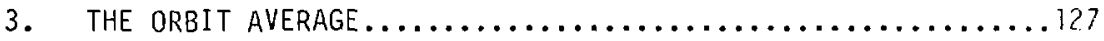

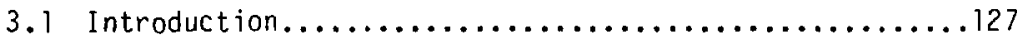

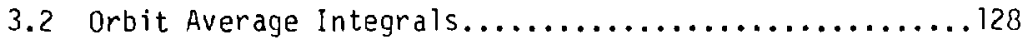

3.3 Harmonic Oscillator Orbit Code.....................144

3.4 Numerical Orbit Average Formulas................ 151

3.5 AV Code Results............................ 177

$3.6 \mathrm{Hi}$ ll's Vortex Orbit Code........................ 190

3.6.1 Investigation Issues..................... 190

3.6.2 Equations of Motion and Method of Solution......191

3.6.3 Axisymmetric Orbit Considerations.............. 195

3.6.4 NEO Drbit Average Results................. 199

3.6.5 Existence of Third Invariants.............204 
3.6.6 Investigation of Conditions for

Stochasticity...........................

4. LARGE LARMOR RADIUS KINETIC EQUATION MODEL............23 I

4.1 Introduction...................................

4.2 Analytic Electric and Magnetic Fields..............232

4.2.1 Zero Radial Electron Velocity...............238

4.2.2 Finite Radial Electron Velocity.............248

4.2.3 Results for Finite Radial Electron Velocity....253

4.3 Kinetic Equation with Large Larmor Radius Effects......260

4.3.1 The Orbit Averaged Kinetic Equation..........263

4.3.2 Poisson Bracket Solution by Eigenfunctions......269

4.3.3 Perturbed Fluid Electron Model.............274

4.3.4 Collision Operator for the Orbit Averaged

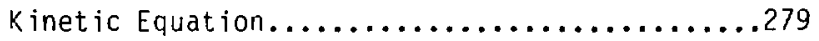

4.3.5 One Dimensional Orbit Averaged Kinetic

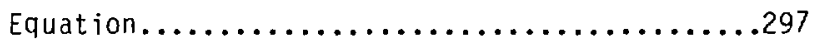

5. SUMMARY AND CONCLUSIONS................................

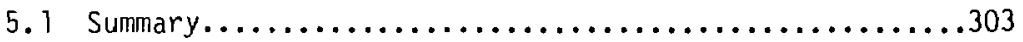

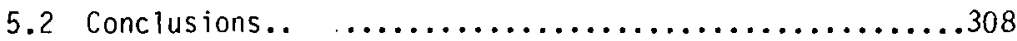

5.3 Future Work.................................

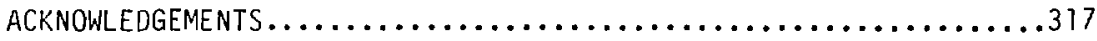

REFERENCES...................................... 
APPENDIX A. DEFINITION OF FUNCTIONS USED FOR ORBIT AVERAGE

FORMULAS...................................

APPENDIX B. COEFFICIENTS FOR THE HILL'S VORTEX POTENTIAL......355 APPENDIX C. INVARIANT POISSON CONSTRAINT EQUATION COEFFICIENTS..357 APPENDIX D. CANONICAL TRANSFORMATION HAMILTONIAN.............36 1 APPENDIX E. RESONANCE CONDITION FOR THE CANONICAL

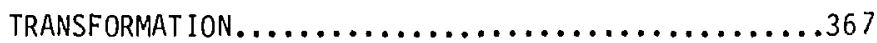

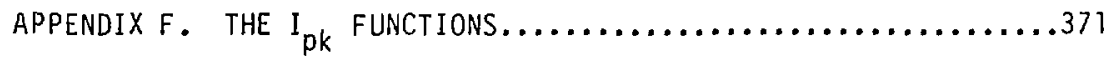




\section{LIST OF FIGURES}

1-1. Cross section of a single mirror cel1.................11

1-2. Minimum B configurations (a) Ioffe bars added to a simple mirror (b) Baseball geometry (c) Schematic of 2XIIB with

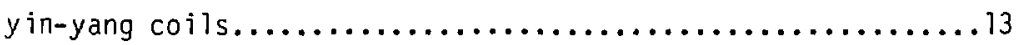

1-3. Tokamak coil geometry with transformer linking plasma......16 1-4. Field reversed mirror resulting from tangential neutral beam

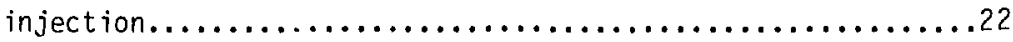

1-5. Homogeneous miagietic field particle orbit encountering 180 degree deflection each cyclotron period.................32

1-6. Trapped particle motion. (a) Bounce and drift motion (b) Azimuthal angle projection showing banana orbit of

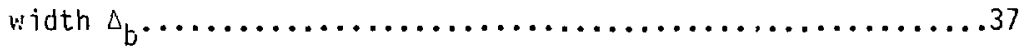

1-7. Particle diffusion coefficient versus collision frequency...39

2-1. Neutral beam injected plasma, $\psi(r, z) .(a) t=0$.

(b) $t=2.8$ milliseconds $\ldots \ldots \ldots \ldots \ldots \ldots \ldots \ldots \ldots \ldots \ldots . . .65$

2-2. Neutral beam injected plasma, $\psi(r, 0)$. (a) $t=0$

(b) $t=2.8 \mathrm{milliseconds}$ .96

2-3. Neutral beam injected plasma, $4 \pi c^{-1} J_{T}(r, z) .(a) t=0$,

(b) $\mathrm{t}=2.8 \mathrm{mi} 11 \mathrm{iseccnds} \ldots \ldots \ldots \ldots \ldots \ldots \ldots \ldots \ldots \ldots \ldots . .97$

2-4. Neutral beam injected plasma, $4 \pi c^{-1} J_{T}(r, 0) .(a) t=0$,

(b) $\mathrm{t}=2.8 \mathrm{mi} 11$ iseconds.

2-5. Neutral beam injected plasma $I_{z}(r) .(a) t=0$,

(b) $\mathrm{t}=2.8 \mathrm{milliseconds.}$ .99 
2-6. Neutral beam injected plasma. (a) $B_{z}(r, 0, t=0)$,

(b) $B_{z}(0, z, t=0)$, (c) $B_{z}(r, 0, t=2.8 \mathrm{msec})$,

(d) $\mathrm{B}_{\mathrm{z}}(0, \mathrm{z}, \mathrm{t}=2.8 \mathrm{msec})$.

2-7. Neutral beam injected plasma. (a) $B_{T}(r, 0, t=0)$,

(b) $B(r, 0, t=0),(c) B_{T}(r, 0, t=2.8 \mathrm{msec})$,

(d) $B(r, 0, t=2.8 \mathrm{msec})$.

2-8. Neutral Beam injected plasma, $n(r, 0)$. (a) $t=0$

(b) $t=2.8$ milliseconds.

2-9. Neutral beam injected plasma, $p(r, 0)$. (a) $t=0$

(b) $\mathrm{t}=2.8 \mathrm{milliseconds.}$

2-10. Neutral beam injected plasma, $T(r, 0) .(a) t=0$,

(b) $t=2.8 \mathrm{milliseconds.}$

2-11. Neutral beam injected plasma entropy profile, $S(\psi)$.

(a) $t=0$, (b) $t=2.8$ milliseconds

2-12. Neutral beam injected plasma. (a) Plasma energy vs time,

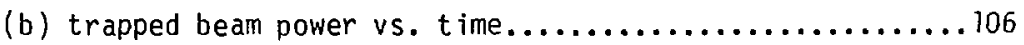

2-13. Neutral beam injected plasma. (a) Radiated power vs. time, (b)

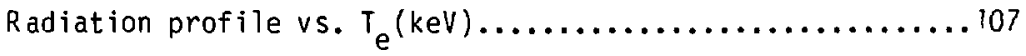

2-14. Neutral beam injected plasma. Fraction of the neutral beam

cuir rent attenuated by the plasma versus time.............108

2-15. Compressed prolate $H i l l$ 's vortex, $\psi(r, z)$. (a) Initial external

current, (b) Final fxternal current...................115

2-16. Compressed prolate Hill's vortex, $\psi(r, 0)$. (a) Initial external

current, (b) Final external current...................116

2-17. Compressed prolate Hill's vortex, $4 \pi c^{-1} J_{T}(r, z),(a)$

Initial external current, (b) Final external current.......117 
2-18. Compressed prolate Hill's vartex, $4 \pi c^{-1} J_{T}(r, 0)$. (a)

Initial external current, (b) Final external current........118

2-19. Compressed prolate Hill's vortex, (a) Initial external current $B_{z}(r, 0)$, (b) Initial external current $B_{z}(0, z)$, (c) Final external current $B_{z}(r, 0)$, (d) Final external

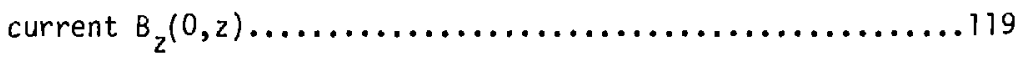
2-20. Compressed prolate Hill's vortex $B(r, z)$. (a) Initial external

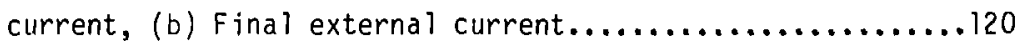

2-21. Compressed prolate Hill's vortex $B(r, 0)$. (a) Initial external current, (b) Final external current...................121

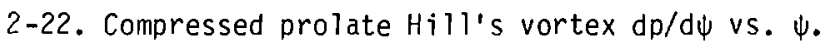

(a) Initial external current, (b) Final external current....122 2-23. Compressed prolate Hill's vortex $p(r, 0)$. (a) Initial external

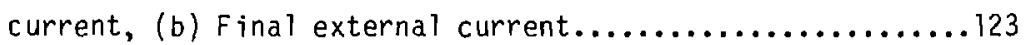
2-24. Compressed prolate Hill's vortex. (a) Plasma energy vs. time, (b) Magnetic energy vs. time. .124

2-25. Compressed prolate Hill's vortex. (a) Poloidal magnetic field energy in the plasma vs. time, (b) Poloidal inagnetic field

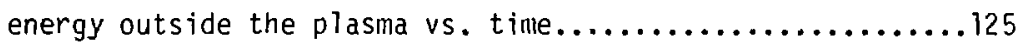

3-1. Local coordinates and grid cell integration path intersections. (a) Bottom to right (b) Bottom to left (c) Bottom to top (d) Top to right (e) Top to left (f) Right to left......153

3-2. Grid cell integration path coordinates for the $\bar{x}^{(6)}$ orbit average. (a) BR contribution (b) BL contribution (c) BT contribution. 158 
3-3. The four regions and coordinate orientations for potential boundary grid cell intersections. (a) BR intersection (b) BL intersection (c) BT intersection to the right (d) BT intersection to the left (e) TR intersection (f) TL intersection (g) RL interseri,ion downward (h) RL intersection

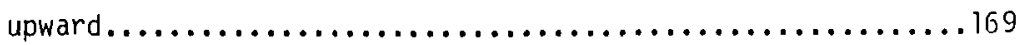

3-4. Orbit with $\omega_{r}=1, \omega_{z}=4, \varphi_{r}=0, \varphi_{z}=0 \ldots \ldots \ldots \ldots \ldots \ldots 182$

3-5. Orbit with $\omega_{r}=1, \omega_{z}=4, \varphi_{r}=.3, \varphi_{z}=.1 \ldots \ldots \ldots \ldots \ldots 183$

3-6. Orbit with $\omega_{r}=.198, \omega_{z}=1.218, \varphi_{r}=.3, \varphi_{z}=.1 \ldots \ldots \ldots 185$

3-7. Potential at $z=0$ versus $r$. (a) $p_{\theta}<-3 / 16$

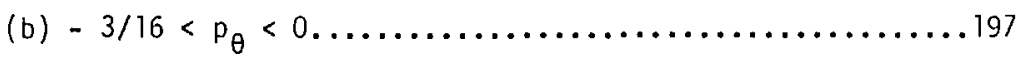

3-8. Orbit positions sampled a long a trajectory with $p_{\theta}=-1.19$, $\nu=.5$. Contours are constant energy values............201

3-9. Velocity direction arrows at orbit positions for

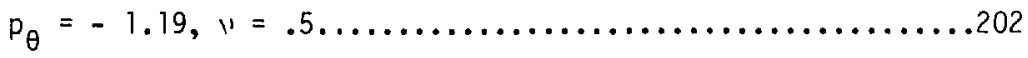

$3-10$. Surface of section for $p_{\theta}=-1.19, v=.5 \ldots \ldots \ldots \ldots \ldots . .203$ 3-11. Invariants versus time for $p_{\theta}=-.2, H \approx V_{0}$. (a) Third order velocity invariant (b) Fourth order velocity

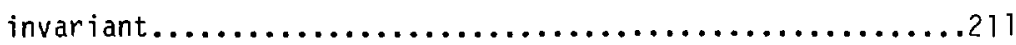

3-12. Surface of section for $p_{\theta}=-.2, H \approx V_{0}$ superposed on a contour $p$ lot of the fourth order velocity invariant.......212

3-13. Four directions shown for velocity direction arrows with

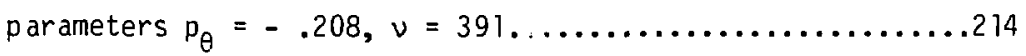

3-i4. Resonances for $p_{0}=-.196(a)(1,-2)(b)(1,-1)$ (c) $(2,-1)$ .227 
3-15. Resonances for $p_{\theta}=-.202$ (a) $(1,-2)$ (b) $(1,-1) \ldots \ldots .228$

3-16. Surface of section for $p_{\theta}=-.196$ exhibiting rippling and structure breakup..............................229

3-17. Surface of section for $p_{\theta}=-.202$ illustrating smooth structure.

4-1. One dimensional equilibrium $\psi(r)$ (a) $v_{r e}=10$,

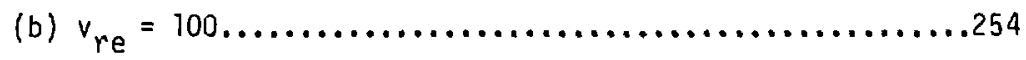

4-2. One dimensional equilibrium $B_{z}(r)(a) v_{r e}=10$,

(b) $v_{\text {re }}=100$.

4-3. One dimensional equilibrium e $\phi(r)(a) v_{r e}=10$,

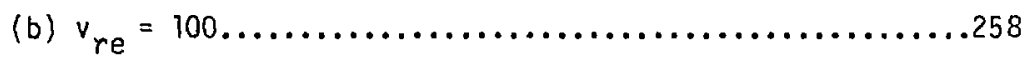

4-4. One dimensioral equilibrium $E_{r}(r)(a) v_{r e}=10$,

(b) $v_{\text {re }}=100$ 


\section{LIST OF TABLES}

2-1 Compressed Prolate Hill's Vortex $I_{j}, I_{f}$ Currents and Positions........................................110

3-1 Orbit Average Values for the AV Code.................. 184

4-1 One Dimens.icnal Analytic E, B Result Parameters.............255 
TRANSPORT AND EQUILIBRIUM IN FIELD REVERSED MIRRORS*

by

John K. Boyd

University of California

Lawrence Livermore National Laboratory

L.ivermore, California 94550

and

Department of Applied Science

Davis, California 95616

September 1982

ABSTRACT

The field reversed mirror is a toroidal, closed magnetic field line plasma confinement device in the compact torus class. It has no conductors linking the plasma as in other toroidal devices such as the tokamak. The Larmor radius in a typical field reversed mirror is $1 / 6$ to $1 / 3$ the piasma radius. In field reversed mirrors fueled and heated by neutral beams, the azimuthal current is principally diamagnetic.

The transport and equilibrium in field reversed mirrors is described in this work by two distinct models. The main differences between the two models are the applicable collision frequency regime, the Larmor radius size and the allowed magnetic fields. In both models the aspect ratio is one and axisymmetry is assumed.

*Work performed under the auspices of the U.S. Department of Energy by the Lawrence Livermore Laboratory under contract number W-7405-ENG-48. 
The first model considers the plasma to have a large collision frequency, and small Larmor radius. The plasma is then modeled as an adiabatic hydromagneitic fluid and both toroidal and poloidal magnetic fields are allowed. The plasma evolves quasi-statically between equilibrium solutions as a result of transport processes or adiabatic external current changes. The computer code and computational methods used to solve the equilibriun and transport equations are described. The computer code results are discussed for several cases considering the effects of neutral beam injection and magnetic field shaping.

The second model considers ihe plasma to have a small collision frequency and a large Larmor radius. The magnetic field in this model only has a poloidal component. The plasma consists of several ion species described by distribution functions which must be calculated and electrons moveled as an inertialess fluid. The ion distribution function for exch species satisfies a kinetic equation formulated in terms of a Poisson bracket with the system Hamiltonian. The ion distribution function is expanded in terins of collision irequency and the kinetic equation then yields a hierarchy of equations. At second order a ..inetic equation for the ime evolution of the zero order distribution function is obtained. This equation is the large Larmor radius analog of the small i srmor radius drift kinetic equation which describes the tokamak neoclassical diffusion regime.

The drift kinetic equation is derived by integrating appropriate equations over a gyro-orbit. In contrast the kinetic 
equation derived in this work requires integration over the actual orbit. To emulate integration over exact orbits without requiring detailed trajectories an orbit average procedure is derived. The orbit average is equivalent to accumulating contributions from integration over exact orbits of an equilibrium distribution. The orbit averaged kinetic equation which is derived at second order in collision frequency then evolves on a slowing down time scale rather than a cyclotron period which is characteristic of standard trajectory following particle codes.

The orbit average procedure is defined in terms of phase space and path integral formulas. Numerical equivalents of these formulas are derived and results of tests in two particle orbit computer codes are presented. The derivation of the orbit average procedure depends on the number of constants of the motion which exist. In axisymmetry with conservative forces there are two constants of the motion. In some circumstances a third constant of the motion may also exist. To explore the existence of a third constant of the motion, several classes of third constant of the motion are examined. Because the examined third constant of the motion does not exist when an orbit is stochastic, consideration is also given to determining parameter regimes where stochastic orbit motion may manifest. 
CHAPTER 1

INTRONUCTION

\subsection{Background}

The field reversed mirror is a closed magnetic field line plasma confinement device studied in the magnetic fusion energy program. Preliminary analysis indicates it has several desirable features. In considering other fusion energy devices, the field reversed mirror offers the advantages of low total structure volume to plasma volume, high fusion power density, and efficient use of magnetic field. In addition, it has no conductors linking the plasma as in other toroidal configurations. In contrast to present commercial energy sources the field reversed mirror has the capability of providing a domestically produced, environmentally safe, inexhaustible energy supply. To motivate the study of the field reversed mirror, a brief review of. 'gy requirements is given followed by a discussion of energy sources, inciuding alternate fusion energy concepts. 


\subsubsection{Energy Requirements}

The pursuit of energy impacts ecology, economics and as a consequence of its international aspects world stability. As the standard of living increases there is a direct effect on the overall energy requirement. From the mid twenties to the inid seventies the United States energy usage increased at an average annual rate of 2.5\% and the gross national product increased at an average annual rate of $3.1 \% .^{[1]}$ Thus on the average the trend of increased economic activity implies increased energy usage. In add ${ }^{+}$ion the United States population increased from 152.3 million to 216.8 million from 1950 to $1976 .{ }^{[2]}$ This is an average annual 1.36\% rate of increase. In the same period energy consumption went from 30.9 quads to 69 quads, [3] where a quad is $10^{15}$ B.T.U. and a B.T.U. is the amount of energy required to raise a pound of water one degree fahrenheit. Thus energy consumption underwent an average annual $3.1 \%$ rate of increase. In addition to the increased energy requirement in relation to economic activity, there is also a trend toward greater per capita energy usage. The global impact of this trend is of concern since of the 69 quads consumed in 1976 only 56.9 were domestically produced. Based on conservative assımptions concerning population increase it is estimated United States energy demand could be reduced to a $1.8 \%$ per capita annual rate of increase. [4] However even with this scenario the projected energy consumption in the year 2000 is 170 quads. All of these factors point toward a continuing reliance on energy and a need to increase the energy supply. 


\subsubsection{Energy Sources}

Present energy supplies may be considered in three categories; renewable, fossil and nuclear.

\section{- Renewable Energy Sources}

In the renewable category there are several energy sources directly or indirectly related to solar energy. Solar energy is derived from radiation emitted by the sun. The sun's radiation may be used for space heating or water heating. In addition focusing collectors may be used to concentrate the heating effect and generate steam from which electricity may be produced. [5] This method requires collectors which track the position of the sun. Direct conversion of radiation to electricity may be achieved by photovoltaic cells. In this case radiation induced electron detachment in a semiconductor material is used to produce an electric current. [6] The greatest utilization of photovoltaics has been for space applications. [7] The main drawback to this technology is the high cost relative to other forms of energy. Solar collection schemes are not as effective at varying latitudes and require expensive energy storage systems to deal with interrupted solar radiation caused by nightfall or weather conditions. Thus development and installation has not been wide spread. A side product of solar radiation is atmospheric pressure differences which cause wind. It has been estimated the potential possible wind energy which may be obtained in the U.S. is 15,000 $\mathrm{GW},{ }^{[8]}$ where the total electric generating capacity is $500 \mathrm{GW}$. 
Wind energy has successfulty been used to pump water and generate electricity, however there are limited locations where siting is possible with reliable prevailing winds. Another form of sclar energy is the temperature difference of different layers in the ocean. The systems which extract this energy are currently experimental and are constrained by the ideal heat engine efficiency which is $1-T_{2} / T_{1}$ where the temperature difference is $T_{2}-T_{1}$. The small anticipated efficiency requires relatively large heat exchangers.

Energy from biomass is renewable since additional organic substances may be grown. Biomass nay be used to obtain methane gas or methyl alcohol, both of which may be burned to yield useful heat. More simply wood, crop debris or municipal refuse may be burned. It is estimated that approximately 2 quads of energy [9] could have been obtained in 7980 from municipal waste alone. Al technology currently exists for biomass energy, its implementation depends on economics of scale, fuel cost due to transportation considerations and separation of unburnable tebris in the case of municipal refuse.

Geotherinal energy is technically not renewable; however, it has been estimated a billion quads of energy exist in the first 5 miles of the earth's crust. [10] This is adequate to supply world needs for a million years. An early exploitation of geotherma 1 energy occurred at Larderello, Italy [1]] where steam was piped to low pressure turbines to produce electricity. A similar installation exists in California near Geyservilie. Unfortunately, these are 
rare sites and the preponderance of potential geothermal energy exists as hot dry rocks or molten lava and magmas. Use of these resources requires developments in the area of drilling techniques, rock fracturing, ano transporting heat to the surface.

Hydroelectric power is a renewable energy source which relies on dammed water flowing through turbines to generate electricity. Most sites where this technology may be implemented have already been utilized. Expansion of existing dums and addition of sinall dams is estimated to add potentially a maximum of $50 \mathrm{GW}^{[1 \%]}$ or $10 \%$ of the total electric generating capacity. This increase would result in water level drawdown and pcssible unfavorable environr. 7tal impacts as well as land use consequences.

\section{- Fossil Fuel Energy Sources}

The main fossil fuels presently used to produce energy are 0il, gas, and coal. These three fuels dominate all other sources of energy used in the United States. In 1976 the percentage of total national energy consumption attribuice to oil, gas, and coal was $47.2 \%, 27.3 \%$, and $18.6 \%$ respectively. ${ }^{[13]} \mathrm{A} 11$ other sources of energy contributed $7 \%$ to the total. Eliminating the $4.1 \%$ contribution of hydroelectric, all remaining alternate energy sources contributed less than $3 \%$. The importance of developing alternate energy sources is emphasized by the obvious reliance on $0 i 7$, and the fact that $43 \%$ of oil used $i: 1976$ was imported. This means $20 \%$ of the total domestic energy supply originated from sources that are not secure or reliable. To alleviate this problem 
consideration has been given tc expanding production of fossil fuels. Estimates of available recoverable reserves indicate the energy equivalent of oil, gas, and coal is 330,421, and 19,000 quads respectively. $[14-16]$ These estimates are conservative since they include only known reserves and extensions to known reserves. No account is made of resources thought to be in unexplored extensions of producing regions, which approximately doubles the stated estimates. These figures show the central domestic fossil fuel energy source is coal. Shais oil is estimated as amounting to a million quads of energy $[17]$; however, economic extraction is a controversial issue. The method of heating shale to about $500^{\circ} \mathrm{C}$ to produce oil fraction, gases and residues has been known for years yet has not been commercially implemented. Coal then whether liquified, gasified, or simply burned is the frontrunner in domestic energy supplies. The drawback of the use of coal centers on the issue of air pollution. Burning coal releases sulfur dioxide, nitrogen oxides, carbon oxides, and poisonous trace elements such as mercury, lead, and arsenic. The overall effect is respiratory and cardiac illness. [18] In addition much debate has occurred over climate impact of increased levels of carbon dioxide, in reference to global heating and cooling. Another threat is the acid rain which has been increasing in the northeast United States. The consequence is ecosystem degradation resulting in aquatic life fatalities and soil nutrient leaching. 


\section{- Nuclear Energy Sources}

The hird category of energy supplies is nuclear. The two approaches in this area are fission and fusion. Fissicr energy is derived from heat obtained from the binding energy of atomic nuclei. Neutrons are used to break a heavy nucleus into several parts and the mass difference of the sum of the parts and original nucleus is made available as heat. In the same way fossil fuel derived heat is used, nuclear reaction heat is used to produce steam and power turbines that produce electricity. Presently there are many light water reactors in commercial operation and there are plans to implement breeder reactors which produce more fuel as they operate. The main disadvantage of fission is the severe safety requirements posed by meltdown and accidental release of radiation, questions of nuclear weapon proliferation, and radioactive waste disposal. Currently there is no long term policy dealing with cisposal of radioactive waste which has a half life of hundreds of years. Public safety has become a promiment issue for nuclear fission in light of the accident at Three Mile Island.

\subsubsection{Fusion Energy}

Based on the need for increasing amounts of energy and the discussio. of energy sources, it is clear a clean, inexhaustible energy source is highly desirable. Fusion has a radioactive waste disposal consideration which is insignificant compared to nuclear fission. It is thus regarded as a clean energy source. The basic fusion reaction consists of combining light nuclei to form a heavier 
nucleus such as the following reaction, [19] involving the combination of deuterium (D) and tritium (T) to form a neutron ans alpha particle.

$$
\mathrm{O}+\mathrm{T} \rightarrow \mathrm{He}^{4}+\mathrm{n}+17.6 \mathrm{Mev}
$$

Tritium is not naturally occurring; however, fission of lithium yields tritium as a reaction product.

$$
\mathrm{Li}^{6}+\mathrm{n}+\mathrm{He}^{4}+\mathrm{T}+4.8 \mathrm{MeV}
$$

Accessible surface sources of 1 ithium represent thousands of years of tritium supply if al: U.S. energy was derived from fusion. Deuterium is a naturally occurring nuciide, found in sea water at a concentration of $.0153 \%$ of the hydrogen, [20] which is adequate to supply millions of years of fusion energy. Thus fusion energy is considered to be inexhaustible and clean, and it is thus a highly desirable energy source.

The reaction described by Eq. (1-1) has a maximum rate at a temperature of approximately $60 \mathrm{keV} .[21]$ The objective of fusion energy research is to design a system in which isotopes of hydrogen at these high temperatures react to yield more energy than required to create conditions in which the reaction can occur. This objective is often stated in terms of the Lawson criteria, [22] which requires $n \tau>10^{14} \mathrm{sec} / \mathrm{cm}^{3}$ for a $D, T$ plasma at reaction temperatures, where $n$ is density and $\tau$ is confinement time. The basic idea is to confine a high temperature plasma long enough at sufficient density such that net energy is produced. A high temperature plasma can not be confined by material walls since either the walis would vaporize or 
the plasma energy would be rapidly lost by contact with cool wall surfaces. To overcome this difficulty plasma containment is achieved by inertial confinement or magnetic confinement.

\section{- Inertial Confinement Fusion}

Inertial confinement fusion relies on a driver such as a laser beam to cause hydrodynamic compression of a pellet to high density. [23] The laser beam pulse is in the neighborhood of nanoseconds and thus the Lawson criteria requires densities greater than $10^{23} / \mathrm{cc}$. For comparison solid DT density is about $10^{22 / c c . ~[24] ~}$ The hydrodynamic compression causes ignition and propagation of a burn wave resulting in fusion energy. Thi $i$ process depends on the driver beam pulse shape and the way the energy couples into the plasma. The time evolution of compression, burn, and energy release is complicated by such effects as ablation, Rayleigh-Taylor instability, temperature gradient instability, and several anomalous phenomena. The largest domestic inertial confinement experiment is the NOVA laser system [25] which is designed to produce as much energy as the laser delivers to the target pellet. Although this is an important step toward a reactor, the reactor goal is yet in the future since useful performance requires the production of 10 or 100 times the delivered laser energy. This requircment is a consequence of laser light iosses and tiie approximate $10 \%$ or less efficiency of converting a broad spectrum of light to the laser light frequency. 


\section{- Magnetic Confinement Fusion}

The magnetic fusion approach seeks to confine plasma by utilizing the properties of a magnetic field to innibit loss or wall contact. The basic motion of a single charged particle subject to a magnetic field is to spiral around the magnetic field line in response to the $V \times B$ force. The consequence is to inhibit motion perpendicular to fieid lines. In practice with a distribution of charged particles, the perpendicular motion is actually diffusive. Directly - long field lines particle motion is unencumbered. To deal with the lack of confinment in the direction of a homogeneous magnetic field there are two distinct magnetic field scometries.

\section{- Open Magnetic Field Line Geometry}

The first geometry is described as open since field lines within the plasma do not connect. This is the geometry of the magnetic mirror and the earliest efforts to prevent loss along field lines in these devices utilized the reflecting effect of an increasing magnetic field along a field line. A schematic of a single mirror cell is shown in Fig. 1-1. The increasing magnetic field strength along a field line is produced by axisymimetric current coils in this case. The maximum magnetic field $B_{\text {max }}$ occurs in the region near coils and the minimum magnetic field is at the device midplane. The basic motion of a single partic ${ }^{7} e$ in this device is to bounce between mirror coils, gyrating or spiraling around field lines and more slowly drifting in the azimuthal symmetry direction. When the change of the magnetic field during a 


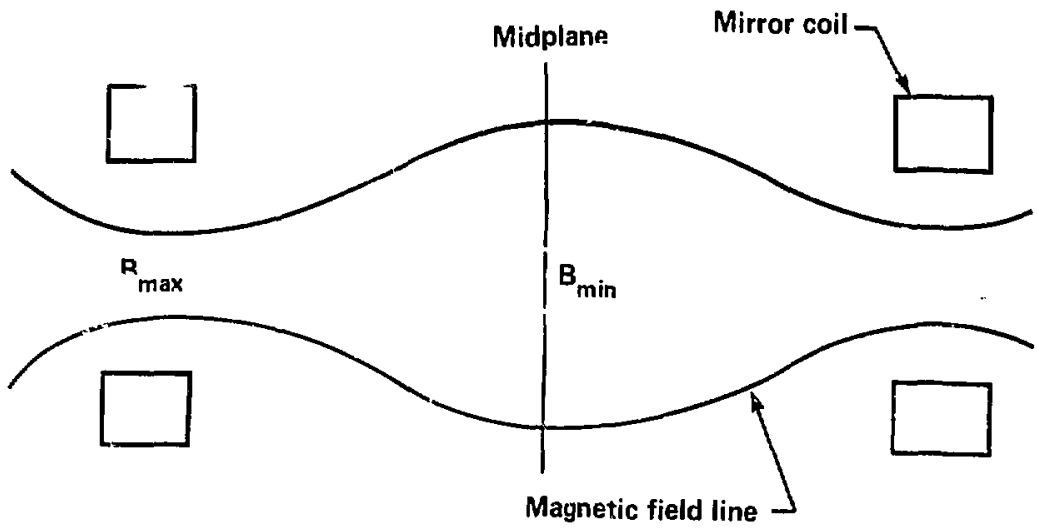

Figure 1-1. Cross section of a single mirror cell. 
gyro-period is much less than the magnetic field magnitude conditions exist for adiabatic behavior and the magnetic moment $H_{\perp} / B$ is approximately constant. [26] In this case non-interacting single particle corfirment occurs for particles with velocity pitch $v_{1} / v_{11}>\left(B_{\min } /\left(B_{\max }-B_{\min }\right)\right)^{1 / 2}$, where the velocities $v_{\perp}, v_{\|}$ refer to directions perpendicular and parallel to a field line at the devir.e midplane and $B_{\min }$ and $B_{\max }$ are the minimum and maximum magnetic field values along the field line. [27] Particles at smaller pitch angles are in the loss cone and are typically lost in a bounce time. In addition there may also be non-adiabatic particles. [28] Early mirror experiments had lifetimes greater than predicted by hydromagnetic instability predictions; ${ }^{[29]}$ however, it was not until experiments by Ioffe $\mathrm{e}^{[30]}$ that a minimum-B geometry was used to obtain greatly enhanced stability. [31] The essential change from the device of Fig. 1-1 was to add current bars in the horizontal or $z$ direction every sixty degrees with alternating current direction. Figure 1-2a shows a schematic of the Ioff $\epsilon$ experiment. Figure 1-?b is a variation known as the Baseball configuration. [32] Figure 1-2c is another minimum $B$ configuration produced with yin yang magnets, representing the $2 X I I B$ experiment. ${ }^{[33]}$ The two new features of this experiment were the microinstability suppression by axial warm plasma stream and sustaining the plasma with neutral beams. Theoretical understanding of microinstabilities $[34,35]$ led to stream stabilization [36] of $r f$ fluctuations associated with the drift cyclotron loss cone mode. [37] The increased plasma lifetime ultimately culminated in a stream stabilized, high beta experiment 
(a)

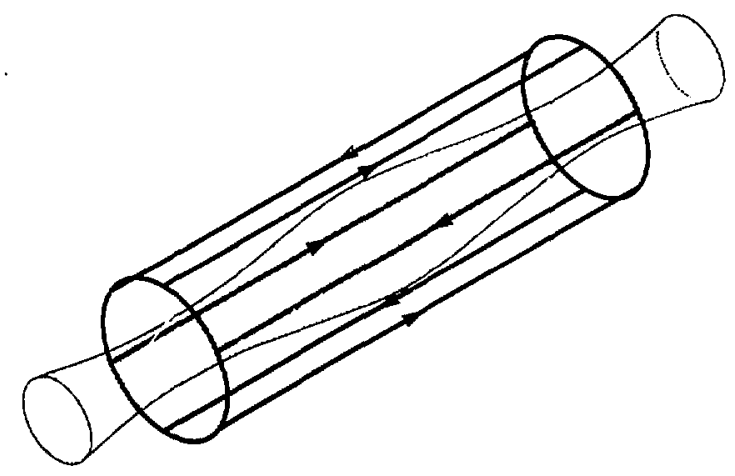

(b)

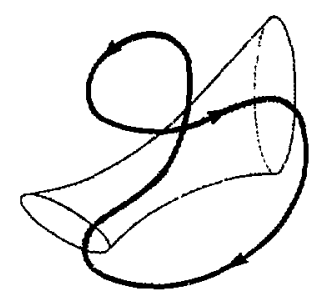

(c)

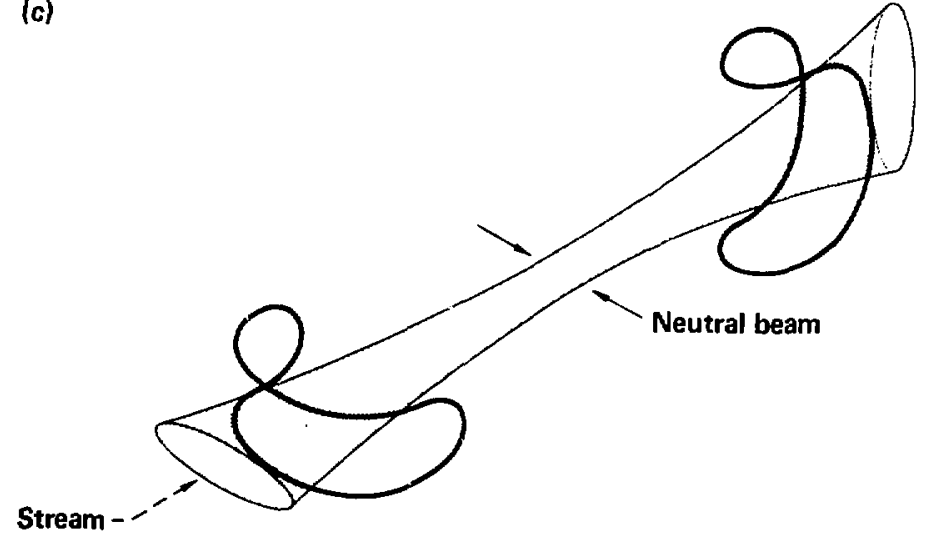

Figure 1-2. Minimum $B$ configurations (a) Ioffe bars added to a simple mirror (b) Baseball geometry (c) Schematic of 2XIIB with yin-yang coils. 
(2XIIB) heated and fueled by neutral beams. [38] As is characteristic of single cell mirrors this experiment was limited by end loss processes. $[39,40]$ particle confinement depends on adiabatic invariance; however, collisional velocity space scattering moves magnetically trapped particles into the loss cone. Because the scattering time goes like the square root of mass, the electrons are rapidly lost until an ambipolar field is generated which maintains approximate charge neutrality and equilibrates net electron and ion loss rates. The result is electrons are low temperature and electrostatically confined, and ions of low energy are lost. The details of this situation have been studied by solving a kinetic equation with a Fokker-Planck collision term. [41]

The inherent presence of an ambipolar field and the success of stream stabilization and neutral beam injection led to a new design designated the tandem mirror. ${ }^{[42,43]}$ The basic idea is to use single cell mirrors as end plugs at both ends of a solenoid. High density end plugs confine the main fusion plasma in the solenoid by high magnetic field and large ambipolar potential. For technological considerations modifications to $t /$ basic design consisting of thermal barriers ${ }^{[4]}$ and $A$ cells ${ }^{[45]}$ are under investigation. These ideas and others are being examined experimentally in the $T M X-U^{[46]}$ device. 
- Closed Magnetic Field Line Geometry

The second basic type of magnetic field geometry used to deal with confinment along field lines is described as closed and is typified by a toroidal geometry. The fundamental configuration difference among toroidal confinement geometries is the presence or absence of material conductors linking the plasma.

\section{- Tokamak Geometry}

Representative of devices, ith a conductor linking the plasina is the tokanak $[47]$ illustrated in Fig. 1-3. A toroidal plasma is confined in a metallic shell wound with poloidal and toi oidal field coils. The toroidal direction is e in Fig. $1-3$ and the poloidal direction $\theta_{p}$, is parallel to the magnetic field direction around the plasma at a fixed $\theta$ value. The plasma acts as the secondary winding of a transformer primary which links the plasma. Ohmic heating is obtained from the toroidal plasma current induced by the transformer. The toroidal plasma current causes a poloidal magnetic field which together with the external toroidal field coils results in helical magnetic field lines. The poloidal angular displacement of a field line after going $2 \pi$ in the toroidal angle is the rotational transform. If the rotational transform is not $2 \pi$ divided by an integer the field lines ergodically fill topologically nested surfaces. For stability [48] reasons, in the large aspect ratio ( $A_{r}=$ major radius/minor radius) limit, the safety factor $q$ must be greater than one. The safety factor is related to the inverse of the rotational transform and for 


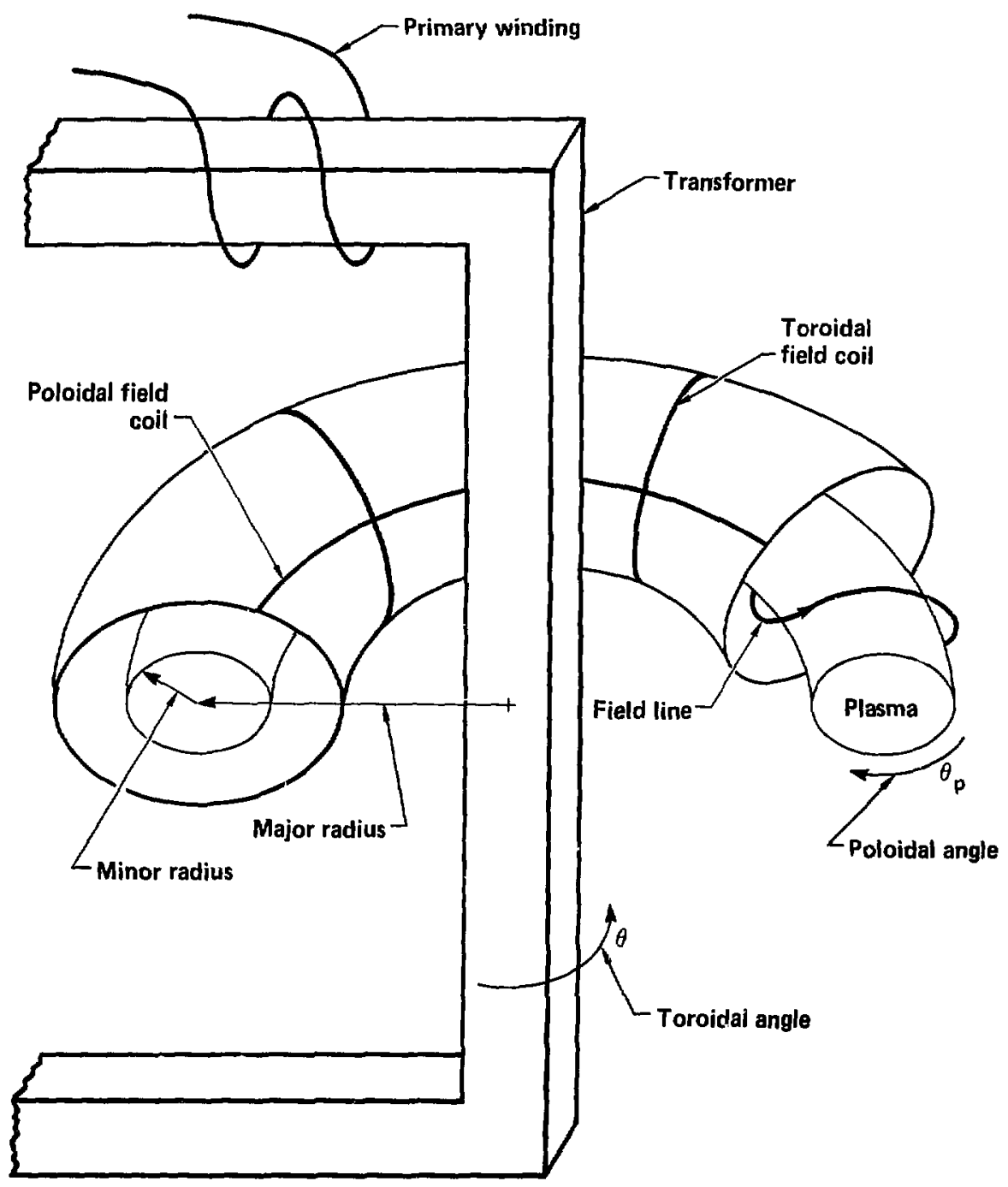

Figure 1-3. Tokamak coil geometry with transformer linking plasma. 
large $A_{r}$ may be written in terms of poloidal field $B_{p}$ and toroidal field $B_{T}$ as follows.

$$
q=B_{T}\left(B_{p} A_{r}\right)^{-1}
$$

Typical values of $A_{r}$ range from $3^{[49]}$ to $8^{[50]}$ and consequent] $y$ for stability a tokanak has $B_{T}>B_{D}$. The confinement of a tokamak plasma is essentially due to the poloidal iield and consequently the $B=8 \pi p /\left(B_{p}+B_{T}\right)^{2}$ is typically a few percent.

\section{- Compact Torus Geometry}

The contrasting class of closed field line confinement devices whic!, do not have plasma linking conductors are described as compact tori. These devices have the advantage of low total structure volume to plasma volume, efficient use of magnetic field, the possibility of high $\beta \sim 1$, and reduced wall surface area to plasma surface area. An added attraction is a higher volume averaged fusion power density compared to a tokamak. A calculation using an idealized compact torus equilibr lum and assuming a $B=.1$ tokamak yields a result of 700 times greater compact torus fusion power density, ${ }^{[51]}$ compared to the tokamak.

Compact torus devices may be grouped into three classes mainly distinguished by the relative magnitude of poloidal and toroidal field and the size of the Larmor radius $\rho_{L}=v_{t h} m c /(e B)$, where $v_{t h}$ is thermal velocity, $m$ is mass, $c$ is the speed of light, $e$ is charge and $B$ is magnetic field. The three classes are particle ring devices, spheromaks and field reversed mirrors or FRM. 


\section{- Particle Ring Devices}

In the class of large Larmor radius devices one of the earliest attempts to improve the conf inement of a mirror device was a particle ring conf inement device known as Astron. [52] The idea was to stack axis encircling relativistic electron rings in a weak mirror field to heat and confine a space charge neutralizing background plasma. The goal was to obtain an intense enough high energy electron ring to cause a current sufficient to reverse the magnetic field inside the ring and result in a closed field line region. Long plasma layers exhibited tearing instabilities ${ }^{[53-55]}$ and field reversal was not reached due to saturation of current buildup during the stacking process. Although reactor designs were proposed assuming these difficulties could be overcome, technological restrictions led to the eventual abandonment of this concept. Later experiments such as RECE-BERTA ${ }^{[56]}$ relied on a single intense burst of high energy relativistic electrons, and achieved field reversal. In this experiment a burst of electrons is perpendicularly injected into a vacuum chamber having an axial magnetic field. In other experiments ${ }^{[57,58]}$ (RECE-CHRISTA) an axial conductor is used to generate a coaxial toroidal field in addition to the axial magnetic field. Axial translation and compression have been achieved and plasma lifetimes up to $1 \mathrm{~ms}$ have been obtained. An alternate approach using magnetically insulated ion sources produces rotating diamagnetic proton layers by axial cusp injection of an annular beam into a magnetic mirror. ${ }^{[59]}$ Axial compression and reflection in a 
magnetic mirror have been demonstrated as well as propagation wit' . space charge disruption.

\section{- Spheromak Compact Torus}

The spheromak is a compact torus device with small Larmor radius and either vanishing poloidal field or poloidal field comparable to toroidal magnetic field in the plasma. External to the plasma the toroidal magnetic field is zero. The limit of an aspect ratio one tokamak, with plasina linking conductors removed, is considered to be a spheromak. The spheromak is an interesting device because force free states, where current is parallel to magnetic field, have been shown by Taylor[60] to be states of minimum energy with the constraint of constant hie?icity $K$,

$$
K=\frac{1}{2} \int \vec{A} \cdot \vec{B} d v
$$

where $\vec{A}$ is vector potential. The magnetic field for these devices satisfies $\nabla \times \vec{B}=\overrightarrow{\alpha B}$ where $\alpha$ is a cunstant. The PS-1 experiment ${ }^{[61]}$ produces a spheromak using external currents in the $z$ and $\theta$ direction in cylindrical coordinates. A $z$ directed current, as in a $z$-pinch discharge, causes a toroidal field in a plasma having an initial axial magnetic field. A $\theta$ current is then initiated with a direction resulting in an axial magnetic field opposing the initial axial field. The result is radial compression and implosion heating with field line reconnection. The final plasma has an essentially force free magnetic field. Formation of a spheromak may also be accomplished on a timescale rapid compared to a resistive diffusion 
time, $4 \pi L^{2}\left(c^{2} n\right)^{-1}$ but slow compared $t r$ an Alfven wave transit time $L(4 \pi \rho)^{1 / 2} B^{-1}$, where $L, c, \eta, \rho, B$ are a length scale, speed of light, resistivity, density and magnetic field respectively. The inductive scheme which achieves this slow formation has been implemented in the Proto-S-1 experiment. [62] In this experiment a supported ring inductively transfers poloidal and toroidal flux to a plasma. Following the formation prucess a spheromak is formed inside of the supported ring in equilibrium with an initial external field.

\section{- Field Reversed Mirror}

The field reversed mirror in contrast to the particle ring and spheromak device has a Larmor radius which is typically $1 / 6$ to $1 / 3$ the plasma radius. Also in devices with neutral beam fueling and heating the azimuthal current is diamagnetic rather than being caused almost totally by an axis encircling species as in a particle ring device. The field reversed theta pinch is considered to be a field reversed mirror without toroidal field. The field reversed theta pinch experiments $F R X-A, F R X-B^{[63]}$ are axially elongated devices with about a 10 to 1 axial to radial plasma length. The plasma formation process begins with a reverse bias field in a preionized plasma. Rapid initiation of a theta current producing an axial magnetic field opposing the initial bias field results in shock heating and field line reconnection yielding a prolate equilibrium having only a poloidal magnetic field.

Experiments in the 2 XIIB single mirror cell resulted in stream stabilized, neutral beam fueled and heated plasma of high 
beta. On the basis of thi. :ess, ${ }^{[64]}$ experiments were conducted with opposing tangential off axis neutral beam injection. Figure $1-4$ is a schenatic of the anticipated field reversed mirror. In the $2 X I I B$ experiment field reversal was not achieved, however the on axis magnetic field was reduced by $90 \%$. Lack of field reversal was attributed to electron currents which have the ability to cancel ion diamagnetic currents, and degradation of plasma currents caused by croling due to external streaming plasma. ${ }^{[65]}$ To avoid the necessity of a transition from open to closed field lines, the Beta II [66] plasma gun experiment was devised. In this experiment a plasma emerges from a Marshal gun ${ }^{[67]}$ and following field line reconnection forms a field reversed plasma in a flux conserver. The goal was to produce a plasma of sufficient lifetime such that neutral beam heating could be applied. It was found plasma lifetime was limited by carbon and oxygen impurity radiation. Approaches to overcome the impurity problem are being researched. [68]

\subsection{Previous Work}

A plasma is subject to many different phenomena occurring on a variety of time scales. The goal is to conf ine a high temperature plasma at sufficient density for long enough time to produce net energy. Experiments applied to this effort have finite volumes and exterior surfaces that are at room temperature. The plasma environment is then one of temperature and density gradients, and possibly magnetic and electric field variations. By Boltzmann's $H$ theorent ${ }^{69]}$ binary collisions cause the plasma distribution function 


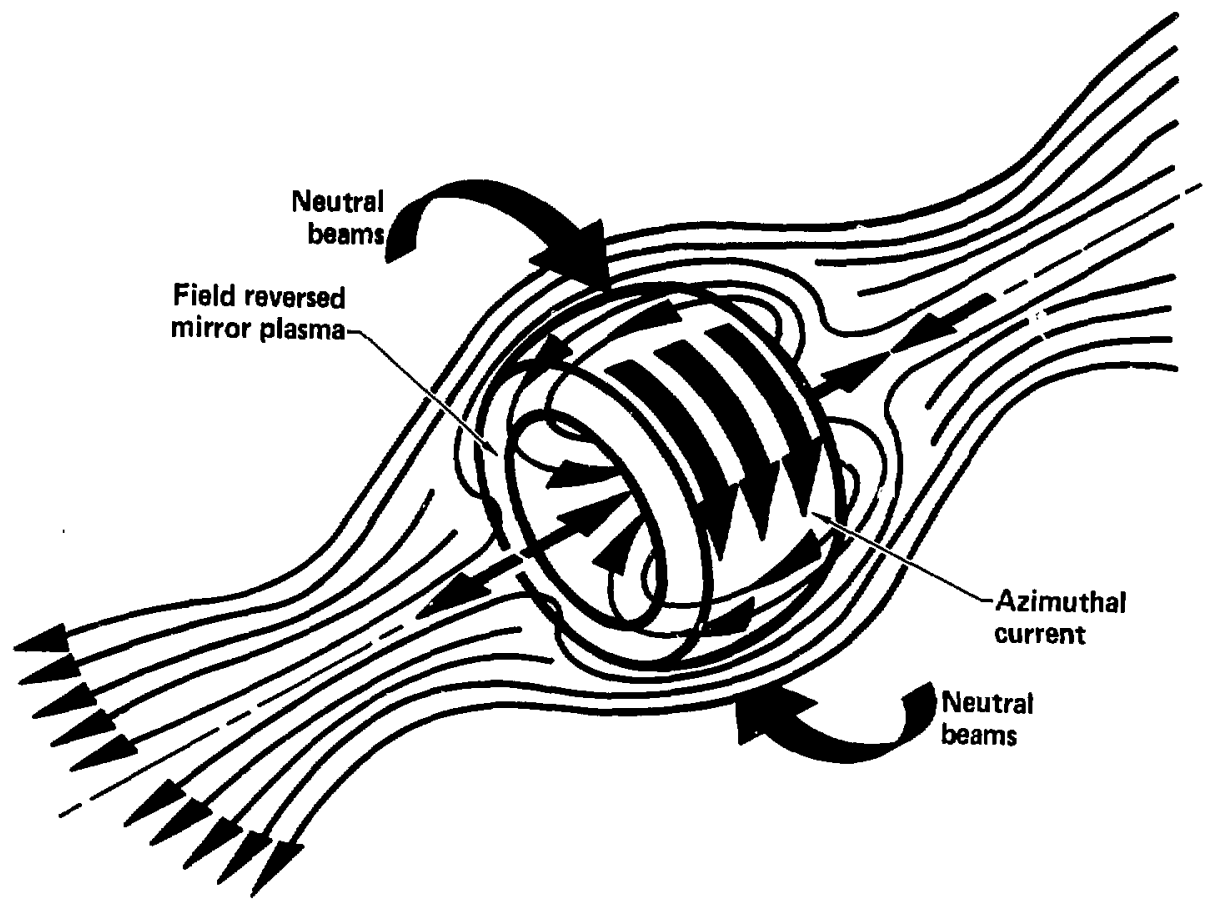

Figure 1-4. Field reversed mirror resulting from tangential neutral beam injection. 
tc become a maxwellian. Consequently the plasma evolves to relax gradients and field variations. When particle sources are present or inappropriate field variations are enforced, macroscopic configurational instability or microscopic velocity space instability may occur. In this work it is assumed such issues are benign. Beyond stability considerations, two other important issues are equilibrium and transport. Equilibrium is concerned with solving equations which arise from minimizing the plasma energy subject to constraints. The energy in this case is thermal and magnetic. Transport considers the slow time evolution of a plasina and the resulting particle and energy diffusion.

\subsubsection{Equilibrium}

The general topic of equilibrium has been reviewed by icNamara. [70] In axisymmetry with scalar pressure, equilibria are determined by solving the magnetostatic equation, $[71]$

$$
\begin{aligned}
& \nabla \cdot \vec{B}=0 \\
& \nabla \times \vec{B}=4 \pi c^{-1} \vec{J} \\
& \vec{J} \times \vec{B}=c \nabla p
\end{aligned}
$$

where $\vec{B}$ is magnetic field, $\vec{J}$ is current density, and $p$ is pressure. The first two equations are from Maxwell's equations with no displacement current and Eq. (1-7) is aither a simplified momentum 
equation or a result of minimizing the energy $w$.

$$
W=\int\left((8 \pi)^{-1} B^{2}+p(\gamma-1)\right) d v
$$

In Eq. $(1-8), \gamma$ is the ratio of specific heats. Direct minimization of $E q .(1-8)$ leads to $B=0, p=0$ and thus Kruskal ard Kulsrud ${ }^{[71]}$ ininimize $W$ for function triples $p, \vec{B}, \psi$ subject to six constraints.

1. $\psi$ has toroidal level surfaces, $\psi=\psi_{W}$ at walls, $\psi_{\min }=0, \psi_{\max }=\psi_{W}$.

2. $V \cdot \vec{B}=0$.

3. $\vec{B} \cdot \nabla \psi=0$.

4. $\int_{\psi \leq C} \vec{B} \cdot \nabla \theta d v=c$.

5. $\int_{\psi \leq c} \vec{B} \cdot(\nabla \psi \times \nabla \theta) d v=h(c)$

6. $\int_{\psi \leq c} p^{1 / Y} d v=M(c)$.

The value $\psi_{W}$ is a constant and $h(c)$ and $M(c)$ are arbitrary functions defined for $0 \leq c \leq \psi_{W}$. A triple $p, \vec{B}, \psi$ subject to the six constraints makes $W$ statiunary if and only if Eq. (1-7) is satisfied and $p$ is only a function of $\psi$.

\section{- Variational Derivation of Equilibrium}

Equilibrium can be determined using methods related to a variational procedure arising from $\mathrm{Eq} .(;-8)$. These techniques are described as waterbag methods. [72] The essential idea is to define a set of contours in two dimensions or a set of surfaces in three dimensions and then move them in a way that reduces energy. The 
state where energy is minimized is then the desirei equilibrium. Typically, surfaces are labelled with a function such as Numerically, a surface is represented by a number cf points having a constant function value such as $p$. The number of $p$ surfaces determines a jrid with varying $p$ values. On a specific $p$ surface there is an additional choice of the distribution of constant $p$ value points, which may be made at any time during the search for an equilibrium. Choices such as equal sparing around a contour have been made; ${ }^{[73]}$ however, it is advantageous to choose a distribution of points so the grid between surfaces is orthogonal. The orthogonal choice increases accuracy by minimizing distance between adjacent $p$ surface prints and also simplifies the representation of equations. [74] In non-orthogonal coordinates extra terins appear. A variational process used to obtain equilibrium in thrie dimensions moves constant $p$ surfaces with displacements proportional to the force acting on a point. [75] This is a steepest descent technique since it yuarantees the change in the potential energy is always negative definite. Each variation step moves closer to the equilibrium state or in some cases no equilibrium is found when an unstable situation results.

\section{- Elliptic Equation Derived Equilibrium}

The alternative method used to obtain equilibrium solutions is to solve an elliptic cquation (the Grad-Shafranov equation discussed in Chapter 2) with a boundary value. [76] Various numerical methods have been employed to invert the elliptic operator 
such as alternating direction implicit, cyclic reduction, relaxation and fast Fourier transforms. [77] The main concern in obtaining a numerical solution is the nonlinearity caused by the source term for the elliptic operator depending on the equilibrium solution. Two types of boundary conditions are usually used. The first is a conducting boundary and the second is an open boundary. In both of these cases the position of the plasma vacuum interface is not known. Part of the nonlinearity difficulty involved in finding a solution is the iterative change of the amount or spatial distribution of current resulting from 2 changing plasma vacuum interface position. The nonlinearity of the formulation necessitates iteration to obtain an equilibrium and iteration invokes questions about uriqueness of solution. In work by Marder and Weitzner ${ }^{[78]}$ a model probiem exhibited bifurcation and it was found two solutions existed for the same boundary conditions. The solutions were distinguished by the amount of current present. The high current case designated, deep and the low current case designated shallow. It was found that a three level iteration scheme resulted in convergence to the shallow solution when a relaxation parameter was chosen in a specific range. Other workers have fixed physical quant ities to obtain convergent solution procedures. In solving for tokamak equilibria ${ }^{[79]}$ the total current has been constrained to remain constant while iterating to a sulution. Solutions of the doublet geometry have been obtained by fixing the position of one point on the contour defining the plasma vacuum interface. [80] 


\section{- Elliptic Equation Boundary Conditions}

In the conducting boundary case the magnetic flux is known to be constant on the boundary and the value is simply applied. In the open boundary case the magnetic flux is not known on the boundary of a finite grid, but may be determined from the current using a Green's function. An alternative technique is to expand the elliptic equation source in terms of Jacobi polynomials and then express the equilibrium solution in spherical coordinates. [81] Callen and Dory[82] solved for equilibrium using successive over-relaxation and applied guard cells of specified value around the computational grid boundary. When the equilibrium solution had small relative changes between iterations the guard cell values were updated by extrapolation. A converged solution was declared when interior values and guard cell values were unchanged by further iteration. Byrne and Klein ${ }^{[83]}$ solve for equilibrium using the alternating direction implicit scheme. The elliptic operator is represented in flux coordinates - id thus it is necessary to generate metric differential coefficients. The required boundary condition is specified by setting the value of magnetic flux paranetrically on the outermost flux surface. The actual constraint is to specify the total current and $f i x$ the location of the outermost flux surface. The relation between current and loop voltage is then used to obtain the boundary value. In other work at Princeton, ${ }^{[84]}$ double cyclic reduction ${ }^{[85]}$ is used to solve the elliptic operator finite differenced on a rectangular grid. Boundary conditions in this case are computed by a Green's function technique in which an integral is 
done ovel the boundary of the computational domain rather than over the plasma current, for the open boundary case. The constraint used to avoid the bifurcation problems is to fix total plasma current. In work by Holmes, Peng and Lynch ${ }^{[86]}$ equilibrium is computed using successive over relaxation to solve the finite differenced elifiptic operator on a rectangular domain. Similar to Callen and Dory flux values are fixed from boundary value considerations and then updated during iteration. Because Holmes, Peng, and Lynch were interested in studying flux-conserving tokamaks they computed equilibrium with fixed magnetic flux difference between the maximum and ninimum. Helton and Wang ${ }^{[87]}$ obtain equilibrium for doublet, droplet, and ellipse plasma cross sections. The solution procedure uses double cyclic reduction with a boundary condition obtained from a Green's function. A limiter is invoked in their case to restrict the free bo:indary position; however, the flux value at the limiter is allowed to change. The main effect of the limiter is to restrict positions where current is defined. To assure convergence the total current is normalized to enforce a constant value. Grad $[88]$ solves equilibrium using cyclic reduction and fixed flux value on a boundary, or periodic in the axial direction for sone cases. Doublet and beit pinch equilibria have been computed in rectangular geometry. $[89,90]$

\section{- Alternate Equilibrium Approache;}

An entirely different approach has been undertaken by Lao. $[91,92]$ He obtains approximate solutions by Fourier expanding 
flux surface coordinates in terms of poloidal angle and minor radius and then solving a set of ordinary differential equations by a variational principle. The object is to compute fourier amplitudes of trigonometric functions of the poloidal angle. The lowest harmonic amplitudes correspond to flux surface shift, minor radius and ellipticity.

Equilibrium soluticns have been obtained from steady state results of the SIJPERLAYER ${ }^{[93]}$ magneto-inductive particle code. In this case fields are obtained by following the motion of representative plasma macro particles.

\subsubsection{Transport}

The general tendency of a plasma is to relax to a maxwellian. The presence of particle and energy sources or fields results in forces which cause fluxes. The forces are of ten given by gradients of quantities such as density and temperature. The associated fluxes are then particles and heat. Due to coupling and the electromagnetic interaction, fluxes of various quantities may depend on several gradients or forces. In the most general formulation geometric considerations must be taken into account. The overall goal of transport studies is to obtain the equations relating fluxes and forces, and the diffusion coefficients or proportionality factors between fluxes and forces taking into account geometry and electromagnetic interaction. An underlying transport consideration is the effect of collisions. The term collision has come to have a very broad meaning. When collisions due to particle-particle interactions are considered the transport is classical or 
neoclassical. When plasma wave-particle interactions effect fluxes of particles, momentum, or energy, the transport is described as anomalous. Only classical or neoclassical transport will be considered so collisions in this work refer only to particle-particle interactions.

\section{- Magnetic Field Free Transport}

Diffusion processes in a magnetic field differ from those in a neutral gas since the magnetic field causes a helical particle path around field lines rather than a straight line path. In a neutral gas, collisions tend to prevent diffusion resulting from free streaming particles. In one dimension with a density gradient the flux $\Gamma$, is proportional to the gradient.

$$
\Gamma=-D_{n} \frac{\partial n}{\partial x}
$$

For collisions of neutral particles or charged particles with neutral particles the diffusion coefficient $D_{n} \approx T /(v m)$ where $T$ is temperature and $v$ is collision frequency. [94] The flux decreases as the inverse of the collision frequency. When charged particles of different mass are diffusing such as ions and electrons, the lighter species is predicted to have a larger flux. However, electric fields which develop when charge imbalance results cause the ion and-electron flux to be equal. This effect is ambipolar diffusion and the overall diffusion coefficient is about tuice the ion diffusion coefficient for equal temperature ions and electrons. 
- Transport with a Magnetic Field

In the presence of a magnetic field $[95]$

$$
\nu_{1}=v^{2} v \omega_{c}^{-2} \approx 3 k T v m c^{2}(Z e B)^{-2}
$$

where $\omega_{c}=2 e B(m c)^{-1}$ and the flux perpendicular to the magnetic field direction increases proportional to the collision frequency. The reason collisions aid diffusion can be seen from the orbit in Fig. 1-5 where a particle in a homogeneous magnetic field encounters a 180 degree collision each cyclotron period. Without collisions the orbit would be a circle of radius $r_{g}=\operatorname{mcv}(Z e B)^{-1}$, where $r_{g}$ is the gyro-radius, $m$ is mass, $c$ is the speed of light, $v$ is velocity, ze is charge and $B$ is magnetic :ield. With collisions the orbit is a cycloid and the particle moves a distance of $r_{g}$ out of the plasina each cyclotron period in the worse case as shown in Fig. 1-5. The actual orbit has collisions anywhere around a circular orbit and thus a particle typically would not go a distance $r_{g}$ toward the plasma boundary each collision. Bohm ${ }^{[96]}$ assumed the collision frequency is equal to the cyclotron frequency and obiained the following diffusion coefficient.

$$
\begin{aligned}
D_{B o h m} & =\omega_{c}(16 v)^{-1} D_{\perp} \\
& \approx 3 c k T(16 \mathrm{Ze} \mathrm{B})^{-1}
\end{aligned}
$$

The important difference between $D_{1}$ and $D_{\text {Bohm }}$ is the field dependence, $D_{1} \sim B^{-2}$ and $D_{B o h m} \sim B^{-1}$. In work by Chandrasekhar ${ }^{[97]}$ the connection was made between diffusion and random walk processes.

$$
D_{r W}=(\Delta x)^{2} / 6 T_{r a n}
$$




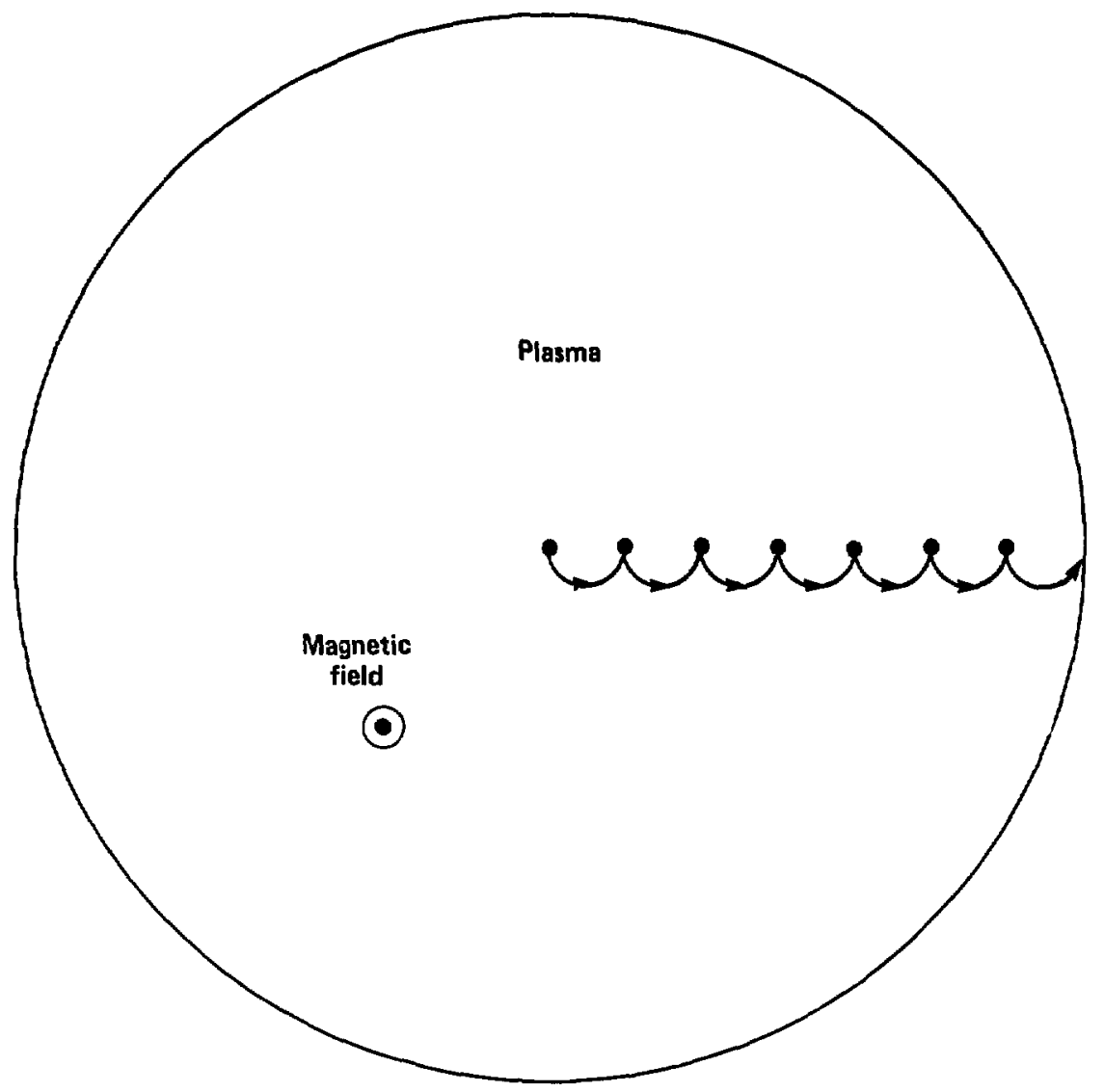

Figure 1-5. Homogeneous magnetic field particle orbit encountering 180 degree deflection each cyclotron period. 
In Eq. (1-12), $\Delta x$ is the random walk step size and $T_{\text {ran }}$ is the time between steps. Using $\Delta x=r_{g}, v^{2}=3 \mathrm{kTm}^{-1}$ and $T_{r \text { an }}=2 \pi / \omega$ the following random walk diffusion coefficient results.

$$
D_{r W}=3 c k T(12 \pi Z e B)^{-1}
$$

This is about .4 times the Bohm estimate.

\section{- Classical Non-Toroidal Transport}

The diffusion coefficient from a clasical treatment by Kruskal and Kulsrud $[71]$ depends on the $p, \vec{B}, \vec{J}$ solution of Eqs. $(1-5),(1-6)$, and $(1-7)$. They consider an isothermal steady state Plasma with source density rate Q slowly diffusing to the walls of a perfectly conducting torus. Admissible $p, \vec{B}, \vec{J}$ solutions must satisfy two auxiliary conditions.

$$
\begin{aligned}
& \int_{p=p} d S|\nabla p|^{-1} \vec{B} \cdot \vec{J}=0 \\
& \int_{p \geq p} d v Q=P \sigma^{-1} \int_{p=p} d S|\nabla p|^{-1} \vec{J} \cdot \vec{J}
\end{aligned}
$$

Given $p, \vec{B}, \vec{J}$ which solve Eqs. $(1-5),(1-6),(1-7)$ and satisfy Eqs. $(1-14),(1-15)$ then two scalar functions a and $\phi$ are obtained from the solution of two magnetic differential equations. The plasma velocity from which a flux can be constructed is then given below.

$$
\vec{v}=|B|^{-2}\left(\nabla \phi-\sigma^{-1} \vec{J}\right) \times \vec{B}+\overrightarrow{a B}
$$

This is a fluid equation treatment of the diffusion problem. 
The fluid equations are derived from moments of the kinetic equation.

$$
\frac{\partial f}{\partial t}+\vec{v} \cdot \nabla f+\vec{a} \cdot \nabla_{v} f=C(f, f)
$$

In Eq. (1-17) $f(\vec{r}, \vec{v}, t) \overrightarrow{d r} d \vec{v}$ is the number of particles in volume $\overrightarrow{d r}$ $\overrightarrow{d v}$ at position $\vec{r}, \vec{v}$ and time $t, \vec{a}$ is acceleration and $c(f, f)$ is the collision operator. Fluid equations for density, momentum and energy result by multiplying Eq. (1-17) by $1, \overrightarrow{m v}, \mathrm{mv}^{2}$ and integrating over velocity. The difficulty in applying these equations is that they are not closed and a complete set only results after taking an infinite number of velocity moments. To avoid this difficulty the true distribution function $f(\vec{r}, \vec{v}, t)$ is approximated as a maxwellian plus a perturbation. The maxwellian is given in terms of $n(\vec{r}, t), T(\vec{r}, t), \vec{v}(\vec{r}, t)$ the local density, temperature and velocity respectively. This approximation has been used by Braginski $i^{[98]}$ to obtain transport coefficients relating fields, $n, \vec{v}, T$ and various gradients to the transfer of momentum force, heat flux, heat and the pressure tensor. The description of Braginsk $i$ is much more complete than that of Kruskal and Kulsrud.

\section{- Toroidal Geometry Transport Effects}

The orbital and confinement geometry have large effects on the classical results. The most fundamental effect results from toroidal geometry. This effect and others are typically analyzed in the literature using large aspect ratio and restricting consideration to various collisionality regimes. Pfirsch and Schluter ${ }^{[99]}$ in 
the highly collisional, large aspect ratio limit obtained an approximately order of magnitude correction to the homogeneous plasma case. The magnetohydromagnetic equations modeled a quasineutral, equilibrium plasma. In addition to Eq. (1-7) they used,

$$
\begin{gathered}
\vec{E}+c^{-1} \vec{\vee} \times \vec{B}=n \vec{J} \\
\nabla \cdot \vec{J}=0 \\
\vec{B}=\left(0, B_{T}, B\left(1-A_{r}^{-1} \cos \theta_{p}\right)\right)
\end{gathered}
$$

where $n$ is resistivity and $\theta_{p}$ is poloidal angle. They angle averaged the flux across a magnetic surface and obtained a safety factor squared correction to the previous cross field diffusion coefficient.

$$
D_{p s}=q^{2} D_{1}
$$

- Trapped Particle Toroidal Geometry Transport Effects

As the collisionality of the plasma decreases, orbit effects and the influence of trapped particles become important. The magnetic field in tokamaks is dominated by the toroidal field which falls off approximately as the inverse distance from the major axis. Particles orbiting along field lines that wind around a torus geometry encounter a magnetic field that increases on the inside of the torus. Assuming constant energy $E$ and magnetic moment $\mu$, the 
velocity along a field line is below.

$$
v_{\|}=(2(E-\mu B) / m)^{1 / 2}
$$

The trapped class of particles has a small enugh $E$ that $v_{1 f}$ goes to zero before they reach the irside of the torus and they thus bounce back and forth on the outside of the torus. In addition to bouncing along field lines there is also a drift velocity across field lines resulting in excursions between flux surfaces. The general motion is shown in Fig. 1-6. The azimuthal drift motion is illustrated in Fig. 1-6a. The projection of the orbit at constant azimuthal angle shows the banana orbit and banana width $\Delta_{b}^{[100]}$ in Fig. 1-6b.

$$
\Delta_{b} \sim A_{r}^{-1 / 2} \operatorname{mv}\left(e B_{p}\right)^{-1}
$$

The fraction of trapped particles are in the region of velocity space where $v_{11} / v \sim A_{r}^{-1 / 2}$ so the effective collision frequency for scattering into an untrapped orbit is $v_{\text {eff }} \sim A_{r} v$. The bounce time of a trapped orbit is $[10]] \tau_{b} \sim A_{r}^{1 / 2}$ (ZeR/V), where $R$ is the plasma major radius. Define $v_{t}=\tau_{b} \nu_{\text {eff }}$, then,

$$
v_{*} \sim A_{r}^{3 / 2} \cup \text { Ze R/v }
$$

where $v_{t h}$ is the thermal velocity. Clearly for $v_{*} \gg A_{r}^{3 / 2}$ particles have collisions before a banana orbit is completed and the issue of trapped particles does not effeci diffusion. 


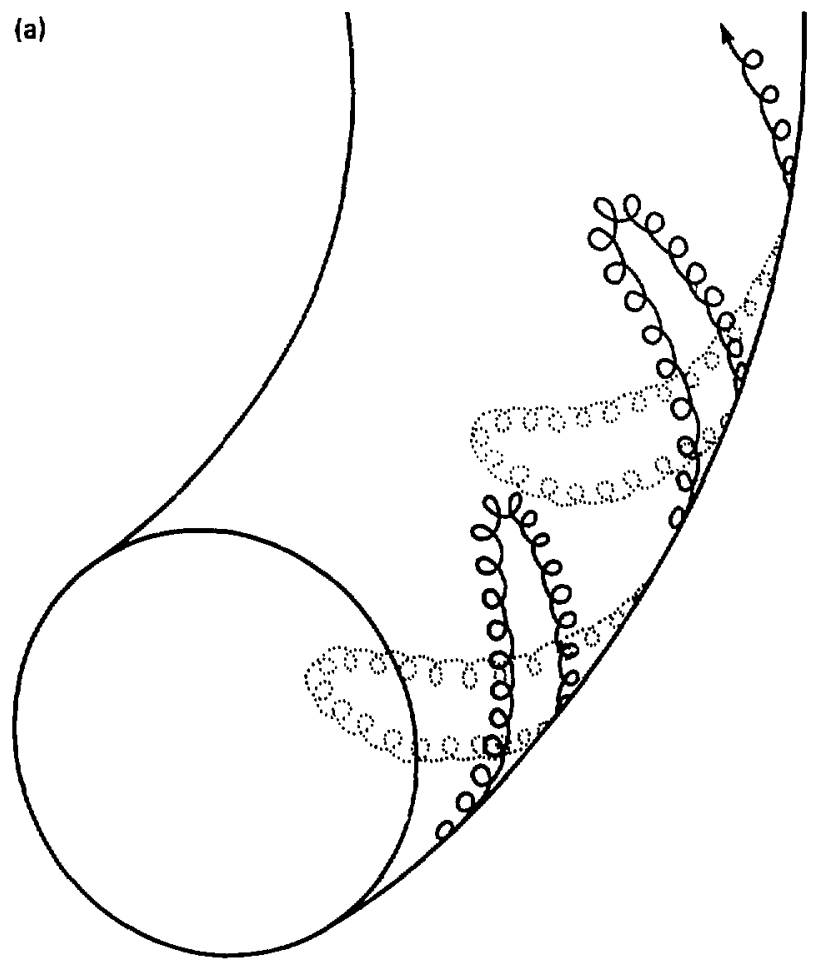

(b)

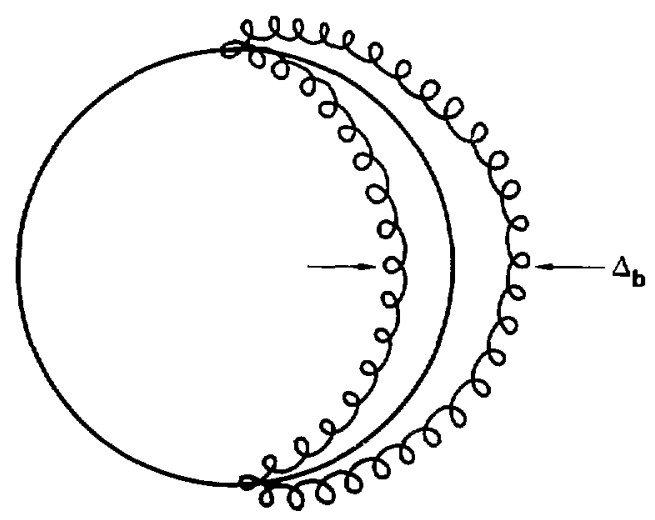

Figure 1-6. Trapped particle motion. (a) Bounce and drift motion (b) Azimuthal angle projection showing banana orbit of width $\Delta_{b}$. 
- Neoclassical Transport

When $v_{*} \ll 1$ trapped particle orbits occur and in the random walk model the step size changes from a gyroradi's to the hanana width. This is the neoclassical diffusion regime. An approximate diffusion coefficient $c$ an be written as follows.

$$
\begin{aligned}
D_{\text {neo }} & \approx \Delta_{b}^{2} \nu_{\text {eff }} A_{r}^{-1 / 2} \\
& =A_{r}^{3 / 2} q^{2} D_{\perp}
\end{aligned}
$$

The neoclassical diffusion regime connects to the plateau regime where che diffusion coefficient is nearly independent of collision frequency. In the plateau regime $1 \ll v_{*} \ll A_{r}^{3 / 2}$. The three diffus: in regimes are shown in Fig. 1-7, where the diffusion coefficient normalized to $D_{\perp}$ is plotted as a function of collision frequency normalized to $v_{t h}(q R)^{-1}$. [102]

- Drift Kinetic Equation

A comprehensive review of toroidal plasma transport has been given by Hinton and Hazeltine. [103] The earliest work that described neoclassical and plateau diffusion was Jone by Galeev and Sagdeev. [104] The essential transpoit solution procedure is to write the kinetic equation in the drift approximation $[105-107]$ and then solve by assuming a perturbed maxwellian distribution function. The drift approximation is based on the gyroracius being sinall compared to a chara: teristic scale length and involves averaging over a Larmor orbit. The drift kinetic equation also has 


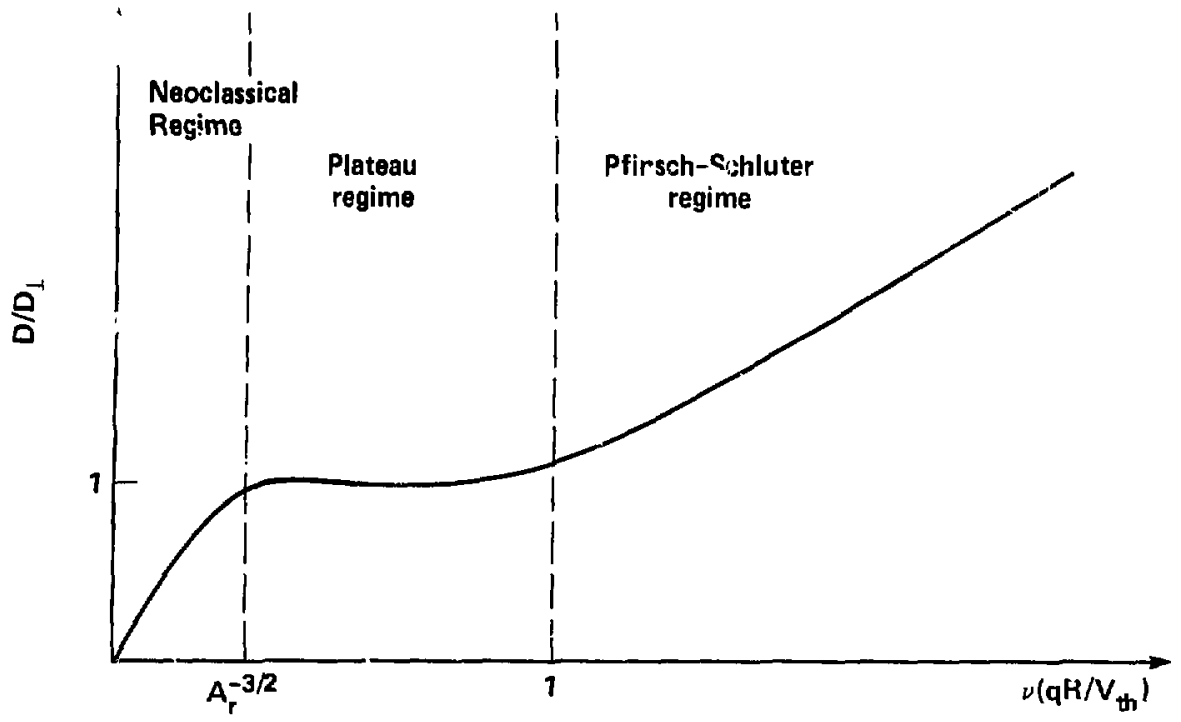

Figure 1-7. Particle diffusion coefficient versus collision frequency. 
an assumed small parameter of collision frequency divided by gyrofrequency used in its derivation. This assumption is related to tokamak ordering where a large toroidal magnetic field is present. The neoclassical, plateau and classical diffusion regimes are studied using the drift kinetic equation by choosing the relative size of two terms. The expansion parameter is collision frequency over transit frequency around a banana orbit. When this parameter is small a term is dropped and the solution of the drift kinetic equation yields neoclassical diffusion as given by Rosenbluth. [108] When the parameter is large, classical Pfirsch-Schluter diffusion results. When the collision frequency is comparable to the transit frequency the solution of the drift kinetic equation yields plateau regime diffusion. The neoc? assical result of Rosenbluth differed from work of Rutherford $[109]$ by including like particle collisions. Both of these results used large aspect ratio, small collision frequency and ignored inductive electric fields. Later the restriction of small collision frequency was removed, $[110]$ and this work was modified to be valid for arbitrary aspect ratio. [111]

\subsection{Scope of the Present Work}

The investigation of transport and equilibrium in field reversed mirrors is approached from two viewpoints. First the plasma is considered to be described as a small Larmor radius, conducting, hydromagnetic fluid. Both toroidal and poloidal magnetic fields are allowed in this case. Timescales of interest and plasma temperatures of interest permit the plasma to be treated 
as adiabatic. There is no restriction made on relative size of toroidal and poloidal magnetic field and the aspect $r$ at io is approximately one. The collision frequency is large as in the Pfirsch-Schluter regime since classical coefficients are used for several transport phenomenon. The state of the plasma is desaribed by the magnetic field structure and the specification of two profiles related to the pressure and toroidal magnetic field. The plasma is assumed to be quasi-static and thus evolves in time between equilibrium solutions as a result of profile changes caused by conduction, radiation, electron ion energy exchange, coulonb friction and neutral beam deposition. In this model the equilibrium is axisymmetric and thus solved in two dimensions. The transport is one dimensional between flux surfaces.

From the second viewpoint the plasma is considered to consist of electrons and several species of ions. The electrons are modeled as an inertialess fluid and the ions are described by a distribution function that must be computed. The magnetic field in this case anly has a poloifal component. The magnetic field is obtained solely from a vector potential and only one component of the vector potential is needed in axisymmetry when the magnetic field is poloidal. The vector potential is obtained from Ampere's law with a curreni which is the difference between the theta velocity moment of the ion distribution function and the density times the electron fluid velocity in the axisymmetry direction. This description includes ions which may have a large Larmor radius. Due to the complexity of this treatment the axisymmetric equilibrium is only 
computed in one dimension. The ion distribution function is expressed as an expansion in collision frequency with the zero order ter $m$ depending only on constants of the motion.

- Magnetohydrodynamic Adiabatic Small Larmor Radius Model

Chapter 2 details the equations which are solved to obtain the small Larmor radius plasma model. The magnetostatic equations with the adiabatic assumption are deduced from the full set of magnetohydrodynamic equations. The flux surface average procedure is defined. The basic equilibrium equation, the Grad-Shafranov equation and its flux surface average are presented with the pr f ile transport equations, and the neutral beam model. The computer code which solves these equations and the computational methods employed are described. The code output and diagnostics are discussed for several cases considering the effects of neutral beam deposition and magnetic field shaping.

\section{- Orbit Average}

Chapter 3 is a preliminary to the large Larmor radius plasma mode1. The ion distribution function satisfies a kinetic equation. Expressing the ion distribution function as an expansion leads to a hierarchy of equations. Integrating over characteristics of these partial differential equations is one method of obtaining a solution. An alternate procedure to integrating over the exact orbit is to average contributions from the exact integration. Chapter 3 describes the orbit average procedure which is equivalent 
to integration over an orbit but does not require a detailed knowledge of the exact orbit. The orbit average procedure is defined in terms of phase space integrals and path integrals. Numerical equivalents of these formulas are derived and tested in two orbit codes. In the first code the orbit is known analytically and many orbit average formulas are compared. The second code requires numerical integration and examines several orbit average formulas in a magnetic field resulting from a solution of the magnetostatic equations. The form of the orbit average integrals varies depending on the number of constants of the motion which exist. To explore the existence of constants of the motion several classes of constants of the motion are examined. Because the examined constants of the motion do not exist when an orbit is stochastic, consideration is also given to determining parameter regimes where stochastic motion is likely in the framework of resonance overlap theory.

- Large Larmor Radius Kinetic Equation Model

In Chapter 4 the large Larmor radius plasma model is presented. Only a poloidal magnetic field is permitted and quasineutrality is assumed. The electrons are considered to be an inertialess fluid and are described by a continuity equation and a momentum equation. The ions are described by a distribution function which satisfies a kinetic equation. The kinetic equation is formulated in terms of a Poisson bracket with the system Hamiltonian. Expressing the ion distribution function as an 
expansion in collision frequency and also expressing the Hamiltonian as an expansion leads to a hierarchy of equations. These equations involve various orders of densities and field potentials. A solution method is implemented using the orbit average derived in Chapter 3. A kinetic equation for the time evolution of the zero order distribution function then results. This equation is analogous to the drift kinetic equation discussed for the small Larmor radius neoclassical diffusion regime. In contrast; however, in this case the actuai orbit has been averaged rather than just averaging over a gyro-orbit. To provide an initiai condition of self consistent electric and magnetic fields the fuli set of equations is solved for model ion distribution functions. Assumptions are made to allow a derivation yielding analytical electric potential and magnetic vector potential. Self-consistent eiectric and magnetic fields are presented for several parameter values. 
CHAPTER 2

ADIABATIC SMALL LARMOR RADIUS MODEL

\subsection{Introduction}

In this chapter the plasma is considered to be a small Larmor radius, conducting, hydromagnetic fluid. The plasma is assumed to be axisymmetric and equilibrium are obtained in cylindrical $r, z$ coordinates. A single fluid model is used for the equilibrium calculation with the magnetic field and two arbitrary profiles determining the state of the plasma. Both poloidal and toroidal magnetic fields are allowed and the profiles are related to pressure and toroidal magnetic field. The profiles are only arbitrary as an initial condition. The plasma evolves between equilibrium states as a result of changes to the vacuum poloidal magnetic field or as a quasi-static evolution of the profiles caused by transport processes. The initial vacuum poloidal magnetic field is varied by 
changes in the currents of external axisymmetric filamert coils of specified radius and axial position. When the plasma evolves in time due to transport processes the ions and electrons are considered separately. Classical transport coefficients are used for conduction, radiation, electron ion energy exchange and neutral beam deposition. Nonlinearities and the complexity of field shape preclude analytic solutions and thus the set of equilibrium and transport equations have been numerically implemented in the $A D B$ computer code. The $A O B$ equilibrium solver and neutral beam package have subsequently been used in conjunction with a more elaborate transport model. [112]

Following a discussion of the equations to be solved, the numerical methods and $A D B$ code initialization are presented. The $A D B$ code output and diagnostics are discussed for several cases which investigate the effects of neutral beam deposition and magnetic field shaping.

\subsection{Equations to be Solved}

\subsubsection{Magnetohydrodynamic Equations}

The single fluid magnetohydrodynamic plasma description is given by the following well known equations. [98] Temperature is in ergs and other units are cgs.

$$
\begin{aligned}
& \frac{\partial n}{\partial t}+\nabla \cdot(\overrightarrow{n u})=0 \\
& m n\left(\frac{\partial \vec{u}}{\partial t}+\vec{u} \cdot \nabla \vec{u}\right)+\nabla p-c^{-1} \vec{J} \times \vec{B}=0
\end{aligned}
$$


47

$$
\frac{\partial p}{\partial t}+\vec{u} \cdot \nabla p+\gamma \nabla \cdot \vec{u}=(\gamma-1) \nabla \cdot(k \nabla)+(\gamma-1) n u^{2}
$$

In Eq. (2-1) to (2-3) quasineutrality is assumed $n_{e}=\sum_{j} n_{j}$ and scalar heat conductivity $k$ and resistivity $n$ are used, $p$ is pressure and $m, n, \vec{u}$ are defined as follows,

$$
\begin{aligned}
m n & =\sum_{j} m_{j} n_{j} \\
\vec{u} & =(m n)^{-1} \sum_{j} m_{j} n_{j} u_{j}
\end{aligned}
$$

where $m_{j}, n_{j}, \vec{u}_{j}$ are species $j$ mass, density and velocity respectively. The Ens. $(2-1)$ to $(2-3)$ are supplemented with Maxwell's equations without displacement current and with electric field $\vec{E}$, eliminated by $0 \mathrm{hm}^{\prime} \mathrm{s}$ law,

$$
\vec{E}+c^{-1} \vec{u} \times \vec{B}=n \vec{J}
$$

which yields,

$$
\begin{aligned}
& \vec{J}=c(4 \pi)^{-1} \nabla \times \vec{B} \\
& \overrightarrow{\partial B}+\nabla \times(\vec{B} \times \vec{u})=-c \nabla \times(n \vec{J}) \\
& \frac{\partial t}{\partial t}+\overrightarrow{ } \\
& \nabla \cdot \vec{B}=0
\end{aligned}
$$

The plasma description is then given by Eqs. (2-1) to (2-3) and Ens. $(2-7)$ to $(2-9)$. There are several approximations of these equations which result in reduced models. The ideal magnetohydrodynamic model 
is obtained by setting $k$ and $n$ to zero. The Pfirsch-Schluter model which led to the $q^{2}$ diffusion coefficient correction results from setting $\overrightarrow{\partial B} / \partial t$ and $m n(\partial \vec{u} / \partial t+\vec{u} \cdot \overrightarrow{\nabla u})$ to zero. The Grad-Hogan model is obtained by only setting $m n(\partial \vec{u} / \partial t+\vec{u} \cdot \overrightarrow{\nabla u})$ to zero. If in addition dissipative terms are ignored $(\kappa=\eta=0)$ the Grad-Hogan formulation becomes the adiabatic model. [114] The equations for the adiabatic model are below.

$$
\begin{aligned}
& \frac{\partial n}{\partial t}+\nabla \cdot(\overrightarrow{n u})=0 \\
& \vec{J} \times \vec{B}=c \nabla p \\
& \vec{j}=c(4 \pi)^{-1} \nabla \times \vec{B} \\
& \vec{B}=\nabla \times(\vec{u} \times \vec{B}) \\
& \frac{\partial \vec{t}}{\vec{B}} \\
& \nabla \cdot \vec{B}=0
\end{aligned}
$$

The adiabatic model is used in this work.

In cylindrical $(r, \theta, z)$ coordinates Eq. (2-14) is satisfied by using the following definition of $\vec{B}$,

$$
\vec{B}=\nabla \psi \times \nabla \theta+f_{T} \nabla \theta
$$

where $\psi$ is related to magnetic flux, $\nabla \theta=1 / r \hat{e}_{\theta}$, where $\hat{e}_{\theta}$ is the unit vector in the $\theta$ direction, and $f_{T}$ is $r$ times the toroidal magnetic field. The relation of $\psi$ to flux is obtained from the flux definition with the area oriented perpendicular to the $z$ axis. 


$$
\begin{aligned}
\text { flux } & =\int_{\vec{B}}^{\vec{B}} \cdot \overrightarrow{d A} \\
& =\int_{0}^{2 \pi} \int_{0}^{r} \operatorname{rdrd\theta }\left(\frac{1}{r} \frac{\partial \psi}{\partial r}\right) \\
& =2 \pi \psi
\end{aligned}
$$

\subsubsection{Grad-Shafranov Equation}

The basic axisymmetric equilibrium equation, the Grad-Shafranov equation, is obtained from the theta component of Eq. $(z-12)$ in combinatien with Eq. $(2-11)$. Substituting Eq. (2-15) into Eq. (2-12) yields,

$$
-\nabla \theta \Delta^{\star} \psi+\nabla f f_{T} \times \nabla \theta=4 \pi c^{-1}\left[\mathrm{~J}_{p^{3}}{ }^{-1} \nabla \psi \times \nabla \theta+r \mathrm{~J}_{T} \nabla \theta\right]
$$

where subscript $p$ denotes puloidal, subscript $T$ denotes toroidal and the Grad-Shafranov operator $\Delta^{*}$ is defined as follows.

$$
\Delta^{*}=r^{2} \nabla \cdot\left(r^{-2} \nabla\right.
$$

The poloidal component of Eq. $(2-17)$ reveals $f_{T}$ is only a function of $\psi$ and thus

$$
J_{p} B_{p}^{-1}=c(4 \pi)^{-1} \frac{d f}{d \psi}
$$

The dot product of $\vec{B}$ with Eq. (2-11) shows $p$ is only a function of \%. Substituting Eq. (2-15) into Eq. (2-11) and using Eq. (2-19) yields,

$$
r J_{T}=r^{2} c \frac{d p}{d \psi}+c(4 \pi)^{-1} f_{T} \frac{d f}{d \psi}
$$


The toroidal component of Eq. (2-17) combined with Eq. (2-20) yields the Grad-Shafranov equation.

$$
\Delta^{*} \psi=-4 \pi r^{2} \frac{d p}{d \psi}-f_{T} \frac{d f_{T}}{d \psi}
$$

When the right side of Eq. $(2-21)$ is specified along with boundary conditions a well posed elliptic operator probiem results.

The integral of Eq. (2-13) over an area, perpendicular to the $z$ axis at position $z=0$, from $r=0$ to the magnetic axis gives,

$$
\frac{\partial}{\partial t}\left(\int \vec{B} \cdot \overrightarrow{d A}\right)=\oint d s(\vec{u} \times \vec{B})
$$

where Stokes' theorem has been used. The magnetic axis is the position where $B_{p}=0$. Using Eq. $(2-16)$ in Eq. $(2-22)$ and setting $\mathrm{B}_{\mathrm{p}}=0$ yields,

$$
\frac{\partial \psi}{\partial t}=\oint d s u_{p} B_{t}
$$

Because $u_{p}=0$ at the magnetic axis, Eq. (2-23) restricts the flux between the separatrix and the magnetic axis to be constant. This means the magnetic axis or 0-point value $\psi=\psi_{0}$ is constant in the adiabatic model. Since a single interior value $\psi_{0}$ is specified, the adiabatic model solution of the Grad-Shafranov equation is non-standard. A technique of enforcing the $\psi_{0}$ value while solving Eq. $(2-21)$ as an elliptic problem has been developed by Grad. [115] The method consists of iterating between the Grad-Shafranov equation and the flux surface average of the Grad-Shafranov equation. The 
flux surface average equation is an ordinary differential equation which admits a boundary value at the magnetic axis and thus incorporates $\psi_{0}$ into its solution. The flux surface average oi a function $\left\langle G_{a}\right\rangle$ is the normalized volume integral of $G_{a}$ over the infinitesimal volume neighboring a flux surface, in the limit as the volume goes to zero.

$$
\left\langle G_{a}>=2 \pi\left(\frac{d V}{d \Psi}\right)^{-1} \oint \frac{d l}{B_{p}} G_{a}\right.
$$

In Eq. (2-24), de is the path in the poloidal plane along a constant $\psi$ contour, $V$ is the volume inside the $\psi$ contour and $d V / d \psi$ is defined as follows.

$$
\frac{d V}{d \psi}=2 \pi \oint \frac{d \ell}{B_{p}}
$$

The basic flux surface average definition with Eq. (2-25) may be used to obtain the following identity.

$$
\left\langle\nabla \cdot \vec{G}_{b}\right\rangle=\frac{d}{d V}\left(\frac{d V}{d \psi}\left\langle\vec{G}_{b} \cdot \nabla \psi\right\rangle\right)
$$

Applying the flux surface average to Eq. (2-21) divider by $r^{2}$ and using Eq. (2-26) yields the average Grad-Shafranov equauian,

$$
\frac{d}{d V}\left(k \frac{d \psi}{d V}\right)=-4 \pi \frac{d p}{d \psi}-f_{T} \frac{d f}{d \psi}\left\langle r^{-2}\right\rangle
$$

where

$$
K=\left\langle|\nabla V|^{2} r^{-2}\right\rangle
$$


52

The flux surface average of transport equations without dissipation can be used to show profiles $S(\psi)$ and $q(\psi)$ are constant in time. These profiles are related to $p$ and $f_{T}$ as follows,

$$
\begin{aligned}
p(\psi) & =s(\psi)\left(\frac{d \psi}{d V}\right)^{\gamma} \\
\left.f_{T}, \psi\right) & \left.=4 \pi^{2} q(\psi)<r^{-2}\right\rangle \frac{d \psi}{d V}
\end{aligned}
$$

where $\gamma$ is the ratio of specific heats. The profiles $S(\psi)$ and $q(\psi)$ are referred to as the entropy and safety factor profile respectively. The right side of Eq. (2-27) can be written in terms of Eqs. $(2-29)$ to $(2-30)$ and their derivatives.

$$
\begin{aligned}
\frac{d}{d V}\left(K \frac{d \psi}{d V}\right)= & -4 \pi \frac{d S}{d V}\left(\frac{d \psi}{d V}\right)^{-1}-4 \pi r^{5}\left(\frac{d \psi}{d V}\right)^{\gamma-2} \frac{d^{2} \psi}{d V^{2}} \\
& -16 \pi^{4} q\left\langle r^{-2}\right\rangle\left(\frac{d c}{d V} \frac{d \psi}{d V}+q \frac{d^{2} \psi}{d V^{2}}\right. \\
& \left.-q\left\langle r^{-2}\right\rangle \frac{d \psi}{d V} \frac{d}{d V}\left\langle r^{-2}\right\rangle\right)
\end{aligned}
$$

Similarly, Eq. $(2-21)$ is rewritten below.

$$
\begin{aligned}
\Delta^{*} \psi & =r^{2}\left(-4 \pi \frac{j}{d V}\left(\frac{d \psi}{d V}\right)^{\gamma-1}-4 \pi \gamma s \frac{d \psi}{d V} \gamma^{\gamma-2} \frac{d^{2} \psi}{d V^{2}}\right) \\
& -16 \pi^{4} q\left\langle r^{-2}\right\rangle^{-2}\left(\frac{d q}{d V} \frac{d \psi}{d V}+q \frac{d^{2} \psi}{d v^{2}}\right. \\
& \left.-q\left\langle r^{-2}\right\rangle^{-2} \frac{d \psi}{d v} \frac{d}{d V}\left\langle r^{-2}\right\rangle\right)
\end{aligned}
$$


To express $d^{2} \psi / d v^{2}$ in terms of first derivatives Fq. $(2-31)$ is expanded and used to solve for $d^{2} \psi / d v^{?}$. Let,

$$
D=K+4 \pi \gamma S\left(\frac{d \psi}{d V}\right)^{\gamma-2}+16 \pi^{4} q^{2}\left\langle r^{-2}\right\rangle^{-1}
$$

then,

$$
\begin{aligned}
\frac{d^{2} \psi}{d V^{2}}= & -D^{-1}\left(\frac{d K}{d V} \frac{d \psi}{d V}+4 \pi \frac{d S}{d V}\left(\frac{d \psi}{d V}\right)^{\gamma-1}\right. \\
& \left.+16 \pi^{4}\left\langle r^{-2}\right\rangle \quad q \frac{d \psi}{d V}\left(\frac{d q}{d V}-\left\langle r^{-2}\right\rangle^{-1} \frac{d}{d V}\left\langle r^{-2}\right\rangle\right)\right)
\end{aligned}
$$

The expression given by Eq. $(2-33)$ is used in Eq. (2-32) so the right side only depends on first derivatives, which are more accurately obtained numerically than $d^{2} \psi / d V^{2}$.

\subsubsection{Boundary Condition for the Grad-Shafranov Equation}

The flux surface averaged Grad-Shafranov equation given by Eq. $(2-31)$ is solved between the magnetic axis and the separatrix. The boundary conditions are then given by $\psi_{0}$ and $\psi_{\text {sep }}$, which are known initial values.

The two dimensional Grad-Shafranov equation given by Eq. (2-32) is solved on a rectangular $r, z$ grid of finite extent. On the edge of the grid two types of boundary conditions are used.

The first type is conducting. The $\psi$ function is decomposed into two parts; $\psi=\psi_{v a c}+\psi_{\text {plasma }}$. The first part, $\psi_{v a c}$, is due to an externally impressed vacuum field. The $\psi_{\text {plasma }}$ pari is 
due to the presence of the plasma. For the conducting boundary condition $\psi_{p l a s m a}$ is set to zero.

The second type of boundary condition is for the open case where there are no conductors around the plasma. In this case the boundary condition is specified using a Green's function technique.

$$
\Psi_{\text {plasma }}(r, z)=\iint d r^{\prime} d z^{\prime} G\left(r^{\prime}, z^{\prime}, r, z\right) J_{T}
$$

In Eq. $(2-34) J_{T}$ is defined by Eq. $(2-20)$ and $G^{[116]}$ is the Green's function,

$$
G\left(r^{\prime}, z^{\prime}, r, z\right)=4 c^{-1}\left(r^{\prime} r k^{-2}\right)^{1 / 2}\left(\left(1-\frac{1}{2} k^{2}\right) k(k)-E(k)\right)
$$

where,

$$
k^{2}=4 r r^{\prime}\left(\left(r^{\prime}+r\right)^{2}+\left(z-z^{\prime}\right)^{2}\right)^{-1}
$$

and $K(k)$ and $E(k)$ in $E q .(2-35)$ are $=11$ iptic integrals of the firs ${ }^{+}$ and second $k$ ind respectively.

The change of $\psi_{1:=c}$ caused by externil coils is calculated from Eq. (2-34) to simulate adiabatic compression. In this case $\mathrm{J}_{T}$ is replaced by a current corresponding to an axisymmetric infinitesima filament coil.

\subsubsection{Transport Equations}

The transport equations are obtained from moments of the kinetic equation as given by Braginskii. [98] The relevant equations 
for this work are the continuity equation,

$$
\frac{\partial n_{j}}{\partial t}+\nabla \cdot\left(n_{j} \vec{u}_{j}\right)=0
$$

and the heat balance equation,

$$
\begin{gathered}
\frac{3}{2} \frac{\partial n_{j}{ }^{\top} j}{\partial t}+\nabla \cdot\left(\frac{3}{2} n_{j}{ }_{j} \vec{u}_{j}\right)+n_{j} T_{j} \nabla \cdot \vec{u}_{j} \\
+\nabla \cdot \vec{q}_{j}=Q_{j}+S_{\text {beam }}^{\prime} \\
\frac{3}{\frac{\partial n_{e}{ }^{\top} e}{\partial t}}+\nabla \cdot\left(\frac{3}{2} n_{e}{ }^{\top} \vec{u}_{e}\right)+n_{e}{ }^{\top} \nabla \cdot \vec{u}_{e} \\
+\nabla \cdot \vec{q}_{e}=Q_{e}-S_{r a d}^{\prime}
\end{gathered}
$$

The continuity equation, Eq. (2-37), is different than Eq. (2-10) since it refers to species $j$ rather than a single fluid. Although neutral beam deposition involves ionization which would appear as a particle source on the right side of Eq. $(2-37)$ the dominant behavior in regimes of interest is charge exchange. Since only charge exchange is treated this is equivalent to equating the ionization rate to the particle loss rate. The effect of the neutral beam is to replace a plasma ion with a hot beam particle. Consequentiy the neutral beam appears as a source $S$ 'beam in the heat balance equation for ions. In the temperature regime of interest only the electrons radiate so a radiation loss term $S^{\prime}{ }_{\text {rad }}$ appears in Eq. (2-39). The off diagonal pressure tensor terms which are 
related to viscosity effects are neglected in Eq. (2-38) and Eq. $(2-39)$. The heat flux vector $q_{j}$ is defined as follows,

$$
\vec{q}_{j}=.5 m_{j} \int\left(\left(\vec{V}-\vec{u}_{j}\right) \cdot\left(\vec{V}-\vec{u}_{j}\right) \vec{V} f_{j} \mathrm{dV}\right.
$$

where $f_{j}$ is the distribution function describing species $j$. The heat generated due to collisions of other species with species $j$ is given below,

$$
Q_{j}=.5 m_{j} \int\left(\vec{V}-\vec{u}_{j}\right) \cdot\left(\vec{V}-\vec{u}_{j}\right) c(f, f) d V
$$

where $C(f, f)$ represents the collision operator.

The adiabatic assumption resulted in dropping the $\overrightarrow{\mathrm{du}} / \mathrm{dt}$ acceleration term in the original momentum equation. This means centrifugal and Coriolis forces are insignificant and any accelerations due to transitory behavior results in sufficiently slow motion that the corresponding accelerations may be neglected. The consequence of these assumptions lead to $f_{T}$ and $p$ being flux surface functions only depending on $\psi$. Changes of $p$ and $f_{T}$ due to adiabatic compression or transport must then be slower than the time for flux surface equilibration. The equilibration time may be estimated as the sound speed divided by the field line connection length $q R$. The slowly changing nature of the adiabatic assumption implies transport is only important between flux surfaces. Consequently transport is one dimensional with coordinate $\psi$ rather than two dimensional in $r$ and $z$. The appropriate one dimensional transport equations are then obtained by flux surface averaging Eq. 
(2-37) to Eq. (2-39). This standard procedure has been carried out in other work. [112] Specializing results of Shumaker to the adiabatic case where the flow velocity is the same as the flux surface velocity yields the flux surface averaged continuity equation,

$$
\frac{d}{d t}\left(n \frac{d V}{d \psi}\right)=0
$$

and the flux surface averaged heat balance equations,

$$
\begin{aligned}
\frac{d}{d t} S_{j}= & -\frac{4 \pi}{3}\left(\frac{d \psi}{d V}\right)^{-2 / 3} \frac{d}{d \psi} \oint \frac{d \ell}{B_{p}} \nabla \psi \cdot \vec{q}_{j} \\
& +s_{b e a m}+\frac{4 \pi}{3}\left(\frac{d \psi}{d V}\right)^{-2 / 3} \oint \frac{d \ell}{B_{p}} Q_{j} \\
\frac{d s_{e}}{d t}= & -\frac{4 \pi}{3}\left(\frac{d \psi}{d V}\right)^{-2 / 3} \frac{d}{d \psi} \oint \frac{d \ell}{B_{p}} \nabla \psi \cdot \vec{q}_{e} \\
& -S_{\text {rad }}+\frac{4 \pi}{3}\left(\frac{d \psi}{d V}\right)^{-2 / 3} \oint \frac{d \ell}{B_{p}} Q_{e}
\end{aligned}
$$

In Eq. (2-42) the density $n$, does not have a species desigriation since quasineutrality is assumed and there is only one type of ions. The heat generated and heat flux vectors have been calculated by Braginskii assuming a local maxwellian distribution. Before describing $\vec{q}$ and $Q$ several definitions are required. The electron and ion collision frequencies are given below,

$$
\begin{aligned}
& \tau_{e}=3.5 \times 10^{5}(\lambda Z n)^{-1} T_{e}^{3 / 2} \\
& \tau_{j}=2.12 \times 10^{7}\left(\lambda z^{3} n\right)^{-1}\left(m_{j} / m_{0}\right){ }^{1 / 2} \mathrm{~T}_{e}^{3 / 2}
\end{aligned}
$$


where $m_{0}$ is proton mass, $Z$ is ion charge state and,

$$
\lambda= \begin{cases}23.4-1.15 \ln (n)+3.45 \ln T_{e} & T_{e}<50 \mathrm{eV} \\ 25.3-1.15 \ln (n)+2.3 \ln T_{e} & T_{e}>50 \mathrm{eV}\end{cases}
$$

The cyclotron frequencies for the electrons and ions have the following definitions.

$$
\begin{aligned}
& \omega_{e}=1.76 \times 10^{7} \mathrm{~B} \\
& v_{j}=9.58 \times 10^{3} \mathrm{ZB} \mathrm{m} / \mathrm{m}_{j}
\end{aligned}
$$

The ion and electron heat flux vectors are then,

$$
\begin{aligned}
& \vec{q}_{j}=-2 n T_{j}\left(m_{j} \omega_{j}^{2} \tau_{j}\right)^{-1} \frac{d T_{j}}{d \psi} \nabla \psi \\
& \vec{q}_{e}=-4.66 n T_{e}\left(m_{e} \omega_{e}^{2} \tau_{e}\right)^{-1} \frac{d T_{e}}{d \psi} \nabla \psi
\end{aligned}
$$

where heat flow along the magnetic field or in the flux surface is ignored. The ion and electron heat generated terms are below.

$$
\begin{aligned}
& Q_{j}=3 m_{e} n_{e}\left(m_{j} \tau_{e}\right)^{-1}\left(T_{e}-T_{j}\right) \\
& Q_{e}=3 m_{e} n_{e}\left(m_{j} \tau_{e}\right)^{-1}\left(T_{j}-T_{e}\right)
\end{aligned}
$$

In Eq. (2-53) the joule heating term $\eta^{2}$ which normally appears is neglected due to the dominance of neutral beam heating and the $\mathrm{T}_{\mathrm{e}}^{-3 / 2}$ dependence of $\mathrm{nJ}^{2}$. 
The radiation term in Eq. (2-44) has the following form,

$$
S_{\text {rad }}=\frac{2}{3}\left(\frac{d V}{d \psi}\right)^{5 / 3} n^{2} \sum_{k} f_{k} L_{k}\left(T_{e}\right)
$$

where the $L_{k}\left(T_{e}\right)$ profile for species $k$ is obtained from corona equilibrium data. [117] In Eq. (2-54) $f_{k}$ is the fraction of the density consisting of a radiating species $k$. Because $f_{k}$ is typically a few percent there is no continuity or heat balance equation for the radiating species.

The neutral beam charge exchange term $S_{\text {beam }}$ in Eq. $(2-43)$ is a flux surface averaged three dimensional deposition. A neutral beam footprint is modeled as a grid of individual beam pencils. The energy deposition is calculated by stepping each beam along a chord passing through the plasma. At each step the local temperature and reaction rate cross section $[118] \overline{\sigma V}$, are used to determine the deposition. To map the three dimensional deposition to axisymmetric flux surfaces the beam deposition is spread in theta by multiplying by a geometric factor $v_{\text {geo }}$ which is the ratio of a beam step volume to the volume of the axisymmetric ring intersecting the deposition location. The deposition is distributed in $r$ and $z$ by flux surface averaging. The attenuation with distance of the bean density $n_{\text {beam }}$ along the deposition path $\ell_{\text {beam }}$ is assumed to be proportional to the plasma density, $\overline{\sigma V}$ and $n_{\text {beam' }}$

$$
\frac{d n_{\text {beam }}}{d l_{\text {beam }}}=-\overline{\sigma V} \cap n_{\text {beam }} v_{\text {beam }}^{-1}
$$

where $V_{\text {beam }}$ is the beam velocity. The deposition at path position $\ell_{\text {beam }}$ is then below, 


$$
\left.\frac{d n_{\text {beam }}}{d \ell_{\text {beam }}}\right|_{\ell_{\text {bearn }}}=-v_{\text {geo }} \overline{\sigma V} n_{\text {Obeam }} v_{\text {beam }}^{-1} e^{-n \overline{\sigma V} \ell_{\text {beam beam }} V^{-1}}
$$

where $n_{\text {0beam }}$ is the initial beam density. The surface average of contributions represented by Eq. $(2-56)$ is weighted with the plasma energy, $E_{\text {plasma }}$ minus beam energy $E_{\text {beam }}$ to account for charge exchange.

$$
S_{\text {beam }}=-\frac{4 \pi}{3}\left(\frac{d V}{d \psi}\right)^{2 / 3}\left(E_{\text {beam }}-E_{p l a s m a}\right) \oint \frac{d \ell}{B_{p}} \frac{d n_{\text {beam }}}{d \ell_{\text {beam }}}
$$

The beam and radiation terms are now defined so the final form of the heat balance equations is obtained by substituting Eq. (2-50) to Eq. (2-53) into Eq. $(2-43)$ and Eq. $(2-44)$.

$$
\begin{aligned}
\frac{d S_{j}}{d t} & =5.457 \times 10^{16}\left(\frac{d \psi}{d V}\right)^{-2 / 3} \frac{d}{d \psi}\left({ }_{n} T_{j} \frac{d T_{j}}{d \psi}\left(Z^{2} \tau_{j} m_{0}\right)^{-1} m_{j} \oint \frac{d \ell}{B_{p}} \frac{|\nabla \psi|^{2}}{B^{2}}\right) \\
& +S_{\text {beam }}+1.82 \times 10^{-27}\left(m_{j} \tau_{e}\right)^{-1}\left(S_{e}-S_{j}\right) \\
\frac{d S}{d t} & =\frac{6.921 \times 10^{13}}{\tau_{e} l}\left(\frac{d \psi}{d V}\right)^{-2 / 3} \frac{d}{d \psi}\left(n T_{e} \frac{d T_{e}}{d \psi} \tau_{e}^{-1} \oint \frac{d \ell}{B_{p}} \frac{|\nabla \psi|^{2}}{B^{2}}\right) \\
& -S_{\text {rad }}-\tau_{e 2}^{-1} S_{e}+1.82 \times 10^{-27}\left(m_{j} \tau_{e}\right)^{-1}\left(S_{j}-S_{e}\right)
\end{aligned}
$$

Two modifications have been made to $\mathrm{Eq} \cdot(2-59)$. A constant $\tau_{\mathrm{e}} \mathrm{l}$ divides the electron conduction term and thus allows conduction to be enhanced over the classical value. Also an empirical term 
$\tau_{\mathrm{e} 2}^{-1} \mathrm{~S}$ e has been added to model an electron profile decay rate with time constant $\tau_{\mathrm{e} 2}$.

\subsection{Numerical Methods for the ADB Code}

The magnetic field structures of $i$ serest and the inherent nonlinearities of the formulation necessitate a numerical solution. The magnetic flux $\psi$ is calculated as a function of volume within a flux surface from the averaged Grad-Shafranov equation and as a function of $r$ and $z$ from the two dimensional Grad-Shafranov equation. A numerical flux surface average is thus required. Numerical methods are required for the averaged Grad-Shatranov equation, the two dimensional Grad-Shafranov equation, the boundary condition and the solution of the transport equation.

\subsubsection{Flux Surface Average}

The flux surface average definition Eq. (2-24) may be written using Eq. (2-25) as follows,

$$
\left\langle G_{a}\right\rangle=\left[G_{a}\right] /[1]
$$

where $\left[G_{a}\right]$ is the un-normalized integral,

$$
\left[G_{a}\right]=\oint \frac{d \ell}{B_{p}} G_{a}
$$

The flux surface average of $G_{a}$ is then determined once $\left[G_{a}\right]$ has been calculated. The functions $G_{a}(r, z), B_{p}(r, z)$ and $\psi(r, z)$ are assumed to be known on a rectangular mesh which may be variable, and are assumed to be symmetric about $z=0$. The computation of $\left[G_{a}\right]$ 
requires the integral of $G_{a} B_{p}^{-1}$ along a constant $\psi$ contour. The most straightforward means of obtaining $\left[G_{a}\right]$ is to solve an initial value problem for the following three ordinary differential equations.

$$
\begin{aligned}
& \frac{d\left[G_{a}\right]}{d \ell}=G_{a} B_{p}^{-1} \\
& \frac{d r}{d l}=B_{r} B_{p}^{-1} \\
& \frac{d z}{d l}=B_{z} B_{p}^{-1}
\end{aligned}
$$

The $r$ position of a specific $\psi$ value at $z=0$ is located and then the $\psi$ contour is followed in $r$ and $z$ while $\left[G_{a}\right]$ is computed. The system of ordinary differential equations given by $\mathrm{Eq} .(2-62)$ is solved using the multistep method described in Chapter 3 . Since $G_{a}$, $B_{r}, B_{z}, B_{p}$ are only defined at grid points and a $\psi$ contour is allowed to cross through a grid cell at any angle it is necessary to fit these functions inside a grid cell. Fitting functions in a grid cell up to third order in $r$ and $z$ provides for continuity of function value, first derivative and second derivative between grid cells. Only $G_{a}$ and $\psi$ are fitted and fields are obtained from the following relations.

$$
\begin{aligned}
& B_{r}=-\frac{1}{r} \frac{\partial \psi}{\partial z} \\
& B_{z}=\frac{1}{r} \frac{\partial \psi}{\partial z} \\
& B_{p}=r^{-1}\left(\left(\frac{\partial \psi}{\partial r}\right)^{2}+\left(\frac{\partial \psi}{\partial z}\right)^{2}\right)^{1 / 2}
\end{aligned}
$$


Fitting $\psi$ to third order yields fields that have continuous first derivatives between grid cells and consequently the solution of Eq. (2-62) is free from discontinuities as a $\psi$ contour is followed between grid cells. Fitting the entire grid and following each desired $\psi$ contour many small steps is a computationally expensive procedure. As a result only a small number of grid cells (typically 10 by 4$)$ are fitted around the magnetic axis. Only in this small region of the grid near the magnetic axis are the differential equations of Eq. (2-62) rigorously solved.

Away from the magnetic axis the actual curved $\psi$ cuntour path is approximated as a number of straight line chords. Each grid cell, for which the $\psi$ value of interest lies between the minimum and maximum grid cell values, is considered to contribute to $\left[G_{a}\right]$. The field line integral path within a grid cell is a straight 1 ine segment between the $\psi$ intersections of the grid cell sides. The $\psi$ intersection and the value of $G_{a} B_{p}^{-1}$ at the intersection position are obtained by fitting a cubic polynomial using the two grid points from the intersected grid cell side and a grid point from the grid cell above and below or right and left depending on whether orientation is vertical or horizontal. It was found linear interpolation gave a poor $\psi$ intersection position when $\psi(r, z)$ was relatively flat. The value of $G_{a} B_{p}^{-1}$ along the straight line path is taken to be the average of the two side intersection values. The contribution of a grid cell to $\left[G_{a}\right]$ is the distance between $\psi$ intersections multiplied by the sum of $G_{a} B_{p}^{-1}$ at the two intersection positions. The factor of $1 / 2$ is absent because $\psi$ 
contours are assumed to be symmetric about $z=0$. The final value of $\left[G_{a}\right]$ for a particular $\psi$ contour is obtained by sumning the contributions from all grid cells. This method of approximating the $\psi$ contour as a straight line chord in a grid celi works well away from the magnetic axis. Near the magnetic axis a $\psi$ contour may intersect a grid cell side twice and the method breaks down completely.

The difficulty of the straight line chord method near the magnetic axis is the reason, in this region, the $\psi$ contour is determined by solving Eq. $(2-62)$. A function such as $K$ which is calculated from $\left[I \nabla V I^{2}{ }^{-2}\right]$ has a sharp peak in $\mathrm{dK} / \mathrm{dV}$ at the $\psi$ contour which is the transition between the two methods of calculating $\left[|\nabla V|^{2} r^{-2}\right]$. To resolve this problem the general field line integral $\left[G_{a}\right]$ is defined to be a weighted sum of the values calculated by the solution of $\mathrm{Eq} .(2-62),\left[G_{a}\right]_{2-62}$ and the values calculated ty the chord method $\left[\mathrm{G}_{\mathrm{a}}\right]_{\text {chord }}$,

$$
\left[G_{\alpha}\right]=e^{\alpha}\left[G_{a}\right]_{2-62}+\left(1-e^{\alpha}\right)\left[G_{a}\right]_{\text {chord }}
$$

where,

$$
\alpha=-\left(\left(\psi-\psi_{0}\right) 5 \psi_{0}^{-1}\right)^{2}
$$

and $\psi_{0}$ is the flux value at the magnetic axis. Using Eq. $(2-66)$, $\left[G_{a}\right]$ is almost entirely $\left[G_{a}\right]_{2-62}$ near the magnetic axis. For $\psi-\psi_{0}>.2 \Psi_{0},\left[G_{a}\right]$ is a imost entirely $\left[G_{a}\right]_{\text {chord }}$ 


\subsubsection{Averaged Grad-Shafranov Equation}

The averaged Grad-Shafranov equation is given by Eq. (2-31). This equation is a second order ordinary differential equation for $\psi$ with volume, $V$ as the independent variable. It is solved on a un:formly spaced volume grid from $V=0$ to the separatrix volume $V=V_{\text {sep }}$. At these two positions the boundary conditions are $\psi=\psi_{0}$ and $\psi=\psi_{\text {sep }}$ which are given constant initial conditions.

The transport equations are set up on a uniform $\psi$ grid because derivatives in those equations involve the $d / d \psi$ operator. Consequently the profiles $S(\psi)$ and $q(\psi)$ in F.q. $(2-31)$ are interpolated to the uniforin volume grid once the $\psi$ values corresponding to the volume grid positions are determined. The volume grid is calculated by numerically computing the volume :ithin given uniform grid $\psi$ values. Once $\psi(V)$ is known $\psi$ values ar obtained on a uniform $V$ grid by interpolation and the volumes are again computed. By a process of iteration $\psi(V)$ on a uniform volume id is established. The surface average functions $k$, and $\left\langle r^{-2}\right\rangle$ are then computed at these $\psi(V)$ values.

It is actually problem dependent whether it is more accurate to interpolate $S(\psi), q(\psi)$ to a uniform volume irid or alternately solve for $\psi(V)$ on a non-uniform volume grid ind use $S(\psi)$ and $q(\psi)$ at the $\psi$ values where they are defined. Consequently Eq. (2-31) is set up to be solved on a non-uniforin volume grid if desired. The left side of Eq. (2-31) is central differenced in a conservative manner. 
66

$$
\begin{aligned}
\frac{d}{d V}\left(K \frac{d \psi}{d V}\right) & =\left(T_{i}^{(1)}+T_{i}^{(3)} \Delta_{1}^{2}\right) \psi_{i+1} \\
& +\left(T_{i}^{(3)}\left(\Delta_{2}^{2}-\Delta_{1}^{2}\right)-T_{i}^{(2)}-T_{i}^{(1)}\right) \psi_{i} \\
& +\left(T_{i}^{(2)}-\Delta_{2}^{2} T_{i}^{(3)}\right) \psi_{i-1}
\end{aligned}
$$

The volume grid position is ciesignated with a subscript $i$ and the local volume spacing between $\psi_{i-1}$ and $\psi_{j}$ is $\Delta_{1}$ and the spacing between $\psi_{i}$ and $\psi_{i+1}$ is $\Delta_{2}$. The $T_{i}^{(j)}$ functions are given below.

$$
\begin{aligned}
& T_{i}^{(1)}=\Delta_{1}^{2}\left(k_{i+1}+k_{i}\right)\left(\Delta_{2}\left(\Delta_{1}^{2} \Delta_{2}+\Delta_{2}^{2} \Delta_{1}\right)\right)^{-1} \\
& T_{i}^{(2)}=\Delta_{2}^{2}\left(k_{i}+k_{i-1} /\left(\Delta_{1}\left(\Delta_{1}^{2} \Delta_{2}+\Delta_{2}^{2} \Delta_{1}\right)\right)^{-1}\right. \\
& T_{i}^{(3)}=2\left(\Delta_{2}^{2}-\Delta_{1}^{2}\right) k_{i}\left(\Delta_{1}^{2} \Delta_{2}+\Delta_{2}^{2} \Delta_{1}\right)^{-2}
\end{aligned}
$$

The right hand side of Eq. (2-31) is written as follows,

$$
\begin{aligned}
R_{i} & =\left(-\Delta_{1} \sigma_{i}-\Delta_{1}^{2} \nu_{i}\right) \psi_{i+1}+\left(-\Delta_{2} \sigma_{i}+\Delta_{2}^{2} v_{i}\right) \psi_{i-1} \\
& +\left(\left(\Delta_{1}+\Delta_{2}\right) \sigma_{i}+\left(\Delta_{1}^{2}-\Delta_{2}^{2}\right) v_{i}\right) \psi_{i}
\end{aligned}
$$

where $\sigma_{i}$ and $v_{i}$ are defined below.

$$
\begin{aligned}
\sigma_{i} & =2\left(\Delta_{1} \Delta_{2}^{2}+\Delta_{\eta}^{2} \Delta_{2}\right)^{-1}\left(4 \pi \gamma S\left(\frac{d \psi}{d \nabla}\right)^{\gamma-2}+16 \pi^{4} q^{2}\left\langle\gamma^{-2}\right\rangle^{-1}\right)_{i} \\
v_{i} & =\left(\Delta_{1}^{2} \Delta_{2}+\Delta_{1} \Delta_{2}^{2}\right)^{-1}\left(4 \pi \frac{\partial S}{\partial V}\left(\frac{d \psi}{d V}\right)^{\gamma-2}+16 \pi^{4} q \frac{d q}{d V}\left\langle r^{-2}\right\rangle^{-1}\right. \\
& \left.-16 \pi^{4} q^{2}\left\langle r^{-2}\right\rangle \frac{d}{d V}\left\langle r^{-2}\right\rangle\right)_{i}
\end{aligned}
$$


67

Using Eq. $(2-67)$ to Eq. (2-73) the differences form of Eq. (2-31) is below,

$$
A_{i} \psi_{i+1}+B_{i} \psi_{i}+C_{i} \psi_{i-1}=0
$$

where,

$$
\begin{aligned}
A_{i} & =T_{i}^{(1)}+T_{i}^{(3)} \Delta_{1}^{2}+\Delta_{1} \sigma_{i}+\Delta_{1}^{2} \nu_{i} \\
B_{i} & =T_{i}^{(3)}\left(\Delta_{2}^{2}-\Delta_{1}^{2}\right)-T_{i}^{(2)}-T_{i}^{(1)} \\
& -\left(\Delta_{1}+\Delta_{2}\right) \sigma_{i}+\left(\Delta_{2}^{2}+\Delta_{1}^{2}\right) \nu_{i} \\
C_{i} & =T_{i}^{(2)}-\Delta_{2}^{2} T_{i}^{(3)}+\Delta_{2} \sigma_{i}-\Delta_{2}^{2} v_{i}
\end{aligned}
$$

Given the boundary conditions $\psi_{0}$, $\psi_{\text {sep }} \mathrm{Eq} .(2-74)$ is solved by the standard tridiagonal algorithm. [119]

$$
\begin{aligned}
& \psi_{i+1}=E_{i} \psi_{i}+F_{i} \\
& E_{i-1}=-C_{i} /\left(A_{i} E_{i}+B_{i}\right) \\
& F_{i-1}=-A_{i} F_{i} /\left(A_{i} E_{i}+B_{i}\right)
\end{aligned}
$$

The solution of Eq. (2-74) must be iterated because $d \psi / d V$ changes and thus at iteration $n$ the coefficients $A_{i}, B_{i}, C_{j}$ actually depend on $\psi^{n-1}$. Nonlinear terms such as $(d \psi / d V)^{\gamma-1}$ are treated as a product of two terms at different iteration levels.

$$
\left[\left(\frac{d \psi}{d V}\right)^{\gamma-1}\right]^{n}=\left[\left(\frac{d \psi}{d V}\right)^{\gamma-2}\right]^{n-1}\left[\frac{d \psi}{d V}\right]^{n}
$$


The functior $k$ has a logarithmic singularity at the separatrix. To avoid the infinity in the $T_{j}^{(1)}$ function, one point from the separatrix, $k_{j+1}+k_{j}$ is replaced by $k_{j+1 / 2}$, obtained from a fit of interior $k$ values assuming the functional form, $[120]$

$$
\begin{aligned}
K & =a_{1} V+a_{2} V+\left(a_{3} V+a_{4} v^{2}\right) \ln (1-V) \\
& +\sum_{j=1}^{\ell} \sin (j \pi V)\left(c_{j} V+d_{j} v^{2}\right)
\end{aligned}
$$

where $V$ is normalized to the volume enclosed by the separtrix and typically $\ell=4$. The analytic derivative of this formula is used for $\mathrm{dK} / \mathrm{dV}$ to obtain a smooth function that properly represents the $(1-V)^{-1}$ singularity.

\subsubsection{Grad-Shafranov Equation}

The two dimensional Grad-Shafranov equation may be written in cylindrical coordinates as follows,

$$
\frac{\partial^{2} \psi}{\partial r^{2}}-\frac{1}{r} \frac{\partial \psi}{\partial r}+\frac{\partial^{2} \psi}{\partial z^{2}}=\mathrm{J}
$$

where $J$ is the right hand side of Eq. (2-32). Central differences are usea $\cdots$ derivatives in Eq. $(2-83)$ to yield,

$$
a_{i, j} \psi_{i+1, j}+\psi_{i, j}+\beta_{i, j} \psi_{i-1, j}+\gamma_{i, j} \psi_{i, j+1}+\lambda_{i, j} \psi_{i, j-1}=\delta_{i, j} j_{i, j}
$$

where $i, j$ designate $r, z$ position and the coefficients are given below, 
69

$$
\begin{aligned}
& \delta_{i, j}=\left[-2\left(\Delta r_{i}+\Delta r_{i+1}\right)\right] R_{i}^{-1} \\
& +\left[\left(\Delta r_{i}\right)^{2}-\left(\Delta r_{i+1}\right)^{2}\right]\left(r_{i} R_{i}\right)^{-1} \\
& \left.-2\left[\Delta z_{j}+\Delta z_{j+1}\right] M_{j}^{-1}\right)^{-1} \\
& \alpha_{i, j}=\delta_{i, j}\left(2 \Delta r_{i} R_{i}^{-1}-\left(\Delta r_{i}\right)^{2}\left(r_{i} R_{i}\right)^{-1}\right) \\
& \beta_{i, j}=\delta_{i, j}\left(2 \Delta r_{i+1} R_{i}^{-1}+\left(\Delta r_{i}\right)^{2}\left(r_{i} R_{i}\right)^{-1}\right) \\
& \gamma_{i, j}=\delta_{i, j} 2 \Delta z_{j} M_{j}^{-1} \\
& \lambda_{i, j}=\delta_{i, j} 2 \Delta z_{j+1} M_{j}^{-1} \\
& R_{i}=\left(\Delta r_{i+1}\right)^{2} \Delta r_{i}+\left(\Delta r_{i}\right)^{2} \Delta r_{i+1} \\
& M_{j}=\left(\Delta z_{j+1}\right)^{2} \Delta z_{j}+\left(\Delta z_{j}\right)^{2} \Delta z_{j+1} \\
& \Delta z_{j}=z_{j}-z_{j-1} \\
& \Delta r_{i}=r_{i}-r_{i-1}
\end{aligned}
$$

Originally Eq. (2-33) was differences on a uniform grid and Fourier techniques were used to obtain solutions. To consider boundaries that are not periodic the alternating direction implicit method was implemented. This method was abandoned due to convergence difficulties. The variable mesh was implemented to increase resolution around the magnetic axis for $\left[G_{a}\right]_{\text {chord }}$ and also to explore the application of distant boundary conditions. 
The solution of Eq. (2-84) with a variable mesh is equivalent to the inversion of a five band matrix with a diagonal band length equal to the number of $r, z$ grid points. The solution is obtained using the Incomplete Cholesky Conjugate Gradient Method (ICCG). [121] This method obtains a solution by expressing the answer in terms of orthonormal vectors. The original matrix problem is pre-conditioned by multiplying by an approximate inverse matrix which has the same sparsity pattern. In theory without roundoff the ICCG method produces an exact answer after equivalently constructing a number of orthonormal vectors equal to the diagonal band length. In practice only a few iterations of the algorithm, usually one thousandth of the theoretical value, are required to obtain an excellent solution. The difference Eq. $(2-84)$ is written as a matrix problem below,

$$
\stackrel{A}{=} \Psi=\underline{J}
$$

where underbar denotes vector and double underbar denotes matrix. The matrix $\underline{A}$ and vector $\underline{J}$ are obtained from $\mathrm{Eq} .(2-84)$ and $\alpha_{i, j}$, $\beta_{i, j}, \gamma_{i, j}, \lambda_{i, j}, \delta_{i, j}$. The problem is to determine $\Psi$ given $\stackrel{A}{=}$ and J. A lower, upper, decomposition of $\underline{A}$ is effected as follows,

$$
\underline{\underline{L}} \underline{\underline{U}} \underline{\underline{U}} \Psi=\underline{\mathrm{J}}
$$

where $\underline{\underline{D}}$ is diagonal and the matrix hands have the definitions below.

$$
\begin{aligned}
& L_{j, i}=A_{j, i}-\sum_{k=1}^{i-1} L_{j, k} U_{k, j} D_{k, k} \\
& U_{i, j}=A_{i, j}-\sum_{k=1}^{i-1} L_{i, k} U_{k, j} D_{k, k}
\end{aligned}
$$


71

$$
D_{i, i}=\left(A_{i, i}-\sum_{k=1}^{i-1} L_{i, k} U_{k, i} D_{k, k}\right)^{-1}
$$

In the case of applying ICCG to a five band matrix $\underline{=}$ it is only necessary to compute $L_{i, i}$. Assuming an $M$ by $N$ grid the bands have the following definitions.

$$
\begin{aligned}
& L_{i, i-1}=A_{i, i-1} \\
& L_{i, i-M}=A_{i, i-M} \\
& L_{i, i}=A_{i, j}-A_{i, i-1}-A_{i, i-M} A_{i-M, i} D_{i-M, i-M} \\
& D_{i, i}=\left(L_{i, i}\right)^{-1} \\
& U_{i, i}=L_{i, i} \\
& U_{i, i+1}=A_{i, i+1} \\
& U_{i, i+M}=A_{i, i+M}
\end{aligned}
$$

Equation (2-99) to Eq. (2-105) are used in the algorithm below. Superscript $i$ designates iteration level.

$$
\begin{aligned}
& \underline{s}^{0}=\underline{J}-\underline{A} \Psi \\
& \underline{c}^{0}=(\underline{\underline{L}} \underline{\underline{0}} \underline{\underline{U}})^{-1} \underline{s}^{0} \\
& \underline{\underline{P}}^{0}=\underline{A}^{T}(\underline{L} \underline{\underline{D}} \underline{\underline{U}})^{-T} \underline{c}^{0} \\
& a^{i}=\left(\underline{P}^{i}, \underline{p}^{i}\right)^{-1}\left(\underline{c}^{i}, \underline{c}^{i}\right) \\
& \Psi^{i+1}=\underline{\Psi}^{i}+a^{i} \underline{\underline{P}}^{i}
\end{aligned}
$$




$$
\begin{aligned}
\underline{s}^{i+1} & =\underline{s}^{i}-a^{i} \underline{A} \underline{p}^{i} \\
\underline{c}^{i+1} & =(\underline{\underline{L}} \underline{\underline{D}} \underline{\underline{U}})^{-1} \underline{s}^{i+1} \\
b^{i} & =\left(\underline{c}^{i}, \underline{c}^{i}\right)^{-1}\left(\underline{c}^{i+1}, \underline{c}^{i+1}\right) \\
\underline{p}^{i+1} & =\underline{A}^{\top}(\underline{L} \underline{D} \underline{U})^{-\top} \underline{c}^{i+1}+b^{i} \underline{p}^{i}
\end{aligned}
$$

There are three initial vectors $\underline{s}^{0}, \underline{c}^{0}, \underline{p}^{0}$ which are needed to begin the ICCG algorithm. The algorithm iterates from Eq. (2-109) to Eq. (2-114) until the magnitude of the $\underline{s}^{i}$ residual vector is sufficiently small. A superscript of $-T$ indicates transpose of the inverse matrix. Brackets around vectors indicate scalar product. Operations such as Eq. (2-112) are performed using back substitution in three steps as follows.

$$
\begin{gathered}
\underline{\underline{L}} \underline{x}=\underline{s}^{i+1} \\
\underline{D} \underline{y}=\underline{x} \\
\underline{\underline{U}} \underline{c}^{i+1}=\underline{y}
\end{gathered}
$$

The algorithm converges in only a few iterations when a close guess is used in Eq. (2-106) since the original equation is almost soived in this case.

\subsubsection{Boundary Condition for the Grad-Shafranov Equation}

The solution of Eq. (2-32) requires a boundry condition for the open case where the plasma is not surrounded by a conductor. A Green's function technique is used to obtain the boundary condition 
by summing the flux due to axisymmetric plasma current rings. Eac:? grid point where the current is non-zero is considered to be a current ring. The flux at an $r, z$ boundary position results from the sum of all current rings which is an approximation to Eq. (2-34).

$$
\Psi_{p \text { lasma }}(r, z)=.25 \sum_{i j}\left[s_{i, j}+s_{i-1, j}+s_{i, j-1}+s_{i-1, j-1}\right]
$$

In Eq. $(2-118) S_{i, j}=\left(J_{T}\right)_{i, j}\left(G\left(r_{i}, z_{j}, r, z\right)+G\left(r_{i},-z_{j}, r, z\right)\right)$ due to symmetry about $z=0$ and $J_{T}$ and $G$ are defined by $E q .(2-20)$ and Eq. (2-35) respectively. The Green's function must be computed for each grid point for all boundary condition positions. This operation is done once for a given specification of $r, z$ dimensions and number of grid points and then $G$ values for Eq. (2-118) are read from a disk file as required. The Green's function values for the boundary point at $z=0$ are stored in computer memory. The $z=0$ boundary point is closest to the plasma and is thus sensitive to changes in current. The change of the flux boundary value at $z=0$ is computed before each two dimensional Grad-Shafranov solution, and all other boundary values are computed only if there is a significant change at $z=0$.

\subsubsection{Transport Equations}

There are three transport equations, Eq. (2-42), Eq. (2-58) and Eq. (2-59). No numerical solution of Eq. (2-42) is required. It is used to determine the density and temperature. 
74

$$
\begin{aligned}
& n(\psi, t)=n\left(\frac{d V}{d \psi}\right)_{t=0}\left(\frac{d \psi}{d V}\right)_{t} \\
& T(\psi, t)=\left(n \frac{d V}{d \psi}\right)_{t=0}^{-1} S(\psi, t)\left(\frac{d \psi}{d \tau}\right)_{t}^{2 / 3}
\end{aligned}
$$

The two heat balance equations are written as a general equation.

$$
\frac{d S_{k}}{d t}=S_{k}^{(1)} \frac{d}{d \psi}\left(S_{k}^{(2)} \frac{d T_{k}}{d \psi}\right)+S_{k}^{(3)} S_{e}+S_{k}^{(4)} S_{j}+S_{k}^{(5)}
$$

For the ions $k=j$ corresponding to Eq. $(2-58)$,

$$
\begin{aligned}
& s_{j}^{(1)}=5.457 \times 10^{16}\left(\frac{d \psi}{d V}\right)^{-2 / 3} \\
& s_{j}^{(2)}=n T_{j}\left(z^{2} \tau_{j} m_{0}\right)^{-1} m_{j} \oint_{\frac{d l}{B_{p}}} \frac{|\nabla \psi|^{2}}{B^{2}} \\
& s_{j}^{(3)}=1.82 \times 10^{-27}\left(m_{j} \tau_{e}\right)^{-1} \\
& s_{j}^{(4)}=-1.82 \times 10^{-27}\left(m_{j} \tau_{e}\right)^{-1} \\
& s_{j}^{(5)}=s_{\text {beam }}
\end{aligned}
$$

and for the electrons $k=$ e corresponding to Eq. (2-59),

$$
\begin{aligned}
& S_{e}^{(1)}=6.92 \times 10^{13} \tau_{e}^{-1}\left(\frac{d \psi}{d V}\right)^{-2 / 3} \\
& S_{e}^{(2)}=n T_{e} \tau_{e}^{-1} \oint_{B_{p}} \frac{d \ell}{B^{2}} \\
& S_{e}^{(3)}=-\tau_{e}^{-1}-1.82 \times 10^{-27}\left(m_{j} \tau_{e}\right)^{-1}
\end{aligned}
$$




$$
\begin{aligned}
& S_{e}^{(4)}=1.82 \times 10^{-27}\left(m_{j} \tau_{e}\right)^{-1} \\
& S_{e}^{(5)}=-S_{\text {rad }}
\end{aligned}
$$

In Eq. (2-122) to Eq. $(2-131) T_{e}$ and $T_{j}$ are understood to be given by $\mathrm{Eq} \cdot(2-120)$ with the $S_{e}$ or $S_{j}$ profile respectively. The general equation has several flux surface average quantities in coefficients $s_{j}^{(2)}, s_{j}^{(5)}$ and $s_{e}^{(2)}$, which are determined by the solution of the two dimensional Grad-Shafranov equation. Also $d \psi / d V$ from the averaged Grad-Shafranov equation is effected by transport. Due to these dependenices, no gain was realized by treating terms on the right side of Eq. (2-121) implicitly. Consequently, a straightforward explicit time difference scheme is employed,

$$
\begin{aligned}
S_{k}^{n+1} & =S_{k}^{n}+\Delta t\left[( \Delta \psi ) ^ { - 2 } \cdot 5 \left(\left(S_{k}^{(2)}(\psi+\Delta \psi)+S_{k}^{(2)}(\psi)\right)\right.\right. \\
& \cdot\left(T_{k}(\psi+\Delta \psi)-T_{k}(\psi)\right)-\left(S_{k}^{(2)}(\psi)+S_{k}^{(2)}(\psi-\Delta \psi)\right) \\
& \left.\cdot\left(T_{k}(\psi)-T_{k}(\psi-\Delta \psi)\right)\right) \\
& \left.+S_{k}^{(3)}(\psi) s_{e}+S_{k}^{(4)} S_{j}+S_{k}^{(5)}\right] n
\end{aligned}
$$

where $\Delta \psi$ is the transport grid spacing. The dominant term is neutral beam deposition and thus $\Delta t$ is chosen so $\left(S_{j}^{n+1}-S_{j}^{n}\right)$ $\left(S_{j}^{n}\right)^{\cdot \cdot 1}$ is no greater than $5 \%$ at any $\psi$ grid position.

\subsection{Initial Conditions}

In Section 2.2 equations were presented which describe the evolution of quasi-static equilibria. To begin the calculation an 
initial condition is required for $\psi$ and the $S(\psi), q(\psi)$ profiles. Analytic $\psi$ have been obtained by assuming various models for $p(\psi)$ and $f_{T}(\psi)$. The $\psi$ solution is then used to calculate $d \psi / d V$ and relations Eq. $(2-29)$ and Eq. $(2-30)$ are used to determine $S(\psi)$ and $q(\psi)$. With $p=n T$ known, assuming a $\psi$ dependence of either $n(\psi)$ or $T(\psi)$ determines the $\psi$ dependence of the other function. The ion $S_{j}(\psi)$ and electron $S_{e}(\psi)$ profiles sum to $S(\psi)$ and thus initially it is assumed $S_{j}=S_{e}=.5 S(\psi)$.

\subsubsection{Weitzner Magnetic Field Model}

The Weitzner ${ }^{[122]}$ model assumes $p(\psi)$ and $f_{T}(\psi)$ are proportional to $\psi$.

$$
\begin{aligned}
& p(\psi)=(4 \pi)^{-1} b \psi \\
& f_{T}(\psi)=a \psi
\end{aligned}
$$

The $\psi$ solution for a spherical plasma of radius $\rho_{\gamma}$ inside the plasma is below.

$$
\begin{aligned}
\psi_{<} & =r^{2} b_{n}\left(-1+k a^{2} \rho_{2}^{-2}\left(\rho_{2}^{-1} \sin \rho_{2}-\cos \rho_{2}\right)\right) \\
\rho_{2} & =a\left(r^{2}+z^{2}\right)^{1 / 2} \\
b_{n} & =a^{-2} b \\
& =-1.5 B_{V}\left(2-\rho_{1}^{2} c^{(1)}\right)^{-1}
\end{aligned}
$$




$$
\begin{aligned}
& c^{(1)}=\left(\rho_{1}^{-2} \cos \left(a \rho_{1}\right)+\sin \left(a \rho_{1}\right)\left(a \rho_{1}^{-1}-\left(a \rho_{1}^{3}\right)^{-1}\right)\right) / c^{(2)} \\
& c^{(2)}=-\cos \left(a \rho_{1}\right)+\left(a \rho_{1}\right)^{-1} \sin \left(a \rho_{1}\right) \\
& k=\rho_{1}^{2} / c^{(2)}
\end{aligned}
$$

The constants in Eq. $(2-137)$ to Eq. $(2-140)$ are expressed in terms of $a, p_{1}$ and $B_{V}$. The uniform vacuum field far from the plasma is $B_{V}$. The boundary of the plasma is at the first zero of

$$
-1+\kappa a^{2} \rho_{1}^{-2}\left(\rho_{1}^{-1} \sin \rho_{1}-\cos \rho_{1}\right)
$$

and thus $a, \rho_{1}$, and $B_{V}$ must be chosen such that there is only one zero within radius $\rho_{1}$. In this model it is not possible to arbitrarily vary pressure and toroidal field.

The external vacuum field which matches onto $\psi_{<}$with continuous value and first derivative is given below.

$$
\psi_{>}=.5 r^{2} B_{V}\left(-\rho_{1}^{3} a^{3} \rho_{2}^{-3}\right)
$$

The magnetic field strength at $r=0, z=0$ is $1.5 B_{V}$ so the field is reversed by a factor of 1.5 times the vacuum field. The toroidal field is caused by plasma currents and thus the toroidal magnetic field is zero outside the plasma. Consequently there are two magnetic field nulls located at $r=0, z= \pm \rho_{1}$. The toroidal current inside the plasma has the form below.

$$
J_{T}=\operatorname{kbc}\left(4 \pi \rho_{2}^{2}\right)^{-1} r\left(\rho_{2}^{-1} \sin \rho_{2}-\cos \rho_{2}\right)
$$




\subsubsection{Spherical Hill's Vortex}

The spherical Hill's vortex ${ }^{[123]}$ is a field reversed plasma model with no toroidal field, $f_{T}=0$. The pressure is assumed to be proportional to $\psi$,

$$
p=-15 B_{V}\left(8 \pi \rho_{1}^{2}\right)^{-1} \psi
$$

The resultant radius $\rho_{1}$ plasma solution with far field $B_{V}$ is as follows,

$$
\begin{aligned}
& \psi_{<}=-.75 B_{V} r^{2}\left(1-\left(r^{2}+z^{2}\right) r_{1}^{-2}\right) \\
& \psi_{s}=.5 B_{V} r^{2}\left(1-\rho_{j}\left(r^{2}+z^{2}\right)^{-3 / 2}\right)
\end{aligned}
$$

\subsubsection{Elliptical Hill's Vortex}

The generalization of the sphericai Hill's vortex is an elliptical shaped plasma region. For this case $f_{T}=0$ as before and pressure is proportional to $\psi$ but with a different constant.

$$
p=-(12+3 E) B_{v}\left(8 \pi p_{1}^{2}\right)^{-1} \psi
$$

The interior plasma solution is easily generalized.

$$
\psi_{<}=-.75 B v^{2}\left(1-\left(r^{2}+E z^{2}\right) 0_{1}^{-2}\right)
$$

The separatrix or $\psi=0$ position is at radial position $p_{1}$ for $z=0$. At. $r=$, the $z$ position of $\psi=0$ is $\rho_{1} E^{-1 / 2}$. For $E<1$ the plasma is prolate and for $E>1$ the plasma is oblate. The magnetic field strength at $r=0, z=0$ is $1.5 B_{V}$ so as in the Weitzner model the 
field is reversed by a factor of 1.5 times the vacuum field value. There are three magnetic field nulls located at $r=0, z= \pm \rho_{1}$ $E^{-1 / 2}$ and $r=2^{-1 / 2} \rho_{1}, z=0$. The toroidal current is 1 inear in $r$,

$$
J_{T}=-c(12+3 E) B_{V}\left(8 \pi \rho_{1}^{2}\right)^{-1} r .
$$

The difficulty in inserting the $E$ coefficient in the $\psi_{<}$formula is that a similar modification to $\psi_{>}$from $E q .(2-146)$ does not yield a vacuum solution $\Delta^{\star} \Psi_{>}=0$. Consequently it is necessary to derive a $\psi_{>}$which matches $\psi_{<}$given by Eq. $(2-148)$ in value and first derivative at $r^{2}+E z^{2}=\rho_{i}^{2}$ and satisfies $\Delta^{\star} \psi_{>}=0$.

The problem of matching exterior solutions nas been considered by Shafranov [124]; however, the form of his solution is computationally cumbersome. At the inception of this work no closed form exterior solutions exister so $\psi_{>}$was derived for the prolate and oblate case. Apparently, jear later the prolate solution was independently derived by Kaneko.[125] The oblate solution is of interest since Rosenbiuth and Bussac ${ }^{[126]}$ showed an oblate spheroidal plasma surrounded by a conducting wall is stable to tilting.

Attempts to obtain $\psi_{>}$first centered around generalizing work bj Strauss ${ }^{[127]}$ in elliptical coordinates. Unfortunately as in the Shafranov work $\psi_{>}$could oniy he expressed in terms of an iniinite series. To resolve this difficulty a transformation is made to prolate sphernidal coordinates $\xi, \eta$. Coordinate $\xi$ is constant on an ellipse and $n$ is an angle like coordinate analogous to $e$ in standard spherical cocrdinates. Irserting prolate spheroidal metric 
differential coefficients into Eq. (2-18) for gradient and divergence yields the Grad-Shafranov operator in prolate spheroidal coordinates,

$$
\Delta * \psi=\left(c_{f}^{2}\left(\xi^{2}-\eta^{2}\right)\right)^{-1}\left(\left(\xi^{2}-1\right) \frac{\partial^{2} \psi}{\partial \xi^{2}}+\left(1-\eta^{2}\right) \frac{\partial^{2} \psi}{\partial \eta^{2}}\right)
$$

where $2 c_{f}$ is the distance between foci. There are four consequences of the form of Eq. (2-150). First the homogeneous equation $\Delta^{*} \psi_{>}=0$ is separable. Second, expressing $\psi_{>}$as a product of functions of $\xi$ and $\eta$ the separated equations for both functions are identical in form. This means $\xi$ and $n$ are represented by the same functions. Third, the following relationships exist,

$$
\begin{aligned}
& \Delta^{\star}\left(c_{f}^{2}\left(\xi^{2}-1\right)\left(1-n^{2}\right) P_{n}^{\prime}(\xi) P_{\ell}^{\prime}(n)\right) \\
& \quad=\left(\xi^{2}-1\right)\left(1-n^{2}\right)\left(\xi^{2}-n^{2}\right)^{-1}(n(n-1)-\ell(\ell-1)) P_{n}^{\prime}(\xi) P_{\ell}^{\prime}(n) \\
& \Delta^{\star}\left(c_{f}^{2}\left(\xi^{2}-1\right)\left(1-n^{2}\right) Q_{n}^{\prime}(\xi) P_{\ell}^{\prime}(n)\right) \\
& =\left(\xi^{2}-1\right)\left(1-n^{2}\right)\left(\xi^{2}-n^{2}\right)^{-1}(n(n-1)-\ell(\ell-1)) Q_{n}^{\prime}(\xi) P_{\ell}^{\prime}(n)
\end{aligned}
$$

where $P_{n}$ is a Legendre polynomial and $Q_{n}$ is a Legendre function of the second kind. The prime indicates derivative with respect to argument. Fourth, homogeneous solutions of $\Delta^{\star} \psi_{>}=0$ are immediately obtained for $n=2$ in Eq. (2-151) and Eq. (2-152). Since $c_{f}^{2}\left(\xi^{2}-1\right)\left(i-n^{2}\right)=r^{2}$ a general exterior solution is given below. 
81

$$
\psi_{>}=r^{2} \sum_{n}\left(a_{n} P_{n}^{\prime}(\xi)+b_{n} Q_{n}^{\prime}(\xi)\right) P_{n}^{\prime}(n)
$$

If an elliptic boundary solution $c$ an be expressed in terms of polynomials in $r$ and $z$ multiplied by $r^{2}$, a matching exterior solution $\psi_{>}$is known once the $\psi_{<} r, z$ polynomial is expressed in terms of Legendre functions. The greatest subscript of $P^{\prime}(\xi)$ in $\psi_{<}$then determines the upper summation range of $n$ in Eq. (2-153).

The interior solution $\psi_{<}$may be written in terms of Legendre functions for the prolate Hill's vortex,

$$
\begin{aligned}
\psi_{<} & =r^{2}\left(\alpha\left(\frac{2}{15} P_{3}^{\prime}(\xi)-\frac{4}{5}\right)\left(\frac{4}{5}-\frac{2}{15} P_{3}(n)\right)\right. \\
& \left.+\alpha E\left(\frac{2}{15} P_{3}^{\prime}(\xi)+\frac{1}{5}\right)\left(\frac{2}{15} P_{3}^{\prime}(n)+\frac{1}{5}\right)-\alpha B^{2}\right)
\end{aligned}
$$

where $\alpha=.75 B_{V} c_{f}^{2} \rho_{j}^{-2}, B^{2}=\rho_{1} C_{f}^{-1}$. The exterior solution is be low.

$$
\psi_{>}=r^{2}\left(a_{1}+b_{1} Q_{j}^{\prime}(\xi)+\left(a_{3} P_{3}^{\prime}(\xi)+b_{3} Q_{3}^{\prime}(\xi)\right) P_{3}^{\prime}(n)\right)
$$

The $a_{n}, b_{n}$ coefficients are determined by the two separatrix $\xi=\xi_{0}=(1-E)^{-1 / 2}$ matching conditions.

$$
\begin{aligned}
& \psi_{<}\left(\xi_{0}, n\right)=\psi_{>}\left(\xi_{0}, n\right) \\
& \frac{\partial \psi_{<}\left(\xi_{0}, n\right)}{\partial \xi_{0}}=\frac{\partial \psi_{>}\left(\xi_{0}, n\right)}{\partial \xi_{0}}
\end{aligned}
$$

The resulting coefficients for Eq. (2-155) follow. 
differential coefficients into Eq. (2-18) for gradient and divergence yields the Grad-Shafranov operator in prolate spheroidal coorcinates,

$$
\Delta^{\star} \psi=\left(c_{f}^{2}\left(\xi^{2}-\eta^{2}\right)\right)^{-1}\left(\left(\xi^{2}-1\right) \frac{\partial^{2} \psi}{\partial \xi^{2}}+\left(1-\eta^{2}\right) \frac{\partial^{2} \psi}{\partial \eta^{2}}\right)
$$

where $2 c_{f}$ is the distance between foc $i$. There are four consequences of the form of Eq. (2-150). First the homogeneous equation $\Delta^{*} \psi_{>}=0$ is separable. Second, expressing $\psi_{>}$as a product of functions of $\xi$ and $n$ the separated equations for both functions are identical in form. This means $\xi$ and $n$ are represented by the same functions. Third, the following relationships exist,

$$
\begin{aligned}
& \Delta^{\star}\left(c_{f}^{2}\left(\xi^{2}-1\right)\left(1-n^{2}\right) P_{n}^{\prime}(\xi) P_{\ell}^{\prime}(n)\right) \\
& \quad=\left(\xi^{2}-1\right)\left(1-n^{2}\right)\left(\xi^{2}-n^{2}\right)^{-1}(n(n-1)-\ell(\ell-1)) P_{n}^{\prime}(\xi) P_{\ell}^{\prime}(n) \\
& \Delta^{\star}\left(c_{f}^{2}\left(\xi^{2}-1\right)\left(1-n^{2}\right) Q_{n}^{\prime}(\xi) P_{\ell}^{\prime}(n)\right) \\
& \quad=\left(\xi^{2}-1\right)\left(1-n^{2}\right)\left(\xi^{2}-n^{2}\right)^{-1}(n(n-1)-\ell(\ell-1)) Q_{n}^{\prime}(\xi) P_{\ell}^{\prime}(n)
\end{aligned}
$$

where $P_{n}$ is a Legendre polynomial and $Q_{n}$ is a Legendre function of the second $k$ ind. The prime indicates derivative with respect to argument. Fourth, homogeneous solutions of $\Delta^{*} \Psi_{>}=0$ are immediately obtained for $n=\ell$ in Eq. $(2-151)$ and $E q \cdot(2-152)$. Since $c_{f}^{2}\left(\xi^{2}-1\right)\left(1-n^{2}\right)=r^{2}$ a general exterior solution is given below. 


$$
\psi_{>}=r^{2} \sum_{n}\left(a_{n} P_{n}^{\prime}(\xi)+b_{n} Q_{n}^{\prime}(\xi)\right) P_{n}^{\prime}(n)
$$

If an elliptic boundary $\psi_{<}$solution can be expressed in terms of polynomials in $r$ and $z$ multiplied by $r^{2}$, a matching exterior solution $\psi_{>}$is known once the $\psi_{<} r, z$ polynomial is expressed in terms of Legendre functions. The greatest subscript of $p$ '( $s)$ $\psi_{<}$then detemines the upper summation range of $n$ in $\mathrm{Eq} .\left(2-1 \mathrm{~b}^{\mathrm{s}}\right.$. The interior solution $\psi_{e}$ may be written in terms of legend: functions for the prolate Hill's vortex,

$$
\begin{aligned}
\psi_{<} & =r^{2}\left(\alpha\left(\frac{2}{15} P_{3}^{\prime}(\xi)-\frac{4}{5}\right)\left(\frac{4}{5}-\frac{2}{15} P_{3}^{\prime}(n)\right)\right. \\
& \left.+\alpha E\left(\frac{2}{15} P_{3}^{\prime}(\xi)+\frac{1}{5}\right)\left(\frac{2}{15} P_{3}^{\prime}(n)+\frac{1}{5}\right)-\alpha \beta^{2}\right)
\end{aligned}
$$

where $\alpha=.75 B_{V} C_{f}^{2} \rho_{f}^{-2}, B^{2}=\rho_{1} c_{f}^{-1}$. The exterior solution is below.

$$
\psi_{>}=r^{2}\left(a_{1}+b_{1} Q_{1}^{\prime}(\xi)+\left(a_{3} P_{3}^{\prime}(\xi)+b_{3} Q_{3}^{\prime}(\xi)\right) P_{3}^{\prime}(n)\right)
$$

The $a_{n}, b_{n}$ coefficients are determined by the two separatrix $\xi=\xi_{0}=(1-E)^{-1 / 2}$ inatching conditions.

$$
\begin{aligned}
& \psi_{<}\left(\xi_{0}, n\right)=\psi_{>}\left(\xi_{0}, \eta\right) \\
& \frac{\partial \psi_{<}\left(\xi_{0}, n\right)}{\partial \xi_{0}}=\frac{\partial \psi_{>}\left(\xi_{0}, n\right)}{\partial \xi_{0}}
\end{aligned}
$$

The resulting coefficients for $\mathrm{Eq} .(2-155)$ follow. 


$$
\begin{aligned}
b_{1}= & \alpha\left(\frac{8}{75}+\frac{2 E}{75}\right)\left(6 P_{3}\left(\xi_{0}\right)-\xi_{0} P_{3}^{\prime}\left(\xi_{0}\right)\right)\left(Q_{1}\left(\xi_{0}\right)-\xi_{0} Q_{1}\left(\xi_{0}\right)\right)^{-1} \\
a_{1}= & -\alpha B^{2}+\left(\frac{E}{25}-\frac{16}{25}\right) \alpha+\alpha\left(\frac{8}{75}+\frac{2 E}{35}\right) P_{3}^{1}\left(\xi_{0}\right)-b_{i} Q_{1}^{1}\left(\xi_{0}\right) \\
b_{3}= & \alpha\left(\frac{8}{75}+\frac{2 E}{75}\right)\left(1-\xi_{0} P_{3}\left(\xi_{0}\right)\left(6 P_{3}\left(\xi_{0}\right)\right)^{-1}\right) \\
& \left(Q_{3}^{\prime}\left(\xi_{0}\right)-Q_{3}\left(\xi_{0}\right) P_{3}^{\prime}\left(\xi_{0}\right)\left(P_{3}\left(\xi_{0}\right)\right)^{-1}\right)^{-1} \\
a_{3}= & \left(\alpha\left(\frac{8}{75}+\frac{2 E}{75}\right)-b_{3} Q_{3}^{\prime}\left(\xi_{0}\right)\right)\left(P_{3}^{\prime}\left(\xi_{0}\right)\right)^{-1}+(E-1) \frac{4 \alpha}{225}
\end{aligned}
$$

In the oblate case $E>1$ and the Grad-Shafranov equation

changes form. The $\xi, \eta$ coordinates now refer to oblate geometry.

$$
\Delta^{*} \psi=\left(c_{f}^{2}\left(\xi^{2}+n^{2}\right)\right)^{-1}\left(\left(\xi^{2}+1\right) \frac{\partial^{2} \psi}{\partial \xi^{2}}+\left(1-n^{2}\right) \frac{\partial^{2} \psi}{\partial n^{2}}\right)
$$

The $n$ product functions remain the same as in the prolate case;

novever, the $\xi$ functions have non-standard definitions. To

zistinguish from the previous standard Legendre functions they are given a subscript $0 b$.

$$
\begin{aligned}
& P_{0 b 0}=1 \\
& P_{0 b 1}(\xi)=\xi \\
& P_{0 b 2}(\xi)=\frac{3}{2}\left(\xi^{2}+\frac{1}{3}\right) \\
& P_{0 b 3}(\xi)=\frac{5}{2}\left(\xi^{3}+\frac{3}{5} \xi\right) \\
& Q_{0 b 1}(\xi)=1-\xi \cot ^{-1} \xi
\end{aligned}
$$


83

$$
\begin{aligned}
& Q_{0 b 2}(\xi)=\frac{3}{2}\left(\xi^{2}+1\right) \cot ^{-1} \xi-\frac{3}{2} \xi \\
& Q_{0 b 3}(\xi)=-\frac{5}{2}\left(\xi^{3}+\frac{3}{5} \xi\right) \cot ^{-1} \xi+\frac{5}{2}\left(\xi^{2}+\frac{1}{3}\right)-\frac{1}{6}
\end{aligned}
$$

Also because the ellipse foci have a vertical orientation

$$
r^{2}=c_{f}^{2}\left(\xi^{2}+1\right)\left(1-\eta^{2}\right)
$$

Similar to the conclusions of Eq. (2-151) and Eq. (2-152) $r^{2} P^{\prime}{ }_{\text {ob }}(\xi) P^{\prime}{ }_{n}(n)$ and $r^{2} Q^{\prime}{ }_{O u n}(\xi) P^{\prime}{ }_{n}(\eta)$ are homogeneous solutions. The interior and exterior solutions are written below for the oblate case.

$$
\begin{aligned}
\psi_{<} & =r^{2}\left(\alpha\left(\frac{2}{15} P_{0 b 3}^{\prime}(\xi)+\frac{4}{5}\right)\left(\frac{4}{5}-\frac{2}{15} P_{3}^{\prime}(n)\right)\right. \\
& \left.+\alpha E\left(\frac{2}{15} P_{0 b 3}^{\prime}(\xi)-\frac{1}{5}\right)\left(\frac{2}{15} P_{3}^{\prime}(n)+\frac{1}{5}\right)-\alpha \beta^{2}\right) \\
\psi_{>} & =r^{2}\left(a_{1}+b_{1} Q_{0 b 1}^{\prime}(\xi)+\left(a_{3} P_{0 b 3}^{\prime}(\xi)+b_{3} Q_{0 b 3}^{\prime}(\xi)\right) P_{3}^{\prime}(n)\right)
\end{aligned}
$$

Applying the matching conditions Eq. (2-156) and Eq. (2-157) at $\xi=\xi_{0}=(E-1)^{-1 / 2}$ yields the oblate spheroidal $a_{n}, b_{n}$ coefficients.

$$
\begin{aligned}
& \mathrm{b}_{1}=\alpha\left(\frac{8}{75}+\frac{2 E}{75}\right)\left(6 P_{0 b 3}\left(\xi_{0}\right)-\xi_{0} P_{0 b 3}^{\prime}\left(\xi_{0}\right)\right)\left(Q_{0 b 1}\left(\xi_{0}\right)-\xi_{0} a_{0 b 1}^{\prime}\left(\xi_{0}\right)\right)^{-1} \\
& a_{1}=-\alpha \beta^{2}+\left(\frac{16}{25}-\frac{E}{25}\right) \alpha+\alpha\left(\frac{8}{75}+\frac{2 E}{75}\right) P_{0 b 3}^{\prime}\left(\xi_{0}\right)-b_{1} Q_{0 b} b_{1}\left(\xi_{0}\right)
\end{aligned}
$$

$(2-173)$ 


$$
\begin{gathered}
b_{3}=-\alpha\left(\frac{8}{75}+\frac{2 E}{75}\right)\left(1-\xi_{0} P_{0 b 3}^{\prime}\left(\xi_{0}\right)\left(6 P_{0 b 3}\left(\xi_{0}\right)\right)^{-1}\right) \\
\left(Q_{0 b 3}^{\prime}\left(\xi_{0}\right)-Q_{0 b 3}\left(\xi_{0}\right) P_{0 b 3}^{\prime}\left(\xi_{0}\right)\left(P_{0 b 3}\left(\xi_{0}\right)\right)^{-1}\right)^{-1} \\
a_{3}=-\left(\alpha\left(\frac{8}{75}+\frac{2 E}{75}\right)+b_{3} Q_{0 b 3}^{\prime}\left(\xi_{0}\right)\right)\left(P_{0 b 3}^{\prime}\left(\xi_{0}\right)\right)^{-1}+(E-1) \frac{4 \alpha}{225}
\end{gathered}
$$

\subsubsection{Variable Coefficient Model}

The Weitzner, spherical, and elliptical Hill's vortex morels a 11 derive the $S(\psi)$ and $q(\psi)$ profiles from assumed forms of $p(\psi)$ and $f_{T}(\psi)$ and the corresponding analytic $\psi(r, z)$. To provide flexibility in the form of $S(\psi)$ and $q(\psi)$ this model represents these profiles as variable coefficient functions.

$$
\begin{aligned}
q(\psi)=q_{1} & +\sum_{i=2}^{6} a_{3 i-4}\left(\frac{\psi}{\psi_{0}}-q_{3 i-3}\right)^{\sigma_{3 i-2}} \\
& +q_{17} \exp \left(a_{18}\left(\frac{\psi}{\psi_{0}}-q_{19}\right)^{q_{20}}\right) \\
S_{i}(i)=s_{1} & +\sum_{i=2}^{6} s_{3 i-4}\left(\frac{\psi}{\psi_{0}}-s_{3 i-3}\right)^{s_{3 i-2}} \\
& -z_{i ;} \leq x p\left(s_{18}\left(\frac{\psi}{\psi_{0}}-s_{19}\right)^{s_{20}}\right)
\end{aligned}
$$

In this model the vacuum flux is specified as follows,

$$
\begin{aligned}
& \psi_{v a c}=B_{V}\left(.25\left(R_{M}+1\right) r^{2}+.5 z_{b} \pi^{-1}\left(R_{M}-1\right)\right. \\
& \left.r I_{b l}\left(\pi r z_{b}^{-1}\right) \cos \left(\pi z_{b}^{-1}\left(z_{b}-z\right)\right)\right)
\end{aligned}
$$


where $R_{M}=B_{z}\left(r=0, z=z_{b}\right) B_{z}^{-1}(r=0, z=0)$ and $I_{b T}$ is the standard Bessel function of imaginary argument, and order one. In the variable coefficient model the analytic $\psi(r, z)$ is not specified so a magnetic axis $\psi_{0}$ value is chosen and an approxinate plasma radius is assumed. The two dimensional Grad-Shafranov equation is then solved iteratively scaling the total toroidal current until a solution evolves with the specified $\psi_{0}$ value. During this initialization phase the plasma configuration may evolve to a prolate or oblate shape.

\subsection{ADB Code Results}

The results of the $A D B$ code with adiabatic compression or transport given by Eq. $(2-42)$, Eq. $(2-58)$, Eq. $(2-59)$, a constant maynetic axis $\psi_{0}$ value, and fixed $q(\psi)$ profile are reasonable for a class of plasma scenarios. A more detailed calculation would yield approximately the same answer if the magnetic flux does not radically decay and neutral beam deposition dominates competing processes.

Recent experiments $[128,129]$ have demonstrated stability of field reversed mirror plasmas on time scales long compared to an Alfven wave transit time. Theoretical investigations $[130,131]$ have also demonstrated stability for various plasma betas and surrounding conducting wall positions. The parameters of these studies are near those used in the results presented here. Consequently a minor configuration change which may be necessary to guarantee stability 
during the time evolution of the plasma would not appreciably effect the results.

The constant magnetic axis flux value is justified by assuming the toroidal electric field is small during the time of a calculation. For a radius $20 \mathrm{~cm}$ plasma at $1 \mathrm{keV}$ the classical energy conf inement time is $4 \mathrm{msec}$. [132] Thus for the implemented model it is reasonable to calculate results out to several milliseconds.

A number of neutral beam deposition simulation and adiabatic compression cases have been run with the $A D B$ code. $[133-136]$ These runs centered around the following issues.

1. Radial versus axial plasma shape changes caused by neutral beam injection.

2. Plasma shaping by adiabatic compression.

3. The effect of different neutral beam energies, currents, and footprint sizes.

4. The question of whether the plasma is heated when energy is supplied by neutral beams and simultaneously lost by impurity radiation or an enforced electron energy decay rate.

The $A D B$ equilibrium solver and beam deposition routines have also been implemented in conjunction with Shumaker $[137-141]$ using a more elaborate transport model.

Two cases run with the ADB code are presented in this section. The first case considers the time evolution of a neutral beam injected plasma with radiation loss and an enforced electron energy decay rate. The $q(\psi)$ profile is held fixed and $S(\psi)$ is evolved in 
accordance with Eq. (2-42), Eq. (2-58), and Eq. (2-59). The second case simulates the adiabatic compression of a prolate shaped plasma. In this case $q(\psi)$ and $S(\psi)$ are both held fixed and the plasma changes as a result of modifications to the vacuum magnetic field.

\subsection{1 simulation of a Neutral Beam Injected Plasma}

Th is example investigates the tille evolution of a plasma subject to competing loss and buildup processes. The losses are bremsstrahiung, line radiation and an enforced electron energy decay rate. The plasma buildup or heating is caused by neutral beam charge exchange. The replacement of plasma ions with hot neutral beam particles causes the ion temperature to increase. The energy exchange of ions with electrons which are loosing energy, drains away the energy coming from the neutral beam. The essential issue is whether or not a credible neutral beam current and energy can heat a plasma with a given radiation impurity level and a specified electron energy decay rate.

An axisymmetric field reversed mirror plasma is simulated in cylindrical $r, z$ coordinates on a computational grid of length $20 \mathrm{cmll}$ in the axial direction and $37.5 \mathrm{~cm}$ in the radial direction. The plasma is assumed to be symmetric about $z=0$, and subject to a uniform $z$ directed $1 \mathrm{kilogauss}$ vacuum magnetic field. Impurity radiation is attributed to oxygen at a concentration of $2 \%$ of the local density. The neutral beam is modeled as a uniform current pencil beam grid impacting the plasma from $z=-7 \mathrm{~cm}$ to $z=7 \mathrm{~cm}$ and $20 \mathrm{~cm}$ radially above and below the $z$ axis. The neutral beam current 
is 400 amps at an energy of $12 \mathrm{keV}$. The magnetic axis flux, $\psi_{0}$ is held constant at $-1 \times 10^{6}$ gauss $-\mathrm{cm}^{2}$. The initial peak temperature and density are $30 \mathrm{eV}$ and $1.6 \times 10^{14} \mathrm{~cm}^{-3}$ respectively. The flux variation of temperature and density is initially $\psi^{1 / 2}$ with the peak value at $\psi_{0}$. The $S(\psi)$ and $q(\psi)$ profiles are chosen to make a toroidal current having a single peak about the $\Psi_{0}$ position. By adjusting the profile coefficients in the model of Section 2.4.4 the total magnetic field at $r=0, z=0$ is set initially to 10 kilogauss, the toroidal current is .4 megamps and the poloidal current is .75 megamps. The non-zei'o coefficients for $[q \cdot(2-176)$ and $\mathrm{Eq} .(2-177)$ are listed below.

$$
\begin{aligned}
& s_{2}=150 . \\
& s_{4}=1 . \\
& q_{1}=.2 \\
& q_{2}=2.5 \\
& q_{3}=.7 \\
& q_{4}=2 . \\
& q_{5}=-130 . \\
& q_{6}=1 . \\
& a_{7}=4 . \\
& q_{17}=.4 \\
& q_{18}=-5 . \\
& q_{19}=.75 \\
& q_{20}=2 .
\end{aligned}
$$

The vacuum field determined by Eq. $(2-178)$ has parameters $B_{V}=1 . \times 10^{3}$ and $R_{m}=1$. 
The case presented here is one of three with identical initial conditions except for $\tau_{\mathrm{e} 2}$ of Eq. (2-59). To study the interaction of neutral beam buildup with energy loss by electrons, the electron energy decay time, $\tau_{e 2}$ was set to 50, 100, and 200 microseconds. The eifect of a decreasing decay time is to reduce the increase of $S_{e}(\psi)$ caused by energy exchange with the ions and also to hold down the ion temperature. The $\tau_{\mathrm{e} 2}=50$ results show the greatest change from the initial conditions. The corresponding results of the 100 and 200 microsecond cases fall between the initial conditions and the 50 microsecond case so only graphs of the 50 microsecond case are shown.

The computational grid boundary at a radial position of $37.5 \mathrm{~cm}$ and axial distance of plis and minus $20 \mathrm{~cm}$ is a conductor so the plasma flux at these positions is set to zero. This boundary condition has the effect of keeping flux surfaces approximately unchanged. Comparing the contour plot of $\psi(r, z)$ in $F i g .2-1$ a at time zero with $\psi(r, z)$ at $2.8 \mathrm{msec}$ in Fig. $2-1 \mathrm{~b}$ a slight nutward radial shift of the magnetic axis is apparent. The separatrix or $\psi=0$ position is at $r=32 \mathrm{~cm}, z=0$ and $r=0, z=18.5 \mathrm{~cm}$ for time zero. At $2.8 \mathrm{msec}$ as shown in Fig. $2-1 \mathrm{~b}$ the separatrix is at $r=33.5 \mathrm{~cm}, z=0$ and $r=0, z=18.5$. The plasma remains oblate during the neutral beam buildup. The single minimum of $\psi(r, 0)$ is preserved as shown in Fig. 2-2. At $r=0, \psi=0$ and at the conducting wall position $\psi=\psi_{\text {vac }}$ which is a fixed value. Thus the $\psi(r, 0)$ plots at time zero in Fig. $2-2 a$ and $2.8 \mathrm{msec}$ in $F i g .2-2 b$ have the same endpoints. The difference between the two plots is mainly the 
magnetic axis position, which is located where $\partial \psi / \partial i^{\circ}=0$. Comparing Fig. 2-2a with Fig. 2-2b it can be seen the magnetic axis has shifted from 20 to $22 \mathrm{~cm}$.

The toroidal current is shown at time zero in Fig. ?-3a and at $2.8 \mathrm{msec}$ in Fig. 2-3b. The initial $J_{T}(r, z)$ with basicaily elliptical contours deve?ops the interior structure shown in $\mathrm{Fig} .2-3 \mathrm{~b}$ inside the 866 contour. The $t=2.8 \mathrm{msec}$ structure is a resuit of $\mathrm{J}_{\mathrm{T}}(r, 0)$ changing from a single peak function to a double peak function as shown by $F i g .2-4$. In Fig. $2-4 \mathrm{a}, \mathrm{J}_{\mathrm{T}}(r, 0)$ at time zero has a single peak and in $F i g .2-4 b J_{T}(r, 0)$ at $t=2.8 \mathrm{msec}$ has a double peak. The total toroidal current rises from an initial value of $3.94 \times 10^{5}$ amps to $4.33 \times 10^{5}$ amps at $t=2.8 \mathrm{msec}$. The initial peak value of $4 \pi \mathrm{c}^{-1}$ $\mathrm{J}_{T}$ of 1400 diminishes to peak values of 1100 and 900 , at $2.8 \mathrm{msec}$. The $I_{z}(r, 0)$ shown $i_{i} F i g .2-5 a$ at tine zero and $F i g .2-5 b$ at time $2.8 \mathrm{msec}$ is a plot of total z current flowirg betwen zero and the $r$ position where an ordinate value is plotted. The peak value at $20 \mathrm{~cm}$ in $\mathrm{Fig} .2-5 \mathrm{a}$ indicates an initial total current of $7.5 \mathrm{x}$ $10^{\overline{5}}$ amps flowing between $r=0$ and the magnetic axis. At $2.8 \mathrm{msec}$ Fig. 2-5b shows the total $z$ directed current between $r=0$ and the magnetic axis is reduced to $6.25 \times 10^{5}$ anps.

The $z$ magnetic field is shown in $F$ ig. $2-b$. At the initial time $R_{z}(r, 0)$ is shown in $F i g .2-6 a$ and $B_{z}(0, z)$ in $F$ ig. 2-6b. A.t 2.8 msec $B_{z}(r, 0)$ is in Fig. 2-6c and $B_{z}(0, z)$ is in Fig. 2-6d. Figure $2 \cdot 6 \mathrm{c}$ shows the iriti=l field peak at $28 \mathrm{~cm}$ has shifted radially outward to $30 \mathrm{~cm}$ and the original peak magnitide of 4 xilogauss becomes .5 kilogauss at $2.8 \mathrm{msec}$. Figure $2-6 d$ shows $B_{z}(0, z)$ 
ranges from -9.5 kilogauss to 1 kilogauss. Initially the field at $r=0, z=0$ is -10 kilogauss. The toroidal field $B_{T}(r, 0)$ is plotted in Fig. 2-7a at time zero and $2.8 \mathrm{msec}$ in Fig. 2-7c. The characteristic single peak of $B_{T}$ is preserved from the initial zondition; however, the maximum value diminishes from $8.5 \mathrm{kilogauss}$ to 7 kilogauss. The total magnetic field magnitude, $B(r, 0)$ is shown in Fig. 2-7b at time zero and in rig. ?-7d at time $2.3 \mathrm{msec}$. The initial peak value of $1.08 \times 10^{4}$ located at $r=8$ is reduced moderately to $.97 \times 10^{4}$ and shifts inward to $r=6$ at $2.8 \mathrm{msec}$. An additional small pes develops at 2.8 insec near $r=30$ with in agnitude 5 kilogauss.

The density $n(r, 0)$ at the initial time is plotted in Fig. 2-3a and the density at $2.8 \mathrm{msec}$ is plotted in Fig. 2-8b. These plots show the density feak fell from $1.65 \times 10^{14} \mathrm{~cm}^{-3}$ to $1.1 \times 10^{14} \mathrm{~cm}^{-3}$. The aumber of farticles is held fixed so the density decrease is a consequence of the expansion of t'e plasma volume. The initial single density prak near the magnetic axis is maintained throughout the time pvolution of the plasma.

The pressure $p(r, 0)$ plotted in $F$ ig. $2-9 a$ at time zero and 2.8 msec in Fig. 2-9b also maintains a single peak near the magnetic axis. Both density and pressure peaks shift outward slightly as the position of the magnetic axis moves from 20 to $22 \mathrm{~cm}$ between the initial time and $2.8 \mathrm{msec}$. The peak pressure goes from an initial value of $1.2 \times 10^{4}$ dyne- $\mathrm{cm}^{-2}$ to $8 \times 10^{5}$ dyne $-\mathrm{cm}^{-2}$. Thus the relative beta for a fixed reference field increases by a factor of 67 and the initially nearly force free plasma evolves toward a finite beta 
state. The toroidal current has a term proportional to $d p / d \psi$. As $c$ an be seen froin Fig. 2-Sa and Fig. 2-sb, taking account of scale, the pressure gradient tands to steepen in the vicinity of $r=14 \mathrm{~cm}$ and $r=27 \mathrm{~cm}$. In these regions tine finite beta current dominates the force free current a: $2.8 \mathrm{msec}$.

The ion temperature $T(r, 0)$ is shown in $F i g .2-10$ a at time zero and in Fig. 2-10b at $2.8 \mathrm{msec}$. The temperature has a double peaked structure with an initial magnitude of $.03 \mathrm{keV}$. After $2.8 \mathrm{msec}$ the peuk temperature increses to $4.7 \mathrm{keV}$. The temperature across the plasma varies from $2.8 \mathrm{keV}$ to $4.7 \mathrm{keV}$.

The $S(\psi)$ entropy profile function is plotted in Fig. 2-1la at time zero and in Fig. 2-1lb at $2.8 \mathrm{msec}$. This is the only profile modified by the neutral beam deposition and transport; $q(\psi)$ is held constant. Initially $S(\psi)$ is a linear function of $\psi$ with a maximum value of 150 , at the magnetic axis. After $2.8 \mathrm{msec}$ the maximum value increases to $2.1 \times 10^{4}$ with the profile monatonically decreasing toward the separatrix. The monotonic behavior and the maximum value at the magnetic axis indicate the neutral beam is penetrating the plasma and there is substantial deposition in the interior.

The energy input to the plasma diminishes as the plasma heats since the source term for the beam is attributed to charge exchange and is thus proportional to the difference between the plasina and beam energy. The plot of total kinetic energy in Fig. 2-12a shows the energy asymptotes to approximately 5 kilojoules. At $2.8 \mathrm{msec}$ Fig. 2-12b shows the trapped beam power has fallen to 2200 kilowatts. 
This is an approximate $54 \%$ decrease from the initial value of 4800 ki lowatts.

The steady state that is eventually reached is mainly a balance between the enforced electron loss term proportional to $\mathrm{T}_{\mathrm{e} 2}^{-1}$, the radiation loss and the incoming trapped neutral beain power. The $L_{k}\left(T_{e}\right)$ radiation profile of $E q \cdot(2-54)$ is plotted as a - function of electron temperature in $\mathrm{keV}$ in $\mathrm{Fig} .2-13 \mathrm{~b}$. The peaks in the radiation profile explain the behavior of the radiated power shown in Fig. 2-13a. Initially the electron temperature is at .03 keV which corresponds to a radiation profile position on the right side of the largest peak in Fig. 2-13b with value $10^{21}$. There are two possibilities. When the electron energy decay and impurity radiation loss dominate the energy obtained from beam heated ions, this causes a decrease in the electron temperature and enhances the radiation loss. When the energy from the bean dominates losses the electron temperature increases which diminishes the impurity radiation and allows for further heating. During the course of the run the initial radiated power was 420 kilowatts. The electron temperature initially decreased and the radiated power rose to a peak value of 700 kilowatts at $.012 \mathrm{msec}$. At this time the beam begins to dominate, the electron temperature increases and the radiated power drops. From $.2 \mathrm{msec}$ to $2.8 \mathrm{msec}$ the radiated pown remains near 10 kilowatts and the electron temperature has an average value across the plasma of .1 keV. This corresponds to the radiation profile position in $F i g .2-13 b$ betweer the large and small peak. 
Depending on parameter values and the amount of expansion of the plasma it is possible for the beam deposition opacity to change during a run. Figure 2-14 shows initially the bean is almost completely absoibed with $99.9 \%$ attenuation. The attenuation falls to $98.7 \%$ by the end of the run. Thus throughout the run almost the entire beam current is absorbed and the diminution of trapped beam power is accounted for by the plasma, beam energy difference factor in Eq. $(2-57)$. 


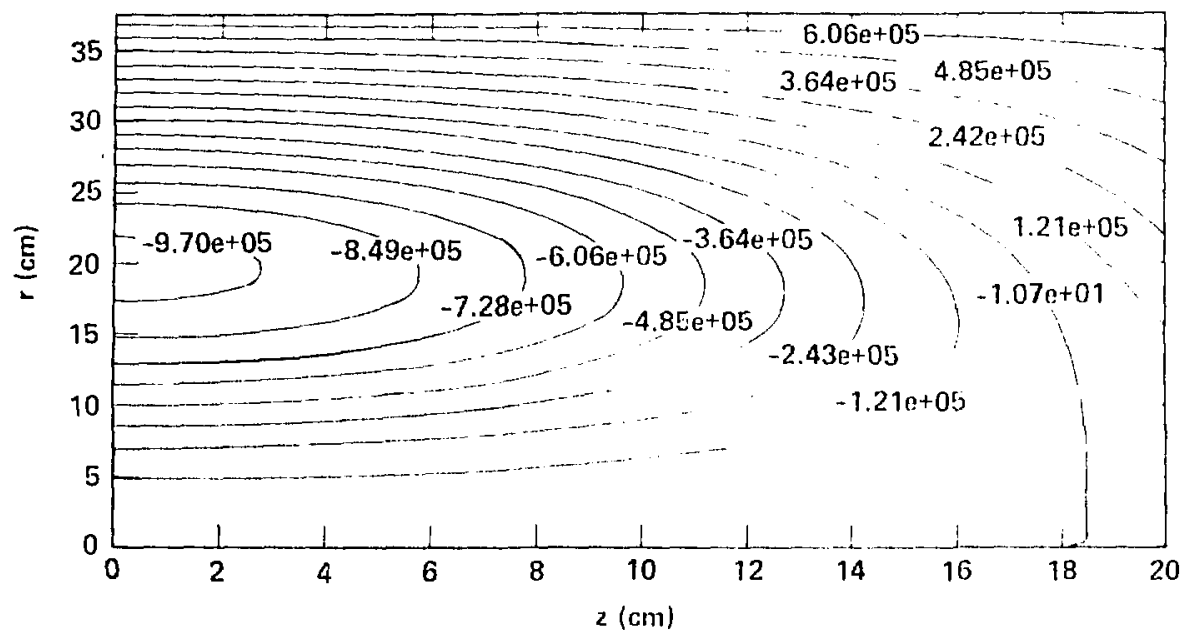

(a)

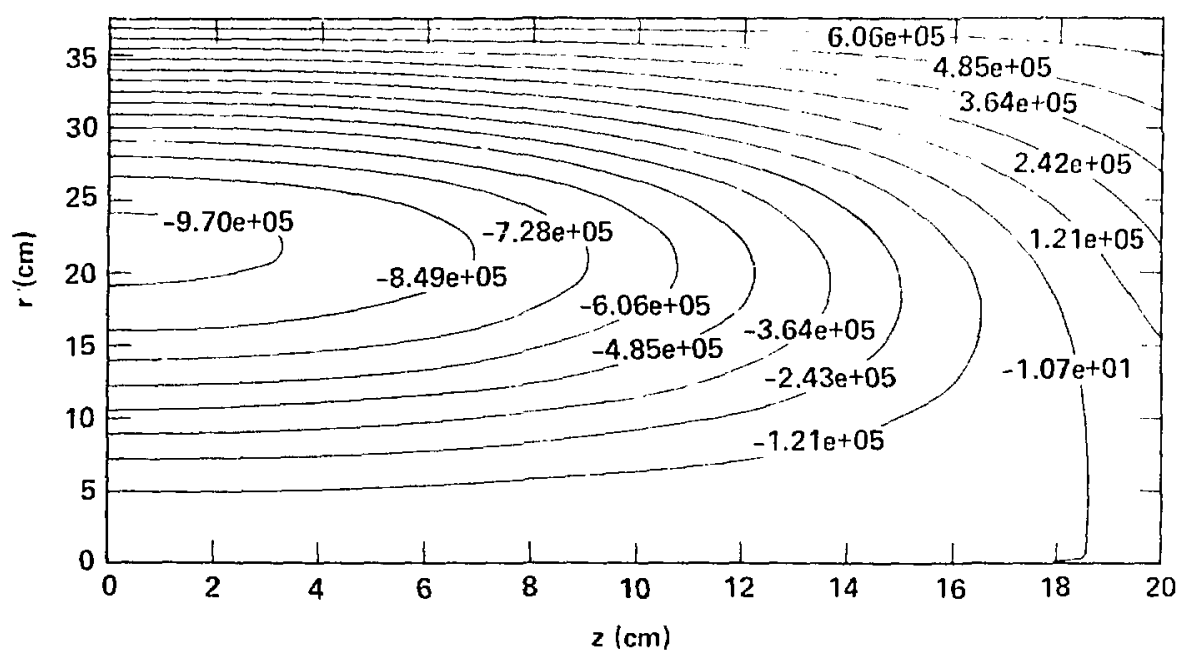

(b)

Figure 2-1. Neutral beam injerted plasma, $\psi(r, z) .(a) t=0$. (b) $\mathrm{t}=2.8 \mathrm{milliseconds.}$ 


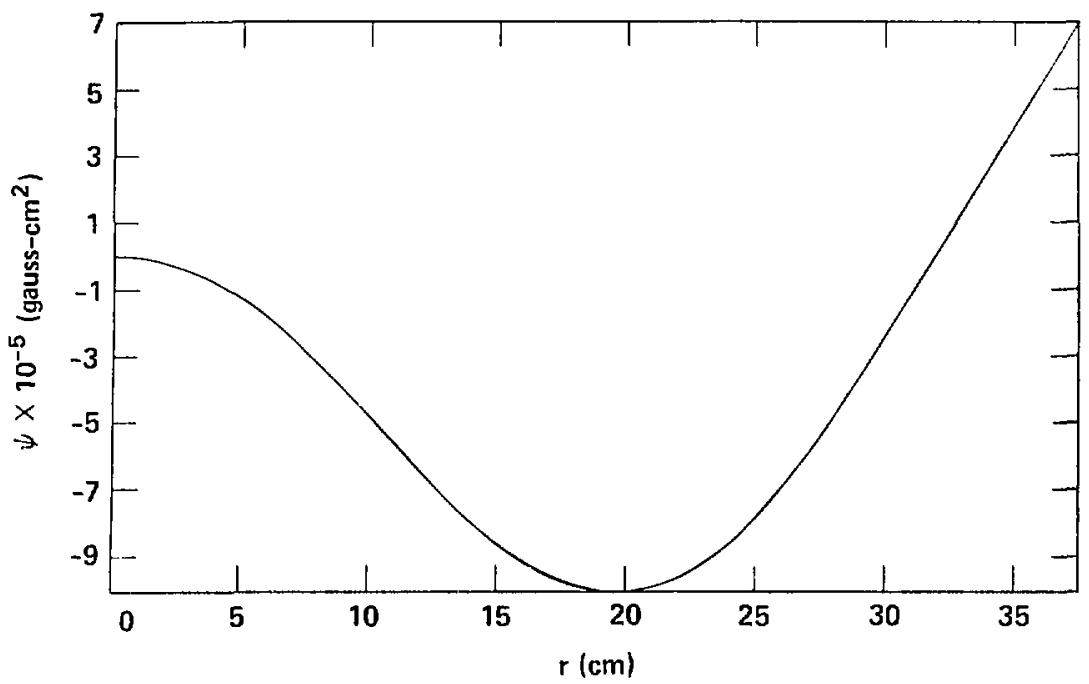

(a)

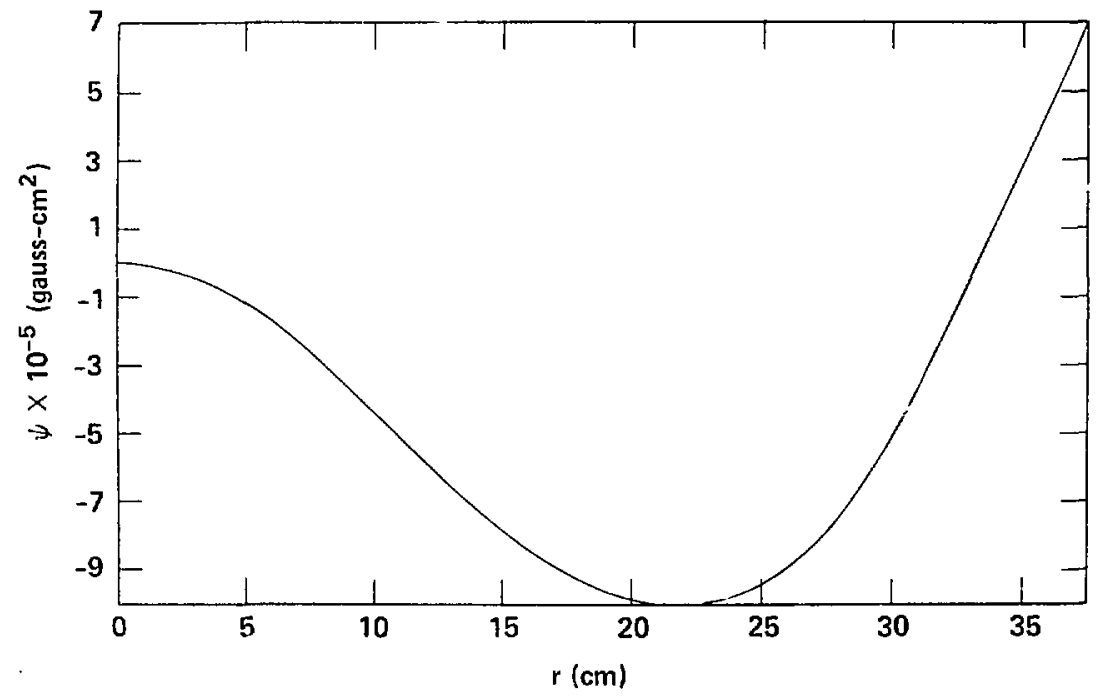

(b)

Figure 2-2. Neutral beam injected plasma, $\psi(r, 0) .(a) t=0$ (b) $t=2.8 \mathrm{milliseconds}$. 


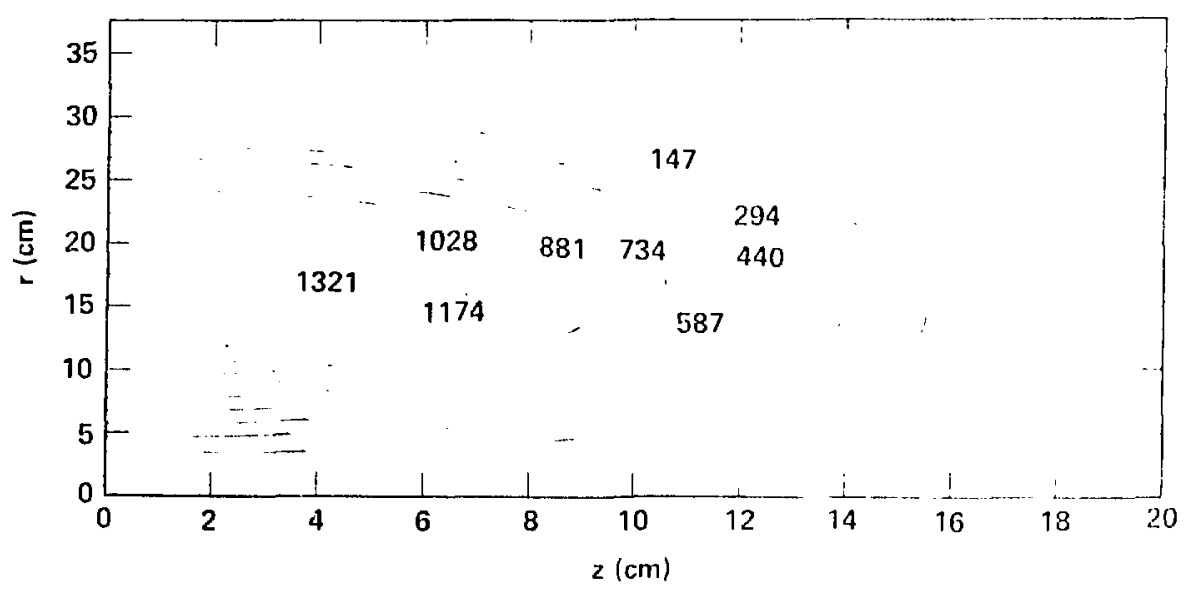

(a)

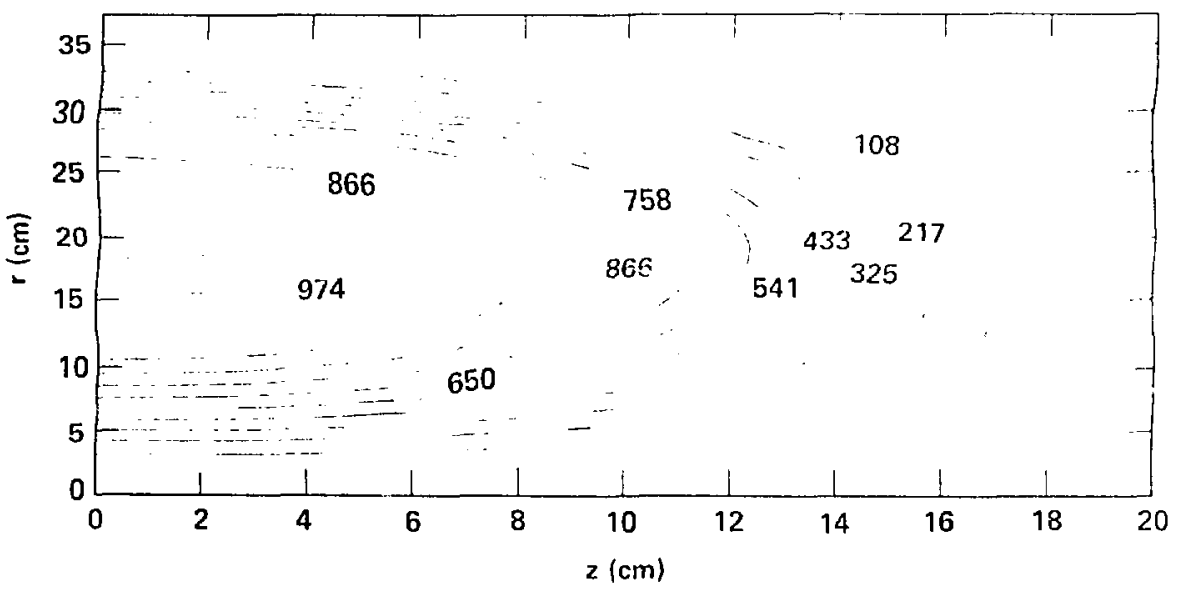

(b)

Figure 2-3. Neutral beam injected plasma, $4 \pi c^{-1} J_{T}(r, z) .(a) t=0$, (b) $t=2.8 \mathrm{milli}$ seconds. 


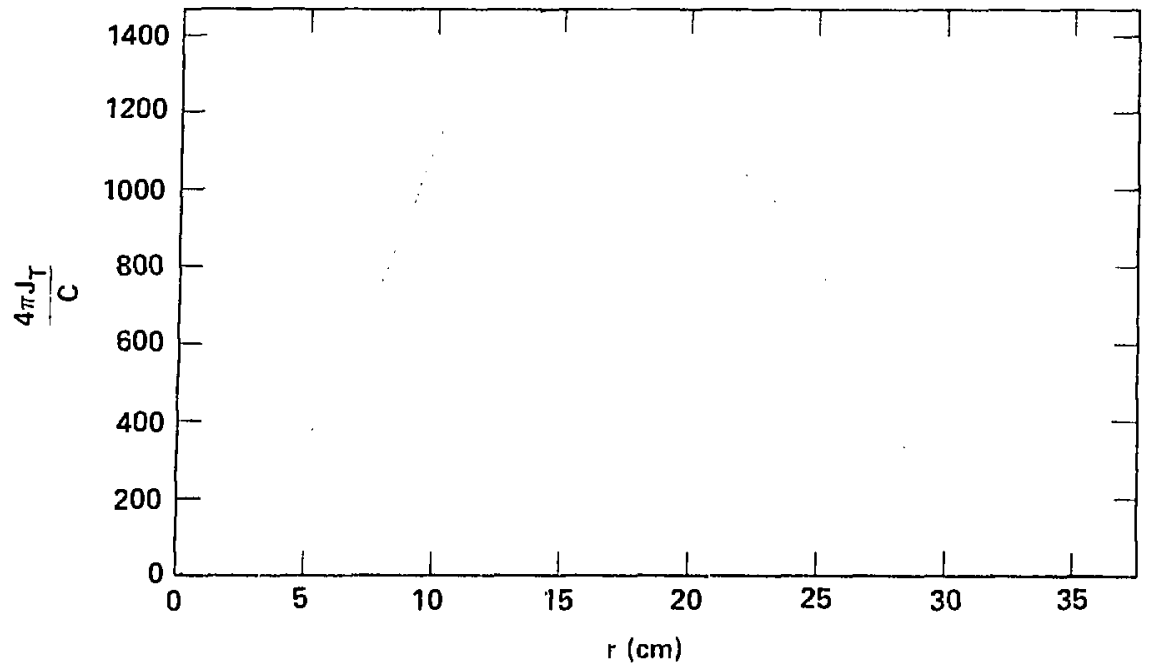

(a)

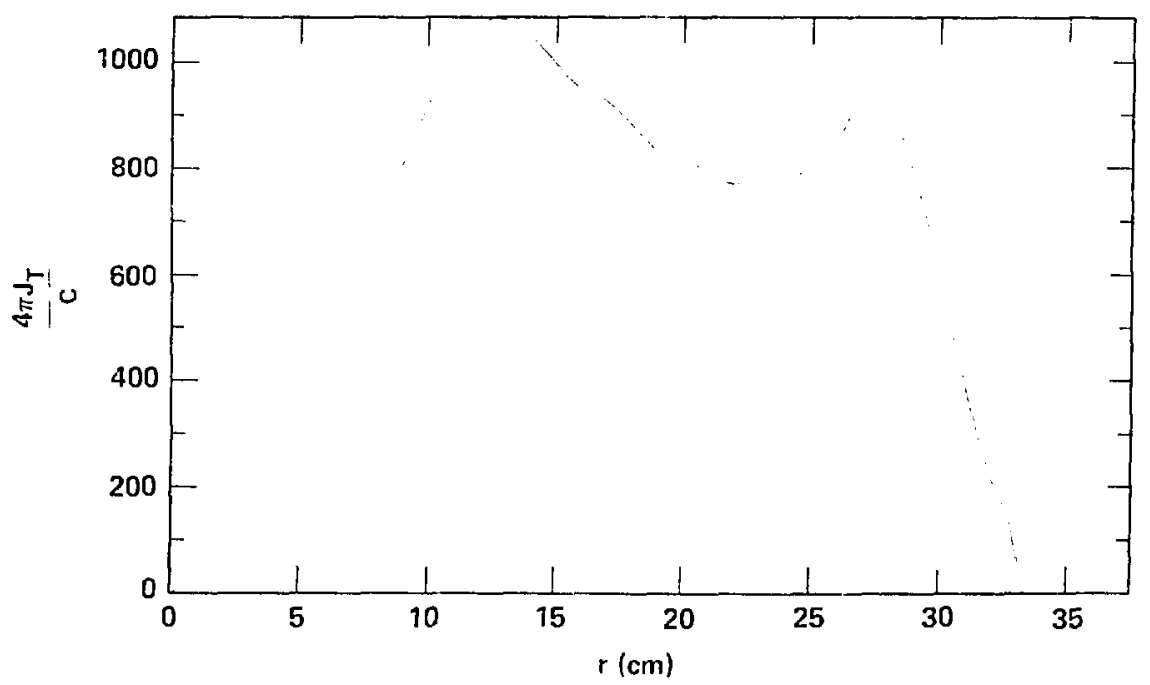

(b)

Figure 2-4. Neutral beam injected $p$ lasma, $4 \pi c^{-1} J_{T}(r, 0) .(a) t=0$, (b) $t=2.8$ milliseconds. 


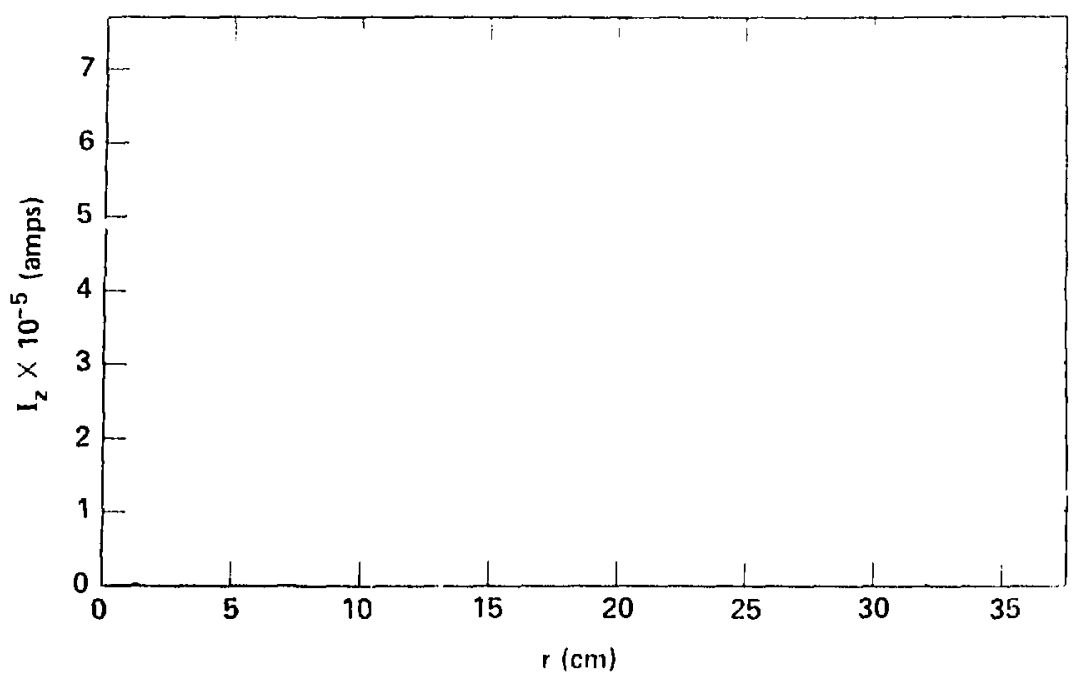

(a)

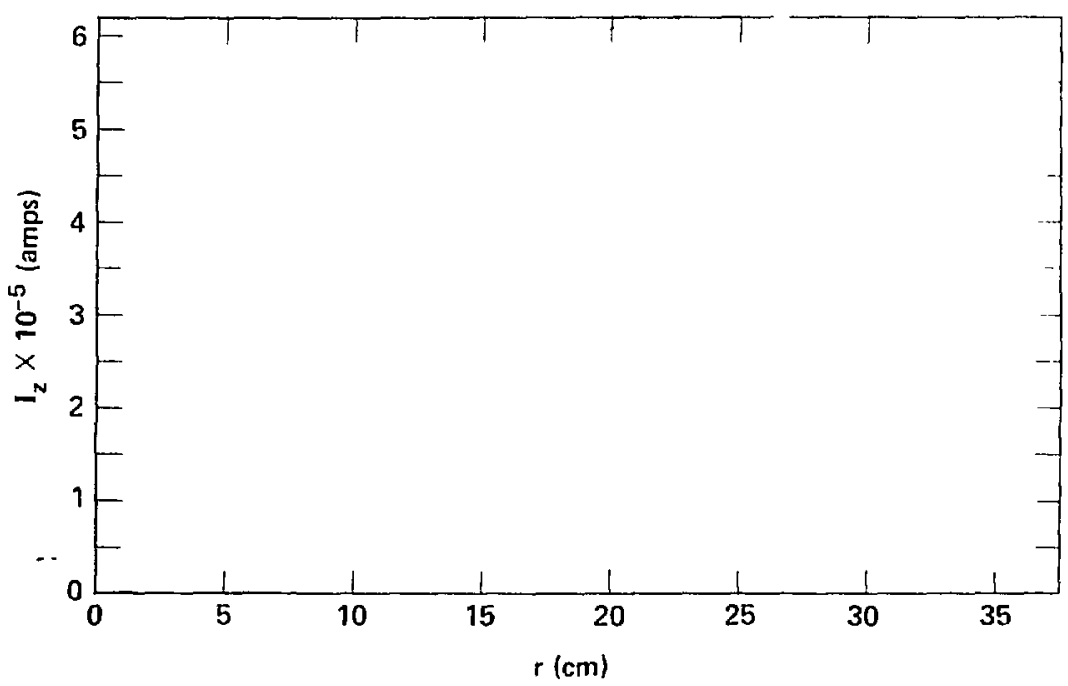

(b)

Figure 2-5. Neu:ral beam injected plasma $I_{z}(r) .(a) t=0$, (b) $t=2.8 \mathrm{milliseconds.}$ 


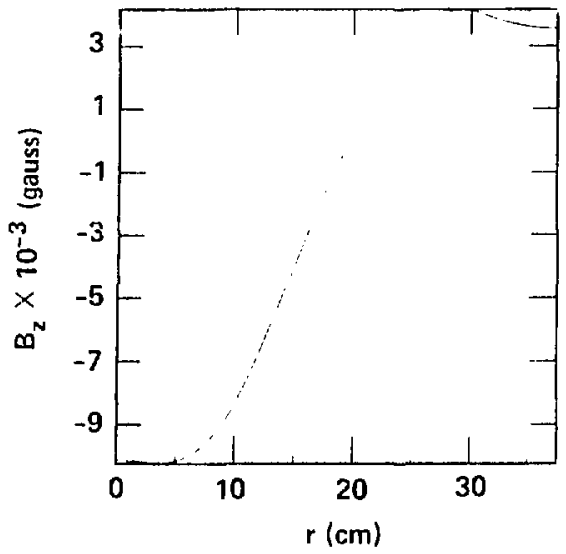

(a)

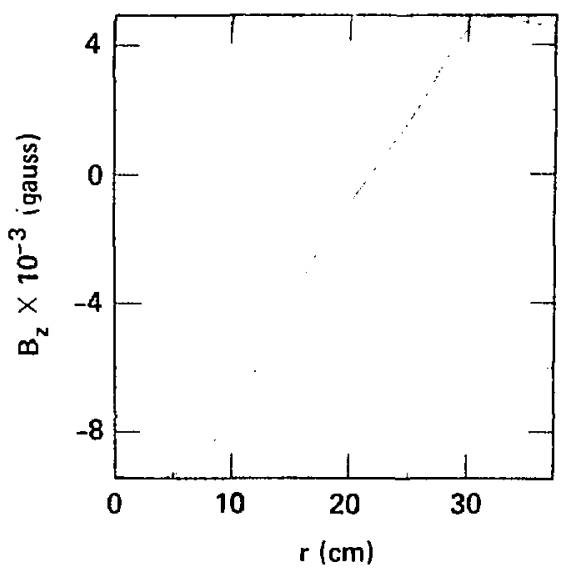

(c)

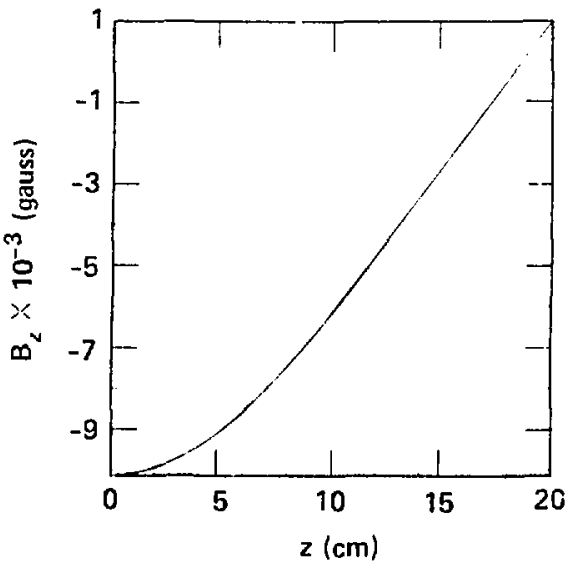

(b)

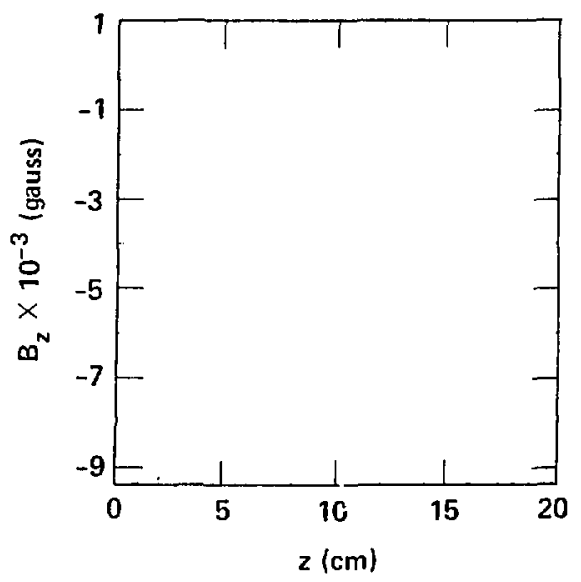

(d)

Figure 2-6. Neutral beam injected plasma. (a) $B_{z}(r, 0, t=0)$, (b) $B_{z}(0, z, t=0)$, (c) $B_{z}(r, 0, t=2.8 \mathrm{msec})$,

(d) $B_{Z}(0, z, t=2.8 \mathrm{msec})$. 


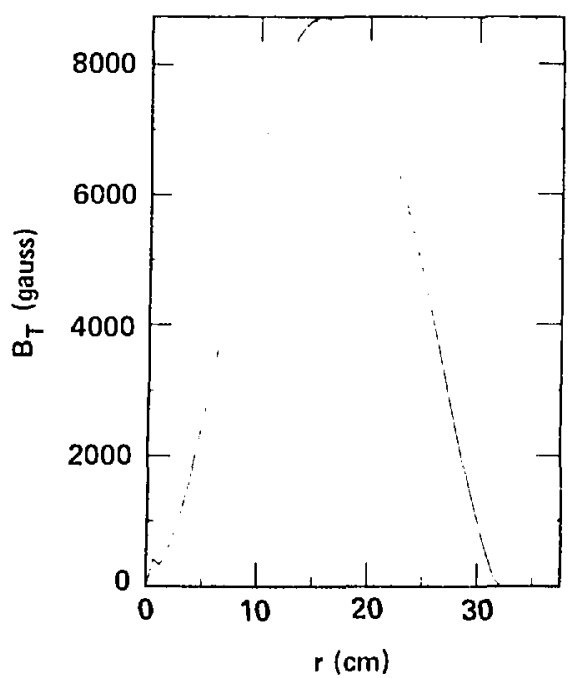

(a)

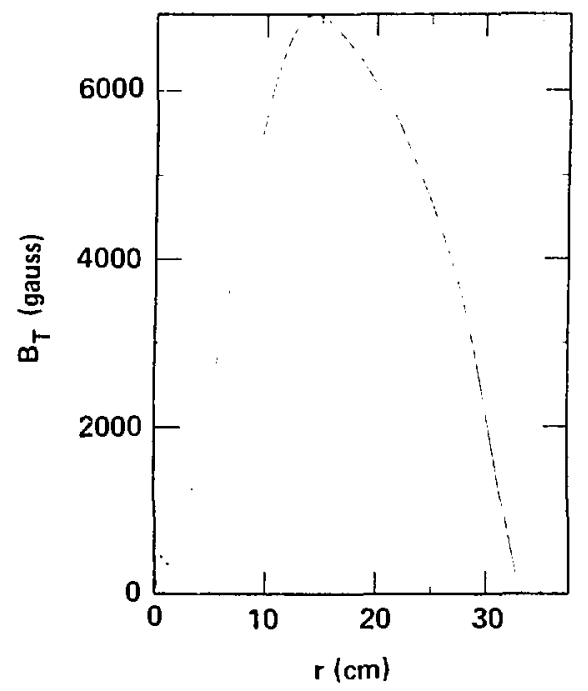

(c)

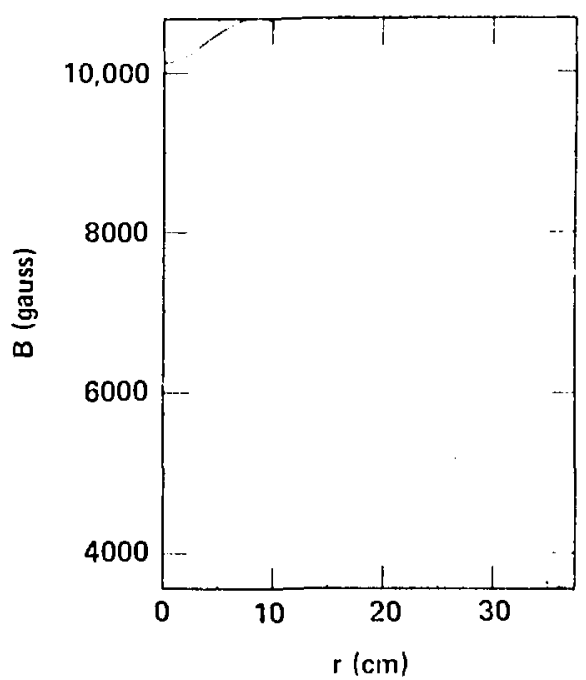

(b)

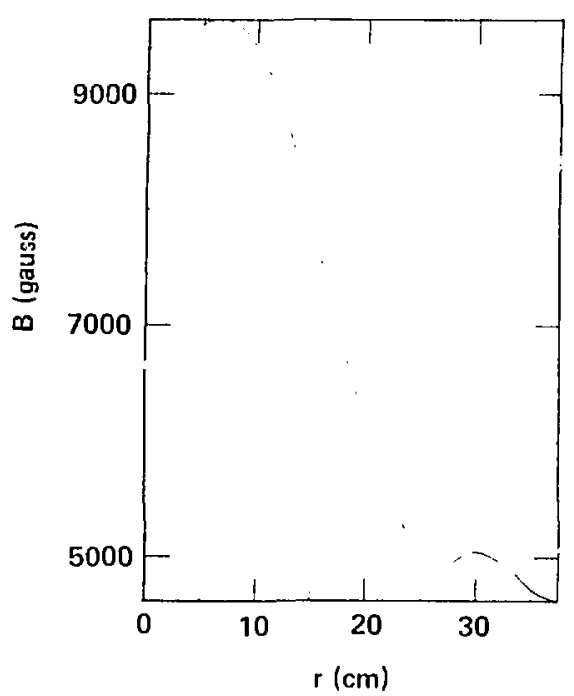

(d)

Figure 2-7. Neutral beam injected plasma. (a) $B_{T}(r, 0, t=0)$,

(b) $B(r, 0, t=0),(c) B_{T}(r, 0, t=2.8 \mathrm{msec})$,

(d) $B(r, 0, t=2.8 \mathrm{msec})$. 


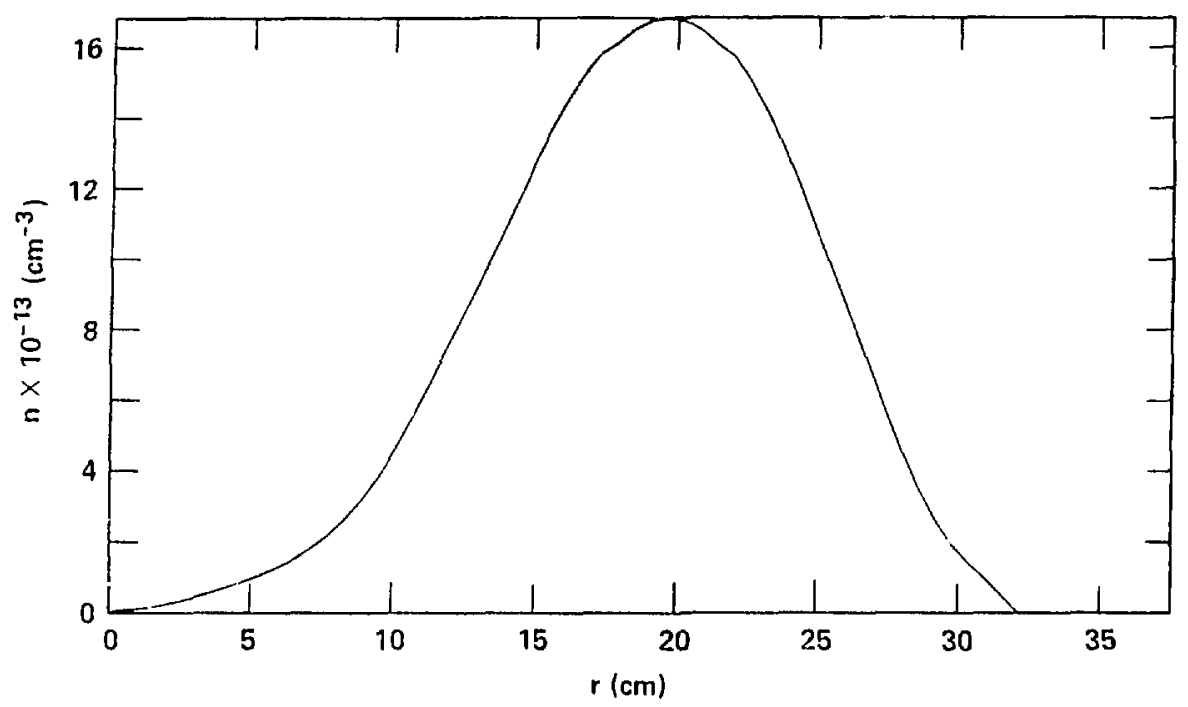

(a)

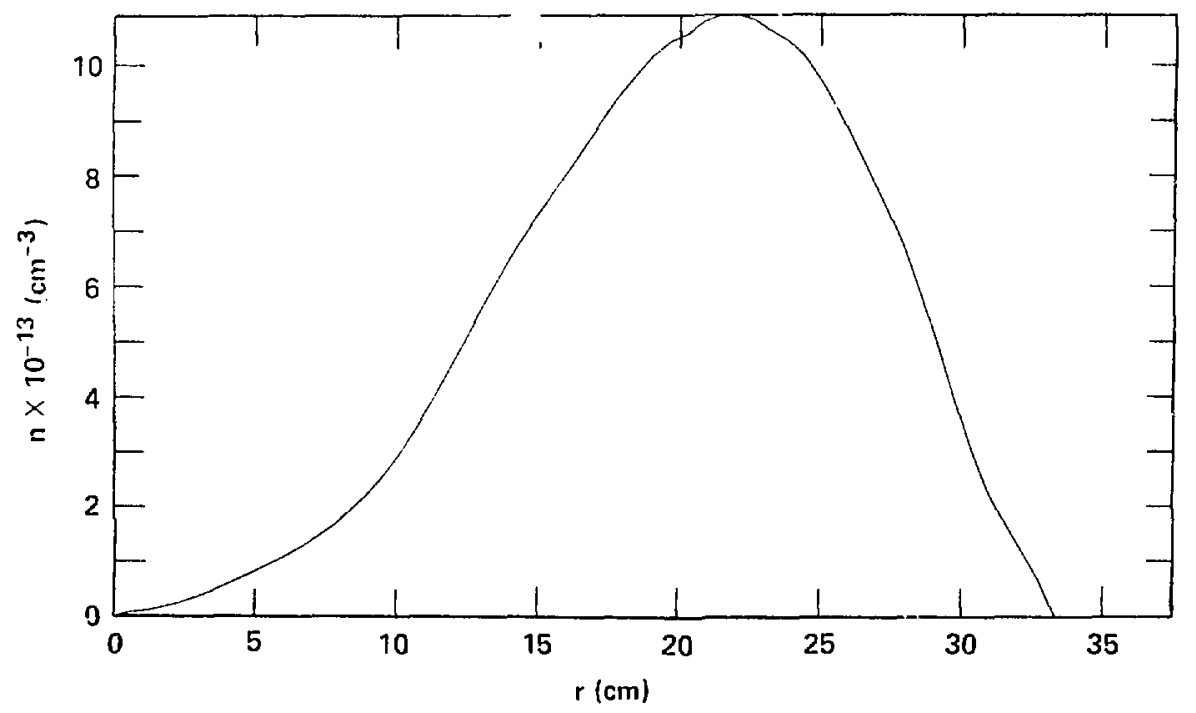

(b)

Figure 2-8. Neutral Beam injected plasma, $n(r, 0)$. (a) $t=0$ (b) $t=2.8$ milliseconds 


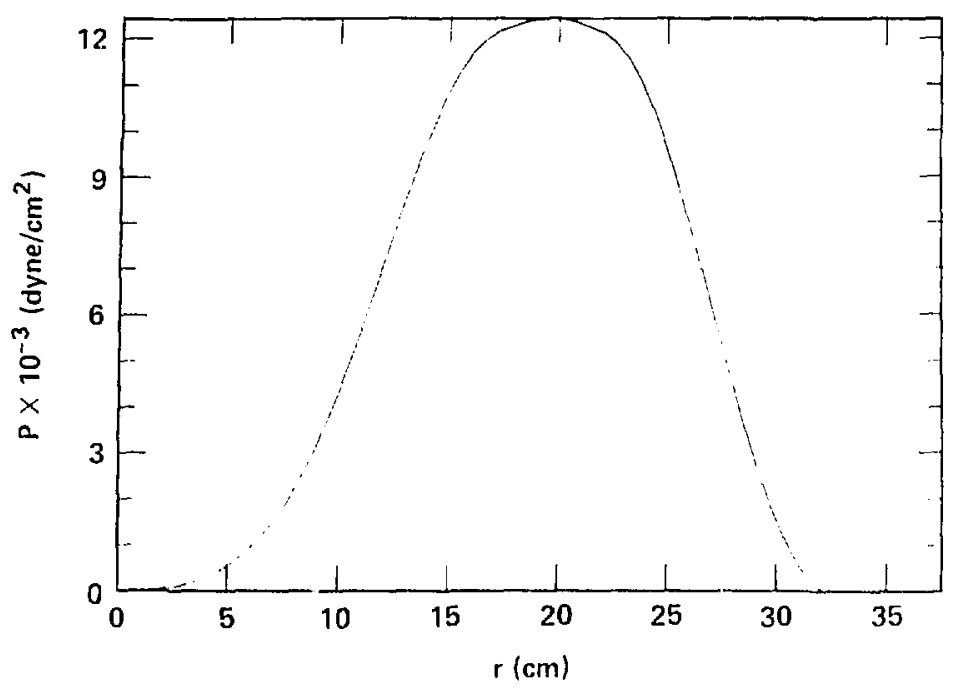

(a)

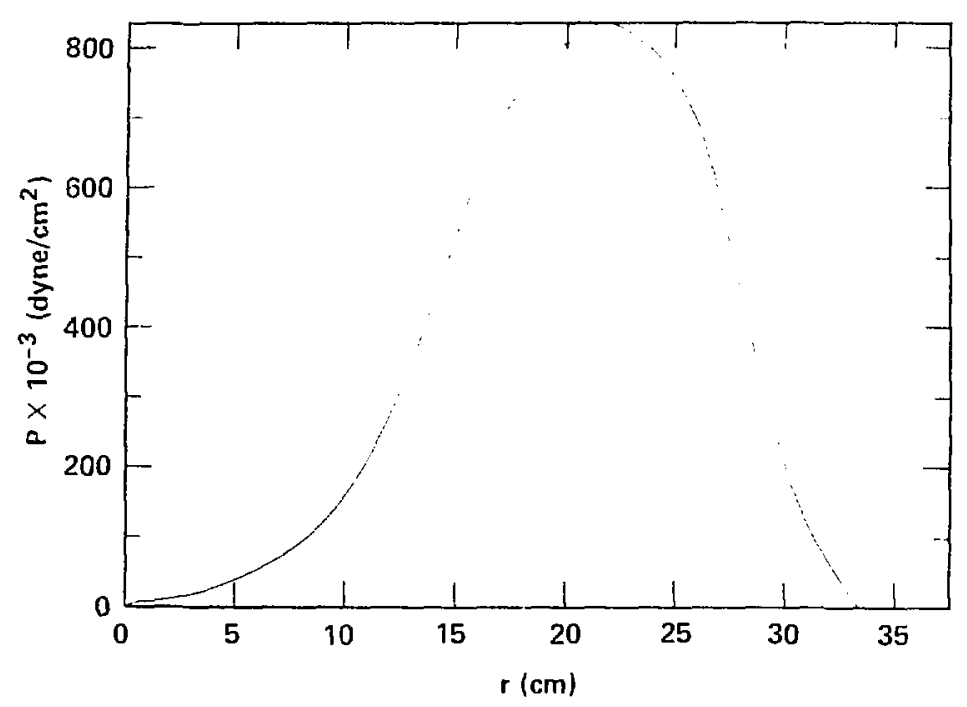

(b)

Figure 2-9. Neutral beam injected plasma, $p(r, 0)$. (a) $t=0$ (b) $t=2.8$ mi iliseconds. 


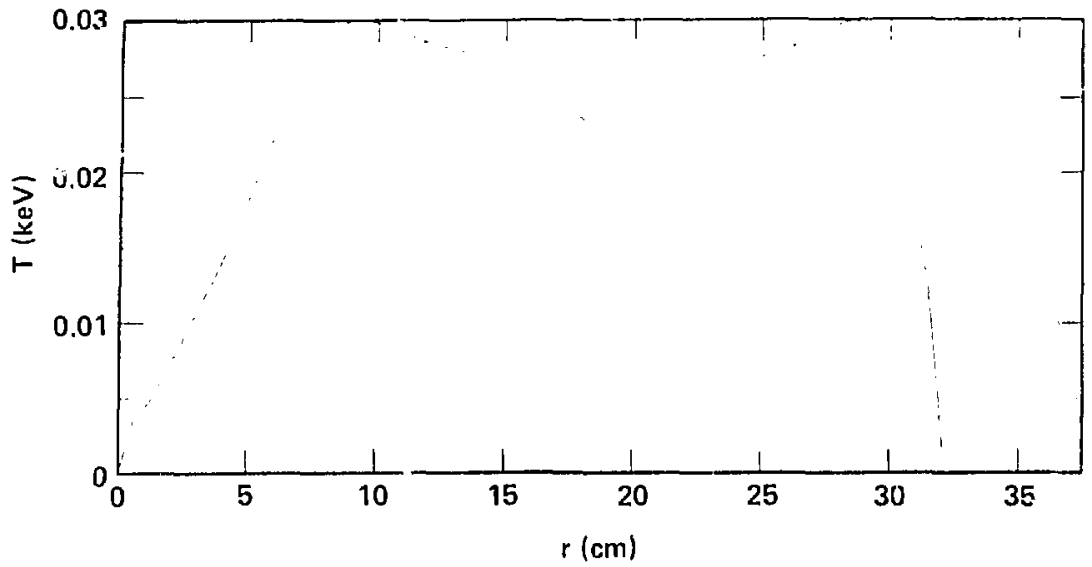

(a)

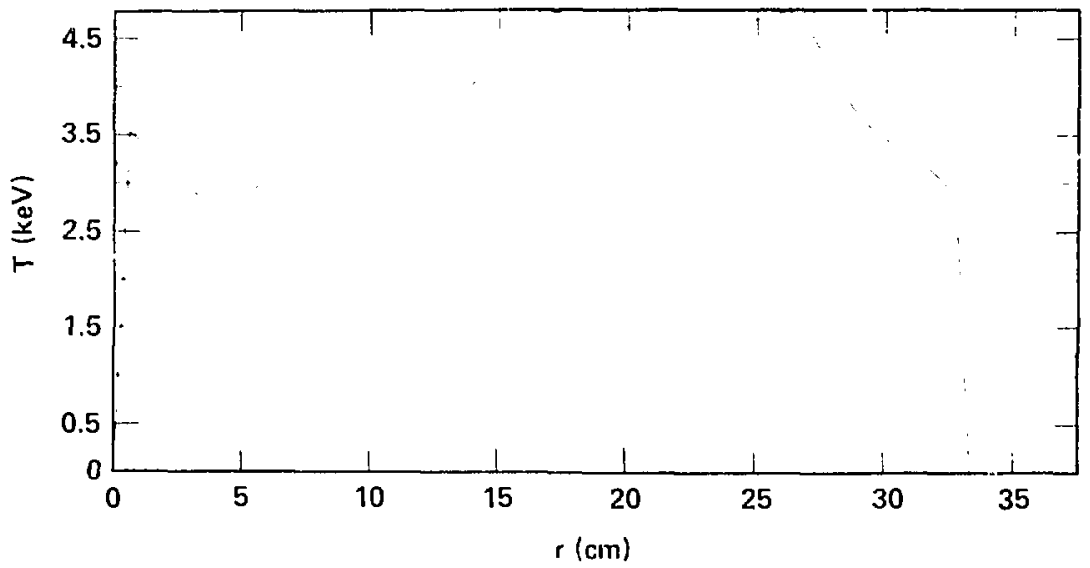

(b)

Figure 2-10. Neutral beam injected plasma, $T(r, 0) .(a) t=0$, (b) $t=2.8 \mathrm{milliseconds}$. 

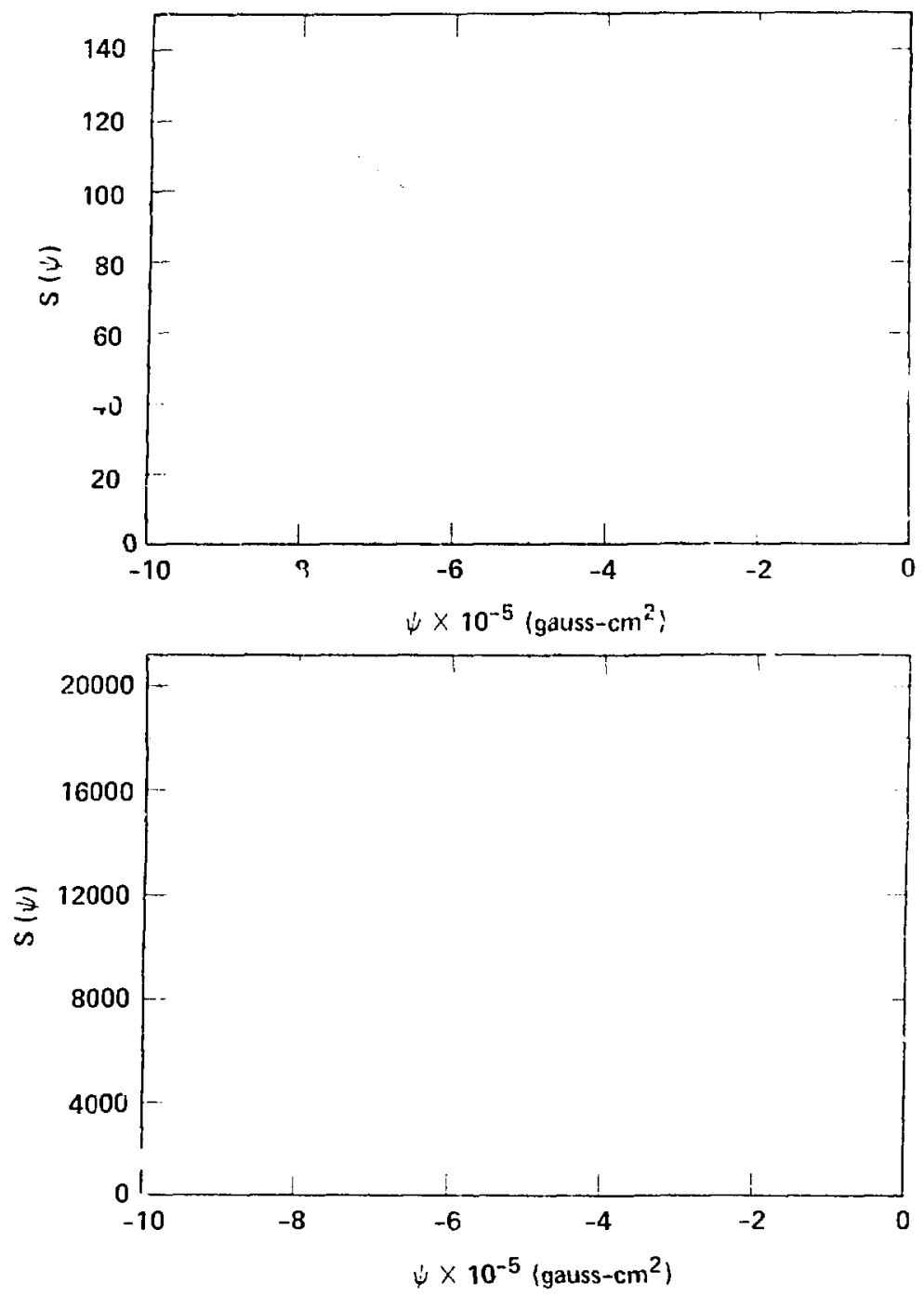

Figure $2-11$. Neutral beam injected plasma entropy profile, $S(\psi)$. (a) $t=0$, (b) $t=2.8$ milliseconds. 


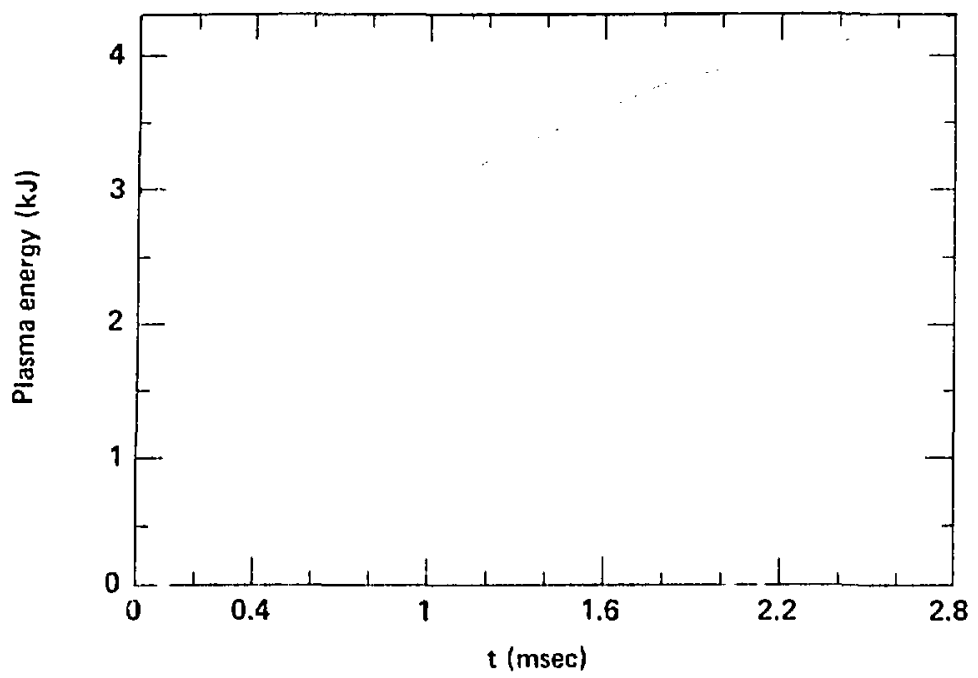

(a)

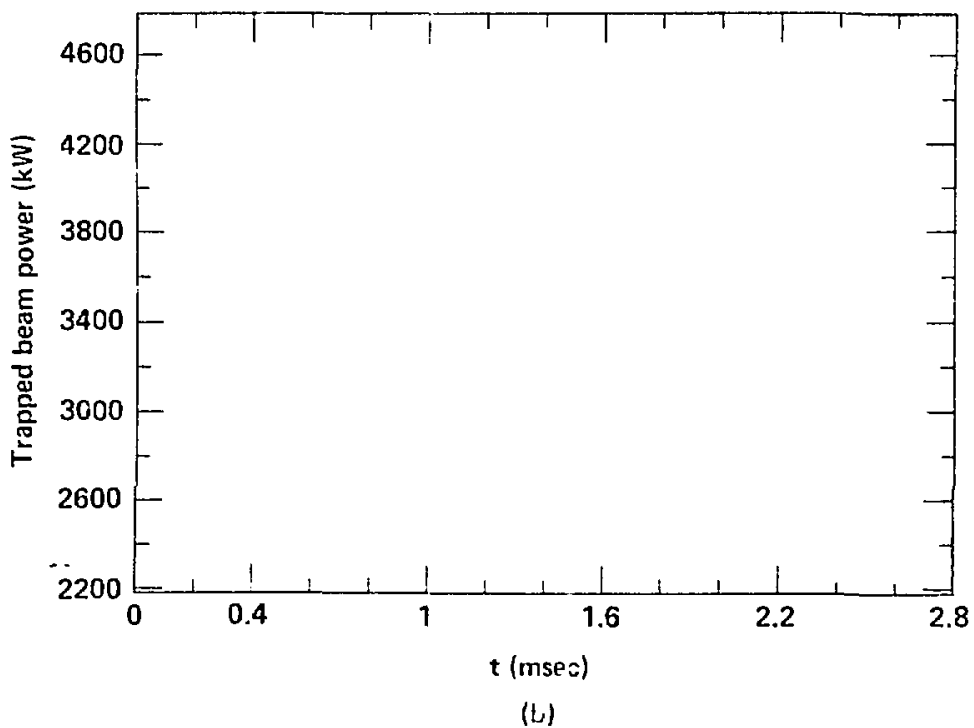

Figure 2-12. Neutral beam injected plasma. (a) Plasma energy vs time, (b) trapped beam power vs. time. 


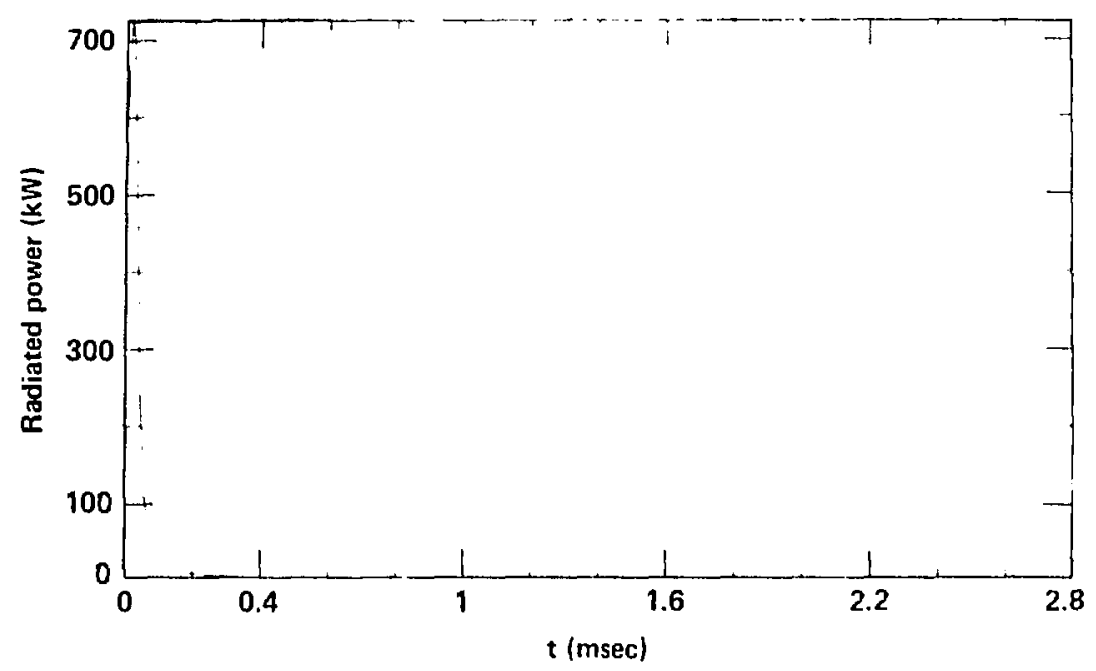

(a)

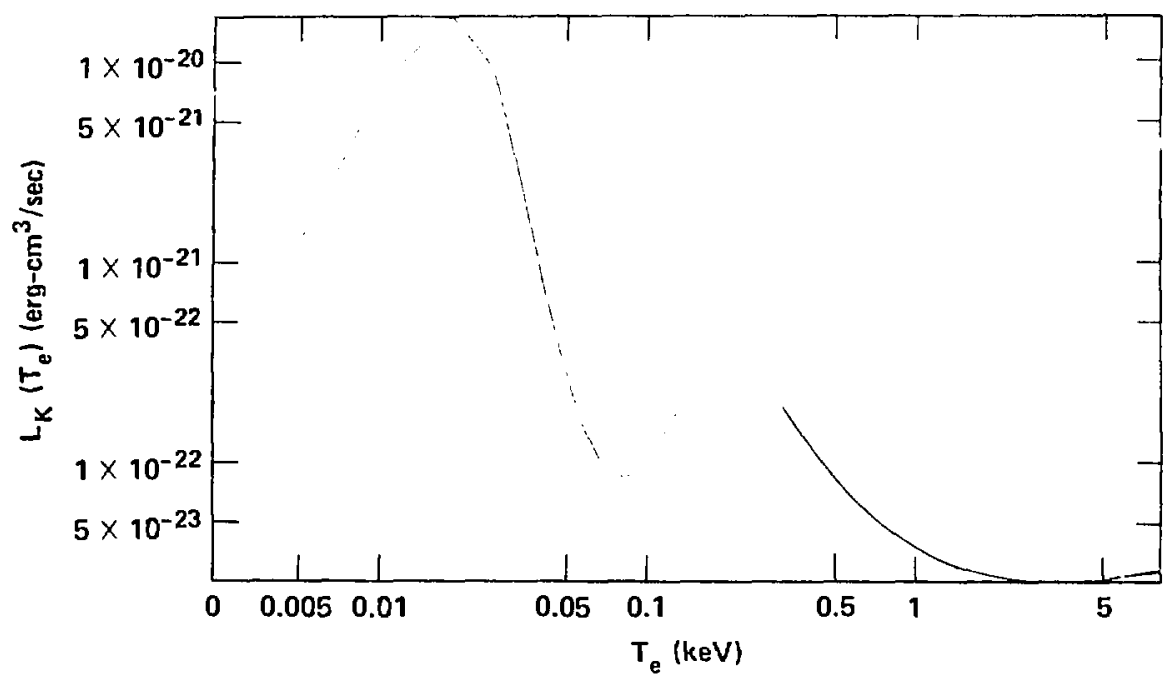

(b)

Figure 2-13. Neutral bean injected plasma. (a) Radiated power vs. time, (b) Radiation profile ys. Te $(\mathrm{keV})$. 


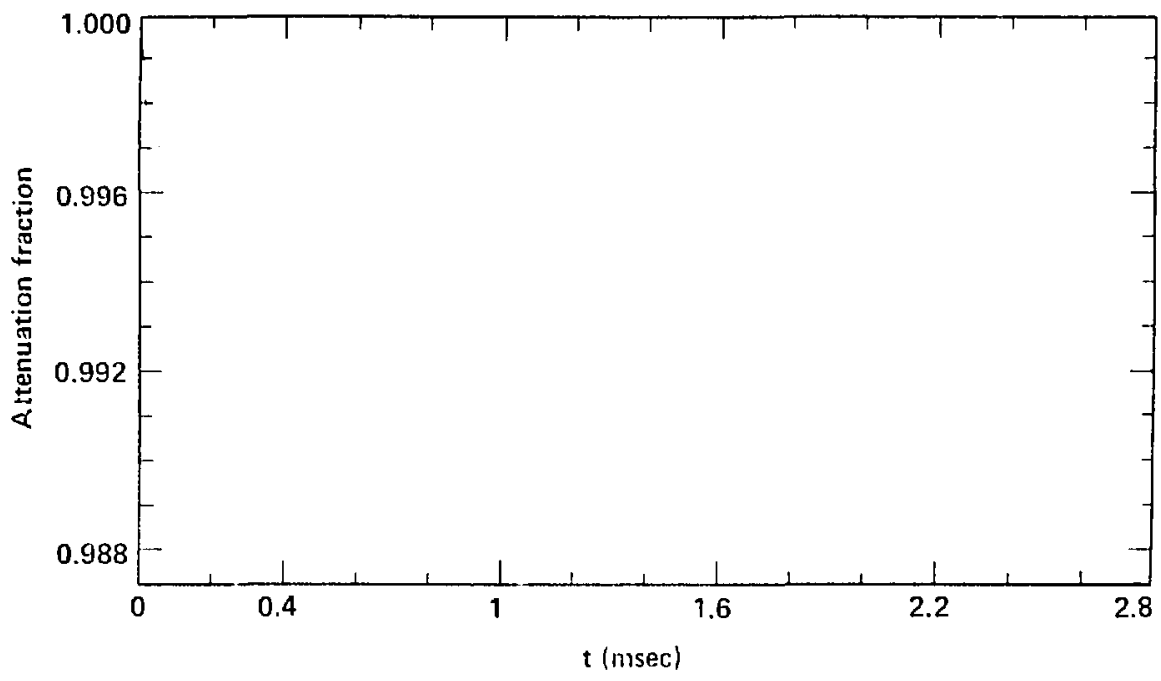

Figure 2-14. Neutral beam injected plasina. Fraction of the neutral beam current attenuated by the plasma versus time. 


\subsubsection{Compressed Prolate Hill's Vortex}

The shape of a plasma is one factor which influences stability. The proximity of conducting walls also plays a role. It was found by Hammer ${ }^{[142]}$ a prolate plasma with an equitorial bulge, referred to as the problimak configuration, is stable to the tilting instability with walls more distant than an oblate shape. This particular geometry is also stable to axial and transvei se displacements. Because these properties are desirable a series of $A D B$ runs were done to attempt to create a problimak by compressing a spherical Hill's vortex. The failure of this effort was one motivation for deriving the external vacuum field which matches an elliptic Hill's vortex plasma. The external vacuum solution for the elliptic Hill's vortex indicates a cusp field is necessary for the elliptic shape. Beginning with an elliptic Hill's vortex a problimak plasma has been successfully generated by the magnetic field caused by six axisymmetric current loops.

The computational grid for this case has an axial length of 20 $\mathrm{cm}$ and a radial extent of $10 \mathrm{~cm}$. The three parameters of the elliptic model given by $E q .(2-148)$ are $B_{V}=6000, E=.25$, and $p_{1}=8 \mathrm{~cm}$. The plasma is initially bounded at $r=8 \mathrm{~cm}, z=0$ and $r=0, z=16 \mathrm{~cm}$. The magnetic axis flux, $\psi_{0}$ is held constant at $-7.2 \times 10^{4}$ gauss- $\mathrm{cm}^{2}$. The toroidal magnetic field is zero so $q(\psi)=0$. The $S(\psi)$ profile is determined from the $d \psi / d \psi$ obtained from the initial analytic $\psi(r, z)$ and rearranging $E q \cdot(2-29)$.

$$
S(\psi)=-(12+3 E) B_{V}\left(8 \pi \rho_{1}^{2}\right)^{-1} \psi\left(\frac{d \psi}{\partial V}\right)^{-5 / 3}
$$


The six external current loops all have a radius of $11.28 \mathrm{~cm}$. The axial positions, initial $I_{j}$, and final $I_{f}$ currents are in Table $2-1$.

\begin{tabular}{|c|c|c|}
\hline$z(\mathrm{~cm})$ & $I_{j}(\mathrm{amps})$ & $I_{f}(\mathrm{amps})$ \\
\hline 2 & -100 & $-3.1 \times 10^{4}$ \\
\hline 6 & 100 & $2.3 \times 10^{4}$ \\
\hline 8 & 100 & $5.8 \times 10^{4}$ \\
\hline 11 & -100 & $-3.5 \times 10^{4}$ \\
\hline 16 & -100 & $-4.7 \times 10^{4}$ \\
\hline 22 & 100 & $6.0 \times 10^{4}$ \\
\hline
\end{tabular}

Table 2-1 Compressed Prolate Hill's Vortex $I_{j}, I_{f}$ Currents and Positions

Positive currents increase the initial magnetic field and negative currents diminish the initia? magnetic field. Due to symmetry about $z=0$ there are also six external coils at reflected $z$ positions with the same initial and final currents. The equilibrium is evolved from the initial state to the final state in 20 steps.

The plasma has open boundary conditions so a plasma flu: value along the grid edge is computed by the Green's function method presented in Section 2.2.3 using Eq. (2-34). The same formulation is also used to compute the flux caused by the external coils. Comparing the contour plot of $\psi(r, z)$ at $I_{j}$ in Fig. 2-15a with $\psi(r, z)$ at $I_{f}$ in Fig. 2-15b shows a pronounced change in the flux shape. 
The initial elliptic shape has evolved into a problimak with a flux surface bulge at $z=0$. The plasma boundary has borome $r=9.2$, $z=0$ and $r=0, z=15.5$. Outward expansion has occurred at $z=0$ and the plasma has contracted axially along the $z$ axis. The new position of the magnetic axis can be seen in Fig. 2-16. The flux $\psi(r, 0)$ at $I_{i}$ is shown in Fig. 2-16a and at $I_{f}$ in Fig. ?-160. The magnetic axis has shifted from $5.7 \mathrm{~cm}$ to $6.5 \mathrm{~cm}$.

The toroidal current $J_{T}(r, z)$ is shown at $I_{i}$ in Fig. ?-17a and at $I_{f}$ in Fig. 2-17b. The initial current is almost proportional to $r$ since the initial external coil currents are small. The final state shown in Fig. 2-17b is approximately proportional to $r$ between $r=0$ and $r=30 \mathrm{~cm}$. For $r>3 \mathrm{~cm}$ a considerable deviation from linear $r$ behavior is apparent. A peak in current develops along the outer radial edge of the plasma, however the total toroidal current is slightly diminished. Initially the area integral of $J_{T}$ is 32,400 amps and in the final state it is 31,500 amps. The radial scan of $\mathrm{J}_{T}(r, 0)$ at $\mathrm{I}_{i}$ in Fig. 2-18a and at $\mathrm{I}_{f}$ in Fig. 2-13b shows the near constancy of total current is a result of a diminution of current between $r=4$ and $r=6$ and an increase for $r>6$. A relatively flat region of $J_{T}$ is evident in Fig. 2-18b and the nitial peak value of 4800 increases to 7000 .

The $z$ magnetic field is shown in Fig. 2-19 with $B_{z}(r, b)$ in Fig. 2-19a at $I_{i}, B_{z}(0, z)$ in Fig. 2-19b at $I_{i}, B_{z}(r, 0)$ in Fig. 2-19C at $I_{f}$ and $B_{z}(0, z)$ in Fig. 2-19d at $I_{f}$. Comparing Fig. 2-19a with Fig. 2-19c, the initial $B_{z}(r, 0$, field maximum of 9000 at $8.2 \mathrm{~cm}$ decreases to 8000 and shifts radially outward to $9.2 \mathrm{~cm}$. Comparing 
Fig. 2-19b with Fig. 2-19d the initial $B_{z}(0, z)$ field maximum of 1000 at $18 \mathrm{~cm}$ increases to 2000 and shifts axially to $20 \mathrm{~cm}$. The value of $B_{z}(0,0)$ remains almost constant at the initial value of 9000 .

The magnitude of the magnetic field $B(r, z)$ is shown in $F i g$. 2-20a at $I_{j}$ and in Fig. 2-20b at $I_{f}$. Initially at $I_{j}$ the following formlila applies inside the plasma.

$$
B(r, z)=.75 B_{V} \rho_{1}^{-2}\left(\left(2 \rho_{1}^{2}-4 r^{2}-2 E z^{2}\right)^{2}+(2 E z r)^{2}\right)^{1 / 2}
$$

At $r=\rho_{1} z^{-1 / 2}, z=0$ and at $r=0, z=\rho_{7^{-1 / 2}}, B(r, z)$ is zero initially. The elliptic envelope boundary between the interior and exterior solution is clearly visible in Fig. 2-20a. In Fig. 2-20b the effect of the full current $I_{f}$ in the external coils is to eliminate the axial field null on the plasma boundary. Also the contours are seen to turn up around $r=10$, and $z$ between 6 and 8 where two coils have large positive currents. An island contour forms at $r=9, z=16$ as a result of the opposite directed current coils at $z=11,16$ and $z=22$. The movement of the field null at $r=\rho_{1} 2^{-1 / 2}, z=0$ can be easily discerned in the $B(r, 0)$ plot at $I_{f}$ in Fig. 2-2lb. Comparing with Fig. 2-2la at $I_{i}$ the field null moves from $r=5.7$ to $r=6.3$. This is the same as the position of the magnetic axis. The initial peak in $B$ at $r=8, z=0$ of magnitude 8900, moves radially outward to $r=9.1$ and decreases to a value of 8000 .

The $\psi$ derivative of pressure $d p / d \psi$ as a function of $\psi$ is plotted in Fig. 2-22a at $I_{j}$ and in Fig. 2-22b at $I_{f}$. The initial 
$d p / d \psi$ is almost a constant at -47.6 and the effect of the small initial c ant in the external coils is to slightly change the magnetic axis value of $d p / d \psi$ to -50 . In the final state shown in Fig. $2-22 b, d p / d \psi$ is reduced by approximately a tactor of two near the magnetic axis and increased in absolute magnitude $50 \%$ at. $\psi=-2.5 \times 10^{4}$. This means the plasma current which is proportional to $d p / d \psi$ is $d i m i n i s h e d$ near the magnetic axis compared to the initial value.

The pressire $p(r)$ is shown in Fig. 2-23a at $I_{j}$ and in Fig. $2-23 b$ at $I_{f}$. The single peak structure in Fig. 2-23a persists ill Fig. 2-23b, huwever the peak in Fig. 2-23b is broader. It is also flatter around the magnetic axis position in correspondence with the diminished $d p / d \psi$ value evidenced in $F i g .2-22 b$. The initial $p(r)$ peak value of $3.4 \times 10^{6}$ increases to $3.7 \times 10^{6}$.

The plasma kinetic energy is plotted as a function of time in Fig. 2-24a. Time is an arbitrary unit which satisfies the adiabatic assumptions. The initial 1.01 kilojoule energy increases to 1.09 kilojoule after the problimak shape is generated. This is approximately an $8 \%$ increase. The magnetic energy in the volume bounded by the computational grid is shown in Fig. 2-24b as a function of time. The initial 14.05 kilojou?e magnetic energy increases to $18.5 \mathrm{kilojoule}$ in the final state. This is a $32 \%$ increase. Most of the magnetic energy change occurs outside of the plasma region. The poloidal magnetic field energy inside the plasma region is plotted as a function of time in Fig. 2-25a. The initial value of $2.55 \mathrm{kilojoules} \mathrm{decreases} \mathrm{to} 3.05$ kilojoules. The poloidal 
magnetic field energy outside the plasma region is shown in Fig. $2-25 b$ as a function of time. The initial value of 10.5 kilojoules increases to 15.5 kilojoules. The majority of the magnetic field energy is exterior to the plasma region. In the plasma region the kinetic energy in the final state is approximately one third the magnetic field energy. 


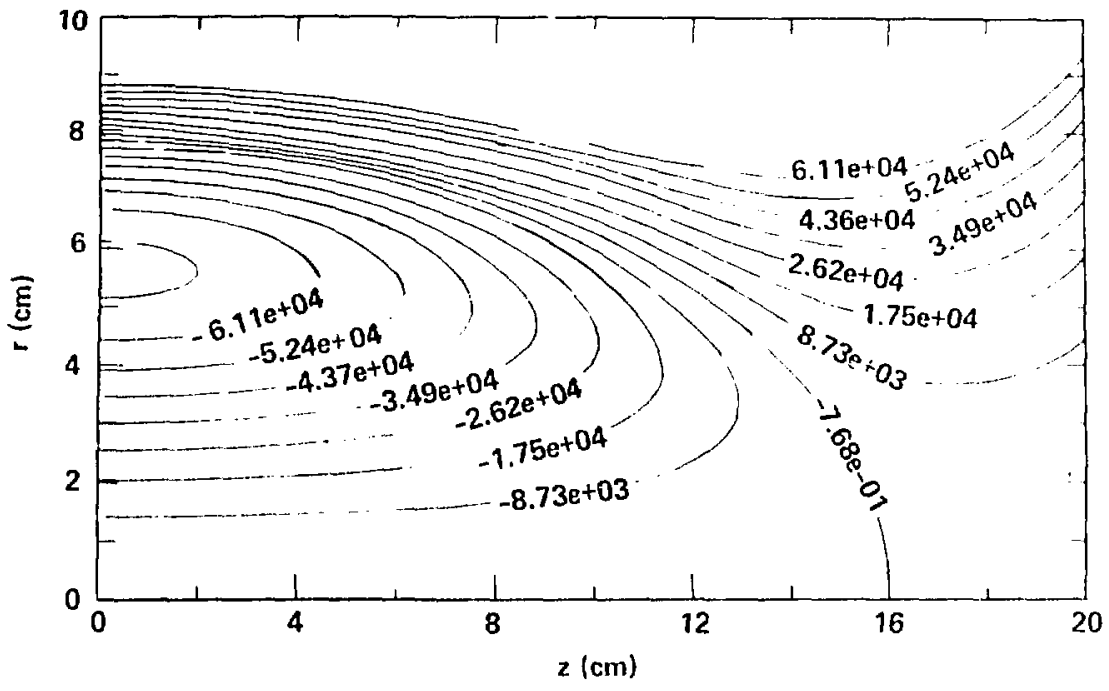

(a)

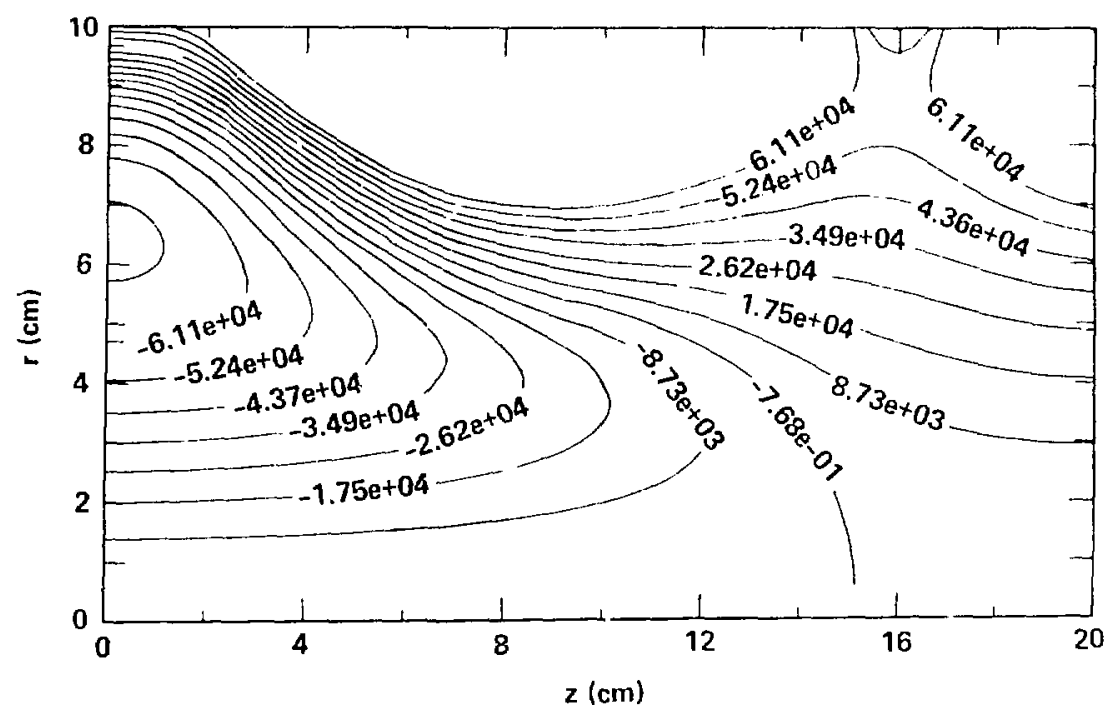

(b)

Figure 2-15. Compressed prolate $H i l l$ 's vortex, $\psi(r, z) .($ a) Initial external current, (b) Final external current. 


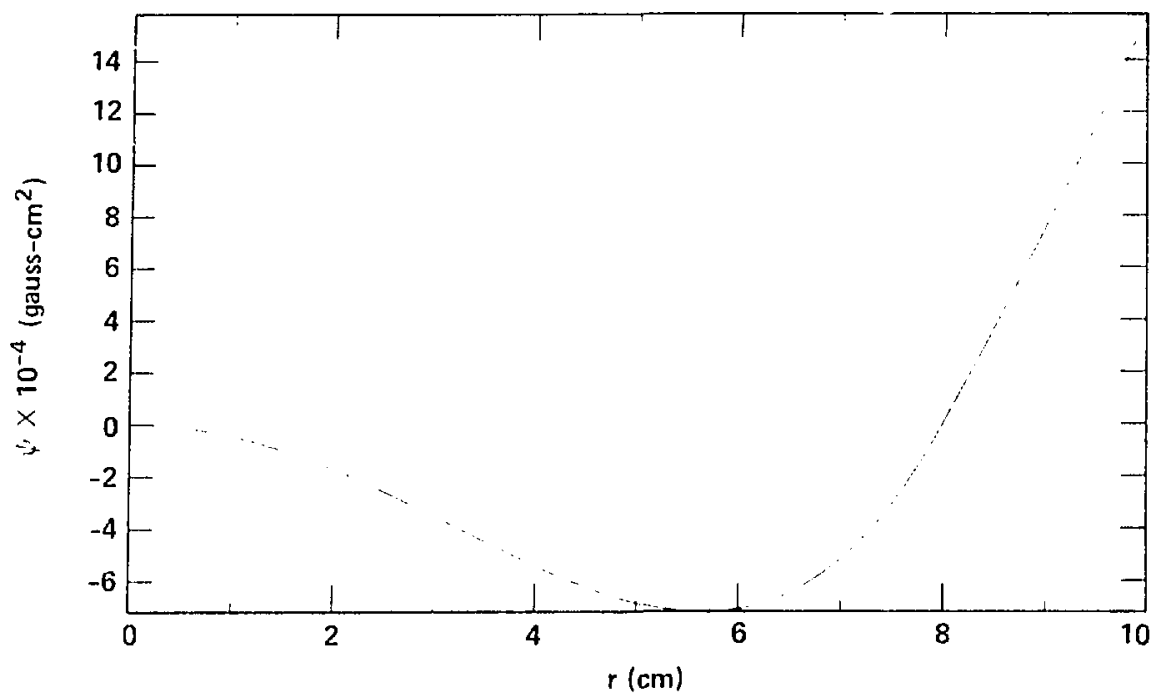

(a)

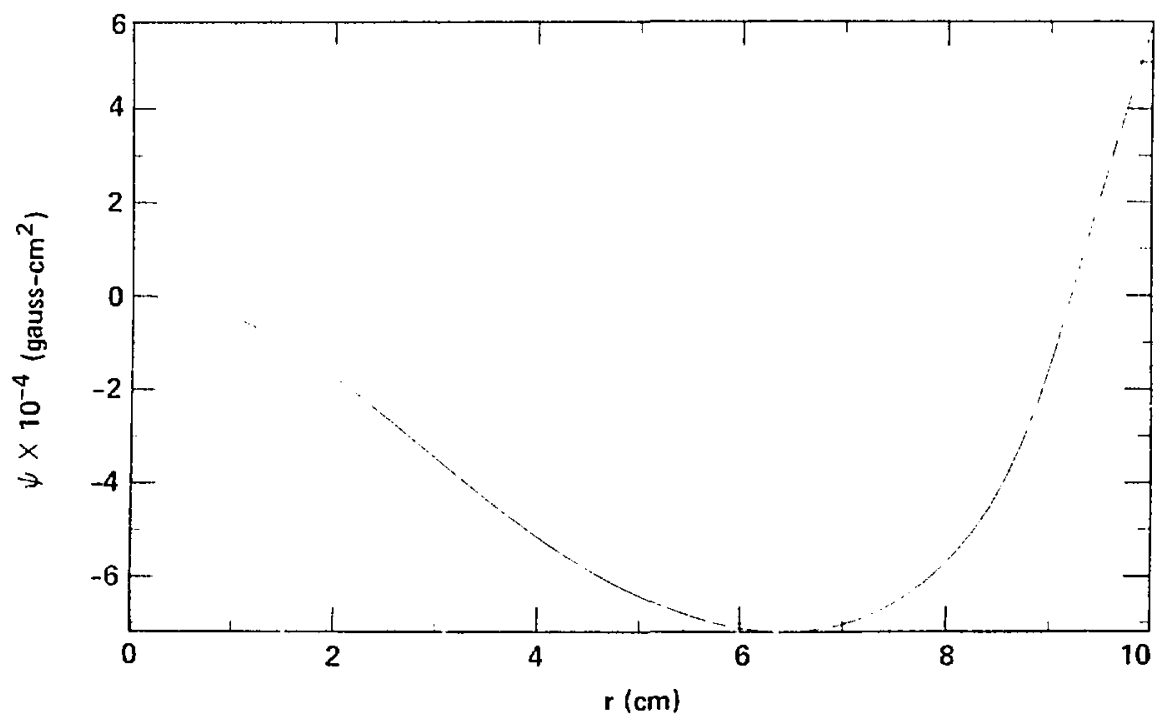

(b)

Figure 2-16. Compressed prolate Hill's vortex, $\psi(r, 0)$. (a) Initial external current, (b) Final external current. 


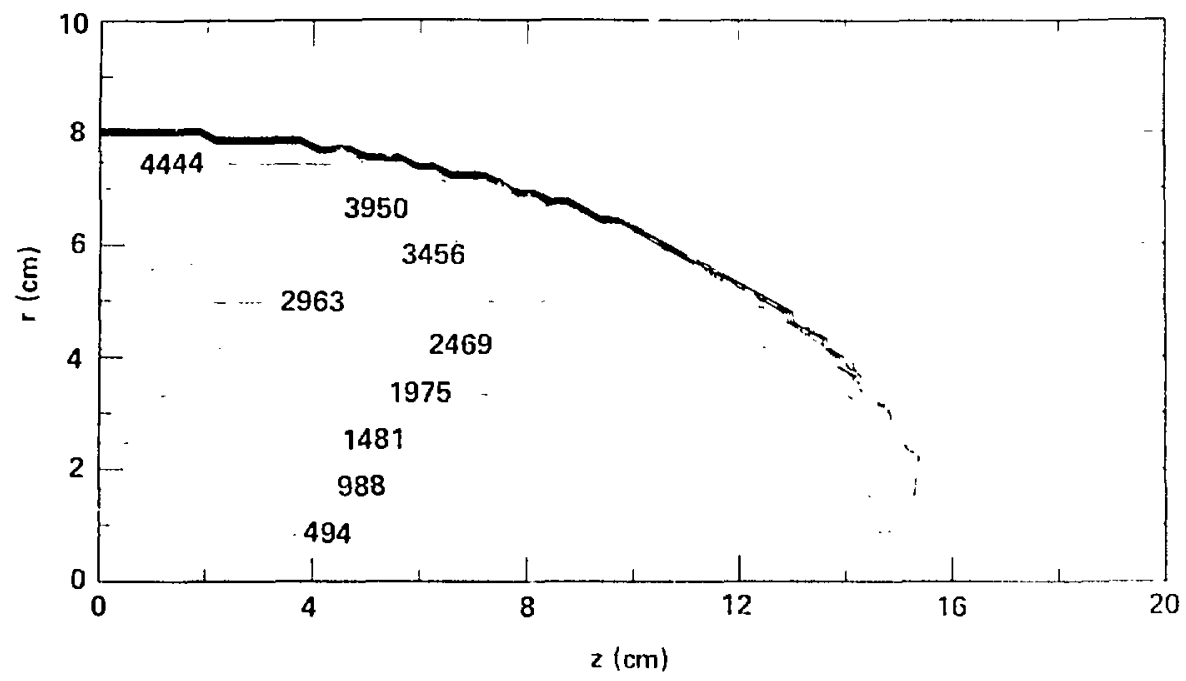

(a)

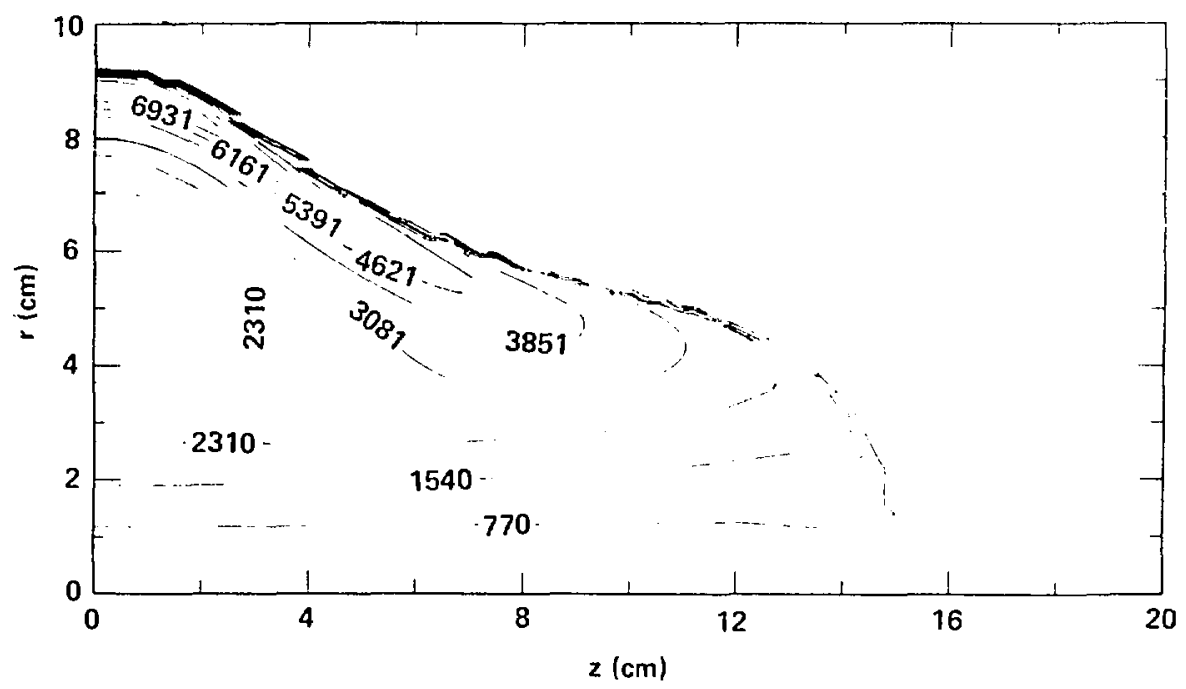

(b)

Figure 2-17. Compressed prolate Hill's vortex, $4 \pi c^{-1} J_{T}(r, z)$. (a) Initial external current, (b) Final external current. 


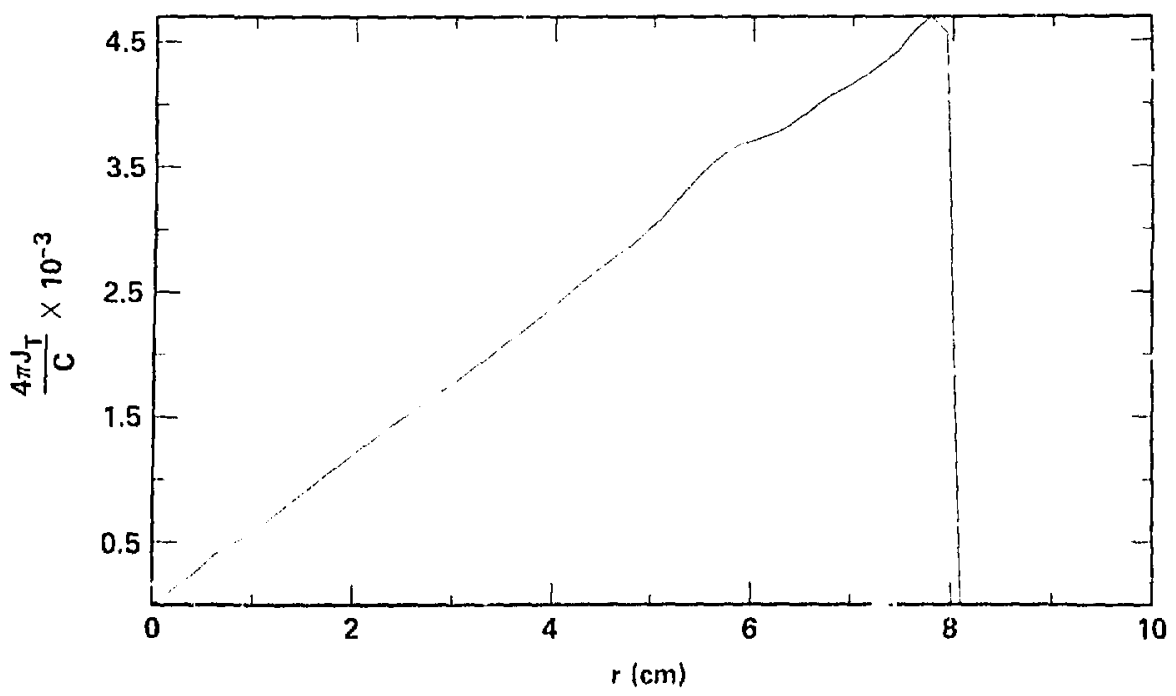

(a)

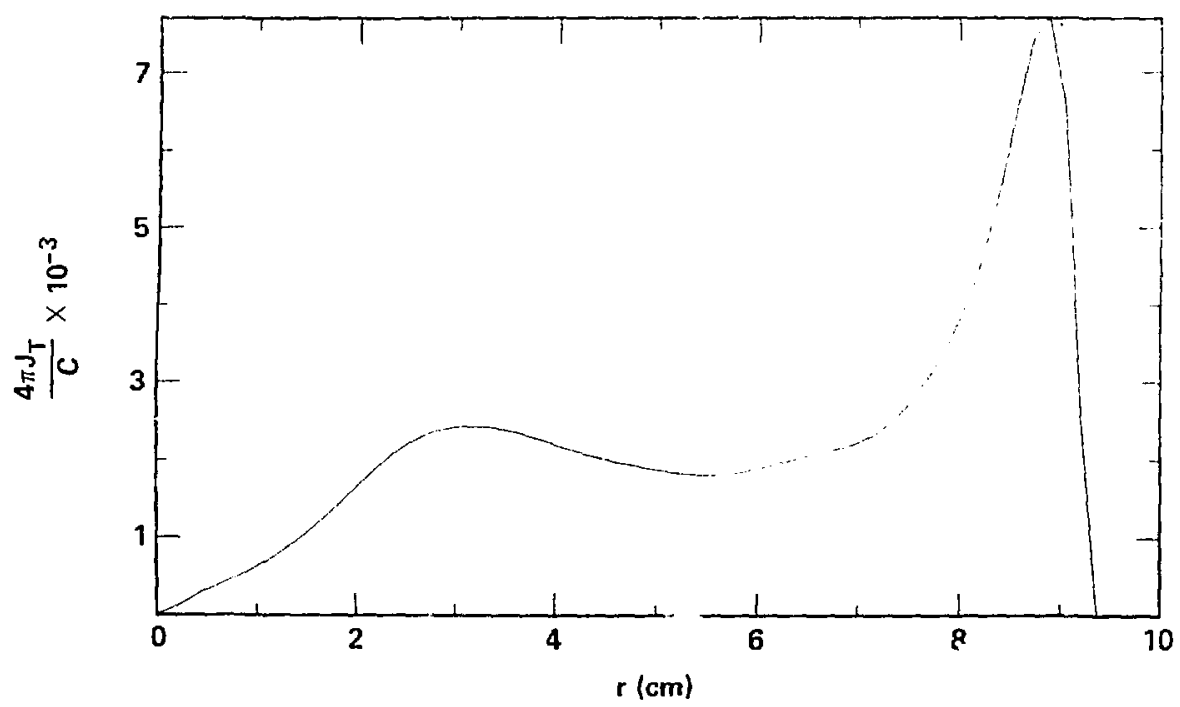

(b)

Figure 2-18. Compressed prolate Hill's vortex, $4 \pi c^{-1} J_{T}(r, 0)$. (a) Initial external current, (b) Final external current. 


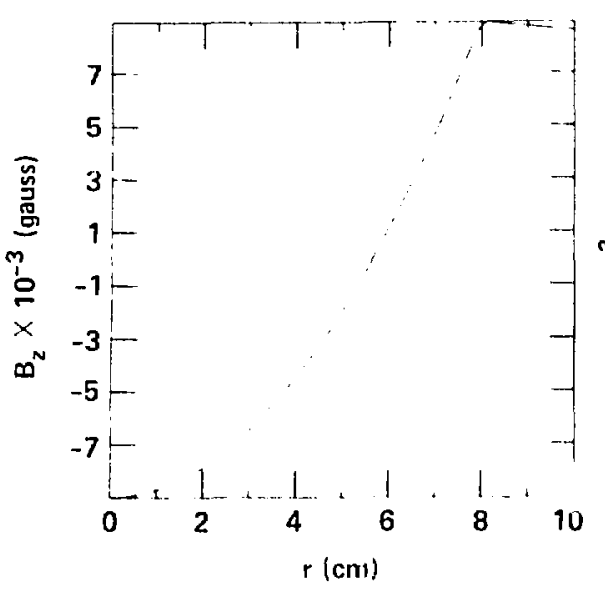

(a)

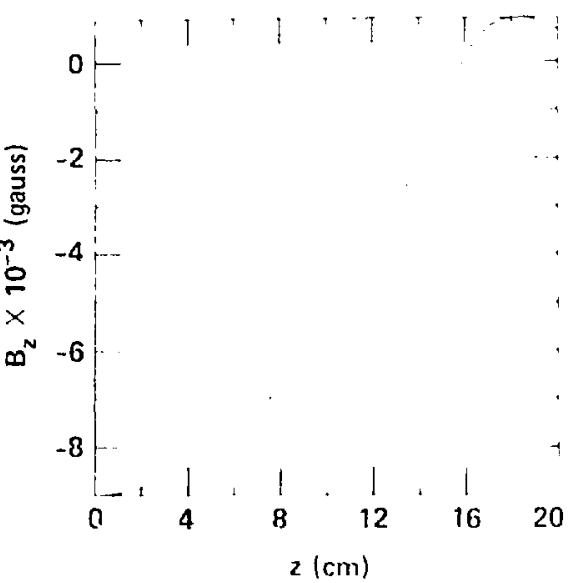

(b)

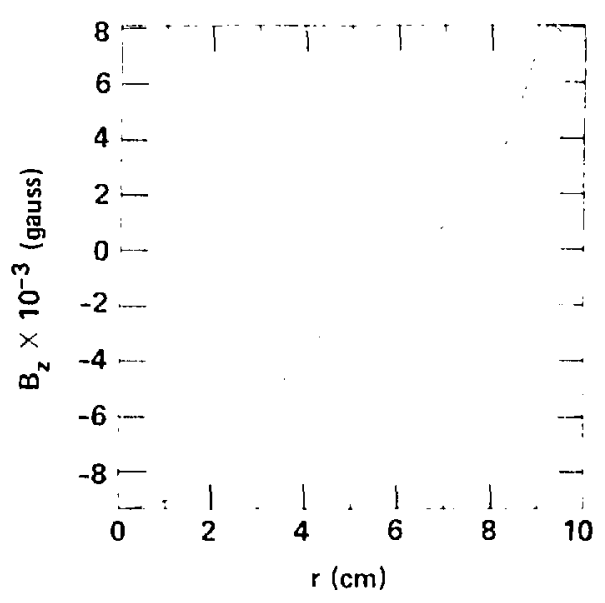

(c)

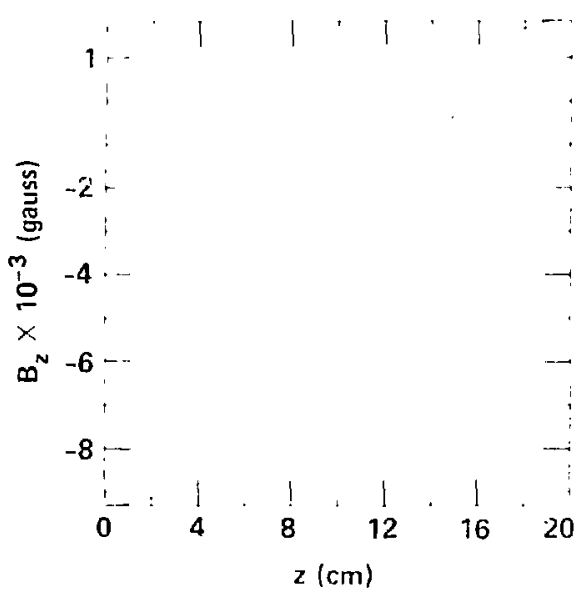

(d)

Figure 2-19. Compressed prolate Hill's vortex. (a) Initial external current $B_{z}(r, 0)$, (b) Initial external current $B_{z}(0, z)$, (c) Final external current $B_{z}(r, 0)$, (d) $F$ inal external current $B_{z}(0, z)$. 


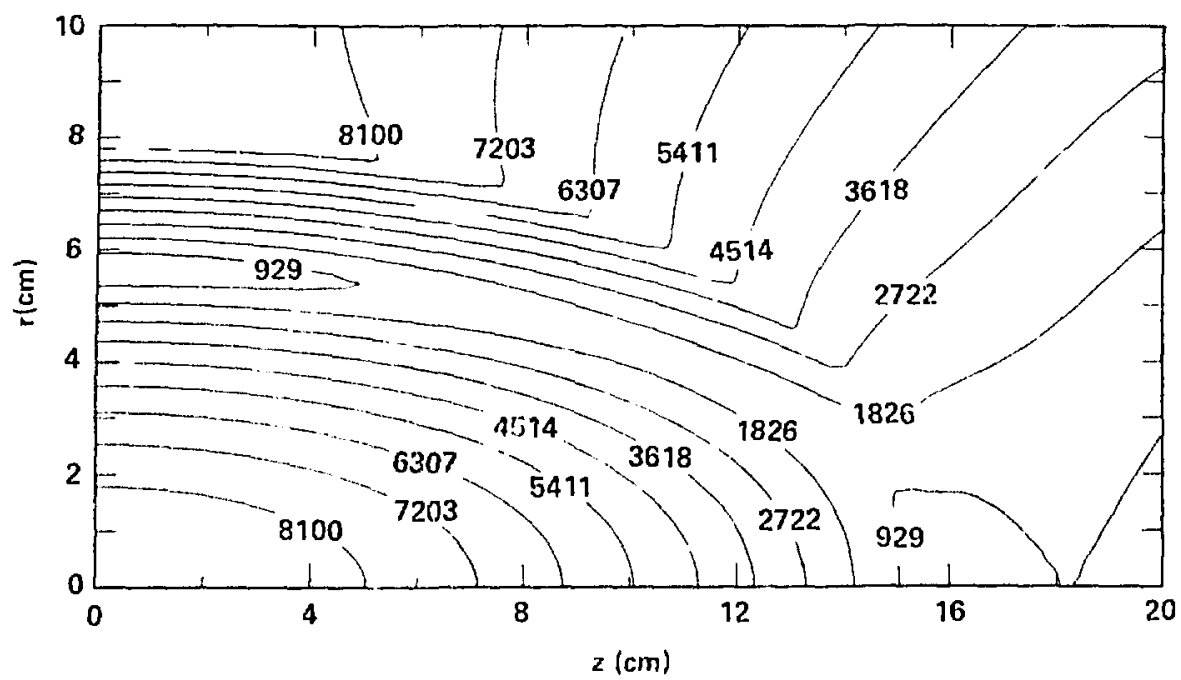

(a)

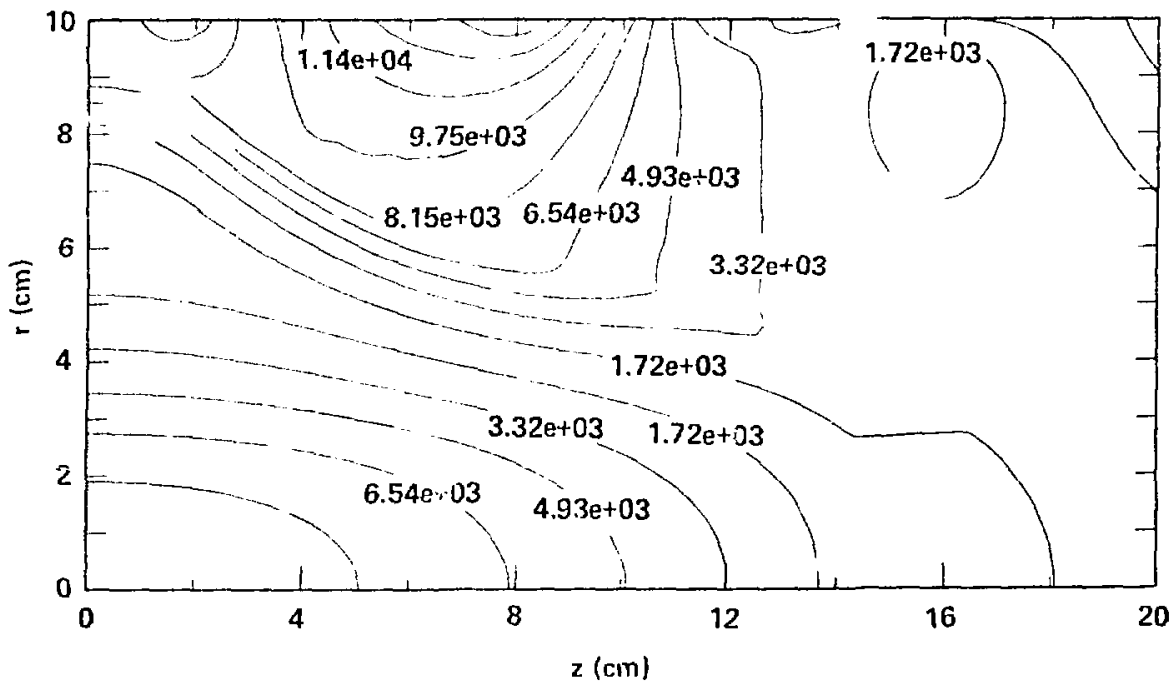

(b)

Figure 2-20. Compressed prolate Hill's vortex $B(r, z) .($ a) Initial external current, (b) Final external current. 


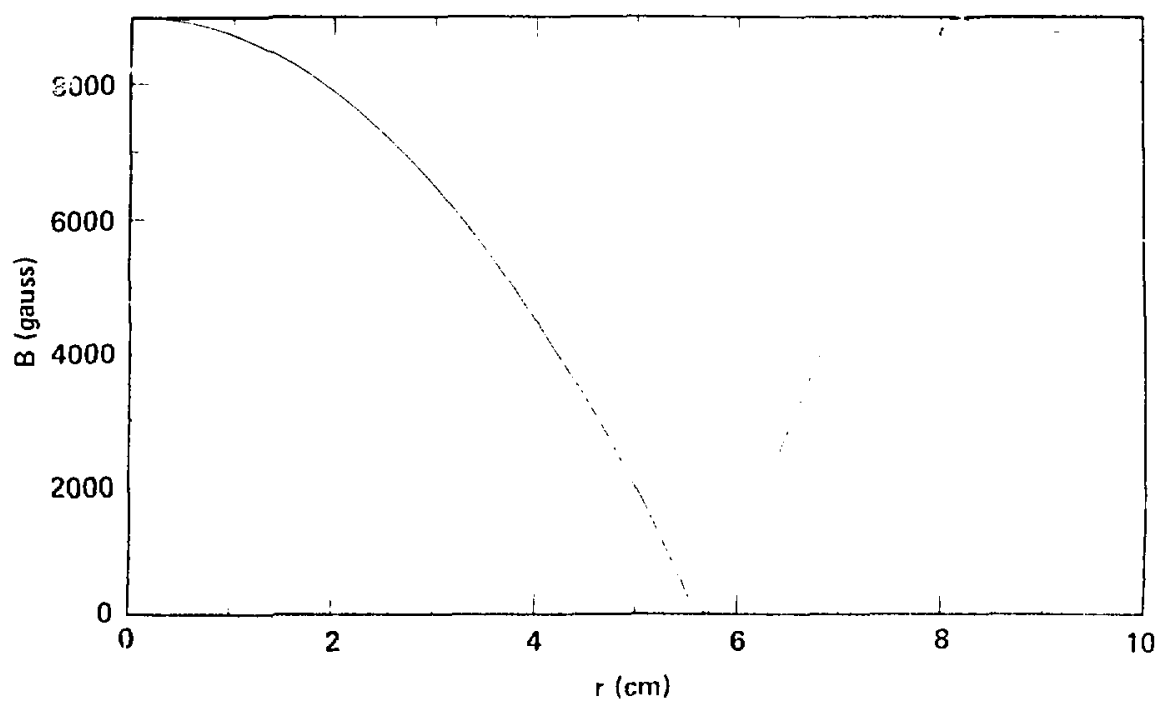

(a)

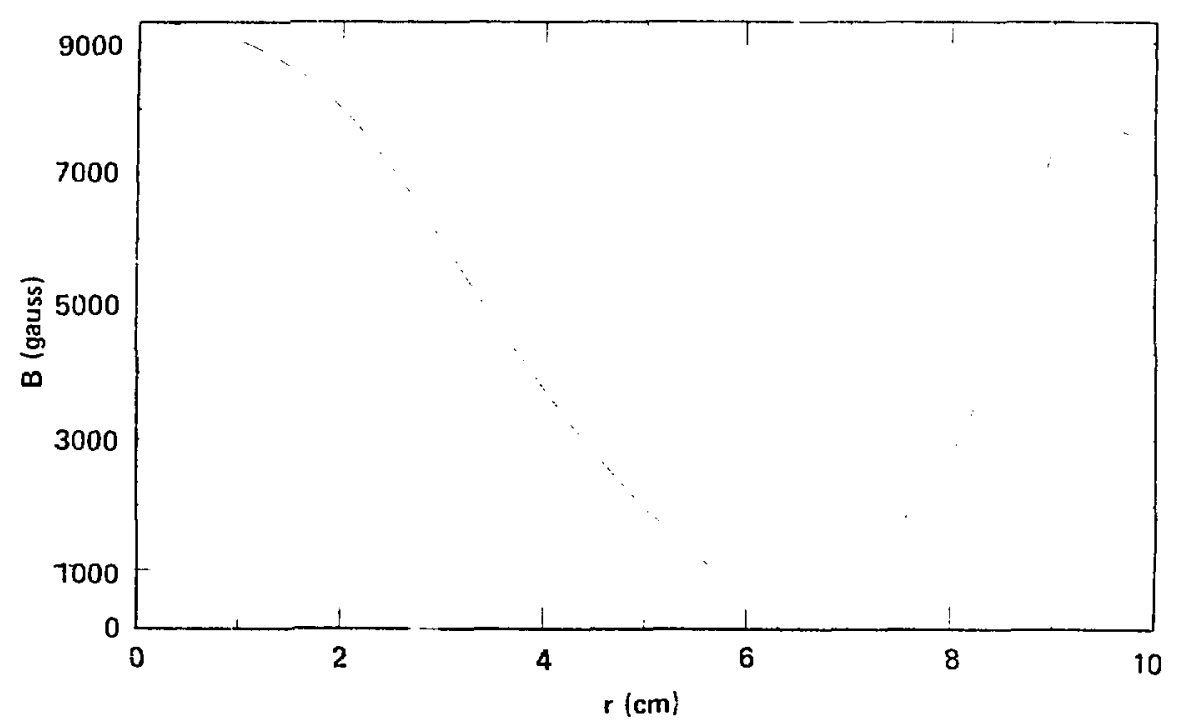

(b)

Figure 2-21. Compressed prolate Hill's vortex $B(r, 0)$. (a) Initial external current, (b) Final external current. 


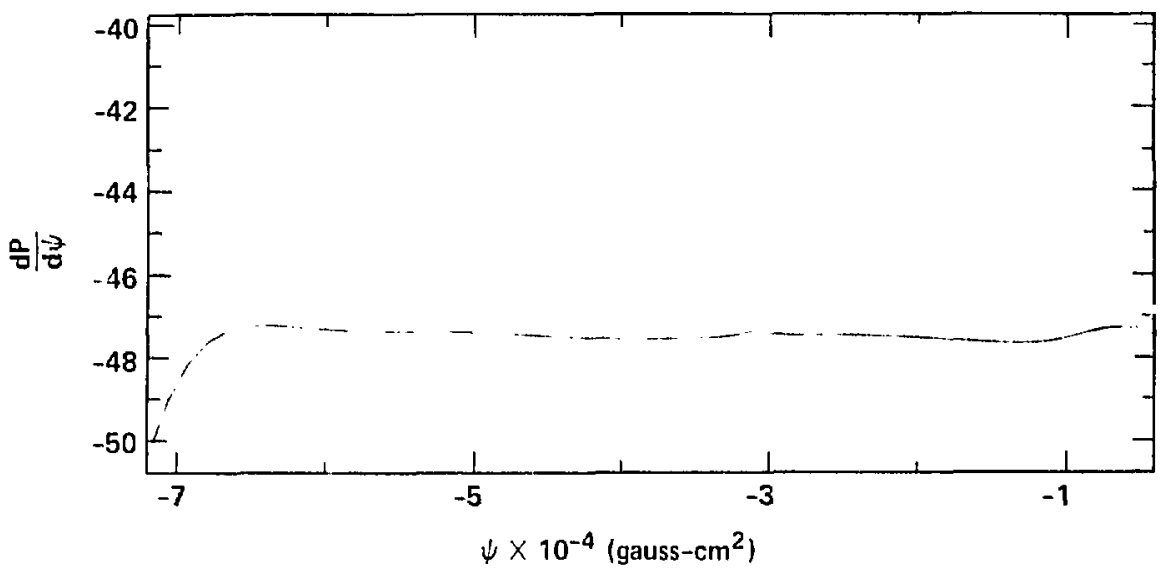

(a)

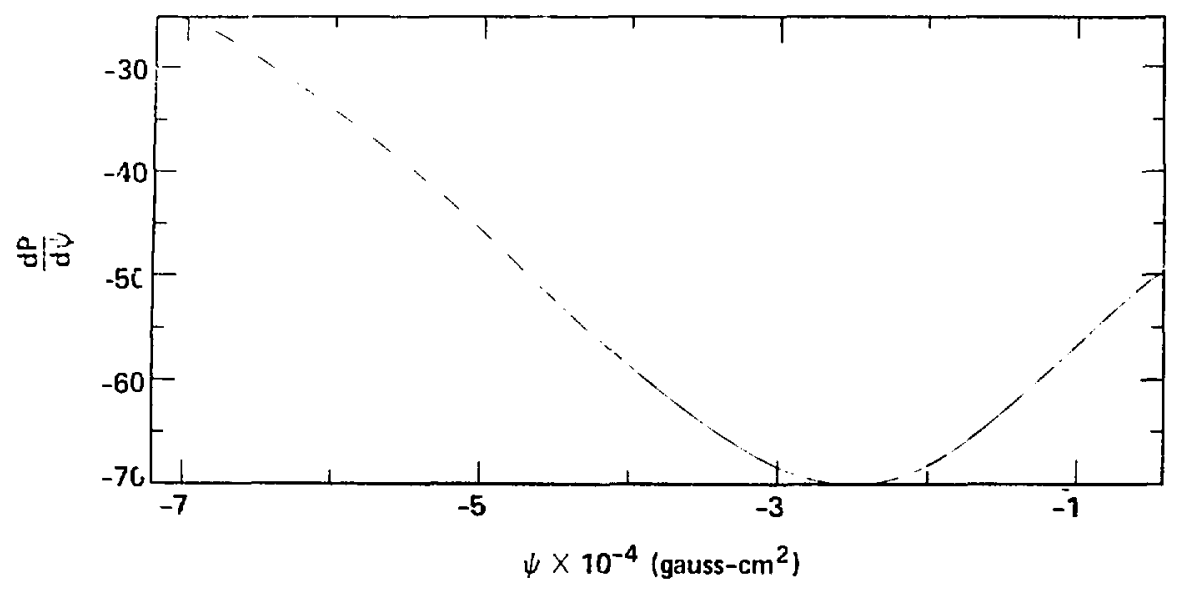

(b)

Figure 2-22. Compressed prolate Hill's vortex dp/d廿 vs. $\psi$. (a) Initial external current, (b) Final external current. 


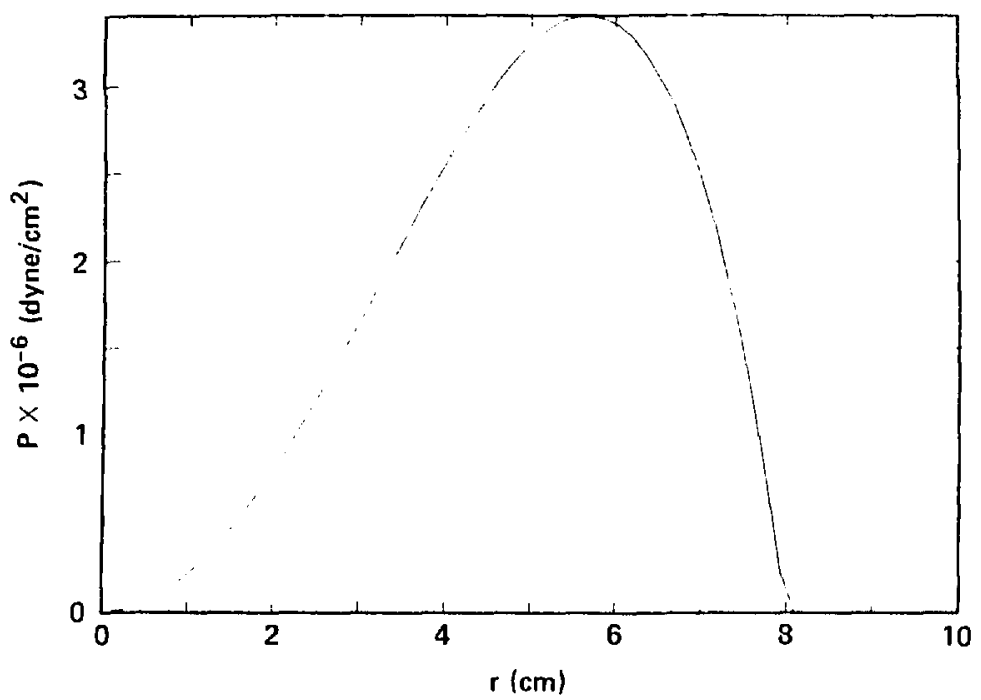

(a)

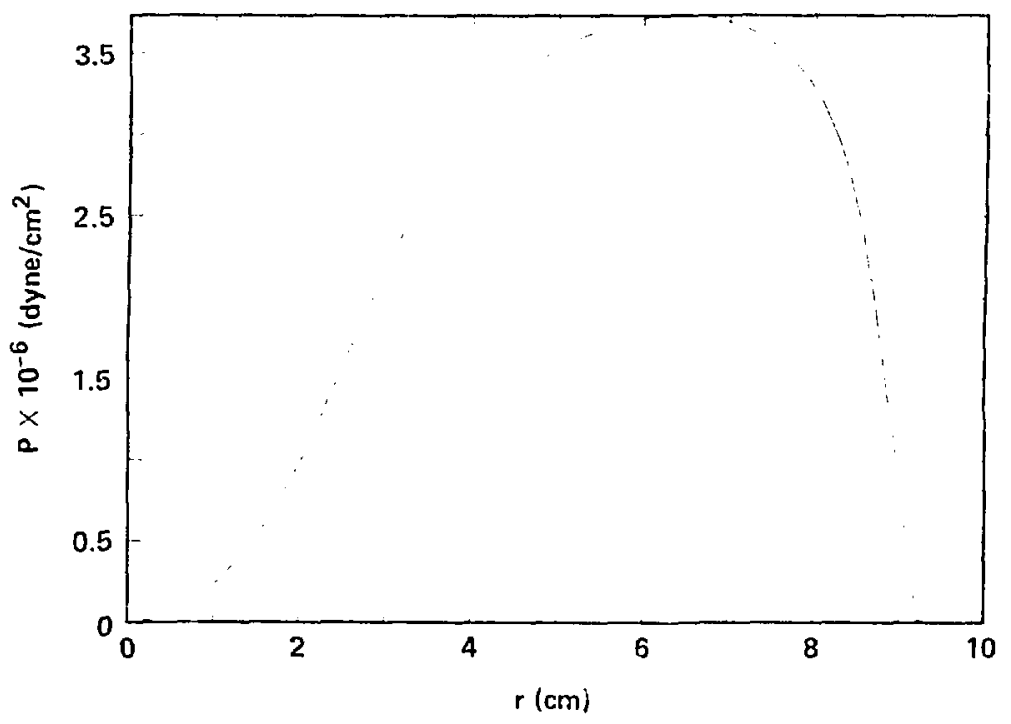

(b)

Figure 2-23. Compressed prolate Hill's vortex $p(r, 0)$. (a) Initial external current, (b) Final external current. 


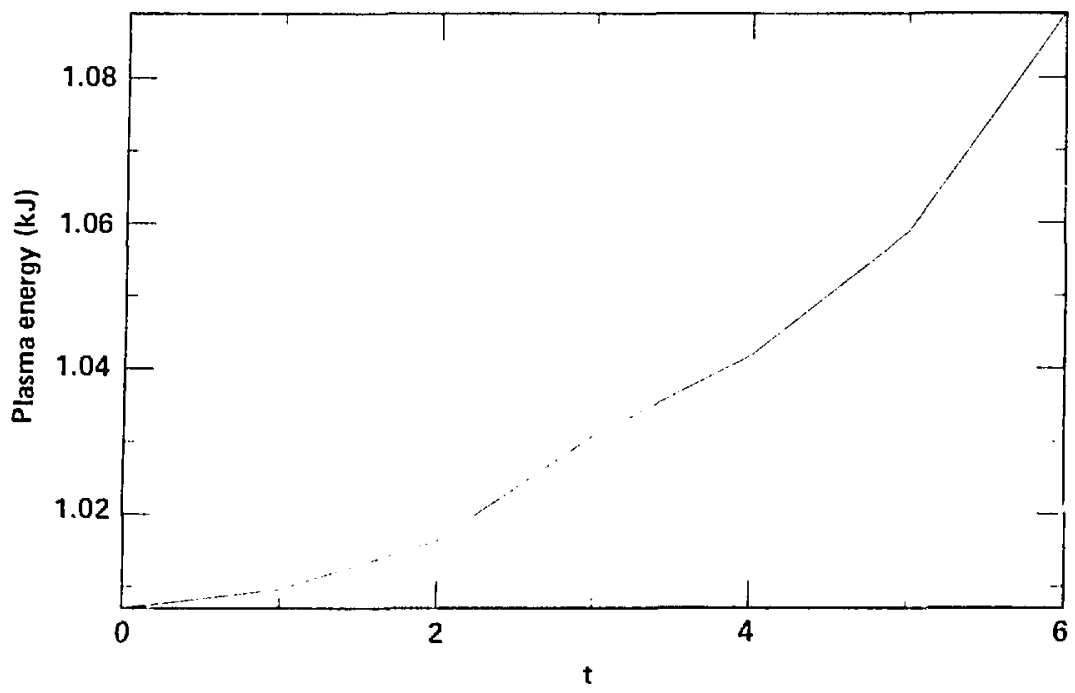

(a)

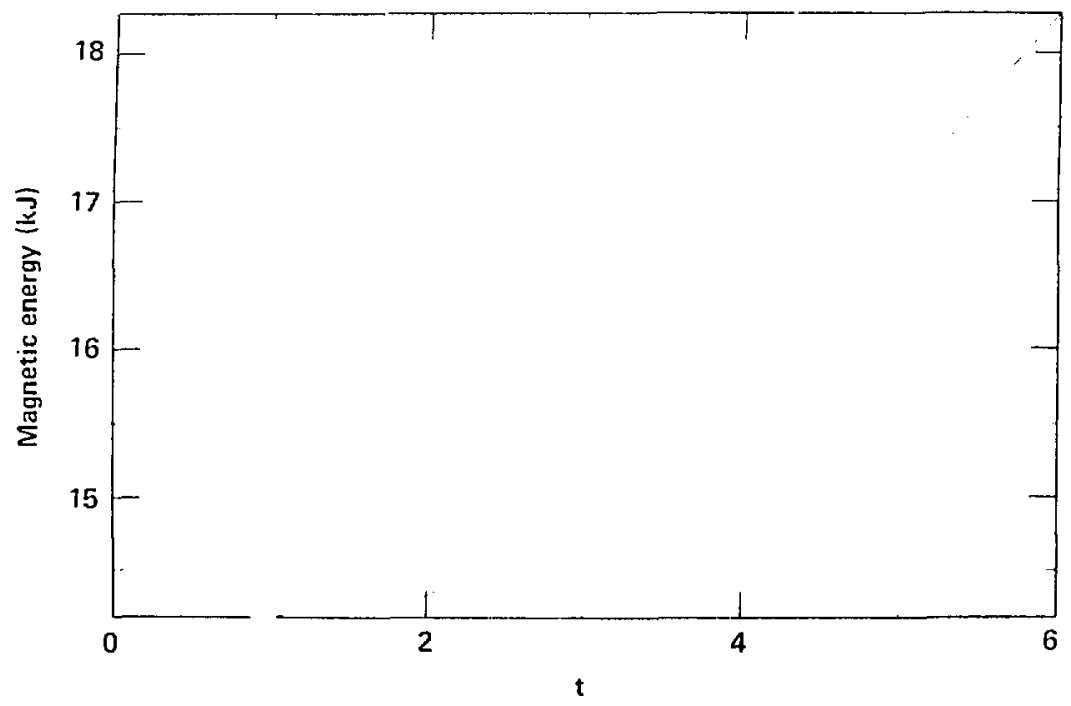

(b)

Figure 2-ż7. Compressed prolate Hill's vortex. (a) Plasma energy vs. time, (b) Magnetic energy vs. time. 


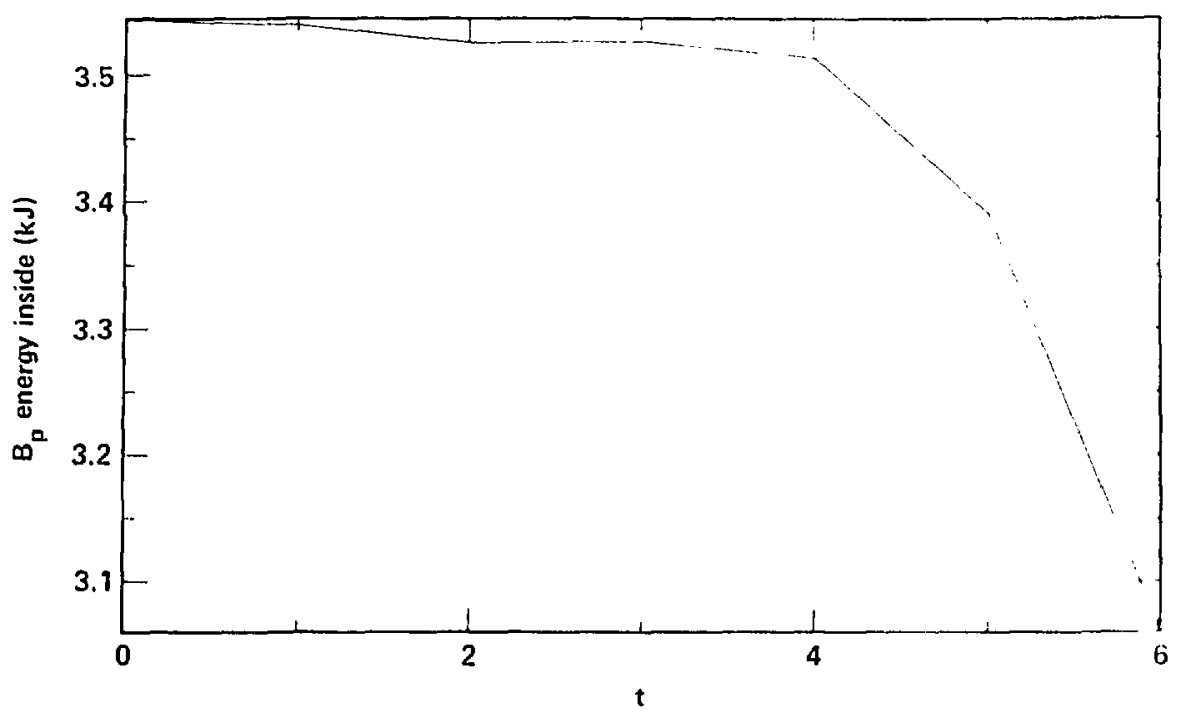

(a)

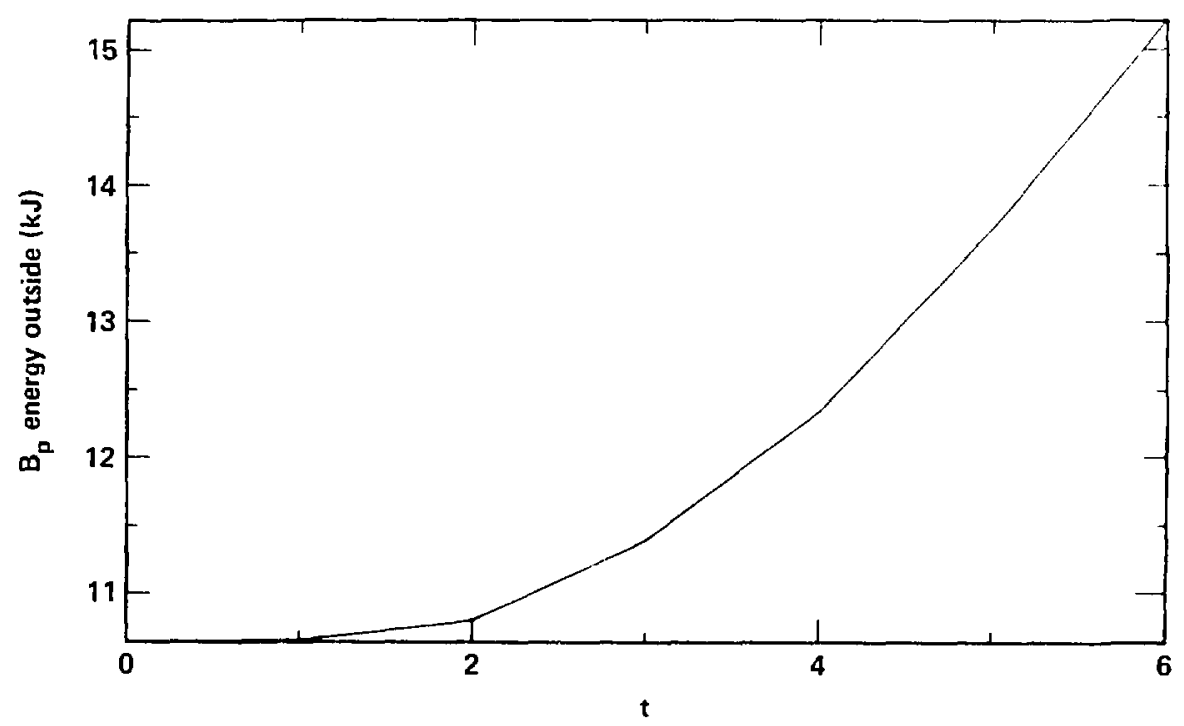

Figure 2-25. Compressed prolate Hill's vortex. (a) Poloidal magnetic field energy in the plasma vs. time, (b) Poloidal magnetic field energy outside the plasma vs. time. 
CHAPTER 3

THE ORBIT AVERAGE

\subsection{Introduction}

The approach used to incorporate finite Larmor radius effects into kinetic theory results in a hierarchy of equations. The method of solving these equations by integrating over characteristics of partial differential equations is equivalent to integrating over an appropriate orbit. The purpose of the orbit average is to provide a procedure by which integration over an orbit is achieved without a detailed knowledge of the orbit.

In this chapter, the orbit average is defined in terms of phase space integrals and path integrals. Numerical equivalents of these formulas are derived and tested in two orbit codes, which follow trajectories in one and two dimensions. The first code, AV, is used to compare orbit average formulas for orbits determined by forces linear in the coordinates. In this case, uncoupled harmonic 
motion results, and the precise orbit is known analytically. The second code, NEO, in which orbit average formulas are investigated, follows trajectories in a Hill's vortex magnetic field. The orbit in this case is obtained by numerical integration.

The form of the orbit average integrals changes when constants of the motion exist. In axisymmetry with conservative forces two constants of the motion exist and this information is used to derive appropriate orbit average formulas. In some circumstances a third constant of the motion nay be found to exist. To explore the possibility of the existence of a third constant of the motion several classes of constants of the motion are examined in the Hill's vortex model. Because no constant of the motion exist when an orbit is stochastic consideration is also given to conditions likely to result in stochastic motion.

\subsection{Orbit Average Integrals}

The orbit average is related to the solution of the kinetic equation obtained by integrating over the orbit. The orbit which is integrated over is the trajectory of a particle representative of an equilibrium distribution function. The kinetic equation solution obtained by integrating over the orbit arises from the convective form of the kinetic equation given below.

$$
\frac{D f}{D t}=c(f, f)
$$

In Eq. (3-1), $f$ is the distribution function and $C(f, f)$ is the 
collision operator. The derivative in Eq. (3-1) represents the time rate of change of $f$ along the orbit and the orbit solution is then obtained by integrating Eq. (3-1).

$$
f(t)=\int_{t\left(l_{0}\right)}^{t(l)} c(f, f) d t+f(t)
$$

In Eq. $(3-2) \ell$ is the trajectory given by the equations of motion and the distribution function at time $t, f(t)$ is then obtained by integrating along $\ell$ and adding the value of $f$ at $t$ ime $t_{0}$. This solution is an example of solving a partial differential equation using the method of characteristics.

Later a hierarchy of ordered equations is derived from Eq. (3-1). The orbit average process has its origin in constraint equations arising from this hierarchy of equation.. At each order there is an equation having the form ${ }^{[143]}$,

$$
L u=v
$$

where $L$ is an operator. The operator $L$ is assumed to have an adjoint $L^{\dagger}$ and an inner product such that,

$$
\left(u_{1}, L u_{2}\right)=\left(L^{\dagger} u_{1}, u_{2}\right)
$$

The existence of the adjoint operator, the property Eq. (3-4), and a homogeneous solution $h, L^{t} h=0$ are used to derive the constraint equations. In general if a solution: exists for Eq. (3-3), then the inner product of $h$ and $v$ must be zero. This $c$ an be demonstrated by taking the inner product of $h$ with the right hand side of Eq. (3-3). 


$$
\begin{aligned}
(h, v) & =(h, L u) \\
& =\left(L^{\dagger} h, u\right) \\
& =0
\end{aligned}
$$

The constraint equation is then the inner product of the right side of an equation obtained from an ordering of Eq. (3-1) with the homogeneous solution of the adjoint operator. The inner product applicable to the hierarchy of equations resulting from Eq. (3-1) is the starting point from which the orbit average is derived.

The object of the orbit average process is to approximate the applicable inner product. The inner product is defined by considering the left side of Eq. (3-1).

$$
\frac{\partial f}{\partial t}=\frac{\partial f}{\partial t}+[f, H]
$$

In Eq. (3-6) $H$ is the Hamiltonian and $[f, H]$ is the Poisson bracket,

$$
[f, H]=\sum_{k}\left(\frac{\partial f}{\partial q_{k}} \frac{\partial H}{\partial p_{k}}-\frac{\partial f}{\partial p_{k}} \frac{\partial H}{\partial q_{k}}\right)
$$

where $p$ and $q$ are phase space coordinates. Equation (3-6) shows $D f / D t$ is an exact differential when $\dot{p}=-\partial H / \partial q$ and $\dot{q}=\partial H / \partial p$, or in other words when the phase space coordinates $p$ and $q$ satisfy the equations of motion. Under this condition the solution given by Eq. (3-2) is immediate. Because later derived constraint equations involve Poisson brackets which are the steady state form of Eq. $(3-6)$, the inner product is taken to be intesrating over the orbit. The orbit average must then be in some sense equivalent to integrating over the orbit. It is also necessary to obtain the 
adjoint of the operation of integrating over the orbit since its homogeneous solution is needed.

The adjoint of the Poisson bracket operator is found by applying the inner product to $g[\mathrm{f}, \mathrm{H}]$. At the limits of integration $f$ and $g$ are assumed to be zero. The integration over the orbit is assumed to be analogous to integration over the phase space coordinates, which appear in the poisson bracket. The inner product operation is manipulated using integration by parts, to obtain $\left(L^{\dagger} g, f\right)$ from $(g, L f)$.

$$
(g, L f) \approx \sum_{k} \int\left(g \frac{\partial f}{\partial q_{k}} \frac{\partial H}{\partial p_{k}}-g \frac{\partial f}{\partial p_{k}} \frac{\partial H}{\partial q_{k}}\right) d p_{k} d q_{k}
$$

Integration by parts with respect to $q_{k}$ is applied to the first term of Eq. (3-8) and integration by parts with respect to $p_{k}$ is applied to the second term of the integrand of $5 q .(3-8)$.

$$
\sum_{k} \int\left(-f \frac{\partial g}{\partial q_{k}} \frac{\partial H}{\partial p_{k}}+f \frac{\partial g}{\partial p_{k}} \frac{\partial H}{\partial a_{k}}\right) d p_{k} d q_{k}
$$

From Eq. (3-9) the adjoint of $[f, H]$ is seen to be $[H, f]$. The adjoint operator of the Poisson bracket with respect to the inner product of integrating over the orbit also has a solution given by integrating over the orbit. The required homogeneous solution of the adjoint operator may then be taken to be any constant of the motion. The orbit average process then reduces to multiplying by a constant and integrating over the orbit, or simply integrating over the orbit. The definition of the orbit average of a general function $x$ is below. 


$$
\bar{x}^{(0)}=\lim _{T \rightarrow \infty} \frac{1}{T} \int_{0}^{T} x d t
$$

The time integration in Eq. (3-10) corresponds to a trajectory given by the equations of motion. The value of $\bar{x}^{-(0)}$ must not depend on the initial position of the trajectory used to solve the equations of motion. This will be true when any particular trajectory encounters all points allowed within the franework of conserved quantities. Under thi condition $\bar{x}^{(0)}$ has a unique value independent of any specific arbit and may be computed without having a detailed knowledge if the actual orbit. The only restriction is the $x$ values used to compute Eq. (3-10) may only be those allowed by any conserved uantities. It is assumed forces are time independent and thus the Hamiltonian $\mathrm{H}$ is conserved. In addition the system under considerat on is cylindrical and axisymmetric and consequently the theta canonical momentum $p_{\theta}$ is conserved. This result follows from the definition,

$$
\frac{\partial p_{\theta}}{\partial t}=-\frac{\partial H}{\partial \ddot{\theta}}
$$

and the fact $H$ does not depend on theta. If $\bar{X}^{(0)}$ is independent of starting position, the integration path or orbit encounters ali positions consistent with conservation of $H$ and $r_{g}$. To satisfy this requirement it is assumed to be sufficient for the orbit to be ergodic. In other words, the orbit approac'es arbitrarily close to each allowed point. The problem of determining he orbit average is then related to the fundamental basis of statistical mechanics. The 
basis of statistical mechanics rests on the equivalency of time averages and quantities derived from ensemble theory. The nhiect of the ensemble theory is to obtain time averages by knowing the probability of allowable configurations, but not naving precise knowledge of any particular system trajectory. A configuration refers to :oordinates describing a system which may include, but is not limited to spatial position and momentum. The goal of the orbit average is to obtain $\bar{\chi}^{(0)}$ without solving the equations of motion and determining the precise orbit. The determination of the cri it may be avoided in two ways. First, using reasoning similar to statistical mechanics the time integral over the orbit in Eq. (3-10) may be cunsidered to be an integral over the allowed phase space volume. Second the basic orbit averaje definition Eq. (3-10) may be converted from a time integral to a path integral and the path integral may be converted to an integral over coordinates. In what follows the orbit average formulas corresponding to derivations based on the first method are superscripted with ps and a number. The orbit average formulas derived from path integrals are superscripted only with a number.

The basic phase space orbit average formula is a normalized integral over phase space coordinates allowed by the conservation of $H$ and $p_{\theta}$.

$$
\bar{x}^{(p s 0)}=\frac{\int \chi W d \Omega}{\int W d \Omega}
$$

In Eq. (3-12) $d \Omega$ is the differential phase space volume element and $W$ 
is a weighting function. Without additional information all allowable phase space points may be assumed to be equally likely and $W$ can be set to one. An additional condition of time invariance of the total allowed phase space volume must be imposed so $\bar{x}^{(p s 0)}$ yields the same result independent of when it is evaluated during a particular system trajectory. The action of a trajectory of points within a phase space volume is such that the volume distorts or appears to flow in phase space with time. From Liouville's theorem $[144]$ the phase space density is incompressibie or the phase space volume is prese ved providing there are no collisions. The orbit average of Eq. $(3-12)$ is then applicable only in the case of no collisions. This is also a condition required to keep $H$ and $p_{\theta}$ constant.

In general, motion in phase spawe for one particle is described by specifying the tive history of six coordinates. In three dimensions there are three spacial coordinates and three velocity coordinates. If a relationship exist between coordinates, it is possible to reduce the number of coordinates by one and introduce a parameter resulting from the relationship. An example is a system in which the Hamiltonian is conserved. A velocity may then be expressed in terms of $H$ and the remaining velocities. In this case, it is desirable to change phase space variables and replace the eliminated velocity by $H$. Hlternatively a system rray be independent of a coordinate. The conjugate momentum is then a constant as in the axisymmetric case where theta is ignorable and $p_{\theta}$ is constant. The motion is then essentially two dimensional, and with $\mathrm{H}$ conserved 
only one additional constant of motion may exist under certain circumstances. The effect on $W$ in Eq. (3-12) in either case is to introduce delia functions which restrict phase space in accordance with constant $H$ and $p_{\theta}$. To simplfy the integration of Eq. (3-12), it is then best to use variables contained within the delta functions as integration variables. More specifically for constant: values $H_{0}$ and $p_{\theta 0}$, the weighting function $W$ is set to ne for allowid phase space points by letting $W$ equal $\delta\left(H-H_{0}\right) \delta\left(p_{\theta}-p_{\theta 0}\right)$. The phase space volume multiplied by $w$ is then given below for cylindrical coordinates, axisymmetry and conserved $H$.

$$
W d \Omega=\delta\left(H-H_{0}\right) \delta\left(p_{\theta}-p_{\theta 0}\right) r d r d \theta d z d v_{r} d v_{\theta} d v_{z}
$$

For ease of integration it is advantageous to convert from $v_{r}$, $v_{\theta}$, $v_{z}$ to $H, p_{\theta}, p_{z}$ coordinates. The new coordinates allon immediate integration of $H$ and $p_{\theta}$. In terms of the new coordinates the phase space volume multiplied by $W$ is given below.

$W d \Omega=\delta\left(H-H_{0}\right) \delta\left(p_{\theta}-p_{\theta 0}\right)\left|m \frac{\partial\left(H, p_{\theta}\right)}{\partial\left(v_{r}, v_{\theta}\right)}\right|^{-1} r d r d \theta d z d H d p d p_{z}$

In Eq. (3-14, the Jacobian is obtained from the three definitions below.

$$
\begin{aligned}
& L=\frac{1}{2} m \dot{q}^{2}-e Z \phi+e z c^{-1} \vec{A} \cdot \vec{q} \\
& p_{i}=\partial L / \partial \dot{q}_{i} \\
& H=\sum p_{i} \dot{q}_{i}-L
\end{aligned}
$$


In Eqs. (3-15) through (3-17) $\mathrm{m}$ is mass, $\mathrm{q}$ is a coordinate, ez is the chir e, $\phi$ is electric potential, $c$ is the speed of light, $\vec{A}$ is the magnetic vector potential, $\dot{q}$ is velocity, $p_{j}$ is canonical momentum, $L$ is the Lagrangian and $H$ is the Hamiltonian. In axisymmetry with cylindrical coordinates,

$$
\begin{aligned}
& p_{r}=m v_{r} \\
& p_{\theta}=m r v_{\theta}+\frac{e z}{c} \psi \\
& p_{z}=m v_{z} \\
& H=(2 m)^{-1}\left(p_{r}^{2}+p_{z}^{2}\right)+\left(2 m r^{2}\right)^{-1}\left(p_{\theta}-\frac{e z}{c} \psi\right)^{2}+e z \phi
\end{aligned}
$$

where $\psi$ is $r A_{\theta}$ and only poloidal field is allowed. Using Eqs. (3-18) to $(3-21)$ the Jacobian can be written as follows.

$$
\left|\frac{\partial\left(H, p_{\theta}\right)}{\partial\left(v_{r}, v_{\theta}\right)}\right|=m^{2} r v_{r}
$$

In Eq. (3-22) mv $v_{r}$ may be expressed in terins of $H, p_{z}, p_{\theta}, \psi$ and $\phi$ using Eq. $(3-21)$.

$$
m v_{r}=\left(2 m(H-e Z \phi)-r^{-2}\left(p_{\theta}-\frac{e Z}{c} \psi\right)^{2}-p_{z}^{2}\right)^{1 / 2}
$$

Equations (3-22) and (3-23) are used in Eq. (3-14) to give,

$$
W d \Omega=\frac{\delta\left(H-H_{0}\right) \delta\left(p_{\theta}-p_{\theta 0}\right) d r d \theta d z d H d p_{\theta} d p_{z}}{m^{2}\left(2 m(H-e Z \phi)-r^{-2}\left(p_{\theta}-c^{-1} e Z \psi\right)^{2}-p_{z}^{2}\right)^{1 / 2}}
$$


Formula 3-24 is substituted into Eq. (3-12) to yield the axisymmetric, cylindrical coordinate, version of $\bar{x}^{(p s 0)}$ assuming equally likely allowed phase space points and conserved $H$ and $p_{\theta}$.

$$
\begin{aligned}
\bar{x}^{-(p s))}= & \int\left(x \delta\left(H-H_{0}\right) \delta\left(p_{\theta}-p_{\theta 0}\right)\right. \\
& \left.\left(2 m(H-e Z \phi)-r^{-2}\left(p_{\theta}-c^{-1} e Z \psi\right)^{2}-p_{z}^{2}\right)^{-1 / 2}\right) \\
& d r d z d H d p_{\theta} d p_{z} \\
& {\left[\int \left(\delta\left(H-H_{0}\right) \delta\left(p_{\theta}-p_{\theta 0}\right)\right.\right.} \\
& \left.\left(2 m(H-e Z \phi)-r^{-2}\left(p_{\theta}-c^{-1} e Z \psi\right)^{2}-p_{z}^{2}\right)^{-1 / 2}\right) \\
& \left.d r d z d H d p_{\theta} d p_{z}\right]^{-1}
\end{aligned}
$$

The above formula for $\bar{x}^{(p s 1)}$ is applicable to a two dimensional $r, z$ case.

For a situation which is one dimensional and radial, $x$ has no $z$ variation and $H$ depends only on $r$. Thus,

$$
\begin{aligned}
\dot{p}_{Z} & =-\partial H / \partial Z \\
& =0
\end{aligned}
$$

and $p_{z}$ is therefore constant. In this case the allowable phase space is further restricted by the one dimensional weighting function below.

$$
W=\delta\left(H-H_{0}\right) \delta\left(p_{\theta}-p_{\theta 0}\right) \delta\left(p_{z}-p_{z 0}\right)
$$


138

Substituting Eq. $(3-27)$ into the $\bar{\chi}^{(p s 0)}$ formula produces the one dimensional analog of $\mathrm{Eq} \cdot(3-25)$.

$$
\begin{aligned}
& \bar{x}^{(p s 2)}= \int\left(x \delta\left(H-H_{0}\right) \delta\left(p_{\theta}-p_{\theta 0}\right) \delta\left(p_{z}-p_{z O}\right)\right. \\
&\left.\left(2 m(H-e Z \phi)-r^{-2}\left(p_{\theta}-c^{-1} e Z \psi\right)^{2}-p_{z}^{2}\right)^{-1 / 2}\right) \\
& d r d H d p_{\theta} d p_{z} \\
& {\left[\int \left(\delta\left(H-H_{0}\right) \delta\left(p_{\theta}-p_{\theta 0}\right) \delta\left(p_{z}-p_{z 0}\right)\right.\right.} \\
&\left.\left(2 m(H-e Z \phi)-r^{-2}\left(p_{\theta}-c^{-1} e Z \psi\right)^{2}-p_{z}^{2}\right)^{-1 / 2}\right) \\
&\left.d r d H d p_{\theta} d p_{z}\right]^{-1}
\end{aligned}
$$

$(3-28)$

To aid the comparison of Ens. (3-25) and (3-28), with orbit average formulas obtained from the time integration orbit average definition $\bar{x}^{(0)}$, the above formulas are rewritten in terms of $v_{r}$.

$$
\bar{x}^{(p s 3)}=\frac{\int x\left(r, z, H_{0}, p_{\theta 0}, p_{z}\right) v_{r}^{-1} d r d z d p_{z}}{\int v_{r}^{-1} d r d z d p_{z}}
$$

In Eq. (3-29) integration is understood to refer to phase space coordinate values allowed within the restriction of constant $H_{0}$, and $P_{\theta 0}$ and thus the delta functions are suppressed. The $v_{r}$ formula for Eq. (3-29) follows.

$$
v_{r}=m^{-1}\left(2 m\left(H_{0}-e Z \phi\right)-r^{-2}\left(p_{\theta 0}-c^{-1} e Z \psi\right)^{2}-p_{z}^{2}{ }^{1 / 2}\right)
$$


The analogous formula for $\bar{x}^{(p s 2)}$ is below.

$$
\bar{x}^{(p s 4)}=\frac{\int x\left(r, H_{0}, p_{B 0}, p_{20}\right) v_{r}^{-1} d r}{\int v_{r}^{-1} d r}
$$

Similar to Eq. (3-29) the integration in Eq. (3-31) refers to phase space coordinate values allowed with in the restriction of constant $\mathrm{H}_{0}, \mathrm{p}_{\theta 0}$, and $\mathrm{p}_{20}$ and the delta functions are again suppressed. The $v_{r}$ formula for Eq. $(3-31)$ follows.

$$
v_{r}=m^{-1}\left(2 m\left(H_{0}-e z \phi\right)-r^{-2}\left(p_{\theta 0}-c^{-1} e z \psi\right)^{2}-p_{20}{ }^{2}\right)^{1 / 2}
$$

Equation (3-32) shows that $p_{20}$ is a parameter in the $\bar{x}^{(p s 4)}$ formula in contrast to the $\bar{x}^{(p s 3)}$ formula where $x$ is integrated over $p_{z}$. A simplification results in Eq. (3-29) when $x$ is not a function of $p_{z}$. In this case, integration over $p_{z}$ yields the following result.

$$
\int v_{r}^{-1} d p_{z}=2 \pi
$$

Equation (3-33) may be used to obtain an orbit average formula for $x$ independent of $p_{Z^{*}}$.

$$
\bar{x}^{(p s 5)}=\frac{\int x\left(r, z, H_{0}, p_{B 0}\right) d r d z}{\int d r d z}
$$

The use of the phase space derived orbit average formulas $\bar{x}^{(p s 3)}, \bar{x}^{(p s 4)}$, and $\bar{x}^{(p s 5)}$ is guided by the corparison with formulas derived from the basic time integral orbit average $\bar{x}^{(0)}$. The procedure used to derive these formulas is to convert $\bar{x}^{(0)}$ from a 
time integral to a path integral and then convert the path integral to an integral over coordinates.

A change of variables is used to convert $\bar{x}^{(0)}$ to a path integral with $\&$ representing distance along the orbit.

$$
\bar{x}^{(1)}=\lim _{\ell \rightarrow \infty} \frac{\int_{0}^{l} x \frac{d t}{d \ell} d \ell}{\int_{0}^{l} \frac{d t}{d \ell} d \ell}
$$

The incremental path length de is expressed in terms of cylindrical coordinates below.

$$
d \ell=\left(d r^{2}+r^{2} d \theta^{2}+d z^{2}\right)^{1 / 2}
$$

The definition of the Hamiltonian, Eq. (3-17), is used with Eq. (3-36) to express the change of variat les Jacobian in terms of a function of spatial conrdinates.

$$
\frac{d t}{d \ell}=(2 / m)^{-1 / 2}(H-e Z \phi)^{-1 / 2}
$$

The path integral orbit average $\bar{x}^{(1)}$ given by Eq. (3-35) may be rewritten using Eq. $(3-37)$.

$$
\bar{x}^{(2)}=\lim _{\ell \rightarrow \infty} \frac{f_{0}^{\ell} x(H-e Z \phi)^{-1 / 2} d \ell}{f_{0}^{\ell}(H-e Z \phi)^{-1 / 2} d \ell}
$$

From Eq. (3-38) it can be seen, with $H$ constant, $\bar{x}^{(2)}$ becomes an average along the path if there is no electric potential. A form of Eq. (3-38) more appropriate to axisymmetry is obtained by projecting the three dimensional orbit onto a poloidal plane. The result of 
projecting onto a poloidal plane is to make the following substitution,

$$
\frac{d \ell}{v}=\frac{d \ell p}{v_{p}}
$$

where $d \ell_{p}$ is incremental path length in a poloidal plane and $v_{p}$ is the poloidal velocity.

$$
v_{p}=1 m^{-1}\left(2 m(H-e Z \phi)-r^{-2}\left(p_{\theta}-c^{-1} e Z \psi\right)^{2}\right)^{1 / 2}
$$

The axisymnetric form of $\mathrm{Eq} .(3-38)$ is then below.

$$
\bar{x}^{(3)}=\lim _{\ell \rightarrow \infty} \frac{\int_{0}^{\ell} x\left(H-e Z \phi-\left(2 m r^{2}\right)^{-1}\left(\rho_{\theta}-c^{-1} e Z \psi\right)^{2}\right)^{-1 / 2} d l_{p}}{f_{0}^{l}\left(H-e Z \phi-\left(2 m r^{2}\right)^{-1}\left(p_{\theta}-c^{-1} e Z \psi\right)^{2}\right)^{-1 / 2} d l_{p}}
$$

Equation (3-4) has been derived without making any assumption about phase space probability. It also shows the orbit average emphasizes values of $x$ when $v_{p}$ becomes small. The orbit average, $\bar{x}^{(3)}$ applies to the axisymmetric two dimensional case. When $x$ has no $z$ variation a further simplification results. The orbit path becomes radial and the relevant velocity is $v_{r}$. This is due to a change of variables Jacobian defined as follows.

$$
\begin{aligned}
(d t / d l) d \ell & =(d t / d r) d r \\
& =v_{r}^{-1} d r \\
& =m\left(2 m(H-e Z \phi)-r^{-2}\left(p_{\theta}-c^{-1} e Z \psi\right)^{2}-p_{z}^{2}\right)^{-1 / 2} d r
\end{aligned}
$$


The one dimensional path integral orbit average is thi:n below.

$$
\bar{x}^{(4)}=\frac{\int x\left(r, H_{0}, p_{\theta 0}, p_{z 0}\right) v_{r}^{-]} d r}{\int v_{r}^{-1} d r}
$$

In the formula for $\bar{x}^{(4)}$ the velocity $v_{r}$ is the same as before and the same assumptions of constant $H, p_{\theta}$, and $p_{2}$ apply. Consequently, the one dimensional path integral $\bar{x}^{(4)}$ is identical to the one dimensional phase space orbit average $\bar{\chi}^{(p s 4)}$. This means a rinange of variables is sufficient to transform the time integration over space. Also for one dimension there is no need to make any assumption about the probability of phase space points, the $W$ weighting function, to obtain the orbit average. As before the range of integration lies within the $H$ and $p_{\theta}$ determined orbit turning points for Eq. (3-43). If the orbit potential well is double valued in $r$ and there are two turning points at $r_{1}$ and $r_{2}$, tren the integration is from $r_{1}$ to $r_{2}$. For a magnetic flux double valued in $r$, at small values of $p_{\theta}$ the potential in $r$ has two disconnected regions each having two turning points. In this case $\bar{x}^{(4)}$ or $\bar{x}^{(p s 4)}$ must be broken into two parts and the integration proceeds between turning points for each region.

In previous work by Lovelace ${ }^{[145]}$, the orbit average is formulated in terms of an integral over a four dimensional space. The function to be averaged is multiplied by a probability function which is ore where the orbit is allowed, within the constraint of constant $H$ and $p_{\theta}$, and zero otherwise. A formula similar to $\bar{x}^{(p s 3)}$ results if the limits of integration are restricted by conservation 
of $H$ and $P_{\theta}$. The orbit is assumed to be ergodic and the probability function is below.

$w_{\ell}=c_{\ell} r^{-1} \delta\left((2 m)^{-1}\left(p_{r}^{2}+p_{z}^{2}\right)+\left(2 m r^{2}\right)^{-1}\left(p_{\theta 0}-c^{-1} e Z \psi\right)^{2}-H_{0}\right)(3-44)$

In Eq. (3-44), $C_{\ell}$ is a constant. The orbit average formula given by Lovelace is then,

$$
\bar{x}^{(\ell)}=\frac{\int x W_{\ell} r d r d z d p_{r} d p_{z}}{\int W_{\ell}{ }^{r} d r d z d p_{r} d p_{z}}
$$

The stability work of Loveiace only requires an orbit average of a function that depends on spatial coordinates. He thus uses $\bar{\chi}^{(\ell)}$ in the following form,

$$
\bar{x}^{(l)}=\frac{\int x d r d z}{\int d r d z}
$$

where the region of integration is restricted by allowed values of $\mathrm{H}$ and $p_{\theta}$. This orbit average is identical to $\bar{x}^{(p 55)}$ derived from phase space considerations. It is thus possible to interpret $W_{\ell}$ as the previous integrand weighting function after a change of variable from $v_{\theta}$ to $p_{\theta}$ and an integration over a delta function in $p_{\theta}$. The difficulty in applying the probability function viewpoint is in the generalization to one dimension. The case of $x$ independent of $z$ in Eq. (3-46) yields the following orbit average.

$$
\frac{\int x d r}{\int d r}
$$


Thus, in considering a function $x$ with no $z$ dependence the previously derived formulas are not recovered and a factor of $v_{r}^{-1}$ is absent.

\subsection{Harmonic Oscillator Orbit Code}

In this section, the AV harmonic oscillator code is described and the orbit average related to the harmonic oscillator Hamiltonian is derived. The objective of the $A V$ code is to examine the orbit average without any coupling terms in the Hamiltonian so the orbit can be determined analytically. To achieve this goal, the orbits in AV are produced by forces proportional to displacement in $r$ and $z$ and the motion is then harmonic oscillation in $r$ and $z$. In the Hamiltonian framework, there is no magnetic field and the electric potential is,

$$
\phi=\frac{1}{2}\left(\omega_{z}^{2} z^{2}+\omega_{r}^{2}\left(r-r_{0}\right)^{2}-r^{-2} p_{\theta 0}^{2}\right)
$$

where $\mathrm{e} Z$ and $\mathrm{m}$ are set to one. In one dimension the electric potential is,

$$
\phi=\frac{1}{2}\left(\omega_{r}^{2}\left(r-r_{0}\right)^{2}-r^{-2} p_{\theta 0}^{2}\right)
$$

As discussed previously theta is ignorable and $p_{\theta}$ is constant. The value of $p_{\theta}$ is taken to be $p_{\theta 0}$ and then the following Hamiltonian results.

$$
H=\frac{1}{2}\left(p_{r}^{2}+p_{z}^{2}+\omega_{z}^{2} z^{2}+\omega_{r}^{2}\left(r-r_{0}\right)^{2}\right)
$$


In one dimension the Hamiltonian is,

$$
H=\frac{1}{2}\left(p_{r}^{2}+p_{z}^{2}+\omega_{r}^{2}\left(r-r_{0}\right)^{2}\right)
$$

The equations of motion in two dimensions are then,

$$
\begin{aligned}
\dot{p}_{r} & =-\frac{\partial H}{\partial r} \\
& =-\omega_{r}^{2}\left(r-r_{0}\right) \\
\dot{p_{z}} & =-\frac{\partial H}{\partial z} \\
& =-\omega_{z}^{2} z
\end{aligned}
$$

and the poloidal orbit is given below.

$$
\begin{aligned}
& r=r_{0}+r_{1} \sin \left(\omega_{r} t+\varphi_{r}\right) \\
& z=z_{1} \sin \left(\omega_{2} t+\varphi_{2}\right)
\end{aligned}
$$

Equations (3-52) and (3-53) describe harmonic nscillation about $r=r_{0}$ and $z=0$. The initial position and velocities follow.

$$
\begin{aligned}
& r=r_{0}+r_{1} \sin \left(\varphi_{r}\right) \\
& z=z_{1} \sin \left(\varphi_{2}\right) \\
& v_{r}=r_{7} \omega_{r} \cos \left(\varphi_{r}\right) \\
& v_{z}=z_{7} \omega_{2} \cos \left(\varphi_{z}\right)
\end{aligned}
$$

Inserting the initial values of $r, z, v_{r}$, and $v_{z}$ into Eq. (3-49) gives the two dimensional energy constant of the motion. 


$$
H_{0}=\frac{1}{2}\left(r_{1}^{2} \omega_{r}^{2}+z^{2} \omega_{z}^{2}\right)
$$

Substituting the intial values of $r, v_{r}$, and $p_{z 0}$; into Eq. (3-50) gives the one dimensional energy constant.

$$
H_{0}=\frac{1}{2}\left(r^{2} \omega_{r}^{2}+p_{z 0}^{2}\right)
$$

The orbit in theta is obtained from $v_{\theta}=r \dot{\theta}$ and a rearrangement of the definition of $p_{\theta}$ in Eq. (3-19).

$$
\dot{\theta}=\frac{p_{00}}{r^{2}}
$$

Taking the initial angle to be zero, substituting tq. (3-52) for $r$, Eq. (3-56) may be integrated to give the following formula for theta.

$$
\theta=\frac{p_{\theta 0}}{r_{0}^{2} \omega_{r}} \int_{\varphi_{r}}^{\omega_{r} t+\varphi_{r}} \frac{d x}{\left(1+r_{0}^{-1} r_{1} \sin (x)\right)^{2}}
$$

The integral in Eq. (3-57) may be put in standard form and theta is then given below.

$$
\begin{aligned}
\theta & =\frac{p_{\theta 0}}{r_{0}^{2} \omega_{r}}\left[\frac{\frac{r_{1}}{r_{0}} \cos (x)}{\left(1-\left(\frac{r_{1}}{r_{0}}\right)\right)\left(1+\frac{r_{1}}{r_{0}} \sin (x)\right)}\right. \\
+ & \left.\frac{2}{\left(1-\left(\frac{r_{1}}{r_{0}}\right)^{2}\right)^{3 / 2}} \tan ^{-1}\left\{\frac{\tan (x / 2)+\frac{r_{1}}{r_{0}}}{\left(1-\left(\frac{r_{1}}{r_{0}}\right)^{2}\right)^{1 / 2}}\right]\right]_{\varphi_{r}}^{\omega_{r} t+\varphi_{r}}
\end{aligned}
$$


The $\tan ^{-1}$ branch on which $\tan (x / 2)$ lies must be used in Eq. (3-58).

The velocity used for the one dimensional formulas $\bar{x}^{(4)}$ or $\bar{x}^{\text {(ps4) }}$ is obtained by rearranging Eq. $(3-50)$ and substitutilin, the one dimensional constant energy from Eq. (3-55).

$$
v_{r}=\omega_{r}^{2}\left(r_{1}^{2}-\left(r \cdot r_{0}\right)^{2}\right)^{1 / 2}
$$

Similarly the velocity used in the path integral orbit average $\bar{x}^{(3)}$ arises from rearranging Eq. (3..49) and inserting the two dimensional constant energy from Eq. (3-54).

$$
v_{p}=\left(\omega_{r}^{2}\left(r_{1}^{2}-\left(r-r_{0}\right)^{2}\right)+w_{z}^{2}\left(z_{i}^{2}-z^{c}\right)\right)^{1 / 2}
$$

In the AV code the Hamiltonian consists of the kinetic energy plus two functions that depend on $r$ or $z$. As a consequence the right side of the equation of motion, Eq. $(3-51)$, for $\dot{p}_{r}$ only depends on $r$ and the right side of the equation for $\dot{p}_{z}$ only depends on $z$. Multiplying both sides of the $\dot{p}_{r}$ equation by $p_{r}$ and the $\dot{p}_{z}$ equation by $p_{z}$, shows the following two quantities are constant in time.

$$
\begin{aligned}
& H_{r}=p_{r}^{2}+\omega_{r}^{2}\left(r-r_{0}\right)^{2} \\
& H_{z}=p_{z}^{2}+\omega_{z}^{2} z^{2}
\end{aligned}
$$

In other words in addition to $H$ being a constant of the motion, the two constituent parts of $\mathrm{H}$ related to $r$ and $z$ motion are also constants of the motion. The orbit average which takes this sitdation into account is a specialization of previous phase space 
formulas. The weighting function in this case consists of three delta functions.

$$
\begin{aligned}
& \text { w } d \Omega=\delta\left(H_{r}-H_{r 0}\right) \delta\left(H_{z}-H_{z 0}\right) \delta\left(p_{\theta}-p_{\theta 0}\right)\left|\frac{\partial\left(H_{r}, H_{z}, P_{\theta}\right)}{\partial\left(v_{r}, v_{z}, v_{\theta}\right)}\right|-1 \\
& r J r d \theta d z d H_{r} d H_{z} d p_{\theta}
\end{aligned}
$$

The Jacobian in Eq. (3-62) is calculated using Eq. (3-21) specialized to the hermonic osciliator.

$$
\left|\frac{\partial\left(H_{r}, H_{z} p_{\theta}\right)}{\partial\left(v_{r}, v_{z}, v_{\theta}\right)}\right|=4 r v_{r} v_{z}
$$

The orbit average obtained from Eq. $(3-12)$ with the phase space volume of $\mathrm{Eq} .(3-62)$ and $x$ independent of $\mathrm{H}_{r}, \mathrm{H}_{z}$, is below.

$$
\bar{x}^{(p s 6)}=\frac{\int x \frac{d r d z}{v_{r} v_{z}}}{\int \frac{d r d z}{v_{r} v_{z}}}
$$

The integration in Eq. (3-64) is over the $r, z$ values allowed by conservation of $\mathrm{H}_{r}, \mathrm{H}_{z}$, and $\mathrm{p}_{\theta}$. The analytic orbit formulas for $r$ a.d $z$ show a specific set of values of $\omega_{r}, \omega_{z}, r_{1}$, and $z_{1}$ result in a trajectory confined to a rectangular box of height $2 r_{1}$ and width $2 z$, . The $\bar{x}^{(p s 6)}$ orbit average then consist of two successive one dimensional orbit averages in a specific reciangular box. In this case the consequence of having three conserved quantities rather than two is to eliminate an integration over rectangular boxes. In this example referring back to Eq. (3-49), it can be seen 
that constant $H$ restricts an orbit to an elliptical region in $r$ and z. There is no particular orbit which can move everywhere in the ellipse as evidenced by the analytic orbit equations. Thus, the $\bar{x}^{\text {(ps5) }}$ orbit average formula, which does integrate over the ellipse, is an equal weighted average over all rectangular boxes, about which the constant $\mathrm{H}$ ellipse may be transcribed. This is demonstrated explictly by integrating the integrand of the numerator and denominator of $\bar{x}^{(p s \sigma)}$ over all possible values of $H_{r O}$. To do this $v_{r}$ is expressed in terins of $\mathrm{H}_{r 0}$,

$$
v_{r}=\left(H_{r 0}-\omega_{r}^{2}\left(r-r_{0}\right)^{2}\right)^{1 / 2}
$$

and $v_{z}$ is expressed in terms of $\mathrm{H}_{0}$ and $\mathrm{H}_{\mathrm{rO}}$,

$$
v_{z}=\left(H_{0}-H_{r_{0}}-\omega_{z}^{2} z^{2}\right)^{1 / 2}
$$

Interpreting $x$ to depend on $\mathrm{H}$ rather than $\mathrm{H}_{r}$ and $\mathrm{H}_{z}$ and restricting the integration to $r z$ values allowed by constant $H$, the averaged $\bar{x}^{(p s 6)}$ becomes,

$$
\overline{x^{-(p s 6)}}=\frac{\int x d r d z \int_{\omega_{r}^{2}\left(r-r_{0}\right)^{2}}^{H} \frac{d x}{\left(x-\omega_{z}^{2} z^{2}\left(r-r_{0}\right)^{2}\right)\left(H_{0}-x-\omega_{z}^{2} z^{2}\right)^{1 / 2}}}{\int d r d z \int_{\omega_{r}^{2}\left(r-\omega_{z}^{2} z^{2}\right.}^{H} \frac{d x}{\left(x-\omega_{r}^{2}\left(r-r_{0}\right)^{2}\right)\left(H_{0}-x-\omega_{z}^{2} z^{2}\right)^{1 / 2}}}
$$

The integral over $x$ in Eq. (3-67) is equal to a constant $\pi$, and as a result, 


$$
\overline{\bar{x}^{(p s 6)}}=\bar{x}^{(p s 5)}
$$

The effect of constant $H_{r}$ and $H_{z}$ on the $\bar{\chi}^{(p s 5)}$ formula is to change the allowed $r, z$ integration area from an ellipse to a rectarigle with weighting function $\left(v_{r_{2}} v_{2}\right)^{-1}$. For the harmonic oscillator Hamiltonian there are analytic formulas for $v_{r}$ and $v_{z}$ in terms of $r$ and $z$. In a more complex case with coupling terms in the Hamiltonian the analytic orbit is unknown and there are in general no formulas for $v_{r}$ and $v_{z}$. It is also frequently the case that the third coriserved quantity in addition to $H$ and $p_{\theta}$ is unknown. Due to these considerations, it is advantageous to approximate the $v_{r} v_{z}$ weighting function. One choice is to assume all allowed values of $v_{r}$ and $v_{z}$ are equally likely and use as a weighting function the average of $v_{r} v_{z}$. Expressing $v_{z}$ in terms of $v_{r}$ and $v_{p}$ which is a function of $H$ and $\phi$ and integrating over allowed $v_{r}$ values yields,

$$
\begin{aligned}
& \overline{v_{r} v_{z}}=v_{p}^{-1} \int_{0}^{v_{p}} v_{r}\left(v_{p}^{2}-v_{r}^{2}\right)^{1 / 2} d v_{r} \\
& =v_{p}^{2}
\end{aligned}
$$

The approximate orbit average is then below.

$$
\bar{x}^{(p s 7)}=\frac{\int x_{p}^{v_{p}^{-2}} d r d z}{\int v_{p}^{-2} d r d z}
$$

The importance of Eq. (3-69) is that the $v_{p}^{-2}$ weighting function only depends on $r$ and $z$ and is thus known when the Hamiltonian is 
given. Equation (3-69) applies to a general Hamiltonian and does not require an analytic orbit.

\subsection{Numerical Orbit Average Formulas}

The orbit average formulas are implemented in the AV code using several numerical approximations. The one dimensional integrals, such as $\bar{x}^{(0)}, \bar{x}^{(p s 4)}$ and $\bar{x}^{(4)}$, are computed using a trapezoidal integration formula.

$$
\int F d x=\frac{1}{2} \Delta x \sum_{i=1}^{N-1}\left(f_{i}+F_{i+1}\right)
$$

In Eq. (3-70) there are $N$ grid points spanning the allowed orbit region. The integrand $F$ represents $x$ or $x / v_{r}$ and $\Delta x$ is the time duration or the length of the orbit region divided by $N-1$.

The two-dimensional orbit-average integrals are approximated by discretizing the allowed orbit region into rectangles and then sumining integrals over grid cells of fitted approximations to the integrand. This procedure integrates over a globally continuous function which has discontinuous first derivatives between grid cells. For several numerical formulas the general case of a non-rectangular orbit region is considered. Rectangular grid cells are also used (in this case) in the interior away from the edge boundary. At the edge, where a curved boundary passes through an otherwise rectangular interior grid cell, the curved boundary is approximated as a straight line chord. The contribution of the cell 
is then a sum of smaller, triangular and rectangular regions. Various formulas which represent numerical orbit averages are expressed in terms of functions designated $I_{n}, T_{n}, N_{n}, U_{n}$, and $Z_{n}$. The definitions of these functions are given in Appendix $A$. There are several different methods used to represent the $\bar{x}^{(3)}$ path integral orbit average in a grid cell. In each approximation the orbit is assumed to pass through the grid cell at all points along the boundary and at all angles. The numerator and denominator integrals of $\bar{\chi}^{(3)}$ are considered separately and each integral is broken into six parts. The six parts consist of the orbit entering the bottom and exiting to the right, entering the bottom and exiting to the left, entering the bottom and exiting the top, entering the top and exiting to the right, entering the top and exiting to the left, and entering on the right and exiting to the left. The six orbit contributions are denoted by subscripts $B R, B L$, $B T, T R, T L$ and $R L$ respectively. Trajectories which go in the reverse direction of those just mentioned are not considered since they cause a factor of two to appear in the numerator and denominator and thus have no effect on the value of the orbit average. Figures 3-la, b, $c, d, e$, and $f$ show the path and local integration coordinates for the $B R, B L, B T, T R, T L$, and $R L$ contributions respectively. The grid cell is $\Delta z$ in width and $\Delta r$ in height. The four corner values $F_{1}, F_{2}, F_{3}$, and $F_{4}$ represent the integrand at the discretized grid points. The local integration coordinates $x, y$ have different origin and meaning for each of the six cases as illustrated in Fig. 3-1. 


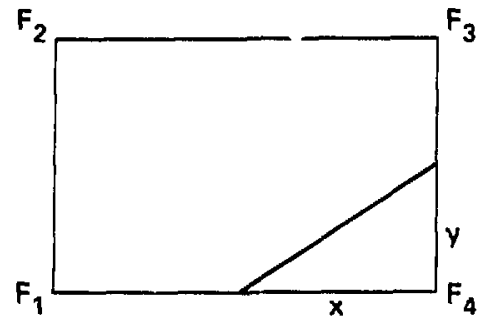

(a)

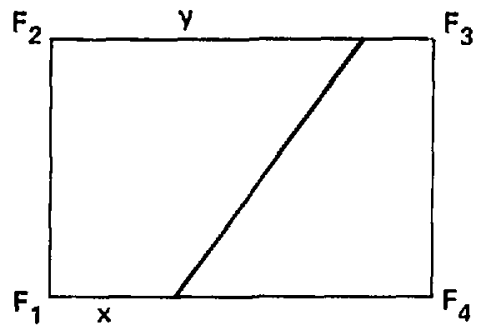

(c)

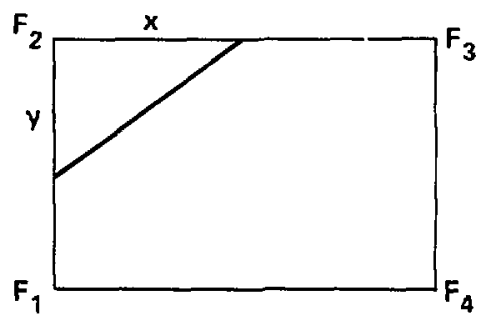

(e)

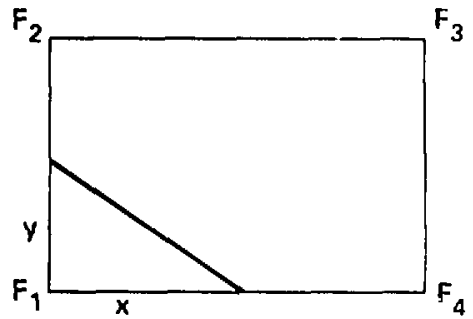

(b)

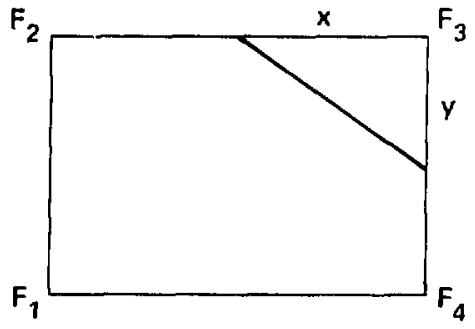

(d)

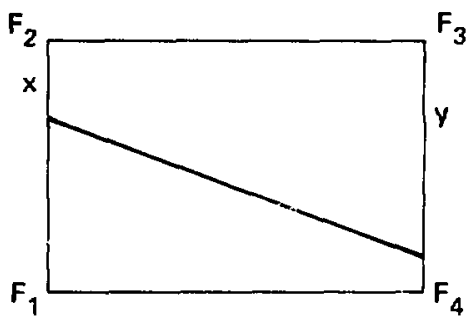

(f)

Fiqure 3-1. Local coordinates and grid coll integration path intersections. (a) Bottom to right (b) Botiom to left (c) Bottom to top (d) Top to right (e) Too to left (f) Right to left. 
The first approximation to $\bar{\chi}^{(3)}$ is to assume the integral is a straight line path length between grid-cell side intersections multiplied by the average of the integrand along the path. The average integrand value is taken to be one half the sum of the integrand values at the grid-cell side intersections. A linear variation of the integrand is assumed along the grid cell sides.

$$
\begin{aligned}
& F(x)=F_{4}+\left(F_{1}-F_{4}\right) x / \Delta z \\
& F(y)=F_{4}+\left(F_{3}-F_{4}\right) y / \Delta r
\end{aligned}
$$

To account for all possible paths, $x$ is integrated from zero to $\Delta z$ and $y$ is integrated from zero to $\Delta r$. The general formula is below.

$\bar{x}_{\text {gen }}^{(5)}=\frac{1}{2} \int_{0}^{\Delta z} d x \int_{0}^{\Delta r} d y(F(x)+F(y))\left(x^{2}+y^{2}\right)^{1 / 2}$

Substituting Eq. (3-71) into Eq. (3-72) yields the BR contribution.

$$
\begin{aligned}
\bar{X}_{B R}^{(5)}=\frac{1}{2} \int_{0}^{\Delta z} d x \int_{0}^{\Delta r} d y\left(2 F_{4}+\left(F_{1}-F_{4}\right) x / \Delta z\right. \\
\left.+\left(F_{3}-F_{4}\right) y / \Delta r\right)\left(x^{2}+y^{2}\right)^{1 / 2} \\
=\frac{1}{2}\left(2 F_{4} T_{1}+T_{2}\left(F_{1}-F_{4}\right) / \Delta z+\left(F_{3}-F_{4}\right) T_{3} / \Delta r\right)
\end{aligned}
$$

For paths from the bottom to the left, the following linear relations for the integrand apply. 
156

$$
\begin{aligned}
& F(x)=F_{1}+\left(F_{4}-F_{1}\right) x / \Delta z \\
& F(y)=F_{1}+\left(F_{2}-F_{1}\right) y / \Delta r
\end{aligned}
$$

Substituting Eq. (3-74) into Eq. (3-72) the BL contribution is obtained.

$$
\begin{aligned}
\bar{x}_{B L}^{(5)=} \frac{1}{2} \int_{0}^{\Delta z} d x \int_{0}^{\Delta r} d y\left(2 F_{1}+\left(F_{4}-F_{1}\right) x / \Delta z\right. \\
\left.\left(F_{2}-F_{1}\right) y / \Delta r\right)\left(x^{2}+y^{2}\right)^{1 / 2} \\
=\frac{1}{2}\left(2 F_{1} T_{1}+T_{2}\left(F_{4}-F_{1}\right) / \Delta z+T_{3}\left(F_{2}-F_{1}\right) / \Delta r\right)
\end{aligned}
$$

For paths from the bottom to the top, the following relations are used.

$$
\begin{aligned}
F(\text { top }) & =F_{2}+\left(F_{3}-F_{2}\right) y / \Delta z \\
F(\text { bottom }) & =F_{1}+\left(F_{4}-F_{1}\right) x / \Delta z
\end{aligned}
$$

Substituting Eq. $(3-76)$ in Eq. $(3-72)$ yields the BT contribution.

$$
\begin{aligned}
\bar{x}_{B T}^{(5)}= & \frac{1}{2} \int_{0}^{\Delta z} d x \int_{0}^{\Delta r} d y\left(F_{1}+F_{2}+\left(F_{4}-F_{1}\right) x / \Delta z\right. \\
& \left.+\left(F_{3}-F_{2}\right) y / \Delta z\right)\left((y-x)^{2}+\Delta r^{2}\right)^{1 / 2} \\
= & \frac{1}{2}\left(\Delta r^{2}\left(\bar{r}_{1}+F_{2}\right) T_{4}+T_{5}\left(F_{3}-F_{2}+F_{4}-F_{1}\right) \Delta r^{2} / \Delta z\right. \\
& \left.+T_{6}\left(F_{3}-F_{2}\right) / \Delta z\right)
\end{aligned}
$$


For paths from the top to the right, the TR contribution is below.

$$
\begin{aligned}
\bar{x}_{T R}^{(5)} & =\frac{1}{2} \int_{0}^{\Delta z} d x\left(\left(2 F_{2}+\left(F_{3}-F_{2}\right) x / \Delta z\right) x^{2} I_{1}(\Delta r / x)\right. \\
& \left.+\left(F_{1}-F_{2}\right)\left(I_{2}(x, \Delta r)-x^{3} / 3\right) / \Delta r\right) \\
& =\frac{1}{2}\left(2 F_{2} T_{1}+\left(F_{3}-F_{2}\right) T_{2} / \Delta z+\left(F_{1}-F_{2}\right) T_{3} / \Delta r\right)
\end{aligned}
$$

For paths from the top to the left, the $\mathrm{TL}$ contribution is below.

$$
\begin{aligned}
\bar{X}_{T L}^{(5)} & =\frac{1}{2} \int_{0}^{\Delta z} d x\left(\left(2 F_{3}+\left(F_{2}-F_{3}\right) x / \Delta z\right) x^{2} I_{1}(\Delta r / x)\right. \\
& \left.+\left(F_{4}-F_{3}\right)\left(I_{2}(x, \Delta r)-x^{3} / 3\right) / \Delta r\right) \\
& =\frac{1}{2}\left(2 F_{3} T_{1}+\left(F_{2}-F_{3}\right) T_{2} / \Delta z+\left(F_{4}-F_{3}\right) T_{3} / \Delta r\right)
\end{aligned}
$$

For paths from the right to the left, the RL contribution is below.

$$
\begin{aligned}
& \bar{X}_{R L}^{(5)=\frac{1}{2}} \int_{0}^{\Delta r}\left(: \int_{0}^{\Delta r} d \jmath\left(F_{2}+F_{3}+\left(F_{1}-F_{2}\right) x / \Delta r\right.\right. \\
& \left.\quad+\left(F_{4}-F_{3}\right) y / \Delta r\right)\left((y-x)^{2}+\Delta z^{2}\right)^{1 / 2} \\
& =\frac{1}{2}\left(\Delta z^{2}\left(F_{2}+F_{3}\right) T_{7}+\Delta z^{2}\left(F_{4}-F_{3}+F_{1}-F_{2}\right) T_{8} / \Delta r\right. \\
& \left.\quad+\left(F_{1}-F_{2}\right) T_{9} / \Delta r\right)
\end{aligned}
$$


The final form of $\bar{x}^{(5)}$ is below,

$$
\bar{x}^{(5)}=\frac{\bar{x}_{\text {num }}^{(5)}}{\bar{x}_{\text {deno }}^{(5)}}
$$

where the numerator consists of six contributions,

$$
\bar{x}_{\text {nuin }}^{(5)}=\bar{x}_{B R}^{(5)}+\bar{x}_{B L}^{(5)}+\bar{x}_{B T}^{(5)}+\bar{x}_{T L}^{(5)}+\bar{x}_{T P}^{(5)}+\bar{x}_{R L}^{(5)}
$$

with $F=x v_{p}^{-1}$. The denominator, $\bar{X}_{\text {deno }}^{(5)}$ is a sum of the same six contributions as in $\bar{x}_{\text {num }}^{(5)}$ however, in this case, $F=v_{p}^{-1}$.

The $\bar{x}^{(5)}$ formulation assumes function values along a path through the grid cell are the average of side intersertion values. The second approximation to $\bar{x}^{(3)}$, denoted $\bar{x}^{(6)}$, obtains a more accurate orbit average by assuming the integrand function has a bilinear variation in a grid cell.

$$
\begin{aligned}
F(r, z)=F_{1} & +\left(F_{2}-F_{1}\right) r / \Delta r+\left(F_{4}-F_{1}\right) z / \Delta z \\
& +\left(F_{1}-F_{2}+F_{3}-F_{4}\right) r z /(\Delta r \Delta z)
\end{aligned}
$$

The trajectory through a grid cell is approximated by a straight line. The grid cell path coordinates for the BR, BL, and $B T$ contribution are shown in Figs. 3-2abc, respectively.

The general $\bar{\chi}^{(6)}$ formula is an integral over all paths of the bilinear integrand formula.

$$
\bar{x}_{\text {gen }}^{(6)}=\int_{0}^{\Delta z} d z_{1} \int_{0}^{\Delta r} d r_{1} \int d \ell F\left(r_{7}, z_{1}\right)
$$




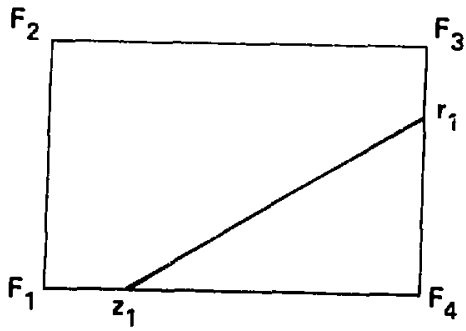

(a)
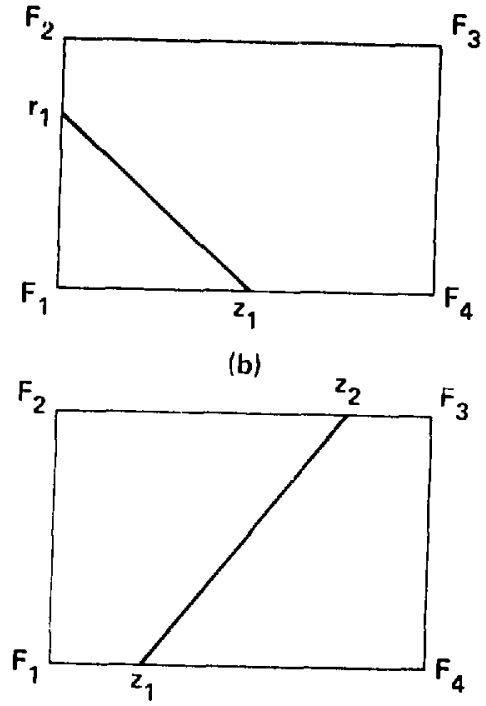

(c) average. (a) $B R$ contribution (b) BL contribution (c) BT contribution. 
Along the bottom right path

$$
z=z_{1}+\left(\Delta z-z_{1}\right) r / r_{1}
$$

and the path integral integration over $\&$ may be expressed in terms of an integration over $r$.

$$
\int d l=\int\left(1+\left(\Delta z-z_{1}\right)^{2} r_{1}^{-2}\right)^{1 / 2} d r
$$

Using Eq. (3-85) in the general formula, Eq. (3-84) yields thr at contribution.

$$
\begin{aligned}
\bar{x}_{B R}^{(6)=} & \int_{0}^{\Delta z} d z_{1} \int_{0}^{\Delta r} d r_{1} \int_{0}^{r} d r\left(1+\left(\Delta z-z_{1}\right)^{2} r_{1}^{-2}\right) \\
& {\left[F_{1}+\left(F_{2}-F_{1}\right) r / \Delta r\right.} \\
& +\left(z_{1} / \Delta_{2}+\left(\Delta z-z_{1}\right) r\right) /\left(r_{1} \Delta z\right)\left(F_{4}-F_{1}\right) \\
& \left.\left.+r\left(z_{1}+\left(\Delta z-z_{1}\right) r / r_{1}\right)\left(F_{1}-F_{2}+F_{3}-F_{4}\right) / \Delta r \Delta z\right)\right] \\
= & F_{4} T_{1}-\left(F_{4}-F_{1}\right) T_{2} /(2 \Delta z)+\left(F_{3}-F_{4}\right) T_{3} /(2 \Delta r) \\
& -\left(F_{1}-F_{2}+F_{3}-F_{4}\right) T 10^{\prime(6 \Delta r \Delta z)}
\end{aligned}
$$

Along the bottom left path shown in Fig. $3-2 b$,

$$
z=z_{1}\left(1-r / r_{1} j\right.
$$

Substituting this relation into Eq. $(3-84)$ yields the $B L$ contribution. 


$$
\begin{aligned}
\bar{x}_{B L}^{(6)}= & \int_{0}^{\Delta z} d z_{1} \int_{0}^{\Delta r} d r_{1} \int_{0}^{r_{1}} d r\left(1+\left(z_{1} / r_{1}\right)^{2}\right)^{1 / 2} \\
& {\left[F_{1}+\left(F_{2}-F_{1}\right) r / \Delta r+\left(1-r / r_{1}\right)\left(F_{4}-F_{1}\right) z_{1} / \Delta z\right.} \\
& \left.+r_{1}\left(1-r_{1} r_{1}\right)\left(F_{1}-F_{2}+F_{3}-F_{4}\right) /(\Delta r \Delta z)\right] d r \\
& =F_{1} T_{1}+\left(F_{4}-F_{1}\right) T_{2} /(2 \Delta z)+\left(F_{2}-F_{1}\right) T_{3} /(2 \Delta r) \\
& +\left(F_{1}-F_{2}+F_{3}-F_{4}\right) T_{10} /(6 \Delta r \Delta z)
\end{aligned}
$$

Along the bottom-to-top path illustrated in Fig. 3-2c,

$$
z=z_{1}+\left(z_{2}-z_{1}\right) r / \Delta r
$$

The BT contribution is obtained by using this relation in Eq. (3-84).

$$
\begin{aligned}
\bar{x}_{B T}^{(6)=} & \int_{0}^{\Delta z} d z_{1} \int_{0}^{\Delta z} d z_{2} \int_{0}^{3 r} d r\left(1+\left(\left(z_{2}-z_{1}\right) / \Delta r\right)^{2}\right)^{1 / 2} \\
& {\left[F_{1}+\left(F_{2}-F_{1}\right) r / \Delta r+\left(z_{1} / \Delta z+r\left(z_{2}-z_{1}\right) /(\Delta r \Delta z)\right)\right.} \\
& \left(F_{4}-F_{1}\right)+\left(r z_{1}+r^{2}\left(z_{2}-z_{1}\right) / \Delta r\right) \\
& \left.\left(F_{1}-F_{2}+F_{3}-F_{4}\right) /(\Delta r \Delta z)\right] \\
= & \frac{1}{2}\left(F_{1}+F_{2}\right) \Delta r^{2} T_{4}+T_{5}\left(F_{3}+F_{4}-F_{1}-F_{2}\right) \Delta r^{2} / \Delta z \\
& +\left(F_{4}-F_{1}-2 F_{2}+2 F_{3}\right) T_{6} /(6 \Delta z)
\end{aligned}
$$

In the same mal.ner that the $B R, B L$, ard $B T$ contributions have been derived, til $T L, T R$ and $R L$ contributions are also derived. The 
top-to-left path formula is below.

$$
\begin{aligned}
\bar{X}_{T L}^{(6)} & =F_{3} T_{1}-\left(F_{3}-F_{2}\right) T_{2} /(2 \Delta z)+\left(F_{4}-F_{3}\right) T_{3} /(2 \Delta r) \\
& -\left(F_{2}-F_{1}+F_{4}-F_{3}\right) T_{10} /(6 \Delta r \Delta z)
\end{aligned}
$$

The top-to-right path formula follows.

$$
\begin{aligned}
\bar{x}_{T R}^{(6)} & =F_{2} T_{1}+\left(F_{3}-F_{2}\right) T_{2} /(2 \Delta z)+\left(F_{1}-F_{2}\right) T_{3} /(2 \Delta r) \\
& +\left(F_{2}-F_{1}+F_{4}-F_{3}\right) T_{10} /(6 \Delta r \Delta z)
\end{aligned}
$$

The right-to-left path formula is below.

$$
\begin{aligned}
\vec{X}_{R L}^{(6)} & =\frac{1}{2}\left(F_{2}+F_{3}\right) \Delta z^{2} T_{7}+\left(F_{4}+F_{1}-F_{3}-F_{2}\right) T_{8} \Delta z^{2} / r \\
& +\left(F_{1}-F_{2}-2 F_{3}+2 F_{4}\right) T_{9} /(6 \Delta r)
\end{aligned}
$$

The $\bar{x}^{(6)}$ orbit average is a ratio of a numerator and denominetor term, each consisting of six contributions,

$$
\bar{x}^{(6)}=\frac{\bar{x}_{\text {num }}^{(6)}}{\bar{x}_{\text {deno }}^{(6)}}
$$

where the numerator is below,

$$
\bar{x}_{n u m}^{(6)}=\bar{x}_{B R}^{(6)}+\bar{x}_{B L}^{(6)}+\bar{x}_{B T}^{(6)}+\bar{x}_{T L}^{(6)}+\bar{x}_{T R}^{(6)} \bar{x}_{R L}^{(6)}
$$

with $F=X v_{p}^{-1}$. The formula for $\bar{X}_{\text {deno }}^{(6)}$ is the same except $F=v_{p}^{-1}$. 
The $\bar{x}^{(5)}$ and $\bar{x}^{(6)}$ orbit averages have singular integrands when $v_{p}$ is zero. The actu=1 orbit average is well behaved so an alternate formula is used in a grid cell where $v_{p}$ goes to zero. In the singular grid cell the general formulas for $\bar{\chi}^{(5)}$ and $\bar{\chi}^{(6)}$ are approximated b. multiplying the grid cell area by the average value of $x_{p}^{-1}$ in the numerator formula and the average value of $v_{p}^{-1}$ in the denominator formula. This approximation resolves the singularity difficulty; however, a consistent formula is not used over the entire mesh. It is desirable to have a consistent formula; thus, a third numerical approximation, $\bar{x}^{(7)}$ is derived for $\bar{x}^{(3)}$. The disadvantage of a consistent treatment is the increased complexity of the iumerical orbit average formula.

The reason the $\bar{x}^{(5)}$ and $\bar{x}^{(6)}$ formulas were singular is because the polvidal velocity in the $r, z$ plane goes to zero at a turning point. To account for this effect, formulas are derived for the squire root singularity displayed below.

$$
\left(2 H-2 \phi(r, z)-p_{\theta}^{2} r^{-2}\right)^{1 / 2}
$$

The $\bar{x}_{\text {gen }}^{(6)}$ forinula is used with $F$ now representing $x$. Also, $F$ is taken ous - the inner path integrai as a constant as the midpoint value given by the bilinear formula as follows,

$$
\begin{aligned}
\bar{X}_{B R}^{(7)}= & \int_{0}^{\Delta z} d z_{1} \int_{0}^{\Delta r} d r_{1} F\left(r_{1} / 2,\left(\Delta z-z_{1}\right) / 2\right) r_{1}-1\left(r_{1}^{2}+\left(\Delta z-z_{1}\right)^{2}\right)^{1 / 2} \\
& \left.\int_{0}^{r_{1}} d r\left(2 H-2 \phi\left(r^{\prime}, z_{1}\right)+\left(\Delta z-z_{1}\right) r / r_{1}\right)-p_{\theta}^{2} r^{-1}\right)^{-1 / 2}
\end{aligned}
$$


where $r^{\prime}$ is the grid radial position plus $r_{1}$. The innermost integral of Eq. (3-95) is done with a trigonometric substitution.

$$
\sin (\theta)=(2 H)^{-1 / 2}\left(2 \phi+p_{0}^{2} r r^{-2}\right)^{1 / 2}
$$

The substitution given by Eq. (3-96) is inserted in Eq. (3-95) by using the following relation.

$$
v_{p}^{-1} d r=(8 H)^{1 / 2} \sin (\theta)\left[\frac{\partial\left(2 \phi+p_{\theta}^{2} r^{-2}\right)}{\partial r}\right]^{-1}
$$

The derivative in Eq. (3-97) is approximated by the difference of $2 \phi+p_{\theta}^{2} r^{-2}$ at the endpoints, divided by the path length. Using Eq. (3-97) in Eq. (3-95), and expressing in terms of $v_{p}$ where the 1 and 2 subscripts on $v_{p}$ refer to the path endpoirits, yields the following result.

$\bar{x}_{B R}^{(7)}=2 \int_{0}^{\Delta z} d z_{1} \int_{0}^{\Delta r} d r_{1} \frac{F\left(r_{1} / 2,\left(\Delta z-z_{1}\right)\left(r_{1}^{2}+\left(\Delta z-z_{1}\right)^{2}\right)^{1 / 2}\right.}{v_{p_{1}}+\frac{v_{p_{2}}}{v_{1}}}$

The averaged function $x$ is assumed to be 1 inear along grid cell sides, and consequently, $v_{p}$ is assumed to have a linear variation a long grid cell sides. In the formulas below, $v_{p}$ is written as $v$ and the subscripts refer to grid cell corners consistent with the way $F$ is subscripted.

$\bar{x}_{B R}^{(7)}=2 \int_{0}^{\Delta z} d z, \int_{0}^{\Delta r} d r_{1}\left(r_{1}^{2}+\left(\Delta z-z_{1}\right)^{2}\right)^{1 / 2}\left(F_{1}+\left(F_{2}-F_{1}\right) r_{1} /(2 \Delta r)\right.$ 


$$
\begin{aligned}
+ & \left(F_{4}-F_{1}\right)\left(\Delta z+z_{1}\right) /(2 \Delta z) \\
+ & \left.\left(F_{1}-F_{2}+F_{3}-F_{4}\right) r_{1}\left(\Delta z+z_{1}\right) /(4 \Delta z \Delta r)\right) \\
& \left(v_{1}+v_{4}+\left(v_{4}-v_{1}\right) z_{1} / \Delta z+\left(v_{3}-v_{4}\right) r_{1} / \Delta r\right)^{-1} \\
= & \left(F_{1}+F_{4}\right) T_{11}+\left(F_{4}-F_{1}\right) T_{12} / \Delta z \\
+ & \left(F_{2}-F_{1}+F_{3}-F_{4}\right) T_{13} /(2 \Delta r) \\
+ & \left(F_{1}-F_{2}+F_{3}-F_{4}\right) T_{14} /(2 \Delta r \Delta z)
\end{aligned}
$$

The bottom-to-left, top-to-right, top-to-left, bottom-to-top, rightto-left path integrals are obtained in a manner similar to the procedure used for the bottom-to-right case.

$$
\begin{aligned}
\bar{x}_{B L}^{(7)}= & \left(F_{1}+F_{4}\right) T_{15}+\left(F_{1}-F_{4}\right) T_{16} / \Delta z \\
& +\left(F_{3}-F_{4}+F_{2}-F_{1}\right) T_{17} /(2 \Delta r) \\
& +\left(F_{4}-F_{3}+F_{2}-F_{1}\right) T_{18} /(2 \Delta r \Delta z) \\
\bar{X}_{T R}^{(7)}= & \left(F_{2}+F_{3}\right) T_{19}+\left(F_{3}-F_{2}\right) T_{20} / \Delta z \\
+ & \left(F_{1}-F_{2}+F_{4}-F_{3}\right) T_{21} /(2 \Delta r) \\
& +\left(F_{2}-F_{1}+F_{4}-F_{3}\right) T_{22} /(2 \Delta r \Delta z)
\end{aligned}
$$




$$
\begin{aligned}
\bar{x}_{T L}^{(7)} & =\left(F_{3}+F_{2}\right) T_{23}+\left(F_{2}-F_{3}\right) T_{24} / \Delta z \\
& +\left(F_{4}-F_{3}+F_{1}-F_{2}\right) T_{25} /(2 \Delta r) \\
& +\left(F_{3}-F_{4}+F_{1}-F_{2}\right) T_{26} /(2 \Delta r \Delta z) \\
\bar{x}_{B T}^{(7)}= & \left(F_{1}+F_{2}\right) T_{27}+\left(F_{4}-F_{1}+F_{3}-F_{2}\right) T_{28} / \Delta z \\
& +\left(F_{4}-F_{1}+F_{3}-F_{2}\right) T_{2 g} /(2 \Delta z) \\
\bar{x}_{R L}^{(7)} & =\left(F_{3}+F_{2}\right) T_{30}+\left(F_{1}-F_{2}+F_{4}-F_{3}\right) T_{31} / \Delta r \\
& +\left(F_{1}-F_{2}+F_{4}-F_{3}\right) T_{32} /(2 \Delta r)
\end{aligned}
$$

The numerical orbit-average approximation to $\bar{\chi}^{(3)}$, which consistently accounts for the velocity singularity, is then given below.

$$
\bar{x}^{(7)}=\frac{1}{2} \frac{\bar{x}_{B R}^{(7)}+\bar{x}_{B L}^{(7)}+\bar{x}_{T L}^{(7)}+\bar{x}_{B T}^{(7)}+\bar{x}_{T R}^{(7)}+\bar{x}_{R L}^{(7)}}{T_{11}+T_{15}+T_{19}+T_{23}+T_{27}+T_{30}}
$$

In addition to the three numerical formulas, $\bar{\chi}^{(5)}, \bar{\chi}^{(6)}$, and $\bar{\chi}^{(7)}$ for the path integral $\bar{\chi}^{(3)}$, there are also three numerical phase-space formulas. The first formula, $\bar{\chi}^{(p s 8)}$ is a numerical implementation of $\bar{x}^{(p s 6)}$. In deriving $\bar{x}^{(p s 8)}$, knowledge of the analytic orbits is used to obtain explicit integration formulas for a grid cell. In two dimensions, the test function which is to be averaged is given below. 
166

$$
x(r, z)=z^{2} e^{\left(\left(r-r_{0}\right) / r_{1}\right)^{2}}
$$

Using the $\bar{x}^{(p s 6)}$ formula, substituting the analytic velocities and Eq. (3-106), the following grid cell formulas result.

$$
\begin{aligned}
& \bar{x}_{\text {deco }}^{(p s 8)}=\left(\omega_{r} \omega_{z}\right)^{-1}\left(\sin ^{-1}\left(z_{B} / z_{1}\right)-\sin ^{-1}\left(z_{A} / z_{1}\right)\right) \\
& \left(\sin ^{-1}\left(\left(r_{B}-r_{0}\right) / r_{1}\right)-\sin ^{-1}\left(\left(r_{A}-r_{0}\right) / r_{1}\right)\right) \\
& \vec{x}_{\text {nun }}^{(p s 8)}=z_{1}^{2}\left(\omega_{r} \omega_{z}\right)^{-1} I_{5}\left(r_{0}, r_{1}, r_{A}, r_{B}\right) \\
& (3-108) \\
& \begin{array}{l}
\left(\sin ^{-1}\left(z_{B} / z_{1}\right)-\sin ^{-1}\left(z_{A} / z_{1}\right)\right. \\
\left.+\left(\sin \left(2 \sin ^{-1}\left(z_{A} / z_{1}\right)\right)-\sin \left(2 \sin ^{-1}\left(z_{B} / z_{1}\right)\right)\right) / 2\right) / 2
\end{array} \\
& \begin{array}{l}
\left(\sin ^{-1}\left(z_{B} / z_{1}\right)-\sin ^{-1}\left(z_{A} / z_{1}\right)\right. \\
\left.+\left(\sin \left(2 \sin ^{-1}\left(z_{A} / z_{1}\right)\right)-\sin \left(2 \sin ^{-1}\left(z_{B} / z_{1}\right)\right)\right) / 2\right) / 2
\end{array}
\end{aligned}
$$

$(3-107)$

In Eq. (3-107) and Eq. (3-108), the grid cell extends radially from $r_{A}$ to $r_{B}$ and axially from $z_{A}$ to $z_{B}$. The numerical orbit-average is then the ratio of contributions from all grid cells.

$$
\bar{x}^{(p s 8)}=\frac{\bar{x}_{\text {mum }}^{(p s 8)}}{\bar{x}_{\text {den }}^{(p s 8)}}
$$

The numerator and denominator of $\bar{x}^{(p s 8)}$ both depend on oscillation frequencies; however $\bar{x}^{(p s 8)}$ itself is independent of the $\omega_{r}$ and $\omega_{z}$ frequencies. The $\bar{x}^{(p s 8)}$ formula is particular to the harmonic oscillator potential and the chosen test function of Eq. (3-106) used 
in the AV code. The main utility of $\bar{\chi}^{(p s 8)}$ is to compare results with the other numerical orbit-average formulas. The frequency independence of the $\bar{x}^{(p s 8)}$ orbit average means the other, more general, orbit-average formulas are not at a disadvantage even though they do not account for this aspect of the precise orbit.

The numerical $\bar{x}^{(p s 5)}$ orbit average, denoted $\bar{x}^{\text {(ps9) }}$, is implemented in a grid cell as an average $x$ value times the grid cell area. The $\bar{x}^{(p s 9)}$ denominator is the grid cell area as shown belo'.

$$
\begin{aligned}
\bar{x}_{\text {deno }}^{(p s 9)} & =\int d r d z \\
& =\Delta r \Delta z
\end{aligned}
$$

The $\bar{x}^{(p s 9)}$ numerator is calculated by assuming a bi iinear $x$ variation in a grid cell.

$$
\begin{aligned}
\bar{x}_{\text {num }}^{(p s 9)} & =\int\left(x_{1}+\left(x_{2}-x_{1}\right) r / \Delta r+\left(x_{4}-x_{1}\right) z / \Delta z\right. \\
& \left.+\left(x_{1}-x_{2}+x_{3}-x_{4}\right) r z /(\Delta r \Delta z)\right) d r d z \\
& =\left(x_{1}+x_{2}+x_{3}+x_{4}\right) \Delta r \Delta z / 4
\end{aligned}
$$

The final forin $0: \bar{x}^{(p s 9)}$ iri a rectangular grid is then a ratio of the sum of numerator and denominator contributions as indicated below. 


$$
\begin{aligned}
\bar{x}^{(p s 9)} & =\sum_{i}\left(\bar{x}_{\text {num }}^{(p s 9)} / \bar{x}_{\text {deno }}^{(p s 9)}\right)_{i} \\
& =\sum_{i}\left(\left(x_{1}+x_{2}+x_{3}+x_{4}\right) / 4\right)_{i}
\end{aligned}
$$

For the case of a potential well, which is not rectangular, the boundary of the allowed orbit region passes through a grid cell. Figures $3-3 a$ to $h$ show the case of the boundary intersecting the bottom and right, the bottom and left, the bottom and top to the right, the bottom and top to the left, the top and right, the top and left, the right and left downward, and the right and left upward grid-cell sides respectively. In assessing the grid cell contribution it must be determined which of the four regions are in the allowed orbit region. Thus, for each grid cell having a boundary intersection, only some of the formulas below are used. The formulas for contributions from regions one-to-four are written in terms of functions $Z_{n}$ and $U_{n}$, with appropriate arguments. The subscript $n$ denotes region one-to-four. In what follows, the $x$ subscript indicates either a numerator (num) or a denominator (deno) contribution on tis left side of the equal sign, or the usua? grid-cell corner values in the arguments of $Z_{n}$ and $U_{n}$. The boundary intersection is contained in the argument with a region designation.

$$
\begin{aligned}
\bar{x}_{\text {num }}^{(p s g)}(B R n)= & z_{n}\left(x_{1},\left(x_{2}-x_{1}\right) / \Delta r,\left(x_{4}-x_{1}\right) / \Delta z,\right. \\
& \left.\left(x_{1}-x_{2}+x_{3}-x_{4}\right) /(\Delta r \Delta z)\right)
\end{aligned}
$$



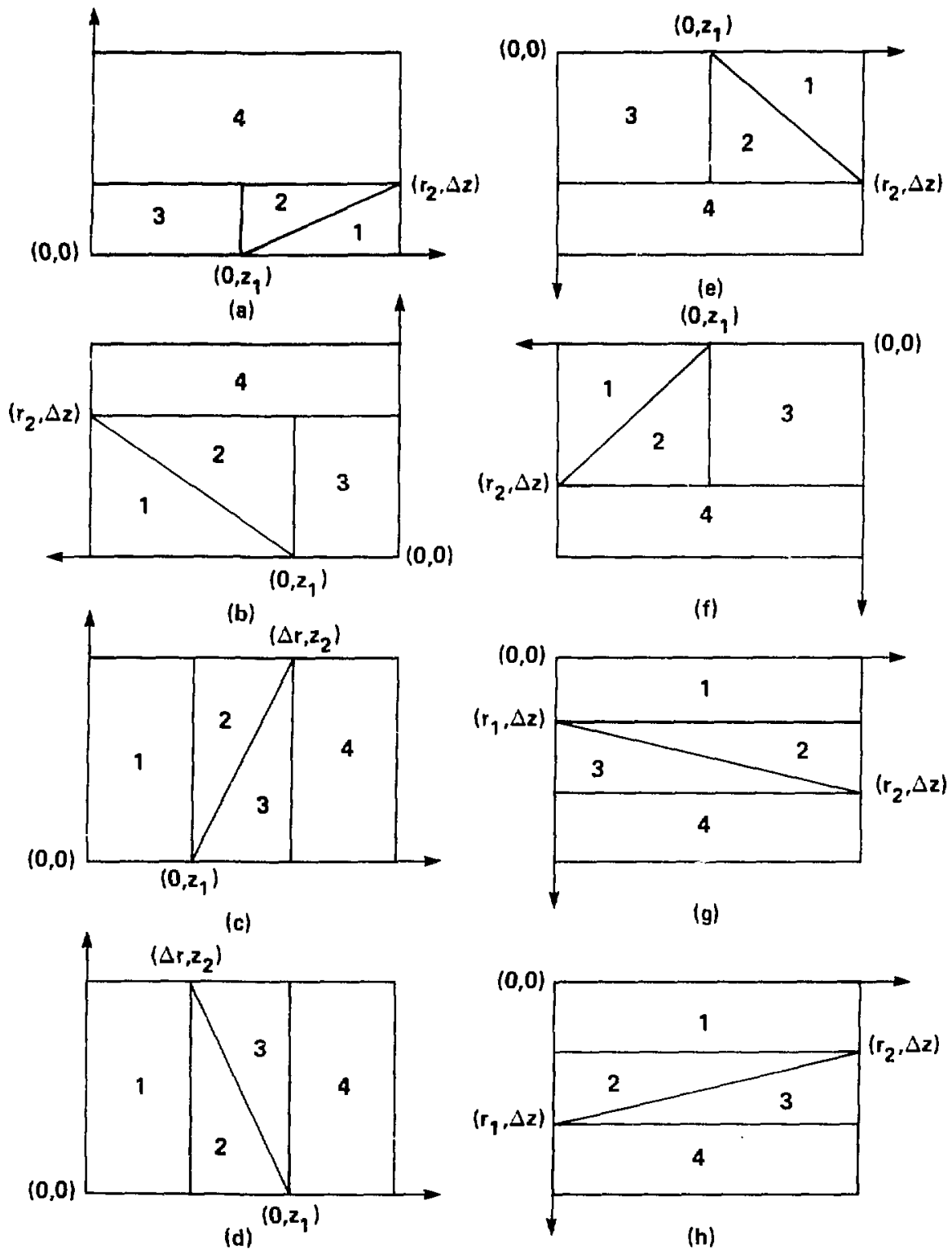

Fiqure $3-3$. The four regions and coordinate orientation for for potential boundary grid cell intersections. (a) BR intersection (b) BL intersection (c) BT intersection to the right (d) BT intersection to the left (e) TR intersection (f) TL intersection (g) RL intersection downward (h) RL intersection upward. 


$$
\begin{aligned}
\bar{x}_{\text {num }}^{(p s g)}(B L n)= & z_{n}\left(x_{4},\left(x_{3}-x_{4}\right) / \Delta r,\left(x_{1}-x_{4}\right) / \Delta z,\right. \\
& \left.\left(x_{4}-x_{3}+x_{2}-x_{1}\right) /(\Delta r \Delta z)\right) \\
\bar{x}_{\text {num }}^{(p s 9)}(T R n)= & z_{n}\left(x_{2},\left(x_{1}-x_{2}\right) / \Delta r,\left(x_{3}-x_{2}\right) / \Delta z,\right. \\
& \left.\left(x_{2}-x_{1}+x_{4}-x_{3}\right) /(\Delta r \Delta z)\right) \\
\bar{x}_{\text {num }}^{(p s 9)}(T L n)= & z_{n}\left(x_{3},\left(x_{4}-x_{3}\right) / \Delta r,\left(x_{2}-x_{3}\right) / \Delta z,\right. \\
& \left.\left(x_{3}-x_{4}+x_{1}-x_{2}\right) /(\Delta r \Delta z)\right)
\end{aligned}
$$

The denominator contributions for $B R_{n}, B L_{n}, T R_{n}$ and $T L_{n}$ are all identical .

$$
\begin{aligned}
& \bar{x}_{\text {deno }}^{(p s 9)}(B R 1)=\bar{\chi}_{\text {deno }}^{(p s 9)}(B L 1)=\bar{x}_{\operatorname{deno}}^{(p s 9)}(T R 1)=\bar{x}_{\text {deno }}^{(p s 9)}(T L 1) \\
& =r_{2}\left(\Delta z-z_{1}\right) / 2 \\
& \bar{x}_{\text {deno }}^{(p s 9)}(B R 2)=\bar{x}_{\text {deno }}^{(p s 9)}(B L 2)=\bar{x}_{\text {deno }}^{(p s 9)}(T R 2)=\bar{x}_{\text {deno }}^{(p s 9)}(T L 2) \\
& =r_{2}\left(\Delta z-z_{7}\right) / 2 \\
& \bar{x}_{\text {deno }}^{(p s 9)}(B R 3)=\bar{x}_{\text {deno }}^{(p s 9)}(B L 3)=\bar{x}_{\text {deno }}^{(p s 9)}(T R 3)=\bar{x}_{\text {deno }}^{(p s 9)}(T L 3) \\
& =r_{2}{ }_{1}
\end{aligned}
$$




$$
\begin{aligned}
\bar{X}_{\text {deno }}^{(p s 9)}(B R 4) & =\bar{\chi}_{\text {deno }}^{(p s 9)}(B L 4)=\bar{\chi}_{\text {deno }}^{(p s 9)}(T R 4)=\bar{\chi}_{\text {deno }}^{(p s 9)}(T L 4) \\
& =\Delta z\left(\Delta r-r_{2}\right)
\end{aligned}
$$

For the potential boundary intersections from the bottom-to-the-top or the right-to-the-left, the following formulas apply.

$$
\begin{aligned}
\bar{x}_{\text {num }}^{(p s 9)}(B T n)= & u_{n}\left(x_{1},\left(x_{2}-x_{1}\right) / \Delta r,\left(x_{4}-x_{1}\right) / \Delta z,\right. \\
& \left.\left(x_{1}-x_{2}+x_{3}-x_{4}\right) /(\Delta r \Delta z), z_{1}, z_{2}, \Delta r\right) \\
\bar{x}_{\text {num }}^{(p s 9)}(R L n)= & u_{n}\left(x_{2},\left(x_{3}-x_{2}\right) / \Delta z,\left(x_{1}-x_{2}\right) / \Delta r,\right. \\
& \left.\left(x_{2}-x_{3}+x_{4}-x_{1}\right) /(\Delta r \Delta z), r_{1}, r_{2} \Delta z\right)
\end{aligned}
$$

The denominators are defined as follows.

$$
\begin{aligned}
& \bar{x}_{\text {deno }}^{(p s 9)}(B T 1)=\Delta r \min \left(z_{1}, z_{2}\right) \\
& \bar{x}_{\text {deno }}^{(p s 9)}(R L 1)=\Delta r \min \left(r_{1}, r_{2}\right) \\
& \bar{x}_{\text {deno }}^{(p s 9)}(B T 2)=\Delta r\left|z_{2}-z_{1}\right| \\
& \bar{x}_{\text {deno }}^{(p s 9)}(R L 2)=\Delta z\left|r_{2}-r_{1}\right| \\
& \bar{x}_{\text {deno }}^{\prime p s 9)}(B T 3)=\Delta r\left|z_{2}-z_{1}\right|
\end{aligned}
$$




$$
\begin{aligned}
& \bar{X}_{\text {deno }}^{(p s 9)}(R L 3)=\Delta z\left|r_{2}-r_{1}\right| \\
& \bar{X}_{\text {deno }}^{(p s 9)}(B T 4)=\Delta r\left(\Delta z-\max \left(z_{1}, z_{2}\right)\right)
\end{aligned}
$$

$$
\bar{x}_{\text {deno }}^{(p s 9)}(R L 4)=\Delta z\left(\Delta r-\max \left(r_{1}, r_{2}\right)\right)
$$

In previous discussion, $\bar{x}^{(p s 7)}$ was derived as an approximation to $\bar{x}^{(p s 6)}$. The difference between these formulas is a replacement of $v_{p}^{?}$ for $v_{r} v_{z}$. The numerical approximation to $\bar{x}^{\langle p s\rangle)}$, denoted $\bar{x}^{(p s 10)}$, is obtained by arcuming a bilinear variation of $x$ and $v_{p}^{2}$ in a rectangular grid cell.

$$
\begin{aligned}
& \bar{x}_{\text {num }}^{(p \log )}=\int_{0}^{\Delta r} \int_{0}^{\Delta z} \frac{g_{1}+g_{2} r+g_{3} z+g_{4} r z}{h_{1}+\frac{h_{2} r+h_{3} z+h_{4} r z}{h_{2}}} d r d z \\
& =\int_{0}^{\Delta r}\left[\left(\frac{g_{1}+g_{2} r}{h_{3}+h_{4} r}-\frac{\left(h_{1}+h_{2} r\right)\left(g_{3}+g_{4} r\right)}{\left(h_{3}+h_{4} r\right)^{2}}\right)\right. \\
& \log \left(1+\frac{h_{3}+h_{4} r}{h_{1}+h_{2} r} \Delta z\right) \\
& \left.+\frac{g_{3}+g_{4} r}{h_{3}+h_{4} r} \Delta z\right] d r \\
& \bar{x}_{\text {deno }}^{(\operatorname{ps~} 10)}=\int_{0}^{\Delta r} \frac{d r}{h_{3}+h_{4} r} \log \left(1+\frac{h_{3}+h_{4} r}{h_{1}+h_{2} r} \Delta z\right)
\end{aligned}
$$


In En. (3-127) and Eq. (3-128), the subscripted $g$ and $h$ parameters are related to $x$ and $v_{p}$ as follows.

$$
\begin{aligned}
& g_{1}=x_{1} \\
& g_{2}=\left(x_{2}-x_{1}\right) / \Delta r \\
& g_{3}=\left(x_{4}-x_{1}\right) / \Delta z \\
& g_{4}=\left(x_{1}-x_{2}+x_{3}-x_{4}\right) /(\Delta r \Delta z) \\
& n_{1}=v_{p_{1}}^{2} \\
& h_{2}=\left(v_{p_{2}}^{2}-v_{p_{1}}^{2}\right) / \Delta r \\
& n_{3}=\left(v_{p_{4}}^{2}-v_{p_{1}}^{2}\right) / \Delta z \\
& n_{4}=\left(v_{p_{1}}^{2}-v_{p_{2}}^{2}+v_{p_{3}}^{2}-v_{p_{4}}^{2}\right) /(\Delta r \Delta z)
\end{aligned}
$$

The orbit average is then a ratio of a sum of numerator and denominator contributions from all grid cells.

$$
\bar{x}^{(p s 10)}=\frac{\bar{x}_{\text {num }}^{(p s 10)}}{\bar{x}_{\text {deno }}^{(p s 10)}}
$$

For the case of an orbit potential passing through a grid cell, formulas are derived with four individual contributions per cell. Figures 3-3a to $h$ depict the various possibilities as discussed for the $\bar{x}^{(p s 9)}$ orbit average. The same rotation is used 
174

for $\bar{x}^{(p s 10)}$ as for $\bar{x}^{(p s 9)}$, and the formulas are expressed in terms of functions $k_{n}^{\text {mum }}, k_{n}^{\text {demo }}, N_{n}^{\text {nun }}$ and $N_{n}^{\text {demo }}$, which are defined in Appendix A.

$$
\begin{aligned}
& \bar{x}_{n u m}^{(p s 10)}(B R n)=k_{n}^{n u m}\left(x_{1},\left(x_{2}-x_{1}\right) / \Delta r,\left(x_{4}-x_{1}\right) / \Delta z,\right. \\
& \left(x_{1}-x_{2}+x_{3}-x_{4}\right) /(\Delta r \Delta z) \\
& v_{p_{1}}^{2},\left(v_{p_{2}}^{2}-v_{p_{1}}^{2}\right) / \Delta r,\left(v_{p_{4}}^{2}-v_{p_{1}}^{2}\right) / \Delta z \\
& \left.\left(v_{p_{1}}^{2}-v_{p_{2}}^{2}+v_{p_{3}}^{2}-v_{p_{4}}^{2}\right) /(\Delta r \Delta z)\right) \\
& \bar{x}_{n u m}^{(p \operatorname{lo})}(B L n)=k_{n}^{n u m m}\left(x_{4},\left(x_{3}-x_{4}\right) / \Delta r,\left(x_{1}-x_{4}\right) / \Delta z\right. \text {. } \\
& \left(x_{4}-x_{3}+x_{2}-x_{1}\right) /(\Delta r \Delta z) \\
& v_{p_{4}}^{2},\left(v_{p_{3}}^{2}-v_{p_{4}}^{2}\right) / \Delta r,\left(v_{p_{1}}^{2}-v_{p_{4}}^{2}\right) / \Delta z, \\
& \left.\left(v_{p_{4}}^{2}-v_{p_{3}}^{2}+v_{p_{2}}^{2}-v_{p_{1}}^{2}\right) /(\Delta r \Delta z)\right) \\
& \bar{x}_{n u m}^{(p s 10)}(T R n)=k_{n}^{n u m}\left(x_{2},\left(x_{1}-x_{2}\right) / \Delta r,\left(x_{3}-x_{2}\right) / n z,\right. \\
& \left(x_{2}-x_{1}+x_{4}-x_{3}\right) /(\Delta r \Delta z) \\
& v_{p_{2}}^{2},\left(v_{p_{1}}^{2}-v_{p_{2}}^{2}\right) / \Delta r,\left(v_{p_{3}}^{2}-v_{p_{2}}^{2}\right) / \Delta z \\
& \left.\left(v_{p_{2}}^{2}-v_{p_{1}}^{2}+v_{p_{4}}^{2}-v_{p_{3}}^{2}\right) /(\Delta r \Delta z)\right)
\end{aligned}
$$


175

$$
\begin{aligned}
& \bar{x}_{n u m}^{(p s 10)}(T L n)=k_{n}^{n u m}\left(x_{3},\left(x_{4}-x_{3}\right) / \Delta r,\left(x_{2}-x_{3}\right) / \Delta z,\right. \\
& \left(x_{3}-x_{4}+x_{1}-x_{2}\right) /(\Delta r \Delta z) \\
& v_{p_{3}}^{2},\left(v_{p_{4}}^{2}-v_{p_{3}}^{2}\right) / \Delta r,\left(v_{p_{2}}^{2}-v_{p_{3}}^{2}\right) / \Delta z \\
& \left.\left(v_{p_{2}}^{2}-v_{p_{1}}^{2}+v_{p_{4}}^{2}-v_{p_{3}}^{2}\right) /(\Delta r \Delta z)\right) \\
& \bar{x}_{\text {deno }}^{(p s 10)}(B R n)=k_{n}^{\text {deno }}\left(v_{p_{1}}^{2},\left(v_{p_{2}}^{2}-v_{p_{1}}^{2}\right) / \Delta,\left(v_{p_{4}}^{2}-v_{p_{1}}^{2}\right) / \Delta,\right. \\
& \left.\left(v_{p_{1}}^{2}-v_{p_{2}}^{2}+v_{p_{3}}^{2}-v_{p_{4}}^{2}\right) /(\Delta r \Delta z)\right) \\
& \bar{x}_{\text {deno }}^{(p s 10)}(B L n)=k_{n}^{\text {deno }}\left(v_{p_{4}}^{2},\left(v_{p_{3}}^{2}-v_{p_{4}}^{2}\right) / \Delta r,\left(v_{p_{1}}^{2}-v_{p_{4}}^{2}\right) / \Delta z,\right. \\
& \left.\left(v_{p_{4}}^{2}-v_{p_{3}}^{2}+v_{p_{2}}^{2}-v_{p_{1}}^{2}\right) /(\Delta r \Delta z)\right) \\
& \bar{x}_{\text {deno }}^{\text {(ps I0) }}(T R n)=k_{n}^{\text {deno }}\left(v_{p_{2}}^{2},\left(v_{p_{1}}^{2}-v_{p_{2}}^{2}\right) / \Delta r,\left(v_{p_{3}}^{2}-v_{p_{2}}^{2}\right) / \Delta z,\right. \\
& \left.\left(v_{p_{2}}^{2}-v_{p_{1}}^{2}+v_{p_{4}}^{2}-v_{p_{2}}^{2}\right) /(\Delta r \Delta z)\right) \\
& \underset{\text { ieno }}{\vdots 1:, 10)}(T L n)=k_{n}^{\text {deno }}\left(v_{p_{3}}^{2},\left(v_{p_{4}}^{2}-v_{p_{3}}^{2}\right) / \Delta r,\left(v_{p_{2}}^{2}-v_{p_{3}}^{2}\right) / \Delta,\right. \\
& \left.\left(v_{p_{2}}^{2}-v_{p_{1}}^{2}+v_{p_{4}}^{2}-v_{p_{3}}^{2}\right) /(\Delta r \Delta z)\right)
\end{aligned}
$$


The $k_{n}^{\text {num }}$ functions are defined in Appendix $A$ with eight consecutive arguments which are $g_{1}, g_{2}, g_{3}, g_{4}, h_{1}, h_{2}, h_{3}$, and $h_{4}$ respectiveiy. The $k_{n}^{\text {deno }}$ functions are defined in Appendix $A$ with four consecutive arguments which are $h_{1}, h_{2}, h_{3}$, and $h_{4}$, respectively. To avoid the zero of $v_{p}^{2}$ at the orbit boundary, an additional parameter $\alpha$ is introduced as the exponent of the bilinear $v_{p}^{2}$ fit. For orbit potential boundary intersections from the bottomto-the-top or from the right-to-the-left, the following formulas are used.

$$
\begin{aligned}
\bar{x}_{n u m}^{(p s 10)}(B T n)= & N_{n}^{n u m}\left(x_{1},\left(x_{2}-x_{1}\right) / \Delta r,\left(x_{4}-x_{1}\right) / \Delta z,\right. \\
& \left(x_{1}-x_{2}+x_{3}-x_{4}\right) /(\Delta r \Delta z), \\
& v_{p_{1}}^{2},\left(v_{p_{2}}^{2}-v_{p_{1}}^{2}\right) / \Delta r,\left(v_{p_{4}}^{2}-v_{p_{1}}^{2}\right) / \Delta z, \\
& \left.\left.\left(v_{p_{1}}^{2}-v_{p_{2}}^{2}+v_{p_{3}}^{2}-v_{p_{4}}^{2}\right) /(\Delta r \Delta z)\right), z_{1}, z_{2}, \Delta r\right) \\
\bar{x}_{d e n 0}^{(p s 10)}(B T n)= & N_{n}^{d e n o}\left(v_{p_{1}}^{2},\left(v_{p_{2}}^{2}-v_{p_{1}}^{2}\right) / \Delta r,\left(v_{p_{4}}^{2}-v_{p}^{2}\right) / \Delta z,\right. \\
& \left.\left(v_{p_{1}}^{2}-v_{p_{2}}^{2}+v_{p_{3}}^{2}-v_{p_{4}}^{2}\right) /(\Delta r \Delta z), z_{1}, z_{2}, \Delta r\right) \\
\bar{x}_{n u m}^{(p s 10)}(R L n)= & N_{n}^{n u m}\left(x_{2},\left(x_{3}-x_{2}\right) / \Delta z,\left(x_{1}-x_{2}\right) / \Delta r,\right. \\
& \left(x_{2}-x_{3}+x_{4}-x_{1}\right) /(\Delta r \Delta z),
\end{aligned}
$$




$$
\begin{aligned}
& v_{p_{2}}^{2},\left(v_{p_{3}}^{2}-v_{p_{2}}^{2}\right) / \Delta z,\left(v_{p_{1}}^{2}-v_{p_{2}}^{2}\right) / \Delta r, \\
& \left.\left(v_{p_{2}}^{2}-v_{p_{3}}^{2}+v_{p_{4}}^{2}-v_{p_{1}}^{2}\right) /(\Delta r \Delta z), r_{1}, r_{2}, \Delta z\right) \\
\bar{X}_{\text {deno }}^{(p s 10)}(R L n)= & N_{n}^{\text {deno }}\left(v_{p_{2}}^{2},\left(v_{p_{3}}^{2}-v_{p_{2}}^{2}\right) / \Delta z,\left(v_{p_{1}}^{2}-v_{p_{2}}^{2}\right) / \Delta r,\right. \\
& \left.\left(v_{p_{2}}^{2}-v_{p_{3}}^{2}+v_{p_{4}}^{2}-v_{p_{1}}^{2}\right) /(\Delta r \Delta z), r_{1}, r_{2}, \Delta z\right)
\end{aligned}
$$

The $N_{n}^{\text {num }}$ functions are defined in Appendix $A$ with eleven consecutive arguments, $g_{1}, g_{2}, g_{3}, g_{4}, h_{1}, h_{2}, h_{3}, h_{4}, z_{1}, z_{c}$, and $z_{3}$, respectively. The $N_{n}^{\text {deno }}$ functions are defined in Appendix $A$ with seven consecutive arguments $h_{1}, h_{2}, h_{3}, h_{4}, z_{1}, z_{2}$, and $z_{3}$, respectively.

\subsection{Av Code Results}

The orbit average formulas are compared numerically against the orbit average definition $\bar{x}^{(0)}$, computed using the trapezoidal integration method. A seven-point integration scheme was checked against the trapezoidal method and it was found $\bar{x}^{(0)}$ did not change up to three decimal places. Thus, in the discussion of results to follow, the $\bar{x}^{(0)}$ orbit average value obtained by the trapezoidal method is taken to be the proper orbit average value.

In one dimension, an exponential $x(r)$ is chosen as the function to be averaged. 


$$
x(r)=e^{\left(r-r_{0}\right) / r_{1}}
$$

The objective in choosing $x(r)$ is to select a smooth function havirig a reasonable variation between $r_{0}-r_{1}$ and $r_{0}+r_{1}$. The AV code was run with the following parameter values: $r_{0}=5, r_{1}=1, \omega_{r}=.198$, $\Delta t=.005, \varphi_{r}=0$. The $r$ grid had 2000 points and the orbit was followed 6348 steps. The frequency $\omega_{r}$ and the number of steps multiplied by $\Delta t$ were chosen so the sine argument of the analytic orbit, $r(t)$, went from zero to $2 \pi$. The oscillation amplitude bounds the orbit between 4 and 6 , and over this range $x(r)$ varies from .37 to 2.72 . The results are $\bar{x}^{(0)}=1.2661$, and $\bar{x}^{(4)}=1.2637$. As verification of the $\bar{\chi}^{(0)}$ value the following trigonometric substitution,

$$
\cos (\theta)=\left(r-r_{0}\right) / r_{1}
$$

is made in $\bar{x}^{(4)}$ to yield,

$$
\bar{x}^{(4)}=\frac{1}{\pi} \int_{0}^{\pi} e^{\cos (\theta)} d \theta
$$

The formula 3-145 is the integral definition of the inaginary Bessel function of order zero and argument one, $I_{0}(1)=1.2661$. The orbit average formula, $\bar{x}^{(4)}$, is thus well-approximated by the numerical integration formula.

In two dimensions the averaged function $\chi(r, z)$, given by formula 3-106, is exponential in $r$ and quadratic in $z$. Due to these symmetries it is only necessary to integrate over one-fourth of the 
allowed orbit region. As in the one dimensional case $\bar{x}^{(0)}$, computed using the trapezoidal integration approximation, is compared with numerical orbit average formulas. As a brief review, the twodimensional numerical orbit-averaged formulas are $\bar{\chi}^{\langle 5\rangle}, \bar{\chi}^{(6)}$, $\bar{x}^{(7)}, \bar{x}^{(p s 8)}, \bar{x}^{\langle p s 9)}$ and $\bar{x}^{(p s 10)}$. The path integral formulas, $\bar{x}^{(5)}, \bar{x}^{(6)}$ and $\bar{x}^{(7)}$, integrate along a straight-l ine path in a grid cell and then integrate over all possible straight-line paths. The path integral formula $\bar{x}^{(5)}$ integrates over a grid cell, assuming a linear variation along grid-cell sides of $x_{p}^{-1}$ for the numerator and $v_{p}^{-1}$ for the denominator. The integration over the path is the path length multiplied by half the sum of $x_{p}^{-1}$ for the numerator and $v_{p}^{-1}$ for the denominator. The integration over the path is the pat'l length multiplied by half the sum of $x_{p}^{-1}$ or $v_{p}^{-1}$ at the grid cell side intersections. The path integral formula, $\bar{\chi}^{(6)}$, integrates over a grid cell assuming a bilinear variation of $x_{p}^{-1}$ for the numerator and $v_{p}^{-1}$ for the denominator. The integral along the path is obtained by using the equation of the path line to convert $\int d \ell$ to $\int(d \ell / d r) d r$. The $\bar{X}^{(7)}$ path integral assumes $x$ has a bilinear variation in a grid cell, but may be taken to be a constant along the path line at the midpoint path value given by the bilinear formula. The path integral in the numerator and denominator contribution is then an integral only over $v_{p}^{-1}$. The path integral is calculated using a trigonometric substitution resulting in the reciprocal of the sum of the path endpoint $v_{p}$ values. The firial form of the $-(7)$ formula is then obtained by assuming $v_{p}$ varies linearly along the path for the integrals over all paths. The phase-space derived 
formulas are $\bar{\chi}^{(p s 8)}$ and $\bar{x}^{(p s 9)}$. The $\bar{\chi}^{(p s 10)}$ formula is a numerical orbit average of the $\bar{x}^{(p s 7)}$ approximation to $\bar{x}^{(p s 6)}$. The $\bar{x}^{\text {(ps8) }}$ formula, except for the $I_{5}$ integral, is essentially analytic in a grid cell. The $x(r, z)$ function to be averaged has been incorporated into $\bar{x}^{(p s 8)}$ as well as the analytic orbits. The $\bar{x}^{(p s 9)}$ formula is an area average of $x(r, z)$ normalized to the total area. Assuming a bilinear $\chi(r, z)$ variation in a grid cell results in a numerator formula which is one-fourth the sum of the four grid-cell corner values. The $\bar{x}^{(p s 10)}$ formula is an integral over the grid cell area, assuming $x(r, z)$ is bilinear and $v_{p}^{2}$ is bilinear.

A 11 of these numerical orbit-average formulas are generally applicable except $\bar{\chi}^{(p s 8)}$, which is particular to the harmonic oscillator potential. In examining the results of a two-dimensional AV-code run, it is not expected $\bar{x}^{(p s 9)}$ would provide a good orbit average value since it assumes a larger orbit region is available. The $\bar{x}^{(p s 9)}$ formula is derived assuming only two constants of the motion exist wher, for the uncoupled two-dimensional oscillator there are, including $p_{\theta}$, three. The object in considering $\bar{x}^{(p s 9)}$ is to gauge the amount of error in applying this formula when a third constant of the motior mxists but is not taken into account. The $\bar{\chi}^{(p s 10)}$ formula is an approximation to $\bar{\chi}^{(p s 8)}$ which does not require the analytic orbits. In a case of basically harmonic motion, where the Hamiltonian has weak coupling or anharmonicity, $\bar{x}^{(p s 10)}$ may be a better approximation than $\bar{x}^{(p s 8)}$. The $\bar{x}^{(p s 5)}, \bar{x}^{(p s 6)}$ and $\bar{x}^{(p s 7)}$ orbit average are evaluated to measure the agreement of $\bar{x}^{(0)}$ with the path integral formulas, which do not include knowledge of the 
exact constants of the motion. In other words, these formulas are the same whether or not there are two or three constants of the motion. All of the numerical orbit-average formulas assume the actual orbit encounters all allowed space at every angle. In the AV code, for a particular orbit, the analytic $r$, and $z$ formulas show the orbit is actually a lissajous figure. This is because, numerically, the frequencies $\omega_{r}$ and $\omega_{z}$ are rational numbers. Consequently, a orbit is periodic and as a result there are always areas that are not encountered. In addition, the analytic positions and velocities may be used to express the velocity ratio in terms of position.

$$
\frac{v_{r}}{v_{z}}= \pm \frac{\omega_{r}}{\omega_{z}}\left(\frac{r_{1}^{2}-\left(r-r_{0}\right)^{2}}{z_{1}^{2}-z^{2}}\right)^{1 / 2}
$$

From Eq. (3-146), it can be seen that a particular orbit can pass through a fixed position at only two specific angles. Figure 3-4 illustrates an orbit with $\omega_{r}=1, \omega_{z}=4, r_{0}=5, r_{1}=1, z_{1}=1$, $\varphi_{z}=0$, and $\varphi_{r}=0$. This shows the orbit periodicity and the occurrence of only two angles at the crossings. The numerical orbitaverage formulas, in assuming the available area is covered, are actually dealing with a distribution of initial phases $\varphi_{r}$ and $\varphi_{z}$ and thus, a range of periodic orbits or equivalently as will be shown later, a nearby orbit having irrational frequency ratio $\omega_{r} / \omega_{z}$, which would then cover the entire area. Figure 3-5 shows an orbit which has the same parameters as that in Fig. $3-4$, except $\varphi_{r}=.3$ and $\varphi_{z}=.1$. Comparing Figs. $3-4$ and $3-5$, it is clear each closed-orbit 


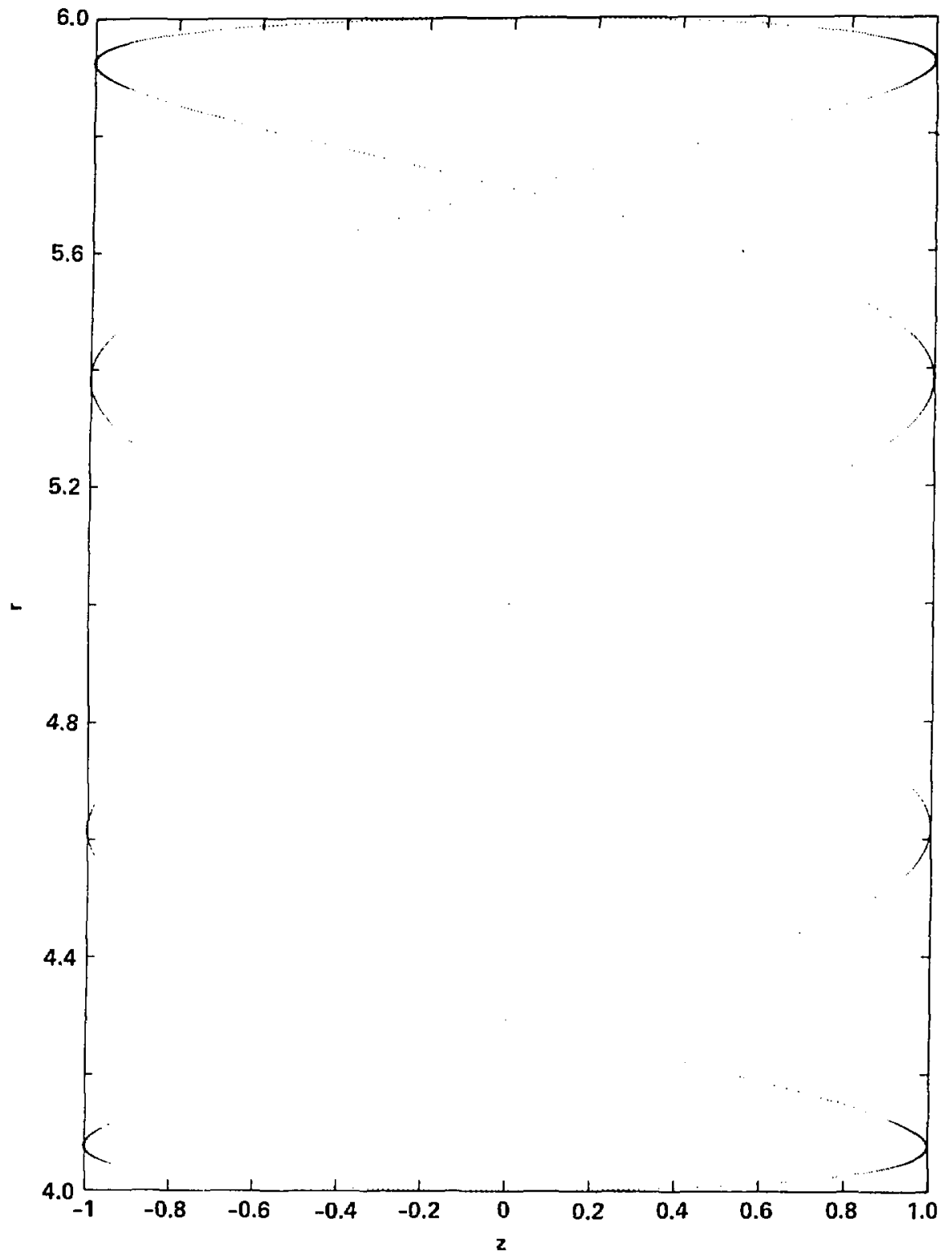

Figure 3-4. Orbit with $\omega_{r}=1, \omega_{z}=4, \varphi_{r}=0, \varphi_{z}=0$. 


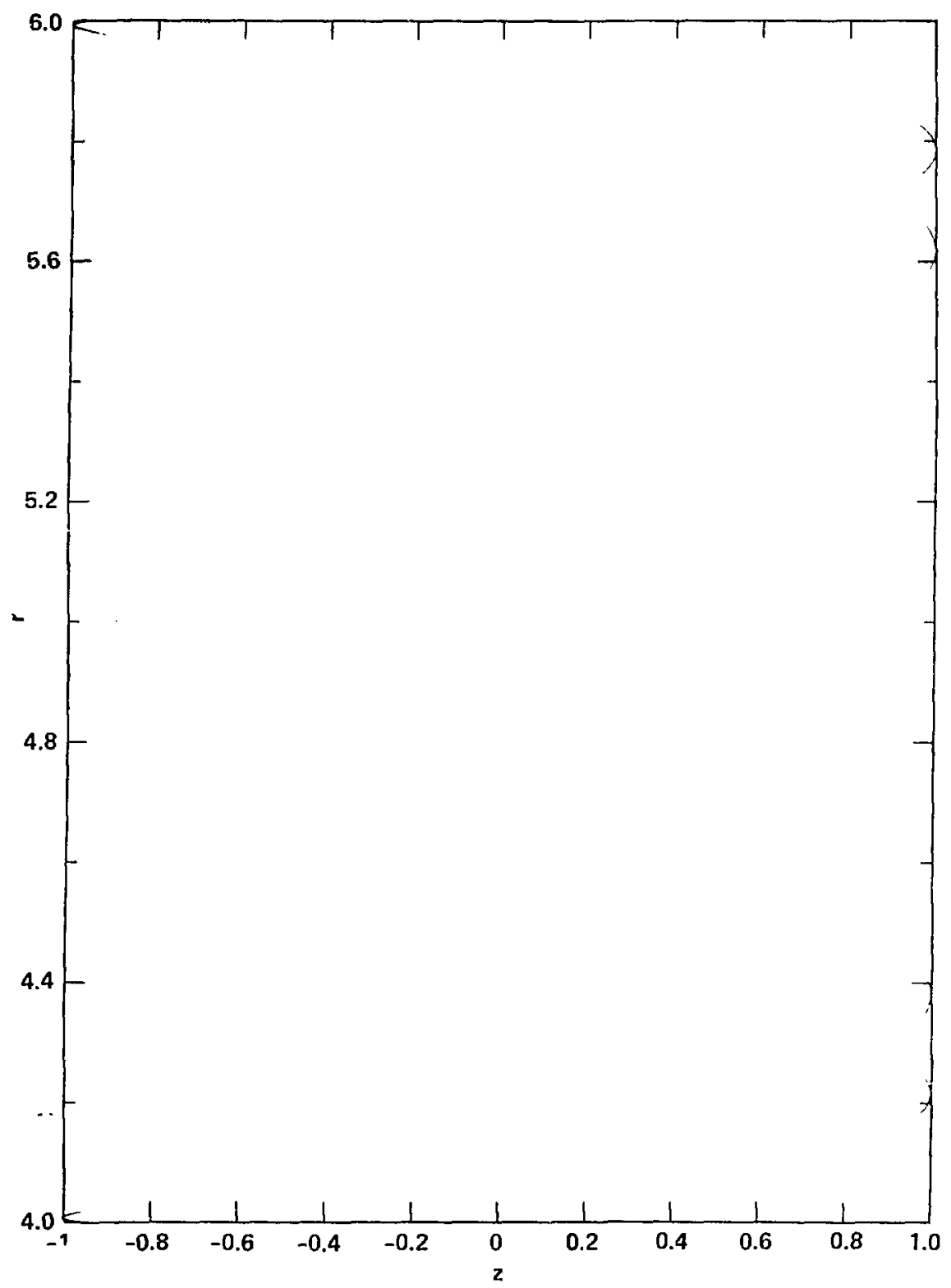

Figure 3-5. Orbit with $\omega_{r}=1, w_{z}=4, \varphi_{r}=.3, \varphi_{z}=.1$. 
having the same $\omega_{r}$ and $\omega_{z}$ encounters very different spatial regions as a consequence of different initial phases. In order to make a comparison of $\bar{x}^{(0)}$ with numerical oribit average formulas, orbit parameters are chosen to cause a dense covering of the allowed orbit region. The objective is to choose oscillation frequencies $\omega_{r}$ and $\omega_{z}$ such that the orbit requires many oscillations before it begins to repeat. The complete $\bar{x}^{(0)}$ value is known after only one orbit period is obtained since the limit of large path length makes the contribution from any partial orbit insignificant.

$$
\bar{\chi}^{(0)}=\frac{\sum \chi d \ell}{\Phi d \ell+\ell \sqrt{n}}+\frac{\int_{0}^{\ell} \chi d \ell}{n d \ell+\ell}
$$

As can be seen from Eq. (3-147), as $n$ becomes large the second term is unimportant. Using oscillation frequencies $\omega_{r}=.198$ and $\omega_{z}=$ 1.218 , the orbit completes after 33 radial oscillations and 203 axial oscillations. Figure 3-6 shows the allowed orbit region is well-covered for this choice of frequencies. The values of orbit average forinulas for 41888 orbit steps at $\Delta t=.025$ used to calculate $\bar{x}^{(0)}$ and a $40 \times 40$ point grid used to calculate all other orbit average formulas are in Table $3-1$.

\begin{tabular}{|l|l|l|l|l|l|l|}
\hline $\bar{x}^{-(0)}$ & $\bar{x}^{(5)}$ & $\bar{x}^{(6)}$ & $\bar{x}^{-(7)}$ & $\bar{x}^{(p s 8)}$ & $\bar{x}^{(p s 9)}$ & $\bar{x}^{(p s 10)}$ \\
\hline .8767 & .7147 & .7264 & .6954 & .8767 & .4874 & .6899 \\
\hline
\end{tabular}

Table 3-1. Orbit Average Values for the AV Code 


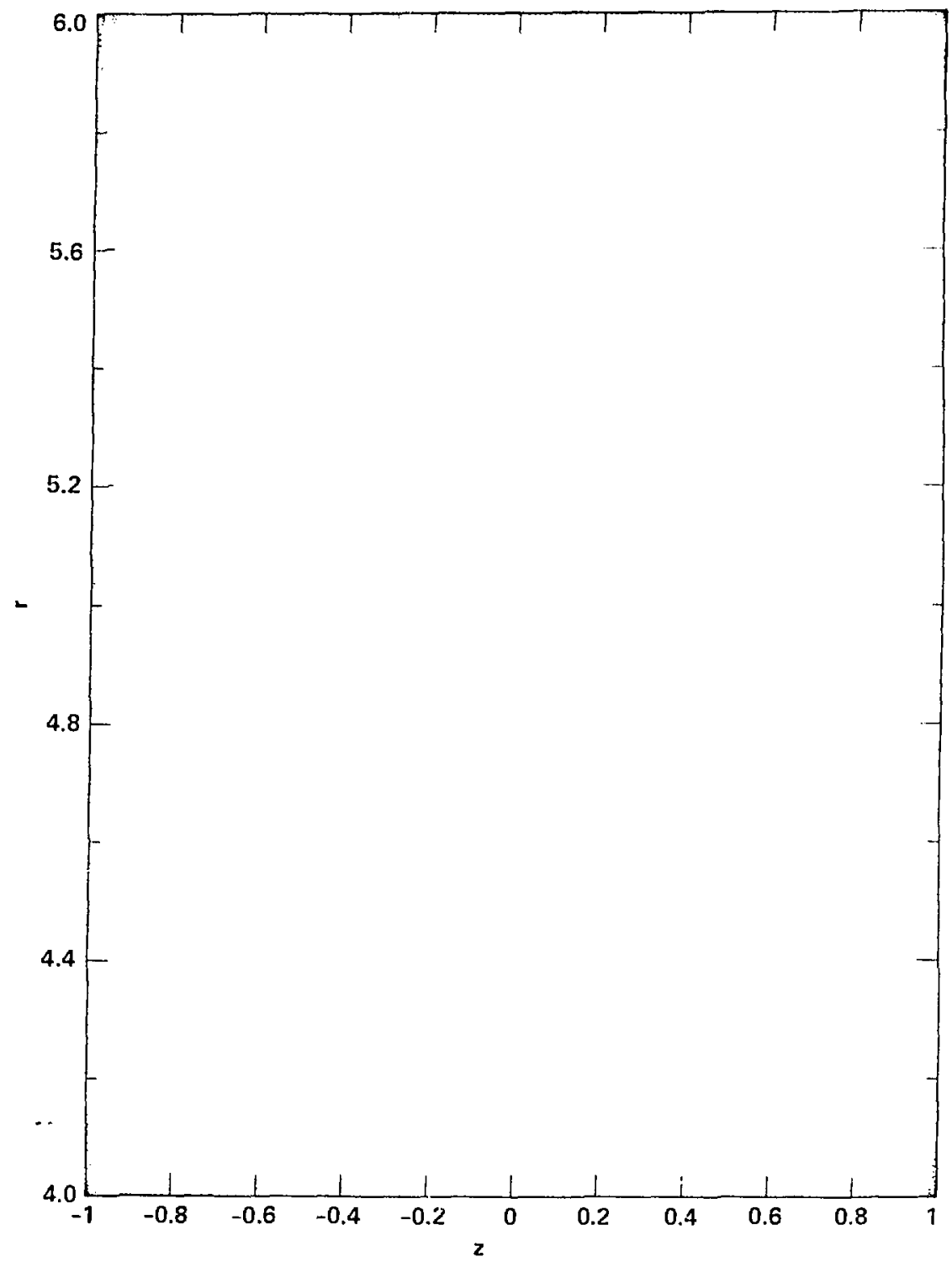

Figure 3-6. Orbit with $\omega_{r}=.198, \omega_{z}=1.218, \varphi_{r}=.3, \varphi_{z}=.1$. 
Changing the grid to $80 \times 80$ results in only a few percent change in $\bar{x}^{(5)}, \bar{x}^{(6)}, \bar{x}^{(7)}, \bar{x}^{(p s 9)}$ and $\bar{x}^{(p s 10)}$. From the table of values it can be seen there is good agreement between $\bar{\chi}^{(0)}$ and $\bar{x}^{(p s 8)}$. The path integral formulas $\bar{x}^{(5)}, \bar{x}^{(6)}, \bar{x}^{(7)}$ are low by $18 \%, 17 \%$ and $20 \%$, respectively. It appears, for this example, there is no gain obtained from the more elaborate $\bar{x}^{(7)}$ formula. The best path integral result in this case is $\bar{\chi}^{(6)}$; however, this may not always be true for orbit averages of other $x(r, z)$ functions. The $\bar{\chi}^{\text {(ps } 9)}$ result demonstrates the hazard of applying a phase-space derived formula without knowledge of the actual constants of the motion. The $\bar{x}^{(p s 9)}$ result differs from $\bar{\chi}^{(0)}$ by $44 \%$, wrich is about twice the error of the other numerical formulas. The $\bar{\chi}^{(p s 10)}$ formula, which is related to $\bar{\chi}^{(p s 9)}$, has a value of .6899 , which is in line with the path integral results.

The numerical orbit-average formulas $\bar{x}^{(5)}, \bar{x}^{(6)}, \bar{x}^{(7)}$, $\bar{x}^{(p s 8)}, \bar{x}^{(p s 9)}$ and $\bar{x}^{(p s 10)}$, all assume the allowed orbit region is covered by the trajectory used with the basic orbit-average definition $\bar{x}^{(0)}$. As was shown by Figs. 3-4 and 3-5, the effect of a distribution of initial phases is to result in a covering of the allowed orbit region. To determine when a single periodic orbit, such as that used to compare the numerical orbit-averaged formulas, represents the orbit average definition, $\bar{x}^{(0)}$ is examined using the analytic orbits. 


$$
\begin{aligned}
\bar{x}^{(0)} & =\lim _{\rightarrow \infty} \frac{1}{T} \int_{0}^{T} z^{2} e^{\left(\left(r-r_{0}\right) / r_{1}\right)^{2}} d t \\
& =\lim _{\rightarrow \infty} \frac{1}{T} \int_{0}^{T} z_{1}^{2} \sin ^{2}\left(\omega_{2} t+\varphi_{z}\right) e^{r^{2} \sin ^{2}\left(\omega_{r} t+\varphi_{r}\right)} d t
\end{aligned}
$$

The half angle formula is used in Eq. (3-148) and the oscillation amplitudes are set to ore to yield,

$$
\bar{x}^{(0)}=\frac{1}{2} e^{1 / 2} \lim _{T \rightarrow \infty} \frac{1}{T} \int_{0}^{T}\left(1-\cos \left(2 \omega_{z} t+2 \varphi_{z}\right)\right) e^{-1 / 2 \cos \left(2 \omega_{r} t+2 \varphi_{r}\right)} d t
$$

The fcimula 3-149 is examined by expanding the exponential in a Taylor series and splitting into two parts. The first part is one times the expanded exponential.

$$
\bar{x}_{1}^{(0)}=\frac{1}{2} e^{i / 2} \lim _{T \rightarrow \infty} \int_{0}^{T} \sum_{r .0}^{\infty} \frac{\left(-\frac{1}{2} \cos \left(2 \omega_{r} t+2 \varphi_{r}\right)\right)^{n}}{n !}
$$

For $n$-odd, the terms vanish in Eq. $(3-150)$ because the integrand is odd over a period. Interchanging the order of integration and summation in Eq. $(3-150)$ and using,

$$
\frac{1}{2 \pi} \int_{0}^{2 \pi} \cos ^{2 n} \theta d \theta=\frac{1 \cdot 3 \cdot 5 \cdots(2 n-1)}{2 \cdot 4 \cdot 6 \cdot \frac{2 n-1)}{2 n}}
$$

yields the following formula. 


$$
\bar{x}_{1}^{(0)}=\frac{1}{2} e^{1 / 2}\left(1+\sum_{n=1}^{\infty} \frac{1}{4^{n}(2 n) !} \frac{1 \cdot 3 \cdot 5 \cdot \cdots(2 n-1)}{2 \cdot 4 \cdot 6 \cdot \cdots 2 n}\right)
$$

Formula 3-152 shows the first contribution to $\bar{x}^{(0)}$ is independent of phase and frequency. The sum of the first few terms of Eq. (3-152) is below.

$$
\bar{x}_{1}^{(0)}=\frac{1}{2} e^{1 / 2}\left(1+\frac{1}{16}+\frac{1}{2024}+\frac{1}{147456}+\frac{1}{37750000}\right)=.87669
$$

To understand why $\bar{x}_{1}^{(0)}$ is essentially equal to $\bar{x}^{(0)}$, the second contribution to $\bar{x}^{(0)}$ must be examined to determine under what conditions it is small. The second contribution to to $\bar{x}^{(0)}$ is $\cos \left(2 \omega_{z} t+2 \varphi_{z}\right)$ times the expanded exponential.

$$
\begin{aligned}
\bar{x}_{2}^{(0)}=-\frac{1}{2} e^{1 / 2} \lim _{+\infty} \frac{1}{T} \int_{0}^{T} \cos \left(2 \omega_{z} t+2 \varphi_{z}\right) \\
\\
\sum_{n=1}^{\infty} \frac{\left.-\frac{1}{2} \cos \left(2 \omega_{r} t+2 \varphi_{r}\right)\right)^{n}}{n !} d t
\end{aligned}
$$

The $n=0$ term vanishes. Expressing cosine as a sum of exponentials, and expanding cosine to the $n$ in a binomial series, and using,

$$
\frac{1}{2 \pi} \int_{0}^{2 \pi} e^{i \alpha t} d t=\delta(\alpha)
$$

yields the following formula.

$$
\bar{x}_{2}^{(0)}=-\frac{1}{2} e^{1 / 2} \sum_{n=0}^{\infty} \sum_{k=0}^{n}\left(\frac{(-1)^{n}}{2^{2 n}(n-k) ! k !}\right.
$$




$$
\begin{aligned}
& \cos \left((2 n-4 k) \varphi_{r}-2 \varphi_{z}\right) \\
& \left.\delta\left((n-2 k) \omega_{r}-\omega_{z}\right)\right)
\end{aligned}
$$

From Eq. (3-155) it can be seen that $\bar{x}_{2}^{(0)}=0$ when $\omega_{z} / \omega_{r}$ is not rational. Thus, for $\omega_{z} / \omega_{r}$ equal to an irrational number $\bar{x}^{(0)}=$ $\bar{x}_{1}^{(0)}$. Also, when $\omega_{z} / \omega_{r}$ is equal to $m$, a rational number, the first non-vanishing term of Eq. $(3-155)$ is bounded by $2^{-2 m / m}$ ! This means that a periodic repeating orbit yields essentially the ergodic orbit average as soon as $m=6$. At $m=6, \bar{x}_{2}^{(0)}$ is down by a factor of 3 $x 10^{-7}$. When $m$ is large there are many axial oscillations for each radial oscillation and the averaged function appears to only have a $z$ variation along the orbit. The same conclusion $c$ an be shown to be true for a general function $x(r, z)$. To do, this the $\bar{x}^{(0)}$ formula is written with $x(r, z)$ represented by a Taylor expansion in $r$ and $z$ expanded about $r_{0}$ and $z_{0}$.

$$
\bar{x}^{(0)}=\lim _{T \rightarrow \infty} \frac{1}{T} \int_{0}^{T} \sum_{n=0}^{\infty} \sum_{m=0}^{\infty}\left(\frac{\partial^{n+m_{2}} x}{a^{n} r_{0} \partial^{m_{z}}}\right) \frac{\left(r-r_{0}\right)^{n}}{n !} \frac{\left(z-z_{0}\right)^{m}}{n ! !} d t
$$

Substituting sinusoids for $r(t)$ and $z(t)$, integrating and expanding the exponential representation of sine in a b.nomial expansion yields the following formula.

$$
\begin{aligned}
\bar{x}^{(0)}= & \sum_{n=0}^{\infty} \sum_{m=0}^{\infty}\left[\left(\frac{\partial^{n+m} x}{a^{n} r_{0} a^{m} z_{0}}\right) \sum_{k=0}^{n} \sum_{\ell=0}^{m} \frac{2^{-(n+m)}}{(n-k) ! k !(m-l) ! \ell !}\right. \\
& \left.e^{i\left(\varphi_{r}(n-2 k)+\varphi_{z}(m-2 l)\right.} \delta\left(\omega_{r}(n-2 k)+\omega_{z}(m-2 \ell)\right)\right]
\end{aligned}
$$


Equation (3-157) shows there are two types of contributions to Eq. (3-156). The first $k$ ind consists of all terms with $n=2 k$ and $m=2 \ell$. These terms are independent of phase and oscillation frequency. Furthermore, if $\omega_{r} / \omega_{z}$ is an irrational number, all other terms vanish. Because the condition of irrational $\omega_{r} / \omega_{2}$ is the condition for an ergodic orbit, the sum of terms with $n=2 k$ and $m=2 l$ is the ergodic contribution to the orbit average. The second $k$ ind of contribution to the orbit average has $\omega_{r} / \omega_{z}$ equal to a rational number. For widely different frequencies these terms are down by a factor $2^{-(n+m)}$, and can then be considered only a small correction to the ergodic contribution to the orbit average. A periodic orbit with widely different radial and axial frequencies is then equivalent to the ergodic orbit average. This conclusion is also valid when the potential is anharmonic, but the orbit is essentially oscillatory. For this case, the expansion analogous to Eq. (3-157) has summations of sinusoids in $r$ and summations of sinusoids in $z$. As before, the ergodic orbit-average contribution consists of terms with irrational frequency ratios, and for widely different frequency ratios, the ergodic orbit average is the dominant contribution.

\subsection{Hilll's Vortex Orbit Code}

\subsubsection{Investigation Issues}

There are essentially three issues in considering the orbit average with the NEO code. First, the application of formulas validated with the AV harmonic oscillator code are examined in 
appropriate $H, P_{\theta}$ regimes. Second, a procedure is applied to determine the existence of the third constant of the motion, $\mathscr{I}$. The existence of $\mathscr{I}$ implies a distribution function depending on $\mathscr{I}$ is possible and thus an orbit average taking into account. 9 v'ould be useful. In the case of low energy with no sharp field gradients or rapid time variations the magnetic monent is a suitable adiabatic constant of the motion. Such a system may then be described in this regime with a distribution function which depends on $H, p_{\theta}$ and the magnetic moment. Third, the question of stochasticity is briefly considered to determine if conditions occur where no constant of the motion exist.

\subsubsection{Equations of Motion and Method of Solution}

The NEO code solves the equations of motion in cylindrical $r$, $\theta, z$ coordinates using magnetic fields derived from the spherical Hill's vortex. The resulting orbits are used to investigate a realistic application of the previously derived numerical orbit averages and to explore the existence of invariants of the motion. As demonstrated in the case of the harmonic oscillator, knowledge of the invariants of the motion lead to specialized forms of the orbit average. Experimentally, it may be possible to design magnetic fields with invariants of the motion that produce beneficial transport properties. The magnetic moment, $H_{1} / 2 B$ which is only an adiabatic invariant $[146,147]$ has been employed to enhance particle confinement 
in magnetic mirror devices. $[148,149]$ Thus the construction of an approximate constant of the motion is also useful.

The equations solved by the NEO code are obtained from the following dimensionless Hamiltonian,

$$
H=\frac{1}{2}\left(p_{r}^{2}+p_{z}^{2}\right)+\frac{1}{2}\left(p_{\theta} r^{-1}-r^{-1} \psi\right)^{2}
$$

where $\psi$ is defined below.

$$
\psi= \begin{cases}\frac{3}{4} r^{2}\left(r^{2}+z^{2}-1\right) & r^{2}+z^{2}<1 \\ \frac{1}{2} r^{2}\left(1-\left(r^{2}+z^{2}\right)^{-3 / 2}\right) & r^{2}+z^{2}>1\end{cases}
$$

Lengths are normalized to the Hill's vortex plasma radius, time is normalized by the uniform field cyclotron frequency ezB/mc, mass and charge are normalized to proton mass and charge, and $p_{\theta}$ is normalized by the product of the proton mass, cyclotron frequency, and plasma radius squared. Since $p_{\theta}$ is a conserved quantity in axisymmetry, it is used to obtain the time derivative of theta. The equations solved by the NEO code are then below.

$$
\begin{aligned}
& \dot{r}=p_{r} \\
& \dot{\theta}=r^{-2}\left(p_{\theta}-\psi\right) \\
& \dot{z}=p_{z} \\
& \dot{p}_{r}=-\left(r^{-1} p_{\theta}-\psi r^{-1}\right)\left(p_{\theta} r^{-2}+\frac{\partial}{\partial r}\left(\psi r^{-1}\right)\right) \\
& \dot{p}_{z}=-\left(r^{-2} p_{\theta}-\psi r^{-2}\right) \frac{\partial \psi}{\partial z}
\end{aligned}
$$


A dot denotes derivative with respect to time. The numerical solution of the system of five first order ordinary differential equations is obtained by the GEAR ${ }^{[150]}$ multistep method employing variable time step and order to guarantee a specified local error criteria is satisfied. For a general first order ordinary differential equation for variable $y$,

$$
\frac{d y}{d t}=\dot{y}(y, t)
$$

the solution method is as follows,

$$
y_{n}=\alpha_{1} y_{n-1}+\Delta t \sum_{j=0}^{k_{1}} \beta_{j} y_{n-j}
$$

where the subscript $n$ represents the time step level. The values of $k_{1}, \alpha_{1}$ and the $B_{j}$ constants have well known values depending on the order of the method. [151] The formula $3-166$ is commonly known as $a k_{1}+1$ order implicit Adams method. It is important from the viewpoint of considering constants of the motion that the orbit position and velocity are accurately known. The formula 3-166 used to solve the set of Eqs. (3-160) through $(3-164)$ has $p_{\theta}$ as a constant since this is enforced in the formulation; however there is no guarantee the energy is numerically constant. There are algorithms, such as the leap frog scheme, ${ }^{[152]}$ which conserve energy exactly. The trouble in using a scheme like leap frog is understood by taking the dot product of both sides of the velocity advance equation,

$$
\vec{v}^{n+3 / 2}=\vec{v}^{n+1 / 2}+\frac{\Delta t}{2}\left(\vec{v}^{n+3 / 2}+\vec{v}^{n+1 / 2}\right) \times \vec{B}^{n+1}
$$


The result is,

$$
\left(v^{n+3 / 2}\right)^{2}=\left(v^{n+1 / 2}\right)^{2}
$$

and consequently the total velocity magnitude is procoryed to within round off error. The difficulty is each velocity component is not known this accurately and aiso $p_{\theta}$ is no longer a constant. To resolve such difficulties, the multistep method is used with an error criteria of $10^{-15}$. To invoke an error criteria as small as $10^{-15}$, it is necessary to use double precision arithmetic. In monitoring the error criteria, it was found the time step must be adjusted in accordance with the local anticipated error rather than the greatest error encountered along an orbit. This consideration arises due to the orbit turning points where the velocities in the $r$ or $z$ direction decrease to zero and then change sign. In general, near a turning point, much smaller time steps are required than during other parts of an orbit. In the most recent ordinary differential equation solver, (LSODE) it is possible to independently set the error criteria for each unknown variable. In the problems of interest here, the theta location is not important so the error criteria for $r, z, p_{r}, p_{z}$ is $10^{-15}$ and the error criteria for $\theta$ is set to one. In practice this means the predicted error caused by the theta equation does not cause the time step to be reduced. 


\subsubsection{Axisymmetric Orbit Considerations}

The reason $\theta$ is not accurately calculated is because only axisymmetry is considered. As discussed previously $p_{\theta}$ is then conserved. The consequence of constant $p_{\theta}$ is that the orbit may be considered to be two dimensional and $v_{\theta}$ is then replaced by a function of $r, z$, and $p_{\theta}$. The Hamiltonian then appears two dimensional with $v_{\theta}$ serving as a potential, $V\left(r, z \mid p_{\theta}\right)$ having a parameter $p_{\theta}$.

$$
v\left(r, z \mid p_{\theta}\right)=\frac{1}{2}\left(p_{\theta} r^{-1}-\psi r^{-1}\right)^{2}
$$

Substituting Eq. (3-169) into Eq. (3-158) recasts the Hamiltoniall as follows.

$$
H=\frac{1}{2}\left(p_{r}^{2}+p_{z}^{2}\right)+V\left(r, z \mid p_{\theta}\right)
$$

From Eq. (3-170) an orbit having $H<V$ is confined to a region of space provided $\mathrm{V}$ has closed contour surfaces. For the Hill's vortex in a range of $p_{\theta}$ values, $v$ does have closed surfaces and thus there is confinement. Physically at the boundary of a confining surface a11 the particle energy is in the theta direction. Thus in two dimensions at the bounding curve $\mathrm{p}_{r}$ and $\mathrm{p}_{z}$ are zero. The confinement of orbits to regions of space as a consequence of the potential $\checkmark$ makes it natural to consider transport properties averaged over these regions. It is then necessary to consider under what conditions $V$ has closed surfaces and potential confinement results. The $\psi$ function is positive outside the plasma region and thus from considering Eq. (3-169) a potential barrier exists when 
$p_{\theta}<0$. There are two distinctive types of orbits that occur for $\mathrm{p}_{\theta}<0$. Examination of Eq. (3-159) shows the maximum value $-\psi$ has inside the plasma is $3 / 16$. Consequently for $p_{\theta}<-3 / 16, v_{\theta}$ is a lways less than zero or in other words the orbit is axis encircling and aiways moves in the negative $\theta$ direction. The consequence for spatial confinement is obtained from the first derivative of $V$ with respect to $r$ inside the plasma.

$$
\frac{\partial V\left(r, z, \mid p_{\theta}\right)}{\partial r}=\left(p_{\theta} r^{-1}+\frac{3}{4} r\left(1-r^{2}-z^{2}\right)\right)\left(-p_{\theta} r^{-2}+\frac{3}{4}\left(1-3 r^{2}-z^{2}\right)\right)
$$

At positive $r$, Eq. $(3-171)$ shows there are three positions where $\partial V / \partial r=0$.

$$
\begin{aligned}
& r_{1}=2^{-1 / 2}\left(1-z^{2}-\left(\left(1-z^{2}\right)^{2}+\frac{16}{3} p_{\theta}\right)^{1 / 2}\right)^{1 / 2} \\
& r_{2}=2^{-1 / 2}\left(1-z^{2}+\left(\left(1-z^{2}\right)^{2}+\frac{16}{3} p_{\theta}\right)^{1 / 2}\right)^{1 / 2} \\
& r_{0}=6^{-1 / 2}\left(1-z^{2}+\left(\left(1-z^{2}\right)^{2}-16 p_{\theta}\right)^{1 / 2}\right)^{1 / 2}
\end{aligned}
$$

When $p_{\theta}<-3 / 16 r_{1}$ and $r_{2}$ are both imaginary. This situation is illustrated qualitatively in Fig. $3-7(a)$. In this case $V$ has a minimum at $r_{0}$. When $-3 / 16<p_{\theta}<0 r_{i}, r_{2}$, and $r_{0}$ are ail real with $r_{1}$ and $r_{2}$ being local minima and $r_{0}$ being a position of local maximum. This case is illustratred qualitatively in Fig. 3-7(b). The basic difference between orbits in these two $p_{\theta}$ regions is for $-3 / 16<p_{\theta}<0$ and $H<V\left(r_{0}, z \mid p_{\theta}\right)$. As shown by Fig. 3-7(b), the 


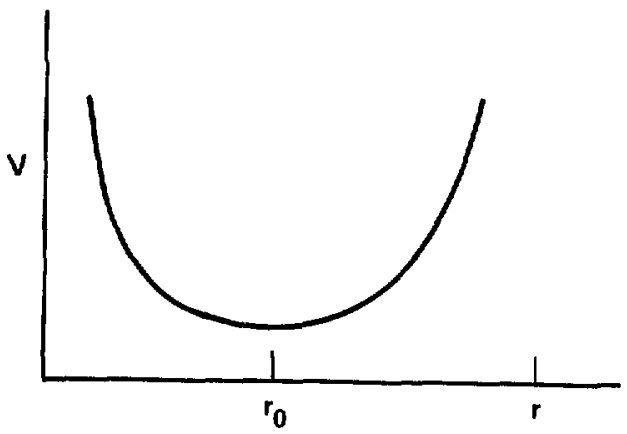

(a)

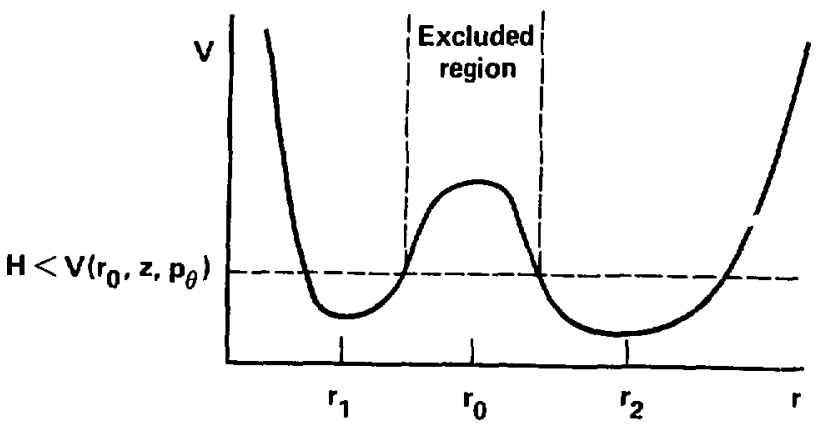

(b)

Figure 3-7. Potential at $z=0$ versus $r$. (a) $p_{\theta}<-3 / 16$ (b) $-3 / 16<p_{\theta}<0$. 
orbit in this case has an excluded region around $r=r_{0}$. In the $r, z$ plane the orbit is then ring like in contrast to $H: V\left(r_{0}, z \mid p_{\theta}\right)$ for which $r_{1}<r<r_{2}$ orbit values are allowed. For $H \approx V\left(r_{1}, z \mid p_{\theta}\right)$ the orbit degenerates to a curve in the $r, z$ plane. If in addition $\mathrm{P}_{\theta} \approx 0$ the curve becomes a constant $\psi$ contour and this class of particles is confined to flux surfaces.

The second parameter which determines when an orbit has potential confinement is $H$. In the radial direction at $z=0$ and large $r, V \sim 1 / 8 r^{2}$. Thus, at large enough $r$ an orbit of any $H$ is confined radially. Typically in practice, a radial wall exists and confinement ceases when the energy is large enough to cause the orbit to intersect the wa11. A sufficient but not necessary condition for radial confinemnt when $r_{0}<?$ is $H<p_{\theta}^{2}$. In this case the orbit only encounters closed field line. For confined orbits which encounter open field lines, loss may occur by intersecting a radial wall or by having an energy large enough so axial loss occurs. The energy threshold for axial loss is derived by considering the $r$ and $z$ derivative of the potential $V$ in the region outside the plasma.

$$
\begin{aligned}
& \frac{d V}{d r}=\frac{1}{r}\left(p_{\theta}-\psi\right)\left(-p_{\theta} r^{-2}-\frac{1}{2}+\frac{1}{2}\left(r^{2}+z^{2}\right)^{-3 / 2}-\frac{3}{2} r^{2}\left(r^{2}+z^{2}\right)^{-5 / 2}\right) \\
& \frac{d V}{d z}=-\frac{3}{2}\left(p_{\theta}-\psi\right) z\left(r^{2}+z^{2}\right)^{-5 / 2}
\end{aligned}
$$

Outside the plasma $\psi$ is positive and $p_{\theta}$ has been chosen negative so the quantity $p_{\theta}-\psi$ is always negative. From Eq. $(3-176), \mathrm{dV} / \mathrm{dz}$ is then always positive, diminishing as $z^{-4}$ as $z$ increases with $r$ 
fixed. This means the potential becomes constant asymptotically in the axial direction. From Eq. $(3-175)$, the second factor on the right indicates at large $z, v$ has a radial minimum at $r^{2}=-2 p_{\theta}$. The energy threshold for axial loss then occurs at $r^{2}=-2 p_{\theta}$ and $z \rightarrow \infty$. Using these values in Eq. (3-169), the anergy threshold for axial loss occurs at $H_{\text {loss }}=-p_{\theta}$. The orbits to be discussed are all energetically confined and thus $p_{\theta}<0$ and $H<-p_{\theta}$. In addition, to limit the discussion to a potential with a single minimum $p_{\theta}$ is further restricted to be less than $-3 / 16$.

\subsubsection{NEO Orbit Average Results}

The $\bar{x}^{(5)}, \bar{x}^{(6)}$, and $\bar{x}^{(7)}$ path integrals are not implemented in the NEO cole. These formilas were derived to be applied to regions determined by a third invariant of the motion, $\mathscr{f}$. The low energy formula derived for Hill's vortex is not readily amenable to this application and consequently only results for $\bar{x}^{-(p s 9)}$ and $\bar{x}^{(p s 10)}$ are presented. The orbits are characterized by the initial positior and velocity. The theta velocity is always selected by specifying $\mathrm{P}_{\theta}$. The remaining velocities may be specified in three ways:

$$
\begin{aligned}
& \text { 1. } H, Y \\
& \text { 2. } p_{r}, p_{Z} \\
& \text { 3. } v, Y
\end{aligned}
$$

where $\cos \gamma=\vec{p} \cdot \vec{B}(p B)^{-1}, v=\left(H-V_{0}\right) / V_{0}$, and $V_{0}$ is the value of the minimum of the potential.

As verified by earlier work [153] when the NEO code is run with $p_{\theta}=-1.19$ and $v=.5$ a stochastic orbit results. The stochastic 
nature of the orbit is evidenced in three ways. First, Fig. 3-8 shows points sampled along the orbit fill, the energetically al lowed region in $r$ and $z$. Second, Fig. 3-9 shows the velocity direction at each point is irregular. Thirf, Fig. 3-10 shows the surface of section is random. The surface of section is the locus of $r, p_{r}$ points at $z=0$. This oroit satisfies the assumptions which were used to derive $\bar{x}^{(p s 9)}$. Using the $x(r, z)$ function given by Eq. $(3-106)$ the orbil was followed 14,000 steps to yield $\bar{x}^{(0)}=$ 526.2. An adaitional run which followed the orbit 28,000 steps changed this result by only $.06 \%$. Calculating $\bar{x}^{(p s 9)}$ ard $\bar{x}^{(p \leqslant 10)}$ on a $30 \times 30$ mesh yields $\bar{x}^{(p s 9)}=470.6$, and $\bar{x}^{(p s 10)}=1019$. The value of $\bar{\chi}^{(p s 9)}$ differs from $\bar{\chi}^{(0)}$ by $11 \%$ and the value of $\bar{x}^{\text {(ps } 10)}$ by $94 \%$. Reasonable agreement is obtained for $\bar{x}^{(p s 9)}$; however, $\bar{\chi}^{-(p s 10)}$ appears to be inappropriate for this case. Apparently the argument used to derive the orbit average $\bar{\chi}^{(0)}$ using anaiytic orbits is not valid for the case of a stuchastic orbit. This is because non-linearities preclude the determination of an analytic orbit and the motion $c$ an not be considered to be in any approximation oscillatory. In the same way the $\bar{x}^{(p s 9)}$ orbit average ignored the constants of the motion in the oscillator case and did not agree with $\bar{x}^{(0)}$, here $\bar{x}^{(p s 10)}$ anticipates oscillatory motion when it is absent and thus does not agree with $\bar{\chi}^{(0)}$ when the orbit is stochastic. These comparisons point to the desirability of knowing when a third constant of the motion exist. 


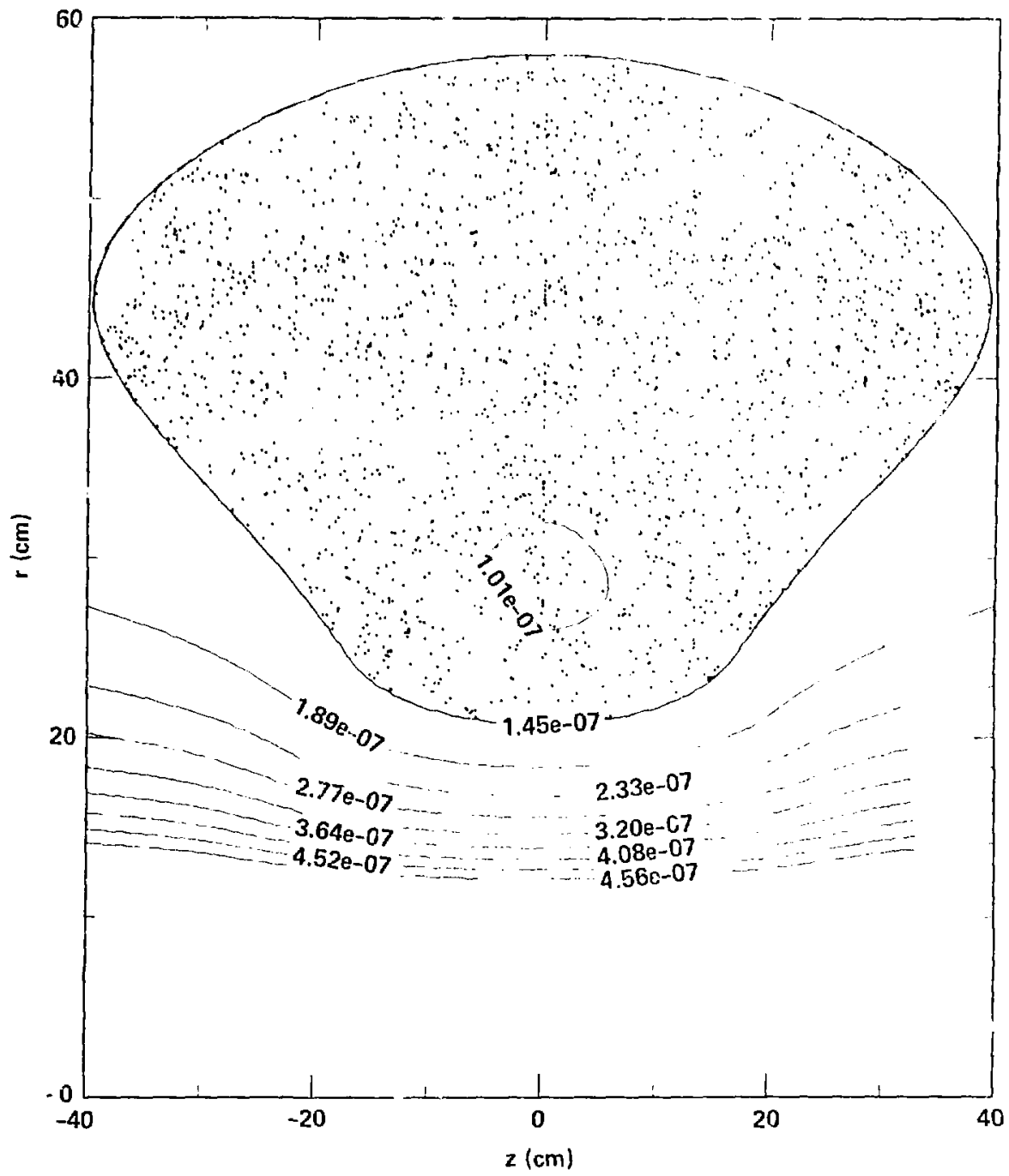

Figure 3-8. 0-bit positions sampled along a trajectory with $p_{\theta}=-1.19, v=.5$. Contours are constant energy values. 


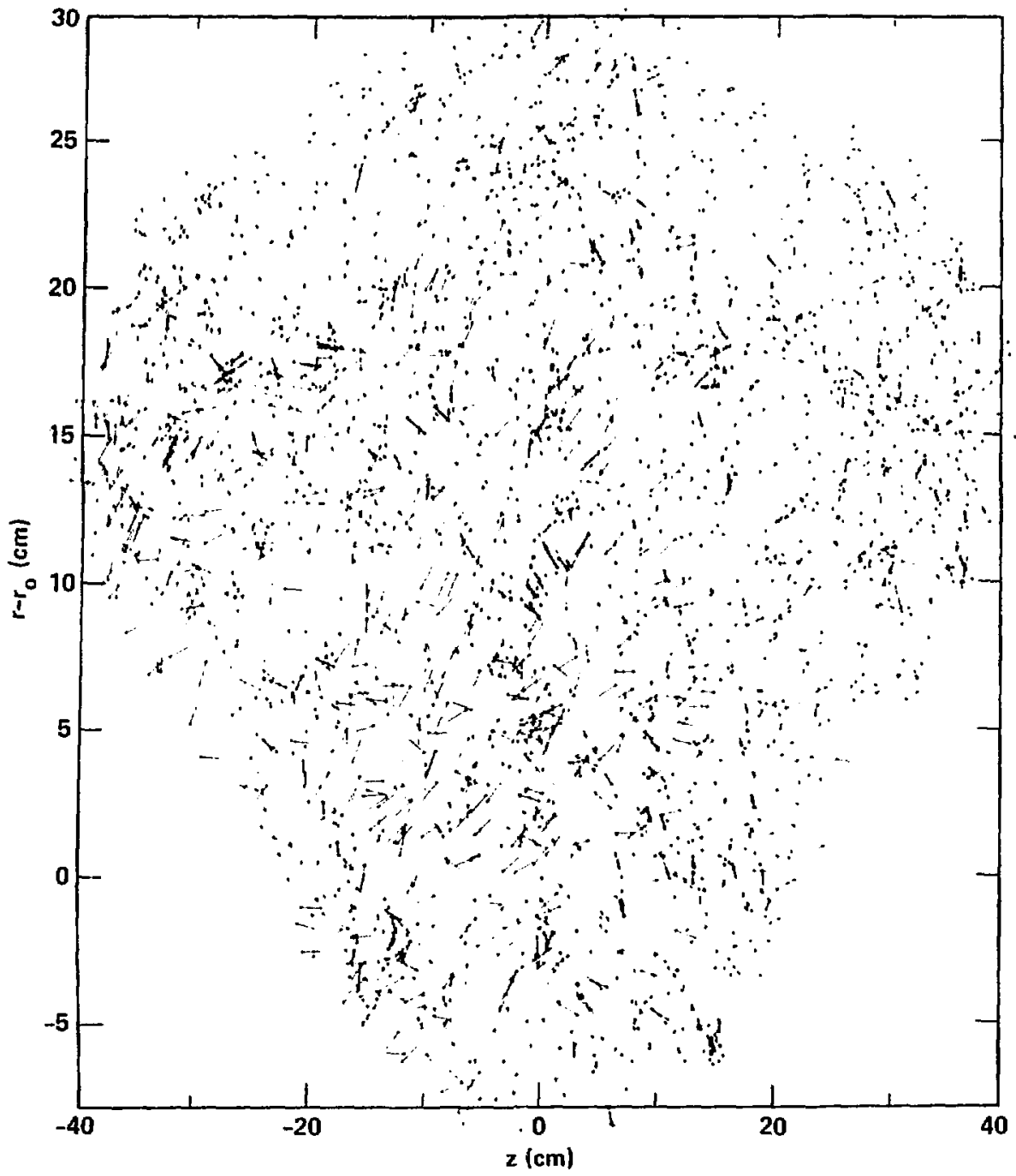

Figure 3-9. Velocity direction arrows at orbit positions for $p_{\theta}=-1.19, v=.5$. 


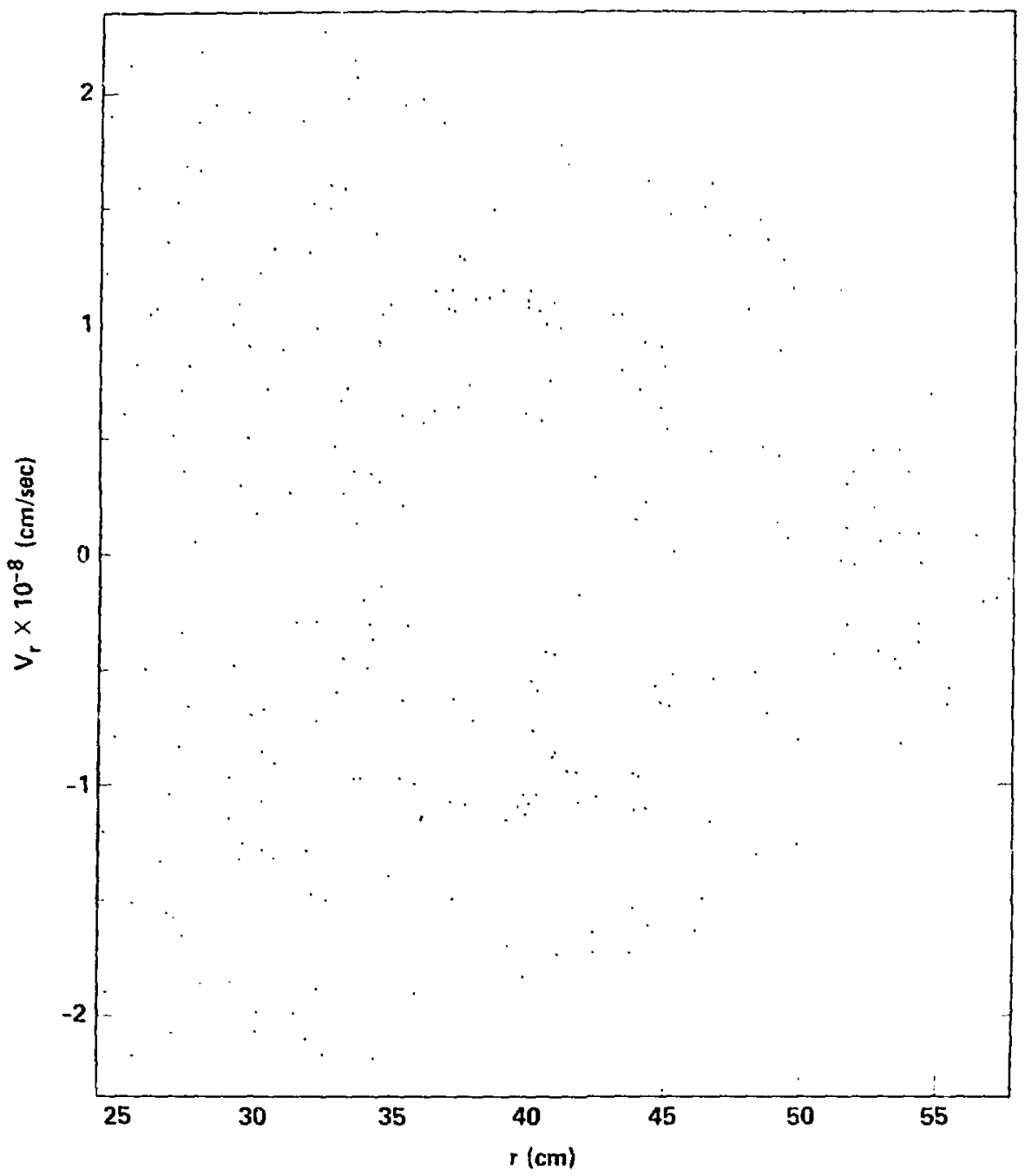

Figure 3-10. Surface of section for $p_{\theta}=-1.19, v=.5$. 


\subsubsection{Existence of Third Invariants}

An early consideration of the conditions for the existence of a third constant of the motion was undertaken by whittaker [154] He determined the conditions for the existence of a constant of the motion which is at most second order in the velocities, but his methodology was incomplete. Hal ${ }^{[155]}$ corrected tinis work by noting velocities are related by conservation of energy, and derived the conditions for the existence of an invariant of $\mathrm{N}^{\text {th }}$ order in the velocity.

$$
\pi_{N}(\dot{z}, \dot{r}, z, r)=\sum_{n=0}^{N} \sum_{m=0}^{n} i_{n i m}\langle z, r) z^{m} \dot{r}^{n-m}
$$

Necessary and sufficient conditions for the existence of $\pi_{N}$ were derived from the expression for " ${ }_{N}$ formed by substituting the equations of motion for $\dddot{z}$ and $\ddot{r}$ and using the relationship between $\dot{z}$ and $\dot{r}$ implied by conservation of energy. The existence conditions arise from requiring coefficients of velocity in $\dot{\%}_{N}$ to vanish. For this work $z$ and $r$ in Eq. (3-177) may alternatively refer to $z$ and $r-r_{0}$, where $r_{0}$ is the radial position of the pot tial minimum defined by Eq. (3-175). In the foliowing discussion attention is restricted to the invariants $\pi_{3}$ and $\pi_{4}$ which are third and fourth order in the velocity respectiveiy.

The application of Hali's theory, for $/ 3$ and $\%$, with the Hill's vortex Hamiltonian reduces to finding a function of space, $\phi$ which satisfies a Poisson equation subject to a constraint condition. The Poisson equation is below. 


$$
\frac{\partial^{2} \phi}{\partial r^{2}}+\frac{\partial^{2} \phi}{\partial z^{2}}=T
$$

In Eq. (3-178), T is twice the kinetic energy $2(E-V)$, which is only a function of space. For $\mathscr{H}_{3}$ the constraint equation is as follows.

$$
\frac{\partial}{\partial z}\left[T\left(\frac{\partial^{2} \phi}{\partial z^{2}}-\frac{\partial^{2} \phi}{\partial r^{2}}\right)\right]-2 \frac{\partial}{\partial r}\left[T \frac{\partial^{2} \phi}{\partial z \partial r}\right]=0
$$

The forinula for $\%_{3}$ in terms of velocity and $\phi$ is below.

$$
\pi_{3}=\left(\frac{\partial^{2} \phi}{\partial r^{2}}-\frac{\partial^{2} \phi}{\partial z^{2}}\right) \dot{z}+2 \dot{r} \frac{\partial^{2} \phi}{\partial z \partial r}+\frac{1}{3} \ddot{z}-\ddot{z} \ddot{r}^{2}
$$

For. $\mathscr{H}_{4}$ the constraint equation is as follows.

$$
\begin{aligned}
\frac{\partial^{2} \phi}{\partial z \partial r}\left(\frac{\partial^{4} \phi}{\partial r^{4}}-\frac{\partial^{4} \phi}{\partial z^{4}}\right) & +2\left(\frac{\partial^{2} \phi}{\partial r^{2}} \frac{\partial^{4} \phi}{\partial r^{3} \partial z}-\frac{\partial^{2} \phi}{\partial z^{2}} \frac{\partial^{4} \phi}{\partial r \partial z^{3}}\right) \\
& +3\left(\frac{\partial^{3} \phi}{\partial r^{3}} \frac{\partial^{3} \phi}{\partial r^{2} \partial z}-\frac{\partial^{3} \phi}{\partial z^{3}} \frac{\partial^{3} \phi}{\partial r \partial z^{2}}\right)=0
\end{aligned}
$$

The formula for. $\%_{4}$ in terms of velocity and $\phi$ is below.

$$
\pi_{4}=\frac{1}{2} F_{0}-\left(\frac{\partial^{2} \phi}{\partial z^{2}}-\frac{\partial^{2} \phi}{\partial r^{2}}\right)\left(\dot{z}^{2}-\dot{r}^{2}\right)+4 \frac{\partial^{2} \phi}{\partial z \partial r} \dot{z} \dot{r}
$$

$$
-8 \dot{z}^{2} \dot{r}^{2}
$$

In Eq. $(3-182\}, F_{0}$ has the following definition, 


$$
\begin{aligned}
F_{0}= & -2 \int d z \frac{\partial^{2} \phi}{\partial z \partial r} \frac{\partial T}{\partial r}-2 \int d r \frac{\partial^{2} \phi}{\partial z \partial r} \frac{\partial T}{\partial z} \\
& +f_{1}(r)+f_{2}(z)
\end{aligned}
$$

where,

$$
\begin{aligned}
f_{2}(z)-f_{1}(r)=4 T\left(\frac{\partial^{2} \phi}{\partial z^{2}}-\frac{\partial^{2} \phi}{\partial r^{2}}\right) & -4 \int d r \frac{\partial^{2} \phi}{\partial z} \frac{\partial T}{\partial r} \\
& -4 \int d z \frac{\partial^{2} \phi}{\partial z \partial r}
\end{aligned}
$$

Both. ${ }_{3}$ and $\mathscr{H}_{4}$ require the solution of the Poisson equation given by Eq. (3-178). Because it is only necessary to obtain a solution $\phi$ which satisfies Eq. (3-178) without regard to boundary conditions, any homogeneous solution may be added to $\phi$. It is then the homogeneous solutions which allow the flexibility needed to satisfy a constraint such as Eq. (3-179) or Eq. (3-191). Since $V(r, z)$ is a polynomial in $r$ and $z$ it is represented as follows.

$$
V(r, z)=r^{-2} p_{\theta}^{2}+\sum_{n=0}^{5} \sum_{m=0}^{n} c_{2 n-2 m, 2 m} r^{2 n-2 m} z^{2 m}
$$

From Eq. $(3-375)$ it can be shown the minimum of the potential $V$ moves outiide the plasma, $r_{0}>1$ when $p_{\theta}<-3 / 2$. Consequently it is necessary to obtain coefficients for Eq. (3-185) using the Hill's vortex formula for the region inside and outside the plasma. Because the exterior solution $\psi_{>}$consist of inverse powers of $r$ and $z$, the expansion in terms of $r$ and $z$ is designed to be accurate near the separatrix. Inside the plasma $V(r, z)$ is exact. To obtain the 
approximate exterior $V(r, z)$ a change of variables is made.

$$
\begin{aligned}
& r=(\rho+1)^{1 / 2} \sin \theta \\
& z=(\rho+1)^{1 / 2} \cos \theta \\
& \rho=r^{2}+z^{2}-1
\end{aligned}
$$

From Eq. (3-186) the expression for $\rho$ shows $\rho=0$ is the lacation of the separatrix and thus $\rho$ serves as an expansion parameter near the separatrix. The Hill's vortex model is written in terins of $p$ and $\theta$ below.

$$
\begin{array}{ll}
\psi_{<}=\frac{3}{4} \sin ^{2} \theta\left(\rho^{2}+\rho\right) & \rho<0 \\
\psi_{>}=\frac{1}{2} \sin ^{2} \theta(\rho+1)\left(1-(\rho+1)^{-3 / 2}\right) & \rho>0
\end{array}
$$

The objective in obtaining the $\mathrm{C}$ coefficients for $\mathrm{Eq} \cdot(3-185)$ is to maintain continuity of value and first derivative across the separatrix. Since it is desired for $V(r, z)$ to be exact for $\rho<0$, $i t$ is necessary to expand $\left(p_{\theta}-\psi_{>}\right)^{2}$ in powers of $\rho$ to the same order as $\left(p_{\theta}-\psi_{<}\right)^{2}$. The expression below indicates $\left(p_{\theta}-\psi_{>}\right)^{2}$ inust be expanded to at least fourth order in $\rho$.

$$
\left(p_{\theta}-\psi_{<}\right)^{2}=p_{\theta}-\frac{3}{2} p_{\theta} \sin ^{2} \theta\left(\rho^{2}+\rho\right)+\frac{9}{16} \sin ^{4} \theta\left(\rho^{4}+2 \rho^{3}+\rho^{2}\right)
$$

To aid in converting from $\rho, \theta$ back to $r, z$ coordinates $\psi_{>}$is expanded in powers of $\rho$ up to fifth order. The quantity $\left(p_{\theta}-\psi_{>}\right)^{2}$ then has $\sin ^{2} \theta$ times powers of $\rho$ up to $\rho^{5}$ and $\sin ^{4} \theta$ times powers of $\rho$ up to 
$\rho^{10}$. The $\rho^{5} \sin ^{2} \theta$ term is replaced by an arbitrary constant times $\rho^{5} \sin ^{2} \theta$ and the $\rho^{5} \sin ^{4} \theta$ and $\rho^{6} \sin ^{4} \theta$ terms are replaced by arbitrary constants times $\rho^{5} \sin ^{4} \theta$ and $\rho^{6} \sin ^{4} \theta$. The substitution is then made that $\rho=\rho^{\prime}-1$, and the arbitrary coefficients are chosen so the polynomial in $\rho$ multiplying $\sin ^{2} \theta$ divided by $\rho^{\prime}$ is a constant plus a polynomial in $\rho^{\prime}$ and the polynomial in $\rho$ multiplying $\sin ^{4} \theta$ divided by $\left(\rho^{\prime}\right)^{2}$ is a constant plus a polynomial in $\rho^{\prime}$. The substitution is then made, $\sin ^{2} \theta=\left(\rho^{\prime}\right)^{-1} r^{2}, \rho^{\prime}=r^{2}+z^{2}$ and $\sin ^{4} \theta=\left(\rho^{\prime}\right)^{-2} r^{4}$. The resulting $C$ coefficients for the $V(r, z)$ expression Eq. (3-185) are listed in Appendix B. Given the expansion formula for $V(r, z)$ then $T=2(E-V)$ is known and a polynomial solution for $\phi$ can be constructed.

$$
\phi=p_{\theta}^{2} \log r+\sum_{n=1}^{6} \sum_{m=0}^{n} A_{n, m} r^{2 n-2 m} z^{2 n}
$$

The $A_{n, m}$ coefficients used in Eq. (3-189) are listed in Appendix $C$. Additional homogeneous solutions may be added to $\phi$ using the following coefficient recursion formula.

$$
A_{n, m+1}=-A_{n, m}(n-m)(2 n-2 m-1)(m+1)^{-1}(2 m+1)^{-1}
$$

However, the upper limit of $n=6$ in Eq. (3-189) is compatible with the upper limit of $n=5$ in the $V(r, z)$ expression given by $E q$. $(3-185)$.

As a preliminary step in constructing $\mathscr{J}_{3}$ and $\mathscr{I}_{4}$ for the Hill's vortex the following potential has been investigated.

$$
V_{H}(r, z)=\frac{1}{2} A z^{2}+\frac{1}{2} B\left(r-r_{0}\right)^{2}+C z^{2}\left(r-r_{0}\right)+\frac{1}{3} D\left(r-r_{0}\right)^{3}
$$


From other work ${ }^{[155]}$, formulas for approximate constants of the motion $h$ been derived for $V_{H}$ with arbitrary $A, B, C, D$ values and exact $c$ nstants of the motion for several special choices. The Hill's vortex, Taylor expanded about $r=r_{0}$ and $z=0$ to third order yields the following coefficient values.

$$
\begin{aligned}
& A=\frac{3}{4}\left(-4 p_{\theta}+3 r_{0}^{4}-3 r_{0}^{2}\right) \\
& B=\frac{3}{8 r_{0}^{4}}\left(16 p_{\theta}^{2}-8 p_{\theta} r_{0}^{4}+45 r_{0}^{8}-36 r_{0}^{6}+3 r_{0}^{4}\right) \\
& C=\frac{9}{4} r_{0}\left(2 r_{0}^{2}-1\right) \\
& D=\frac{3}{4 r_{0}^{5}}\left(-16 p_{\theta}^{2}+45 r_{0}^{8}-18 r_{0}^{6}\right)
\end{aligned}
$$

The special coefficient values for constants of the motion are $A=B$, $C=D$ for exact $\%_{2}, C=0$ for exact $\%_{2}, 6 C=0$ for exact $. \%_{2}, 16 A=B$, $16 C=D$ for exact $\% 4$ and $A=B, C=-D$ for approximate $\% 3$. From Eq. (3-194) and Eq. (3-175) it can be shown $C=0$ for $p_{\theta}=-3 / 16$. Thus, the approximate third order fit to the Hill's vortex has an exact constant of the motion when $p_{\theta}=-3 / 16$. In general, for $-3 / 2<p_{\theta}<-3 / 16$ the ratio $B / A$ varies from 4 to $5, D / C$ varies from $-5 \times 10^{-5}$ to -3 and $6 C / D$ varies from $-1.2 \times 10^{5}$ to -2 . There is then no apparent exact invariant and approximate invariant formulas have been implemented for $\pi_{3}$ and $\pi_{4}$. The main result of the approximate $\mathscr{J}_{3}$ and $\mathscr{J}_{4}$ invariant formulas for the $V_{H}$ potential having coefficients given by Eq. $(3-192)$ is that $\%_{3}$ is not 
appropriate for the Hill's vortex. This is illustrated by Figs. $3-11(a)$ and $3-11(b)$. For an orbit with $p_{\theta}=-.2$ and $H \approx V_{0}, F i g$.

$3-11$ (a) shows $\mathscr{H}_{3}$ changes by $100 \%$ varying in sign and magnitude. Figure $3-11$ (b) shows $\mathscr{H}_{4}$ has a $.6 \%$ variation and thus demonstrates reasonable constancy. Furthermore, the surface of section plot in Fig. 3-12 is superpojed a contour piot of $\mathscr{H}_{4}\left(r, z=0, v_{r}\right.$, $\left.v_{z}\left(v_{r}\right)\right)$ and good ayseement is apparent. The reason behind the constancy of $\% 4$ is understood by examining an alternate form of the Eq. $(3-181)$ constraint equation.

$$
\begin{aligned}
& \frac{\partial}{\partial r}\left(2 \frac{\partial T}{\partial z}\left(\frac{\partial^{2} \phi}{\partial z^{2}}-\frac{\partial^{2} \phi}{\partial r^{2}}\right)-4\left(\frac{\partial T}{\partial r}\right) \frac{\partial^{2} \phi}{\partial r \partial z}\right. \\
& \left.+T \frac{\partial}{\partial z}\left(\frac{\partial^{2} \phi}{\partial z^{2}}-\frac{\partial^{2} \phi}{\partial r^{2}}\right)-2 T \frac{\partial^{3} \phi}{\partial z \partial r^{2}}\right) \\
& \frac{\partial}{\partial z}\left(2 \frac{\partial T}{\partial r}\left(\frac{\partial^{2} \phi}{\partial z^{2}}-\frac{\partial^{2} \phi}{\partial r^{2}}\right)+4\left(\frac{\partial T}{\partial z}\right) \frac{\partial^{2} \phi}{\partial z \partial r}\right. \\
& \left.+T \frac{\partial}{\partial r}\left(\frac{\partial^{2} \phi}{\partial z^{2}}-\frac{\partial^{2} \phi}{\partial r^{2}}\right)+2 T \frac{\partial^{3} \phi}{\partial r \partial z^{2}}\right)=0
\end{aligned}
$$

Equation (3-196) shows when $T$ is the sum of a function of $r$ and a function of $z$ then the constraint becomes,

$$
\begin{aligned}
0 & =\frac{\partial T}{\partial z} \frac{\partial^{3} \phi}{\partial r^{3}}-\frac{\partial T}{\partial r} \frac{\partial^{3} \phi}{\partial z^{3}} \\
& =0
\end{aligned}
$$

and thus the constraint equation for $\mathscr{I}_{4}$ is satisfied identically. This means a small coupling coefficient for terms involving powers of both $r$ and $z$ in $V(r, z)$ causes the constraint equation to be 

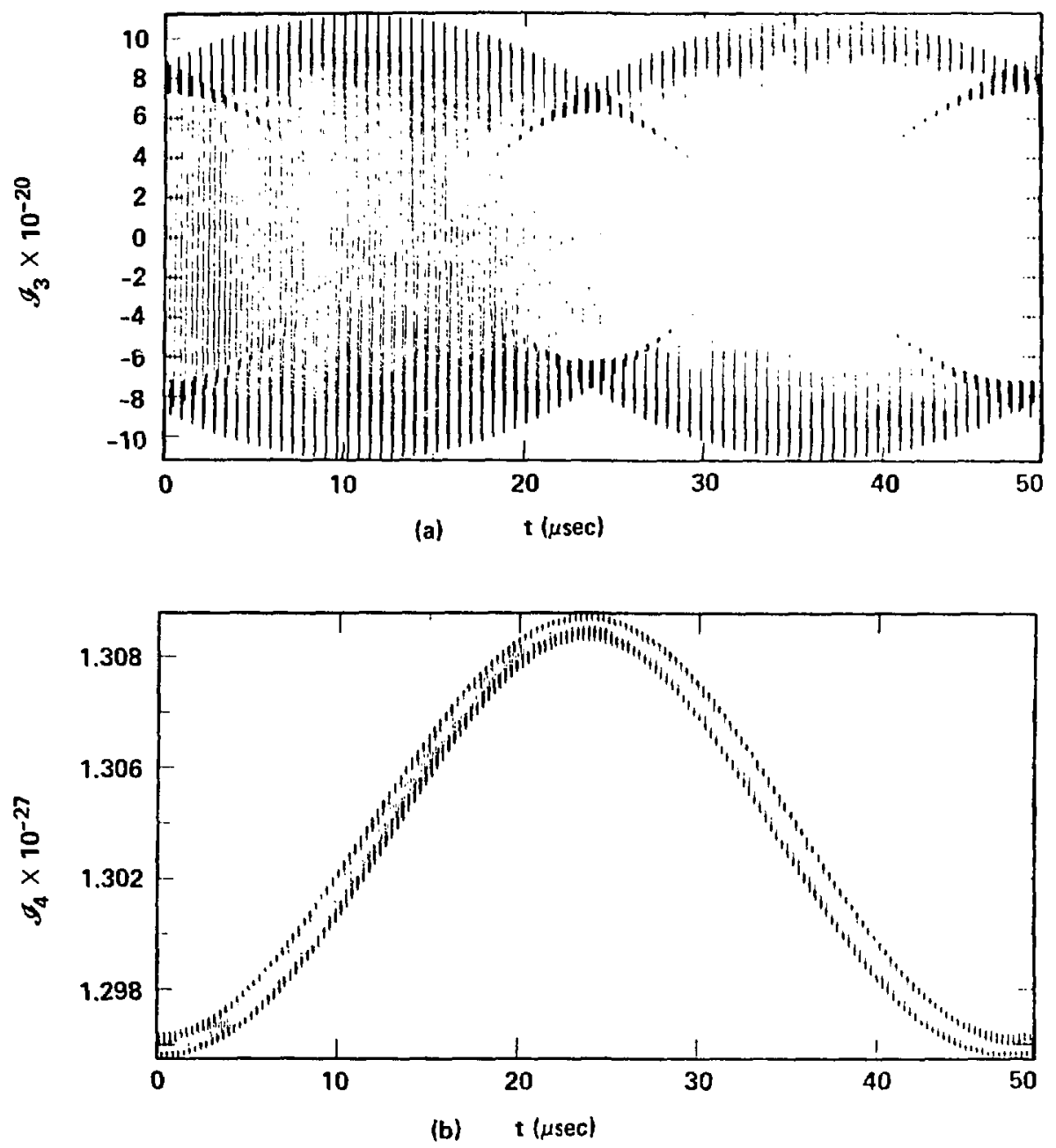

Figure 3-11. Invariants versus $t$ ime for $p_{\theta}=-.2, H \approx V_{0}$. (a) Third order velocity invariant (b) Fourth order velocity invariant. 


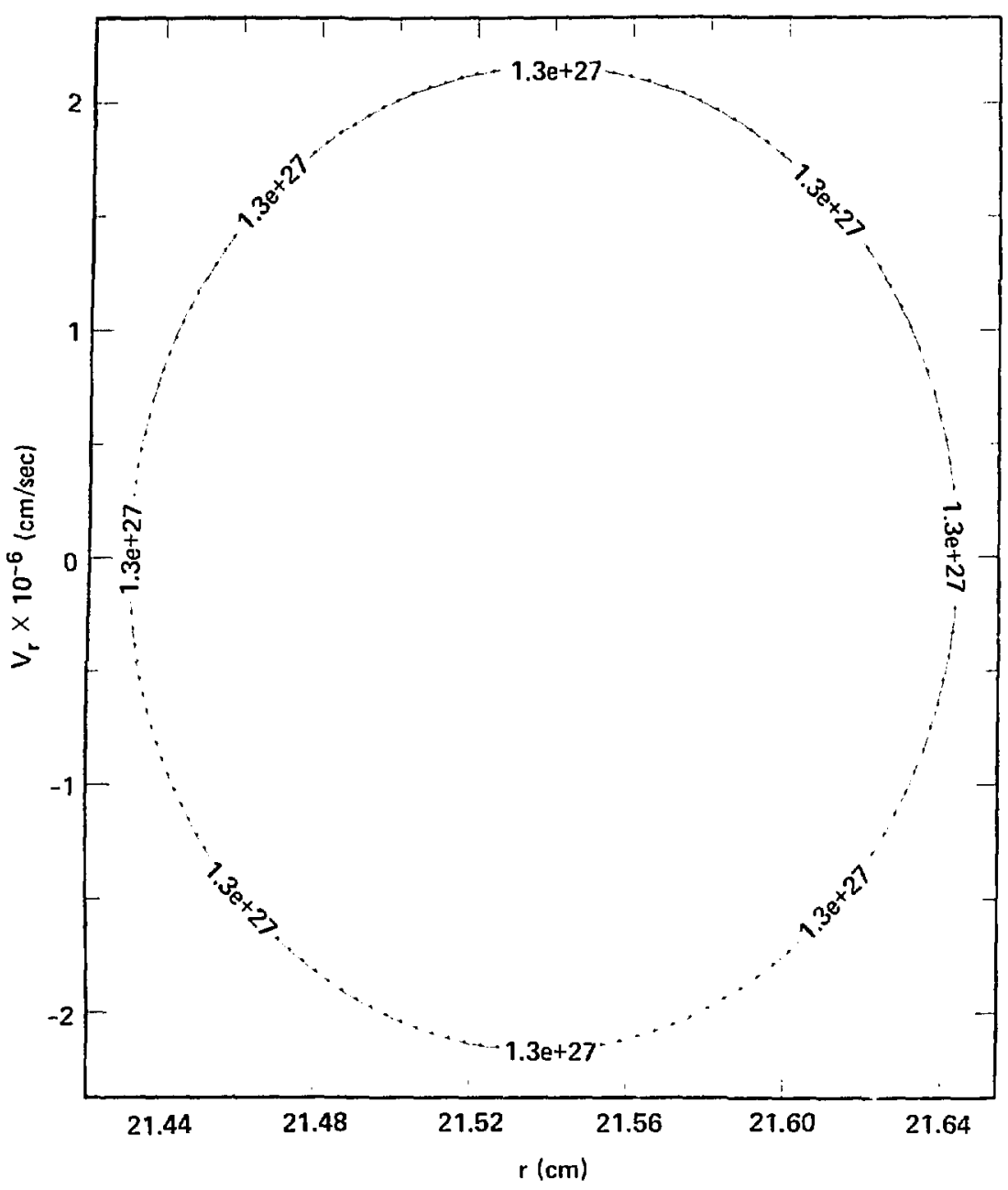

Figure 3-12. Surface of section for $p_{\theta}=-.2, H \approx V_{C}$ superposed on a contour plot of the fourth order velocity invariant. 
closer to being satisfied. In the case of $/ 3$ there is no automatic soiution of the Eq. (3-179) constraint equation.

For the complete Hill's vortex potential oniy. 4 has been implemented as a conjequence of experience gained with the approximate $V_{H}$ pritential. Additional motivation for the appropriateness of $\%$ is provided by arrow plots of particle orbits. Along an orbit at chosen time intervals the particle velocities may be recorded and an arrow of fixed length plotted with direction $\tan ^{-1}\left(v_{r} v_{z}^{-1}\right)$ at the corresponding coordinate position. An example is shown in Fig. 3-13. This is an orbit with $v=391$ and $p_{\theta}=-.208$. The arrows in this plot indicate at orbit crossings there are four directions of the motion. Consequently the constant of the motion must be at least fourth order in the velocity. This fact is related to the invariance of $v^{n} e^{i n \theta}$ for $n$ different values of $\theta$ from zero to $2 \pi$. For $n=4$ there are then four different directions allowed which leave $\left(v_{z}+i v_{r}\right)^{4}$ invariant.

The previously derived $\phi$ formula given by Eq. $(3-189)$ san be seen to have six arbitrary coefficients $A_{1,1}, A_{2,2}, A_{3,3}, A_{4,4}, A_{5,5}$, $A_{6,6}$. Because all other coefficients have been selected so the Poisson equation is satisfied, these six coefficients may be chosen to sotisfy the constraint equation for \% . A substitution of $\phi$ into the $\%_{4}$ constraint equation yields a polynomial in $r$ and $z$ which has more than six terms. It is not then possible to satisfy the constraint equation with only six arbitrary coefficients. Adding more homogeneous solutions to $\phi$ does not . lp since additional homogeneous solutions are of higher order and thus add liore terms 


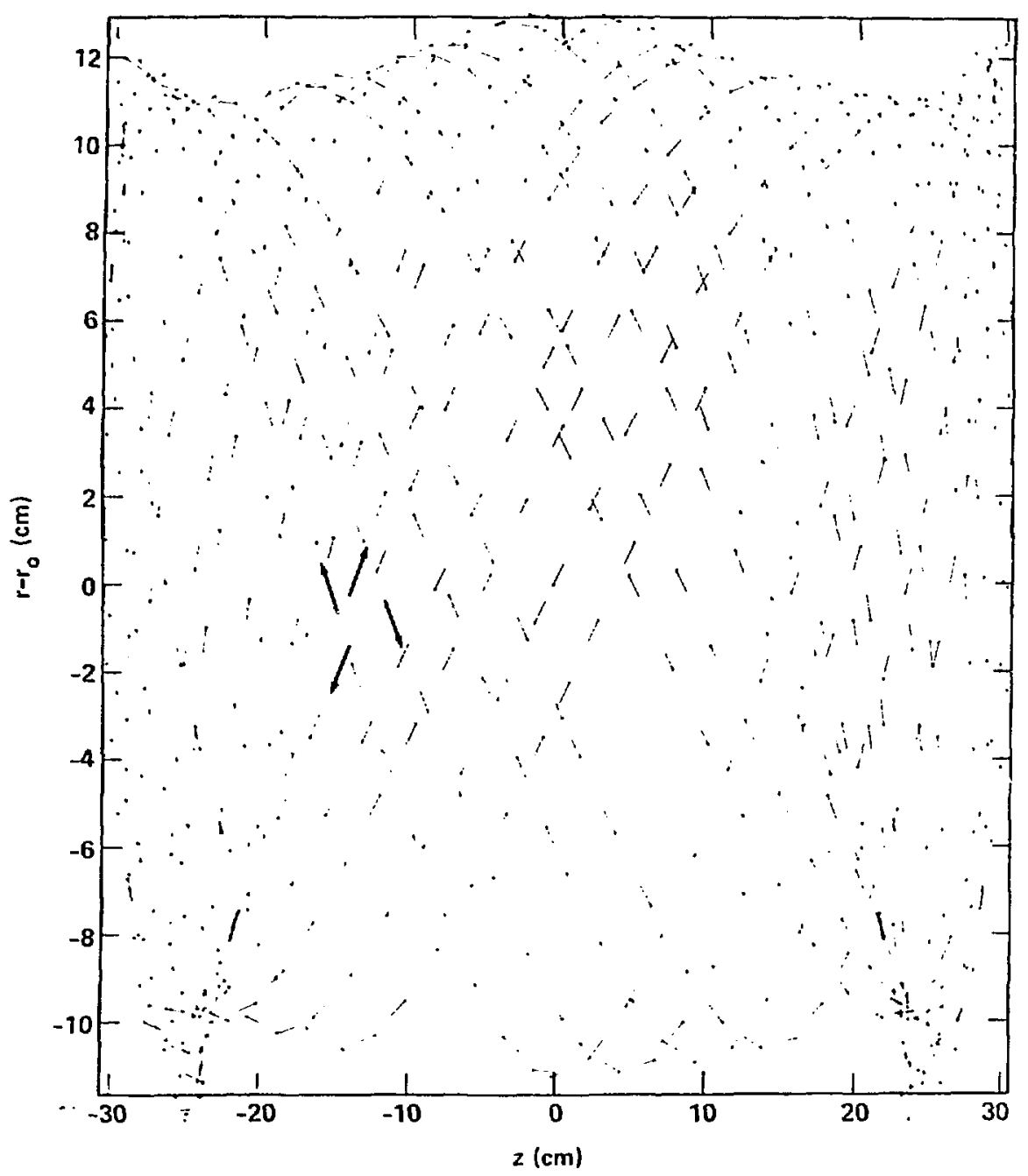

Figure 3-13. Four directions shown for velocity direction arrows with parameters $p_{\theta}=-.208, v=391$. 
than can be eliminated. The approach that has been implemented to achieve the best. $\mathscr{H}_{4}$ invariant is to expand the constraint equation polynomial, written in terms of $r^{2}$ and $z^{2}$, about $r^{2}=r^{2}$ and $z^{2}=0$. It is then possible to use the six arbitrary coefficients to elimirate the constant, linear and quadratic terms in the expansion. This procedure has been accomplished using th? REDUCE ${ }^{[156]}$ algebraic manipulation program. REOUCE generates subroutines which complite the coefficients of a system of six equations that are liviear in the six arbitrary coefficients. A standard decomposition of a $\tilde{\sigma}$ by 6 matrix into the usual LU product then yields the unknown coefficients. ihe result is a formula for $\%_{4}$ for which $\phi$ satisfies the Poisson equation and the constraint equation is satisfied to second order near $r^{2}=r_{0}^{2}$ and $z=0$. The constraint equation may be guaranteed to higher order by adding more hornogeneous solutions. In general, to go from satisfying the cons'raint equation at $n^{\text {th }}$ order to $n+1$ order requires adding $n+1$ homogeneous solutions to $\phi$. The actual " 4 formula with the constraint equation satisfied to second order is many pages lony and thus not written here. It is however known and is available to be used to calculate a change of variables Jacobian which could be implemented to produce an orbit average formula particular to $\%$. Such a formula would however only apply to low ene:"gy. Using the $\%_{4}$ formula with the constraint equation guaranteed to second order for this purpose is betier than using the $\mathscr{O}_{4}$ formula based on the $V_{H}$ potential with fitted Hill's vortex coefficients. Low energy $H \approx V_{0}$ orbits show the former formuia is superior. An orbit with $p_{\theta}=-.2, v=.0$ ? had a variation of less 
than a percent for the former formula, but a variation greater than $5 \%$ in the latter case.

Adding in more homogeneous solutions better satisfies the constraint equation, however this process is at best asymptotic. 0therwise in the case of a potential having stachastic orbits it would be possible to construct an invariant. The term stochastic here refers to an orbit for which correlations diminish in time and neighboring orbits diverge exponentially.

\subsubsection{Investigation of Conditions for Stochasticity}

Complementary to the effort directed at constructing constants of the motion a consideration has been given to the conditions under which an orbit is stochastic. One means of examining stochasticity is to transform to canonical variables ${ }^{[157]}$ and express the Hamiltonian in terms of a function only of momenta and a perturbing function which may depend on all coordinates. $[158,159]$ The reason for this approach is the resulting form of the equations of motion.

$$
\begin{aligned}
& H=G_{i}\left(P_{i}\right)+\varepsilon G_{2}\left(Q_{i}, P_{i}\right) \\
& \dot{P}_{\mathbf{i}}=-\varepsilon \frac{\partial G_{2}}{\partial Q_{i}} \\
& \dot{Q}_{i}=\frac{\partial \dot{G}_{1}}{\partial P_{i}}
\end{aligned}
$$

Since $\varepsilon$ is a small parameter Eq. (3-199) implies $P_{i}$ is almost constant. If $\varepsilon=0$ then the particle orbit is a point in $P_{i}$ momenta space. For non-zero $\varepsilon$ the orbit tends to fluctuate near the 
$\varepsilon=0$ point. When the small part of the Hamiltonian $G_{2}$ can be written as follows,

$$
G_{2}=\sum_{k \ell} G_{k \ell}\left(P_{i}\right) \sin \left(k Q_{1}+\ell Q_{2}\right)
$$

then the fluctuations can be large if the argument of the sine is slowly varying, or when the resonance condition $k \dot{Q}_{1}+2 \dot{Q}_{2}=0$ is satisfied. The magnitude of the fluctuation near the resonance is 1 imited because $Q$ depends on $P$ and thus the resonance condition is detuned as $P$ changes. The fluctuation magnitude or resoilance width is approxinated by Chirikov [159] from a pendulum Hamiltonian obtained by expanding $H$ about a resonance and then only keeping the resonant term in $G_{2}$. The resonance width is then taken to be the momentum range between the separatrix which separates oscillating and circulating orbits.

$$
\Delta \nabla\left(\varepsilon G_{k \ell}\right)^{1 / 2}
$$

The momenta and hence the particle orbit behaves stochastically when the widths of two resonances over lap. In other work ${ }^{[160-163]}$ the Hamiltonian given by $E q .(3-198)$ has an $E$ independent of $P$ and $Q$ and may therefore be used to determine a stochasticity th^eshold. For example in the work of Cohen, $[160]$ the basic motion is axial and radial bouncing and the perturbation is the effect of a quadrupole field. The parameter $\varepsilon$ then measures the strength of the quadrupole field and indicates when this effect leads to stochasticity. In the case of Hill's vortex there is no independent 
$\varepsilon$ parameter. The origin of stcchastic behavior for Hill's voriex is understood by examining a canonical transformation of a separable Hamiltonian. In a separable two dimensional Hamiltonian the potential consist of two functions which each only depend on one coordinate,

$$
H=\frac{1}{2}\left(F_{r}^{2}+p_{z}^{2}+G_{3}(r)+G_{4}(z j)\right.
$$

In principle the solution of the equations of motion corresponding to Eq. $(3-203)$ can be written as a quadrature by introducing the following generating funcrion. [157]

$$
\begin{aligned}
F(q, P) & =\int d r\left(2 P_{1}-G_{3}(r)\right)^{1 / 2} \\
& +\int d z\left(2 P_{2}-G_{4}(z)\right)^{1 / 2}
\end{aligned}
$$

The generating function in Eq. (3-204) consist of old coordinates and new momenta and the following relations thus obtain.

$$
\begin{aligned}
p_{r} & =\frac{\partial F}{\partial r} \\
& =\left(2 P_{1}-G_{3}(r)\right)^{1 / 2} \\
p_{z} & =\frac{\partial F}{\partial z} \\
& =\left(2 P_{2}-G_{4}(z)\right)^{1 / 2} \\
Q_{1} & =\frac{\partial F}{\partial P_{1}} \\
& =\int d r\left(2 P_{1}-G_{3}(r)\right)^{-1 / 2}
\end{aligned}
$$




$$
\begin{aligned}
Q_{2} & =\frac{\partial F}{\partial P_{2}} \\
& =\int d z\left(2 P_{2}-G_{4}(z)\right)^{-1 / 2}
\end{aligned}
$$

Substituting Eqs. (3-205) and (3-206) into Eq. (3-203) yields,

$$
H=P_{1}+P_{2}
$$

and the new Hamiltonian does not depend on coordinates. Thus, $p_{1}$ and $P_{2}$ are constants and $p_{z}^{2}+G_{4}$ and $p_{r}^{2}+G_{3}$ are also constant. As usual $\dot{Q}=$ aH/aP so $Q_{1}=t+c_{1}$ and $Q_{2}=t+c_{2}$, and $r(t)$ and $z(t)$ are implicitly defined by Eqs. (3-207) and (3-208). In the case of Hill's vortex there are terms in Eq. (3-185) which are products of powers of $r$ and $z$, and the Hamiltonian can not be written as Eq. (3-203). It is then these coupling terms which prevent an obvious canonical transformation resulting in a Hamiltonian depending only on momenta, which would then be constant. In general, methods exist to transform a Hamiltonian to normal form. In this case the Hamiltonian is only a function of actions which are $\pi\left(P_{i}^{2}+Q_{i}^{2}\right)$. Birkhoff $\left[{ }^{[164]}\right.$ has derived a procedure which cast a Hamiltonian inte normal form to a given order in the new canonical variables. As the order goes to infinity the normal form is obtained; however, there are questions of convergence of the procedure. Also Birkhoff requires the frequencies of the linearized motion to be incommensurate. In more recent work ${ }^{[165]}$ a normalization method has been derived which allows commensurate frequencies, including zero frequency, but even obtaining normal form at small order can 
require an immense amount of algebra. Typically, algebraic operitions are done on a computer.

An alternative approach is to seek a generating function which transforms the Hamiltonian to the form of Eq. (3-198), with $G_{2}$ similar to that of Eq. (3-201). The Hamiltonian valid for both $\psi_{>}$ and $\psi_{<}$with the appropriate $C_{n, m}$ coefficients is as follows.

$$
\begin{aligned}
H & =\frac{1}{2}\left(p_{r}^{2}+p_{z}^{2}\right)+\frac{1}{2} r^{-2}\left(p_{\theta}-\psi\right)^{2} \\
& =\frac{1}{2}\left(p_{r}^{2}+p_{z}^{2}\right)+\frac{1}{2} r^{-2} p_{\theta}^{2}+\frac{1}{2} \sum_{n=1}^{5} \sum_{m=0}^{n} c_{2 n-2 m, 2 m} r^{2 n-2 m z} 2 m
\end{aligned}
$$

The goal of the canonical transformation is to obtain new momenta or actions which account for the zero order particle motion. Thus $H$ is considered in an expansion about $r^{2}=r_{0}^{2}$ and $z^{2}=0$. From Eq. (3-210) it can ve seen the $r^{-2} p_{e}^{2}$ term, which is present for all magnetic fields, causes the expansion to have an infinite number of terms. The lowest order terms in the expansion are $4 \alpha^{2}\left(r^{2}-r_{0}^{2}\right)$ and $4 \beta^{2} z^{2}$ where,

$$
\begin{aligned}
& \alpha^{2}=\frac{1}{4}\left(\frac{\partial H}{\partial r^{2}}\right) r^{2}=r_{0}^{2} \\
& \beta^{2}=\frac{1}{4}\left(\frac{\partial H}{\partial z^{2}}\right) z^{2}=0
\end{aligned}
$$

A generating function is then constructed similar to Eq. (3-204) taking account of all contribusions from $r^{-2} p_{\theta}^{2}$ and considering 
only $4 \alpha^{2}\left(r^{2}-r_{0}^{2}\right)$ and $4 \beta^{2} z^{2}$,

$$
\begin{aligned}
F(q, p) & =\int d r\left(8 \alpha^{2} \bar{p}_{1}-4 \alpha^{2} r^{-2} \bar{p}_{\theta}-4 \alpha^{2} r^{2}\right)^{1 / 2} \\
& +\int d z\left(8 \beta^{2} \bar{p}_{2}-4 \beta^{2} z^{2}\right)^{1 / 2}
\end{aligned}
$$

where,

$$
\begin{aligned}
& \bar{p}_{1}=\alpha^{-1} p_{1} \\
& \bar{p}_{2}=\beta^{-1} p_{2} \\
& \bar{p}_{\theta}=p_{\theta}^{2} /\left(4 \alpha^{2}\right)
\end{aligned}
$$

The canonical variables are $P_{1}$ and $P_{2}$, and the barred variables $\bar{P}_{1}, \bar{P}_{2}$ are convenient normalizations. The following definitions were obtained similar to the manner in which Eqs. (3-205) to (3-208) were derived.

$$
\begin{aligned}
& Q_{1}=\sin ^{-1}\left(\left(r^{2}-\bar{p}_{1}\right)\left(\bar{P}_{1}^{2}-\bar{p}_{\theta}\right)^{-1 / 2}\right) \\
& P_{1}=\left(p_{r}^{2}+4 \alpha^{2} r^{-2} \bar{p}_{\theta}+4 \alpha^{2} r^{2}\right) /(8 \alpha) \\
& Q_{2}=2 \sin ^{-1}\left(2\left(2 \bar{p}_{2}\right)^{-1 / 2}\right) \\
& P_{2}=\left(p_{z}^{2}+4 \beta^{2} z^{2}\right) /(8 \beta) \\
& r^{2}=\bar{P}_{1}+\left(\bar{p}_{1}^{2}-\bar{p}_{\theta}\right)^{1 / 2} \sin Q_{1}
\end{aligned}
$$




$$
z^{2}=\bar{P}_{2}\left(1-\cos Q_{2}\right)
$$

The Hamiltonian which results from substituting Eq. (3-217) to Eq. (3-222) for $p_{r}, p_{z}, r^{2}$ and $z^{2}$ is in Appendix 0 . Considering as before the resonance condition $k \dot{Q}_{1}+\ell \dot{Q}_{2}=0$ the relevant $(k, \ell)$ values are $(1,1),(1,-1),(2,1),(2,-1),(1,2),(1,-2),(1,0)$, and $(0,1)$. The zero order Hamiltoniar, ito, is assumed to consist of terms that only depend on momenta and are no higher power than cubic. Inside the plasma region this is exact; however, outside the plasma this is an approximation. The resulting coordinate time derivatives are below.

$$
\begin{aligned}
\dot{\mathrm{Q}}_{1} & =\frac{1}{\alpha} \frac{\partial \mathrm{H}_{0}}{\partial \overline{\mathrm{P}}_{1}} \\
& =\frac{1}{2 \alpha}\left(C_{2,0}+4 \alpha^{2}+\frac{15}{8} c_{10,0} \overline{\mathrm{p}}_{\theta}^{2}-\frac{3}{2} C_{6,0} \overline{\mathrm{p}}_{\theta}\right) \\
& +\frac{1}{\alpha}\left(\frac{3}{2} C_{4,0}-\frac{15}{4} C_{8,0} \overline{\mathrm{p}}_{\theta}\right) \overline{\mathrm{P}} \\
& +\frac{1}{2 \alpha}\left(C_{2,2}-\frac{3}{2} C_{6,2} \overline{\mathrm{p}}_{\theta}\right) \overline{\mathrm{P}}_{2} \\
& +\frac{3}{2 \alpha}\left(\frac{5}{2} C_{6,0}-\frac{35}{4} c_{10,0} \bar{p}_{\theta}\right) \overline{\mathrm{P}}_{1}^{2} \\
& +\frac{1}{2 \alpha}\left(\frac{3}{2} C_{2,4}-\frac{9}{4} C_{6,4} \bar{p}_{\theta}\right) \bar{P}_{2}^{2}
\end{aligned}
$$




$$
\begin{aligned}
+ & \frac{1}{\alpha}\left(\frac{3}{2} C_{4,2}-\frac{15}{4} C_{8,2} \bar{p}_{\theta}\right) \bar{P}_{1} \bar{P}_{2} \\
\dot{Q}_{2}= & \frac{1}{\beta} \frac{\partial H_{0}}{\partial \bar{P}_{2}} \\
& =\frac{1}{2 B}\left(C_{0,2}+4 \beta^{2}+\frac{3}{8} C_{8,2} \bar{p}_{\theta}^{2}-\frac{1}{2} C_{4,2} \bar{p}_{\theta}\right) \\
& +\frac{1}{\beta}\left(\frac{3}{2} C_{0,4}-\frac{3}{4} C_{4,4} \bar{p}_{\theta}\right) \bar{P}_{2} \\
& +\frac{1}{2 B}\left(C_{2,2}-\frac{3}{2} C_{6,2} p_{\theta}\right) \bar{P}_{1} \\
+ & \frac{3}{2 B}\left(\frac{5}{2} C_{0,6}-\frac{5}{4} C_{4,5} \bar{p}_{\theta}\right) \bar{P}_{2}^{2} \\
& +\frac{1}{2 B}\left(\frac{3}{2} C_{4,2}-\frac{15}{4} C_{8,2} \bar{p}_{\theta}\right) \bar{P}_{1}^{2} \\
& +\frac{1}{B}\left(\frac{3}{2} C_{2,4}-\frac{9}{4} C_{6,4} \bar{p}_{\theta}\right) \bar{P}_{1} \bar{P}_{2}
\end{aligned}
$$

The resonance condition determines the resonance curves in momentum space. The excursions about the resonance curves are obtained by expanding $\mathrm{H}_{0}$ about a harmonic oscillator at position $\mathrm{P}_{10}, \mathrm{P}_{20}$. The resonance condition and $h_{1}, h_{2}, h_{3}, h_{4}, h_{5}$ are written out in Appendix E. The expanded $\mathrm{H}_{0}$ is then below.

$$
\begin{aligned}
H_{0} & =H_{0}\left(P_{10}, P_{20}\right)+h_{1}\left(P_{1}-P_{10}\right)+h_{2}\left(P_{2}-P_{20}\right) \\
& +h_{3}\left(P_{1}-P_{10}\right)\left(P_{2}-P_{20}\right)+h_{4}\left(P_{1}-P_{10}\right)^{2}+h_{5}\left(P_{2}-P_{20}\right)^{2}
\end{aligned}
$$


Each resonance is examined separately by considering the Hamiltonian to consist of $\mathrm{H}_{0}$ and one trigonometric term. A canonical transformation is made to make the argument of the trigonometric function a single variable. The momentum conjugate to the cyclic angle is then constant. The resonance width is then given by the separatrix in the pendulum Hamiltonian. For the general sin $(k)_{1}+$ $\mathrm{lQ}_{2}$ ) term the resonance condition is as previously mentioned $k \dot{Q}_{1}+$ $\ell \dot{Q}_{2}=0$ and the following generating function is used to make $Q_{2}$ cyclic.

$$
F(Q, \pi)=\left(k Q_{1}+\ell Q_{2}\right) \pi_{1}+Q_{1} P_{10}+Q_{2}\left(\pi_{2}+P_{20}\right)
$$

The transformed coordinates are below,

$$
\begin{aligned}
& Q_{j}^{\prime}=k Q_{1}+\ell Q_{2} \\
& Q_{2}^{\prime}=Q_{2} \\
& \pi_{1}=\left(P_{1}-P_{10}\right) / k \\
& \pi_{2}=P_{2}-P_{20}-\ell\left(P_{1}-P_{10}\right) / k
\end{aligned}
$$

Considering $H_{0}$ expanded about $P_{10}, P_{20}$, neglecting constants and other trigonometric terms the Hamiltonian is as follows.

$$
\begin{aligned}
H=\left(k h_{1}+l h_{2}\right) \pi_{1}+ & \left(h_{3} k l+h_{4} k^{2}+h_{5} \ell^{2}\right) \pi_{1}^{2} \\
& +G_{k l} \sin Q_{1}
\end{aligned}
$$

The coefficient $G_{k l}$ is the coefficient of $\sin \left(k Q_{1}+\ell Q_{2}\right)$ written in Appendix $D$, expressed in terms of the expansion position variables 
$P_{10}, P_{20}$. The resonance width is then obtained from the followirig relations.

$$
\begin{aligned}
& P_{1}=P_{10} \pm k\left(2 G_{k \ell}\right)^{1 / 2}\left(h_{3} k \ell+h_{4} k^{2}+h_{5} \ell^{2}\right)^{1 / 2} \\
& P_{2}=P_{20} \pm \ell\left(2 G_{k \ell}\right)^{1 / 2}\left(h_{3} k \ell+h_{4} k^{2} i h_{5} \ell^{2}\right)^{1 / 2}
\end{aligned}
$$

The above formulas and the relevant resonance conditions for the Hill's vortex have been plotted as a function of $p_{\theta}$. For $-.202<$ $p_{\theta}<0$ there are three resonance conditions which are satisfied, $(1,-1),(2,1)$ and $(1,-2)$. For $-.325<p_{\theta}<-.202$ there are two resonance conditions satisfied, $(1,-1)$ and $(1,-2)$. For $p_{\theta}<-.325$ there is one resonance condition satisfied, $(1,-2)$. The plots of resonance conditions show ranges of $P_{1}$ and $P_{2}$ which correspond to overlap and thus an orbit which has these values would be predicted to exhibit stochastic behavior. The resonance condition predictions have been explored with corresponding orbits in the NEO code. It was found orbits that exhibited excurions between three resonances appeared to be regular. Also, no stochastic behavior was evident in surface of section plots. These results occurred for $P_{1}>1, P_{2}$ $>1$ and consequently in this range the underlying assumption that $G_{k \ell}$ is small compared to $H_{0}$ is violated. An alternate symptom that an assumption has been violated is that the resonance widths are of the same order as $P_{10}, P_{20}$. The difficulty stems from the nonconstancy of $P_{1}$ and $P_{2}$ which indicates these variables are not adequately describing the zero order motion. In a different regime 
where $P_{1}<.11$ and $P_{2}<.1$, the resonance widths are smaller than $p_{10}, P_{20}$. The resonance curves for $p_{\theta}=-.196$ and $p_{\theta}=-.202$ ire shown in Figs. 3-14 and 3-15 respectively. At $p_{\theta}=-.196$, there are three resonances and for $P_{2} \approx 0, P_{1} \approx .1$ the $(1,-1)$ and $(2,-1)$ branches are nearby. At $p_{\theta}=-.202$, the $(2,-1)$ resonance coridition is not satisfied and for $P_{2} \approx 0, p_{1} \approx .1$ there is only the $(1,-1)$ resonance. To investigate the effect of the resonances two orbits were followed. The first at $p_{\theta}=-.196$ and the secend at $p_{\theta}=-.202$. In each case the energy was chosen as $6 \%$ of the axial loss value. For $p_{\theta}=-.196, .098<p_{1}<.1, .01<p_{2}<.045$. For $p_{\theta}=-.202$, $.1<P_{1}<.11, .01<P_{2}<.037$. In both cases it can be seen $P_{1}$ is nearly a constant. The surface of section $v_{r}$ versus $r$ at $z=0$ is qualitatively different for the two orbits. The $p_{\theta}=-.196$ surface of section shown in Fig. 3-16 exhibits rippling and begins to show the breakup of a smooth structure. This is the case where the orbit has $P_{1}, P_{2}$ values that intersect two resonance curves. In Fig. 3-17 for $p_{\theta}=-.202$, the orbit is only under the influence of one resonance and the surface of section is regular and very smooth. A change of only $3 \%$ in the value of $p_{\theta}$ results in an irregular surface of section. The small $p_{\theta}$ change brings in another resonance curve and this is responsible for the irregular surface of section. At small values of $P_{1}, P_{2}$ the interaction of several resonances with an orbit causes surface of section irregularity; however, the total lack of structure characteristic of stochasticity has not been observed. At large values of $P_{1}, P_{2}$ where the orbits are stochastic, this implementation of resonance overlap theory does not apply since as discussed previously the coordinates are inappropriate in this regime. 

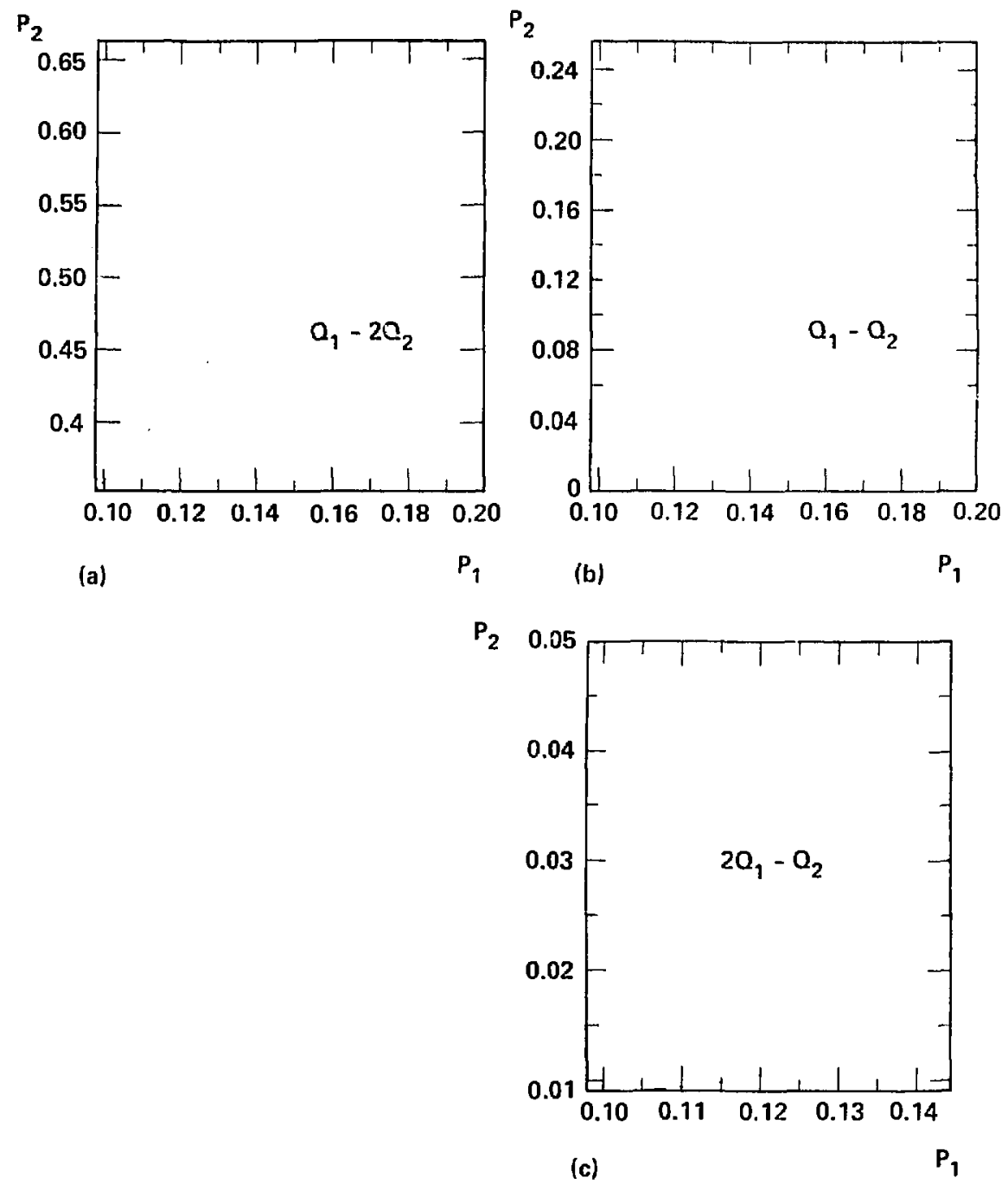

Figure 3-14. Resonances for $p_{\theta}=-.196(a)(1,-2)$ (b) $(1,-1)$ (c) $(2,-1)$ 


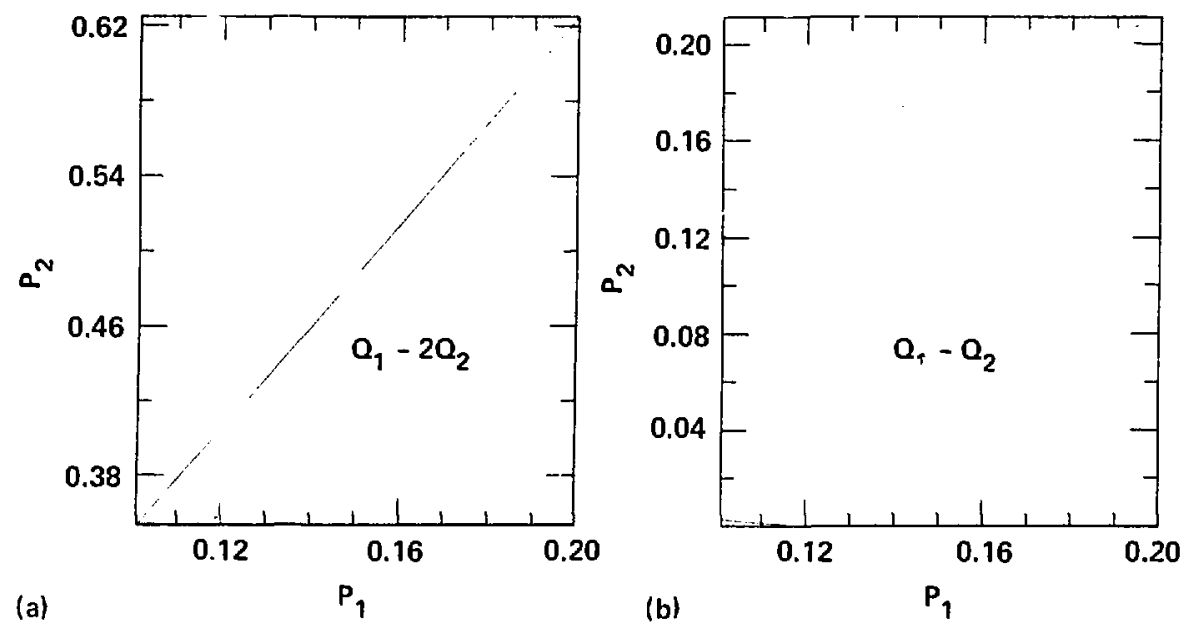

Figure 3-15. Resonances for $p_{\theta}=-.202(a)(1,-2)(b)(1,-1)$. 


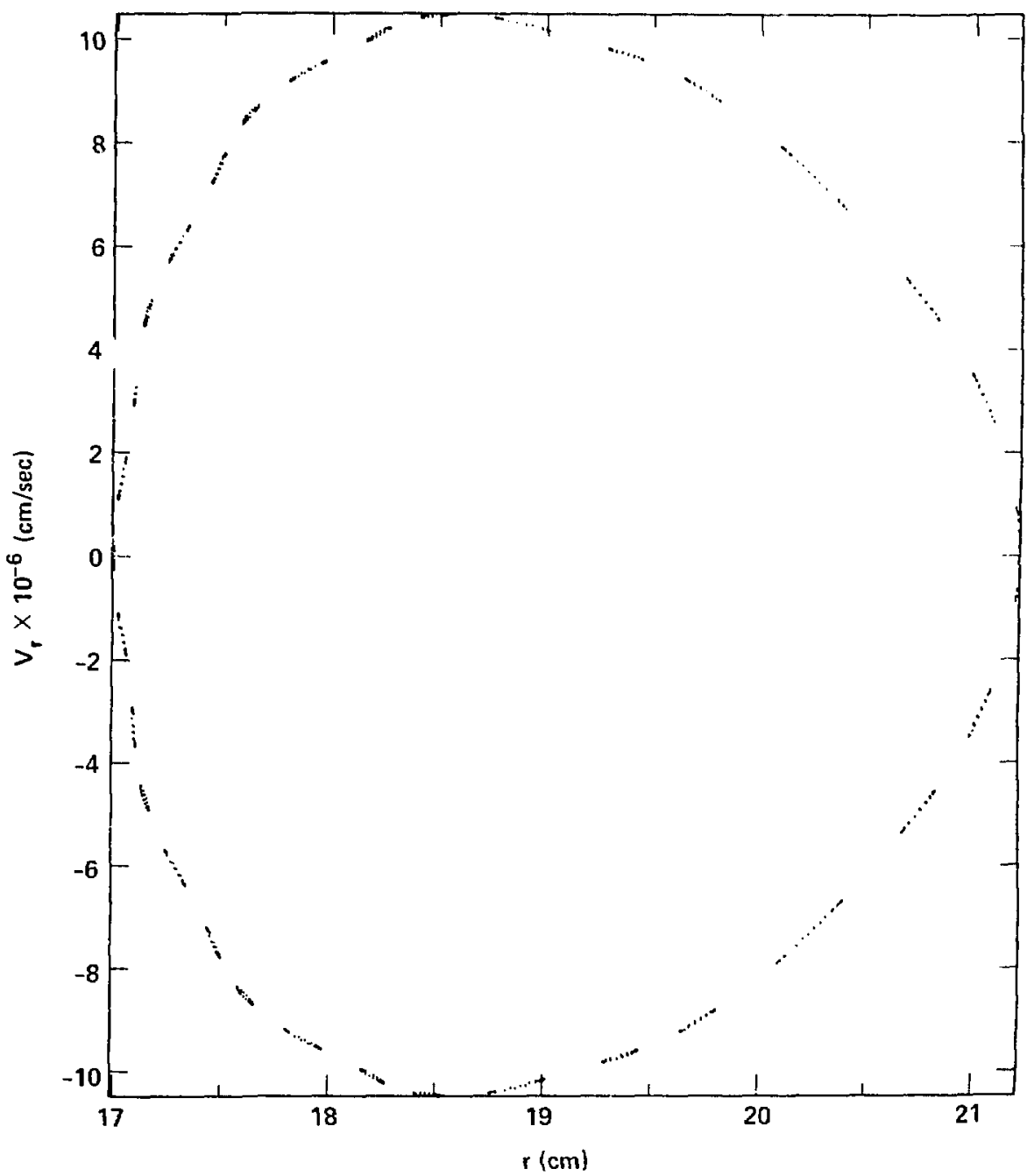

Figure 3-16. Surface of section for $p_{\theta}=-.196$ exhibiting rippling and structure breakup. 


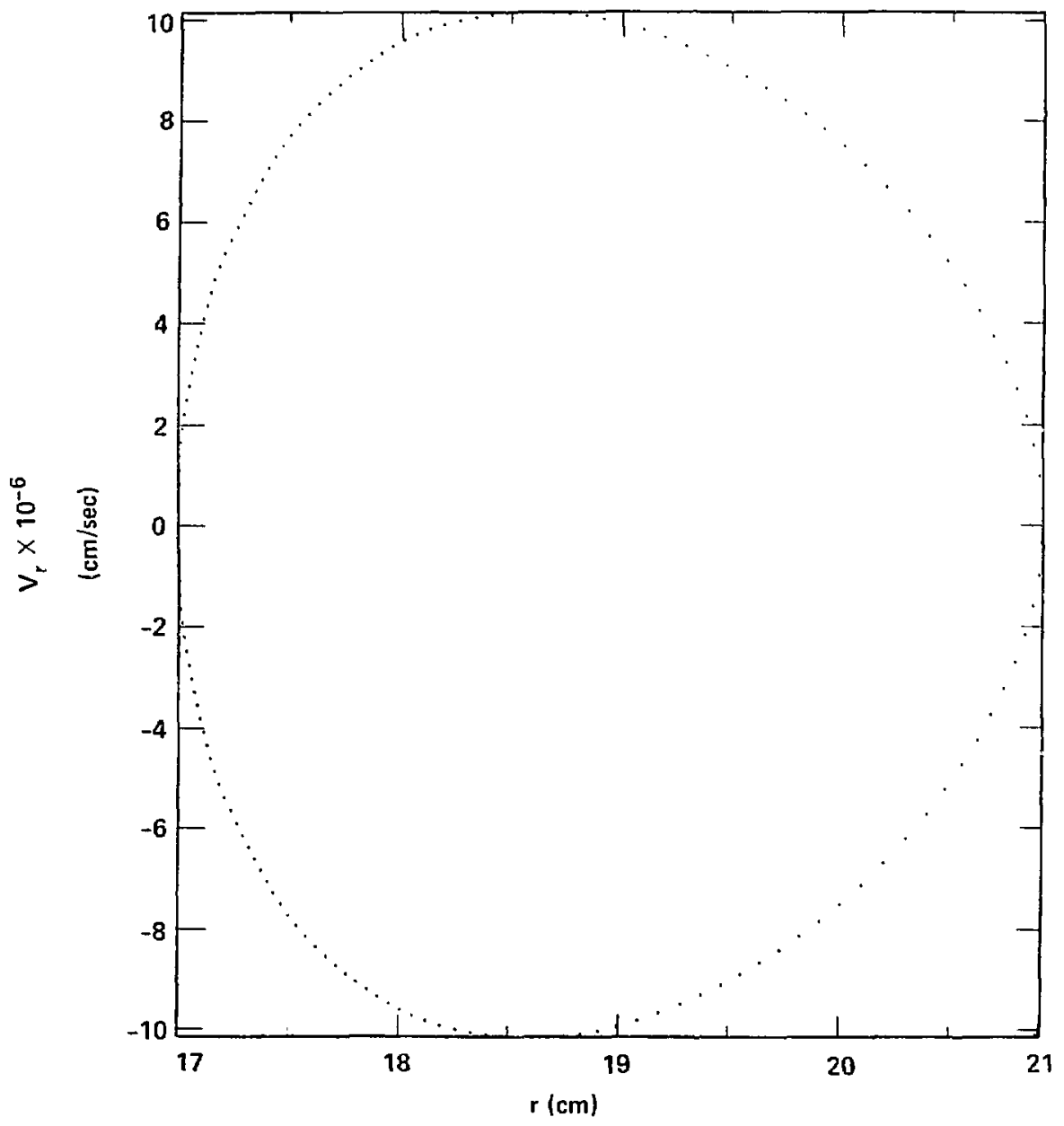

Figure 3-17. Surface of section for $p_{\theta}=-.202$ illustrating smooth structure. 
CHAPTER 4

LARGE LARMOR RADIUS KINETIC EQUATION MODEL

\subsection{Introduction}

The large Larmor radius effects are incorporated in the plasma description by considering the electrons as an inertialess fluid and modeling the ions with a distribution function. The electrons are governed by a continuity and momentum equation, and quasineutrality is assumed. The ion distribution function is required to satisfy a kinetic equation. Oue to the complexity of the problem axisymmetry is assumed and only a radial spatial variation is allowed. The formulation is thus one dimensional.

The plasma is subject to a magnetic field obtained from the cylindrical coordinate theta component of the vector potential. This restricts the magnetic field to be poloidal and in one dimension there is only a $B_{z}$ magnetic field. The vector potential is obtained by solving Ampere's law with a current which is the 
difference between the theta veiocity moment of the ion distribution function and the density times the theta electron fluid velocity. The kinetic equation is formulated in terms of a Poisson bracket with the system Hamiltonian. The Hamilton distrioution function are expanded using collision frequency as an expansion parameter. By applying the orbit average to the hierarchy of resulting equations a kinetic equation for the zero order inn distribution function is obtained. This procedure is similar to the method used to derive neoclassical diffusion however, the actual orbit has been averaged rather than just the gyro-orbit.

The time evolution of the zero order ion distribution function requires an initial condition. To generate self-consistent electric and magnetic fields, the electron equations have been solved in conjunction with an idealized ion distribution function. Assumptions have been made to generate completely analytic self-consistent electric and magnetic fields. More realism is possible by relaxing assumptions and proceeding with a numerical solution however, the analytic solution shows the effect of the radial electron velocity and the resulting electric and magnetic fields.

\subsection{Analytic Electric and Magnet ic Fields}

The off axis tangential neutral beam injection experiments in 2XIIB diu not achieve field reversal as discussed in Chapter 1 . It was proposed that electron dynamics were responsible for this result. The hypothesis was the electrons cancelled the intended dominant ion current by electron ion drag and drifts caused by radial electric 
fields. A model to study the electron effects was proposed by Baldwin and Rensink ${ }^{[166]}$ and forms the basis of the electron mode 1 in this analysis. Their electron model consists of quasineutrality,

$$
0=\sum_{j} n_{j} z_{j}-n_{e}
$$

and the electron momentum equation,

$$
\begin{aligned}
0 & =-e\left(\vec{E}+c^{-1} \vec{v}_{e} \times \vec{B}\right)-n_{e}^{-1} \nabla n_{e}^{\top} e \\
& +m_{e} v_{e} i_{e}^{-1} \sum_{j} n_{j} z_{j}^{2}\left(\vec{v}_{j}-\vec{v}_{e}\right)
\end{aligned}
$$

where $j$ is the ion species subscript, e is the magnitude of the charge of the electron, subscript e refers to electrons, $z_{j}$ is the ion charge state and the electron ion collision frequency is defined be low.

$$
v_{e i}=4 n_{e} 2^{1 / 2} \pi e^{4} \times\left(3 m_{e}^{1 / 2} T_{e}^{3 / 2}\right)^{-1}
$$

In Eq. (4-3), $\lambda$ is the coulomb logarithm given by Eq. (2-47). The collision term in Eq. (4-2) is treated as 1 inear in the velocity difference. This approximation has been derived by Braginski $i^{[167]}$ by expanding relative electron ion velocity in terms of ion velocity in the collision operator, neglecting terms of order $\mathrm{m}^{\mathrm{m}^{-1}} \mathrm{j}^{-1}$, and approximating the electron distribution as a perturbed maxwellian. The other feature of Eq. (4-2) is the absence of the $m_{e} d v_{e} / d t$ inertia term, which implies the electron mass is assumed small or the 
electron response time is instantaneous. This is the inertialess fluid approximation.

An additional equation beyond the Baldwin, Rensink model which applies to the electrons is the continuity equation.

$$
\frac{\partial n_{e}}{\partial t}+\nabla \cdot\left(n_{e} \vec{v}_{e}\right)=s(e)
$$

In Eq. (4-4) $\mathrm{S}^{(e)}$ is the electron source function.

Maxwell's equations without displacement current are used to solve for $\vec{E}$ and $\vec{B}$. The magnetic field only has a poloidal component.

$$
\begin{aligned}
& \vec{B}=\nabla \psi \times \nabla \theta \\
& B_{r}=-\frac{1}{r} \frac{\partial \psi}{\partial z} \\
& B_{z}=\frac{1}{r} \frac{\partial \psi}{\partial r}
\end{aligned}
$$

In Eq. (4-5) as before $\psi=r A_{\theta}$ where $A_{\theta}$ is the theta component of the vector potential. Ampere's law neglecting displacement current then yields the equation below.

$\nabla \times(\nabla \psi \times \nabla \theta)=4 \pi c^{-1} \vec{\jmath}$

Faraday's law yields an electric field of the following form,

$$
\vec{E}=-c^{-1} \nabla \theta \frac{\partial \psi}{\partial t}-\nabla \phi
$$

where $\phi$ is the electric field scalar potential. The electron momentum equation, $\mathrm{Eq} .(4-2)$ is written in terms of resistivity $n$ and 
235

current using Eq. $(4-9)$ for $\vec{E}$.

$$
\begin{aligned}
0 & =c^{-1} \frac{\partial \psi}{\partial t} \nabla \theta+\nabla \phi-c^{-1} v_{e} \times \vec{B}-\left(e_{e}\right)^{-1} m_{e} e_{e} \\
& +n(\vec{J}-\vec{j} \text { Ohkawa) }
\end{aligned}
$$

In Eq. $(4-10)$ the following definitions are used,

$$
\begin{aligned}
& \vec{j}=e \sum_{j} \vec{n}_{j} v_{j} z_{j}-e n_{e} \vec{v}_{e} \\
& \left.\vec{j} \text { Ohkawa }=e \sum_{j} n_{j} v_{j} z_{j}\left(1-z_{j}<Z\right\rangle^{-1}\right) \\
& n=m_{e} v_{e i}\langle Z\rangle\left(e^{2} n_{e}\right)^{-1} \\
& \langle Z\rangle=r_{e}^{-1} \sum_{j} n_{j} z_{j}^{2}
\end{aligned}
$$

In a steady state, axisymmetric, one dimensional system the electrons are modeled by six equations, with six unknowns " $e$, $s^{(e)}, \psi, \phi, v_{r e}, v_{\theta e}$. The electron temperature $T_{e}$ is actually specified by a heat or energy equation, however for the purposes of this model $T_{e}$ is given an assumed functional form or used as a free parameter. Introducing another equation would bring in the heat flux which would then have to have some assumed form. The ion quantities in the six equations, such as $n_{j}, v_{r j}, v_{\theta j}$ are assumed to be obtained from moments of a known ion distribution function. The electron density is defined in terms of ion densities and charge states by the quasineutrality condition, Eq. (4-1). 


$$
n_{e}=\sum_{j} n_{j} z_{j}
$$

The continuity equation, Eq. (4-4) in steady state is used as a means of determining $s^{(e)}$ after $n_{e} v_{r e}$ is known. This is actually a consistency condition because usually $S^{(e)}$ is defined initially and $n_{e} v_{r e}$ is determined. The equation below defines a source function which must have existed to generate the $n_{e} v_{r e}$ which is really constrained by the choice of ion distribution function.

$$
s^{(e)}=\frac{1}{r} \frac{\partial}{\partial r}\left(r n_{e} v_{r e}\right)
$$

The theta component of Ampere's ?aw in Eq. $(4-8)$ is used to deterimine $\psi$

$$
\frac{\partial}{\partial r}\left(\frac{1}{r} \frac{\partial \psi}{\partial r}\right)=-4 \pi c^{-1} J_{\theta}
$$

The radial component of the electron momentum equation, Eq. (4-10) provides an equation for $\phi$.

$$
\begin{aligned}
\frac{\partial \Phi}{\partial r} & =(r c)^{-1} v_{\theta e} \frac{\partial \psi}{\partial r}+\left(e_{e}\right)^{-1} \frac{\partial}{\partial r}\left(n_{e} T_{e}\right) \\
& -\eta\left(J_{r}-J_{r}^{\text {Ohkawa }}\right)
\end{aligned}
$$

The radial electron velocity $v_{r e}$ is obtained from a rearrangement of the radial component of Ampere's law, Eq. (4-19).

$$
\begin{aligned}
& J_{r}=0 \\
& v_{r e}=n_{e}^{-1} \sum_{j} n_{j} v_{r j} z_{j}
\end{aligned}
$$


The theta electron velocity results from the theta component of the electron momentum equation,

$$
\frac{\partial \psi}{\partial t}+v_{r e} \frac{\partial \psi}{\partial r}=-r c n\left(J_{\theta}-J_{\theta}^{0 h k a w a}\right)
$$

written in steady state.

$$
J_{\theta}=-v_{r e}(r c n)^{-1} \frac{\partial \psi}{\partial r}+J_{\theta}^{0 h k a w a}
$$

The first term on the right side of Eq. (4-22) is analogous to the bootstrap current $[168]$ in tokamak theory. The second term is the Ohkawa current ${ }^{[169]}$ which was first studied in an application related to neutral beam injection of tokamaks. The ohkawa current is relevant in this application since Eq. (4-22) shows near the field null $(\partial \psi / \partial r=0) J_{\theta}$ vanishes without an Ohkawa current. From Eq. (4-12) it can be seen $J_{\theta}^{\text {Ohkawa }}$ is zero if all ion species have the same average velocity or charge state. Thus, it is necessary to have ion species of different charge states or average velocities to maintain a current at the field null. Baldwin and Rensink argue that an assumed field variation of $B=\rho^{\alpha}$, gives $J_{\theta}=\rho^{\alpha-1}$ from Ampere's law where $\rho$ is distance from the field null and Eq. (4-22) then implies $v_{r e}=\rho^{-1}$ if $J_{\theta}^{\text {Ohkawa }}=0$. It is then necessary to have an Ohkawa current to avoid a singular electron velcoity at the field null. They further conclude from the diffusion character of Eq. (4-21) an initially nonreversed field can not become field reversed without an Ohkawa current. Substituting Eq. $(4-11)$ and Eq. $(4-12)$ into Eq. $(4-22)$ yields the equation for $v_{\theta e}$ * 


$$
v_{\theta e}=\left(e n_{e}\right)^{-1}\left(v_{r e}(r c n)^{-1} \frac{\partial \psi}{\partial r}+e \sum_{j} n_{j} v_{\theta j} z_{j}^{2}\langle z\rangle^{-1}\right)
$$

It can be seen from Eq. (4-23) that near the field null $v_{\theta e}$ is almost totally determined by the second term on the right side. From Eq. (4-22) this is a statement that the current $J_{\theta}$ is essentially equal to $J_{0}^{\text {Oill. awa }}$.

\subsubsection{Zero Radial Electron Velocity}

The condition $J_{\theta}=J_{\theta}^{\text {Ohkawa }}$ is satisfied at the field null. Assuming $v_{r j}=0$ then from Eq. $(4-20), v_{r e}=0$ and $J_{\theta}=J_{\theta}^{0 h k a ́ r a ~}$ everywhere. Any function of the constants of the motion satisfies the vlasov equation and as shown in a later section it is thus reasonable to assume the ion distribution function depends on $\mathrm{H}$, $p_{\theta}$ and $p_{Z}$. The Hamiltonian $H$ is even in $p_{r}$ so the $p_{r}$ velocity moment of the ion distribution function is zero and $v_{r j}=0$. The radial electron velocity is thus set to zero and $v_{\theta e}$ is obtained from Eq. (4-23), substituting the definition of $\langle Z>$ from Eq. $(4-14)$.

$$
v_{\theta e}=\left(\sum_{j} n_{j} z_{j}^{2}\right)^{-1} \sum_{j} n_{j} v_{\theta j} z_{j}^{2}
$$

The condition $v_{r j}=0$ also changes Eq. $(4-18)$ as follows.

$$
\frac{\partial \phi}{\partial r}=(r c)^{-1} v_{\theta e} \frac{\partial \psi}{\partial r}+\left(e n_{e}\right)^{-1} \frac{\partial}{\partial r}\left(n_{e}{ }_{e}\right)
$$

Solving Eq. (4-25) requires the ion distribution $f_{j}$ to obtain $n_{j} v_{\theta j}$. It is then possible to calculate $J_{\theta}$ and solve for $\psi$ by $E q$. (4-17). In general $J_{\theta}$ depends on $\phi$ and $\psi$, and Eq. (4-25) must be 
solved simultaneously with $\mathrm{Eq} .(4-17)$. The coupling between these equations is avoided using a formulation by Marx ${ }^{[170]}$ as extended by Post.[171] The distribution function is assumed to be a delta function in $H$ and $p_{\theta}$ and constant in $p_{z}$.

$$
f_{j}=R_{0} n_{0 j}\left(\pi m_{j}\right)^{-1} \delta\left(H-H_{0 j}\right) \delta\left(p_{\theta}-p_{0 j}\right)
$$

In Eq. (4-26) $R_{0}$ is the position where density $n_{0 j}$ occurs. The density is obtained from a velocity space integration over $f_{j}$.

$$
n_{j}=\int f_{j} d p_{r} d p_{\theta}^{\prime} d p_{z}
$$

In Eq. (4-27),

$$
\begin{aligned}
p_{\theta}^{\prime} & =m v_{\theta} \\
& =r^{-1}\left(p_{\theta}-e z_{j} c^{-1} \psi\right)
\end{aligned}
$$

where $p_{\theta}$ is the usual canonical monentum. To integrate $f_{j}$ which is a function of $H, p_{\theta}, P_{z}$ a change of variables Jacobian is used.

$$
\left|\frac{\partial\left(H, p_{\theta}\right)}{\partial\left(p_{r}, p_{\theta}^{\prime}\right)}\right|=m_{j}^{-1} r p_{r}
$$

Expressing $p_{r}$ in terms of $H, p_{\theta}, p_{z}$ using Eq. (3-21), and substituting in Eq. (4-27) yields the following equation.

$$
n_{j}=r^{-1} m_{j} \int \frac{f_{j} d H d p_{\theta} d p_{z}}{\left(2 m_{j}\left(H-e z_{j} \phi\right)-r^{-2}\left(p_{\theta}-e z_{j} c^{-1} \psi\right)^{2}-p_{z}^{2}\right)^{1 / 2}}
$$


Let,

$$
\alpha_{j}=\left(2 m_{j}\left(H_{0 j}-e z_{j} \phi\right)\right)^{1 / 2}
$$

and substituting $E_{4} \cdot(4-26)$ in $E q .(4-30)$ yields,

$$
\begin{aligned}
n_{j} & =n_{0 j} R_{0}(\pi r)^{-1} \int d p_{z}\left(\alpha_{j}^{2}-r^{2}\left(p_{0 j}-e z_{j} c^{-1} \psi\right)-p_{z}^{2}\right)^{-1 / 2} \\
& =n_{0 j} R_{0} r^{-1}
\end{aligned}
$$

In Eq. (4-32) the limits of integration are

$$
\pm\left(\alpha_{j}^{2}-r^{-2}\left(p_{0 j}-e z_{j} c^{-1} \psi\right)^{2}\right)^{1 / 2} \text {. }
$$

The current for species $j$ is obtained from a velocity space inteyration over $v_{\theta} f_{j}$.

$$
J_{\theta j}=e z_{j} \int v_{\theta} f_{j} d p_{r} d p_{\theta}^{\prime} d p_{z}
$$

Changing variables to $H, P_{\theta}, P_{z}$ yields,

$$
\begin{aligned}
J_{\theta j} & =e z_{j} r^{-2} \int \frac{\left(p_{\theta}-e z_{j} c^{-1} \psi\right) f_{j} d H d p_{\theta} d p_{z}}{\left(z_{j}\left(H-e z_{j} \phi\right)-r^{2}\left(p_{\theta}-e z_{j} c^{-1} \psi\right)^{2}-p_{z}^{2}\right)^{1 / 2}} \\
& =e z_{j}{ }^{n} 0 j^{R} R_{0}\left(m_{j} r^{2}\right)^{-1}\left(p_{0 j}-e z_{j} c^{-1} \psi\right)
\end{aligned}
$$

From $J_{\theta}=J_{\theta}^{\text {Ohk awa }}$ and $E q \cdot(4-12)$ with $E q \cdot(4-34)$ the current is below.

$$
J_{\theta}=\sum_{j} e z_{j}{ }_{0 j}{ }^{R} 0\left(m_{j} r^{2}\right)^{-1}\left(p_{0 j}-e z_{j} c^{-1} \psi\right)\left(1-z_{j}<z>\right)
$$


The Post ${ }^{[171]}$ result solved for $\psi$ with no electric field $\phi=0$. Comparing the current in the work of Post with Eq. (4-35) it can be seen the functional dependence is identical and only constant factors differ. Thus, the previous $\phi=0$ solutions obtained for $\psi$ by Post are still applicable with modified constants for this case which includes an electric field. Because Eq. (4-35) does not depend on $\phi, E q .(4-17)$ used to solve for $\psi$ is uncoupled from Eq. (4-25) used to solve for $\phi$. Thus, $\psi$ may be assumed to be a known function of $r$ and Eq. (4-25) may be integrated after obtaining an expression for $v_{\theta e}$,

$$
\begin{aligned}
\sum_{j} n_{j} z_{j}^{2} & =R_{0} r^{-1} s^{(2)} \\
\sum_{j} n_{j} z_{j} v_{\theta j} & =\sum_{j} e^{-1} z_{j} J_{\theta j} \\
& =r^{-2}\left(c R_{0} s^{(0)}-c R_{0} s^{(5)} \psi s^{(2)}\right. \\
\langle r c)^{-1} v_{\theta e} & =r^{-2}\left(s^{(0)}-s^{(5)} \psi\right)
\end{aligned}
$$

The definition of the $s^{(i)}$ constants follow.

$$
\begin{aligned}
& s^{(0)}=\left(s^{(2)} c\right)^{-1} \sum_{j} m_{j}^{-1} z_{j}^{2} p_{0 j} n_{0 j} \\
& s^{(1)}=\sum_{j} n_{0 j} z_{j} \\
& s^{(2)}=\sum_{j} n_{0 j} z_{j}^{2} \\
& s^{(3)}=e^{-1} \sum_{j} n_{0 j} H_{0 j} z_{j}
\end{aligned}
$$




$$
\begin{aligned}
& s^{(4)}=s^{(3)} / s^{(2)} \\
& s^{(5)}=\left(e s^{(2)} c^{2}\right)^{-1} \sum_{j} z_{j}^{3} n_{0 j} m_{j}^{-1} \\
& \left.s^{(6)}=.5 \pi \sum_{j} n_{0 j} H_{0 j}\left(1-z_{j}<z\right\rangle^{-1}\right) \\
& \langle z\rangle=s^{(2) / s^{(1)}}
\end{aligned}
$$

Substituting Eq. (4-38) and Eq. (4-32) into Eq. (4-25) yields the following equation.

$$
\begin{aligned}
\frac{\partial \phi}{\partial r} & =s^{(0)}\left(\frac{\partial}{\partial r}\left(r^{-2} \psi\right)+2 r^{-3} \psi\right)-s^{(5)}\left(\frac{\partial}{\partial r}\left(.5 r^{-2} \psi^{2}\right)+r^{-3} \psi^{2}\right) \\
& +e^{-1}\left(\frac{\partial T}{\partial r}-r^{-1} T_{e}\right)
\end{aligned}
$$

Integrating Eq. (4-47) gives an expression for $\phi$.

$$
\begin{aligned}
\phi & =s^{(0)} r^{-2} \psi-.5 s^{(5)} r^{-2} \psi^{2}+e^{-1} T_{e} \\
& +2 s^{(0)} \int r^{-3} \psi d r-s^{(5)} \int r^{-3} \psi^{2} d r \\
& -e^{-1} \int r^{-1} T_{e} d r
\end{aligned}
$$

Assuming a radial variation of $T_{e}$ and using the Post formulas for $\psi$ it is possible to calculate $\phi$. A numerical solution is possible, however analytic progress is inhibited since $\psi$ is expressed in terms of modified Bessel functions and it is thus necessary to integrate products of modified Bessel functions. It is however possible to gain information from Eq. (4-48) concerning the cross field variation 
of density. Assume $T_{e}$ is constant and rewrite Eq. (4-48).

$$
\begin{aligned}
\text { eф } T_{e}^{-1} & =\ln n_{e}+e S^{(0)} \psi r^{-2} T_{e}^{-1}-.5 e j^{(5)} \psi^{2} r^{-2} T_{e}^{-1} \\
& +2 S^{(0)} T_{e}^{-1} e \int \psi r^{-3} d r-S^{(5)} T_{e}^{-1} \int e \psi^{2} r^{-3} d r
\end{aligned}
$$

The magnitude of the various terms can be estimated for typical parameters. The first terin on the right side of Eq. $(4-49)$ is of the Boltzmann form and leads to the relationship $n=n_{0} e^{e \phi / T} e$. The scaling of the first term is approximately 10. The other four terms on the right side of Eq. (4-49) are a consequence of the $v \times B$ term in the momentum equation. The second and fourth terms scale as $e_{\theta} \psi\left(L^{2} m_{j} c T_{e}\right)^{-1}$. Approximating $\psi$ as $B L^{2}, T_{e}$ as $m_{j} v^{2} / 3\left(T_{e} / T_{j}\right)$ and $p_{\theta}$ as $m_{j} v L+e B L^{2} / c$ yields the following result,

$$
\mathrm{ep}_{\theta} \psi\left(r^{2} \mathrm{~m}_{j} c T_{e}\right)^{-1}=\left(L+L^{2} \bar{a}_{i}^{-1}\right) 3 a_{i}^{-1}\left(T_{i} / T_{e}\right)
$$

where $a_{i}$ is ion gyroradius and $L$ is a scale length. Usually $a_{j}$ is half or a third $L$ so the second and fourth terms are about 20 $T_{i} / T_{e}$. For ions one to ten times hotter than electrons these terms are $: 3$ to 200 times larger than the Boltzmann term. The third and fifth term scale as $e^{2} \psi^{2}\left(m c^{2} L^{2} T_{e}\right)^{-1}$. Using the previous assumptions yields the following result.

$$
e^{2} \psi^{2}\left(m c^{2} L^{2} T_{e}\right)^{-1}=3 L^{2} a_{i}^{-2} T_{i} / T_{e}
$$

The scaling in Eq. (4-51) is the same as Eq. (4-50) so again the tnird and fifth terms are 20 to 200 times larger than the Boltzmann 
term. Thus, it is inappropriate to assume the Boltzmann relätionship for density across the magnetic field.

The formula in Eq. (4-48) is not tractable analytically using the $\psi$ from the Post solution. Also as can be seen from $E q .(4-32)$ the density has a singularity at $r=0$. To resolve these difficulties an alteriate ion distribution function has been devised.

$$
f_{j}=c n_{0 j}\left(e \pi\left(\psi_{0}+\psi_{c}\right) m_{j} z_{j}\right)^{-1} \delta\left(p_{2}\right) \delta\left(H-H_{0 j}\right)\left(p_{\theta}+e z_{j} c^{-1} \psi_{c}\right)
$$

In Eq. (4-52), $n_{0 j}$ is the density at $\psi=\psi_{0}$ which is the magnetic axis position. It follows from the definition of $B$, that $\psi_{0}<0$ and $\psi_{C}$ is a constant chosen less than zero so $\psi_{0}+\psi_{C}<0$. Consequently to maintain a positive $f_{j}$ it is necessary to require $p_{\theta}+$ $e Z_{j} c^{-1} \psi_{c}<0$. This restricts the class of particles mainly to those which are absolutely confined or confined by the orbit potential as explained in Chapter three. Using formulas given by Eq. (4-28), Eq. (4-30), and Eq. (4-31) yields the followine formula for the densit:y.

$$
\begin{aligned}
n_{j} & =c n_{0 j} \pi\left(\psi_{0}+\psi_{c^{\prime}} e z_{j}{ }^{-1} \int \frac{\left(m_{j} \theta_{\theta}^{r}+e z_{j} c^{-1}\left(\psi+\psi_{c}\right)\right) r d\left(m_{j} v_{\theta}\right)}{a_{j}\left(1-m_{j}^{2} v_{\theta}^{2} \alpha_{j}^{-2}\right)^{1 / 2}}\right. \\
& =n_{0 j}\left(\psi+\psi_{c}\right)\left(\psi_{0}+\psi_{c}\right)^{-1}
\end{aligned}
$$

In Chapter 2 in the magnetohydrodynamic limit of small Larmor radius it was found pressure and density were functions of $\psi$. In the ion distribution function description which allows large Larmor radius particles it is unusual to obtain a result like Eq. (4-53). The 
current for species $j$ is obtained in a manner similar to the derivation of Eq. $(4-34)$,

$$
\begin{aligned}
J_{\theta j} & =c n_{0 j}\left(\pi\left(\psi_{0}+\psi_{c}\right) m_{j} r\right)^{-1} \int \frac{m_{j} v_{\theta}\left(m_{j} v_{\theta}^{r}+e z_{j} c^{-1}\left(\psi+\psi_{c}\right)\right) r d\left(m_{j} v_{\theta}\right)}{\alpha_{j}\left(1-m_{j}^{2} v_{\theta}^{2} \alpha_{j}^{-2}\right)^{T / 2}} \\
& =c r_{0 j}\left(\psi_{0}+\psi_{c}\right)^{-1}\left(H_{0 j}-e z_{j} \phi\right) r
\end{aligned}
$$

In contrast to $\mathrm{Eq} \cdot(4-34), \mathrm{J}_{\theta j}$ in Eq. (4-54) depends on $\phi$ which has the potential of coupling the equations used to solve for $\psi$ and ф. From $\mathrm{J}_{\theta}=\mathrm{J}_{\theta}^{\text {Ohk awa }}$ and Eq. (4-12) with Eq. (4-54) the expression for $J_{\theta}$ is derived,

$$
\begin{aligned}
J_{\theta} & =c r\left(\psi_{0}+\psi_{c}\right)^{-1} \sum_{j}\left(H_{0 j}-e z_{j} \phi\right)\left(1-z_{j}\langle z\rangle^{-1}\right) \\
& =2 c s^{(6)}\left(\pi\left(\psi_{0}+\psi_{c}\right)\right)^{-1} r
\end{aligned}
$$

As a result of the following relationship there is no coupling.

$$
\sum_{j} n_{0 j} z_{j}\left(1-z_{j}<z>^{-1}\right)=0
$$

Using Eq. (4-53) and Eq. (4-54) the relationships below are derived.

$$
\begin{aligned}
& \sum_{j} n_{j} z_{j}^{2}=s^{(2)}\left(\psi_{0}+\psi_{c}\right)^{-1}\left(\dot{\psi}+\psi_{c}\right) \\
& \sum_{j} n_{j} z_{j}^{2} v_{\theta j}=\sum_{j} e^{-1} z_{j} J_{\theta j} \\
& =r c\left(\psi_{0}+\psi_{c}\right)^{-1}\left(S^{(3)}-S^{(2)} \phi\right) \\
& (r c)^{-1} v_{\theta e}=\left(\psi+\psi_{c}\right)^{-1}\left(s^{(4)}-\phi\right)
\end{aligned}
$$


Substituting Eq. (4-59) and Eq. (4-53) into Eq. (4-25) yields the following equation.

$$
\frac{\partial \phi}{\partial r}=\left(\psi+\psi_{c}\right)^{-1}\left(S^{(4)}-\phi\right) \frac{\partial \psi}{\partial r}+e^{-1}\left(\psi+\psi_{c}\right)^{-1} \frac{\partial}{\partial r}\left(\psi+\psi_{c}\right) T_{e}
$$

From the form of Eq. (4-60) if $T_{e}$ is assumed to be a function of $\psi$ then $\phi$ may be assumed to be a function of $\psi$ and the $r$ derivatives are changed to $\psi$ derivatives.

$$
\frac{\partial \phi}{\partial \psi}=\left(\psi+\psi_{c}\right)^{-1}\left(S^{(4)}-\phi\right)+e^{-1}\left(\psi+\psi_{c}\right)^{-1} \frac{\partial}{\partial \psi}\left(\psi+\psi_{c}\right) T_{e}
$$

Using $\psi+\psi_{C}$ as an integrating factor and then integrating Eq. (4-61) yields an expression for $\phi$.

$$
\phi=s^{(4)} \psi\left(\psi+\psi_{c}\right)^{-1}+e^{-1} T_{e}
$$

The solution in Eq. (4-62) is for the plasma region $r<R_{\text {sep }}$, inside of the separatrix. Outside the plasma $\phi$ satisfies Laplace's equation.

$$
\frac{1}{r} \frac{\partial}{\partial r}\left(r \frac{\partial \phi}{\partial r}\right)=0
$$

The general solution of Eq. (4-63) has two constants $c_{p l}, c_{p 2}$.

$$
\phi\left(r>R_{\text {sep }}\right)=c_{p 1}+c_{p 2} \ln r
$$

The corstants $c_{p l}, c_{p 2}$ are chosen for continuity of $\phi$ and $\partial \phi / \partial r$ at $r=R_{\text {sep }}$ or if the plasma is in a conductor the constants can be chosen for the potential value at the conductor and at $r=R_{\text {sep }}$. 
247

The magnetic field and $\psi$ are obtained from Eq. (4-17) with $\mathrm{J}_{\theta}$ given by $\mathrm{Eq} .(4-55)$.

$$
\frac{\partial}{\partial r}\left(\frac{1}{r} \frac{\partial \psi}{\partial r}\right)=-8 s^{(6)}\left(\psi_{0}+\psi_{c}\right)^{-1} r
$$

The solution of Eq. (4-65) introduces an arbitrary constant $B_{0}$.

$$
\psi=-.5 B_{0} r^{2}-s^{(6)}\left(\psi_{0}+\psi_{C}\right)^{-1} r^{4}
$$

For consistency it is necessary that $\psi$ have the value $\psi_{0}$ at the magnetic axis $r_{0}$. For $s^{(6)}>0$ and $\psi_{0}+\psi_{c}<0$ the following formula is obtained.

$$
r_{0}=\left(-B_{0}\left(\psi_{0}+\psi_{c}\right) /\left(4 S^{(6)}\right)\right)^{1 / 2}
$$

The constant $B_{0}$ is then determined,

$$
B_{0}=4\left(s^{(6)} \psi_{0}\left(\psi_{0}+\psi_{c}\right)^{-1}\right)^{1 / 2}
$$

Substituting Eq. (4-68) into Eq. (4-66) gives the $\psi$ solution.

$$
\psi=-2\left(s^{(6)} \psi_{0}\left(\psi_{u}+\psi_{c}\right)^{-1}\right)^{1 / 2} r^{2}-s^{(6)}\left(\psi_{0}+\psi_{c}\right)^{-1} r^{4}
$$

The exterior or vacuum solution satisfies

$$
\frac{\partial}{\partial r}\left(\frac{1}{r} \frac{\partial \psi_{v a c}}{\partial r}\right)=0
$$

which has a solution with two constants.

$$
\psi_{v a c}=c_{1}+c_{2} r^{2}
$$


Requiring continuity of $\psi$ and $\partial \psi / \partial r$ at $r=R_{\text {sep }}$ determines $c_{1}$ and $c_{2} \cdot$

$$
\psi_{v a c}=2\left(s^{(6)} \psi_{0}\left(\psi_{0}+\psi_{c}\right)^{-1}\right)^{1 / 2} \cdot\left(r^{2}-2 r_{0}^{2}\right)
$$

From Eq. (4-69) and $\psi\left(R_{\text {sep }}\right)=0$, and Eq. (4-67) and Eq. (4-68) the following relations are obtained

$$
\begin{aligned}
r_{0} & =\left(\psi_{0}\left(\psi_{0}+\psi_{c}\right) / s^{(6)}\right)^{1 / 4} \\
R_{\text {sep }} & =2^{1 / 2} r_{0}
\end{aligned}
$$

The total current per unit length in amps is obtained by multiplying the right side of Eq. $(4-65)$ by $-10(4 \pi)^{-1}$ and integrating from 0 to $r=R_{\text {sep }}$.

$$
I_{\text {total }}=\frac{20}{\pi}\left(\frac{s^{(6)} \psi_{0}}{\psi_{0}+\psi_{c}}\right)^{1 / 2}
$$

\subsubsection{Finite Radial Eiectron Velocity}

The self consistent $\phi$ and $\psi$ solutions in Eq. (4-62) and Eq. (4-69) were derived assuming $J_{\theta}=J_{\theta}^{0 h k a w a}$. To examine the region of validity of this assumption it is necessary to estimate the deviation of $J_{\theta}$ from $J_{\theta}^{0 h k a w a}$. An approximate formula for $v_{r e}$ has been derived in other work ${ }^{[172]}$ related to the 2 XIIB experiment.

$$
v_{r e}=300\left(r-r_{0}\right)
$$

The speed of light times resistivity is below with $T_{e}$ in eV. 


$$
c \eta=4.2 \times 10^{-3} \mathrm{~T}_{\mathrm{e}}^{-3 / 2} \mathrm{~cm}-\text { statohm }
$$

The current difference is obtained from Eq. (4-22), using Eq. (4-77) for $\mathrm{C} \eta$, and $E q .(4-76)$ for $v_{r e}$.

$$
J_{\theta}^{\text {Ohkawa }}-J_{\theta}=7 \times 10^{4}\left(r-r_{0}\right) B_{z} T^{3 / 2}
$$

The right side of Eq. (4-65) multiplied by $c(4 \pi)^{-1}$ gives an estimate of $\mathrm{J}_{\theta}^{\text {Ohk awa }}$ in terms of density and energy in $\mathrm{eV}$.

$$
J_{\theta}^{\text {Ohk awa }}=4.8 \times 10^{-6} \mathrm{nH}
$$

Assuming a magnetic field of 2 kilogauss, a product of $n \mathrm{H}$ of $10^{16}$ and $r-r_{0}=10$ gives the following relationship.

$$
\left(J_{\theta}-J_{\theta}^{\text {Ohk awa }}\right) / J_{\theta}^{\text {Ohk awa }}=.015 \mathrm{~T}_{\mathrm{e}}^{3 / 2}
$$

Thus the $J_{\theta}=J_{\theta}^{\text {Onkawa }}$ results apply when the electron temperature is sma11. At $5 \mathrm{eV}, \mathrm{Eq} .(4-80)$ gives a value of .17 and at $100 \mathrm{eV}$, a value of 15 .

Allowing a finite $v_{r e}$ requires the ion distribution function to have a nonzero radial velocity moment. This means $f_{j}$ in Eq. (4-52) must have a correction added to it which yields a finite $v_{r j}$. This correction is small and it is assumed Eq. $(4-20)$ is satisfied, but the $v_{r j}=0$ formula is used for $n_{j}$.

The theta electron velocity is obtained from $\mathrm{Eq} .(4-23)$ which now includes the $v_{r e}$ term. The equation for $\phi$ is obtained by substituting Eq. (4-23) into Eq. (4-18). 


$$
\begin{aligned}
\frac{\partial \phi}{\partial r} & =B_{z}\left(c n_{e}\right)^{-1}\left(v_{r e} B_{z}(c n e)^{-1}+\langle z\rangle^{-1} \sum_{j} n_{j} v_{\theta j} z_{j}^{2}\right) \\
& +n_{e}^{-1} \frac{\partial}{\partial r}\left(n_{e} T_{e}\right) \\
& +n e\langle z\rangle-1 \sum_{j} n_{j} z_{j}^{2}\left(v_{i \cdot e}-v_{r j}\right)
\end{aligned}
$$

The previous equation for $\psi$, Eq. (4-65) is modified by a term linear in $v_{r e}$.

$$
\frac{\partial B}{\partial r}=-8 S^{(6)}\left(\psi_{0}+\psi_{c}\right)^{-1} r+4 \pi v r_{z}{ }^{B}\left(n c^{2}\right)^{-1}
$$

Assuming $v_{r e}$ is a known function of $r$, the solution to Eq. (4-82) may be written as two quadratures.

$$
\begin{aligned}
B_{z}= & -8 S^{(6)}\left(\psi_{0}+\psi_{c}\right)^{-1} e^{\int 4 \pi v} r e^{\left(n c^{2}\right)^{-i}} d r \\
& -B_{7.0} e^{\int 4 \pi v} r e^{\left(n c^{2}\right)^{-1}} d r
\end{aligned}
$$

Further progress taward an analytic solution requires a model for $T_{e}(r)$ and $v_{r e}(r)$. The electron temperature is assumed to be constant. The equilibration around a flux surface is much more rapid than across a flux surface so the electrons are lost by moving away from the magnetic axis. A step model is the first approximation to this behavior and thus the radial electron velocity is $v_{\text {re }}=v_{0}$ when $B_{z}>0$ and $v_{\text {re }}=-v_{0}$ when $B_{z}<0$. 
In the remainder of this section subscript - denotes below and subscript + denotes above the magnetic axis or field null. The exponent integral in Eq. (4-83) has two formulas,

$$
\int 4 \pi v_{r e}\left(\pi c^{2}\right)^{-1} d r= \begin{cases}-\alpha r & r<r_{0} \\ \alpha r-2 \alpha r_{0} & r>r_{0}\end{cases}
$$

where $\alpha$ is the absolute value of $4 \pi v_{0}\left(n c^{2}\right)^{-1}$. Substituting Eq. (4-84) into Eq. (4-83) gives the magnetic field solution.

$$
\begin{aligned}
& B_{z-}=-8 S^{(6)}\left(\psi_{0}+\psi_{c}\right)^{-1}\left(r \alpha^{-1}-\alpha^{-2}+e^{\alpha\left(r_{0}-r\right)}\left(\alpha^{-2}-r_{0} \alpha^{-1}\right)\right) \\
& B_{z+}=-8 S^{(6)}\left(\psi_{0}+\psi_{c}\right)^{-1}\left(e^{\alpha\left(r-r_{0}\right)}\left(\alpha^{-2}+r_{0} \alpha^{-1}\right)-r \alpha^{-1}-\alpha^{-2}\right)
\end{aligned}
$$

It is necessary that $B_{z-}\left(r_{0}\right)=0$ and this condition expresses the arbitrary $B_{20}$ constant in Eq. (4-83) in terms of $S^{(6)}, \psi_{0}, \psi_{C}$, $\alpha$, and $r_{0}$.

$$
B_{z 0}=85^{(6)}\left(\psi_{0}+\psi_{c}\right)^{-1}\left(r_{0} \alpha^{-1}+\alpha^{-2}\left(e^{-\alpha r_{0}}-1\right)\right) e^{\alpha r_{0}}
$$

The formulas for $\psi$ are obtained by multiplying Eq. (4-85) and Eq. (4-86) by $r$ and integrating with respect to $r$.

$$
\begin{aligned}
\psi_{-} & =-8 s^{(6)}\left(\psi_{0}+\psi_{c}\right)^{-1} r^{3}(3 \alpha)^{-1}-r^{2} \alpha^{-2} / 2 \\
& \left.-\left(r e^{\alpha r_{\alpha}-1}+\alpha^{-2}\left(e^{-\alpha r}-1\right)\right)\left(\alpha^{-2}-r_{0} \alpha^{-1}\right) e^{\alpha r_{0}}\right)
\end{aligned}
$$


252

$$
\begin{aligned}
\psi_{+} & =\psi_{0}-8 S^{(6)}\left(\psi_{0}+\psi_{c}\right)^{-1}\left(\left(r_{0}^{3}-r^{3}\right)(3 \alpha)^{-1}+\left(r_{0}^{2}-r^{2}\right)\left(2 \alpha^{2}\right)^{-1}\right. \\
& \left.+\left(\alpha^{-3}+r_{0} \alpha^{-2}\right)\left(r^{\alpha\left(r-r_{0}\right)}-r_{0}+\alpha^{-1}\left(1-e^{\alpha\left(r-r_{0}\right)}\right)\right)\right)
\end{aligned}
$$

The $\psi$ and $B_{z}$ solutions are used in Eq. (4-81) to solve for $\phi$. Using Eq. (4-59), Eq. (4-82), and Eq. (4-53) for $n_{j}$, in Eq. (4-81) yields,

$$
\begin{aligned}
\frac{\partial \phi}{\partial r} & =\left(\psi_{0}+\psi_{C}\right)\left(e 4 \pi S^{(1)}\left(\psi+\psi_{C}\right)\right)^{-1} B_{z}\left(\frac{\partial B_{z}}{\partial r}+8 S^{(6)} r\left(\psi_{0}+\psi_{C}\right)^{-1}\right) \\
& +\left(\psi+\psi_{C}\right)^{-1} \frac{\partial \psi}{\partial r}\left(S^{(4)}-\phi\right)+e^{-1} T_{e}\left(\psi+\psi_{C}\right)^{-1} \frac{\partial}{\partial r}\left(\psi+\psi_{C}\right) \\
& \pm S_{\text {sum }}\left(\psi+\psi_{C}\right)
\end{aligned}
$$

where $+S_{\text {sum }}$ is used for $r>r_{0}$ and $-S_{\text {sum }}$ for $r<r_{0}$.

$$
S_{s u m}=n e\left(s^{(2)}\left(\psi_{0}+\psi_{c}\right)\right)^{-1} \sum_{j} n_{0 j} z_{j}^{2}\left(\sum_{k} n_{0 k} z_{k}\left(v_{r k}-v_{r j}\right)\right)
$$

Multiplying Eq. (4-90) by $\psi+\psi_{c}$, and integrating gives the following solution.

$$
\begin{aligned}
& \phi=e^{-1} T_{e}+S^{(4)}+2 S^{(6)}\left(S^{(1)} e \pi\right)+c_{p h i}\left(\psi+\psi_{c}\right)^{-1} \\
& +B_{2}^{2}\left(\psi_{0}+\psi_{c}\right)\left(\left(\psi+\psi_{c}\right) S^{(1)} e 8 \pi\right)^{-1} \\
& +S_{\text {sum }}\left(\psi+\psi_{c}\right)^{-1}\left(H_{s}\left(r-r_{0}\right) \sum_{k=2}^{g} I_{p k}-\left(1-H_{s}\left(r-r_{0}\right)\right) I_{p l}\right)
\end{aligned}
$$


In Eq. (4-92), $C_{\text {phi }}$ is a constant and $H_{s}$ is the step function.

$$
H_{s}(x)= \begin{cases}1 & x>0 \\ 0 & x>0\end{cases}
$$

The $I_{p k}$ functions are related to the integral of flux squared.

$$
\begin{aligned}
& I_{p 1}=\int_{0}^{r}\left(\psi_{c}+\psi_{c}\right)^{2} d r \\
& \sum_{k=2}^{9} I_{p k}=\int_{r_{0}}^{r}\left(\psi_{>}+\psi_{c}\right)^{2} d r+\int_{0}^{r_{0}}\left(\psi_{<}+\psi_{c}\right)^{2} d r
\end{aligned}
$$

The formulas for $I_{p k}$ are in Appendix F. In Eq. (4-92) the $\psi$ function is understood to be $\psi_{-}$for $r<r_{0}$ and $\psi_{+}$for $r>r_{0}$.

\subsubsection{Results for Finite Radial Electron Velocity}

The formulas for $\phi, E_{r}, \psi$, and $B_{z}$ derived in Section 4.2 .2 are plotted for two cases to illustrate the effect of the magnitude of $v_{r e}$. For the step model the variable that is changed between the two cases is $v_{0}$. In the first case $v_{0}=10 \mathrm{~cm} / \mathrm{sec}$ and in the second case $v_{0}=100 \mathrm{~cm} / \mathrm{sec}$. The complete set of parameters for the two cases are listed in Table 4-1. A charge state two, impurity at 5\% the main density and one sixth the main species energy yields an effective charge state of 1.09 .

The total current per length of -457 amps for Case $I$ and -470 amps for Case II is almost the same. As a result the plot of $\psi(r)$ in Fig. 4-1 a, for Case I and in Fig. 4-1b for Case II are nearly identical. The magnetic axis position is at $r=10 \mathrm{~cm}$ and the 


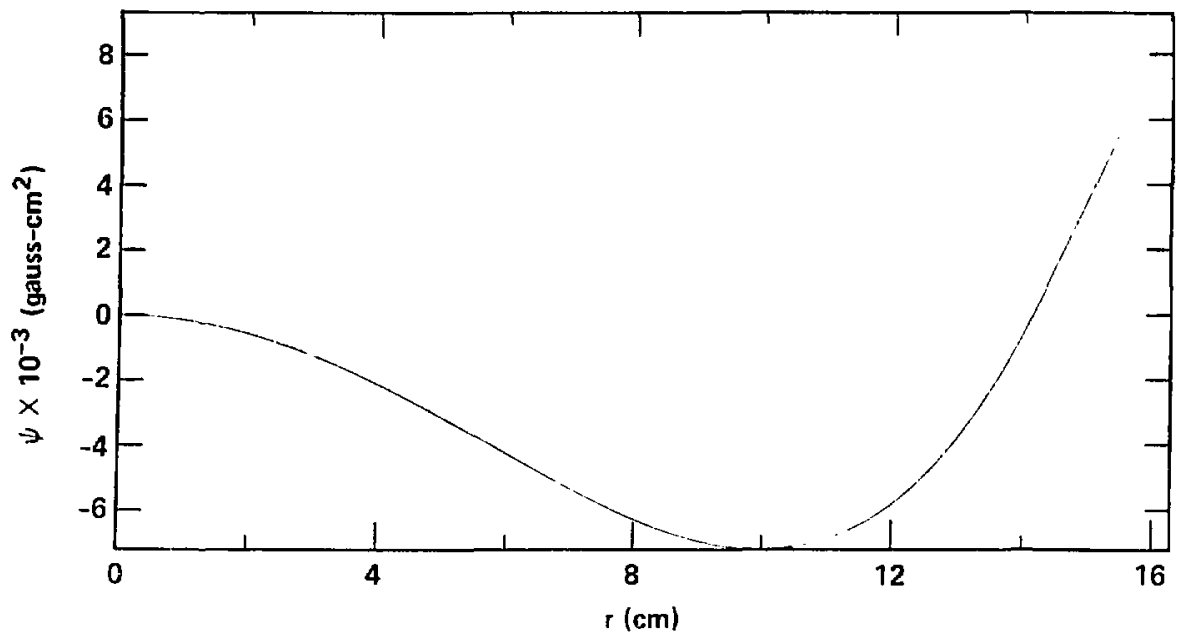

(a)

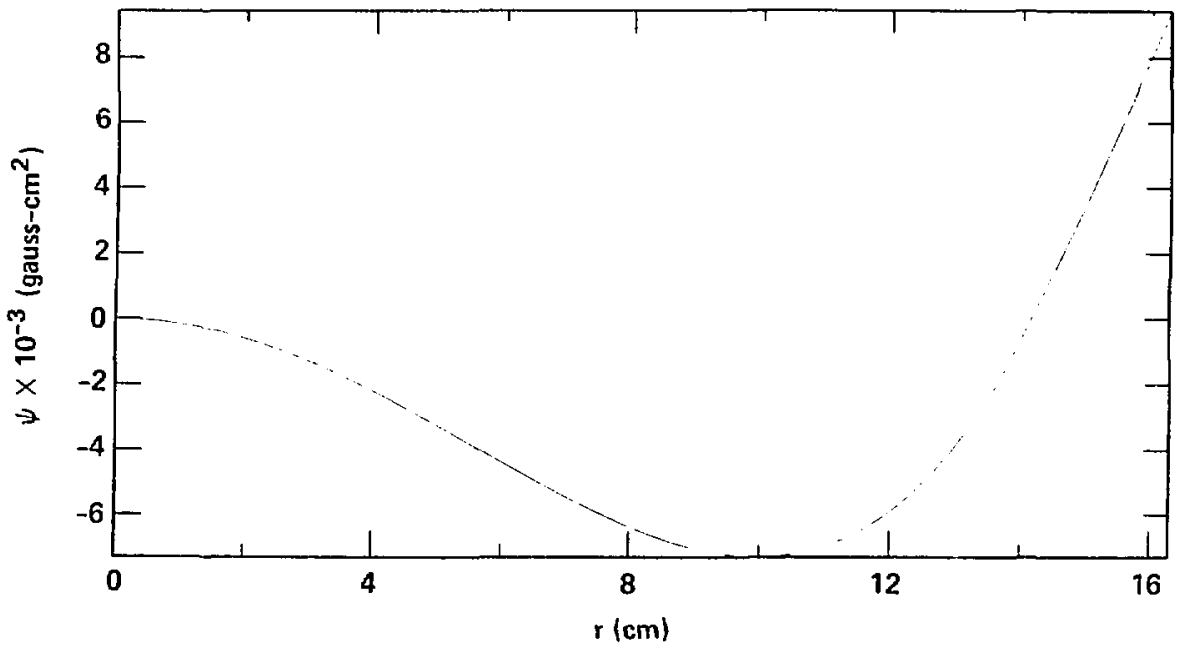

(b)

Figure 4-1. One dimensional equilibrium $\psi(r)$ (a) $v_{r e}=10$, (b) $v_{\text {re }}=100$. 


\begin{tabular}{|c|c|c|}
\hline & $I$ & $I I$ \\
\hline$n_{1}$ & $1 \times 10^{13} \mathrm{~cm}^{-3}$ & $1 \times 10^{13} \mathrm{~cm}^{-3}$ \\
\hline$n_{2}$ & $5 \times 10^{11} \mathrm{~cm}^{-3}$ & $5 \times 10^{11} \mathrm{~cm}^{-3}$ \\
\hline$z_{1}$ & 1 & 1 \\
\hline$z_{2}$ & 2 & 2 \\
\hline$H_{01}$ & $3 \mathrm{keV}$ & $3 \mathrm{keV}$ \\
\hline$H_{02}$ & $.5 \mathrm{keV}$ & $.5 \mathrm{keV}$ \\
\hline$v_{r 1}$ & $10.05 \mathrm{~cm} / \mathrm{sec}$ & $10.05 \mathrm{~cm} / \mathrm{sec}$ \\
\hline$v_{r 2}$ & $9.502 \mathrm{~cm} / \mathrm{sec}$ & $99.95 \mathrm{~cm} / \mathrm{s} \cdot$ \\
\hline$<z>$ & 1.09 & 1.09 \\
\hline$I$ & $-457 \mathrm{amps}$ & $-470 \mathrm{amps}$ \\
\hline$T_{e}$ & $.1 \mathrm{keV}$ & $.1 \mathrm{keV}$ \\
\hline$v_{r e}$ & $10 \mathrm{~cm} / \mathrm{sec}$ & $100 \mathrm{~cm} / \mathrm{sec}$ \\
\hline$\alpha$ & $9.13 \times 10^{-4}$ & $9.13 \times$ \\
\hline
\end{tabular}

Table 4-1. One Dimensional E, B Result Parameters 
separatrix is at $r=14 \mathrm{~cm}$ for both cases. The $\psi_{0}$ magnetic axis value for Case I is -7214 gauss $-\mathrm{cm}^{2}$ and -7298 gauss $-\mathrm{cm}^{2}$ for Case II. The edge flux $\psi_{C}$ is -800 gauss $-\mathrm{cm}^{2}$ for both cases.

The magnetic field is shown in Fig. 4-2a for Case I and in Fig. 4-2b for Case II. For both cases the general behavior is positive $B_{z}$ for $r>r_{0}$ and negative $B_{z}$ for $r<r_{0}$. The field goes to zero at the $r_{0}$ magnetic axis position in both cases. As in the $\psi$ plots the $B_{Z}$ plots are nearly the same. The magnetic field at $r=0$ is -290 gauss for Case I and -302 gauss for Case II. The magnetic field at the separatrix is 289 gauss for Case I and 292 for Case II.

The plots of e $\phi(r)$ in Fia, 4-3a for Case I and in Fig. 4-3b for Case II indicate the change of $v_{\text {re }}$ has a more pronounced effect on $\phi$ than $\psi$. For $v_{\text {re }}=10 \mathrm{~cm} / \mathrm{sec}$ Fig. $4-3 a$ shows $e \phi$ is nearly constant at $2.64 \mathrm{keV}$. For $v_{\text {re }}=100 \mathrm{~cm} / \mathrm{sec} F i g .4-3 \mathrm{~b}$ shows e $\phi$ ranges from 2.63 to $2.74 \mathrm{keV}$. The larger variation of $\phi$ then appears as an increased electric field.

The electric fieid for Case I is plotted in Fig. 4-4a and for Case II in Fig. 4-4b. The Case I result in Fig. 4-4a shows $E_{r}(r)$ has a minimum of $-.5 \mathrm{volt} / \mathrm{cm}$ and a maximum of $3.5 \mathrm{volt} / \mathrm{cm}$. The effect of increased $v_{\text {re }}$ is shown in Fig. 4-4b as a peaking of the electric field near $r=0$ and the separatrix. A change of $v_{r e}$ from 10 to $100 \mathrm{~cm} / \mathrm{sec}$ results in an increase in the maximum electric fieid from 3.5 to 50 volts $/ \mathrm{cm}$. 


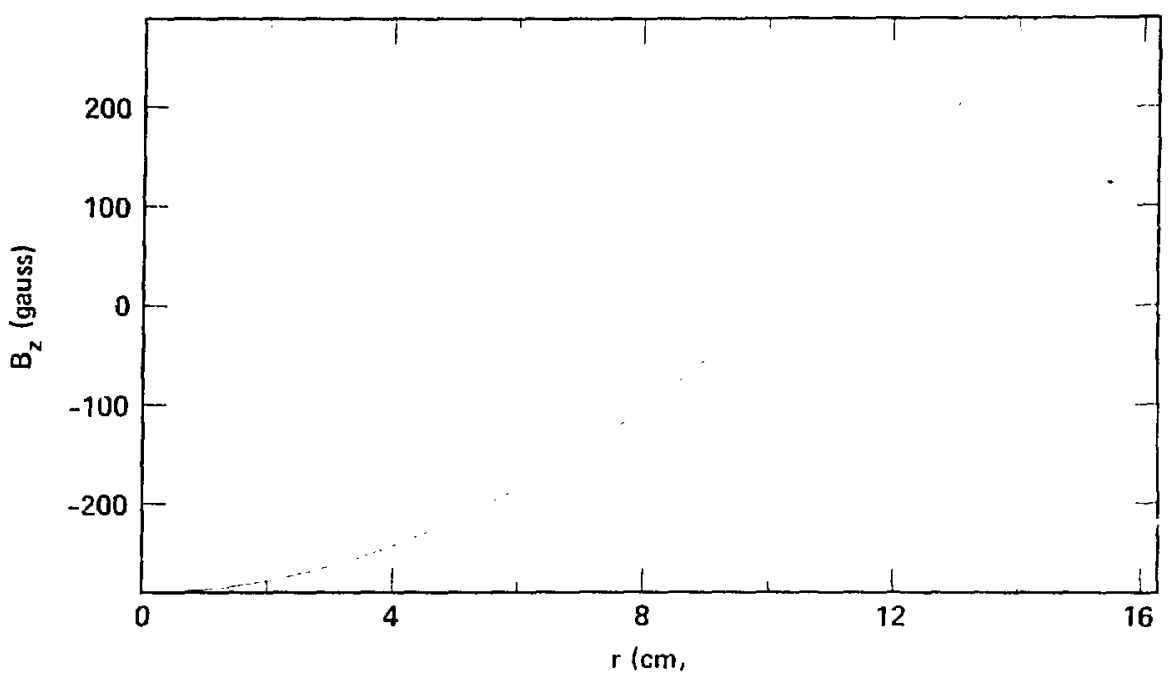

(a)

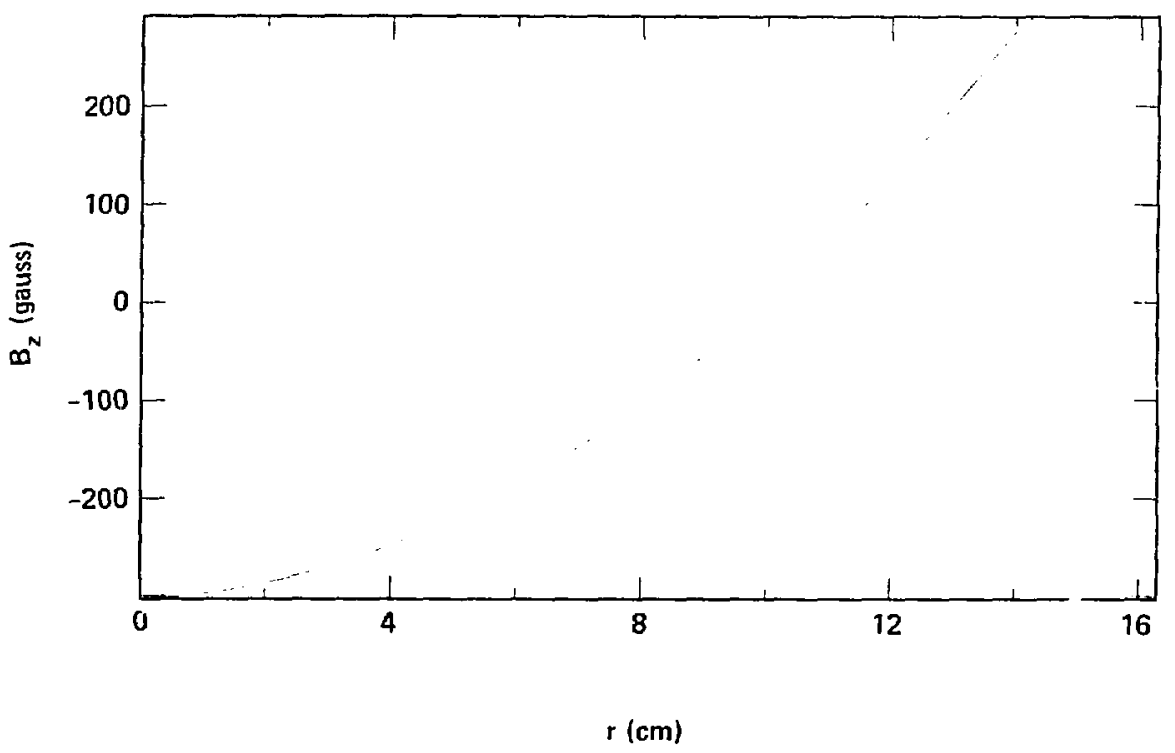

(b)

Figure 4-2, One dimensional equilibrium $B_{2}(r)(a) v_{r e}=10$, (b) $v_{\text {re }}=100$. 


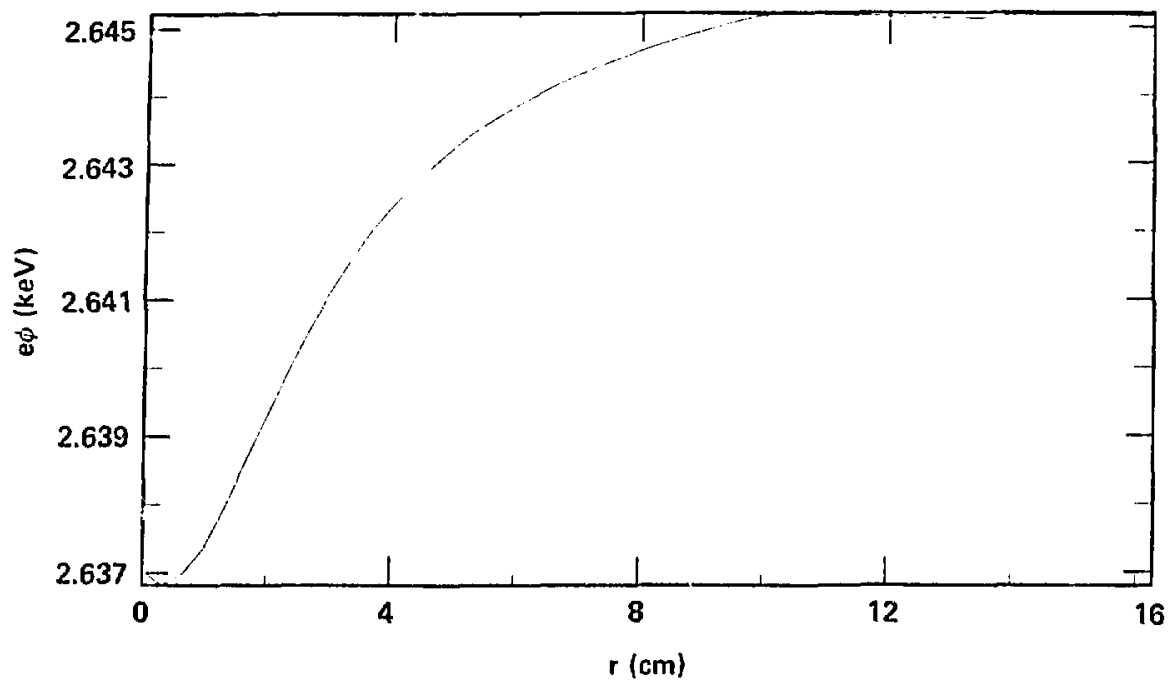

(a)

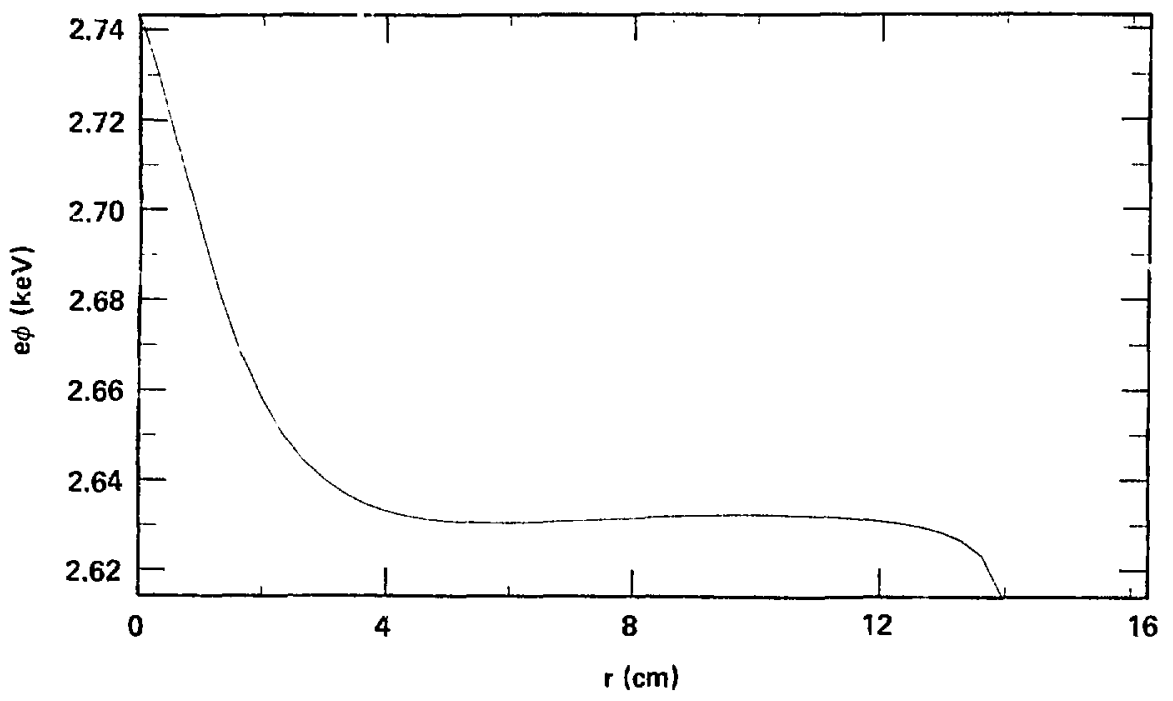

(b)

Figure 4-3. One dimensional equilibrium e $\phi(r)(a) v_{r e}=10$, (b) $v_{\text {re }}=100$. 


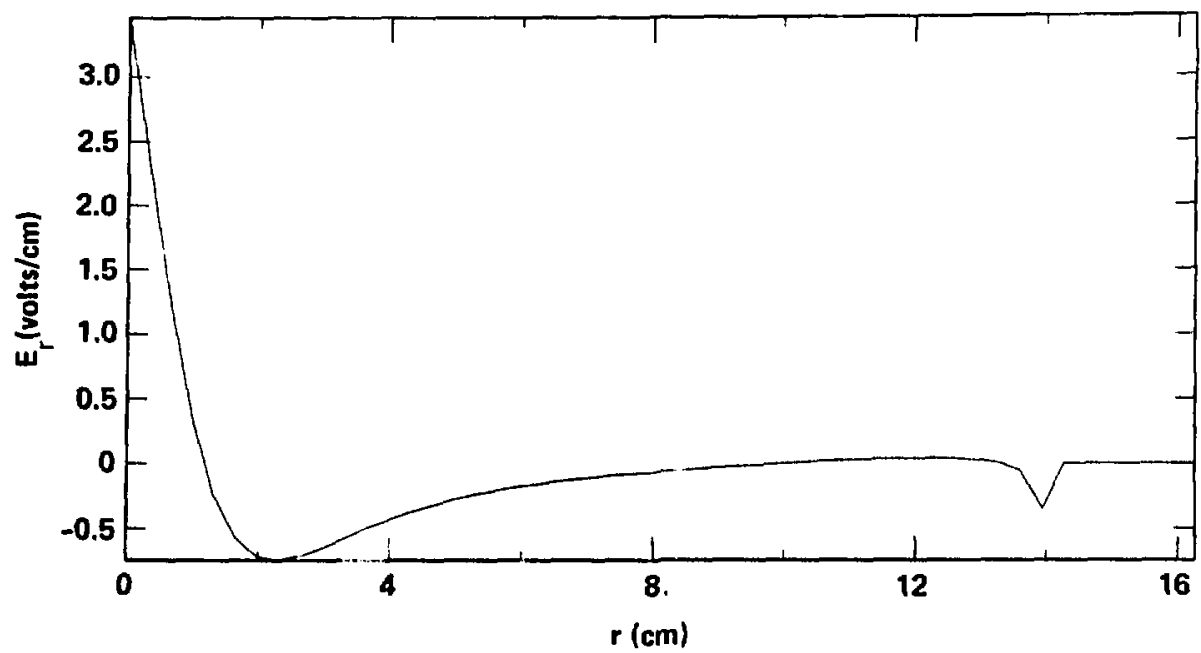

(a)

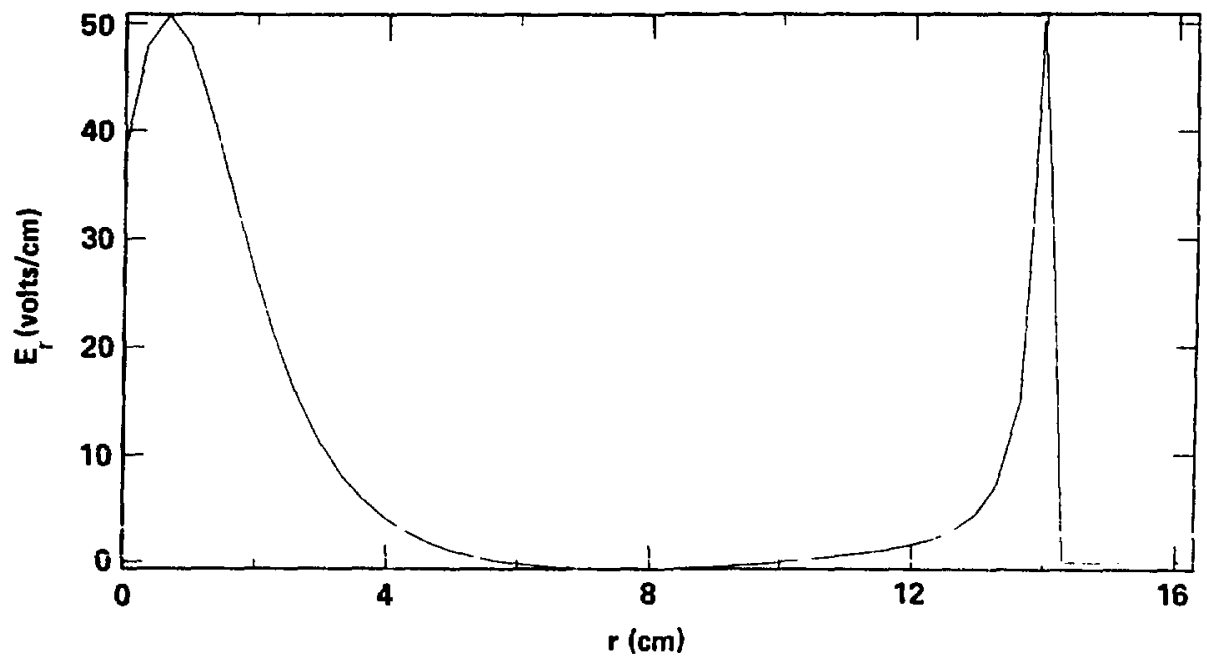

(b)

Figure 4-4. One dimensional equilibrium $E_{r}(r)(a) v_{r e}=10$,
(b) $v_{r e}=100$. 


\subsection{Kinetic Equation with Large Larmor Radius Effects}

The generation of energetic fusion products or the injection of high energy neutral beams in a plasma creates a population of large Larmor radius ions. These ions cause modifications to the results obtained in Chapter 2 which relied on the magnetohydrodynamic theory. Technically the magnetohydrodynamic theory of Chapter 2 is applicable to zero Larmor radius and is cansidered to be a limit for the desired small Larmor radius plasma. One of the earliest treatments where finite Larmor radius corrections are incorporated into the magnetahydrodynamic theory was work by Roberts and Taylor. [173]

Finite Larmor radius corrections for transport effects were considered by Nocentini and Engelman. [174] In the collision dominated regime they obtained results indicating a much reduced ion heat flux compared to the Pfirsch-Schluter zero Larmor radius magnetohydrodynamic theory. The heat flux reduction is atiributed to poloidal variation of density and ion temperature. This is in direct contrast to the Chapter 2 mode 1 where transport occurs between flux surfaces. The Nocentini results are not directly applicable to field reversed mirrors since he assumed low beta, concentric circular flux surfaces and large aspect ratio. It is however true the consideration of ion orbits restricted by $\mathrm{H}$ and $p_{\theta}$ in a field reversed mirror does lead to transport variation around flux surfaces.

Another method of accounting for large Larmor radius effects, without magnetohydrodynamic theory, has been :mplemented by Haldy 
and Ligou. [175] They consider the problem of energetic ions caused by fusion products or beams, interacting with a plasma in thermal equilibrium. The energetic ion population is described by a distribution function which obeys a kinetic equation. The distribution function is approximately constructed from its first few moments. [176] The results of this formulation are obtained only for an infinite homogeneous plasma without electromagnetic fields, which permits a tractable solution.

The inclusion of geometry or restricting the plasma to a finite size leads to the necessity of averaging over orbits. The usual procedure is to average the distribution function kinetic equation over the gyro-orbit to obtain neoclassical theory. The drift kinetic equation derived in this case is accurate to first order in the ratio of Larmor radius to gradient scale length. [177] Retaining extra terms $c$ an lead to a second order accurate drift kinetic equation. [178-180]

In the field reversed mirror the orbit is not adequately described by a drift and a rapid gyration about a field line. It is then necessary to integrate over the exact orbit. Boff $i$ and Molinari[181] derive an integral transport theory which formally includes this orbit integration, uses a separable isotropic scattering kernal, but finally only allows a constant magnetic field.

In the derivation in this section the magnetic field is a function of the radius. The ions are described by a distribution function expressed as an exparision in collision frequency and the electrons are modeled as an inertialess fluid. At zero order this 
is similar to the vlasov fluid model used for stability studies.[182-184] In the vlasov fluid model the ions are treated as collisionless and the electrons are an inertialess fluid. In this section the kinetic equation collision operator is considered to be first order in collision frequency which yields a zero order equation with no collision operator contribution. The zero order distribution function equation is then solved by any function of the constants of the motion.

A one dimensional kinetic ion model for a neutral beam injected field reversed mirror has been developed by Haill. [185] This model has been used in conjunction with a fluid model for electrons and low energy ions by Stark. [186] A multigroup kinetic treatment ${ }^{[187]}$ of high energy ions is used to account for large Larmor radius. The energetic ions are described by groups in energy and $p_{\theta}$ constants of the motion. Electron collisions and the inductive electric field move particles to adjacent groups. The energy loss, ion current and density are obtained from profiles derived from the average of the ion orbit over a radial bounce.

The same physics issues and geometry are considered in the large Larmor radius model derived in this work. There are however three major contrasts with the Stark model. First the orbit average obtained from discussions in Chapter 3 is used rather than a weighted bounce average. Second, the electron fluid equations are based on the Onkawa current model previously discussed. Third, the ion distribution function is expressed as an expansion in collision frequency and an orbit averaged kinetic equation analogous to the neoclassical drift kinetic equation is derived. 


\subsubsection{The Orbit Averaged Kinetic Equation}

The kinetic equation for the ion distribution function is written in terms of a Poissun bracket.

$$
\frac{\partial f}{\partial t}+[f, H]=C(f, f)+S
$$

The collision operator is represented by $C(f, f)$ and $S$ accounts for sources or losses. The distribution function $f$, the Hamiltonian $H$, the collision operator $C$ and sources $S$ are expanded in terms of collision frequency $v$ which is considered to be a small parameter.

$$
\begin{aligned}
& f=\sum_{i} v^{i} f(i) \\
& H=\sum_{i} v^{i} H^{(i)} \\
& c=\sum_{i} v^{i} C^{(i)} \\
& s=\sum_{i} v^{i} S^{(i)}
\end{aligned}
$$

The time scale for change of $f$ is assumed to be $v^{2}$. Substituting Eq. (4-96) to Eq. (4-99) into Eq. (4-95) yields the results below to second order.

$$
\begin{aligned}
v^{2} \frac{\partial f}{\partial t}[(0) & \left.+v_{f}^{(0)}, H^{(0)}\right]+v\left[f^{(1)}, H^{(0)}\right]+v^{2}\left[f^{(2)}, H^{(0)}\right] \\
& +v\left[f^{(0)}, H^{(1)}\right]+v^{2}\left[f^{(1)}, H^{(1)}\right]+v^{2}\left[f^{(0)}, H^{(2)}\right] \\
& =c^{(0)}+v c^{(1)}+v^{2} c^{(2)}+s^{(0)}+v S^{(1)}+v^{2} s^{(2)}
\end{aligned}
$$


A hierarchy of equations results from Eq. (4-100) by requiring that the coefficient of each power of $v$ vanishes. The zero, first and second order equations are below.

$$
\begin{aligned}
{\left[f^{(0)}, H^{(0)}\right] } & =c^{(0)}+s^{(0)} \\
{\left[f^{(1)}, H^{(0)}\right] } & =c^{(1)}+s^{(1)}-\left[f^{(0)}, H^{(1)}\right] \\
{\left[f^{(2)}, H^{(0)}\right] } & =c^{(2)}+S^{(2)}-\frac{\partial f^{(0)}}{\partial t} \\
& -\left[f^{(1)}, H^{(1)}\right]-\left[f^{(0)}, H^{(2)}\right]
\end{aligned}
$$

As explained in Chapter 3 and shown in Eq. (3-5) a constraint equation results from each of Eq. (4-101) to Eq. (4-103). The inner product of the rights sides of these equations with the homogeneous adjoint operator solution yields the constraint equation. The inner product is the Chapter 3 orbit average and the homogeneous solution is a constant of the motion. The constraint equations are then the orbit averages of the right sides of Eq. (4-101) to Eq. (4-103).

$$
\begin{aligned}
& \overline{c^{(0)}}+\overline{s^{(0)}}=0 \\
& \overline{c^{(1)}}+\overline{s^{(1)}}-\overline{\left.f^{(0)}, H^{(1)}\right]}=0 \\
& \overline{c^{(2)}}+\overline{s^{(2)}}-\frac{\partial f^{(0)}}{\partial t}-\overline{\left[f^{(1)}, H^{(1)}\right]}-\overline{\left[f^{(0)}, H^{(2)}\right]}=0
\end{aligned}
$$

The orbi: averaged kinetic equation is derived from $E q$. (4-106) $\therefore$ irig several assumptions and an operator which integrates the : :suating part of a function along the orbit. This operater is 
designated with a caret and is defined below for a general function $x \cdot$

$$
\hat{x}=\int(x-\bar{x}) d t
$$

An expression enclosed in parenthes is super-scripted by 'indicates the caret operator is to be applied to the entire expression. From the Eq. (4-107) and Eq. (3-10) definitions, the following relations are obtained for a function of the coustants of the motion $F$ and a general function $G$. [188]

$$
\begin{aligned}
& ([F, G])^{\prime}=[F, G] \\
& {[F, G]=[F, \bar{G}]}
\end{aligned}
$$

The caret operator is an indefinite integral along the orbit. Since integration along the orbit inverts the Poisson bracket of a function with $\mathrm{H}$, then the caret operator plus any function of the constants of the motion solves the expressions in Eq. $(4-101)$ to Eq. $(1-103)$. This is true because Eq. (3-5) can be written for a general function $G$ which may be the $n^{\text {th }}$ order distribution function.

$$
\overline{\left[G, H^{(0)}\right]}=0
$$

The solution for $f^{(0)}$ and $f^{(1)}$ are then written in terms of the caret operator.

$$
f^{(0)}=\hat{c}^{(0)}+\hat{s}^{(0)}+F_{0}
$$




$$
f^{(1)}=\hat{c}^{(1)}+\hat{s}^{(1)}-\left(\left[f^{(0)}, H_{1}\right]\right)^{\wedge}+F_{1}
$$

In Eq. (4-111) and Eq. (4-112) $F_{0}$ and $F_{7}$ are functions of the constants of the motion. Substituting Eq. (4-111) and Eq. (4-112) into Eq. (4-106) yields the orbit average kinetic equation in terms of $f^{(0)}$.

$$
\begin{aligned}
\overline{\frac{\partial f(0)}{\partial t}=} & \left.\left.\bar{c}^{(2)}+\bar{s}^{(2)}-\overline{\left[\hat{c}^{(1)}, H^{(1)}\right.}\right]-\overline{\left[\hat{s}^{(1)}, H^{(1)}\right.}\right] \\
& +\overline{\left[\left(\left[f^{(0)}, H^{(1)}\right]\right)^{\wedge}, H^{(1)}\right]-\left[F_{1}, H^{(1)}\right]} \\
& -\overline{\left[\hat{c}^{(0)}, H^{(2)}\right]}-\overline{\left[\hat{s}^{(0)}, H^{(2)}\right]}-\left[F_{0}, H^{(2)}\right]
\end{aligned}
$$

Equation (4-113) provides the orbit average of the time derivative of $f^{(0)}$. To obtain a kinetic equation for $f^{(0)}$ it is necessary to derive an equation for $\partial f^{(0)} / \partial t$. This is possible using Eq. (4-113) if,

$$
\hat{c}^{(0)}+\hat{s}^{(0)}=0
$$

In this case $f^{(0)}$ is only a function of the constants of the motion and the following identity results.

$$
\overline{\frac{\partial f(0)}{\partial t}}=\frac{\partial f(0)}{\partial t}
$$


Because $f^{(0)}$ is now considered to be a function of the constants of the motion $F_{1}$ is absorbed into the definition of $f^{(0)}$. The following orbit averaged kinetic equation for $f^{(0)}$ then results from Eq. $(4-113)$.

$$
\begin{aligned}
\frac{\partial f(0)}{\partial t} & =\bar{c}^{(2)}+\bar{s}^{(2)}-\overline{\left[c^{(1)}, H^{(1)}\right]}-\overline{\left[\hat{S}^{(1)}, H^{(1)}\right]} \\
& +\overline{\left[\left[f^{(0)}, \hat{H}^{(1)}\right], H^{(1)}\right]}-\left[f^{(0)}, \bar{H}^{(1)}\right]
\end{aligned}
$$

Equation (4-116) has been derived assuming only that $f^{(0)}$ is a function of the constants of the motion, which is implied by Eq. (4-114). Further modifications occur to Eq. (4-116) when axisymmetry is invoked. A general function $F\left(I_{1}, I_{2}, I_{3}\right)$ where $I_{7}, I_{2}, I_{3}$ are constants of the motion has the following Poisson bracket with function $G$.

$$
[F, G]=\frac{\partial F}{\partial I_{1}}\left[I_{1}, G\right]+\frac{\partial F}{\partial I_{2}}\left[I_{2}, G\right]+\frac{\partial F}{\partial I_{3}}\left[I_{3}, G\right]
$$

In axisymmetry $I_{1}$ is $H_{3} I_{2}$ is $p_{\theta}$ and there may be a third invariant $I_{3}$ depending on the fields. Thus Eq. $(4-117)$ in axisymmetry becomes,

$$
[F, G]=\frac{\partial F}{\partial H}[H, G]+\frac{\partial F}{\partial I_{3}}\left[I_{3}, G\right]
$$

The orbit average of Eq. (4-118) is obtained using Eq. $(4-109)$ and Eq. $(4-110)$.

$$
\begin{aligned}
{[\overline{F, G]}} & =[F, \bar{G}] \\
& =\frac{\partial F}{\partial I_{3}}\left[I_{3}, \bar{G}\right]
\end{aligned}
$$


A similar relation applies to the caret operator.

$$
\begin{aligned}
([F, G]) & =[F, \hat{G}] \\
& =\frac{\partial F}{\partial I}\left[I_{3}, G\right]
\end{aligned}
$$

The axisyminetric version of Eq. $(4-116)$ is obtained using Eq. $(4-119)$ and Eq. $(4-120)$.

$$
\begin{aligned}
& \frac{\partial f(0)}{\partial t}=\bar{c}^{(2)}+\bar{s}^{(2)}-\overline{\left[\dot{c}^{(1)}, H^{(1)}\right]}-\overline{\left[\bar{s}^{(1)}, H^{(1)}\right]} \\
& +\left[\overline{\left.\frac{\partial f}{\partial I_{3}}\left[I_{3}, H^{(1)}\right], H^{(1)}\right]}-\frac{\partial f^{(0)}}{\partial I_{3}}\left[I_{3}, \vec{H}^{(1)}\right]\right.
\end{aligned}
$$

In the one dimensional $\bar{c}$ isymmetric case the second term of Eq. $(4-118)$ becomes,

$$
\begin{aligned}
\frac{\partial F}{\partial I_{3}}\left[I_{3}, G\right] & =\frac{\partial F}{\partial p_{2}} \frac{\partial G}{\partial z} \\
& =0
\end{aligned}
$$

since there is no $z$ dependence. In the two dimensional axisymmetric case with no third constant of the motion $\partial F / \partial I_{3}$ is zero. In both of these cases Eq. (4-118) becomes,

$$
[F, G]=\frac{\partial F}{\partial H}[H, G]
$$

Equation (4-123) with Eq. (4-110) yields the following result,

$$
\overline{\left[f^{(0)}, G\right]}=0
$$


The following thr. conditions,

1. $f^{(0)}$ only a function of the constants of motion,

2. axisymmetry,

3. two dimensional with no third invariant or one dimensional, $\therefore$ : 1 the three constraint equations from Eq. (4-101) to Eq. (4-103).

$$
\begin{aligned}
& \bar{c}^{(0)}+\bar{s}^{(0)}=0 \\
& \bar{c}^{(1)}+\bar{s}^{(1)}=0 \\
& \bar{\tau}^{(2)}+\bar{s}^{(2)}-\frac{\partial f^{(0)}}{\partial t}-\overline{\left[f^{(1)}, y^{(1)}\right]}=0
\end{aligned}
$$

The first order distribution function becomes,

$$
f^{(1)}=\hat{C}^{(1)}+S^{(1)}-\frac{\partial f^{(0)}}{\partial H} H^{(1)}
$$

and the orbit averaged $k$ inetic equation is then below.

$$
\frac{\partial f^{(0)}}{\partial t}=\bar{c}^{(2)}+\bar{s}^{(2)}-\overline{\left[\hat{c}^{(1)}, H^{(1)}\right]}-\overline{\left[\hat{s}^{(1)}, H^{(1)}\right]}
$$

Equation (4-129) is a kinetic equation in $H, P_{\theta}, P_{Z}$.

\subsubsection{Poisson Bracket Solution by Eigenfunctions}

The orbit average kinetic equation in Eq. (4-129) applies to one and two dimensions in axisymmetry. The primary reason only the one dimensional radial geometry is cunsidered in this chapter is a consequence of the formula for $f^{(1)}$ in Eq. (4-128). The orbit average has been derived for one dimension, $r$ or two dimensions, $r, z$ 
in Chapter 3; however, the $X$ operation of Eq. (4-107) is difficult to obtain in two dimensions. The integrals $\hat{c}^{(1)}$ and $\hat{S}^{(1)}$ are integrals of the variable part of $C^{(1)}$ and $S^{(1)}$ along the orbit from a starting position to the orbit position at which $f^{(1)}$ is to be evaluated. An integral of tilis type requires a detailed knowledge of the orbit and is thus impractical. The function which is actually needed is $f^{(1)}$ and the particular solution technique of integrating along the orbit necessitated calculating $\dot{C}^{(1)}$ and $s(1)$. An alternate approach due to Lewis and Symon $[189]$ is to obtain $\mathrm{f}^{(7)}$ by setting it equal to $\mathrm{H}^{(1)}$ af $(0) / 2 \mathrm{H}$ plus an expansion of eigenfunctions of the Poisson bracket. This method may ultimately be a means of resolvi.ig the $r, z$ orbit following problem. In this section the method is presented for the one dimensional case.

The first order distribution function $f^{(1)}$ previousiy given by Eq. $(4-128)$ is represented by eigenfunctions $w_{\alpha}$ below.

$$
f^{(1)}=H^{(1)} \frac{\partial f(0)}{\partial H}+\sum_{\gamma} \gamma_{\alpha} w_{\alpha}
$$

The $W_{\alpha}$ satisfy the relation below,

$$
\left[W_{\alpha}, H\right]=i \mu_{\alpha} W_{\alpha}
$$

where $\mu_{\alpha}$ is the eigenvalue. To obtain $W_{\alpha}$ the orbit is projected into the nonignorable region of phase space, $r, p_{r}$. An orbit is determined by setting the zero order Hamiltonian equal to a constant $\varepsilon$.

$$
H\left(r, p_{r}, p_{\theta}, p_{z}\right)=\varepsilon
$$


The orbit label implied by Eq. (4-132) is $\varepsilon, p_{\theta}, p_{z}$, a where a distinguishes between orbits in $r, p_{r}$ space that are discontinuous. A discontinuous orbit corresponds to the $p_{\theta}>-3 / 16$ case discussed in the axisymmetric orbit considerations section of Chapter 3 . Let $\tau$ be the time measured along the orbit, then Hamilton's equation along the orbit is given below.

$$
\frac{\partial r}{\partial \tau}=\left.\frac{\partial H}{\partial p_{r}}\right|_{p_{r}=p_{r}\left(r, a, E, p_{\theta}, p_{z}\right)}
$$

Using the definition of $\mathrm{H}, \mathrm{Eq} .(4-133)$ becomes,

$$
\frac{\partial r}{\partial \tau}=m^{-1}\left(2 m(\varepsilon-e Z \phi)-p_{z}^{2}-r^{-2}\left(p_{\theta}-e z c^{-1} \psi\right)^{2}\right)^{1 / 2}
$$

where Eq. (4-134) refers to an orbit labelled a. The time along the orbit is then obtained in terms of $r, a, \varepsilon, p_{\theta}, p_{z}$ by solving $E q$. $(4-134)$,

$$
\tau=\ln \int d r\left(2 m(\varepsilon-e z \phi)-p_{z}^{2}-r^{-2}\left(p_{\theta}-e z c^{-1} \psi\right)^{2}\right)^{-l / 2}
$$

with the integration range given by the $\varepsilon, p_{\theta}$ determined accessible orbit region. The Poisson bracket is now written in terms of $\tau, \varepsilon$ instead of $r, p_{r}$.

$$
\left[, \therefore j=\frac{\partial}{\partial \tau}+\frac{\partial H}{\partial p_{z}} \frac{\partial}{\partial z}+\frac{\partial H}{\partial p_{\theta}} \frac{\partial}{\partial \theta}\right.
$$

In Eq. (4-1.36) the ignorable coordinates $\theta, z$ appear because $\partial / \partial \tau$ is at constant $\varepsilon, \theta, z, p_{\theta}, p_{z}$ and along the orbit $\theta$ and $z$ vary. 
The derivatives $\partial H / \partial p_{Z}$ and $\partial H / \partial p_{\theta}$ in Eq. (4-136) are computed taking $H$ to be a function of $r, p_{r}, p_{\theta}, p_{z}$ and then expressing the result in terms of $\tau, a, \varepsilon, p_{\theta}, p_{z}$. Equation (4-136) is then,

$$
[, H]=\frac{\partial}{\partial \tau}+m^{-1} p_{z} \frac{\partial}{\partial z}+\left(m r^{2}\right)^{-1}\left(p_{\theta}-e z c^{-1} \psi\right) \frac{\partial}{\partial \theta}
$$

where $r, \psi$ must be el iminated in favor of $\tau, \varepsilon$, a using Eq. (4-132) and Eq. (4-135). In the form of Eq. $(4-137),[, H]$ commutes with $H$, $p_{\theta}, p_{z}, \partial / \partial z$ and $\partial / \partial \theta$. Consequent $l y$, eigenfunctions may be chosen that are simultaneously eigenfunctions of all the commuting operators. This means $W_{\alpha}$ is a product of eigenfunctions of $\varepsilon, p_{\theta}$, $p_{z}, \partial / \partial \theta, \partial / \partial z$ multiplied by a function of $\tau$. The eigenfunction of a coordinate such as momentum in momentum space is obtained by considering the quantum analogy of conjugate coordinates. For the case of position and momentum, in position space the momentum operator is $-i(2 \pi)^{-1} h \partial / \partial x$. The projection of the operator into momentum space is the Fourier integral of $e^{i k x}$ which is the eigenfunction of $-i(2 \pi)^{-1} h \partial / \partial x$. The momentum space eigenfunction is $(2 \pi)^{-1} h \delta\left(k-k^{\prime}\right)$.

By similar reasoning $W_{\alpha}$ is a product of delta functions for $H, p_{\theta}, p_{z} \cdot$

$$
\begin{gathered}
W_{\alpha}=\delta_{\dot{a} a^{\prime}} \delta\left(\varepsilon-\varepsilon^{\prime}\right) \delta\left(p_{\theta}-p_{\theta}^{\prime}\right) \delta\left(p_{z}-p_{z}^{\prime}\right) \\
e^{i k_{\theta} \theta} e^{i k_{z} z} u_{\alpha}(\tau)
\end{gathered}
$$

The function $u_{\alpha}(\tau)$ is found by substituting Eq. (4-138) into Eq. $(4-131)$, using Eq. $(4-137)$ for $[, \mathrm{H}]$. Note in Eq. $(4-138) k_{\theta}$ is 
an integer to ensure $\theta$ periodicity.

$$
\frac{d u_{\alpha}}{d \tau}+i\left(m^{-1} p_{z} k_{z}+k_{\theta}\left(m r^{2}\right)^{-1}\left(p_{\theta}-e z c^{-1} \psi\right)-u_{\alpha}\right) u_{\alpha}=0
$$

The solution of Eq. $(4-139)$ with $d_{\alpha}$ as a normalization constant is,

$$
u_{\alpha}=d_{\alpha} e^{i\left(\mu_{\alpha} \tau-G_{\alpha}(\tau)\right)}
$$

where,

$$
\begin{aligned}
& G_{\alpha}=\int_{0}^{\tau}\left(m^{-1} p_{z} k_{z}+k_{\theta}\left(m r^{2}\right)^{-1}\left(p_{\theta}-e z c^{-1} \psi\right)\right) d \tau \\
& \tau_{b}=m \int p_{r}^{-1} d r \\
& B_{\alpha}=\tau_{b}^{-1} \int_{0}^{\tau_{b}} k_{\theta}\left(m r^{2}\right)^{-1}\left(p_{\theta}-e z c^{-1} \psi\right) d \tau+m^{-1} k_{z} p_{z} \\
& \mu_{\alpha}=\beta_{\alpha}+\tau_{b}^{-1} 2 \pi k
\end{aligned}
$$

with $k_{\tau}$ an integer and $\mu_{\alpha}$ chosen to guarantee periodicity. The e igenfunction label $\alpha$ is $a^{\prime}, \varepsilon^{\prime}, p_{\theta}^{\prime}, p_{z}^{\prime}, k_{\theta}, k_{z}, \mu_{\alpha}$ and the orthnormality condition requires $d_{\alpha}=(2 \pi)^{-2}$. The eigenfunction $w_{\alpha}$ is then,

$$
\begin{aligned}
W_{\alpha}= & (2 \pi)^{-2} \delta_{a a^{\prime}} \delta\left(\varepsilon-\varepsilon^{\prime}\right) \delta\left(p_{\theta}-p_{\theta}^{\prime}\right) \delta\left(p_{z}-p_{z}^{\prime}\right) \\
& e^{i k_{\theta}^{\theta}} e^{i k_{z} z} e^{i\left(\mu_{\alpha} \tau-G_{\alpha}(\tau)\right)}
\end{aligned}
$$

The method of expanding $f^{(1)}$ in terms of $w_{\alpha}$ means it is necessary to express $C^{(1)}$ and $S^{(1)}$ in terms of the $w_{\alpha}$ eigenfunctions. 
This procedure is an alternative to the integral along an orbit which is used in Section 4.3.4 to compute quancities needed in the Eq. $(4-128) f^{(1)}$ definition.

\subsubsection{Perturbed Fluid Electron Model}

The solution of Eq. (4-129) requires the perturbed Hamiltonian. The perturbed potentials which occur in the perturbed Hamiltonian are obtained from the fluid electron model which includes Ampere's and Faraday's law.

The perturbed Hamiltonian results from substituting collision frequency expansions of $\phi$ and $\psi$ into $\mathrm{Eq} .(3-21)$.

$$
\begin{aligned}
H=(2 m)^{-1}\left(p_{r}^{2}+p_{z}^{2}\right) & +\left(2 m r^{2}\right)^{-1}\left(p_{\theta}-e z c^{-1} \sum_{i} v_{\psi}^{i_{\psi}(i)}\right)^{2} \\
& +e z \sum_{i} \nu_{\phi}^{i_{\phi}(i)}
\end{aligned}
$$

To second order the Eq. (4-146) Hamiltonian is below.

$$
\begin{aligned}
H=(2 m)^{-1}\left(p_{r}^{2}+p_{z}^{2}\right) & +\left(2 m r^{2}\right)^{-1}\left(p_{\theta}^{2}-2 e z c^{-1}\left(p_{\theta} \psi^{(0)}\right.\right. \\
& \left.+v p_{\theta} \psi^{(1)}+v^{2} p_{\theta} \psi^{(2)}\right)+e^{2} z^{2} c^{-2}\left(\psi^{(0) 2}\right. \\
& \left.\left.+2 v \psi^{(0)} \psi^{(1)}+2 v^{2} \psi^{(0)} \psi^{(2)}+v^{2} \psi^{(1) 2}\right)\right) \\
& +e z\left(\phi(0)+v \phi(1)+v_{\phi}^{2}(2)\right)
\end{aligned}
$$

As a consequence of $\mathrm{Eq} .(4-147)$ the following definitions result. 


$$
\begin{aligned}
& H^{(0)}=(2 m)^{-1}\left(p_{r}^{2}+p_{z}^{2}\right)+\left(2 m r^{2}\right)^{-1}\left(p_{\theta}-e z c^{-1} \psi^{(0)}\right)^{2}+e z \phi(0) \\
& H^{(1)}=\left(m r^{2}\right)^{-1} e^{-1} c^{-1}\left(-p_{\theta} \psi^{(1)}+e z c^{-1} \psi^{(0)} \psi(1)\right)+e z \phi(1) \\
& H^{(2)}=\left(m r^{2}\right)^{-1} e z c^{-1}\left(-p_{\theta} \psi^{(2)}+e z c^{-1}\left\langle 2 \psi^{(0)} \psi^{(2)}+\psi^{(1) 2}\right\rangle\right) \\
& +e Z_{\phi}^{(2)}
\end{aligned}
$$

The solution of Eq. $(4-129)$ requires $H^{(1)}$ and implicitly $H^{(0)}$. From Eq. $(4-148)$ and Eq. $(4-149)$ it is then necessary to derive equations which determine $\psi^{(0)}, \psi^{(1)}, \phi^{(0)}$, and $\phi^{(1)}$. The equations used in Section 4.2 are written below with $v$ as an order parameter which will later be set to one. The quasineutrality condition came from the divergence equation below.

$$
\nabla \cdot \vec{E}=4 \pi e\left(\sum_{j} n_{j} z_{j}-n_{e}\right)
$$

On the basis of a Boltzmann distribution the density has an exponential dependence, with exponent $-\epsilon \phi T_{e}^{-1}$. Assuming this quantity is small the exponential can be expanded and Eq. (4-151) is below,

$$
\frac{1}{r} \frac{\partial}{\partial r}\left(r \frac{\partial \phi}{\partial r}\right)=\lambda_{D}^{-2} \phi
$$

where $\lambda_{D}$ is the Debye length. The content of Eq. (4-152) is that the $r_{3}$ it side of Eq. $(4-157)$ scales like $\lambda_{D}^{-2}$. Since $\lambda_{0}$ is small compared to a system scale length this means tremendous electric fields result from small density differences. This is then the 
motivation for the quasineutrality assumption. Consistent with this assumption and in recognition of the presence of a low energy ion background $e \phi$ is assumed to be comparable to temperature. As a consequence $\phi^{(0)}$ is assumed to be zero. The electron model equations with $v$ inserted are then below.

$$
\begin{aligned}
& n_{e}=\sum_{j} n_{j} z_{j} \\
& v v_{r e}=n_{e}^{-1} v \sum_{j} n_{j} v_{r j} z_{j} \\
& v e n_{e} n v_{\theta e}=(r c)^{-1} v v_{r e} \frac{\partial \psi}{\partial r}+v e n<z>^{-1} \sum_{j} n_{j} z_{j}^{2} v_{\theta j} \\
& \qquad+v^{2}(r c)^{-1} \frac{\partial \psi}{\partial t} \\
& \frac{\partial \phi}{\partial r}=(r c)^{-1} v_{\theta e} \frac{\partial \psi}{\partial r}+\left(e n_{e}\right)^{-1} \frac{\partial n_{e} e^{\top}}{\partial r}-v e n\left(J_{r}-J_{r}^{0 h k a w a}\right) \\
& \frac{\partial}{\partial r}\left(r \frac{\partial \psi}{\partial r}\right)=-c^{-1} 4 \pi e\left(\sum_{j} n_{j} v_{\theta j} z_{j}-n_{e} v_{\theta e}\right)
\end{aligned}
$$

The quasineutrality condition gives Eq. (4-153). The radial component of Ampere's $i$ jives Eq. $(4-154)$. Since $f^{(0)}$ is a function of $H, p_{\theta}, p_{z}$, the radial ion velocity is zero in zero order and Eq. $(4-154)$ is order $v$. In Eq. $(4-155) \pi$ is a cullision frequency quantity and it is thus given a $v$ coefficient. The time evolution of $\psi$ is assumed to be caused by the change of $f^{(0)}$ and it is thus order $v^{2}$. As discussed, $\phi^{(0)}$ is zero so $\partial \phi / \partial r$ in Eq. (4-156) is order $v$. The five functions $n_{e}, v_{r e}, v_{\theta e}, \phi, \psi$ are obtained from 
Eq. (4-153) to Eq. (4-157) at each order by substituting the following expansions in those equations.

$$
\begin{aligned}
& n_{e}=\sum_{i} v^{i} n_{e}^{(i)} \\
& v_{r e}=\sum_{i} v^{i} v_{r e}^{(i)} \\
& v_{\theta e}=\sum_{i} v^{i} v_{\theta e}^{(i)} \\
& \phi=\sum_{i} v^{i}{ }_{\phi}^{(i)} \\
& \psi=\sum_{i} v^{i} \psi^{(i)}
\end{aligned}
$$

At order zero the following equations apply.

$$
\begin{aligned}
& n_{e}^{(0)}=\sum_{j} n_{j}^{(0)} z_{j} \\
& v_{r e}^{(0)}=0
\end{aligned}
$$$$
v_{\theta e}^{(0)}=-c r\left(e n_{e}^{(0)} \frac{\partial \psi^{(0)}}{\partial r}\right)^{-1} \frac{\partial}{\partial r}\left(n_{e}^{(0)} T_{e}\right)
$$$$
\phi^{(0)}=0
$$

$$
\frac{\partial}{\partial r}\left(\frac{1}{r} \frac{\partial \psi^{(0)}}{\partial r}\right)=-4 \pi e c^{-1}\left(\sum_{j} n_{j}^{(0)} v_{\theta j}^{(0)}-n_{e}^{(0)} v_{g e}^{(0)}\right)
$$

Near the field null $\partial y / \partial r$ goes to zero and a singularity develops in the coefficient on the right side of $\mathrm{Eq} \cdot(4-165)$. The singularity is resolved by solving for $r$ as a function of $\psi$ near the field null 
and then changing the $r$ derivative to a $\psi$ derivative.

$$
v_{\theta e}^{(0)}=-\operatorname{cr}\left(e n_{e}^{(0)}\right)^{-1} \frac{\partial}{\partial \psi}\left(n_{e}^{(0)} T_{e}\right)
$$

In Eq. $(4-168) n_{e}^{(0)} T_{e}$ is considered to be a function of $\psi$.

At first order the following equations are obtained.

$$
\begin{aligned}
& n_{e}^{(1)}= \sum_{j} n_{j}^{(1)} z_{j} \\
& v_{r e}^{(1)}=\left(n_{e}^{(0)}\right)^{-1} \sum_{j} n_{j}^{(0)} v_{r j}^{(1)} z_{j} \\
& v_{\theta e}^{(1)}=\left(e n_{e}^{(0)}\right)^{-1}\left(e n_{e}^{(1)} v_{\theta e}^{(0)}+(r c)^{-1} v_{r e}^{(1)} \frac{\partial \psi}{\partial r}\right. \\
&\left.+\langle z\rangle^{-1} \text { en } \sum_{j} n_{j}^{(0)} z_{j}^{2} v_{\theta j}^{(0)}\right)
\end{aligned}
$$

$$
\begin{aligned}
\frac{\partial \phi^{(1)}}{\partial r} & =(r c)^{-1}\left(v_{\theta e}^{(1)} \frac{\partial \psi^{(0)}}{\partial r}+v_{\theta e}^{(0)} \frac{\partial \psi^{(1)}}{\partial r}\right) \\
& +\left(e n_{\mathrm{e}}^{(0)}\right)^{-1} \frac{\partial}{\partial r}\left(n_{\mathrm{e}}^{(1)} T_{\mathrm{e}}\right)
\end{aligned}
$$$$
\frac{\partial}{\partial r}\left(\frac{1}{r} \frac{\partial \psi^{(1)}}{\partial r}\right)=-4 \pi e^{-1} \quad\left(\sum_{j}\left(n_{j}^{(0)} v_{\theta j}^{(1)}+n_{j}^{(1)} v_{\theta j}^{(0)}\right) z_{j}\right.
$$$$
\left.-n_{e}^{(0)} v_{\theta e}^{(1)}-n_{e}^{(1)} v_{\theta e}^{(0)}\right)
$$

The ion quantities in Eq. (4-153) to Eq. (4-173) are obtained from moments of the ion distribution function as follows.

$$
n_{j}^{(i)}=r^{-1} m_{j} \int f_{j}^{(i)} p_{r}^{-1} d H d p_{\theta} d p_{z}
$$


In Eq. (4-174) superscript (i) refers to 0 or 1.

$$
\begin{aligned}
& n_{j}^{(0)} v_{\theta j}^{(0)}=r^{-2} \int f_{j}^{(0)} p_{r}^{-1}\left(p_{\theta}-e z_{j} c^{-1} \psi\right) d H d p_{\theta} d p_{z} \\
& n_{j}^{(0)} v_{r j}^{(1)}=r^{-1} \int f_{j}^{(1)} d H d p_{\theta} d p_{z} \\
& n_{j}^{(0)} v_{\theta j}^{(1)}+n_{j}^{(1)} v_{\theta j}^{(0)}=r^{-2} \int f_{j}^{(1)} p_{r}^{-1}\left(p_{\theta}-e z_{j} c^{-1} \psi\right) d H d p_{\theta} d p_{z}
\end{aligned}
$$

\subsubsection{Collision Operator for the Orbit Averaged Kinetic}

\section{Equation}

The solution of Eq. $(4-129)$ requires $\bar{C}^{(2)}$ anc $i(1)$. The usual approximation is made that large angle scattering due to collisions results from many small angle scatters. The collision operator $C(f, f)$ is then taken to be the Fokker-Planck collision operator, $[190]$

$$
c(f, f)=\sum_{\alpha} \Gamma_{j}\left[-\frac{\partial}{\partial v_{\mu}}\left(f_{j} \frac{\partial h_{\alpha}}{\partial v_{\mu}}\right)+\frac{1}{2} \frac{\partial^{2}}{\partial v_{\mu} \partial v_{\gamma}}\left(f_{j} \frac{\partial^{2} g_{\alpha}}{\partial v_{\mu} \partial v_{\gamma}}\right)\right]
$$

where Eq. (4-178) models collisions of species $j$ with all species $\alpha$ and the following definitions are used.

$$
\begin{aligned}
& r_{j}=m_{j}^{-2} 4 \pi z_{j}^{2} z_{\alpha}^{2} \\
& h_{\alpha}=\lambda_{\alpha^{r^{\prime \prime} \alpha}}^{r^{-1}}\left(m_{j}+m_{\alpha}\right) \int f_{\alpha^{\prime}}\left(\overrightarrow{v^{\prime}}\right)\left|\vec{v}-\overrightarrow{v^{\prime}}\right|-1 d^{3} v^{\prime} \\
& g_{\alpha}=\lambda_{\alpha} \int f_{\alpha}\left(\overrightarrow{v^{\prime}}\right)\left|\vec{v}-\vec{v}^{\prime}\right| d^{3} v^{\prime}
\end{aligned}
$$


The expression in Eq. (4-178) is in terms of cartesian velocities which may be $v_{r}, v_{\theta}, v_{z}$. Equation (4-178) is written in terms of $v_{r}, v_{\theta}, v_{z}$ below.

$$
\begin{aligned}
& c(f, f)=\sum_{\alpha} \Gamma_{j}\left(\sum_{i=1}^{3} \Omega_{j}^{(n)}+\frac{1}{2} X_{\mu, \gamma}{ }_{\mu, \gamma}^{(n)}\right) \\
& \Omega_{1}^{(n)}=-\frac{\partial}{\partial v_{r}}\left(f(n) \frac{\partial h_{\alpha}}{\partial v_{r}}\right) \\
& \Omega_{2}^{(n)}=-\frac{\partial}{\partial v_{\theta}}\left(f_{j}(n) \frac{\partial h_{\alpha}}{\partial v_{\theta}}\right) \\
& \Omega_{3}^{(n)}=-\frac{\partial}{\partial v_{z}}\left(f_{j}^{(n)} \frac{\partial h_{\alpha}}{\partial v_{2}}\right) \\
& x_{1,1}=\frac{\partial^{2}}{\partial v_{r}^{2}} \\
& x_{1,2}=\frac{\partial^{2}}{\partial v_{r} \partial v_{\theta}} \\
& x_{1,3}=\frac{\partial^{2}}{\partial v_{r} \partial v_{2}} \\
& x_{2,1}=x_{1,2} \\
& x_{2,2}=\frac{\partial^{2}}{\partial v_{\theta}^{2}} \\
& x_{2,3}=\frac{\partial^{2}}{\partial v_{\theta}^{\partial v} z} \\
& x_{3,1}=x_{1,3}
\end{aligned}
$$


281

$$
\begin{aligned}
& x_{3,2}=x_{2,3} \\
& x_{3,3}=\frac{\partial^{2}}{\partial v_{2}^{2}} \\
& \frac{Y(n)}{\mu, \gamma}=f_{j}^{(n)} x_{\mu, \gamma^{9} \alpha}
\end{aligned}
$$

The orbit averaged kinetic equation, Eq. $(4-129)$ is in coordinates $\mathrm{H}, \mathrm{p}_{\theta}, \mathrm{p}_{z}$. To put the collision operator in the same variables the relations below are used.

$$
\begin{aligned}
& H_{\theta l}=2 m_{j}\left(H-\left(2 m_{j} r^{2}\right)^{-1}\left(p_{\theta}-e z_{j} c^{-1} \psi\right)^{2}-\left(2 m_{j}\right)^{-1} p_{z}^{2}-e z_{j} \phi\right) \\
& H=\left(2 m_{j}\right)^{-1} H_{\theta 1}+\left(2 m_{i} r^{2}\right)^{-1}\left(p_{\theta}-e z_{. j} c^{-1} \psi\right)^{2} \\
& +\left(2 m_{j}\right)^{-1} p_{z}^{2}+e z_{j} \phi \\
& \left.\left.\left.\frac{\partial}{\partial v_{r}}\right)_{v_{\theta}, v_{z}}=\frac{\partial H}{\partial v_{r}}\right)_{v_{\theta}, v_{z}} \frac{\partial}{\partial H}\right)_{p_{\theta}, p_{z}} \\
& \left.=H_{\theta l}^{1 / 2} \frac{\partial}{\partial H}\right)_{p_{\theta}, p_{z}} \\
& \left.\left.\left.\left.\left.\frac{\partial}{\partial v_{\theta}}\right)_{v_{r}, v_{z}}=\frac{\partial H}{\partial v_{\theta}}\right)_{v_{r}, v_{z}} \frac{\partial}{\partial H}\right)_{p_{\theta}, p_{z}}+\frac{\partial p_{\theta}}{\partial v_{\theta}}\right)_{v_{r}, v_{z}} \frac{\partial}{\partial p_{\theta}}\right)_{H}, p_{z} \\
& \left.\left.=r^{-1}\left(p_{\theta}-e z_{j} c^{-1} \psi\right) \frac{\partial}{\partial H}\right)_{p_{\theta}, p_{z}}+m_{j} r \frac{\partial}{\partial p_{\theta}}\right)_{H, p_{Z}}
\end{aligned}
$$


282

$$
\begin{aligned}
\left.\frac{\partial}{\partial v_{z}}\right)_{v_{r}, v_{\theta}} & \left.\left.\left.\left.=\frac{\partial H}{\partial v_{z}}\right)_{v_{r} v_{\theta}} \frac{\partial}{\partial H}\right)_{p_{\theta}, p_{z}}+\frac{\partial p_{z}}{\partial v_{z}}\right)_{v_{r}, v_{\theta}} \frac{\partial}{\partial p_{z}}\right)_{H, p_{\theta}} \\
& \left.\left.=p_{z} \frac{\partial}{\partial H}\right)_{p_{\theta} p_{z}}+m_{j} \frac{\partial}{\partial p_{z}}\right)_{H, p_{\theta}}
\end{aligned}
$$

Using Eq. (4-198) to Eq. $(4-200)$ in Eq. $(4-182)$ to Eq. $(4-194)$ yields Eq. (4-182) terms in $H, p_{\theta}, p_{z}$ coordinates.

$$
\begin{aligned}
& \Omega_{1}^{(n)}=-H_{\theta l}^{1 / 2} \frac{\partial}{\partial H}\left(f_{j}^{(n)} H_{\theta l}^{1 / 2} \frac{\partial h_{\alpha}}{\partial H}\right) \\
& \Omega_{2}^{(n)}=-r^{-2}\left(p_{\theta}-e z_{j} c^{-1} \psi\right)^{2} \frac{\partial}{\partial H}\left(f_{j}^{(n)} \frac{\partial h_{\alpha}}{\partial H}\right) \\
& -n_{j}\left(p_{\theta}-e z_{j} c^{-1} \psi\right) \frac{\partial}{\partial H}\left(f_{j}^{(n)} \frac{\partial h_{\alpha}}{\partial p_{\theta}}\right) \\
& -m_{j} \frac{\partial}{\partial p_{\theta}}\left(\left(p_{\theta}-e z_{j} c^{-1} \psi\right) f \underset{j}{(n)} \frac{\partial h_{\alpha}}{\partial H}\right) \\
& -m_{j}^{2} r^{2} \frac{\partial}{\partial p_{\theta}}\left(f(n) \frac{\partial h_{\alpha}}{\partial p_{\theta}}\right) \\
& \Omega_{3}^{(n)}=-p_{z}^{2} \frac{\partial}{\partial H}\left(f(n) \frac{\partial h^{\alpha}}{\partial H}\right)-m_{j} p_{z} \frac{\partial}{\partial H}\left(f(n) \frac{\partial h \alpha}{\partial p_{z}}\right) \\
& -m_{j} \frac{\partial}{\partial p_{z}}\left(p_{z} f_{j}^{(n)} \frac{\partial h}{\partial H}\right)-m_{j}^{2} \frac{\partial}{\partial p_{z}}\left(f_{j}^{(n)} \frac{\partial h}{\partial p_{z}}\right) \\
& x_{1,1}=H_{\theta 1}^{1 / 2} \frac{\partial}{\partial H}\left(H_{\theta 1}^{1 / 2} \frac{\partial}{\partial H}\right. \\
& x_{1,2}=H_{\theta 1}^{l / 2} r^{-1}\left(p_{\theta}-e z_{j} c^{-1} \psi\right) \frac{\partial^{2}}{\partial H^{2}}+H_{\theta l}^{1 / 2} m_{j} r \frac{\partial^{2}}{\partial H \partial p_{\theta}}
\end{aligned}
$$


283

$$
\begin{aligned}
& x_{1,3}=H_{\theta 1}^{1 / 2} p_{z} \frac{\partial^{2}}{\partial H^{2}}+H_{\theta 1}^{1 / 2} m_{j} \frac{\partial^{2}}{\partial H^{2} \partial p_{2}} \\
& x_{2,1}=x_{1,2}
\end{aligned}
$$

$$
\begin{aligned}
x_{2,2} & =r^{-2}\left(p_{\theta}-e z_{j} c^{-1} \psi\right)^{2} \frac{\partial^{2}}{\partial H^{2}}+m_{j}\left(p_{\theta}-e z_{j} c^{-1} \psi\right) \frac{\partial^{2}}{\partial H \partial p_{\theta}} \\
& +m_{j} \frac{\partial}{\partial p_{\theta}}\left(p_{\theta}-e z_{j} c^{-1} \psi\right) \frac{\partial}{\partial H}+m_{j}^{2} r^{2} \frac{\partial^{2}}{\partial p_{\theta}^{2}}
\end{aligned}
$$

$$
\begin{aligned}
x_{2,3} & =r^{-1}\left(p_{\theta}-e z_{j} c^{-1} \psi\right) p_{z} \frac{\partial^{2}}{\partial H^{2}}+m_{j} r^{-1}\left(p_{\theta}-e z_{j} c^{-1} \psi\right) \frac{\partial^{2}}{\partial H \partial p_{z}} \\
& +m_{j} r p_{z} \frac{\partial^{2}}{\partial p_{\theta} \partial H}+m_{j}^{2} r \frac{\partial^{2}}{\partial p_{\theta}^{\partial p_{z}}} \\
x_{3,1} & =x_{1,3} \\
x_{3,2} & =x_{2,3} \\
x_{3,3} & =p_{z}^{2} \frac{\partial^{2}}{\partial H^{2}}+m_{j} p_{z} \frac{\partial^{2}}{\partial H \partial p_{z}}+m_{j} \frac{\partial}{\partial p_{z}} p_{z} \frac{\partial}{\partial H}+m_{j}^{2} \frac{\partial^{2}}{\partial p_{z}^{2}}
\end{aligned}
$$

The $C^{(1)}$ term requires $\bar{C}^{(i)}$ which from Eq. $(3-31)$ and $E q$. (3-32) is defined below.

$$
\begin{aligned}
& \bar{c}^{(1)}=\left(\int_{r_{\min }}^{r \max } d r p_{r}^{-1}\right)^{-1} \int_{r_{\min }}^{r_{\max }} d r p_{r}^{-1} \sum_{\alpha} \\
& \left(\Gamma_{j}\left(\sum_{i=1}^{3} \Omega_{i}^{(0)}+\frac{i}{2} x_{\mu, \gamma}{ }_{\mu, \gamma}^{(0)}\right)\right)
\end{aligned}
$$


In Eq. (4-213) the $r_{\min }$, $r_{\max }$ integral limits are determined implicitly for specific $H, p_{\theta}, p_{z}$ values from the relation below.

$$
H=\left(2 m_{j}\right)^{-1} p_{z}^{2}+\left(2 m_{j} r^{2}\right)^{-1}\left(p_{\theta}-e z_{j} c^{-1} \psi\right)^{2}+e z_{j} \phi
$$

The allowed orbit region determined by Eq. (4-214) may lead to multiple disconnected regions. In this case the integrals in Eq. $(4-213)$ are understood to be the sum of integrations between the $r_{\min }$ and $r_{\max }$ of each disconnected region.

There are two simplicities that result in the evaluation of terms in Eq. (4-213). The first is that $f_{j}^{(0)}$ commutes with the orbit average and caret operator. The second is the ortit average and caret iperator commute with $\partial / \partial H, \partial / \partial p_{\theta}$ and $\partial / \partial p_{z}$. It appears the orbit average and caret operator do not commute with these derivatives since the integral limits depend on $H, p_{\theta}$, and $p_{z}$. The basic definitions with time parameterizing a trajectory and integrating over many bounce periods do commute with the orbit average and caret operator. Thus an interchange may be made between the basic definition and a derivative and then the orbit average or caret operator may be converted to its equivalent single bounce period definition.

The $\hat{c}^{(1)}$ term is similar to Eq. $(4-213)$,

$$
\begin{aligned}
\hat{c}^{(1)} & =m_{j} \int_{r_{\min }}^{r} d r p_{r}^{-1}\left(\sum _ { \alpha } \left[\Gamma _ { j } \left(\sum_{i=1}^{3} \Omega_{j}^{(0)}\right.\right.\right. \\
& \left.\left.\left.+\frac{1}{2} X_{\mu, \gamma}{ }_{\mu, \gamma}^{(0)}\right)\right]-\bar{c}^{(1)}\right)
\end{aligned}
$$


h.s for Eq. (4-213) the $r_{\min }$ in Eq. $(4-215)$ is determined by Eq. $(4-214)$. In the case of multiple disconnected regions the integral in Eq. $(4-215)$ is a sum of integrations over regions bounded by $r$ values less than the minimum of the region containing the $r$ position where $\hat{c}^{(1)}$ is to be evaluated plus the integral from $r_{\text {min }}$ to $r$ in the region of interest.

The orbit average or caret of terms on the right side of Eq. (4-213) and Eq. (4-215) are obtained using the commuting properties and the operator relation below.

$$
g(x) \frac{\partial}{\partial x} \mid=-\frac{\partial g(x)}{\partial x}+\frac{\partial}{\partial x}[g(x)
$$

The relation in Eq. $(4-216)$ is used to express the terins as derivatives of orbit averaged or careted quantities. The terins in Eq. (4-213) are similar to those of Eq. $(4-215)$ and thus only the terms in Eq. (4-275) are presented.

The Eq. (4-201) definition is written using Eq. (4-216) as follows.

$$
\begin{aligned}
\Omega_{1}^{(0)} & =\left(\frac{\partial H_{\theta l}^{1 / 2}}{\partial H}\right)\left(f_{j}^{(0)} H_{\theta l}^{1 / 2} \frac{\partial h_{\alpha}}{\partial H}\right)-\frac{\partial}{\partial H}\left(f_{j}^{(0)} H_{\theta l} \frac{\partial h_{\alpha}}{\partial H}\right) \\
& =m_{j} f j \\
j & \frac{\partial h_{\alpha}}{\partial H}+\frac{\partial}{\partial H}\left(2 m_{j} f_{j}^{(0)} h_{\alpha}-f_{j}^{(0)} \frac{\partial}{\partial H}\left(H_{\theta 1} h_{\alpha}\right)\right)
\end{aligned}
$$

The caret of $E q .(4-217)$ is then below.

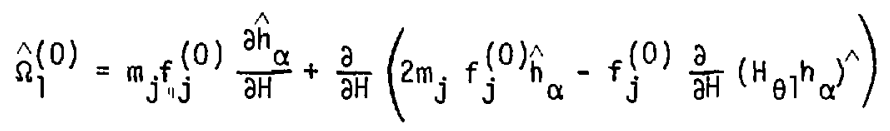

The other terms in Eq. (4-215) are evaluated in a similar manner. 
286

$$
\begin{aligned}
& \hat{\Omega}_{2}^{(0)}=-\frac{\partial}{\partial H}\left(f _ { j } ^ { ( 0 ) } \frac { \partial } { \partial H } \left(p_{\theta}^{2}\left(r^{-2} h_{\alpha}\right)^{\wedge}-2 e z_{j} p_{\theta} c^{-1}\left(r^{-2} \psi h_{\alpha}\right)^{\wedge}\right.\right. \\
& +\left(e z_{j} c^{-1}\right)^{2}\left(r^{-2} \psi^{2} h_{\alpha} \hat{j}\right) \\
& +m_{j} \frac{\partial}{\partial H}\left(f_{j}^{(0)} \hat{h}_{\alpha}-f_{j}^{(0)} \frac{\partial}{\partial p_{\theta}}\left(p_{\theta} \hat{h}_{\alpha}-e z_{j} c^{-1}\left(\psi h_{\alpha}\right)^{\wedge}\right)\right) \\
& -m_{j} \frac{\partial}{\partial p_{\theta}}\left(f(0) p_{\theta} \frac{\partial \hat{h_{\alpha}}}{\partial H}-e z_{j} c^{-1} f_{j}^{(0)} \frac{\partial}{\partial H}\left(\psi h_{\alpha}\right)^{\wedge}\right) \\
& -m_{j}^{2} \frac{\partial}{\partial p_{\theta}}\left(f_{j}^{(0)} \frac{\partial}{\partial p_{\theta}}\left(r^{2} h_{\alpha}\right)\right) \\
& \dot{\Omega}_{3}^{(0)}=-p_{z}^{2} \frac{\partial}{\partial H}\left(f(0) \frac{\partial h_{\alpha}}{\partial H}\right)-m_{j} p_{z} \frac{\partial}{\partial H}\left(f_{j}^{(0)} \frac{\partial \hat{h} \alpha}{\partial p_{z}}\right) \\
& -m_{j} \frac{\partial}{\partial p_{z}}\left(p_{z} f(0) \frac{\partial \hat{h}}{\partial H}\right)-m_{j}^{2} \frac{\partial}{\partial p_{z}}\left(f(0) \frac{\partial \hat{h}}{\partial p_{z}}\right) \\
& X_{,, Y} Y_{i, 1}^{(0)}=H_{\theta 1}^{1 / 2} \frac{\partial}{\partial H}\left(H_{\theta 1}^{1 / 2} \frac{\partial}{\partial H}\left(f_{j}^{(0)} H_{\theta 1}^{1 / 2} \frac{\partial}{\partial H}\left(H_{\theta 1}^{i / 2} \frac{\partial g_{\alpha}}{\partial H}\right)\right)\right)
\end{aligned}
$$

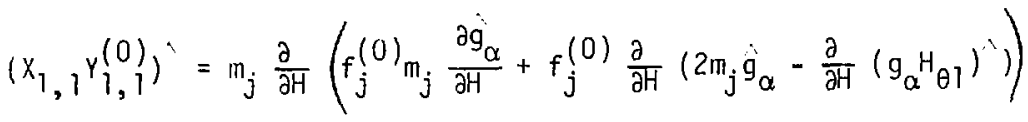

$$
\begin{aligned}
& +\frac{\partial}{\partial H}\left[-2 m_{j} f(0)\left(-m_{j} \frac{\partial \hat{g}_{\alpha}}{\partial H}+\frac{\partial}{\partial H}\left(-2 m_{j} \hat{g}_{\alpha}+\frac{\partial}{\partial H}\left(g_{\alpha} H_{\theta l}\right)\right)\right)\right. \\
& +\frac{\partial}{\partial H}\left(-3 m_{j} f_{j}^{(0)}\left(-2 m_{j} \hat{g}_{\alpha}+\frac{\partial}{\partial H}\left(g_{\alpha} H_{\theta l}\right)\right)\right. \\
& \left.\left.\left.+f_{j}^{(0)} \frac{\partial}{\partial H}\left(-4 m_{j}\left(g_{\alpha} H_{\theta 1}\right) \hat{}+\frac{\partial}{\partial H}\left(g_{\alpha} H_{\theta 1}^{2}\right)\right)\right)\right)\right]
\end{aligned}
$$


287

$$
\begin{aligned}
& X_{1,2}{ }_{1,2}^{(0)}=\left(H_{\theta 1}^{1 / 2} r^{-1}\left(p_{\theta}-e Z_{j} c^{-1} \psi\right) \frac{\partial^{2}}{\partial H^{2}}+m_{j} H_{\theta 1}^{1 / 2} r \frac{\partial^{2}}{\partial H_{\partial p_{\theta}}}\right) \\
& \left(f \underset{j}{(0)} H_{\theta l}^{1 / 2} r^{-1}\left(p_{\theta}-e Z_{j} c^{-1} \psi\right) \frac{\partial^{2} g_{\alpha}}{\partial H^{2}}+m_{j} f_{j}^{(0)} H_{\theta l}^{1 / 2} r \frac{\partial^{2} g_{a}}{\partial H_{\partial p_{\theta}}}\right)
\end{aligned}
$$

In the formulas that follow $v_{m \theta}=r^{-1}\left(p_{\theta}-e z_{j} c^{-1} \psi\right)$

$$
\begin{aligned}
& \left(x_{1,2} Y_{1,2}^{(0)}\right)^{:}=-m_{j}^{2} f_{j}^{(0)}\left(8 m _ { j } ^ { 2 } \left(p_{\theta}^{2}\left(r^{-2_{H}}{ }_{\theta l}^{-3} g_{\alpha}\right)-2 e z_{j} c^{-1} p_{\theta}\left(r^{-2} H_{\theta l}^{-3} \psi q_{\alpha}\right)\right.\right. \\
& +\left(e z_{j} c^{-1}\right)^{2}\left(r^{-2} H_{\theta 1}^{-3} \psi^{2} g_{\alpha}\right) \\
& +4 m_{j} \frac{\partial}{\partial H}\left(p_{\theta}^{2}\left(r^{-2} H_{\theta l}^{-2} g_{\alpha}\right)-2 e z_{j} c^{-1} p_{\theta}\left(r^{-2} H_{\theta l}^{-2} \psi g_{\alpha}\right)\right. \\
& \left.+\left(e z_{j} c^{-1}\right)^{2}\left(r^{-2} H_{\theta 1}^{-2} \psi^{2} g_{\alpha}\right)\right) \\
& +\frac{\partial^{2}}{\partial H^{2}}\left(p_{\theta}^{2}\left(r^{-2} H_{\theta 1}^{-1} g_{\alpha}\right)-2 e z_{j} c^{-1} p_{\theta}\left(r^{-2} H_{\theta l}^{-1} \psi g_{\alpha}\right)\right. \\
& \left.+\left(e z_{j} c^{-1}\right)^{2}\left(r^{-2} H_{\theta 1}^{-1} \psi^{2} g_{\alpha}\right) i\right) \\
& -2 m_{j} \frac{\partial}{\partial H} f_{j}^{(0)} \frac{\partial^{2}}{\partial H^{2}}\left(v_{m \theta}^{2} g_{\alpha}\right)^{r} \\
& +\frac{\partial^{2}}{\partial H^{2}}\left(f _ { j } ^ { ( 0 ) } \left(-4 m_{j} \frac{\partial}{\partial H}\left(v_{m \theta}^{2} g_{\alpha}\right)\right.\right. \\
& \left.+\frac{\partial^{2}}{\partial H^{2}}\left(v_{m \theta}^{2} g_{\alpha}\right)\right)
\end{aligned}
$$


288

$$
\begin{aligned}
& -m_{j}^{3} f_{j}^{(0)}\left(-8 m_{j}\left(v_{m \theta}^{2} H_{\theta 1}^{-3} g_{\alpha}\right)^{\wedge}-2 m_{j}\left(H_{\theta 1}^{-2} g_{\alpha}\right)^{\wedge}\right. \\
& +2 m_{j} \frac{\partial}{\partial p_{\theta}}\left(H_{\theta l}^{-2} r v_{m \theta} g_{\alpha}\right)^{\wedge} \\
& +\frac{\partial}{\partial H}\left(-2\left(H_{\theta 1}^{-2} v_{m \theta}^{2} g_{\alpha}\right)^{\wedge}-\left(H_{\theta 1}^{-1} g_{\alpha}\right)^{\wedge}+\frac{\partial}{\partial p_{\theta}}\left(H_{\theta 1}^{-1} r v_{m \theta} g_{\alpha}\right)^{\wedge}\right) \\
& -2 m_{j}^{2} \frac{\partial}{\partial H}\left(f_{j}^{(0)} \frac{\partial}{\partial H}\left(-\hat{g}_{\alpha}+\frac{\hat{\partial}}{\partial p_{\theta}}\left(r v_{m \theta} g_{\alpha} \hat{)}\right)\right)\right. \\
& +m_{j} \frac{\partial^{2}}{\partial H^{2}} f_{j}^{(0)}\left(2 m_{j} \hat{g}_{\alpha}-2 m_{j} \frac{\partial}{\partial p_{\theta}}\left(r v_{m \theta} g_{\alpha}\right)^{\wedge}\right. \\
& +\frac{\partial}{\partial H}\left(-2\left(r^{-1} v_{m \theta} g_{\alpha}\right) \hat{-}-\left(H_{\theta 1} g_{\alpha}\right)+\frac{\partial}{\partial p_{\theta}}\left(r v_{m \theta} H_{\theta 1} g_{\alpha}\right)\right) \\
& +m_{j}^{2} f_{j}^{(0)}\left(8 m_{j}^{2}\left(H_{\theta 1}^{-3} v_{m \theta}^{2} g_{\alpha}\right)+4 m_{j} \frac{\partial}{\partial H}\left(v_{m \theta}^{2} H_{\theta 1}^{-2} g_{\alpha}\right)^{\wedge}\right. \\
& \left.+\frac{\partial^{2}}{\partial H^{2}}\left(v_{m \theta}^{2} H_{\theta 1}^{-1} g_{\alpha}\right)^{\wedge}\right) \\
& +m_{j}^{2} \frac{\partial}{\partial p_{\theta}}\left(f(0) \frac{\partial^{2}}{\partial H^{2}}\left(r v_{m \theta} g_{\alpha}\right)^{\wedge}\right) \\
& +m_{j} \frac{\partial}{\partial H}\left(f_{j}^{(0)} \frac{\partial^{2}}{\partial H^{2}}\left(v_{m \theta}^{2} g_{\alpha}\right)^{\lambda}\right) \\
& +m_{j} \frac{\partial}{\partial p_{\theta}}\left(f_{j}^{(0)}\left(-4 m_{j} \frac{\partial}{\partial H}\left(r v_{m \theta} g_{\alpha}\right)^{\wedge}+\frac{\partial^{2}}{\partial H^{2}}\left(r v_{m \theta} H_{\theta 1} g_{\alpha}\right) \hat{)}\right)\right. \\
& +m_{j}^{3} f_{j}^{(0)}\left(-8 m_{j}\left(r^{-1} v_{m \theta} H_{\theta 1}^{-3} g_{\alpha}\right)^{\wedge}-2 m_{j}\left(H_{\theta l}^{-2} g_{\alpha}\right)^{\wedge}\right.
\end{aligned}
$$


289

$$
\begin{aligned}
& +2 m_{j} \frac{\partial}{\partial p_{\theta}}\left(r v_{m \theta} H_{\theta l}^{-2} g_{\alpha}\right)^{\prime} \\
& +\frac{\partial}{\partial H}\left(-2\left(v_{m \theta}^{2} H_{\theta 1}^{-2} g_{\alpha}\right)-\left(H_{\theta 1}^{-1} g_{\alpha}\right) \dot{+}+\frac{\partial}{\partial p_{\theta}}\left(H_{\theta 1}^{-1} r v_{m \theta} g_{\alpha}\right)\right) \\
& +m_{j}^{3} \frac{\partial}{\partial p_{\theta}}\left(f_{j}^{(0)} \frac{\partial^{2}}{\partial H \partial p_{\theta}}\left(r^{2} g_{\alpha}\right)\right) \\
& +m_{j}^{2} \frac{\partial}{\partial H}\left(f_{j}^{(0)} \frac{\partial}{\partial H}\left(-g_{\alpha}+\frac{\partial}{\partial p_{\theta}}\left(r V_{m \theta} g_{\alpha}\right)\right)\right. \\
& +m_{j}^{2} \frac{\partial}{\partial p_{\theta}}\left(f _ { j } ^ { ( 0 ) } \left(-2 m_{j} \frac{\partial}{\partial p_{\theta}}\left(r^{2} g_{\alpha}\right)-2 \frac{\partial}{\partial H}\left(r v_{m \theta} g_{\alpha}\right)\right.\right. \\
& \left.+\frac{\partial}{\partial p_{\theta}}\left(H_{\theta 1} g_{\alpha}\right)\right) \\
& X_{1,3} Y_{l, 3}^{(0)}=\left(H_{\theta l}^{1 / 2} p_{z} \frac{\partial^{2}}{\partial H^{2}}+i n_{j} H_{\theta l}^{1 / 2} \frac{\partial^{2}}{\partial H_{\partial p_{z}}}\right)\left(f _ { j } ^ { ( 0 ) } \left(p_{z} H_{\theta l}^{1 / 2} \frac{\partial^{2} g_{\alpha}}{\partial H^{2}}\right.\right. \\
& \left.\left.+m_{j} H_{\theta l}^{1 / 2} \frac{\partial^{2} g_{\alpha}}{\partial H \partial p_{z}}\right)\right) \\
& =\left(-m_{j} p_{z} \frac{\partial}{\partial H} H_{\theta l}^{-1 / 2}+m_{j} \frac{\partial^{2}}{\partial H_{\partial-}} H_{\theta l}^{l / 2}+p_{z} \frac{\partial^{2}}{\partial H^{2}} H_{\theta l}^{l / 2}\right. \\
& \left.-m_{j}^{2} \frac{\partial}{\partial p_{z}} H_{\theta l}^{-1 / 2}\right)\left(f_{j}^{(0)}\left(p_{z} H_{\theta l}^{1 / 2} \frac{\partial^{2} g_{\alpha}}{\partial H^{2}}+m_{j} i_{\theta l}^{1 / 2} \frac{\partial^{2} g_{c}}{\partial H \partial p_{z}}\right)\right) \\
& \left(X_{l, 3} Y_{i, 3}^{(0)}\right)^{\wedge}=-m_{j} p_{Z}^{2} \frac{\partial}{\partial H}\left(f(0) \frac{\partial^{2} \hat{g_{\alpha}}}{\partial H^{2}}\right)-m_{j}^{2} p_{z} \frac{\partial}{\partial H}\left(f(0) \frac{\partial^{2} \hat{g}_{\alpha}}{\partial H \partial p_{z}}\right) \\
& +m_{j} \frac{\partial^{2}}{\partial H \partial p_{z}}\left(p_{z} f_{j}^{(0)}\left(H_{\theta 1} \frac{\partial^{2} g_{\alpha}}{\partial H^{2}}\right)+m_{j}^{2} \frac{\partial^{2}}{\partial H \partial p_{z}}\left(f_{j}^{(0)}\left(H_{\theta l}^{1 / 2} \frac{\partial^{2} g_{\alpha}}{\partial H \partial p_{z}}\right)\right)\right.
\end{aligned}
$$




$$
\begin{aligned}
& +p_{z}^{2} \frac{\partial^{2}}{\partial H^{2}}\left(f_{j}^{(0)}\left(H_{\theta 1} \frac{\partial^{2} g_{\alpha}}{\partial H^{2}}\right)\right)+m_{j} p_{z} \frac{\partial^{2}}{\partial H^{2}}\left(f_{j}^{(0)}\left(H_{\theta T} \frac{\partial^{2} g_{\alpha}}{\partial H_{\partial} p_{z}}\right)\right) \\
& -m_{j}^{2} \frac{\partial}{\partial p_{z}}\left(p_{z} f_{j}^{(0)} \frac{\partial^{2} \hat{g}_{\alpha}}{\partial H^{2}}\right)-m_{j}^{3} \frac{\partial}{\partial p_{z}}\left(f(0) \frac{\partial^{2} \hat{g}_{\alpha}}{\partial H \partial p_{z}}\right) \\
& \left(X_{2,1} Y_{2,1}^{(0)}\right) \hat{}=\left(X_{1,2} Y_{1,2}^{(0)}\right) \hat{~} \\
& x_{2,2} \gamma_{2,2}^{(0)}=\left(v_{m \theta}^{2} \frac{\partial^{2}}{\partial H^{2}}+m_{j} \frac{\partial}{\partial H}\left(-r+\frac{\partial}{\partial p_{\theta}} r v_{m \theta}\right)+m_{j}^{2} r^{2} \frac{\partial^{2}}{\partial p_{\theta}^{2}}\right) \\
& \left(f _ { j } ^ { ( 0 ) } \left(v_{m \theta}^{2} \frac{\partial^{2} g_{\alpha}}{\partial H^{2}}+m_{j} \frac{\partial}{\partial H}\left(-r g_{\alpha}+\frac{\partial}{\partial p_{\theta}}\left(r v_{m \theta^{9} \alpha}\right)\right)\right.\right. \\
& \left.\left.+m_{j}^{2} r^{2} \frac{\partial^{2} g_{\alpha}}{\partial p_{\theta}^{2}}\right)\right) \\
& \left(X_{2,2} Y_{2,2}^{(0)} \hat{)}=\frac{\partial^{2}}{\partial H^{2}}\left(f(0) \frac{\partial^{2}}{\partial H^{2}}\left(v_{m \theta^{2}}^{4} \hat{)}\right)+m_{j}^{2} \frac{\partial^{2}}{\partial H^{2}}\left(f \underset{j}{(0)}\left(r^{2} v_{m \theta}^{2} \frac{\partial^{2} g_{\alpha}}{\partial p_{\theta}^{2}}\right)\right)\right.\right. \\
& +m_{j} \frac{\partial^{2}}{\partial H^{2}}\left(f _ { j } ^ { ( 0 ) } \frac { \partial } { \partial H } \left(-\left(r g_{\alpha} v_{m \theta}^{2} \hat{)}+\left(v_{m \theta}^{2} \frac{\partial}{\partial p_{\theta}}\left(r v_{m \theta} g_{\alpha}\right)\right)\right)\right.\right. \\
& +m_{j} \frac{\partial}{\partial H}\left(-f_{j}^{(0)} \frac{\partial^{2}}{\partial H^{2}}\left(r v_{m \theta}^{2} g_{\alpha}\right)+\frac{\partial}{\partial p_{\theta}}\left(f_{j}^{(0)} \frac{\partial^{2}}{\partial H^{2}}\left(r v_{m \theta}^{3} g_{\alpha}\right)\right)\right) \\
& +m_{j}^{2} \frac{\partial}{\partial H}\left(f_{j}^{(0)} \frac{\partial}{\partial H}\left(\left(r g_{\alpha}^{2}\right)^{\wedge}-\frac{\partial}{\partial p_{\theta}}\left(r^{2} v_{m \theta} g_{\alpha}\right)^{\wedge}\right)\right) \\
& +m_{j}^{2} \frac{\partial^{2}}{\partial H_{\partial p_{\theta}}}\left(f_{j}^{(0)} \frac{\partial}{\partial H}\left(-\left(r^{2} v_{i n \theta} g_{\alpha}\right)^{\wedge}+\left(r v_{m \theta} \frac{\partial}{\partial p_{\theta}}\left(r v_{m \theta} g_{\alpha}\right)\right)\right)\right.
\end{aligned}
$$




$$
\begin{aligned}
& +m_{j}^{3} \frac{\partial}{\partial H}\left(-f_{j}^{(0)} \frac{\partial^{2}}{\partial p_{\theta}^{2}}\left(r^{3} g_{\alpha}\right)+\frac{\partial}{\partial p_{\theta}}\left(f(0)\left(r^{3} v_{m \theta} \frac{\partial^{2} g_{\alpha}}{\partial p_{\theta}^{2}}\right)\right)\right) \\
& +m_{j}^{2} \frac{\partial^{2}}{\partial p_{\theta}^{2}}\left(f(0)\left(r^{2} v_{m \theta}^{2} \frac{\partial^{2} g_{\alpha}}{\partial H^{2}}\right)\right)+m_{j}^{4} \frac{\partial^{2}}{\partial p_{\theta}^{2}}\left(f_{j}^{(0)} \frac{\partial^{2}}{\partial p_{\theta}^{2}}\left(r^{4} g_{\alpha}\right)\right) \\
& +m_{j}^{3} \frac{\partial^{2}}{\partial p_{\theta}^{2}}\left(f_{j}^{(0)} \frac{\partial}{\partial H}\left(-\left(r g_{\alpha}^{3}\right)+\frac{\partial}{\partial p_{\theta}}\left(r^{3} v_{m \theta}{ }^{g}\right)^{2}\right)\right) \\
& x_{2,3}{ }_{2,3}^{(0)}=\left(v_{m \theta} p_{z} \frac{\partial^{2}}{\partial H^{2}}+m_{j} v_{m \theta} \frac{\partial^{2}}{\partial H_{\partial} p_{z}}+m_{j} p_{z} r \frac{\partial^{2}}{\partial p_{\theta} \partial H}+m_{j}^{2} r \frac{\partial^{2}}{\partial p_{\theta} \partial p_{z}}\right) \\
& \left(f \underset{j}{(0)}\left(V_{m \theta} p_{z} \frac{\partial^{2} g_{\alpha}}{\partial H^{2}}+m_{j} V_{m \theta} \frac{\partial^{2} g_{\alpha}}{\partial H \partial p_{z}}+m_{j} p_{z} r \frac{\partial^{2} g_{\alpha}}{\partial p_{\theta} \partial H}+m_{j}^{2} r-\frac{\partial^{2} g_{\alpha}}{\partial p_{\theta} \frac{\partial p_{z}}{\partial p}}\right)\right) \\
& \left(x_{2,3} Y_{2,3}^{(0)}\right)=\frac{\partial^{2}}{\partial H^{2}}\left(f f _ { j } ^ { ( 0 ) } \left(p_{z}^{2} \frac{\partial^{2}}{\partial H^{2}}\left(v_{m \theta}^{2} g_{\alpha}\right)+m_{j} p_{z} \frac{\partial^{2}}{\partial H_{\partial p_{z}}}\left(v_{m \theta^{2}}^{2}\right)\right.\right. \\
& \left.+m_{j} p_{z}^{2}\left(r v_{m \theta} \frac{\partial^{2} g_{\alpha}}{\partial p_{\theta} \partial h^{-}}\right)+m_{j}^{2} p_{z}\left(r v_{m \theta} \frac{\partial^{2} g_{\alpha}}{\partial p_{\theta} \partial p_{z}}\right)\right) \\
& +m_{j} \frac{\partial^{2}}{\partial H \partial p_{z}}\left(f _ { j } ^ { ( 0 ) } \left(p_{z} \frac{\partial^{2}}{\partial H^{2}}\left(v_{m \theta} g_{\alpha}\right)^{\prime}+m_{j} \frac{\partial^{2}}{\partial H \partial p_{z}}\left(v_{m \theta}^{2} g_{\alpha}\right)^{\prime}\right.\right. \\
& \left.+m_{j} p_{z}\left(r v_{m \theta} \frac{\partial^{2} g_{\alpha}}{\partial p_{\theta} \partial H}\right)+m_{j}^{2}\left(r v_{m \theta} \frac{\partial^{2} g_{\alpha}}{\partial p_{\theta} \partial p_{z}}\right)\right) \\
& +\left(m_{j}^{2} \frac{\partial^{\ddot{i}}}{\partial p_{\theta} \partial p_{z}}+m_{j} p_{z} \frac{\partial^{2}}{\partial p_{\theta} \partial H}\right)\left(f ( 0 ) \left(p_{z} \frac{\partial^{2}}{\partial H^{2}}\left(r v_{m \theta^{9}}\right)^{\prime}\right.\right.
\end{aligned}
$$




$$
\begin{aligned}
& \left.\left.+m_{j} \frac{\partial^{2}}{\partial H \partial p_{z}}\left(r v_{m \theta} g_{\alpha}\right)^{\wedge}+m_{j} p_{z} \frac{\partial^{2}}{\partial p_{\theta} \partial H}\left(r^{2} g_{\alpha}\right)+m_{j}^{2} \frac{\partial^{2}}{\partial p_{\theta} \partial p_{z}}\left(r^{2} g_{\alpha}\right)\right)\right) \\
& \left(x_{3,1} y_{3,1}^{(0)}\right)^{\wedge}=\left(x_{1,3} Y_{1,3}^{(0)}\right)^{\wedge} \\
& \left(x_{3,2} Y_{3,2}(0)\right)^{\wedge}=\left(X_{2,3} Y_{2,3}(0) \wedge\right. \\
& \left(x_{3,3} Y_{3,3}^{(0)}\right)=\left(p_{z}^{2} \frac{\partial^{2}}{\partial H^{2}}+m_{j} p_{z} \frac{\partial^{2}}{\partial H^{2} p_{z}}+m_{j} \frac{\partial}{\partial p_{z}} p_{z} \frac{\partial}{\partial H}+m_{j}^{2} \frac{\partial^{2}}{\partial p_{z}^{2}}\right) \\
& \left(f_{j}^{(0)}\left(p_{z}^{2} \frac{\partial^{2}}{\partial H^{2}}+m_{j} p_{z} \frac{\partial^{2}}{\partial H \partial p_{z}}+m_{j} \frac{\partial}{\partial p_{z}} p_{z} \frac{\partial}{\partial H}+m_{j}^{2} \frac{\partial^{2}}{\partial p_{z}^{2}}\right) g_{\alpha}\right)
\end{aligned}
$$

Using terms defined in Eq. $(4-218)$ to $\mathrm{Eq} \cdot(4-234) \hat{\mathrm{C}}^{(1)}$ is constructed from the following formula.

$$
\hat{c}^{(1)}=\sum_{\alpha} \Gamma_{j}\left(\sum_{i=1}^{3} \hat{\Omega}_{i}^{(0)}+\frac{1}{2}\left(x_{\mu, \gamma}{ }_{\mu, \gamma}^{(0)}\right)\right.
$$

The orbit average of $c^{(2)}$ is defined below.

$$
\bar{c}^{(2)}=\sum_{\alpha} \Gamma_{j}\left(\sum_{i=1}^{3} \bar{\Omega}_{i}^{(1)} \frac{1}{\frac{1}{2} \bar{\mu}_{\mu, \gamma} Y_{\mu, \gamma}^{(T)}}\right)
$$

The bar orbit average operator in $\mathrm{Eq} .(4-236)$ refers to the integral operation discussed in relation to Eq. (4-213). The terms in Eq. (4-236) require integrals of more complicated functions than $c(1)$ because $C^{(2)}$ involves $f_{j}^{(1)}$ rather than $f_{j}^{(0)}$ which is a function only of the constants of motion. From Eq. (4-128) which defines $f_{j}^{(1)}$, 
it is clear $f_{j}^{(l)}$ depends on $H, p_{\theta}, p_{z}$, and $r$. Consequently, in the terms derived below for Eq. (4-236) $f_{j}^{(1)}$ is included in the orbit average evaluation.

$$
\begin{aligned}
& \overline{\Omega_{T}^{(1)}}=m_{j} \overline{f_{j}^{(1)} \frac{\partial h_{\alpha}}{\partial H}}+\frac{\partial}{\partial H}\left(2 m_{j} \overline{f_{j}^{(l)_{h}}}-\overline{f_{j}^{(l)} \frac{\partial}{\partial H}\left(H_{\theta 1} l_{\alpha}\right)}\right) \\
& \overline{\Omega_{2}^{(1)}}=-\frac{\partial}{\partial H}\left(v_{m \theta}^{2} \overline{f_{j}^{(1)} \frac{\partial h_{\alpha}}{\partial H^{\alpha}}}+\overline{m_{j} r v_{m \theta} f_{j}^{(1)} \frac{\partial h_{\alpha}}{\partial p_{\theta}}}\right) \\
& -m_{j} \frac{\partial}{\partial p_{\theta}}\left(\overline{r v_{m \theta} f_{j}^{(1)} \frac{\partial h_{\alpha}^{\alpha}}{\partial H^{\prime}}}+m_{j} \overline{r^{2} f_{j}^{(1)} \frac{\partial h_{\alpha}}{\partial p_{\theta}}}\right) \\
& \overline{\Omega_{3}(T)}=-p_{z} \frac{\partial}{\partial H}\left(p_{z} \overline{f_{j}^{(l)} \frac{\partial h_{\alpha}}{\partial H^{\prime}}}+m_{j} \overline{f_{j}^{(l)} \frac{\partial h_{\alpha}}{\partial p_{z}}}\right) \\
& -m_{j} \frac{\partial}{\partial p_{z}}\left(j_{z} \overline{f_{j}^{(1)} \frac{\partial h_{\alpha}}{\partial H}}+m_{j} \overline{f_{j}^{(1)} \frac{\partial h_{\alpha}}{\partial p_{z}}}\right) \\
& \overline{x_{1,1} Y_{i, 1}^{(1)}}=m_{j} \frac{\partial}{\partial H}\left(\overline{m_{j} f_{j}^{(1)} \frac{\partial g}{\partial H}}+\overline{f_{j}^{(1)} \frac{\partial}{\partial H}\left(2 m_{j} g_{\alpha}-\frac{\partial}{\partial H}\left(g_{\alpha}{ }_{\alpha \theta T}\right)\right)}\right) \\
& \left.+\frac{\partial}{\partial H}\left(-2 m_{j}\left(-m_{j} f_{j}^{(1)} \frac{\partial g_{\alpha}}{\partial \dot{H}}+\overline{f_{j}^{(1)} \frac{\partial}{\partial H}\left(-2 m_{j} g_{\alpha}+\frac{\partial}{\partial H}\left(g_{\alpha}{ }^{H} \theta 1\right)\right.}\right)\right)\right) \\
& +\frac{\partial}{\partial H}\left(-3 m_{j}\left(-2 m_{j} \overline{f_{j}^{(1)} g_{\alpha}}+\overline{f_{j}^{(1)} \frac{\partial}{\partial H}\left(g_{\alpha} H_{\theta T}\right)}\right)\right. \\
& +\overline{\left.f_{j}^{(1)} \frac{\partial}{\partial H}\left(-4 m_{j} g_{\alpha} H_{\theta l}+\frac{\partial}{\partial H}\left(g_{\alpha} H_{\theta \top}^{2}\right)\right)\right)}
\end{aligned}
$$


$\overline{x_{1,2}{ }_{1,2}^{(1)}}=\overline{H_{\theta 1}^{1 / 2} v_{m \theta} \frac{\partial^{2}}{\partial H^{2}}\left(f_{j}^{(1)} H_{\theta 1}^{1 / 2} v_{m \theta} \frac{\partial^{2} g_{\alpha}}{\partial H^{2}}\right)}$

$+H_{\theta 1}^{1 / 2} r v_{m \theta} m_{j} \frac{\partial^{2}}{\partial H^{2}}\left(f_{j}^{(1)} H_{\theta 1}^{1 / 2} \frac{\partial^{2} g_{\alpha}}{\partial H \partial p_{\theta}}\right)$

$+m_{j} H_{\theta 1}^{1 / 2} r \frac{\partial^{2}}{\partial H \partial p_{\theta}}\left(f_{j}^{(1)} H_{\theta 1}^{1 / 2} v_{m \theta} \frac{\partial^{2} g_{\alpha}}{\partial H^{2}}\right)$

$+m_{j}^{2} H_{\theta 1}^{1 / 2} r^{2} \frac{\partial^{2}}{\partial H_{\partial p_{\theta}}}\left(f_{j}^{(1)} H_{\theta 1}^{1 / 2} \frac{\partial^{2} g_{\alpha}}{\partial H_{\partial} p_{\theta}}\right)$

$\overline{X_{1,3} Y(1)}=-m_{j} p_{z}^{2} \frac{\partial}{\partial H}\left(\overline{f_{j}^{(1)} \frac{\partial^{2} g_{\alpha}}{\partial H^{2}}}\right) \cdot m_{j}^{2} p_{z} \frac{\partial}{\partial H}\left(\overline{f(1) \frac{\partial^{2} g_{\alpha}}{\partial H \partial p_{z}}}\right)$

$+m_{j} \frac{\partial^{2}}{\partial H \partial p_{z}}\left(\overline{p_{z} f j} \overline{f_{j}^{(1)} H_{\theta 1} \frac{\partial^{2} g_{\alpha}}{\partial H^{2}}}\right)+m_{j}^{2} \frac{\partial^{2}}{\partial H_{\partial p_{z}}}\left(\overline{f_{j}^{(1)} H_{\theta 1} \frac{\partial^{2} g_{\alpha}}{\partial H_{\partial z}}}\right)$

$+p_{z}^{2} \frac{\partial^{2}}{\partial H^{2}}\left(\overline{f_{j}^{(1)} H_{\theta 1} \frac{\partial^{2} g_{\alpha}}{\partial H^{2}}}\right)+\min _{j} p_{z} \frac{\partial^{2}}{\partial H^{2}}\left(\overline{f_{j}^{(1)} H_{\theta 1} \frac{\partial^{2} g_{\alpha}}{\partial H \partial p_{z}}}\right)$

$-m_{j}^{2} \frac{\partial}{\partial p_{z}}\left(p_{z} \overline{f(1) \frac{\partial^{2} g_{\alpha}}{\partial H^{2}}}\right)-m_{j}^{3} \frac{\partial}{\partial p_{z}}\left(\overline{f_{j}^{(1)} \frac{\partial^{2} g_{\alpha}}{\partial H \partial p_{z}}}\right)$

$X_{2,1} Y_{2,1}^{(T)}=X_{1,2} Y_{1,2}^{(1)}$ 


$$
\begin{aligned}
& \overline{x_{2,2} Y_{2,2}^{(1)}}=\frac{\partial^{2}}{\partial H^{2}}\left(\overline{f_{j}^{(1)} \frac{\partial^{2}}{\partial H^{2}}\left(v_{m \theta}^{4} g_{\alpha}\right)}\right)+m_{j}^{2} \frac{\partial^{2}}{\partial H^{2}}\left(\overline{f_{j}^{(1)} r^{2} v_{m \theta}^{2} \frac{\partial^{2} g_{\alpha}}{\partial p_{\theta}^{2}}}\right) \\
& +m_{j} \frac{\partial^{2}}{\partial H^{2}}\left(-\overline{f_{j}^{(1)} \frac{\partial}{\partial H}\left(r g_{\alpha} v_{m \theta}^{2}\right)}+\overline{f_{j}^{(1)} u_{m \theta}^{2} \frac{\partial^{2}}{\partial H \partial p_{\theta}}\left(r v_{m \theta} g_{\alpha}\right)}\right) \\
& +m_{j} \frac{\partial}{\partial H}\left(\overline{-f_{j}^{(l)} \frac{\partial^{2}}{\partial H^{2}}\left(r v_{m}^{2} g_{\alpha}\right)}+\frac{\partial}{\partial p_{\theta}}\left(\overline{f_{j}^{(l)} \frac{\partial^{2}}{\partial H^{2}}\left(r v_{i n \theta}^{3} g_{\alpha}\right)}\right)\right) \\
& +m_{j}^{2} \frac{\partial}{\partial H}\left(\overline{f_{j}^{(1)} \frac{\partial}{\partial H}\left(r^{2} g_{\alpha}\right)}-\overline{f_{j}^{(1)} \frac{\partial^{2}}{\partial H^{2} \partial p_{\theta}}\left(r^{2} v_{m \theta} g_{\alpha}\right)}\right) \\
& +m_{j}^{2} \frac{\partial^{2}}{\partial H \partial p_{\theta}}\left(\overline{-f_{j}^{(1)} \frac{\partial}{\partial H}\left(r^{2} v_{m \theta} g_{\alpha}\right)}+\overline{f_{j}^{(1)} r v_{m \theta} \frac{\partial^{2}}{\partial H \partial p_{\theta}}\left(r v_{m \theta} g_{\alpha}\right)}\right) \\
& +m_{j}^{3} \frac{\partial}{\partial H}\left(\overline{-f_{j}^{(l)} \frac{\partial^{2}}{\partial p_{\theta}^{2}}\left(r^{3} g_{c}\right)}+\frac{\partial}{\partial p_{\theta}}\left(\overline{f(l)} r^{3} v_{m \theta} \frac{\partial^{2} g_{\alpha}}{\partial p_{\theta}^{2}}\right)\right) \\
& +m_{j}^{2} \frac{\partial^{2}}{\partial p_{\theta}^{2}}\left(\overline{f_{j}^{(1)} r^{2} v_{m \theta}^{2} \frac{\partial^{2} g_{\alpha}}{\partial H^{2}}}\right)+m_{j}^{4} \frac{\partial^{2}}{\partial p_{\theta}^{2}}\left(\overline{f_{j}^{(1)} \frac{\partial^{2}}{\partial p_{\theta}^{2}}\left(r^{4} g_{\alpha}\right)}\right) \\
& +m_{j}^{3} \frac{\partial^{2}}{\partial p_{\theta}^{2}}\left(\overline{f_{j}^{(1)} \frac{\partial}{\partial H}\left(-r^{3} g_{\alpha}+\frac{\partial}{\partial p_{\theta}}\left(r^{3} v_{m \theta} g_{\sigma_{\alpha}}\right)\right)}\right)
\end{aligned}
$$

$\overline{x_{2,3} y_{2,3}^{(1)}}=\left(p_{z} \frac{\partial^{2}}{\partial H^{2}}+m_{j} \frac{\partial^{2}}{\partial H \partial p_{z}}\right)\left(\overline{\left(f_{j}^{(1)} p_{z} \frac{\partial^{2}}{\partial H^{2}}\left(V_{m \theta} g_{\alpha}\right)\right.}+\right.$ 


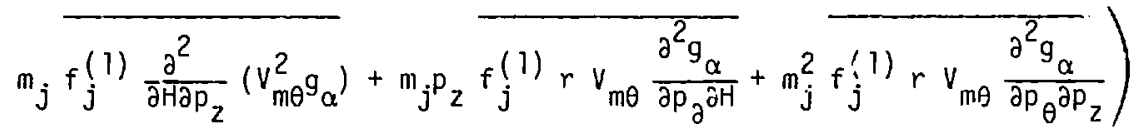

$$
\begin{aligned}
& +\left(m_{j}^{2} \frac{\partial^{2}}{\partial p_{\theta} \partial p_{z}}+m_{j} p_{z} \frac{\partial^{2}}{\partial p_{\theta} \partial H}\right)\left(\overline{p_{z} f_{j}^{(1)} \frac{\partial^{2}}{\partial H^{2}}\left(r v_{m \theta} g_{\alpha}\right)}+\right. \\
& m_{j} f_{j}^{(1)} \frac{\partial^{2}}{\partial H \partial p_{z}}\left(r v_{m e} g_{\alpha}\right) \\
& \left.+m_{j} p_{z} f_{j}^{(1) \frac{\partial^{2}}{\partial p_{\theta} \partial H}\left(r^{2} g_{\alpha}\right)}+m_{j}^{2} \overline{f(1) \frac{\partial^{2}}{\partial p_{\theta} \partial p_{z}}\left(r^{2} g_{\alpha}\right)}\right) \\
& \overline{X_{3,1} Y_{3,1}^{(i)}}=\overline{X_{1,3} Y_{1,3}^{(1)}} \\
& \overline{X_{3,2} Y_{3,2}^{(1)}}=\overline{X_{2,3} Y_{2,3}^{(1)}} \\
& \overline{x_{3,3} Y_{3,3}^{(1)}}=\left(p_{z}^{2} \frac{\partial^{2}}{\partial H^{2}}+m_{j} p_{z} \frac{\partial^{2}}{\partial H \partial p_{z}}+m_{j} \frac{\partial}{\partial p_{z}} p_{z} \frac{\partial}{\partial H}+m_{j}^{2} \frac{\partial^{2}}{\partial p_{z}^{2}}\right) \\
& \left(p_{z}^{2} f_{j}^{(1)} \overline{\frac{\partial^{2} g_{\alpha}}{\partial H^{2}}}+m_{j} p_{z} f \bar{j} \overline{\frac{\partial^{2} g_{\alpha}}{\partial H \frac{\partial p_{z}}{1}}}\right. \\
& +m_{j} f(1) \frac{\partial}{\partial p_{z}}\left(p_{z} \frac{\partial g_{\alpha}}{\partial H}\right)+m_{j}^{2} \overline{\left.f_{j}^{(1)} \frac{\partial^{2} g_{\alpha}}{\partial \mu_{z}^{2}}\right)}
\end{aligned}
$$




\subsubsection{One Dimensional Mrbit Averagcc Kinetic Equation}

The orbit averaged kinetic equation in Eq. (4-129) can be solved given $\mathrm{c}^{(1)}, \overrightarrow{\mathrm{c}}^{(2)}, \mathrm{s}^{(1)}, \mathrm{s}^{(2)}$. The sour.es $\mathrm{s}^{(1)}$ and $\mathrm{s}^{(2)}$ are obtained from physical processes in a particular problem. The constraint equation, Eq. (4-126) which involves the orbit average of $s^{(1)}$ and $C^{(1)}$ may be interpreter in two ways. The $C^{(1)}$ collision operator involves $f_{j}^{(0)}$ and correspondingly the constraint equation may be interpreted as a restriction on the functional dependence of $f_{j}^{(U)}$. This viewpoint considers the zero order distribution to be consirained by the sources. Alternatively an initial $f_{j}^{(U)}$ is chosen and che sources required for $f_{j}^{(0)}$ to exist and for the time evolution to be described by Eq. (4-129) are determined frum $\bar{s}^{(1)}=-\bar{c}^{(1)}$. in this interpretation an assumed $f_{j}^{(0)}$ evolving in accordance with Eq. (4-129) defines sources which are required to make the model consistent.

Assuming sources are known the one dimensional orbit averaged kinetic equation is constructed using the Eq. (4-749), Eq. (4-235), Eq. $(4-236)$ definitions of $H^{(1)}, C^{(1)}$, anc $\bar{C}^{(2)}$ respectively. In the derivation of the orbit avarage and caret of $\Omega_{j}$ and $x_{\mu, \gamma}{ }_{\mu, \gamma}$, $\mathrm{p}_{z}$ was assumed to be a constant of the motion and thus the derived formulas only apply to the one dimensional radial case.

The Poisson bracket reduces to derivatives with respect to $r$ and $P_{r}$. The first order Hamiltonian does not depend on $p_{r}$ and thus the first Poisson bracket on the right side of Eq. (4-129) reduces to the expression below.

$$
\left[\dot{C}^{(1)}, H_{4}(1)\right]=-m_{j}^{-1}\left(\frac{\partial H(1)}{\partial r}\right) H_{\theta 1}^{1 / 2} \frac{\partial \dot{C}^{(1)}}{\partial H}
$$


Similarly the second Poisson bracket is as follows,

$$
\left[\hat{S}^{(1)}, H^{(1)}\right]=-m_{j}^{-1}\left(\frac{\partial H^{(1)}}{\partial r}\right) H_{\theta}^{1 / 2} \frac{\partial \hat{S}^{(1)}}{\partial H}
$$

Using Eq. (4-249) and Eq. (4-250) in Eq. (4-129) and substituting expressions for $\vec{c}^{(2)}, \hat{c}^{(1)}$, and $H^{(1)}$ yields the one dimensional orbit averaged kinetic equation.

$$
\begin{aligned}
& \frac{\partial f(0)}{\partial t}=\sum_{\alpha} \Gamma_{j}\left(\sum_{i=1}^{3} \overline{\Omega_{i}^{(I)}}+\frac{1}{2} \overline{X_{\mu, \gamma}{ }_{\mu, \gamma}^{(T)}}\right)+\vec{s}^{(2)} \\
& +m_{j}^{-2} e z_{j} H_{\theta 1}^{1 / 2} \frac{\partial}{\partial r}\left(r^{-2} c^{-1}\left(-p_{\theta} \psi^{(1)}+e z_{j} c^{-1} \psi^{(0)} \psi^{(1)}\right)+\phi^{(1)}\right) \frac{\partial \hat{S}^{(1)}}{\partial H} \\
& +\sum_{\alpha} r_{j}\left(m_{j}^{-2} e z_{j} \overline{H_{\theta l}^{1 / 2} \frac{\partial}{\partial r}\left(r^{-2} c^{-1}\left(-p_{\theta} \psi^{(1)}+e z_{j} c^{-1} \psi^{(0)} \psi^{(1)}\right)+\phi(1)\right.}\right) \\
& \overline{\sum_{i=1}^{3} \frac{\partial \hat{\Omega}_{i}^{(0)}}{\partial H}}
\end{aligned}
$$

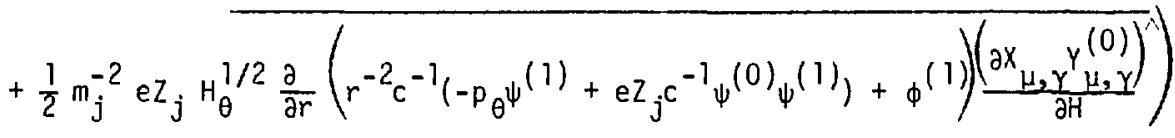$$
\text { (4-251) }
$$

In Eq. $(4-251)$ the $\psi^{(0)}, \psi^{(1)}$, and $\phi^{(1)}$ potentials are obtained from the electron model equations in Section 4.3.3. The $\left(\Omega_{j}^{(0)}\right)^{\wedge}, \bar{\Omega}_{j}^{(1)}$, $\left(x_{\mu, \gamma} \gamma_{\mu, \gamma}^{(0)}\right)$, and $\overline{\left(x_{\mu, \gamma} \gamma_{\mu, \gamma}^{(1)}\right)}$ functions have been defined in section 4.3.4. 
The field reversed mirror scenario discussed earlier consisted of sustaining an already field reversed plasma with neutral beams. In this case the neutral beam injection is considered to be the source which causes a large Larmor radius ion population. These ions are modeled by the orbit averaged kinetic equation in Eq. (4-251). The large Larmor radius ions could also be considereu to be fusion products such as alpha particles. The cooler ions are modeled by the classical Braginskii equations discussed in Chapter 2. A small population of large Larmor radius ions is then considered to be immersed in a background of cool ions described by a maxwellian. The orbit averaged kinetic equation gives the cool ion heating rate caused by the large Larmor radius ions. The plasma evolution can then be calculated on a slowing down time rather than on a cyclotron period time which is characteristic of standard particle codes. The Eq. $(4-125)$ constraint is satisfied since $c^{(0)}=0$ and there is no zero order $s^{(0)}$ source required. If the majority of ions are non-maxwellian $S^{(0)}$ is necessary because $c^{(0)}$ is not zero. In the case under discussion the large Larmor radius ion source enters the formulation at first order as $S^{(1)}$ and represents a replacement of diffusion losses. To include the large Larmor radius ions in the cool ion transport eciuations the $H, p_{\theta}$ coordinates in Eq. (4-251) must be viewed as an $r, v_{r}, v_{\theta}, v_{z}$ dependence using the definition of the Hamiltonian, $p_{r}, p_{\theta}$, and $p_{z}$ in terms of $v_{r}, v_{\theta}$, and $v_{z}$. It is then possible to take moments following the standard procedure used by Braginskii to derive the cool ion transport equations. The flux surface average of transport equations derived in this fashion 
from Eq. (4-251) provides terms accounting for large Larmor radius effects on the right side of the continuity, momentum and energy transport equations.

The moment or integral over velocity of the one dimensional orbit averaged kinetic equation, Eq. (4-251) is below.

$$
\frac{\partial M_{\ell}}{\partial t}=N_{\ell}+\vec{v}_{\psi} \cdot \nabla M_{\ell}
$$

The partial derivative with respect to $t$ is at constant $\psi$ in Eq. (4-252) rather than constant position as in $\mathrm{Eq} \cdot(4-251)$. The $\mathrm{M}_{\ell}$, $\mathrm{N}_{\ell}$ functions have the following derinitions.

$$
\begin{aligned}
& M_{\ell}=\int f_{j}^{(0)}\left(\vec{v}-\overrightarrow{v_{i n}}\right)^{\ell l} d v_{r} d v_{\theta} d v_{z} \\
& v_{\ell}=\int \frac{\partial f_{j}^{(0)}}{\partial t}\left(\vec{v}-\vec{v}_{\mathrm{In}}\right)^{\ell} d v_{r} d v_{\theta} d v_{z}
\end{aligned}
$$

In Eq. (4-253) and Eq. (4-254) \& is zero for the continuity equation and two for the energy equation. The velocity moment is taken with respect to the random velocity $\vec{v}-\vec{v}_{m}$, where $\vec{v}_{m}$ is the average velocity. The $M_{\ell}$ moment is density or pressure and $N_{\ell}$ is the moment of the right side of Eq. (4-251). The general time rate of change of a flux surface average quantity $G_{a}$ is below, ${ }^{[83]}$

$$
\frac{d}{d t}\left(\left\langle G_{a}\right\rangle \frac{d V}{d \psi}\right)=\left\langle\frac{\partial G}{\partial t}\right\rangle \frac{d V}{d \psi}+\frac{\partial}{\partial \psi}\left\langle G_{a} \vec{v}_{\psi} \cdot \nabla V\right\rangle
$$

where $\vec{v}_{\psi}$ is the velocity of a flux surface. Substituting Eq. (4-252) into Eq. (4-255) gives the large Larmor radius correction to the Chapter 2 flux surface averaged transport equations. 


$$
\frac{d}{d t}\left(\left\langle M_{\ell}\right\rangle \frac{d V}{d \psi}\right)=\left\langle N_{\ell}+\vec{v}_{\psi} \cdot \nabla M_{\ell}\right\rangle \frac{d V}{d \psi}+\frac{\partial}{\partial \psi}\left\langle M_{\ell} V_{\psi} \cdot \nabla V\right\rangle
$$

The flux surface average in Eq. $(4-256)$ has the same definition as in Chapter 2; however, because density and other functions are one dimensional the flux surface average reduces to the expression below.

$$
\left\langle G_{a}\right\rangle=\left(G_{a}\left(r_{1}\right) B_{z}^{-1}\left(r_{1}\right)+G_{a}\left(r_{2}\right) B_{z}^{-1}\left(r_{2}\right)\right)\left(B_{z}^{-1}\left(r_{1}\right)+B_{z}^{-1}\left(r_{2}\right)\right)
$$

In Eq. (4-257) $\psi$ is assumed to be only double valued in $r$ and $\psi\left(r_{1}\right)=\psi\left(r_{2}\right)$, where $\left\langle G_{a}\right\rangle$ is evaluted at $\psi$. 
CHAPTER 5

SUMMARY AND CONCLUSIONS

\subsection{Summary}

Two plasma models relevant to compact torus research have been developed to study transport and equilibrium in field reversed mirrors. In the first model for small Larmor radius and large collision frequency, the plasma is described as an adiabatic hydromagnetic fluid. In the second model for $1 ;$ ge Larmor radius and small collision frequency, a kinetic theory description has been developed. Various aspects of the two models have been studied in five computer codes ADB, AV, NEO, OHK, RES. The ADB code computes two dimensional equilibrium and one dimensional transport in a flux coordinate. The AV code calculates orbit average integrals in a harmonic oscillator potential. The NEO code follows particle trajectories in a Hill's vortex magnetic field to study stochasticity, invariants of the motion, and orbit average 
formulas. The OHK code displays analytic $\psi(r), B_{z}(r), \phi(r), E_{r}(r)$ formulas developed for the kinetic theory description. The RES code calculates resonance curves to consider overlap regions relevant to stochastic orbit behavior.

In the first model both poloidal and toroidal magnetic fields are allowed and the plasma evolves quasi-statically between equilibrium solutions as a result of transport processes or adiabatic external current changes. The equilibrium plasma solution is described by the magnetic field structure and the specification of two profiles related to pressure and toroidal magnetic field. When the plasma evolves due to adiabatic external current changes the two profiles are held constant and the plasma evolution occurs as a result of modifications to the vacuum magnetic field. When the plasma is subject to transport processes changes result due to alterations of the pressure related profile.

The adiabatic fluid model is deduced from the full set of magnetohydrodynamic equations by neglecting dissipation. The resulting force balance equation and Anpere's law without displacement current yield the Grad-Shafranov equation which determines the two dimensional equilibrium. Because the Eq. (2-18) $\Delta^{*}$ operator is elliptic a boundary condition in addition to the $J_{T}$, right side of the equilibrium equation, specifies an equilibrium solution. The adiabatic assumption fixes a flux value at the magnetic axis, which in general can not be guaranteed by the two dimensional equilibrium solution. Consequently, it is also necessary to solve the flux surface averaged Grad-Shafranov equation. 
The equilibration time in a flux surface is much shorter tr.an between Ix surfaces. Consequently, the braginskij classical transpu.t equations are flux surface averaged. The transport is then one dimensional with coordinate $\psi$. The transport prncesses considered are conduction, radiation, electron ion energy exchanne, coulomb friction, and neutral beam deposition. The neutral beam deposition is modeled in three dimensions and then mapped to the $\psi$ transport coordinate by a geometric volume ratio and a flux surface эverage.

The computation of transport and equilibrium begins from a given initial condition. The initial condition is determined by specifying $\psi(r, z)$ and the $S(\psi), q(\psi)$ profiles. These functions are detarmined in the ADB code by four models. The first model is the Weitzner field which assumes $p(\psi), f_{T}(\psi)$ are proporticnal to $\psi$. The second model is the spherical Hill's vortex which assumes $p(\psi)$ is proportional to $\psi$ and $f_{T}(\psi)=0$. The third model is the elliptical Hill's vortex. This model is similar to spherical Hill's vortex except the separatrix is oblate or prolate. For this model exterior vacuum region solutions were derived. The fourth model represents the $S(\psi)$ and $q(\psi)$ profiles as variable coefficient functions.

Many neutral bean deposition simulation and adiabatic compression cases have been run with the $A D B$ code. The focus of these investigations has been in the areas of radial versus axial plasma shape change caused by neutral beam injection, plasma shaping by adiabatic compression, the effect of varying neutral beam energies 
and currents, and the energy balance between neutral beam heating and radiation energy loss.

In the second field reversed mirror model, the magnet ic field is restricted to a poloidal component. The large Larmor radius effects are incorporated in the plasma description by considering the electrons as an inertialess fluid and modeling the ions with a distribution function. This model only allows a radial spatial variation and is thus one dimensional.

The electrons are governed by fluid continuity and momentum equations and quasineutrality is assumed. This electron description with Ampere's law without displacement current has been solved analytically to obtain an initial condition for a diffusion problem. The analytic model provides self consistent electric and magnetic fields based on an idealized ion distribution function.

A more realistic ion distribution function satisfies a kinetic equation which accounts for large Larmor radius effects. The ion distribution function and Hamiltonian are expanded in terms of collision frequency and the kinetic equation then yields a hierarchy of equations. At second order a kinetic equation for the zero order ion distribution $i$. - on is obtained. This equation is the large Larmor radius analog of the 711 Larmor radius drift kinetic equation of standard neoclassical theory. At first order a constraint equation determines the first order ion distribution function in terms of the first order source, collision operator and Hamiltorian.

The expression for the first order Hamiltonian is obtained by substituting collision frequency expansions of $\psi$ and $\phi$ into the 
definitinn of the Hamiltonian. The potentials which occur in the first order Hamiltonian are obtaines: from the perturbation expansion of the electron fluid equations. The ion quantities required for the perturbed electron fluid equations are obtained from velocity moments of the zero and first order ion distribution function.

The orbit averaged kinetic equation involves the collision operator acting on the zero and first order distribucion function. In this work, collisions are represented by the Fokkfr-Planck operator. Since the orbit averaged kinetic equation is in $H$, $\mathrm{p}_{0}, \mathrm{p}_{\mathrm{z}}$ coordinates the standard Fokker-Planck operator has also been converted to $H, \mathrm{P}_{\theta}, \mathrm{P}_{z}$. Orbit average or caret operatur expressions have been derived for required collision operator terms.

The neoclassical drift kinetic equation is derived by integrating appropriate equations over a gyro-orbit. The orbit averaged kinetic equation requires integration over the complete orbit. The orbit average operation provides a procedure by which integration over an orbit is performed without a detailed knowledge of the orbit. The orbit average operation is the method used to derive the orbit averaged kinetic equation. The orbit average has been derived in terms of phase space integrals and path integrals.

The orbit averaged kinetic equation is derived up to a point assuming $f^{(0)}$ is a function of the constants of the motion, axisymmetry, and one dimensional or two dimensional with only two constants of the motion. Further progress has been made by specializing to one dimension. The benefit of the one dimensional assumption is that $p_{z}$ is then a constant of the motion. As a 
direct result the orbit average formula is simpler and also some complexity is removed from the collision operator expressions.

\subsection{Conclusions}

The field reversed mirror has been modeled in the large collision frequency, small Lärmor radius limit using a reduced set of magnetohydrodynamic equations. The adiabatic assumption is appropriate for time evolution of a plasma in parameter regions of interest. The central issues investigated were adiabatic compression and neutral beam heating.

In this model equations are solved in $r, z$ to obtain $\psi(r, z)$ and in a volume coordinate to obtain $\psi(V)$. The Grad methodology of iterating between the flux surface averaged Grad-Shafranov equation and the two dimensional Grad-Shafranov equation was found to be successful except in one case. When large currents deve1, n near the separatrix a numerical instability can be driven if the boundary condition is updated during the iteration. This difficulty can occur during strong auiabatic compression. The numerical instability is avoided in the case of a conductor around the plasma where the boundary condition is fixed at $\psi_{\text {plasma }}=0$.

The implementation of the ICCG method to solve the two dimensional Grad-Shafranov equation was prompted by the desire to allow the flexibility of a variable mesh spacing. The initial motivation was twofold. First it was desirable to cluster grid cells near the magnetic axis to improve the accuracy of the chord $\cdots: t i n d$ flux surface average. Second distant homogeneous holindary 
conditions were an alternative means of dealing with a plasma having no surrounding conductor. The Green's function method of specifying boundary conditions proved to be a viable alternative to using variable grid spacins as a means of applying the known $\psi_{\text {plasma }}=0$ at infinity boundary condition. To economically implement the Green's function technique it is necessary to only update boundary grid points in the event a monitored boundary point undergoes a significant change of value.

The chord method of computing the flux surface average near the magnetic axis ultimately proved unsatisfactory even with increased grid resolution using a variable mesh. This difficulty was risolved by following constant $\psi$ contours by solving the governing ordinary differential equations using an error controlled multistep method. This method necessitated the cubic spline fitting of the $\psi(r, z)$ function near the magnetic axis.

In addition to the equilibrilum solution, the large collision frequency, small Larmor radius model also requires numerical solution of transport equations. The transport equations have coefficients which contain flux surface average functions which are deterinined by solution of the two dimensional Grad-Shafranov equation. Consequently, no gain was realized by treating terms implicitly and thus a straightforward explicit time difference scheme has been used.

The equilibrium transport solution begins from a given initial condition. The most commonly used initial anditions in this work are the Hill's vortex or variable coefficient profile model. The 
Weitzner magnetic field is useful since it incluues poloidal and toroidal magnetic fields in contrast $10 \mathrm{Hill's}$ vortex which only has poloidal field. Unfortunately, it is restricted since in the Weitzner model it is not possible to arbitrarily vary pressure and toroidal field and also maintain a flux function that is at most double valued. The greatest flexibility for tailoring the initial condition is realized with the variable coefficient model in terms of shaping profiles. When it is crucial to have a specific shape such as oblate or prolate the Hill's vortex may be used.

Two ADB code results have been presented. The first casc considers neutral beam deposition with an enforced lectron onergy decay rate and radiation loss. The second case simulates adiabatic compression of a prolate plasma.

The first case addressed the issue of whether or not a realicable neutral beam current and energy could heat a plasma with radiation and supplemental electron energy loss. - $t$ is clear from this example that a density which allows the neutral beam to penetrate into the plasma interior is important in the eventual time evolution. The character of the beam poiletration is evidenced in the flux dependence of the $S(\psi)$ profile. Another factor which is infiuential is that the neutrdl beam cnergy input is proportional to the difference between the plasma energy and beam energy. This is a consequence of charge exchange. As the plasma heats, the neutral beam becones less effective at addin. udditional energy. The radiation loss was attributed to oxygen which has a power loss as a function of electron temperature which has two peaks. The main 
conclusion to be drawn from this example is that beginning from an initial state with temperature chosen to cause large radiation loss the neutral beam is able to dominate and the plasma heats. It should be noted that in this example a conoucting bourdary condition vias used and consrquently the amount of possible plasma expansion was limited. This fact contributes to the large amount of neutral beam ahsorption which at $2 n$, ent of $2.8 \mathrm{msec}$ was $98.7 \%$.

The second ADB code result investigated a particular plasma shaping consideration. The proximitv of conducting walls influonces plasina stability. It was found bj other workers plasma stalility is enhanced with distant walls by causing an snuatorial bulge in a prolate plasma. The $A D B$ code sucressfully qenerated the appropriate thape after a judicious chcice of currents in six external coils. The initial condition used in this result was a prolate Hill's vortex. The final shape is must expediently obtained beginning with a prolate qeometry; however, a spherical qeometry may be used as we 11. To change a spherical geometry to prolate geonetry coils must be added to generate a cusp field. The final currents that were used in conjunction with the 'rolate Hill's vortex to sbtain the desired shape were positive and negative. This means the plasma was compressed at some axiai positions and expanded at others. The overall eifect seems to be a near constancy of bulk properties. It is reasonable to conclude the desired final state was more of a rearrangement than an alterstion of the plasma. The toroidal current went from 32,400 amps to 31,500 amps. Also the kinetic energy only increased by $8 \%$. 
The second basic model of the field reversed mirror involves 1 arge Larmor radius and small collision frequency. Since the ions in this model are described by a distribution function the principle problem is to derive a kinetic equation for the ion distribution function which incorporates large Larmor radius effects. Fundamental to deriving the kinetic equation is the issue of generating a procedure that is equivalent to following orbits but which does not require a detailed knowledge of trajectories. The procedure that has been derived to satisfy this need is the orbit average. Beginning from the definition of the orbit average, which actually amounts to integrating along a known trajectury, equivalent phase space and path integral formulas have been derived. In one dimension the path integral and phase space formula are the same. Also the caret operator which is a partial integral along an orbit has been derived. Consequently, equations for the one dimensional case have been written down for the orbit averaged kinetic equation. Also analytic solutions have been derived for an initial condition using an idealized ion distribution function. For the scenario of a large Larmor radius population of hot ions in a cooler background the means by which the usual transport equations are modified has been indicated.

In two dimensions the orbit averaged kinetic equation is more difficult to derive for two reasons. First no equivalent form of the caret operator has been derived in which a detailed knowledge of a trajectory is not required. Second the existence of a third constant of the motion changes the form of the orbit average. The 
advantage of the one dimensional treatment is that $p_{z}$ is known to be the third constant of the motion. In two dimensions only the governing equation and constraint equation are known for various classes of constants of the motion. Thus it is not known for a general potential, especially when it is only defined numerically, whether or not a third constant of the motion even exists. The existence of a third invariant alters the Wds formula in $5 q .(2-14)$ by adding another delta function in the third invariant variable and also changes the coordinate transformation Jacobian. The effect in the phase space formulas is to change the integration weighting function. Depending on the form of the invariant the accessible orbit average volume may also be changed. Since this change affects transport a third invariant which is fourth order in the velocity has been constructed for the Hill's vortex in the low transverse energy limit. The functional form of this invariant determines the orbit average formula near the ortit potential well minimum.

In the case of the AV harmonic oscillator code the third invariant is known. The radial and axial energies are conserved. The resulting orbit average formula is given by Eq. (3-64). It was shown that the conditions for a comparison of a single orbit and this formula are an axial to radial oscillation frequency ratio of six. With this restriction excellent agreement is obtained. It has been shown the integral over $H_{r}$ values yields the orbit average formula which is derived assuming only $H$ and $p_{\theta}$ are constants of the motion. The conclusion is that if a third constant of the motion exists and the particle distribution is a function of the third 
constant of the motion the orbit average accessible volume and change of variables jucobian should take this into account. If however the third constant $0^{-}$the motion does not exist or the distribution function does not depend on $i t$, the orbit average is the same as the formula with only $H$ and $p_{\theta}$ as constants.

Implementing the orbit average which assumes only $H$ and $p_{\theta}$ are constant gave good agreenent with a stochastic orbit generated using the NEn code. In the stachastic orbit regime there is no third constant of the motion and the orbit average is then known. It is thus important to find paraneter ranges where stochastic motion occurs. In this work stochasticity has been examined by expressing the Hamiltonian in transformed canonical variables which separate into a function of momenta and a function of momenta and coordinates. Ideally the function of momenta and coordinates is a perturbation and resonance overlap in momenta space indicates stochasticity. The check of predicted stochastic orbits in the NEO code showed for the selected canonical coordinates the resonance overlap theory can only be applied at low energy. For small change of $p_{\theta}$ near $p_{\theta}=-.2$ an irregular surface of section results; however, the total lack of surface of section structure characteristic of stochasticity has not been observed.

\subsection{Future Work}

The equilibrium solver which forms the basis of the $A D B$ code has been merged with a more elaborate transport model in the Shumaker FRT code. Improvements to better simulate field reversed mirrors 
assuming small Larmor radius should thus be addressed to upgrading the FRT code. It would be useful to implement an additional initial condition which allows a selection of geometry and flexibility in the determination of the $S(\psi)$ and $q(\psi)$ profiles. Originally the ICCG method was implemented to allow the use of a variable mesh. In computer calculations in which the mesh is uniform, it would be desirable to have an option to solve the two dimensional Grad-Shafranov equation using cyclic reduction. The advantage in using cyclic reduction is that the iteration necessary in the ICCG mechod is avoided.

The fundamental requirement necessary to calculate a two dimensional orbit averaged kinetic equation is a practical definition of the caret operator in two dimensions. The precise type of averaging of orbits leading to a given $r, z$ point which yields the caret operator of a quantity at that point is not understood at this time. The Poisson bracket eigenfunction expansion may lead to a resolution of this problem.

The orbit average procedure is well defined in one dimension. In two dimensions a formula exists for constant $H$ and $p_{\theta}$. The specialization required in the event of the occurrence of a third invariant dependent distribution function has been discussed. The search for third invariants of various orders appears to be a fruitful area of research. The ultimate goal is to understand which potentials may be beneficial to transport considerations. The asymptotic polynomial approximation procedure may be extended to higher order or other techniques may be employed to solve the relevant equations. A least squares fit or application of the Rayleigh-Ritz principle may generate useful approximate invariants. 
The numerical implementation of various orbit averages in this work has relied largely on bilinear fits. Improved accuracy could be realized with bicubic b-splines or more elaborate quadrature methods. The issue of integrating near the boundary of the accessible orbit region has been handled by a chord approximation to the curved boundary. Increased accuracy at the cost of added complexity is possible by integrating along low order curve fits to the actual boundary.

The question of how to delineate parameter regimes where stochasticity occurs is unanswered globally. In a case where a third invariant does exist there is a possibility it may break down for some parameter ranges. It is then desirable to determine the phase space boundary between regions where the third invariant determined orbit average is applied and the stochastic orbit average is applied.

The one jimensional orbit averaged kinetic equation has been derived and terms have been formulated in $H, p_{\theta}, p_{z}$ space for the Fokker-Planck collision operator. The next step is to describe the sources for a particular problem and numerically implement the electron fluid equation and the orbit averaged kinetic equation. Reasonable initial conditions can be obtained from the analytic model to begin the one dimensional calculation. A more realistic choice of distribution function and electron radial velocity would be advantareous. Using the sane distribution function the electron radial velocity may be modeled as linear in radius and error function expressions may be derived for the initial $\psi$ and $\phi$. Beyond this approximation a full numerical treatment is required. 
ACKNOWLEDGEMENTS

Gratitude is expressed to Professor John Killeen for serving as thesis committee chairman and academic adviser. Also for useful ongoing project review and for teaching courses at the Department of Applied Science.

Brendan McNamara is thanked for providing overall project supervision and oversight. Following attendance at the author's first conference in New York he encouraged graduate schnol attendance which has culminated in this manuscript. Between trips to England, Italy, South America and other places which shall go nameless he has aided and directed this project with useful suggestions and often lively discussions. His influence on this work can only be realised by a careful reading.

Appreciation is extended to Harry Dwyer for his part on the thesis committee and also for participating as a member of the oral examination panel. As a result the Nusselt number will always be remembered as the ratio of thermal transfer to thermal conduction. Guidance given in the area of transport by Dan Shumaker is appreciated. Explanation concerning invariants of the motion by L. S. Hall is gratefully acknowledged. Clarification of fine points in the use of ordinary differential equation solution software by Alan Hindmarsh has been indispensable. The outcome of conversations with Harold Grad and Don Stevens concerning two dimensional equilibrium solution is evidenced in this work. 
Help with computer software by P. A. Willmann and useful discussions with D. V. Anderson, S. P. Auerbach, H. L. Berk, Yu-Jiuan Chen, B. I. Cohen, R. H. Cohen, R. P. Freis, J. M. Gilmore, J. H. Hammer, C. W. Hartman, T. B. Kaiser, L. L. LoDestro, Y. Matsuda, M. G. McCoy, A. A. Mirin, W. A. Newcomb, R. F. Post, D. S. Prono, M. E. Rensink, T. D. Rognlien, A. I. Shestakov, G. R. Smith, J. J. Stewart, and W. C. Turner are appreciaced.

The aid provided by the M-Division staff, T. K. Fowler, M. A. Harrison, 0. G. Jones, J. E. Bailey, B. L. Sanger, F. L. Faria; the computer staff, N. Maron, T. A. Brengle, D. W. Smith, E. C. Patterman, D. L. Jones, and the National Magnetic Fusion Energy Computer Center is acknowledged. The effect of conversations with B. C. Curtis and L. G. Berdahl can not be overstated. Recognition is given to the University of California at Davis Graduate Division and the Department of Applied Science Chairman Professor F. Wooten and staff, Kathryn Smith, Donna Clifford, Donna Parley and previous staff, Jane Ellis, Nancy Barnes, and Leah Hanson for attention to prolific but necessary details.

The professors of the Department of Applied Science, A. Goldberg, W. Hoover, J. DeGroot, K. Marx, S. Bloom, W. Talley, J. Shaner, and those at U.C. Berkeiey, A. Lichtenberg, C. Birdsall, M. Lieberman, J. Whinnery, S. Silver, P. Pagni, A. Kaufman, H. Cordes, W. Kunke1, 0. Chamberlain, B. Noyer, S. Davis, and many others are thanked for providing the instruction required for this work.

The support of the Lawrence Livermore National Laboratory, the Art Division, the Technical Information Division, and the Magnetic Fusion Librarian C. J. Crone have been invaluable. 
This manuscript has been typed by Jan Wikkerink who steadfastly carried this work forward even during times when the facilities could only be entered by thwarting demenstrators. Sincere gratitude for perseverance beyond the call of duty.

To assist those that were unable to understand the reason for granuate work and in acknowledgement of a need to assuage a quote by Isaac Newton is included. "I do not know what I may appear to the world; but to myse if I seem to have been only like a boy, playing on the sea-shore, and diverting myseif, in now and then finding a smoother pebble or a prettier shell than ordinary, while the great ocean of truth lay all undiscovered before me."

To all those who have read this far and not found their name specifically mentioned, this opportunity is taken to thank everyone who has contributed. A deep sincere heartfelt thank yuu to those who provided the source of strength which made this project possible.

I) I INIR

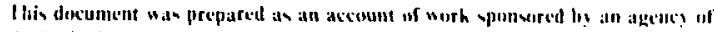

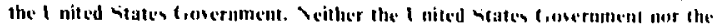

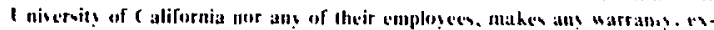

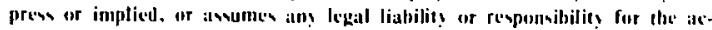
curasy. cunpletelles, or usefulness of ang information, apparatus, product, or prosen diactemed, or cepresent that its use nould not infringe privately anned rights. Reference herein (1) all! specific commercial products, process, of errics

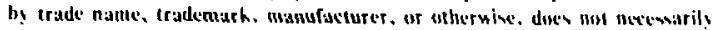
constitute or imply it coldorsement. recommendation, or favoring by the I nited

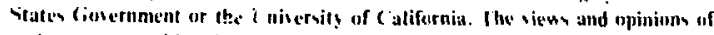
authots esprested herein do not necestarily state ar reflece these af the I nited retess cionemment thereof. and wall nut be aned for adsertiving or product entdorsement purperes.
\end{abstract}




\section{REFERENCES}

[1] S. H. Schurr, EPRI Journal (May 1978).

[2] U.S. Bureau of the Cellsus, "Statistical Abstract of the IJnited States 1976" (Washington, D.C., GP0, 1976).

[3] Uirited Nations, "World Energy Supplies," 1950-1974 Series J, No. 19 (New York, UN, 1976).

[4] E. Teller, Energy, Report to the energy Panel of the Commission on Critical Choices for Anericans, New York 1975.

[5] H.J. Killian, G. L. Dugger, J. Grey, eds. Solar Energy for Earth, an AIAA Assessinent, New York, American Institute of Aeronautics and Astronautics, 1975.

[6] W. Ramsay, "Electricity from New Technologies, "National Energy Strategies Project, Washington, D.C., Resources for the Future, June 1978.

[7] P.E. Glasser, "Solar Power from Satellites," Physics Today, Vol. 30, No. 2, Feb. 1977.

[8] B. Sorenson, "Dependability of Wind Fnergy Generators with Short Term Energy Storage," Science, Vol. 194, No. 4268, Nov. 1976, and "Wind Energy," Bulletin of the Atomic Scientists, Vol. 32, No. 7, September 1976.

[9] L.L. Anderson, "Energy Potential from Organic Waste: A Review of the Quantities and Sources", U.S. Bureau of Mines Information Circular 8549, Washington, D.C., GPO, 1972. 
[10] D.F. White, D.L. Williams, eds, "Assessment of Geothermal Resources of the United States-1975", U.S. Geological Survey Circular 726, washington, D.C., GPO 1975.

[11] E. Barbier, M. Fanelli, "Overview of Geothermal Exploration and Development in the World", Pisa, National Research Council, 1973.

[12] U.S. Army Corps of Engineers, Institute for Water Resources, "Estimate of National Hydroelectric Power Potential at Existing Dams," Alexandria, Virginia, July 1977.

[13] S.H. Schurr, Energy in America's Future, John Hopk ins University Press, Baltimore, 1980, p. 71.

[14] P. Averitt, "Coal Resources of the United States", January 7, 1974, USGS Bulletin 1412, Washington, D.C., GPO (1974).

[15] B. Miller, "Geological Estimates of Undiscovered Recoverable 0 il and Gas Resources in the United States", IISGS., Circular 725, Reston, Virginia, USGS 1975.

[16] B. Miller, $0 i 1$ and Gas Journal, Vol. 76, No. 16, April 1978, p. 53 .

[17] D.C. Duncan, "Organic-Rich Shale of the United States and World Land Areas", U.S. Geological Survey, Circular 523, Washington, D.C., GPO, 1965.

[18] L.B. Lave, "Air Pollution and Human Health," Science, Vol. 169, August 1970.

[19] R.G. Mills, Ed., "A Fusion Plant", MATT-1050, Princeton, NJ., Plasma Physics Laboratory, Princeton University, August 1974, p. 20 . 
[20] D.J. Rose, M.C.Clark, Plasmas and Controlled Fusion, John Wiley and Sons, NY, 1961.

[21] R.F. Post, Revs. Modern Phys., 28, 338 (1956).

[22] J.D. Lawson, Proc. Roy. Soc., London B70, 6 (1957).

[23] Energy and Technology Review, UCRL 52000-77-8, August 1977.

[24] M.O. Fiske and W.W. Havens, Jr., eds., Physics and the Energy Problem, American Institute of Physics, 1974, p. 260.

[25] Energy and : achnology Review, UICRL 52000-81-12, necember 1980, p. 1 .

[26] T.G. Northrup, The Adiabatic Motion of Charged Particlus, Interscience, New York.

[27] R.F. Post, Proc. Seconı U.N. Int'l. Conf. on Peacefu? Uses of Atomic Energy, Geneva, 1958, Vol. 1, p. 154.

[28] R.H. Cohen, G. Rowlands, J.H. Foore, "Nonadiabaticity in Mirror Machines," Lawrence Livermore National Laboratory Report, UCRL-78889 (1976).

[29] M.N. Rosenbluth, C. Longmire, Ann. Phys., New York, 1, 120 (1957).

[30] Y.B. Gott, M.C. Ioffe, V.G. Telkovsky, Proc. Conf. on Plasma Physics and Controlled Nuclear Fusion Research, Saizburg, Part 3, p. 1045 (1951)

[31] 3.8. Taylor, Phys. Fluids 7, 767 (1964).

[32] K.H. Berkner, et. al., Proc. Fourth Conf. Plasma Phys. and Cont. Nucl. Fus. Res., Vol. 2, p. 707, IAEA, Vienna, 1971. 
[33] F.H. Coensgen, et. al., Proc. Sixth Conf. Plasma Phys. and Cont. Nuc. Fus. Res., Vol. 3, p. 135, IREA, Viennic, 1976.

[34] M.N. Rosenbluth, R.F. Post, Phys. Fluids $\underline{8}, 547$ (1965).

[35] H.L. Berk, L.D. Pearlstein, J.D. Callen, C.W. Horton, M.N. Rosenbluth, Phys. Rev. Lett. 22, 876 (1969).

[36] F.H. Coensgen, W.F. Cummins, B.G. Logan, A.W. Molvik, W.E. Nexsen, T.C. Simonen, B.W. Stallard, W.C. Turner, Phys. Rev. Lett., 35, 1501 (1975).

[37] M.J. Gerver, Phys. Fluids 19, 1581 (1976).

[38] W.C. Turner, J.F. Clauser, F.H. Coensgen, D.L. Correll, W.F. Cummins, R.P. Freis, R.K. Goodman, A.L. Hunt, T.E. Kaiser, G.M. Melin, W.E. Nexsen, T.C. Simonen, B.W. Stallard, Nuclear Fusion, 19, 10111 (1979).

[39] D.E. 'aldwir, Rev. Mod. Phys., Vol. 49, No. 2, 317 (1977).

[40] R.H. Cohen, M.E. Rensink, T.A. Cutler, A.A. Mirin, Nuc. Fusic 1 18, 1229 (1978).

[41] J.Killeen, A.A. Mirin, M.E. Rensink, Methods in Computational Physics, Vol. 16 (1976).

[42] T.K. Fowler, B.G. Logan, Comments Plasma Physics 2, 167 (1977).

[43] G.i. Dimov, V.V. Zakaidakov, M.E. Kishinevskii, Fizika Plasma 2, $597(1976)$.

[44] D.E. Baldwin, B.G. Logan, Phys. Rev. Lett. 43, $13: 8$ (1979).

[45] B.G. Logan, A.A. Mirin, M.E. Rensink, T.K. Fowler, Lawren Livermore National Laboratory Report, UCRL 80552 (1978). 
[46] F.H. Coensgen, T.C. Simonen, A.K. Charqin, B.G. Logan, Lawrence Livermore National Laboratory, Proposal 172, April (1980).

[47] H.P. Furth, Nucl. Fusion 15, 48 ? (1975).

[48] J.L. Johnson, C.R. Oberman, R.M. Kulsrud, F.A. Frieman, A.E.C. Report No. NY0-7904 (PM-S-34, 1958).

[49] "Summary Report on Tokamak Conf inement Experiments, " U.S. Dept. of Fnerq.y Report DOE/ER-0122, March (19:32).

[50] „.L. Dimock, Nuc. Fusion, i3, $271(1973)$.

[51] R.F. Post, Proc. of International School of Plasina Physic: Varenna, Italy (1979).

[52] N.C. Christofilos, Proc. Secona United Nations Intern. Conf. Peaceful Uses of Atomic Energy, Geneva, 32, 279 (1958).

[53] N.C. Christofilos, Proc. of the Nuc. Fusion Reactors Conf. Culham, England, 173 (1969).

[54] D.V. Anderson, G.A. Benford, M. Brettschneider, J. Killeen, W.A. Newcomb, A.A. Mirin, and M.E. Rensink, Proc. Fourth Conf. Plasma Phys. and Cont. Nuc. Fus. Res., Vol 1, p. 137, IAEA, Vienna, 1971.

[55] N.C. Christofilos, et. al., Pror Fourth Conf. Plasma Phys. and Cont. Nuc. Fus. Res., Vol. 1, p. 119, IAEA, Vienna, 1971.

[56] S.C. Luckhardt, H.H. Fleischmann, Phys. Rev. Lett., 39, 743 (1977).

[57] D.J. Rej, H.A. Davis, H.H. Fleischmann, Bullet in of the AM. Physical Soc., 22, No. 9, 1069 (1977).

[58] M. Tuszewski, D.J. Rej, H.H. Fleischmann, Phys. Rev. Lett. 43, 449 (1979). 
[59] J.B. Greeniy, F.L. Dreike, D.A. Hammer, P.M. Lyster, Y. Nakagawa, R.N. Sudan, Proc. Third Symposium on Physics and Technology of Compact Toroids, Los Alamos Scientific Laboratory, LA-8700-C, p. 36 (1980).

[60] J.B. Taylor, Phys. Rev. Lett. 33, 1139 (1974).

[6I] G.C. Goldenbaum, J.H. Irby, Y.P. Chong, G.W. Hart, Phys. Rev. Letts. 44,393 (1980).

[62] M. Yamada, R.A. Ellis, H.P. Furth, M. Okabayashi, G. Sheffield, J. Sinnis, T.H. Stix, A.M. Todd, Bulletin Am. Phys. Soc. 24, 1022 (1979).

[63] W.T. Armstrong, ?.K. Linford, J. Lipson, E.G. Sherwood, Phys. Fluids, 24, 2068 (1981).

[64] T.C. Simonen, J.F. Clauser, F.H. Coensgen, D.L. Correll, W.F. Cummins, R.P. Orike, J.H. Foote, A.H. Futch, R.K. Goodman, D.P. Grubb, A.L. Hunt, G.M. Melin, A.W. Molvik, W.E. Nexsen, B.W. Stallard, W.C. Turner, Proc. Seventh Conf. Plasma Physics and Cont. Nucl. Fus. Res., Voi. 2, p. 389, IAEA, Vienna, 1978.

[65] L.D. Pearlstein, D.V. Anderson, D.E. Baldwin, J.A. Byers, B.I. Cohen, R.H. Cohen, W.C. Condit, T.K. Fowler, R.P. Freis, T.B. Kaiser, R.F. Post, M.E. Rensink, G.R. Smith, A.H. Boozer, Proc. Seventh Conf. Plasma Physics and Cont. Nucl. Fus. Res., Vol. 2, p. 457, IAEA, Vienna, 1978.

[66] W.C. Turrier, G.C. Goldenbaum, E.H.A. Granneman, J.H. Hammer, C.W. Hartman, D.S. Prono, J. Taska, A.C. Smith, Proc. Third Symp. on Physics and Tech. of Compact Toroids, Los Alamos, LA-8700-C, p. 13 (1980). 
[67] J. Marshal, Phys. Fluids $\underline{3}, 134$ (1960).

[68] R.F. Post, W.C. Turner, Proc. Fourth Symposium on the Physics and Technology of Compact Toroids, Lawrence Livermore National Laboratory, CONF-811087, October (1981).

[69] 5. Chapman and T.G. Cowling, The Mathematical Theory of Non-Uniform Gases, Cambridge, London, 1952.

[70] B. McNamara, Methods in Computational Physics, Vol. 16, Academic Press, New York (1976).

[71] M.D. Kruskal and R.M. Kulsrud, Phys. Fluids 1, 265 (1958).

[7^] D. Potter, Methods in Computational Physics, Vol. 16, Academic Press, New York (1976).

[73] R. White, D. Monticello, M.N. Rosenbluth, H. Strauss, B.B. Kadometsev, Proc. Fifth Conf. Plasma Phys, and Cont. Nuc. Fus. Res., Vol. 1, p. 495, IAEA, Vienna, 1974.

[74] D.E. Potter and G.H. Tuttle, J. Comp. Phys. 13, 483 (1973).

[75] 0. Betancourt and P. Garabedian, Am. Phys. Soc. Conf. Plasma Phys. (1974).

[76] V.O. Shafranov, Sov. Phys. JETP $\underline{8}, 494$ (1958).

[77] D. Potter, Computational Physics, John Wiley and Sons, New York, 1973.

[78] M.B. Marder and H. Weitzner, Plasma Phys. 12, 435 (1970).

[79] M.S. Chance, et. al., Proc. Fifth Conf. Plasma Phys. Cont. Nuc. =us. Res., Vol. 1, p. 463, IAEA, Vienna, 1974.

[80] M.S. Chu, D. Dobrott, T.H. Jensen, T. Tamano, Phys. Fluids 17, 1183 (1974).

[81] K. Lackrer, J. Geophys. Res. 75, 16 (1970). 
[82] J.D. Callen and R.A. Dory, Phys. Fluids 15, 1523 (1972).

[83] R.i Byrne and H.H. Klein, J. Comp. Phys. 26, 352 (1978).

[84] J.L. Johnson, H.E. Dalhed, J.M. Greene, R.C. ârimm, Y.Y. Hsieh, S.C. Jardin, J. Manikam, M. Okabayashi, R.G. Storer, A.M.M. Todd, D.E. Voss, K.E. Weimer, J. Comp. Phys. 32, 212 (1979).

[85] 0. Buneman, Stanford University Institute for Plasma Research, Rpt. SUIPR-294 (1968).

[86] J.A. Holmes, Y.K.M. Peng, S.J. Lynch, J. Comp. Phys. 36, 35 (1980).

[87] F.J. Helton, T.S. Wang, Nuc. Fusion 18, 1523 (1978).

[88] H. Grad, "Survey of 1-1/2 D Transport Codes, Courant Institute, New York, Report MF-93 (1978).

[89] D.C. Stevens, H. Grad, P.N. Hu, Bulletin Am. Phiys. Soc., 19, $865(1974)$.

[90] H. Grad, P.N. Hu, D.C. Stevens, Proc. Nat. Acad. Sci., 72, $3789(1975)$.

[91] L.L. Lao, S.P. Hirshman, R.M. Wieland, Phys. Fluids 24, 1431 (1981).

[92] L.L. Lao, R.M. Wieland, W.A. Houlberg, S.P. Hirshman, ORNL/TM-7871, Feb. (1982).

[93] J.A. Byers, Phys. Rev. Lett. 39, 1476 (1977).

[94] E.H. Holt and R.E. Haskell, Plasma Dynamics, MacMillan Co., New York, p. 185 (1968).

[95] ibid, p. 190 
[96] D. Bohm, The Characteristics of Electrical Discharges in Magnetic Fields, (A. Gutherie, R.K. Wakerling, eds) McGraw-Hill, New York, p. 173 (1949).

[97] S. Chandrasekhar, Rev. Mod. Phys. 15, I (1943).

[98] S.I. Braginskii, Reviews of Plasma Physics (M.A. Leonovich, ed.) Consultants' Bureau, New York, Vol. 1 (1965).

[99] D. Pfirsch, A. Schluter, Max Planck Institute Report MPI/PA/7/62, Munich (1962).

[100] M.N. Rosenbluth, Fusion (E. Teller, ed) Vol. I, Acadenic Press, New York (1981).

[101] ibid

[102] B.B. Kadomtsev and 0.P. Pogutse, Nuc. Fus. 11, 67 (1971).

[103] F.L. Hinton and R.D. Hazeltine, Rev. Mod. Phvs. 48, 239 (1975).

[104] A.A. Galeev, R.Z. Sagdeev, Sov. Phys. JETP 26, 233 (1968).

[105] R.J. Hastie, J.B. Taylor, F.A. Haas, Ann. Phys. New York 41, $303(1967)$.

[106] E.A. Freiman, Phys. Fluids 13, 490 (1970).

[107] A.I. Morozov, L.S. Solov'ev, Reviews of Plasma Physics, (M.A. Leonovich, ed) Consultants Bureau, New York, Vol. 2 (1965).

[108] M.N. Rosenbluth, R.D. Hazeltine, F.L. Hinton, Phys. Fluids $\underline{15}$, $116(1972)$.

[109] P.H. Rutherford, Phys. Fluids 13, 482 (1970).

[110] F.L. Hinton and M.N. Rosenbluth, Phys. Fluids 16, 836 (1973).

[111] R.D. Hazeltine, F.L. Hinton, M.N. Rosenbluth, Phys. Fluids 16, 1645 (1973). 
[112] D.E. Shumaker, J.K. Boyd, S.P. Auerbach, and B. McNamara, J. Comp. Phys., 42, 266 (19:32).

[113] H. Grad, J. Hogan, Phys. Rev. Lett., 24, 1377 (1970).

[114] H. Grad, P.N. Hu, and D.C. Stevens, Proc. Nat. Acad. Sci., 72, 3789 (1975).

[115] H. Grad, Survey of 1-1/20 Transport Codes, Courant Institute, NY, Report MF-93, p. 6 (1978).

[116] W.R. Smythe, Static and Dynamic Electricity, McGraw-Hi11, New York, NY (1968).

[117] D.E. Post, R.V. Jensen, C.B. Tarter, W.H. Grasberger, and W.A. Lokke, At. Data Nucl. Data Tables, 20, 297 (1977).

[118] R.S. Devoto and J.D. Hanson, Lawrence Livermore National Laboratory, Rept. UCRL 52559 (1978).

[119] R.D. Richtmeyer and K.W. Morton, Difference Miethods for Initial Value Problems, Second Edition, Interscience, NY (1967).

[120] S.P. Auerbach, private communication.

[127] D.S. Kershaw, J. Comp. Phys. 26, 43 (1978).

[122] H.L. Berk, J.H. Hammer, and H. Weitzner, Phys. Fluids 24, $1758(1981)$.

[123] M.J. Hi11, Phil. Trans. Royal Soc (A), Part I, 214 (1894).

[124] V.D. Shafranov, Sov. Phys. JETP 10, 775 (1960).

[125] S. Kaneko, K. Chiyoda, and I. Hirota, J. Phys. Soc. Japan Lett. 50,359 (1981).

[126] M.N. Rosenbluth and M.N. Bussac, Nucl. Fusion, 19, 489 (1979).

[127] H.R. Strauss, Phys. Rev. Lett. 26, 616 (1971). 
[128] T.R. Jarboe, I. Henins, H.W. Hoida, R.K. Linford, J. Marshall, D.A. Platts, and A.R. Sherwood, Phys. Rev. Lett., 45, $1264(1980)$.

[129] G.C. Goldenbaum, J.H. Irby, Y.P. Chong, and G.W. Hart, Phys. Rev. Lett., 44, 393 (1980).

[130] H.E. Dalhed, Bullet in Am. Phys. Soc., Vol. 25, No. 8, 3025, October $(1980)$.

[131] H.E. Dalhed, Proc. Annual Controlled Fusion Theory Conf., Austin, Texas (1981).

[132] S.P. Auerbach and W. C. Condit, Lawrence Livermore National Laboratory Rept. UCRL 85112 (1980).

[133] J.K. Boyd, S.P. Auerbach, H.L. Eerk, B. McNamara, and P.A. Willmann, "Adiabatic Compression and Neutral Beam Buildup in a Fiedi Reversed Mirror Configuration," Proc. Sherwood Meeting on Theoretical Aspects of Controlled Thermonuclear Research, Tucson, Arizona (1980).

[134] J.K. Boyd, B. McNamara, P. A. Willmann, and S.P. Auerbach, "Computational Methods for Axisymmetric Equilibria," Proc. Ninth Conf. on Numerical Simulation of Plasmas, North Western University, Evanston, I1 linois (1980).

[135] J.K. Boyd, S.P. Auerbach, H.L. Berk, B. McNamara, and P.A. Willmann, "Neutral Beam Injection and Adiabatic Compression in a Field Reversed Mirror," Bullet in Am. Phys. Soc., Vol. 25, No. 8, October (1980).

[136] B. McNamara, S.P. Auerbach, and J.K. Boyd, "SC.ling Laws for Beam Heated Field Reversed Mirrors, "Proc. Annual Controlled Fusion Theory Conference, Austin, Texas (1981). 
[137] D.E. Shumaker, B. McNamara, and J.K. Boyd, "Field Reversed Mirror Transport Code," Bulletin Am. Phys. Soc., Vol. 24, Ho. 8, October (1979).

[138] D.E. Shumaker, J.K. Boyd, S.P. Auerbach, H.L. Berk, and B. McNamara, "Field Reversed Mirror Transport Code," Proc. Sherwood Meeting on Theoretical Aspects of Controlled Thermonuclear Research, Tuscon, Arizona (1980).

[139] D.E. Shumaker, J.K. Boyd, S.P. Auerbach, and B. McNamara, "Transport in Field Reversed Mirror," Bullet in Am. Phys. Soc., Vol. 25, No. 8, October (1980).

[140] D.E. Shumaker, J.K. Boyd, S.P. Auerbach, and B. MCNamara, "Nunerical Simulation of the Beta II Experiment," Proc. Annual Controlled Fusion Theory Conference, Austin, Texas (1981).

[141] D.E. Shumaker, J.K. Boyd, B. McNamara, and W.C. Turner, "Numerical Simulation of a Bean Heated Compact Torus," Bullet in Am. Phys. Soc., Vol. 26, No. 7 (1981).

[142] J.H. Hamner, "The Prohlimak: A Non-Tilting Field Reversed Equilibrium," Proc. Sherwood Meeting on Theoretical Aspects of Controlled Thermonuclear Research, Tucson, Arizona (1980).

[143] A.H. Glasser, W.B. Thompson, Phys. Fluids 16, No. 1, 1973.

[144] R. Balescu, Stati-ical Mechanics of Charged Particles, Interscience, New York, 1963.

[145] R.V. Lovelace, Phys. Fluids 21, No. 8, 1978.

[146] R. Kulsrud, Phys. Rev., 106, 205 (1957). 
[147] M. Kruskal, J. Math. Phys., 3, 860 (1962).

[148] B. I. Cohen, "Status of Mirrur Fusion Research," UCAR 10049-80, Rev. 1.

[149] R. F. Post, "Experimental Base of Mirror Confinement Physics, " UCRL-84220, 1980.

[150] A. C. Hindnarsh, "GEAR: Ordinary Differential Equation System Solver, " UCID-30001, December 197.?.

[151] C. W. Gear, Numerical Initial Value Problems in Ordinary Ditferential Equations, Prentice-Ha11, 1971.

[152] J. M. Dawson, H. Okuda, B. Rosen, "Collective Transport in Plasmas," Methods in Computational Physics, 16, 282 (1976).

[153] J. M. Finn, Plasma Phys. 21, 405 (1979).

[154] E. T. Whittaker, A Treatise on the Analytical Dynamics of Particles and Rigid Bodies (4th ed., Cambridge University Press, London 1937).

[155] L. S. Hall, "On the Existence of a Last Invariant of Conservative Motion," UCIO-18980, Rev. I., (to be published Annàls of Physics).

[156] A. C. Hearn, M. L. Griss, REDUCE2, LSERS MANUAL, 2nd edition, UCP-19 (1975).

[157] H. Goldstein, Classical Mechanics, Addison Wesley, Men 10 Park, CA, 2nd edition, 1981.

[158] G. M. Zaslavskii, B. V. Chirikov, Sov. Phys. Uspekhi, Vol. 14, No. 5, 549 (1972).

[159] B. V. Chirikov, Physics Reports 52, 263 (1979). 
[l60] R. H. Cohen, Conments Plasma Phys. Cont. Fusion, Vol. 4, No. o, $157(1979)$.

[161] R. H. Cohen, D. V. Anderson, C. B. Sharp, Phys. Rev. Lett. 41, 1304 (1978).

[162] G. R. Smith, A. N. Kaufman, Phys. Fluids, 21, 2230 (1978).

[163] G. R. Smith, J. A. Byers, L. LoDestro, Phys. Fluids, 23, 278 (1980).

[164] G. D. Birkhoff, Dynanical Systems, American Mathematica] Society Colloquium Publications Vol. 9, 1927.

[165] J. M. Finn, Ph.d Thesis, University of Maryland, 1974.

[166] D.E. Baldwin and M.E. Rensink, Conments Plasma Phys., Vo]. 4, No. 2, $55(1978)$.

[167] S.I. Braginskii, Reviews in Plasma Physics, (M. A. Leonovich, ed.) Consultants Bureau, New York, Vol. 1, 238 (1965).

[168] R.J. Bickerton, J.W. Conner, and J.B. Taylor, Nature Physical Science 229, $110(1971)$.

[169] T. Othawa, Nuc. Fus., 10, 185 (1970).

[170] K.D. Marx, Phys. Fluids, 11, 357 (1968).

[171] R.F. Post, Fourth International Conference on Driven Magnetic Fusion Reactors, Erice, Italy, September (1978).

[172] D.E. Baldwin and T.K. Fowler, Lawrence Livermore National Laboratory Rept. UCID 17691, December (1977).

[i73] K.V. Roberts and J.B. Taylor, Phys. Rev. Lett. 8,197 (1977).

[174] Y. Nocentini and F. Engelmann, Nuc. Fusion 17, 761 (1977).

[175] P.A. Haldy and J. Ligou, Nuc. Fusion 17, 1225 (1977).

[176] L.V. Spencer, Phys. Rev. 98, 1597 (1955). 
[177] R.D. Hazeltine, Plasma Phys. 15, $\underline{77}$ (1973).

[178] W.I. Van Rij, H.K. Meier, C.o. Beasley, and J.E. McCune, Oak Ridge National Laboratory Rept. ORNL/TM-5376.

[179] L. Cheung, Phys. Rev. A 13, 1209 (1976).

[180] R.D. Hazeltine and A.A. Ware, Plasma Phys. 20, 673 (1978).

[181] V.C. Boffi and V.G. Molinari, Nuovo Cimento, 496, 77 (1979).

[182] J.P. Friedberg, Phys. Fluids 15, 1102 (1972).

[183] J.P. Friedberg, L.O. Pearlstein, Phys. Fluids 2], 1207 (197i3).

[184] L.0. Pearlstein and J.P. Friedberg, Phys. Fluids 2], 1218 (1978).

[185] T.A. Hai11, R.A. Stark, and G.H. Miley, Trans. Am. Nuc. Soc. 39, 491 (1981).

[186] R.A. Stark, Proc. Annual Controlled Fusion Theory Coní., Santa Fe, New Maxico, April (1032).

[187] H.J. Willenberg, J. Comp. Phys. 34, 330 (1980).

[188] B.McNamara and K.J. Whiteman, J. Math. Phys. 8, 2029 (1967).

[189] H.R. Lewis, K.R. Symon, J. Math. Phys. 20, 413 (1979).

[190] M.N. Rosenbluth, W.M. MacDonald, and 0. Judd, Phys. Rev. 102, 1 (1957). 


\section{APPENDIX A}

Definition of Functions Used for Orbit Average Formulas

The In functions

$$
\begin{aligned}
I_{1}= & \int\left(1+x^{2}\right)^{1 / 2} d x \\
= & \frac{1}{2} x\left(1+x^{2}\right)^{1 / 2}+\frac{1}{2} \log \left(x+\left(1+x^{2}\right)\right) \\
I_{2}(x, y)= & \int y\left(x^{2}+y^{2}\right)^{1 / 2} d y \\
= & \frac{1}{3}\left(x^{2}+y^{2}\right)^{3 / 2}(A-1) \\
I_{3}(a, b, c, x) & =\int d x\left(x^{2}+c\right)(a x+b)^{-1} \\
& =a^{-1}\left(\left(x^{2}+c\right)^{1 / 2}-(b / a) \log \left(2\left(\left(x^{2}+c\right)^{1 / 2}+x\right) / a\right)\right. \\
& -\left(c+(b / a)^{2}\right)^{l / 2} \log \left(\left(2\left(c+(b / a)^{2}\right)^{1 / 2}\left(x^{2}+c\right)^{1 / 2}\right.\right. \\
& \left.\left.\left.-2 b x x^{\prime} a+2 c\right)(a x+b)^{-1}\right)\right)
\end{aligned}
$$

In the case when $c=0$ in $A-3$, a different formula is required.

$$
\begin{aligned}
& I_{3}(a, b, 0, x)=x / a-b a^{-2} \log (x+b / a) \\
& I_{4}(a, b, c, x)=\int x\left(x^{2}+c\right)^{1 / 2}(a x+b)^{-1} d x
\end{aligned}
$$




$$
\begin{aligned}
& =a^{-1} \int\left(x^{2}+c\right)^{1 / 2} d x-(b / a)\left(x^{2}+c\right)^{1 / 2}(a x+b) d x \\
& =(c / a) I_{1}\left(x / c^{1 / 2}\right)-(b / a) I_{3}(a, b, c, x)
\end{aligned}
$$

In the case when $c=0$ in $A-5$, the following formula applies.

$$
\begin{aligned}
& I_{4}(a, b, 0, x)=x^{2} /(2 a)-(b / a) I_{3}(a, b, 0, x) \\
& I_{5}\left(r_{0}, r_{1}, r_{A}, r_{B}\right)=\int_{\theta_{1}}^{\theta_{2}} e^{r_{1}^{2} \sin ^{2} \theta} d \theta
\end{aligned}
$$

where,

$$
\begin{aligned}
& \theta_{1}=\sin ^{-1}\left(\left(r_{A}-r_{0}\right) / r_{1}\right) \\
& \theta_{2}=\sin ^{-1}\left(\left(r_{B}-r_{0}\right) / r_{1}\right)
\end{aligned}
$$

The $K_{n}$ functions

$$
\begin{aligned}
k_{1}^{n u m} & =\int_{z_{1}}^{\Delta z} \int_{0}^{-\frac{h_{1}+h_{3} z}{h_{2}+h_{4} z}} \frac{g_{1}+g_{2} r+g_{3} z+g_{4} r z}{\left(h_{1}+h_{2} r+h_{3} z+h_{4} r z\right)^{\alpha}} d r \\
& =\int_{z_{1}}^{\Delta z} d z\left[-\frac{\left(g_{1}+g_{3} z\right)\left(h_{1}+h_{3} z\right)^{1-\alpha}}{(1-\alpha)\left(h_{2}+h_{4} z\right)}\right. \\
& \left.+\frac{\left(g_{2}+g_{4} z\right)\left(h_{1}+h_{3} z\right)^{2-\alpha}}{(1-\alpha)(2-\alpha)\left(h_{2}+h_{4} z\right)^{2}}\right]
\end{aligned}
$$




$$
\begin{aligned}
& k_{1}^{\text {deno }}=\int_{z_{1}}^{\Delta z}-\frac{\left(h_{1}+h_{3} z\right)^{1-\alpha}}{(1-\alpha)\left(h_{2}+h_{4} z\right)} d z \\
& k_{2}^{\text {num }}=\int_{z_{1}}^{\Delta z} d z \int_{-\frac{h_{1}+h_{3} z}{h_{2}+h_{4} z}}^{\Delta r} d r \frac{g_{1}+g_{2} r+g_{3} z+g_{4} r z}{\left(h_{1}+h_{2} r+h_{3} z+h_{4} r z\right)^{\alpha}} \\
& =\int_{z_{1}}^{\Delta z} d z\left[\frac{\left(g_{1}+g_{3} z\right)\left(h_{1}+h_{3} z+\left(h_{2}+h_{4} z\right) \Delta r\right)^{1-\alpha}}{(1-\alpha)\left(h_{2}+h_{4} z\right)}\right. \\
& \left(g_{2}+g_{4} z\right)\left(\frac{\Delta r\left(h_{1}+h_{3} z+\left(h_{2}+h_{4} z\right) \Delta r\right)^{l-\alpha}}{(1-\alpha)\left(h_{2}+h_{4} z\right)}\right. \\
& \left.\left.-\frac{\left(h_{1}+h_{3} z+\left(h_{2}+h_{4} z\right) \Delta r\right)^{2-\alpha}}{(1-\alpha)(2-\alpha)\left(h_{2}+h_{4} z\right)^{2}}\right)\right] \\
& k_{2}^{\text {deno }}=\int_{z_{1}}^{\Delta z} d z \frac{\left(h_{1}+h_{3} z+\left(h_{2}+h_{4} z\right) \Delta r\right)^{1-\alpha}}{(1-\alpha)\left(h_{2}+h_{4} z\right)} \\
& k_{3}^{\text {num }}=\int_{0}^{z_{1}} d z \int_{0}^{r_{2}} d r \frac{g_{1}+g_{2} r+g_{3} z+g_{4} r z}{\left(h_{1}+h_{2} r+h_{3} z+h_{4} r z\right)^{\alpha}} \\
& =\int_{0}^{z} 1 z\left[( g _ { 1 } + g _ { 3 } z ) \left(\frac{\left(h_{1}+h_{3} z+\left(h_{2}+h_{4} z\right) r_{2}\right)^{1-\alpha}}{(1-\alpha)\left(h_{2}+h_{4} z\right)}\right.\right. \\
& \left.-\frac{\left(h_{1}+h_{3} z\right)^{1-\alpha}}{(1-\alpha)\left(h_{2}+h_{4} z\right)}\right)
\end{aligned}
$$


340

$(A-13)$

$$
\begin{aligned}
& +\left(g_{2}+g_{4} z\right)\left(\frac{r_{1}\left(h_{1}+h_{3} z+\left(h_{2}+h_{4} z\right) r_{2}\right)^{1-\alpha}}{(1-\alpha)\left(h_{2}+h_{4} z\right)}\right. \\
& +\frac{\left(h_{1}+h_{3} z\right)^{2-\alpha}}{(1-\alpha)(2-\alpha)\left(h_{2}+h_{4} z\right)^{2}} \\
& \left.\left.-\frac{\left(h_{1}+h_{3} z+\left(h_{2}+h_{4} z\right) r_{2}\right)^{2-\alpha}}{(1-\alpha)(2-\alpha)\left(h_{2}+h_{4} z\right)^{2}}\right)\right] \\
& k_{3}^{\text {deno }}=\int_{0}^{z} d z \frac{\left(h_{1}+h_{3} z+\left(h_{2}+h_{4} z\right) r_{2}\right)^{7-\alpha}-\left(h_{1}+h_{3} z\right)^{l-\alpha}}{(1-\alpha)\left(h_{2}+h_{4} z\right)} \\
& (A-14) \\
& k_{4}^{\text {numi }}=\int_{0}^{\Delta z} d z \int_{r_{2}}^{\Delta r} d r \frac{g_{1}+g_{2} r+g_{3} z+g_{4} r z}{\left(h_{1}+h_{2} r+h_{3} z+h_{4} r z\right)^{\alpha}} \\
& =\int_{0}^{\Delta z} d z\left[\frac{g_{1}+g_{3} z}{(1-\alpha)\left(h_{2}+h_{4} z\right)}\left(\left(h_{1}+h_{3} z+\left(h_{2}+h_{4} z\right) \Delta r\right)\right]^{1-\alpha}\right. \\
& \left.-\left(h_{1}+h_{3} z+\left(h_{2}+h_{4} z\right) r_{2}\right)^{1-\alpha}\right) \\
& +\frac{g_{2}+g_{4} z}{\Pi-\alpha)\left(h_{2}+h_{4} z\right)}\left(\Delta r\left(h_{1}+h_{3} z+\left(h_{2}+h_{4} z\right) \Delta r\right)^{1-\alpha}\right. \\
& -r_{2}\left(h_{1}+h_{3} z+\left(h_{2}+h_{4} z\right) r_{2}\right)^{7-\alpha} \\
& +\frac{1}{(z-\alpha)\left(h_{2}+h_{4} z\right)}\left(\left(h_{1}+h_{3} z+\left(h_{2}+h_{4} z\right) r_{2}\right)^{2-\alpha}\right. \\
& \left.\left.\left.-\left(h_{i}+h_{3} z+\left(h_{2}+n_{4} z\right) \Delta r\right)^{2-\alpha}\right)\right)\right]
\end{aligned}
$$




$$
\begin{aligned}
k_{4}^{\text {deno }} & =\int_{0}^{\Delta z} d z\left(\left(h_{1}+h_{3} z+\left(h_{2}+h_{4} z\right) \Delta r\right)^{l-\alpha}\right. \\
& \left.-\left(h_{1}+h_{3} z+\left(h_{2}+h_{4} z\right) r_{2}\right)^{l-\alpha}\right) \frac{1}{(1-\alpha)\left(h_{2}+h_{4} z\right)}
\end{aligned}
$$

The $N_{n}$ functions

$$
\begin{aligned}
& N_{1}^{\text {num }}=\int_{0}^{\min \left(z_{1}, z_{2}\right)} d z \int_{0}^{z_{3}} d r \frac{g_{1}+g_{2} r+g_{3} z+g_{4} r z}{\left(h_{1}+h_{2} r+h_{3} z+h_{4} r z\right)^{\alpha}} \\
& =\int_{0}^{\min \left(z_{1}, z_{2}\right)} d z\left[\frac{g_{1}+g_{3} z}{(1-\alpha)\left(h_{2}+h_{4} z\right)}\right. \\
& \left(\left(h_{1}+h_{3} z+\left(h_{2}+h_{4} z\right) z_{3}\right)^{1-\alpha}\right. \\
& \left.-\left(h_{1}+h_{3} z\right)^{1-\alpha}\right) \\
& +\frac{g_{2}+g_{4} z}{(1-\alpha)\left(h_{2}+h_{4} z\right)}\left(z_{3}\left(h_{1}+h_{3} z+\left(h_{2}+h_{4} z\right) z_{3}\right)^{7-\alpha}\right. \\
& +\frac{1}{(2-\alpha)\left(h_{2}+h_{4} z\right)}\left(\left(h_{1}+h_{3} z\right)^{2-\alpha}\right. \\
& \left.\left.\left.-\left(h_{1}+h_{3} z+\left(h_{2}+h_{4} z\right) z_{3}\right)^{2-\alpha}\right)\right)\right] \\
& N_{1}^{\text {deno }}=\int_{0}^{\min \left(z_{1}, z_{2}\right)} d z\left(\left(h_{1}+h_{3} z+\left(h_{2}+h_{4} z\right) z_{3}\right)^{1-\alpha}\right.
\end{aligned}
$$$$
\left.-\left(h_{1}+h_{3} z\right)^{1-\alpha}\right) \frac{1}{(1-\alpha)\left(h_{2}+h_{4} z\right)}
$$ 
342

Regions two and three have different formulas depending on the relative size of $z_{1}$ and $z_{2}$. For $z_{2}>z_{1}$ the following formulas apply.

$$
\begin{aligned}
& N_{2}^{\text {nom }}=\int_{z_{1}}^{z_{2}} d z \int_{-\frac{h_{1}+h_{3} z}{h_{2}+h_{4} z}}^{z_{3}} d r \frac{g_{1}+g_{2} r+g_{3} z+g_{4} r z}{\left(h_{1}+h_{2} r+h_{3} z+h_{4} r z\right)^{\alpha}} \\
& =\int_{z_{1}}^{z_{2}} d z\left[\frac{g_{1}+g_{3} z}{(1-\alpha)\left(h_{2}+h_{4} z\right)}\left(h_{1}+h_{3} z+\left(h_{2}+h_{4} z\right) z_{3}\right)^{1-\alpha}\right. \\
& \frac{g_{2}+g_{4} z}{(T-\alpha)\left(h_{2}+h_{4} z\right)}\left(z_{3}\left(h_{1}+h_{3} z+\left(h_{2}+h_{4} z\right) z_{3}\right)^{1-\alpha}\right. \\
& \left.\left.-\frac{\left(h_{1}+h_{3} z+\left(h_{2}+h_{4} z\right) z_{3}\right)^{2-\alpha}}{(2-\alpha)\left(h_{2}+h_{4} z\right)}\right)\right] \\
& N_{2}^{\text {deco }}=\int_{z_{1}}^{z_{2}} d z \frac{\left(h_{1}+h_{3} z+\left(h_{2}+h_{4} z\right) z_{3}\right)^{l-\alpha}}{(1-\alpha)\left(h_{2}+h_{4}\right)^{2}} \\
& N_{3}^{\text {nun }}=\int_{z_{1}}^{z_{2}} d z \int_{0}^{-\frac{h_{1}+h_{3} z}{h_{2}+h_{4} z}} d r \frac{g_{1}+g_{2} r+g_{3} z+g_{4} r z}{\left(h_{1}+h_{2} r+h_{3} z+h_{4} r z\right)^{\alpha}} \\
& =\int_{z_{1}}^{z_{2}} d z\left(\frac{g_{1}+g_{3} z}{(1-\alpha)\left(h_{2}+h_{4} z\right)}\left(-\left(h_{1}+h_{3} z\right)^{1-\alpha}\right)\right. \\
& \left.+\frac{g_{2}+g_{4} z}{(1-\alpha)(2-\alpha)\left(h_{2}+h_{4} z\right)^{2}}\left(h_{1}+h_{3} z\right)^{2-\alpha}\right)
\end{aligned}
$$




$$
N_{3}^{\text {deno }}=\int_{z_{1}}^{z_{2}} d z \frac{-\left(h_{1}+h_{3} z\right)^{1-\alpha}}{(1-\alpha)\left(h_{2}+h_{4} z\right)}
$$

For $z_{2}<z_{1}$ the following formulas apply for regions two and three.

$$
\begin{aligned}
& N_{2}^{n u m}=\int_{0}^{z} d z\left(\frac{g_{1}+g_{3} z}{(1-\alpha)\left(h_{2}+h_{4} z\right)}\left(-\left(h_{1}+h_{3} z\right)^{1-\alpha}\right)\right. \\
& \left.+\frac{g_{2}+g_{4} z}{(1-\alpha)(2-\alpha)\left(h_{2}+h_{4} z\right)^{2}}\left(h_{1}+h_{3} z\right)^{2-\alpha}\right) \\
& N_{2}^{\text {deno }}=\int_{z_{2}}^{z} d z \frac{-\left(h_{1}+h_{3} z\right)^{1-\alpha}}{(T-\alpha)\left(h_{2}+h_{4} z\right)} \\
& N_{3}^{\text {num }}=\int_{z_{2}}^{z} 1 d z\left[\frac{g_{1}+g_{3} z}{(T-\alpha)\left(h_{2}+h_{4} z\right)}\left(h_{1}+h_{3} z+\left(h_{2}+h_{4} z\right) z_{3}\right)^{l-\alpha}\right. \\
& +\frac{9_{2}+g_{4} z}{\left.\prod-\alpha\right)\left(h_{2}+h_{4} z\right)}\left(z_{3}\left(h_{1}+h_{3} z+\left(h_{2}+h_{4} z\right) z_{3}\right)^{1-\alpha}\right. \\
& \left.\left.-\frac{\left(h_{1}+h_{3} z+\left(h_{2}+h_{4} z\right) z_{3}\right)^{2-\alpha}}{(z-\alpha)\left(h_{2}+h_{4} z\right)}\right)\right] \\
& N_{3}^{\text {deno }}=\int_{z_{2}}^{z_{1}} d z \frac{\left(h_{1}+h_{3} z+\left(h_{2}+h_{4} z\right) z_{3}\right)^{1-\alpha}}{(1-\alpha)\left(h_{2}+h_{4} z\right)} \\
& N_{4}^{n u m}=\int_{\max \left(z_{1}, z_{2}\right)}^{\Delta r \Delta z / z_{3}} d z \int_{0}^{z_{3}} \frac{g_{1}+g_{2} r+g_{3} z+g_{4} r z}{\left(h_{1}+h_{2} r+h_{3} z+h_{4} r z\right)^{\alpha}} d r
\end{aligned}
$$




$$
\begin{aligned}
= & \int_{\max \left(z_{1}, z_{2}\right)}^{\Delta r \Delta z / z_{3}} d z\left[\frac{g_{1}+g_{3} z}{(1-\alpha)\left(h_{2}+h_{4} z\right)}\right. \\
& \left(\left(h_{1}+h_{3} z+\left(h_{2}+h_{4} z\right) z_{3}\right)^{l-\alpha}-\left(h_{1}+h_{3} z\right)^{1-\alpha}\right) \\
+ & \frac{g_{2}+g_{4} z}{(T-\alpha)\left(h_{2}+h_{4} z\right)}\left(z_{3}\left(h_{1}+h_{3} z+\left(h_{2}+h_{4} z\right) z_{3}\right)^{T-\alpha}\right. \\
+ & \frac{1}{(2-\alpha)\left(h_{2}+h_{4} z\right)}\left(\left(h_{1}+h_{3} z\right)^{2-\alpha}\right. \\
& \left.\left.\left.-\left(h_{1}+h_{3} z+\left(h_{2}+h_{4} z\right) z_{3}\right)^{2-\alpha}\right)\right)\right] \\
N_{4}^{\text {deno }}= & \int_{\max \left(z_{1}, z_{2}\right)}^{\Delta r \Delta z / z_{3}} d z \frac{\left(h_{1}+h_{3} z+\left(h_{2}+h_{4} z\right) z_{3}\right)^{1-\alpha}-\left(h_{1}+h_{3}\right)^{1-\alpha}}{(1-\alpha)\left(h_{2}+h_{4} z\right)}
\end{aligned}
$$

The $T_{n}$ functions

$$
\begin{aligned}
& T_{1}=\int_{0}^{\Delta z} x^{2} I_{1}(\Delta r / x) d x \\
& T_{2}=\int_{0}^{\Delta z} x^{3} I_{1}(\Delta r / x) d x \\
& T_{3}=\int_{0}^{\Delta z} I_{2}(x, \Delta r) d x-\Delta z^{4} / 12 \\
& T_{4}=\int_{0}^{\Delta z} d x\left(I_{1}((\Delta z-x) / \Delta r)-I_{1}(-x / \Delta r)\right)
\end{aligned}
$$




$$
\begin{aligned}
& T_{5}=\int_{0}^{\Delta z} x\left(I_{1}((\Delta z-x) / \Delta r)-I_{1}(-x / \Delta r)\right) d x \\
& T_{6}=\int_{0}^{\Delta z} d x\left(I_{2}(\Delta r, \Delta z-x)-I_{2}(\Delta r,-x)\right) \\
& T_{7}=\int_{0}^{\Delta r} d x\left(I_{1}((\Delta r-x) / \Delta z)-I_{j}(-x / \Delta z)\right) \\
& T_{8}=\int_{0}^{\Delta r} d x\left(I_{1}((\Delta r-z) / \Delta z)-I_{1}(-x / \Delta z)\right) x \\
& T_{9}=\int_{0}^{\Delta r} d x\left(I_{2}(\Delta z, \Delta r-x)-I_{2}(\Delta z, x)\right) \\
& T_{10}=\int_{0}^{\Delta z} d x I_{2}(x, \Delta r) x-\Delta z^{5} / 15 \\
& T_{17}=\int_{0}^{\Delta z} d x\left(I _ { 3 } \left(\left(v_{3}-v_{4}\right) / \Delta r, v_{1}+v_{4}\right.\right. \\
& \left.+\left(v_{4}-v_{1}\right) x / \Delta z,(\Delta z-x)^{2}, \Delta r\right) \\
& \left.-I_{3}\left(\left(v_{3}-v_{4}\right) / \Delta r, v_{1}+v_{4}+\left(v_{4}-v_{7}\right) x / \Delta z,(\Delta z-x)^{2}, 0\right)\right) \\
& T_{12}=\int_{0}^{\Delta z} d x\left(I _ { 3 } \left(\left(v_{3}-v_{4}\right) / \Delta r, v_{1}+v_{4}\right.\right.
\end{aligned}
$$


346

(A-40)

$$
\begin{aligned}
& \left.+\left(v_{4}-v_{1}\right) x / \Delta z,(\Delta z-x)^{2}, \Delta r\right) \\
& \left.-I_{3}\left(\left(v_{3}-v_{4}\right) / \Delta r, v_{1}+v_{4}+\left(v_{4}-v_{1}\right) x / \Delta z,(\Delta z-x)^{2}, 0\right)\right) x \\
T_{13} & =\int_{0}^{\Delta z} d x\left(I _ { 4 } \left(\left(v_{3}-v_{4}\right) / \Delta r, v_{1}+v_{4}\right.\right. \\
& \left.+\left(v_{4}-v_{1}\right) x / \Delta z,(\Delta z-x)^{2}, \Delta r\right) \\
& \left.+I_{4}\left(\left(v_{3}-v_{4}\right) / \Delta r, v_{1}+v_{4}+\left(v_{4}-v_{1}\right) x / \Delta z,(\Delta z-x)^{2}, 0\right)\right) \\
& =I_{0}\left(\left(v_{2}-v_{4}\right) x / \Delta z,(\Delta z-x)^{2}, \Delta r\right) \\
& \left.\left.+\left(v_{3}-v_{4}\right) / \Delta r, v_{1}+v_{4}+\left(v_{4}-v_{1}\right) x / \Delta z,(\Delta z-x)^{2}, 0\right)\right) x \\
& =\int_{0}^{\Delta z} d x\left(I _ { 4 } \left(\left(v_{3}-v_{4}\right) / \Delta r, v_{1}+v_{4}\right.\right. \\
& \left.=I_{3}\left(\left(v_{2}-v_{1}\right) / \Delta r, v_{1}+\left(v_{1}-v_{4}\right) x / \Delta z,(\Delta z-x)^{2}, 0\right)\right) \\
& \left.=(A z-x)^{2}, \Delta r\right)
\end{aligned}
$$

(A-41) 


$$
\begin{aligned}
T_{16} & =\int_{0}^{\Delta z} d x\left(I _ { 3 } \left(\left(v_{2}-v_{1}\right) / \Delta r, v_{1}+v_{4}\right.\right. \\
& \left.+\left(v_{1}-v_{4}\right) x / \Delta z,(\Delta z-x)^{2}, \Delta r\right) \\
& \left.-I_{3}\left(\left(v_{2}-v_{1}\right) / \Delta r, v_{1}+v_{4}+\left(v_{1}-v_{4}\right) x / \Delta z,(\Delta z-x)^{2}, 0\right)\right) x \\
T_{17} & =\int_{0}^{\Delta z} d x\left(I _ { 4 } \left(\left(v_{2}-v_{1}\right) / \Delta r, v_{1}+v_{4}\right.\right. \\
& \left.+\left(v_{1}-v_{4}\right) x / \Delta z,(\Delta z-x)^{2}, \Delta r\right) \\
T_{19} & =\int_{0}^{\Delta z} d x\left(I _ { 3 } \left(\left(v_{4}-v_{3}\right) / \Delta r, v_{2}+v_{3}\right.\right. \\
& \left.\left.+v_{3}-v_{2}\right) x / \Delta z,(\Delta z-x)^{2}, \Delta r\right) \\
& \left.-I_{4}\left(\left(v_{2}-v_{1}\right) / \Delta r, v_{1}+v_{4}+\left(v_{1}-v_{4}\right) x / \Delta z,(\Delta z-x)^{2}, 0\right)\right) \\
& +v_{18}=\int_{0}^{\Delta z} d x\left(I_{4}\left(\left(v_{2}-v_{1}\right) / \Delta r, v_{1}+v_{4}\right) x / \Delta z,(\Delta z-x)^{2}, \Delta r\right) \\
& \left.\left.\left.=v_{1}\right) / \Delta r, v_{1}+v_{4}+\left(v_{1}-v_{4}\right) x / \Delta z,(\Delta z-x)^{2}, 0\right)\right) x
\end{aligned}
$$




$$
\begin{aligned}
& \left.-I_{3}\left(\left(v_{4}-v_{3}\right) / \Delta r, v_{2}+v_{3}+\left(v_{3}-v_{2}\right) x / \Delta z,(\Delta z-x)^{2}, 0\right)\right) \\
T_{20} & =\int_{0}^{\Delta z} d x\left(I _ { 3 } \left(\left(v_{4}-v_{3}\right) / \Delta r, v_{2}+v_{3}\right.\right. \\
& \left.+\left(v_{3}-v_{2}\right) x / \Delta z,(\Delta z-x)^{2}, \Delta r\right)
\end{aligned}
$$$$
\left.-I_{3}\left(\left(v_{4}-v_{3}\right) / \Delta r, v_{2}+v_{3}+\left(v_{3}-v_{2}\right) x / \Delta z_{1}(\Delta z-x)^{2}, 0\right)\right) x
$$$$
T_{21}=\int_{0}^{\Delta z} d x\left(I _ { 4 } \left(\left(v_{4}-v_{3}\right) / \Delta r, v_{2}+v_{3}\right.\right.
$$$$
\left.+\left(v_{3}-v_{2}\right) x / \Delta z,(\Delta z-x)^{2}, \Delta r\right)
$$$$
\left.-I_{4}\left(\left(v_{4}-v_{3}\right) / \Delta r, v_{2}+v_{3}+\left(v_{3}-v_{2}\right) x / \Delta,(\Delta z-x)^{2}, 0\right)\right)
$$$$
T_{22}=\int_{0}^{\Delta z} d x\left(I _ { 4 } \left(\left(v_{4}-v_{3}\right) / \Delta r, v_{2}+v_{3}\right.\right.
$$$$
\left.+\left(v_{3}-v_{2}\right) x / \Delta z,(\Delta z-x)^{2}, \Delta r\right)
$$

$$
\left.-I_{4}\left(\left(v_{4}-v_{3}\right) / \Delta r, v_{2}+v_{3}+\left(v_{3}-v_{2}\right) x / \Delta z,(\Delta z-x)^{2}, 0\right)\right) x
$$


349

(A-51)

$$
\begin{aligned}
& T_{23}=\int_{0}^{\Delta z} d x\left(I _ { 3 } \left(\left(v_{1}-v_{2}\right) / \Delta r, v_{2}+v_{3}\right.\right. \\
& \left.+\left(u_{2}-v_{3}\right) x / \Delta z,(\Delta z-x)^{2}, \Delta r\right) \\
& \left.-I_{3}\left(\left(v_{1}-v_{2}\right) / \Delta r, v_{2}+v_{3}+\left(v_{2}-v_{3}\right) x / \Delta z,(\Delta z-x)^{2}, 0\right)\right) \\
& T_{24}=\int_{0}^{\Delta z} d x\left(I _ { 3 } \left(\left(v_{1}-v_{2}\right) / \Delta r, v_{2}+v_{3}\right.\right. \\
& \left.+\left(v_{2}-v_{3}\right) x / \Delta z,(\Delta z-x)^{2}, \Delta r\right) \\
& \left.-I_{3}\left(\left(v_{1}-v_{2}\right) / \Delta r, v_{2}+v_{3}+\left(v_{2}-v_{3}\right) x / \Delta z,(\Delta z-x)^{2}, 0\right)\right) x \\
& T_{25}=\int_{0}^{\Delta z} d x\left(I _ { 4 } \left(\left(v_{1}-v_{2}\right) / \Delta r_{2} v_{2}+v_{3}\right.\right. \\
& \left.+\left(v_{2}-v_{3}\right) x / \Delta z,(\Delta z-x)^{2}, \Delta r\right) \\
& \left.-1_{4}\left(\left(v_{1}-v_{2}\right) / \Delta r, v_{2}+v_{3}+\left(v_{2}-v_{3}\right) x / \Delta z,(\Delta z-x)^{2}, 0\right)\right) \\
& T_{26}=\int_{0}^{\Delta z} d x\left(I _ { 4 } \left(\left(v_{1}-v_{2}\right) / \Delta r, v_{2}+v_{3}\right.\right. \\
& \left.+\left(v_{2}-v_{3}\right) x / \Delta z,(\Delta z-x)^{2}, \Delta r\right)
\end{aligned}
$$


350

(A-54)

$$
\begin{aligned}
& \left.-I_{4}\left(\left(v_{1}-v_{2}\right) / \Delta r, v_{2}+v_{3}+\left(v_{2}-v_{3}\right) x / \Delta z,(\Delta z-x)^{2}, 0\right)\right) x \\
T_{27} & =\int_{0}^{\Delta z} d x\left(I _ { 3 } \left(\left(v_{3}-v_{2}\right) / \Delta z, v_{1}+v_{2}\right.\right. \\
& \left.+\left(v_{4}-v_{1}+v_{3}-v_{2}\right) x / \Delta z, \Delta r^{2}, \Delta z-x\right) \\
& -I_{3}\left(\left(v_{3}-v_{2}\right) / \Delta z, v_{1}+v_{2}\right. \\
& \left.\left.+\left(v_{4}-v_{1}+v_{3}-v_{2}\right) x / \Delta z, \Delta r^{2},-x\right)\right) \\
& -I_{4}\left(\left(v_{3}-v_{2}\right) / \Delta z, v_{1}+v_{2}\right. \\
& =\int_{0}^{\Delta z} d x\left(I _ { 3 } \left(\left(v_{3}-v_{2}\right) / \Delta z, v_{1}+v_{2}\right.\right. \\
& \left.+\left(v_{4}-v_{1}+v_{3}+v_{3}-v_{2}\right) x / \Delta z, \Delta r^{2}, \Delta z-x\right) \\
& \left.+\left(v_{4}-v_{1}+v_{3}-v_{2}\right) x / \Delta z, \Delta r^{2}, \Delta z-x\right) \\
& -I_{3}\left(\left(v_{3}-v_{2}\right) / \Delta z, v_{1}+v_{2}\right. \\
&
\end{aligned}
$$


351

$$
\begin{aligned}
& \left.\left.+\left(v_{4}-v_{1}+v_{3}-v_{2}\right) x / \Delta z, \Delta r^{2},-x\right)\right) \\
T_{30} & =\int_{0}^{\Delta r} d x\left(I _ { 3 } \left(\left(v_{4}-v_{3}\right) / \Delta r, v_{3}+v_{2}\right.\right. \\
& \left.+\left(v_{1}-v_{2}+v_{4}-v_{3}\right) x / \Delta r, \Delta z^{2}, \Delta r-x\right) \\
& -I_{3}\left(\left(v_{4}-v_{3}\right) / \Delta r, v_{3}+v_{2}\right. \\
& \left.\left.+\left(v_{1}-v_{2}+v_{4}-v_{3}\right) x / \Delta r, \Delta z^{2},-x\right)\right) \\
& \left.\left.+\left(v_{1}-v_{2}+v_{4}-v_{3}\right) x / \Delta r, \Delta z^{2},-x\right)\right) \\
T_{31} & =\int_{0}^{\Delta r} d x\left(I _ { 3 } \left(\left(v_{4}-v_{3}\right) / \Delta r, v_{3}+v_{2}\right.\right. \\
& \left.\left.+\left(v_{1}-v_{2}+v_{4}-v_{3}\right) / \Delta r, v_{3}+v_{2}-v_{3}\right) x / \Delta r, \Delta z^{2}, \Delta r-x\right) \\
& \left.\left.+\left(v_{1}-v_{2}+v_{4}-v_{3}\right) x / \Delta r, \Delta z^{2}, \Delta r-x\right)\right) \\
& -I_{3}\left(\left(v_{4}-v_{3}\right) / \Delta r, \cdot 3+v_{2}\right.
\end{aligned}
$$

(A-58)

(i-59)

(A-60) 
The $U$ functions

$$
\begin{aligned}
U_{1}(a, b, c, d, f, g, h) & =\int_{0}^{\min (f, g)} d z \int_{0}^{h}(a+b r+c z+d r z) d r \\
& =\left(a h+b h^{2} / 2\right) \min (f, g) \\
& +\left(c h+d h^{2} / 2\right)(\min (f, g))^{2} / 2 \\
U_{2}(a, b, c, d, f, g, h) & =\int_{0}^{h} d r \int_{\min (f, g)}^{f+(g-f) r / h}(a+b r+c z+d r z) d z \\
& =a(f-\min (f, g)) h+a(g-f) h / 2 \\
& +b\left(f-\min (f, g !) h^{2} / 2+b(g-f) h^{2} / 3\right. \\
& +\left(g^{3}-f^{3}\right) h c /(6(g-f))-c h(m i n(f, g))^{2} / 2 \\
& +d h^{2}\left(g^{3} /(6(g-f))-\left(g^{4}-f^{4}\right) /\left(24(g-f)^{2}\right)\right. \\
& \left.+(\min (f, g))^{2} / 4\right)
\end{aligned}
$$

For $f>g U_{3}$ has the definition given below.

$$
\begin{aligned}
U_{3}(a, b, c, d, f, g, i) & =\int_{g}^{f} d z \int_{(z-f) h /(g-f)}^{h}(a+b r+c z+d r z) d r \\
& =a h(f-g) / 2+c h\left(2 f^{2}-f g-g^{2}\right) / 6 \\
& -b h^{2}(g-f) / 3+d h^{2}\left(5 f^{2}-3 g^{2}-2 f g\right) / 24
\end{aligned}
$$


353

The $U_{3}$ function is defined below for the case of $f<g$.

$$
\begin{aligned}
U_{3}(a, b, c, d, f, g, h) & =\int_{f}^{g} d z \int_{0}^{h(z-f) /(g-f)}(a+b r+c z+d r z) d r \\
& =a h(g-f) / 2+c h\left(2 g^{2}-f g-f^{2}\right) / 6 \\
& +b h^{2}(g-f) / 6+d h^{2}\left(g(g-f)-(g-f)^{2} / 4\right) / 6 \\
(A-64) & \\
U_{4}(a, b, c, d, f, g, h)= & \int_{\max (f, g)}^{\Delta r \Delta z / h} d z \int_{0}^{h}(a+b r+c z+d r z) d r \\
& =\left(a h+b h^{2} / 2 ; r \wedge z / h-\max (f, g,)\right) \\
& +\left(c h+d h^{2} / 2\right)\left((\Delta r \Delta z / h)^{2}-(\max (f, g, g))^{2}\right) / 2
\end{aligned}
$$

The $Z_{n}$ functions

$$
\begin{aligned}
z_{1}(a, b, c, d) & =\int_{z_{1}}^{\Delta z} d z \int_{0}^{r_{2}\left(z-z_{1}\right)\left(\Delta z-z_{1}\right)^{-1}} d r(a+b r+c z+d r z) \\
& =a_{2}\left(\Delta z-z_{1}\right) / 2+c r_{2}\left(2 \Delta z^{2}-z_{1} \Delta z-z_{1}^{2}\right) / 6 \\
& +b r_{2}^{2}\left(\Delta z-z_{1}\right) / 6 \\
& +d r_{2}^{2}\left(\Delta z\left(\Delta z-z_{1}\right)-\left(\Delta z-z_{1}\right)^{2} / 4\right) / 6
\end{aligned}
$$

$(A-66)$ 
354

$$
\begin{aligned}
z_{2}(a, b, c, d) & =\int_{0}^{r_{2}} d r \int_{z_{1}}^{z_{1}+r\left(\Delta z-z_{1}\right) / r_{2}}(a+b r+c z+d r z) d z \\
& =\left(\Delta z-z_{1}\right)\left(a r_{2}+b r_{2}^{2} / 3+c r_{2}\left(\Delta z+2 z_{1}\right) / 6\right. \\
& \left.=d r_{2}^{2}\left(5 z_{1}+3 \Delta z\right) / 24\right) \\
z_{3}(a, b, c, d) & =\int_{0}^{21} d z \int_{0}^{r_{2}}(a+b r+c z+d r z) d r \\
= & \left(a r_{2}+b r_{2}^{2} / 2\right) z_{1}+\left(c r_{2}+d r_{2}^{2} / 2\right) z_{1}^{2} / 2 \\
& +\left(c\left(\Delta r-r_{2}\right)+d\left(\Delta r^{2}-r_{2}^{2}\right) / 2\right) \Delta z^{2} / 2 \\
& =\left(a\left(\Delta r-r_{2}\right)+b\left(\Delta r^{2}-r_{2}^{2}\right) / 2\right) \Delta z \\
z_{4}(a, b, c, d) & =\int_{0}^{\Delta z} d z r_{2}^{\Delta z}(a+b r+c z+d r z) d r
\end{aligned}
$$




\section{APPENDIX B}

Coefficients for the Hill's Vortex Potential

Inside the separatrix the following coefficients are non-zero:

$$
\begin{aligned}
& c_{0,0}=3 p_{\theta} / 2 \\
& c_{0,2}=-3 p_{\theta} / 2 \\
& c_{2,0}=-3 p_{\theta} / 2+9 / 76 \\
& c_{2,2}=-9 / 8 \\
& c_{2,4}=9 / 16 \\
& c_{4,0}=-9 / 8 \\
& c_{4,2}=9 / 8 \\
& c_{6,0}=9 / 16
\end{aligned}
$$

Outside the separatrix the following coefficients are non-zero:

$$
\begin{aligned}
& c_{0,0}=1027 p_{\theta} / 128 \\
& c_{0,2}=-693 p_{\theta} / 32 \\
& c_{0,4}=1485 p_{\theta} / 64 \\
& c_{0,6}=-385 p_{\theta} / 32 \\
& c_{0,8}=375 p_{\theta} / 128 \\
& c_{2,0}=-693 p_{\theta} / 32+1149 / 256
\end{aligned}
$$




$$
\begin{aligned}
& c_{2,2}=1485 p_{\theta} / 32-987 / 64 \\
& c_{2,4}=1155 p_{\theta} / 32+2547 / 128 \\
& c_{2,6}=315 p_{\theta} / 128-735 / 64 \\
& c_{2,8}=645 / 256 \\
& c_{4,0}=1485 p_{\theta} / 64-987 / 64 \\
& c_{4,2}=-2310 p_{\theta} / 64+2547 / 64 \\
& c_{4,4}=945 p_{\theta} / 64-2205 / 64 \\
& c_{4,6}=645 / 64 \\
& c_{6,0}=-385 p_{\theta} / 32+2547 / 128 \\
& c_{6,2}=315 p_{\theta} / 32-2205 / 64 \\
& c_{6,4}=1935 / 128 \\
& c_{8,0}=315 p_{\theta} / 128-1470 / 128 \\
& c_{8,2}=645 / 64 \\
& c_{10,0}=645 / 256 \\
& c_{10}
\end{aligned}
$$


357

APPENDIX C

Invariant Poisson Constraint Equation Coefficients

Coefficients for the solution of the poisson equation are as follows:

$$
\begin{aligned}
& A_{1,0}=-A_{1,1}+E-\frac{1}{2} C_{0,0} \\
& A_{2,1}=-\frac{1}{2} C_{0,2}-6 A_{2,2} \\
& A_{2,0}=-\frac{1}{12} C_{2,0}-\frac{1}{6} A_{2,1} \\
& A_{3,2}=-\frac{1}{2} C_{0,4}-15 A_{3,3} \\
& A_{3,1}=-\frac{1}{12} C_{2,2}-A_{3,2} \\
& A_{3,0}=-\frac{1}{30} C_{4,0}-\frac{1}{15} A_{3,1} \\
& A_{4,3}=-\frac{1}{2} C_{0,6}-28 A_{4,4} \\
& A_{4,0}=-\frac{1}{56} C_{6,0}-\frac{1}{28} A_{4,1} \\
& A_{4,2}=-\frac{1}{12} C_{2,4}-\frac{5}{2} A_{4,3} \\
& A_{4,1}=-\frac{1}{30} C_{4,2}-\frac{2}{5} A_{4,2} \\
& A_{5,4}=-\frac{1}{2} C_{0,8}-45 A_{5,5} \\
& A_{2,6} C_{2,6}-\frac{14}{3} A_{5,4}
\end{aligned}
$$


358

$$
\begin{aligned}
& A_{5,2}=-\frac{1}{30} C_{4,4}-A_{5,3} \\
& A_{5,1}=-\frac{1}{56} C_{6,2}-\frac{3}{14} A_{5,2} \\
& A_{5,0}=-\frac{1}{50} C_{8,0}-\frac{1}{45} A_{5,1} \\
& A_{6,5}=-\frac{1}{2} C_{0,10}-66 A_{6,6} \\
& A_{6,4}=-\frac{1}{12} C_{2,8}-\frac{15}{2} A_{6,5} \\
& A_{6,3}=-\frac{1}{30} C_{4,6}-\frac{28}{15} A_{6,4} \\
& A_{7,1}=-\frac{1}{11} A_{7,2} \\
& A_{6,2}=-\frac{1}{56} C_{6,4}-\frac{15}{28} A_{6,3} \\
& A_{7,6}=-\frac{1}{31} A_{7,7} \\
& A_{6,1}=-\frac{1}{90} C_{8,2}-\frac{2}{15} A_{6,2} \\
& A_{7,4}=-\frac{1}{132} C_{10,0}-\frac{2}{66} A_{6,7} \\
& A_{7,4} A_{7,5} \\
& A_{7,3}=-11 A_{7,6} \\
& A_{7,3}
\end{aligned}
$$




$$
\begin{aligned}
& A_{7,0}=-\frac{1}{91} A_{7,1} \\
& A_{8,7}=-120 A_{8,8} \\
& A_{8,6}=-\frac{91}{6} A_{8,7} \\
& A_{8,5}=-\frac{22}{5} A_{8,6} \\
& A_{8,4}=-\frac{45}{28} A_{8,5} \\
& A_{8,3}=-\frac{28}{45} A_{8,4} \\
& A_{8,2}=-\frac{5}{22} A_{8,3} \\
& A_{8,1}=-\frac{6}{91} A_{8,2} \\
& A_{8,0}=-\frac{1}{120} A_{8,1} \\
& A_{9,8}=-153 A_{9,9} \\
& A_{9,6}=-\frac{91}{15} A_{9,7} \\
& A_{9,5}=-\frac{33}{14} A_{9,6} A_{9,4} \\
& A_{9,4}=-A_{9,5} \\
& A_{9,8}
\end{aligned}
$$


360

$$
\begin{aligned}
& A_{9,2}=-\frac{15}{91} A_{9,3} \\
& A_{9,1}=-\frac{1}{20} A_{9,2} \\
& A_{9,0}=-\frac{1}{153} A_{9,1} \\
& A_{10,9}=-190 A_{10,10} \\
& A_{10,8}=-\frac{51}{2} A_{10,9} \\
& A_{10,7}=-83 A_{10,8} \\
& A_{10,6}=-\frac{91}{28} A_{10,7} \\
& A_{10,5}=-\frac{22}{15} A_{10,6} \\
& A_{10,4}=-\frac{15}{22} A_{10,5} \\
& A_{10,3}=-\frac{28}{91} A_{10,4} \\
& A_{10,2}=-\frac{1}{8} A_{10,3} \\
& A_{10,1}=-\frac{2}{51} A_{10,2} \\
& A_{10,0}=-\frac{1}{190} A_{10,1}
\end{aligned}
$$

(C-44)

(C-46)

(C-47)

$(C-43)$

(C-49)

(C-50)

(C-5)

(C-52)

(C-53)

(C-54)

(C-55) 
361

APPENDIX O

Canonical Transformation Hamiltonian

$$
\begin{aligned}
& H=\frac{1}{2}\left[c_{0,0}+\frac{3}{8} c_{8,0} \bar{p}_{\theta}^{2}-\frac{1}{2} c_{4,0} \bar{p}_{\theta}\right. \\
& +\left(C_{2,0}+4 \alpha^{2}+\frac{15}{8} c_{10,0} \bar{p}_{\theta}^{2}-\frac{3}{2} C_{6,0} \bar{p}_{\theta}\right) \bar{p}_{1} \\
& +\left(C_{0,2}+4 \beta^{2}+\frac{3}{8} C_{3,2} \bar{p}_{\theta}^{2}-\frac{C_{42}}{2} \vec{p}_{\theta}\right) \bar{p}_{2} \\
& +\left(\frac{3}{2} c_{0,4}-\frac{3}{4} c_{4,4} \bar{p}_{\theta}\right) \bar{p}_{2}^{2}+\left(\frac{3}{2} c_{4,0}-\frac{15}{4} c_{8,0} \bar{p}_{\theta}\right) \bar{p}_{1}^{-2} \\
& +\left(c_{2,2}-\frac{3}{2} c_{6,2} \bar{p}_{\theta}\right) \bar{P}_{1} \bar{p}_{2} \\
& +\left(\frac{5}{2} C_{6,0}-\frac{35}{4} C_{10,0} \bar{p}_{\theta}\right) P_{1}^{3}+\left(\frac{5}{2} C_{0,6}-\frac{5}{4} C_{4,6} \bar{p}_{\theta}\right) \bar{P}_{2}^{3} \\
& +\left(\frac{3}{2} c_{2,4}-\frac{9}{4} c_{6,4} \bar{p}_{\theta}\right) \bar{p}_{1} \bar{p}_{2}^{2}+\left(\frac{3}{2} c_{4,2}-\frac{15}{4} c_{8,2} \bar{p}_{\theta}\right) \bar{p}_{1}^{2} \bar{p}_{2} \\
& +\frac{35}{8} c_{8,0} \bar{p}_{1}^{4}+\frac{5}{2} c_{6,2} \bar{P}_{1}^{3} \bar{p}_{2}+\frac{5}{2} c_{2,6} \bar{p}_{1} \bar{p}_{2}^{3} \\
& +\frac{9}{4} c_{4,4} \bar{p}_{1}^{2} \bar{p}_{2}^{2}+\frac{35}{8} c_{0,8} \bar{p}_{2}^{4} \\
& +\frac{\mathrm{b} 3}{8} c_{0,10} \overline{\mathrm{P}}_{2}^{5}+\frac{35}{8} c_{2,8} \overline{\mathrm{P}}_{1} \overline{\mathrm{P}}_{2}^{4}+\frac{35}{8} c_{8,2} \overline{\mathrm{P}}_{1}^{4} \overline{\mathrm{P}}_{2} \\
& +\frac{15}{4} c_{4,6} \bar{p}_{1}^{2} \bar{p}_{2}^{3}+\frac{15}{4} c_{6,4} \bar{p}_{1}^{3} \bar{p}_{2}^{2}+\frac{63}{8} c_{10,0} \bar{p}_{1}^{5}
\end{aligned}
$$




$$
\begin{aligned}
& +\sin \left(Q_{1}+Q_{2}\right) \frac{1}{8}\left(\left(6 C_{6,4} \bar{P}_{2}^{2}+\left(12 C_{8,2} \bar{P}_{1}+3 C_{6,2}\right) \bar{P}_{2}\right) \bar{p}_{\theta}\left(\bar{P}_{1}^{2}-\bar{p}_{\theta}\right)^{1 / 2}\right. \\
& +\left(-28 C_{2,8} P_{2}^{4}+\left(-30 C_{4,6} P_{1}-15 C_{2,6}\right) P_{2}^{3}\right. \\
& +\left(-30 c_{6,4} \bar{P}_{1}^{2}-16 c_{4,4} \bar{P}_{1}-8 c_{2,4}\right) \bar{P}_{2}^{2} \\
& \left.+\left(-28 c_{8,2} \bar{P}_{1}^{3}-15 c_{6,2} \bar{P}_{1}^{2}-8 c_{4,2} \bar{P}_{1}-4 c_{2,2}\right) \bar{P}_{2}\right)\left(\bar{P}_{1}^{2}-\bar{p}_{\theta}\right)^{1 / 2} \\
& \left.+\sin \left(Q_{1}-Q_{2}\right) \text { (coeff as for } \sin \left(Q_{1}+Q_{2}\right)\right) \\
& +\cos \left(2 Q_{1}+Q_{2}\right) \frac{1}{16}\left(4 c_{8,2} \bar{P}_{2} \bar{p}_{\theta}^{2}+\left(-15 c_{4,6} \bar{p}_{2}^{3}\right.\right. \\
& +\left(-24 C_{6,4} \bar{P}_{1}-8 C_{4,4}\right) \bar{P}_{2}^{2}+\left(-32 c_{8,2} \bar{P}_{1}^{2}-12 c_{6,2} \bar{P}_{1}\right. \\
& \left.\left.-4 c_{4,2}\right) \bar{P}_{2}\right) \bar{p}_{\theta}+15 c_{4,6} \bar{p}_{1}^{2} \bar{p}_{2}^{3} \\
& +\left(24 C_{6,4} \bar{P}_{1}^{3}+8 C_{4,4} \bar{P}_{1}^{2}\right) p_{2}^{2} \\
& \left.+\left(28 C_{8,2} \bar{P}_{1}^{4}+12 C_{6,2} \bar{P}_{1}^{3}+4 C_{4,2} \bar{P}_{1}^{2}\right) \bar{P}_{2}\right) \\
& +\cos \left(2 Q_{1}-Q_{2}\right)\left(\text { same coeff as } \cos \left(2 Q_{1}+Q_{2}\right)\right. \\
& +\sin \left(Q_{1}+2 Q_{2}\right)\left(-\frac{1}{16}\right)\left(\bar{P}_{1}^{2}-\bar{p}_{\theta}\right)^{1 / 2}\left(3 C_{6,4} \bar{P}_{2}^{2}\left(\bar{P}_{1}^{2}-\bar{p}_{\theta}\right)^{1 / 2} \bar{p}_{\theta}\right. \\
& +\left(-28 c_{2,8} \vec{P}_{2}^{4}+\left(-24 c_{4,6} \bar{P}_{1}-12 c_{2,6}\right) P_{2}^{3}\right. \\
& \left.+\left(-15 r_{\bar{b}, 4} \bar{P}_{1}^{2}-8 C_{4,4} \bar{P}_{1}-4 C_{2,4}\right)\left(P_{2}^{2}\right)\right)
\end{aligned}
$$


363

$+\sin \left(Q_{1}-2 Q_{2}\right)\left(\right.$ same coeff as $\left.\sin \left(Q_{1}+2 Q_{2}\right)\right)$

$$
\begin{aligned}
& +\sin Q_{1}\left(5 C_{10,0}\left(\bar{p}_{1}^{2}-\bar{p}_{\theta}\right) \bar{p}_{\theta}^{2}+\left(-9 C_{6,4} \bar{P}_{2}^{2}\right.\right. \\
& +\left(-24 C_{8,2} \bar{p}_{1}-6 C_{6,2}\right) \bar{P}_{2}-70 C_{10,0} \bar{p}_{1}^{2}
\end{aligned}
$$

$$
\left.-24 C_{8,0} \vec{p}_{1}-6 C_{6,0}\right)\left(\bar{P}_{1}^{2}-\bar{p}_{\theta}\right)^{1 / 2} \bar{p}_{\theta}
$$

$+\left(35 c_{2,8} \bar{p}_{2}^{4}+\left(40 C_{4,6} \bar{p}_{1}+20 c_{2,6}\right) \bar{p}_{2}^{3}\right.$

$+\left(45 C_{6,4} \bar{p}_{1}^{2}+24 C_{4,4} \bar{p}_{1}+12 C_{2,4}\right) P_{2}^{2}$

$+\left(56 c_{8,2} \bar{p}_{1}^{3}+30 c_{6,2} \bar{p}_{1}^{2}+16 c_{4,2} \bar{P}_{1}+8 c_{2,2}\right) \bar{P}_{2}$

$+105 c_{10,0} \bar{P}_{1}^{4}+56 c_{8,0} \bar{P}_{1}^{3}+30 c_{6,0} \bar{P}_{1}^{2}+8\left(c_{2,0}-4 \alpha^{2}\right)$

$\left.\left.+16 r_{4,0} \overrightarrow{\mathrm{P}}_{1}\right)\left(\overline{\mathrm{P}}_{1}^{2}-\bar{p}_{\theta}\right)^{1 / 2}\right) \frac{1}{8}$

$$
\begin{aligned}
& +\cos 2 Q_{1}\left(-\frac{1}{4}\right)\left(\left(2 c_{8,2} \bar{p}_{2}+10 c_{10,0} \bar{p}_{1}+2 c_{8,0}\right) \bar{p}_{\theta}^{2}\right. \\
& +\left(-5 c_{4,6} \bar{P}_{2}^{3}+\left(-9 c_{6,4} \bar{p}_{1}-3 c_{4,4}\right) \bar{P}_{2}^{2}\right. \\
& +\left(-16 c_{8,2} \bar{p}_{1}^{2}-6 c_{6,2} \bar{P}_{1}-2 c_{4,2}\right) \bar{P}_{2} \\
& \left.+40 c_{10,0} \bar{P}_{1}^{3}-16 c_{8,0} \bar{p}_{1}^{2}-6 c_{6,0} \bar{p}_{1}-2 c_{4,0}\right) \bar{p}_{\theta}
\end{aligned}
$$


364

$$
\begin{aligned}
& +5 c_{4,6} \bar{P}_{1}^{2} \bar{P}_{2}^{3}+\left(9 c_{6,4} \bar{P}_{1}^{3}+3 c_{4,4} \bar{P}_{1}^{2}\right) \bar{P}_{2}^{2} \\
& +\left(14 c_{8,2} \bar{P}_{1}^{4}+6 c_{6,2} \bar{P}_{1}^{3}+2 c_{4,2} \bar{P}_{1}^{2}\right) \bar{P}_{2} \\
& \left.+30 c_{10,0} \bar{P}_{1}^{5}+14 c_{8,0} \bar{P}_{1}^{4}+6 c_{6,0} \bar{P}_{1}^{3}+2 c_{4,0} \bar{P}_{1}^{2}\right) \\
& +\sin 3 Q_{1}\left(-\frac{1}{16}\right)\left(5 c_{10,0}\left(\bar{p}_{1}^{2}-\bar{p}_{\theta}\right)^{1 / 2} \bar{p}_{\theta}^{2}+\left(-6 c_{6,4^{2}} \bar{p}^{2}\right.\right. \\
& +\left(-16 c_{8,2} \bar{F}_{1}-4 c_{6,2}\right) \bar{P}_{2}-50 c_{10,0} \bar{F}_{1}^{2}-16 c_{8,0} \bar{p}_{1} \\
& \left.-4 c_{6,0}\right)\left(\bar{p}_{1}^{2}-\bar{p}_{\theta}\right)^{1 / 2} \bar{p}_{\theta}+\left(6 c_{6,4} \bar{p}_{1}^{2} \bar{p}_{2}^{2}\right. \\
& +\left(16 c_{8,2} \bar{p}_{1}^{3}+4 c_{6,2} \bar{p}_{1}^{2} \bar{p}_{2}+45 c_{10,0} \bar{p}_{1}^{4}\right. \\
& \left.\left.+16 c_{8,0} \bar{P}_{1}^{3}+4 c_{6,0} \bar{P}_{1}^{2}\right)\left(\bar{P}_{1}^{L}-\vec{p}_{\theta}\right)^{1 / 2}\right) \\
& +\cos 4 Q_{1}\left(\frac{1}{8}\right)\left(\left(C_{8,2} \bar{P}_{2}+5 C_{10,0} \bar{P}_{1}+C_{8,0}\right) \bar{P}_{\theta}^{2}\right. \\
& +\left(-2 c_{8,2} \bar{P}_{1}^{2} \bar{P}_{2}-10 c_{10,0} \bar{P}_{1}^{3}-2 c_{8,0} \bar{P}_{1}^{2}\right) \bar{P}_{\theta} \\
& \left.+c_{8,2} \bar{P}_{1}^{4} \bar{P}_{2}+5 c_{10,0} \bar{P}_{1}^{5}+c_{8,0} \bar{p}_{1}^{4}\right) \\
& +\sin 5 q_{1}\left(\frac{1}{16}\right)\left(\bar{p}_{1}^{2}-\vec{p}_{\theta}\right)^{1 / 2}\left(c_{10,0} \vec{p}_{\theta}^{-2}-2 c_{10,0} p_{1}^{-2} \vec{p}_{\theta}+c_{10,0} \bar{p}_{1}^{4}\right. \\
& +\cos Q_{2}\left(-\frac{1}{8}\right)\left(3 c_{8,2} \bar{P}_{2} \bar{p}_{\theta}^{2}+\left(-15 c_{4,6} \bar{P}_{2}^{3}+\left(-24 c_{6,4} \bar{P}_{1}\right.\right.\right.
\end{aligned}
$$


365

$$
\begin{aligned}
& \left.\left.-8 C_{4}, \cdot\right) \bar{P}_{2}^{2}+\left(-30 C_{8,2} \bar{P}_{1}^{2}-12 C_{6,2} \bar{P}_{1}-4 C_{4,2}\right) \bar{P}_{2}\right) \bar{p}_{\theta} \\
& +105 C_{0,10} P_{2}^{5}+\left(56 C_{2,8} P_{1}+56 C_{0,8}\right) P_{2}^{4} \\
& +\left(45 C_{4,6} P_{1}^{2}+30 C_{2,6} F_{1}+30 C_{0,6}\right) P_{2}^{3}+8\left(C_{0,2}-4 \beta^{2}\right) F_{2} \\
& +\left(40 C_{6,4} \bar{p}_{1}^{3}+24 C_{4,4} \bar{p}_{1}^{2}+16 C_{2,4} \bar{p}_{1}+16 C_{0,4}\right) \bar{p}_{2}^{2} \\
& \left.+\left(35 C_{8,2} P_{1}^{4}+20 C_{6,2} \bar{P}_{1}^{3}+12 C_{4,2} F_{1}^{2}+8 C_{2,2} \bar{P}_{1}\right) \bar{P}_{2}\right) \\
& +\cos 2 Q_{2}\left(-\frac{1}{4}\right)\left(\left(3 C_{4,6} \bar{p}_{2}^{3}+\left(3 C_{6,4} \bar{p}_{1}+C_{4,4}\right) \bar{p}_{2}^{2}\right) \bar{p}_{\theta}\right. \\
& -30 c_{0,10} \bar{P}_{2}^{5}+\left(-14 C_{2,8} \bar{p}_{1}-14 C_{0,8}\right) P_{2}^{4} \\
& +\left(-9 c_{4.5} \bar{p}_{1}^{2}-6 c_{2,6} \bar{p}_{1}-6 c_{0,6}\right) F_{2}^{3} \\
& +\left(-5 c_{6,4} \bar{P}_{1}^{3}-3 c_{4,4} \bar{P}_{1}^{2}-2 c_{2,1} \bar{p}_{1}-2 c_{0,4}\right) \bar{P}_{2}^{2} \\
& +\cos 3 Q_{2}\left(\frac{1}{16}\right)\left(2 C_{4,6} \bar{P}_{2}^{3} \bar{p}_{\theta}-45 c_{0,10} \bar{P}_{2}^{5}+\left(-16 c_{2,8} \bar{P}_{1}\right.\right. \\
& \left.\left.-16 c_{0,8}\right) \bar{P}_{2}^{4}+\left(-6 c_{4,6} \bar{P}_{1}^{2}-4 c_{2,6} \bar{P}_{1}-4 c_{0,6}\right) \bar{P}_{2}^{3}\right) \\
& +\cos 4 Q_{2}\left(5 C_{0,10} \bar{P}_{2}^{5}+\left(C_{2,8} \bar{P}_{1}+C_{0,8}\right) \bar{P}_{2}^{4}\right) \frac{1}{8}
\end{aligned}
$$


366

$$
+\cos 5 Q_{2}\left(-\frac{C_{0,10} \bar{P}_{2}^{5}}{16}\right)
$$

+ resonances not included on the inside] 
$3 E 7$

APPENDIX E

Resonance Condition for the Canonical Transformation

$$
\begin{aligned}
& \dot{\mathrm{Q}}_{1}+\dot{\ell Q}_{2}=0 \\
& {\left[\frac{3 k}{2 \alpha}\left(\frac{5}{2} C_{6,0}-\frac{35}{4} C_{10,0} \bar{p}_{\theta}\right)+\frac{\ell}{2 \beta}\left(\frac{3}{2} C_{4,2}-\frac{15}{4} C_{3,2} \bar{P}_{\theta}\right)\right] \bar{p}_{1}^{2} \quad(E-1)} \\
& +\left[\frac{k}{\alpha}\left(\frac{3}{2} C_{4,0}-\frac{15}{4} C_{8,0} \bar{p}_{\theta}\right)+\frac{\ell}{2 \beta}\left(C_{2,2}-\frac{3}{2} C_{6,2} \bar{p}_{\theta}\right)\right] \bar{p}_{1} \\
& +\left[\frac{k}{2 \alpha}\left(c_{2,0}+4 \alpha^{2}+\frac{15}{8} c_{10,0} \bar{p}_{\theta}^{2}-\frac{3}{2} c_{6,0} \bar{p}_{\theta}\right)\right. \\
& \left.+\frac{\ell}{2 \beta}\left(C_{0,2}+4 \beta^{2}+\frac{3}{8} C_{8,2} \bar{p}_{\theta}^{2}-\frac{C_{42}}{2} \bar{p}_{\theta}\right)\right] \\
& {\left[\frac{k}{2 \alpha}\left(\frac{3}{2} c_{2,4}-\frac{9}{4} c_{6,4} \bar{p}_{\theta}\right)+\frac{3 l}{2 \beta}\left(\frac{5}{2} c_{0,6}-\frac{5}{4} c_{4,6} \bar{p}_{\theta}\right)\right] \bar{p}_{2}^{2}} \\
& +\left[\frac{k}{2 \alpha}\left(c_{2,2}-\frac{3}{2} C_{6,2} \bar{p}_{\theta}\right)+\frac{\ell}{\beta}\left(\frac{3}{2} C_{0,4}-\frac{3}{4} C_{4,4} \bar{p}_{\theta}\right)\right] \bar{P}_{2} \\
& +\left[\frac{k}{\alpha}\left(\frac{3}{2} C_{4,2}-\frac{15}{4} C_{8,2} p_{\theta}\right)+\frac{\ell}{\beta}\left(\frac{3}{2} C_{2,4}-\frac{9}{4} C_{6,4} \bar{p}_{\theta}\right)\right] \bar{p}_{1} \bar{P}_{2}=0 \\
& h_{1}=\frac{7}{2 \alpha}\left(C_{2,0}+4 \alpha^{2}+\frac{15}{8} c_{10,0} \vec{p}_{\theta}^{2}-\frac{3}{2} C_{6,2}, \vec{p}_{\theta}\right. \\
& (E-2) \\
& +\frac{2}{\alpha}\left(\frac{3}{\sigma_{4}} \mathrm{C}_{4,0}-\frac{15}{4} C_{8,0} \bar{p}_{\theta}\right) P_{10} \\
& +\frac{1}{\beta}\left(c_{2,2}-\frac{3}{2} c_{6,2} p_{\theta}\right) P_{20}+\frac{3}{\alpha^{2}}\left(\frac{5}{2} C_{6,0}-\frac{35}{4} C_{10,0} \bar{p}_{\theta}\right) P_{10}^{2}
\end{aligned}
$$


368

$$
\begin{aligned}
& +\frac{1}{\beta^{2}}\left(\frac{3}{2} c_{2,4}-\frac{9}{4} c_{6,4} \bar{p}_{\theta}\right) P_{20}^{2} \\
& \left.+\frac{2}{\alpha \beta}\left(\frac{3}{2} c_{4,2}-\frac{15}{4} C_{8,2} \bar{p}_{\theta}\right) P_{10} P_{20}\right) \\
& h_{2}=\frac{1}{2 \beta}\left(c_{0,2}+4 \beta^{2}+\frac{3}{8} c_{8,2} \bar{p}_{\theta}^{2}-\frac{C_{42}}{2} \bar{p}_{\theta}\right. \\
& +\frac{2}{\beta}\left(\frac{3}{2} C_{0,4}-\frac{3}{4} C_{4,4} \bar{p}_{\theta}\right) P_{20}+\frac{1}{\alpha}\left(C_{2,2}-\frac{3}{2} C_{6,2} \bar{p}_{\theta}\right) P_{10} \\
& +\frac{3}{\beta^{2}}\left(\frac{5}{2} C_{0,6}-\frac{5}{4} C_{4,6} \bar{p}_{\theta}\right) P_{20}^{2} \\
& +\frac{2}{\alpha \beta}\left(\frac{3}{2} c_{2,4}-\frac{9}{4} c_{6,4} \bar{p}_{\theta}\right) P_{10} P_{20}+\frac{1}{\alpha^{2}}\left(\frac{3}{2} c_{4,2}-\frac{15}{4} c_{8,2} \bar{p}_{\theta}\right) P_{10}^{2} \\
& h_{3}=\frac{1}{2 \alpha^{3}}\left(c_{2,2}-\frac{3}{2} c_{6,2} \bar{p}_{\theta}+\frac{2}{\beta}\left(\frac{3}{2} c_{2,4}-\frac{9}{4} c_{6,4} \bar{p}_{\theta}\right)_{20}\right. \\
& \left.+\frac{2}{\alpha}\left(\frac{3}{2} c_{4,2}-\frac{15}{4} c_{8,2} \bar{p}_{\theta}\right) P_{10}\right) \\
& h_{4}=\frac{1}{\alpha^{2}}\left(\left(\frac{3}{2} C_{0,0}-\frac{15}{4} C_{8,0} \bar{p}_{\theta}\right)\right. \\
& +\frac{3}{\alpha}\left(\frac{5}{2} c_{6,0}-\frac{35}{4} c_{10,0} \bar{p}_{\theta}\right) P_{10} \\
& \left.+\frac{1}{\beta}\left(\frac{3}{2} c_{4,2}-\frac{15}{4} c_{8,2} \bar{p}_{\theta}\right) P_{20}\right) \\
& h_{5}=\frac{1}{\beta^{2}}\left(\frac{3}{2} c_{0,4}-\frac{3}{4} c_{4,4} \bar{p}_{\theta}\right.
\end{aligned}
$$




$$
\begin{aligned}
& +\frac{3}{\beta}\left(\frac{5}{2} c_{0,6}-\frac{5}{4} c_{4,5} \bar{p}_{\theta}\right) P_{20} \\
& \left.+\frac{1}{\alpha}\left(\frac{3}{2} c_{2,4}-\frac{9}{4} c_{6,4} \bar{p}_{\theta}\right) P_{10}\right)
\end{aligned}
$$


371

APPENDIX F

The I pk functions

In this appendix the following definitions are used for $\alpha_{2}, B, C$.

$$
\begin{aligned}
& \alpha_{2}=\left(\alpha^{-3}-r_{0} \alpha^{-2}\right) e^{\alpha r_{0}} \\
& B=8 S^{(6)}\left(\psi_{0}+\psi_{c}\right)^{-1} \\
& C=\alpha^{-3}+r_{0} \alpha^{-2} \\
& =\left(20 B^{2} r^{7} \alpha^{-2}-70 B^{2} r^{6} \alpha^{-3}+63 B^{2} r^{5} \alpha^{-4}\right. \\
+ & 210 B^{2} r^{4} \alpha_{2} \alpha^{-2}-420 B^{2} r^{3} \alpha^{-3} \alpha_{2} \\
+ & 1260 B^{2} r \alpha_{2}^{2} \alpha^{-2}-210 B r^{4} \alpha^{-1} \psi_{c} \\
+ & 3360 B^{2} r^{3} \alpha_{2} \alpha^{-3}-1260 B^{2} r^{2} \alpha_{2} \alpha^{-4} \\
+ & 420 B r^{3} \alpha^{-2} \psi_{c}-2520 B r \alpha_{2} \alpha^{-1} \psi_{c} \\
+ & 1260 r \psi_{c}^{2}+e^{-\alpha r}\left(840 B^{2} r^{4} \alpha_{2} \alpha^{-2}\right. \\
& -420 B^{2} r^{3} \alpha^{-3} \alpha_{2}-1260 B^{2} r^{2} \alpha^{-2} \alpha_{2} \\
&
\end{aligned}
$$

$(\mathrm{F}-\mathrm{l})$ 
372

$$
\begin{aligned}
& -2520 \mathrm{~B}^{2} r \alpha_{2} \alpha^{-5}+2520 \mathrm{~B}^{2} \alpha_{2}^{2} \alpha^{-3} \\
& -2520 B \alpha_{2} \alpha^{-2} \psi_{C}+10080 B^{2} r^{2} \alpha_{2} \alpha^{-4} \\
& \left.+17640 B^{2} r \alpha_{2} \alpha^{-5}+15120 B^{2} \alpha_{2} \alpha^{-6}\right) \\
& +\mathrm{e}^{-2 \alpha r}\left(-630 B^{2} r^{2} \alpha_{2}^{2} \alpha^{-1}-1890 B^{2} r \alpha_{2}^{2} \alpha^{-2}\right. \\
& \left.-1575 B^{2} \alpha_{2}^{2} \alpha^{-3}\right) / / 1260 \\
& I_{p 2}=e^{-\alpha r_{0}} \alpha^{-2}\left(-12 e^{\alpha r} \alpha B C r\left(\psi_{0}+\psi_{C}\right)+24 e^{\alpha r} B C\left(\psi_{0}+\psi_{C}\right)\right. \\
& +12 e^{\alpha r_{0}} \alpha^{2} B C r\left(r_{0}-\alpha^{-1}\right)+12 e^{\alpha r_{0}} \alpha^{2} B C r r_{0} \psi_{C} \\
& -12 e^{\alpha r_{0}} \alpha B C r \psi_{C}+e^{\alpha r_{0}} \alpha B r^{4}\left(\psi_{0}+\psi_{C}\right) \\
& +2 e^{\alpha r_{0}} B r^{3}\left(\psi_{0}+\psi_{c}\right)-4 e^{\alpha r_{0}} \alpha B r r_{0}^{3} \psi_{0} \\
& -6 e^{\alpha r_{0}} B r r_{0}^{2} \psi_{0}-4 e^{\alpha r_{0}} \alpha B r r^{3} \psi_{C} \\
& -6 \mathrm{e}^{\alpha r_{0}} \mathrm{Brr}_{0}^{2} \psi_{\mathrm{c}}+12 \mathrm{e}^{\alpha r_{0}} \alpha^{2} r_{0}^{2} \\
& \left.+24 e^{\alpha r_{0}} \alpha^{2} r \psi_{0} \psi_{c}+12 e^{\alpha r_{0}} \alpha^{2} r \psi_{c}^{2}\right) / 12 \\
& I_{p 3}=-B e^{-\alpha r_{0}} \alpha^{-6}\left(168 e^{\alpha r} \alpha^{4} B C r^{3}\left(r-\alpha^{-1}\right)-168 e^{\alpha r} \alpha^{4} B C r_{0}^{3}\left(r-\alpha^{-1}\right)\right. \\
& -672 \mathrm{e}^{\alpha r} \alpha^{3} \mathrm{BCr} r^{3}+504 \mathrm{e}^{\alpha r} \alpha^{2} \mathrm{BCr}{ }^{2}
\end{aligned}
$$


373

$$
\begin{aligned}
& +168 \mathrm{e}^{\alpha r^{r}} \alpha^{3} B C r_{0}^{3}+2016 \mathrm{e}^{\alpha r} \alpha^{2} \mathrm{BCr^{2 }} \\
& \text { - } 1008 e^{\alpha r} \alpha B C r-4032 e^{\alpha r} a B C r \\
& +5040 e^{\alpha r} B C-42 e^{\alpha r_{0}} 0 \alpha^{5} \operatorname{SLr}^{4} r_{0} \\
& +42 e^{\alpha r_{0}} \alpha^{4} B C r^{4}+168 e^{\alpha r_{0}} \alpha^{5} \mathrm{BCrr}_{0}^{4} \\
& -168 e^{\alpha r_{0}} \alpha^{4} B C r r_{0}^{3}-8 e^{\alpha r_{0}} \alpha^{4} B r^{7} \\
& -14 e^{\alpha r_{0}} \alpha^{3} B r^{6}+28 e^{\alpha r_{0}} \alpha^{4} B r^{4} r_{0}^{3} \\
& +21 e^{\alpha r_{0}} \alpha^{3} B r^{4} r_{0}^{2}+28 e^{\alpha r_{0}} \alpha^{3} B r^{3} r_{0}^{3} \\
& -56 e^{\alpha r_{0}} \alpha^{4} B r r_{0}^{6}-84 e^{\alpha r_{0}} \alpha^{3} B r r_{0}^{5} \\
& \left.-42 e^{\alpha r_{0}} \alpha^{5} r^{4}\left(\psi_{0}+\psi_{c}\right)+168 e^{\alpha r_{0}} \alpha^{5} r_{0}^{3} r\left(\psi_{0}+\psi_{c}\right)\right) / 504 \\
& I_{p 4}=-B e^{-\alpha r} 0 \alpha^{-6}\left(180 e^{\alpha r} \alpha^{3} B C r^{2}\left(r-\alpha^{-1}\right)-180 e^{\alpha r} \alpha^{3} B C r_{0}^{2}\left(r-\alpha^{-1}\right)\right. \\
& -540 e^{\alpha r} \alpha^{2} B C r^{2}+360 e^{\alpha r} \alpha B C r \\
& +180 e^{\alpha r} \alpha^{2} \mathrm{BCr}_{0}^{2}+1080 \mathrm{e}^{\alpha r} \alpha \mathrm{BCr} \\
& -1440 e^{\alpha r} B C-60 e^{\alpha r} 0 \alpha^{4} B C r^{3} r_{0} \\
& +60 e^{\alpha r_{0}} \alpha^{3} \mathrm{BC} r^{3}+180 e^{\alpha r_{0}} \alpha^{4} \mathrm{BC} r r_{0}^{3}
\end{aligned}
$$


374

$-180 e^{\alpha r_{0}} \alpha^{3} B C r r_{0}^{2}-10 e^{\alpha r_{0}} \alpha^{3} B r^{6}$

$-18 e^{\alpha r_{0}} \alpha^{2} B r^{5}+15 e^{\alpha r_{0}} \alpha^{3} B r^{4} r_{0}^{2}$

$+20 e^{\alpha r_{0}} \alpha^{3} B r^{3} r_{0}^{3}+60 e^{\alpha r_{0}} \alpha^{2} B r^{3} r_{0}^{2}$

$-60 e^{\alpha r_{0}} \alpha^{3} B r r_{0}^{5}-90 e^{\alpha r_{0}} \alpha^{2} \mathrm{err} r_{0}^{4}$

$\left.-60 e^{\alpha r_{0}} \alpha^{4} r^{3}\left(\psi_{0}+\psi_{c}\right)+180 e^{\alpha r_{0}} \alpha^{4} r r_{0}^{2}\left(\psi_{0}+\psi_{c}\right)\right) / 360$

(F-7)

$$
\begin{aligned}
& I_{p 5}=-B C e^{-\alpha r_{0}} \alpha^{-4}\left(-4 e^{\alpha r} \alpha B r^{3}-6 e^{\alpha r} B r^{2}+4 e^{\alpha r} \alpha B r_{0}^{3}\right. \\
& +6 e^{\alpha r} B r_{0}^{2}-12 e^{\alpha r} \alpha^{2}\left(\psi_{b}+\psi_{c}\right)+12 e^{\alpha r} B r^{2} \\
& +12 e^{\alpha r} B \alpha^{-1}-24 e^{\alpha \alpha^{-1}} B r-12 e^{\alpha r} \alpha^{-2} B \\
& +24 e^{\alpha r_{B} \alpha^{-2}}-e^{\alpha r_{0}} \alpha^{3} B r^{4} r_{0}+e^{\alpha r_{0}} \alpha^{2} B r^{4} \\
& -2 e^{\alpha r_{0}} \alpha^{2} B r^{3} r_{0}+2 e^{\alpha r_{0}} \alpha B r^{3}+4 e^{\alpha r_{0}} \alpha^{3} B r r_{0}^{4} \\
& +6 e^{\alpha r_{0}} \alpha^{2} B r r_{0}^{3}-4 e^{\alpha r_{0}{ }^{2} B r r_{0}^{3}} \\
& -6 e^{\alpha r_{0}} \alpha \beta r r_{0}^{2}-12 e^{\alpha r_{0}} \alpha^{4} r r_{0} \psi_{0}+12 e^{\alpha r_{0}} \alpha^{3} r \psi_{0} \\
& \left.-12 e^{\alpha r_{0} 4} r r_{0} \psi_{c}+12 e^{\alpha r_{0}}{ }_{\alpha}^{3} r \psi_{c}\right) / 12
\end{aligned}
$$


375

$$
\begin{aligned}
& I_{p 6}=B^{2} C^{2} e^{-2 \alpha r_{0}} \alpha^{-1}\left(e^{2 \alpha r_{\alpha}-2}+4 e^{\alpha\left(r+r_{0}\right)} \alpha^{-1}\left(r_{0}-\alpha^{-1}\right)\right. \\
& +2 e^{2 \alpha r_{0}} \alpha r r_{0}^{2}-4 e^{2 \alpha r_{0}} r r_{0} \\
& \left.+2 e^{2 \alpha r_{0}} \alpha^{-1} r\right) / 2 \\
& I_{p 7}=B^{2} e^{-2 \alpha r_{0}} \alpha^{-2} c^{2}\left(-2 r e^{\alpha r}+\alpha^{-1} e^{L^{r}}-4 e^{\alpha r_{0}} \alpha r r_{0}\right. \\
& \left.+4 r e^{\alpha r_{0}}+4 r_{0} e^{\alpha r_{0}}-4 \alpha^{-1} e^{\alpha r} 0\right) / 2 \\
& I_{p 8}=-B C e^{\alpha\left(r-r_{0}\right)} \alpha^{-5}\left(2 \alpha^{3} B r^{4}+3 \alpha^{2} B r^{3}-2 \alpha^{3} B r r_{0}^{3}\right. \\
& -3 \alpha^{2} B r r_{0}^{2}+6 \alpha^{4} r\left(\psi_{0}+\psi_{C}\right) \\
& -8 \alpha^{2} B r^{3}+16 \alpha B r^{2}+2 \alpha^{2} B r_{0}^{3} \\
& +3 \alpha B r_{0}^{2}-6 \alpha^{3}\left(\psi_{0}+\psi_{C}\right) \\
& -30 \mathrm{Br}-66 \mathrm{Ba}^{-1} / / 6 \\
& I_{p 9}=B^{2} c^{2} e^{2 \alpha\left(r-r_{0}\right)}\left(2 \alpha^{2} r^{2}-2 \alpha r+1\right) /\left(4 \alpha^{3}\right)
\end{aligned}
$$

